\title{
32nd International Symposium on Intensive Care and Emergency Medicine
}

\author{
Brussels, Belgium, 20-23 March 2012
}

Published: 20 March 2012

P1 Impaired innate and adaptive immunity of accelerated-aged Klotho mice in sepsis

S Inoue, K Suzuki-Utsunomiya, K Suzuki-Utsunomiya, T Sato, T Chiba, K Hozumi

Tokai University, Kanagawa, Japan

Critical Care 2012, 16(Suppl 1):P1 (doi: 10.1186/cc10608)

Introduction Sepsis is primarily a disease of the aged and $60 \%$ of sepsis occurs in patients older than 65 years, $80 \%$ of deaths due to sepsis occur in this age group. Klotho knockout mice (Klotho mice) develop a syndrome resembling human aging, and exhibit shortened life spans (8 weeks); however, details regarding the immunity of and immunological changes in Klotho mice after sepsis are still unclear. The purpose of the study is to elucidate the immunological changes that occur in Klotho mice after sepsis in order to identify therapeutic targets for sepsis that occurs in aged individuals.

Methods (1) Survival study: cecum ligation puncture (CLP) was performed to Klotho and wild-type (WT) mice and 4-day survivals were compared. (2) Cell analysis study: mice were sacrificed at 8 hours post CLP or sham surgery. Spleens, thymus, and serum were harvested for FACS analysis using caspase 3 as a marker for apoptosis, and blood for serum cytokine assay. Bacterial colony count in peritoneal lavage was also analyzed.

Results (1) Klotho septic mice started to die from 8 to 12 hours after CLP, and final survival of Klotho mice with CLP was significantly lower than that of WT with CLP $(0 \%$ vs. $100 \%, P<0.01)$. (2) Increased bacterial count in peritoneal cavity and decreased recruitment of neutrophils and macrophages to the peripheral cavity were observed in Klotho-CLP mice. Serum concentration of IL-6, TNF, and IL-10 were significantly higher in Klotho-CLP mice than those in the WT-CLP mice. A dramatically increased caspase 3 positive proportion in Klotho-CLP mice was observed in both flow cytometric and immunohistological analysis $(P<0.01)$.

Conclusion Poor survival in Klotho-septic mice may be associated with impaired bacterial clearance with decreased recruitment of neutrophils/macrophages in peritoneal cavity, elevated cytokines in serum, and increased apoptosis in thymus and spleen, following to impaired innate and adaptive immunity.

P2

IL-17A rs 1974226 GG genotype is associated with increased susceptibility to Gram-positive infection and mortality of severe sepsis

TNakada, J Russell, J Boyd, K Walley

University of British Columbia, Vancouver, Canada

Critical Care 2012, 16(Suppl 1):P2 (doi: 10.1186/cc10609)

Introduction IL-17A plays a key role in host defense against microbial infection including Gram-positive bacteria. Genetic factors contribute to the host defense. Whether genetic variation of IL-17A is associated with altered clinical outcome of severe sepsis is unknown.

Methods We tested for genetic association of IL-17A SNPs with susceptibility to infection and clinical outcome of severe sepsis using two cohorts of European ancestry (St Paul's Hospital (SPH) derivation cohort, $n=679$; Vasopressin and Septic Shock Trial (VASST) validation cohort $n=517$ ). The primary outcome variable was susceptibility to Gram-positive bacterial infection. The secondary outcome variable was 28-day mortality.

Results Of four tested tag SNPs (rs4711998, rs8193036, rs2275913, rs 1974226) in the IL-17A gene, rs1974226 SNP was associated with altered susceptibility to Gram-positive bacterial infection in the derivation cohort (corrected $P=0.014$ ). Patients who have the GG genotype of the rs1974226 SNP were more susceptible to Grampositive bacterial infection, compared to the AG/AA genotype in the two cohorts of severe sepsis (SPH, $P=0.0036$; VASST, $P=0.011$ ) and in the subgroup having lung infection $(P=0.017)$. Furthermore, the $\mathrm{G}$ allele of the IL-17A rs1974226 SNP was associated with increased 28day mortality in two cohorts (SPH, adjusted OR $1.44,95 \% \mathrm{Cl} 1.04$ to $2.02, P=0.029$; VASST, adjusted OR $1.67,95 \% \mathrm{Cl} 1.17$ to $2.40, P=0.0052$ ). Conclusion IL-17A genetic variation is associated with altered susceptibility to Gram-positive infection and 28-day mortality of severe sepsis. References

1. Puel A, et al:: Chronic mucocutaneous candidiasis in humans with inborn errors of interleukin-17 immunity. Science 2011, 332:65-68.

2. Cho JS, et al: IL-17 is essential for host defense against cutaneous Staphylococcus aureus infection in mice. J Clin Invest 2010, 120:1762-1773.

P3

Prevalence of TLR4 single nucleotide polymorphisms (ASP299GLY, THR399ILE) in healthy subjects and septic patients, and association with outcome

T Mohovic', R Salomao ${ }^{2}$, E Nogueira²

'Albert Einstein Hospital, São Paulo, Brazil;' 2UNIFESP, São Paulo, Brazil

Critical Care 2012, 16(Suppl 1):P3 (doi: 10.1186/cc10610)

Introduction Our study aimed to determine the prevalence of functional SNPs (Asp299Gly, Thr399lle) of TLR4 receptors, in healthy volunteers and septic patients in a Brazilian population and to correlate the presence of these polymorphisms in septic patients with clinical outcome.

Methods We verified the presence of polymorphisms ASP299GLY, THR399 ILE by PCR-restriction fragment length polymorphism followed by digestion with enzymes Ncol for SNP 299 and Hinfl for SNP399 followed by electrophoresis for identification of alleles.

Results We observed a statistically significant difference between the genotypes of the Thr399lle polymorphism and respiratory dysfunction, indicating a higher frequency than wild-type genotype in subjects with respiratory dysfunction than those without this condition $(P=0.001)$. We also observed a statistically significant difference between genotype groups formed by the Asp299Gly and Thr399lle polymorphisms and respiratory dysfunction more often featuring group 299Selv/399Selv grupo $299 \mathrm{Het} / 399 \mathrm{Het}$ and less frequently in individuals with respiratory dysfunction than those without this condition $(P=0.003)$. 
Conclusion Our study shows for the first time an assessment of the prevalence of polymorphisms of TLR4 Asp299Gly and Thr399lle considering its cosegregation in healthy individuals and septic patients. And that septic patients who develop respiratory dysfunction have more presence and genotypes 399Selv 299Selv/399Selv and less the presence of genotype $299 \mathrm{Het} / 399 \mathrm{Het}$, featuring a protective effect of the polymorphism Thr399lle.

References

1. Lorenz E, Mira JP, Cornish KL, Arbour NC, Schwartz DA: A novel polymorphism in the toll-like receptor 2 gene and its potential association with staphylococcal infection. Infect Immun 2000, 68:6398-6401.

2. Janeway CA, Jr, Medzhitov R: Introduction: the role of innate immunity in the adaptive immune response. Semin Immunol 1998, 10:349-350.

P4

Modelling immune responses in sepsis

R Grealy', M White ${ }^{2}$, M O'D wyer ${ }^{2}$, P Stordeur ${ }^{3}$, DG Doherty ${ }^{1}$, R McManus', TRyan ${ }^{2}$

'Trinity College Dublin, Ireland; '2St James's Hospital, Dublin, Ireland; ${ }^{3}$ Hopital d'Erasme, Bruxelles, Belgium

Critical Care 2012, 16(Suppl 1):P4 (doi: 10.1186/cc10611)

Introduction The onset and evolution of the sepsis syndrome in humans is modulated by an underlying immune suppressive state [1,2]. Signalling between immune effector cells plays an important part in this response. The objective of this study was to investigate peripheral blood cytokine gene expression patterns and serum protein analysis in an attempt to model immune responses in patients with sepsis of varying severity. We hypothesised that such immunologic profiling could be of use in modelling and prediction of outcomes in sepsis in addition to the evaluation of future novel sepsis therapies.

Methods A prospective observational study in a mixed medical/ surgical ICU and general wards of a large academic teaching hospital was undertaken. Eighty ICU patients with a diagnosis of severe sepsis, 50 patients with mild sepsis (bacteraemia not requiring ICU admission) and 20 healthy controls were recruited. Gene expression analysis by qPCR for INFY, TNFa, IL-2, IL-7, IL-10, IL-23, IL-27 on peripheral blood mononuclear cells (PBMCs) and serum protein analysis for IL-6 was performed. Multivariate analysis was used to construct a model of gene expression based on cytokine copy numbers alone and in combination with serum IL-6 levels.

Results Sepsis was characterised by decreased IL-2, IL-7, IL-23, INFY and greater TNFa, IL-10 and IL-27 gene expression levels compared to controls. Severe sepsis differed from mild sepsis by a decreased INFy and increased IL-10 gene expression $(P<0.0001)$. A composite cytokine gene expression score differentiated controls from mild sepsis and mild sepsis from severe sepsis $(P<0.0001)$. A model combining these cytokine gene expression levels and serum IL- 6 protein levels distinguished sepsis from severe sepsis with an ROC value of 0.89 .

Conclusion Accurate modelling of patient response to infection is possible using peripheral blood mononuclear cell gene expression and serum protein analysis. Molecular biological techniques provide a robust method of such profiling. This approach may be used to evaluate novel sepsis therapies.

References

1. O'Dwyer et al:: The occurrence of severe sepsis and septic shock are related to distinct patterns of cytokine gene expression. Shock 2006, 26:544-550.

2. O'Dwyer et al:: The human response to infection is associated with distinct patterns of interleukin 23 and interleukin 27 expression. Intensive Care Med 2008, 34:683-691.

P5

Decreased peripheral $\mathrm{CD}^{+} / \mathrm{CD}^{+}$lymphocytes and poor prognosis in aged sepsis

S Inoue, K Utsunomiya-Suzuki, S Morita, TYamagiwa, S Inokuchi

Tokai University, Kanagawa, Japan

Critical Care 2012, 16(Suppl 1):P5 (doi: 10.1186/cc10612)

Introduction Aging is a significant factor and is associated with a poor prognosis in sepsis; however, the mechanism of immunological changes in aged sepsis is still unclear. The purpose of this study was to clarify the immunological changes in sepsis of aged patients.

Methods Forty-four septic patients and 48 gender-matched healthy volunteers were prospectively enrolled in the study, which included the following investigations: (1) The SOFA score and clinical outcome were compared between adult sepsis ( $<65$ years of age) and older adult sepsis ( $\geq 65$ years of age). (2) Blood samples were collected from septic and control volunteers. Separated peripheral blood mononuclear cells were stained with CD4, CD8, programmed death-1 (PD-1), CD28, and CD62L antibodies and analyzed by flow cytometry, and serum was used to measure cytokine concentrations by using multiplex bead assay. Values were compared among four groups: normal adult $(<65$ years of age), normal older adult ( $\geq 65$ years of age), adult sepsis ( $<65$ years of age), and older adult sepsis ( $\geq 65$ years of age) groups.

Results (1) No differences in SOFA scores were observed between adult sepsis ( $n=19,39$ years) and older adult sepsis ( $n=25,78$ years), but 3-month survival in older adult sepsis was significantly decreased compared with that in adult sepsis ( $36 \%$ vs. $4 \%, P<0.05)$. (2) Population of $\mathrm{CD}^{+} \mathrm{T}$ cells in normal older adults was significantly less than that in normal adults $\left(1.5 \times 10^{5}\right.$ vs. $\left.5.7 \times 10^{4} / \mathrm{ml}, P<0.01\right)$, and percentage of PD- $1^{+} \mathrm{CD} 8^{+} \mathrm{T}$ cells in the older adult sepsis group was significantly greater than that in the normal older adult group ( $40 \%$ vs. $29 \%$, $P<0.01$ ). Population of $\mathrm{CD}^{+}, \mathrm{CD} 2 \mathrm{~L}^{+} \mathrm{CD} 4^{+}$, and $\mathrm{CD} 28^{+} \mathrm{CD} 4^{+} \mathrm{T}$ cells in the older adult sepsis group was significantly less than that in the normal older adult group ( $n=26,80$ years) $\left(1.8 \times 10^{5} \mathrm{vs.} 5.9 \times 10^{4} / \mathrm{ml}, 1.6 \times 10^{5} \mathrm{vs}\right.$. $5.4 \times 10^{4} / \mathrm{ml}$, and $1.6 \times 10^{5}$ vs. $4.4 \times 10^{4} / \mathrm{ml}$, respectively; $P<0.01$ ); however, these values did not differ between the adult sepsis and normal adult ( $n=22,39$ years) groups. Serum IL-12 level in older adult sepsis was increased when compared with that in the other three groups $(P<0.01)$. Conclusion Poor prognosis in older adult sepsis may be related to both preexisting decrease of $C D 8^{+} T$ cells with aging and loss of $C D 4^{+} T$ cells with sepsis.

\section{P6}

Homeostatic pulmonary microenvironment is responsible for alveolar macrophages resistance to endotoxin tolerance

F Philippart' ${ }^{1}$, C Fitting ${ }^{2}$, B Misset ${ }^{1}$, J Cavaillon ${ }^{2}$

'Groupe Hospitalier Paris Saint Joseph, Paris, France; 'Institut Pasteur de Paris, Paris, France

Critical Care 2012, 16(Suppl 1):P6 (doi: 10.1186/cc10613)

Introduction Endotoxin tolerance (ET) is a modification of immune response to a second challenge with lipopolysaccharide (LPS), which results in a decreased production of proinflammatory cytokines, and is considered partly responsible for the susceptibility to infectious processes in hospitalized patients [1]. We previously observed an absence of ET of alveolar macrophages (AM) to LPS in an ex vivo murine model of endotoxin tolerance [2]. We hypothesized that this singularity could be mediated by granulocyte-macrophage colonystimulating factor (GM-CSF) (known to be predominantly produced by type II pneumocytes) and interferon-gamma (INFץ), two cytokines known to prevent the occurrence of ET [3]. The objectives were to confirm the absence of tolerance of AM to LPS and to assess the respective roles of GM-CSF and INFY in this phenomenon and the cellular origin of INFY.

Methods We used different wild-type mice strains (BALB/C, C57BL/6,129SV), and KO mice lacking different leukocytes subset

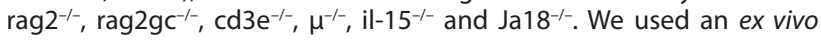
model consisting of intravenous injection of LPS 20 hours prior to an in vitro stimulation of $\mathrm{AM}$, peritoneal macrophages and monocytes with LPS. We pretreated the wild-type mice with anti-cytokines antibodies, and KO mice with B cells and NK cells adoptive transfer.

Results We confirmed the absence of AM tolerance to endotoxin in all the strain of wild-type mice. Inhibiting either GM-CSF or INFY in vivo at homeostasis led to a decrease in TNF production by AM during the in vitro stimulation by LPS, suggesting the involvement of these cytokines in the prevention of tolerance within the lungs. The fact that AM from rag2 $2^{-1}$, rag $2 \mathrm{gc}^{-1}, \mu^{---}$could be tolerated, the fact that adoptive transfer of $B$ lymphocytes in these deficient mice restores the wild-type response, and the presence of INFY mRNA in the lungs at homeostasis in wild-type mice and before and after adoptive B-lymphocyte transfer 
in KO mice demonstrated the involvement of these cells in the wildtype phenotype.

Conclusion We confirm the resistance of $\mathrm{AM}$ to endotoxin tolerance. Both GM-CSF and INFY within the lung microenvironment at homeostasis are involved in this phenomenon. B lymphocytes play a key role in the local expression of INFY.

References

1. Cavaillon JM, et al.: Bench Crit Care 2006, 10:233.

2. Fitting C, et al:: I Infect Dis 2004, 189:1295-1303.

3. Adib-Conquy M, et al.: I Biol Chem 2002, 277:27927-27934.

P7

In vivo natural killer and natural killer T-cell depletion affects mortality in a murine pneumococcal pneumonia sepsis model E Christaki', E Diza', SM Opal², A Pistiki', DI Droggiti ${ }^{3}$, DP Carrer ${ }^{3}$ M Georgitsi' ${ }^{3}$, N Malisiovas', P Nikolaidis', EJ Giamarellos-Bourboulis ${ }^{3}$ 'Aristotle University of Thessaloniki, Greece; ${ }^{2}$ Memorial Hospital of RI, Alpert School of Medicine of Brown University, Providence, RI, USA; ${ }^{3}$ University of Athens, Medical School, Athens, Greece

Critical Care 2012, 16(Suppl 1):P7 (doi: 10.1186/cc10614)

Introduction Apart from macrophages and neutrophils, natural killer (NK) and natural killer T (NKT) cells have been found to play a role in the early stages of bacterial infection. In this study, we investigated the role of NK and NKT cells in host defense against Streptococcus pneumoniae, using a murine pneumococcal pneumonia sepsis model. Our hypothesis was that NK and NKT cells play an immune-regulatory role during sepsis and thus in vivo depletion of those cell populations may affect mortality.

Methods We used four groups of C57BL/ 6 mice (A, B, C and D, $n=10$ mice/group). Animals were infected intratracheally with $50 \mu \mathrm{l}$ of S. pneumoniae suspension $\left(10^{6} \mathrm{cfu}\right)$. Twenty-four hours prior to bacterial inoculation, Group A received $50 \mu \mathrm{l}$ of anti-asialoGM1 rabbit polyclonal antibody (Wako Chemicals $\mathrm{GmbH}$, Neuss, Germany) intravenously (i.v.) to achieve in vivo NK cell inactivation; in Group B, NKT cell depletion was performed by targeting the CD1d receptor using $2 \mathrm{mg} / \mathrm{kg}$ of the monoclonal antibody anti-CD1d, clone 1B1 (BD Pharmingen, San Diego, CA, USA) i.v.; Group C (control) received an equivalent amount of isotype antibody control (nonspecific Ig). Group D received sham intratracheal installation of normal saline. Animals were observed daily for 7 days and deaths were recorded. The survival analysis was plotted using the Kaplan-Meier method and differences in survival between groups were compared with the log-rank test.

Results We found that in vivo NK cell depletion improved survival after pneumococcal pneumonia and sepsis in the group of mice that received the anti-asialoGM1 antibody when compared with animals that received nonspecific IgG antibody $(P=0.041)$ (Figure 1). Nevertheless, when NKT cell depletion was attempted, survival worsened compared to the control group; however, that difference did not reach statistical significance $(P=0.08)$ (Figure 2$)$.

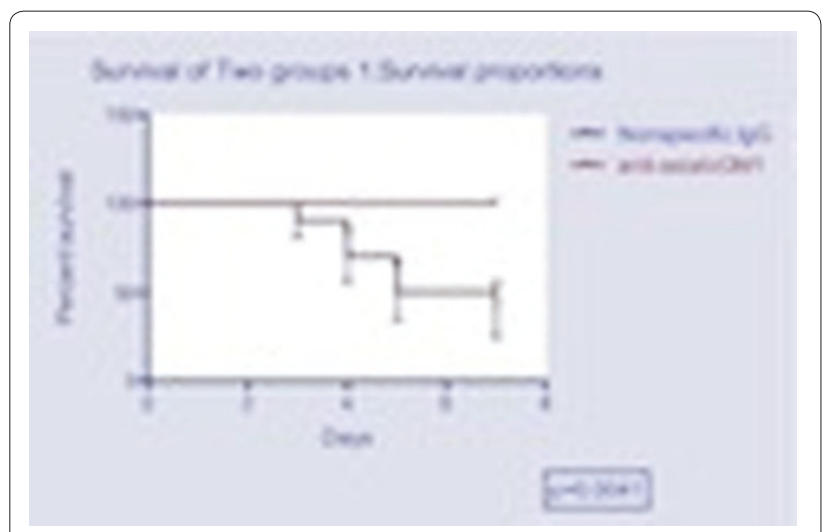

Figure 1 (abstract P7). Kaplan-Meier survival curve of groups A and C.

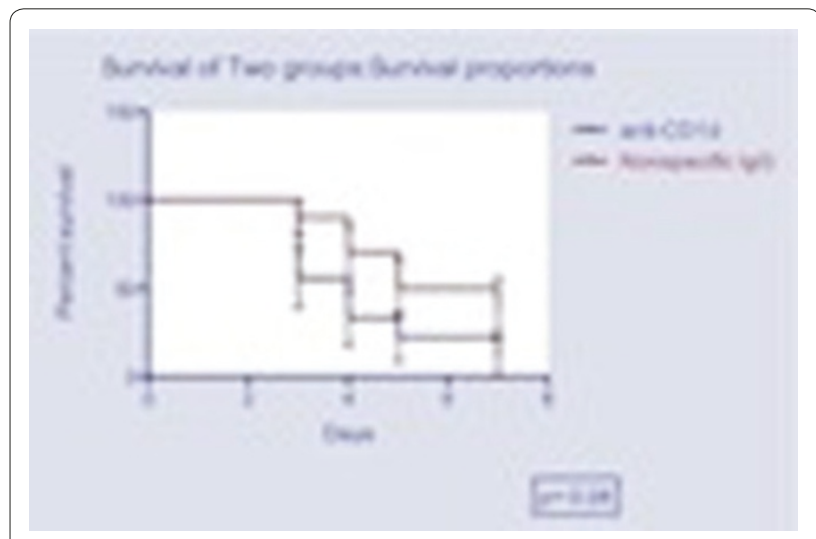

Figure 2 (abstract P7). Kaplan-Meier survival curve of groups B and C.

Conclusion Our study has shown that NK cells appear to contribute to mortality in pneumococcal pneumonia. More research is needed to explore their role in host response to bacterial infection and sepsis.

P8

Mobilization of hematopoietic and nonhematopoietic stem cell subpopulations in sepsis: a preliminary report.

T Skirecki', U Zielińska-Borkowska', M Złotorowicz', M Złotorowicz', J Kawiak², G Hoser ${ }^{1}$

'Medical Center of Postgraduate Education, Warsaw, Poland; ${ }^{2}$ Polish Academy of Science, Warsaw, Poland

Critical Care 2012, 16(Suppl 1):P8 (doi: 10.1186/cc10615)

Introduction Sepsis and septic shock lead to the multiorgan damage by extensive release of inflammatory mediators. Regenerative mechanisms include such regimens as stem cells which differentiate towards specific tissues. Also, in the course of the systemic inflammation the disruption of various regulatory axes occurs, including chemokines (VEGF, HGF) and complement proteins $(\mathrm{C} 5 \mathrm{a}, \mathrm{C} 3 \mathrm{a})$. Among other functions these axes maintain stem cell circulation and recruitment [1]. The aim of the study was to evaluate circulating stem cells in the peripheral blood of septic patients.

Methods Blood samples were obtained from five patients with sepsis or septic shock on the second day after diagnosis. Blood from five healthy volunteers served as control. Samples were stained with the panel of antibodies against: CD45, lineage markers (Lin), CD34, CD133, VEGFR2 and isotypic controls. Cells were analyzed by flow cytometry and the total cell count per milliliter was calculated.

Results On the basis of cell surface phenotype the following stem cell subpopulations were distinguished: hematopoietic stem cells (HSCs) CD $34^{+} \mathrm{CD} 133^{+} \mathrm{CD} 45^{+} \mathrm{Lin}^{-}$, endothelial progenitor cells (EPCs) CD34+CD133+VEGFR2'; and primitive nonhematopoietic stem cells. In the blood of septic patients we found: HSCs (5/5), median level 96/ml; EPCs (5/5), median 48/ml; and nonhematopoietic stem cells $(4 / 5)$, median $48 / \mathrm{ml}$. Whereas in the control group the results were as follow: HSCs (5/5), median level 644/ml; EPCs (5/5), median 70/ml; and nonhematopoietic stem cells $(0 / 5)$. Two of five patients died of septic shock. A trend to lower number of HSCs in nonsurvivors was observed. Conclusion Stem cells can be identified phenotypically in the blood of septic patients and healthy volunteers. However, the circulating primitive nonhematopoietic stem cells could not be detected under physiological conditions. Furthermore, we suggest that stem cells analysis may have serve as prognostic tool in the future.

Acknowledgements Supported by EU Structural Funds, 'Innovative Methods of Stem Cells Applications in Medicine', Innovative Economy Operational Programme, POIG 01.02-00-109/09.

\section{Reference}

1. Ratajczak MZ, et al:: Leukemia 2010, 24:1667-1675. 
P9

Blunted IL-17 responses early after advent of multiple injuries M Paraschos' ${ }^{1}$, M Patrani', A Pistiki ${ }^{2}$, J Van der Meer ${ }^{3}$, M Netea ${ }^{3}$,

E Giamarellos-Bourboulis², K Mandragos

'Korgialeneion Benakeion Hospital, Athens, Greece; 'University of Athens, Medical School, Athens, Greece; ${ }^{3}$ UMC St Radboud, Nijmegen, the Netherlands Critical Care 2012, 16(Suppl 1):P9 (doi: 10.1186/cc10616)

Introduction To define the impact of multiple injuries without the presence of sepsis in IL-17 responses.

Methods A total of 32 patients and 17 healthy volunteers were enrolled. All patients were bearing: multiple injuries necessitating ICU admission with an injury severity score more than 16; and systemic inflammatory response syndrome. Patients with infections upon ICU admission were excluded from the study. Heparinized venous blood was sampled within the first 24 hours after ICU admission. Peripheral blood mononuclear cells (PBMCs) were isolated after gradient centrifugation of whole blood over Ficoll. They were incubated for 5 days in RPMI 1640 supplemented with $2 \mathrm{mM}$ glutamine and 10\% FBS in the presence of $10 \mathrm{ng} / \mathrm{ml}$ lipopolysaccharide (LPS) of Escherichia coli $\mathrm{O} 55: \mathrm{B} 5$; of $5 \mu \mathrm{g} / \mathrm{ml}$ phytohemmaglutin (PHA); of $5 \times 10^{5} \mathrm{cfu} / \mathrm{ml}$ of heatkilled Candida albicans (HKCA), of Pseudomonas aeruginosa (HKPA) or of Staphylococcus aureus (HKSA). IL-17 was measured in supernatants by an enzyme immnunoassay.

Results Mean APACHE II score of patients was 14. Release of IL-17 by PBMCs of patients was significantly lower compared to controls, as shown in Figure 1. $P$ values refer to comparisons between controls and patients.

Conclusion The presented findings show that early upon advent of multiple injuries IL-17 responses are blunted. This may corroborate with the susceptibility of patients for superinfections.

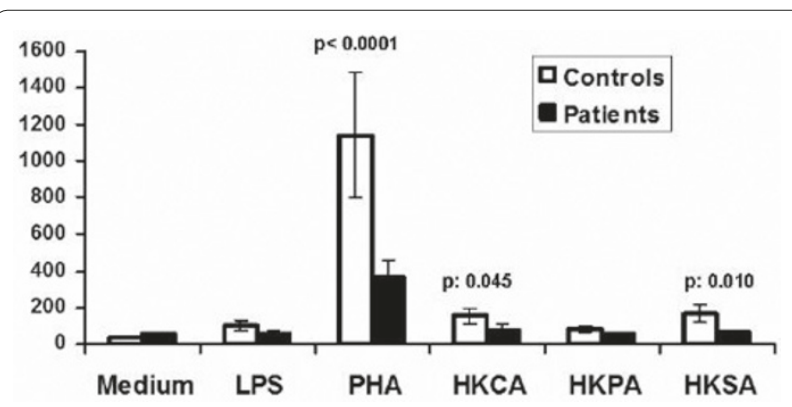

Figure 1 (abstract P9). Release of IL-17 by PBMCs of controls and of patients.

P10

Apoptosis of neutrophils, expression of TREM-1 on neutrophils and IL-17 responses in experimental burn in injury are related to the type and time of burn exposure

A Alexis ${ }^{1}$, D Carrer ${ }^{1}$, A Pistiki', K Louis' ${ }^{1}$, D Droggiti' , J Van der Meer², M Netea², E Giamarellos-Bourboulis ${ }^{1}$

'University of Athens, Medical School, Athens, Greece; '2UMC St Radboud, Nijmegen, the Netherlands

Critical Care 2012, 16(Suppl 1):P10 (doi: 10.1186/cc10617)

Introduction To define inflammatory responses in experimental burn injury in relation with the type and time of burn exposure.

Methods Burn injury was induced in $110 \mathrm{C} 57 / \mathrm{B} 6$ male mice after time exposure of their back as follows: group 0 , sham; group $\mathrm{A}, 60^{\circ} \mathrm{C}$ for 60 seconds; group $\mathrm{B}, 60^{\circ} \mathrm{C}$ for 45 seconds and $4^{\circ} \mathrm{C}$ for 45 seconds; group $C, 75^{\circ} \mathrm{C}$ for 60 seconds; group $\mathrm{D}, 90^{\circ} \mathrm{C}$ for 5 seconds; and group $\mathrm{E}$, $4^{\circ} \mathrm{C}$ for 45 seconds and $60^{\circ} \mathrm{C}$ for 45 seconds. Mice were sacrificed at 24 and 48 hours. Tissues were cultured and splenocytes were isolated and stimulated with heat-killed Staphylococcus aureus and Candida albicans for 5 days for release of IL-17. Neutrophil apoptosis and expression of TREM-1 were determined after staining for ANNEXIN-V, PI and antiTREM-1-PE and flow cytometry analysis.

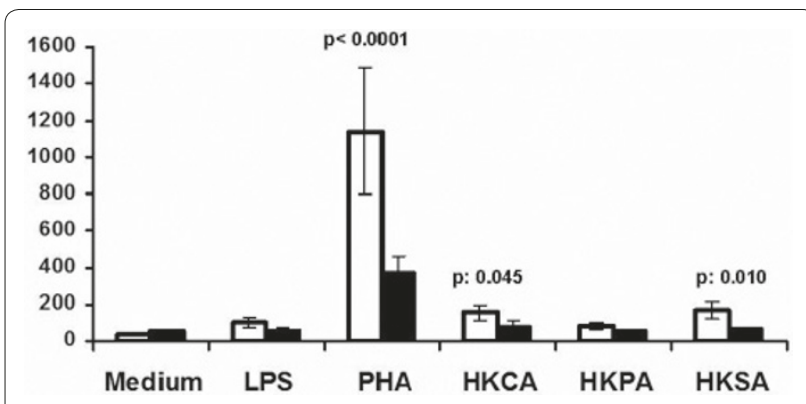

Figure 1 (abstract P10). Release of IL-17 by mice splenocytes in relation to the type of thermal injury.

Results Mean respective apoptosis of groups $0, A, B, C, D$ and E at 24 hours were $37.9 \%, 77.6 \%, 81.9 \%, 73.8 \%, 83.6 \%$ and $75.4 \%$; and at 48 hours $78.5 \%, 79.4 \%, 77.7 \%, 78.2 \%, 81 \%$ and $84.9 \%(P<0.05$ group 0 vs. others). Mean respective MFI of TREM- 1 of groups $0, A, B, C, D$ and $E$ at 24 hours were $2.4,4.4,3.4,3,3.2$ and 3 ; and at 48 hours $2.7,2.8,2.8$, $2.6,2.8$ and $2.7(P<0.05$ group 0 vs. others). Tissue cultures were sterile. Release of IL-17 was greater by splenocytes of group D (Figure 1).

Conclusion Increased neutrophil apoptosis and TREM-1 expression and modulated IL-17 responses are found within burn injury.

P11

Insufficient autophagy relates to mitochondrial dysfunction, organ failure and adverse outcome in an animal model of critical illness J Gunst, I Derese, A Aertgeerts, EJ Ververs, A Wauters, G Van den Berghe, IVanhorebeek

Katholieke Universiteit Leuven, Belgium

Critical Care 2012, 16(Suppl 1):P11 (doi: 10.1186/cc10618)

Introduction Increasing evidence implicates mitochondrial dysfunction in the pathogenesis of critical illness-induced multiple organ failure. We previously demonstrated that prevention of hyperglycemia limits mitochondrial damage in vital organs $[1,2]$, thereby reducing morbidity and mortality [3]. We now hypothesize that inadequate activation of mitochondrial repair processes (mitochondrial clearance by autophagy, mitochondrial fusion and fission, and biogenesis) may contribute to accumulation of mitochondrial damage, persistence of organ failure and adverse outcome of critical illness.

Methods We addressed this hypothesis in a rabbit model of critical illness. First, we studied whether vital organ mitochondrial repair pathways are differentially affected in surviving and nonsurviving hyperglycemic animals, in relation to mitochondrial and organ function. Next, we investigated whether preventing hyperglycemia with insulin affects mitochondrial repair over time. We quantified mRNA/protein levels of key players of these processes. Activities of respiratory chain complexes I to $\mathrm{V}$ were measured spectrophotometrically. Plasma transaminases and creatinine were measured as markers of liver, respectively kidney, dysfunction.

Results In the liver and kidney of nonsurviving hyperglycemic rabbits, molecular markers of insufficient autophagy were evident, including accumulation of p62 protein (but no increase of p62 mRNA) and decreases in the autophagosome-associated protein LC3-II (microtubule-associated protein light chain 3). These changes were less prominent in surviving animals and correlated with impaired mitochondrial and organ function. In contrast, key players in mitochondrial fusion, fission or biogenesis were not affected by survival status. Therefore, we focused on autophagy to study the impact of preventing hyperglycemia. Both after 3 and 7 days of illness, autophagy was better preserved in normoglycemic than in hyperglycemic rabbits, which correlated strongly with improved mitochondrial and organ function.

Conclusion These findings put forward insufficient autophagy as a potentially important contributor to mitochondrial and organ dysfunction in critical illness, and open perspectives for therapies that activate autophagy during critical illness. 


\section{References}

1. Vanhorebeek I, et al:: Lancet 2005, 365:53-59.

2. Vanhorebeek I, et al: Kidney Int 2009, 76:512-520,

3. Van den Berghe G, et al:. N Engl J Med 2001, 345:1359-1367.

P12

Modulation of mediators derived from whole blood or monocytic cells stimulated with lipopolysaccharide reduces endothelial cell activation

A Schildberger, T Stoifl, D Falkenhagen, V Weber

Danube University Krems, Austria

Critical Care 2012, 16(Suppl 1):P12 (doi: 10.1186/cc10619)

Introduction Modulation of inflammatory mediators with specific or selective adsorbents may represent a promising supportive therapy for septic patients. The aims of this study were to modulate mediator concentrations from lipopolysaccharide (LPS)-stimulated whole blood or monocytic THP-1 cells with specific or selective adsorbents and to compare the influence on endothelial cell activation.

Methods Whole blood or THP- 1 cells $\left(1 \times 10^{6}\right.$ cells per $\mathrm{ml}$ medium containing $10 \%$ human plasma) [1] were stimulated with $10 \mathrm{ng} / \mathrm{ml}$ LPS from Escherichia coli for 4 hours. Mediator modulation was performed with either a specific adsorbent for TNFa which was based on sepharose particles functionalized with anti-TNFa antibodies, or with a selective albumin-coated polystyrene divinylbenzene copolymer (PS-DVB) [2]. Human umbilical vein endothelial cell (HUVEC) activation was monitored for 15 hours by measuring secretion of IL- 6 and IL-8, as well as surface expression of the adhesion molecules ICAM- 1 and E-selectin. Results Conditioned media derived from whole blood (CMB) or THP1 cells (CMT) both contained approximately $1,300 \mathrm{pg} / \mathrm{ml}$ TNFa which is known to be an important stimulator for HUVEC $[1,2]$. However, CMB led to a significantly higher HUVEC activation as compared to CMT, as indicated by increased secretion of IL-6 and IL-8 (IL-6: 52,000 vs. $2,000 \mathrm{pg} / \mathrm{ml}$; IL-8: $295,000 \mathrm{vs.} 43,000 \mathrm{pg} / \mathrm{ml}$ ), as well as significantly increased E-selectin surface expression (50 vs. 12 mean fluorescence intensity for CMP and CMT, respectively). Adsorption of inflammatory mediators from the conditioned medium of whole blood or THP-1 cells either with the specific TNFa adsorbent or with the selective PS-DVB beads resulted in decreased endothelial cell activation, as shown by statistically significant reduction of IL-6 and IL-8 secretion from HUVEC, as well as statistically significant reduction of surface expression of the adhesion molecules ICAM- 1 and E-selectin. The reduction of HUVEC activation was more pronounced when applying the selective adsorbent showing that the modulation of more than one cytokine is more effective than removing TNFa alone.

Conclusion Inflammatory mediator modulation with specific or selective adsorbents reduces endothelial cell activation and thus may support the development of new therapies for sepsis.

\section{References}

1. Schildberger et al: Innate Immun 2010, 16:278-287.

2. Schildberger et al.: Blood Purif 2011, 32:286-295.

\section{P13}

A/H1N1 infection: immunological parameters in ICU patients

I Zykova, P Sedlák, T Zajíc, A Vitouš, F Stejskal

Regional Hospital Liberec, Czech Republic

Critical Care 2012, 16(Suppl 1):P13 (doi: 10.1186/cc10620)

Introduction The outbreak of influenza A/H1N1 2009 had influenced ICUs all over the world. In the season 2009/10 we admitted to intensive care 13 patients with $\mathrm{A} / \mathrm{H} 1 \mathrm{~N} 1$ infection in our regional hospital. In the next season 2010/11 another outbreak of A/H1N1 infection was predicted. We decided to study the immunological profiles of these patients and its development in time.

Methods We conducted a prospective study on patients admitted to our hospital with $\mathrm{A} / \mathrm{H} 1 \mathrm{~N} 1$ infection in the season 2010/11. The diagnosis was confirmed by RT-PCT from nasopharyngeal smear or bronchoalveolar lavage in all patients. Immunological parameters (leukocyte count, lymphocyte count, CD19, CD4, CD8, immunoregulatory index, NK cells) were analysed on admission and 3 weeks after admission.
Results In season 2010/11 only six patients with a confirmed A/H1N1 infection required admission to intensive care ( $47 \%$ of all patients with a confirmed A/H1N1 infection admitted to our hospital). All patients required ventilation. Median APACHE II score was 18.2. Median ICU stay was 18.5 days. Median number of ventilator days was 14 . No patient died, both 28-day and 3-month mortality was $0 \%$. Total leukocyte count was without substantial differences, but there was a prominent lymphopenia at the time of admission ( 0.05 to $0.22 \%$ of total leukocyte count) as has been described in similar studies. All lymphocyte populations were decreased but a most prominent decrease was in CD4 (T-helpers) and CD8 (T-suppressors), CD19 (B-lymphocytes) and NK cells were less decreased. Comparison of the admission sample and the second sample taken 21 days after admission: both CD4 and CD8 were most decreased at admission, immunoregulatory index had a shift to positive values in the admission sample.

Conclusion Our small sample of intensive care patients with a confirmed A/H1N1 infection supports the scarce published data about the early immunological profile of these patients. All our patients had a prominent lymphopenia with a most significant decrease in CD4 and CD8 cells. Due to the number of patients in the season 2010/11 and the survival of all patients we could not analyse the relation of survival and the change in time of immunological profile in this unique and probably already extinct group of patients.

Acknowledgements Our study was supported by a grant from Scientific Board of Regional Hospital Liberec.

References

1. Shapovalov KG, et al:. Immunological and bacteriological monitoring of patients with pneumonia and influenza A/H1N1 infection. Zh Mikrobiol Epidemiol Immunobiol 2011, 1:79-82

2. Kim JE, et al.: $\mathrm{CD}^{+} / \mathrm{CD} 8^{+} \mathrm{T}$ lymphocytes imbalance in children with severe 2009 pandemic influenzaA/H1N1 pneumonia. Korean J Pediatr 2011, 54:207-211.

P14

Time of course CD64, a leukocyte activation marker, during extracorporeal circulation

S Djebara, P Biston, F Emmanuel, A Daper, M Joris, P Cauchie,

M Piagnerelli

CHU Charleroi, Belgium

Critical Care 2012, 16(Suppl 1):P14 (doi: 10.1186/cc10621)

Introduction CD64 is a high-affinity leukocyte receptor for the Fc portion of IgG [1]. As CD64 expression on neutrophil cells (PMNs) is upregulated specifically after bacterial stimulation, it could be used to discriminate inflammatory states from bacterial infections [1-3]. The objective was a comparison of the time course of CD64 expression on PMN cells between patients undergoing cardiac surgery with extracorporeal circulation (ECC) with septic patients.

Methods Prospective study realized in the ICU of CHU Charleroi (Belgium). Thirty-nine patients scheduled for a cardiac surgery with ECC (coronary, valvular or mixed surgery) (ECC group) and 11 patients with severe sepsis or septic shock (septic group) were included. The CD64 expression on PMNs was quantified by the hematologic Cell Dyn Sapphire method (Abotte US) before T0, at ICU admission (T1) and postoperatively on day 1 (T2) and day 5 (T3) for the ECC group and on days 0,1 and 5 for the septic group. Values are expressed as median (25th to 75 th) percentiles Results Fifty patients were included among which 39 in the ECC group (nine valvular, 20 coronary artery bypass grafting and 10 mixed surgery). As expected, the inflammatory parameters were significantly increased in septic patients compared to the ECC group except on day

Table 1 (abstract P14)

\begin{tabular}{lccc}
\hline & ECC & Sepsis & $P$ value \\
\hline T0 & $0.8(0.6$ to 1.08$)$ & $3.24(1.9$ to 7.8$)$ & $<0.001$ \\
T1 & $0.9(0.6 \text { to } 1.14)^{\dagger}$ & Not available & \\
T2 & $1.3(0.77 \text { to } 1.8)^{* * * *}$ & $4.4(2.63 \text { to } 6.7)^{*}$ & $<0.001$ \\
T3 & $1.1(0.74 \text { to } 1.4)^{\ddagger}$ & $1.3(0.74$ to 1.4$)$ & 0.16 \\
\hline
\end{tabular}

${ }^{*} P<0.05$ T2 vs. T3, ${ }^{* *}$ T2 vs. T0, ${ }^{\dagger}$ T2 vs. T1, ${ }^{\ddagger}$ T3 vs. T0. ANOVA tests. 
5 (for example, CRP: 0.2 (0.1 to 0.6 ) vs. 12.5 (5.7 to 26.9 ) mg/dl; WBC 6.5 (5.2 to 8.7 ) vs. 19.5 (12 to 20.5$) 10^{3} / \mathrm{mm}^{3}$; for respectively ECC and septic group at T0, $P<0.001)$. The CD64 expression increased significantly in both groups but index values were lower in the ECC compared to the septic group except on T3 (Table 1).

Conclusion ECC modifies the inflammatory parameters, including the expression of the CD64 on PMNs but this one presents the best specificity to diagnose an infection. Thus, CD64 expression could be proposed as a promising marker in the early diagnosis of the infection. References

1. Ioan-Fascinay A, et al.: Immunity 2002, 16:391-402.

2. Nuutila J, et al:. I Immunol Methods 2007, 328:189-200.

3. Qureshi SS, et al.: Clin Exp Immunol 2001, 125:258-265.

P15

Oral neutrophil quantitation in patients undergoing elective cardiopulmonary bypass

ME Wilcox', P Perez ${ }^{2}$, C DosSantos ${ }^{3}$, M Glogauer ${ }^{1}$, E Charbonney ${ }^{3}$,

A Duggal ${ }^{2}$, S Sutherland ${ }^{2}$, G Rubenfeld ${ }^{2}$

'University of Toronto, Canada; '2Sunnybrook Health Sciences Centre, Toronto,

Canada; ${ }^{3}$ St Michael's Hospital, Toronto, Canada

Critical Care 2012, 16(Suppl 1):P15 (doi: 10.1186/cc10622)

Introduction Recent research suggests that the oral cavity may provide an early opportunity to monitor the innate immune system; an oral rinse assay was found to be a reliable predictor of bone marrow engraftment and neutrophil recovery in patients undergoing bone marrow transplantation [1]. Multiorgan failure may be mediated by neutrophil extravasation and aggregation [2] in highly inflammatory states, such as cardiopulmonary bypass (CPB). The objective of this novel pilot study was to determine whether the kinetics of oral neutrophil recovery postCPB surgery reflect systemic immune activation.

Methods Samples [3] from four-quadrant mucosal swabs and oral cavity rinses were obtained from 41 patients undergoing on-CPB elective cardiac surgery preoperatively ( $t-1)$ and postoperatively upon arrival to the CVICU (t0), at 12 to 18 hours (t1), and on day 3 (t2). Oral neutrophil counts $(/ \mathrm{ml})$ were determined by hemacytometry and validated by an electronic cell counter. Concurrent blood samples were collected for measurement of IFNa, interleukins (IL-1 $1 \beta$, IL-6, IL-8 and IL-10), chemokine C-C motif ligand 4 (CCL-4) and Th1 and Th2 cytokines using a 10-plex human cytokine mediator panel. Continuous variables were summarized with means (standard deviation). Preoperative and postoperative oral neutrophil counts were compared using paired $t$ tests.

Results Patients were 65 (10.6) years old; $78 \%$ male; $51 \%$ had significant co-morbidities (25\% diabetes); $54 \%$ took a statin; APACHE II score was 22 (4.4); and multiorgan dysfunction score (MODS) was highest on hospital day $1(6.2 ; 2.2)$. Mean delta oral neutrophil count by oral swab (between $\mathrm{t}-1$ and t0) was $1.7 \times 10^{6}\left(2.0 \times 10^{6}\right)$. A significant difference was seen in the absolute neutrophil counts (oral swab) between $\mathrm{t}-1\left(1.7 \times 10^{6}\right.$ $\left.\left(1.3 \times 10^{6}\right)\right)$ and to $\left(3.4 \times 10^{6}\left(2.7 \times 10^{6}\right) ; P<0.001\right)$, but not between $\mathrm{t}-1$ and t1 $\left(2.0 \times 10^{6}\left(1.7 \times 10^{6}\right) ; P=0.14\right)$ or t2 $\left(6.6 \times 10^{5}\left(1.1 \times 10^{6}\right) ; P=0.14\right)$. Similar results were obtained by oral cavity rinse.

Conclusion An oral swab assay has the potential to provide rapid, riskfree, and early data on neutrophil activation and chemotactic defects in response to CPB, obviating the need for invasive sampling. This method could provide a new perspective on the systemic inflammatory response in surgery, traumatic injury, burns, and sepsis.

References

1. Cheretakis C, et al:: Bone Marrow Transplant 2005, 36:227-232.

2. Fung YL, et al:. I Crit Care 2008, 23:542-549.

3. Wright DG, et al.: Blood 1986, 67:1023-1030

P16

C13-pyruvate administration revealed differential metabolism between heart, liver and red blood cells and improved heart

function during endotoxemia

RM Bateman

Keio University, Tokyo, Japan

Critical Care 2012, 16(Suppl 1):P16 (doi: 10.1186/cc10623)

Introduction The systemic inflammatory response to bacterial infection, or sepsis, results in a hypermetabolic state; yet, systemic metabolic changes in metabolism and the metabolic interaction between tissues and red blood cells are not well understood. The objective of this study was to assess changes in intermediary metabolism during the onset of an animal model of sepsis by determining glycolytic, TCA and PPP metabolites, amino acids and ATP levels in heart, liver and red blood cells.

Methods C57BL/6 mice (30 to $35 \mathrm{~g}$ ) were injected intraperitoneally with lipopolysaccharide (LPS, $40 \mathrm{mg} / \mathrm{kg}$ ) to induce endotoxemia. Six hours post LPS, C13-pyruvate (a key intermediate metabolite) was administered subcutaneously for fluxome analysis of intermediate metabolites. At 20, 40 and 60 minutes, heart, liver and red blood cells were collected and stored at $-80^{\circ} \mathrm{C}$. Labeled metabolites were measured using capillary electrophoresis-mass spectrometry, quantified by calculating the AUC/t0-60 and expressed relative to control. Heart function was monitored by echocardiography.

Results Red blood cells preferentially metabolized pyruvate (ninefold increase) compared to heart (1.2-fold increase) or liver (-2.1-fold decrease), and were a net lactate source (2.1-fold increase). Glycolytic intermediates increased in the heart, but decreased in red blood cells, while TCA intermediates decreased in the heart and amino acids increased in the liver. Under the hypoglycemic conditions of the animal model, red blood cells were found to accumulate glycerol-3-phosphate (red cell glycerol flux remained normal) and 2,3BPG following C13pyruvate injection. ATP was stable in the heart, but decreased in the liver and red blood cells. Echocardiography revealed a transient recovery of left ventricular function that correlated with shifts in red blood cell metabolism.

Conclusion Metabolic investigation of different septic tissues revealed shifts in metabolism between organs, suggesting that sepsis induces complex metabolic shifts in response to changing nutrient availability and cell function; moreover, enhancing red blood cell metabolism may be beneficial to depressed organ function during the onset of endotoxemia.

Acknowledgements Supported by the Ministry of Education, Culture, Sports, Science and Technology, Japan, Global COE Program.

P17

AMP-activated protein kinase controls liposaccharide-induced hyperpermeability

D Castanares-Zapatero', M Overtus², D Communi', M Horckmans's, L Bertrand ${ }^{2}$, C Oury ${ }^{4}$, C Lecut ${ }^{4}$, P Laterre', S De man ${ }^{2}$, C Sommereyns ${ }^{2}$,

S Horman², C Beauloye ${ }^{2}$

'Université catholique de Louvain, Cliniques universitaires Saint Luc,

Brussels, Belgium; ${ }^{2}$ Université catholique de Louvain, Institut de Recherche Expérimentale et Clinique, Brussels, Belgium: ${ }^{3}$ Université libre de Bruxelles, Institut de Recherche Interdisciplinaire en Biologie humaine et moléculaire, Brussels, Belgium; ${ }^{4}$ Université de Liège, Groupe Interdisciplinaire de Génoprotéomique Appliquée, Liège, Belgium

Critical Care 2012, 16(Suppl 1):P17 (doi: 10.1186/cc10624)

Introduction Organ dysfunction determines the severity of sepsis and is correlated to mortality. Endothelial increased permeability contributes to the development of organ failure. AMP-activated protein kinase (AMPK) has been shown to modulate cytoskeleton and could mediate endothelial permeability. Our hypothesis is that AMPK controls sepsis-induced hyperpermeability in the heart and is involved in septic cardiomyopathy.

Methods Sepsis was induced by intraperitoneal injection of liposaccharide, 10 mg/kg (LPS). Alpha-1 AMPK knockout mice (a1KO) were compared with wild-type. Vascular permeability was characterized by Evans blue extravasation. Inflammatory cytokine mRNA expression was determined by qPCR analysis. Left ventricular mass was assessed by echocardiography. In addition, to emphasize the beneficial role of AMPK on heart vascular permeability, AMPK activator (acadesine) was administered to C57BI6 mice before LPS injection. The ANOVA test with Bonferroni's post hoc test and the log-rank test were used. $P<0.05$ was considered as significant.

Results Increased cardiac vascular permeability was observed in the LPS group in comparison to untreated animals $(2.5 \%$ vs. $16 \% ; P<0.05)$. The a1KO mice exhibited an increase vascular permeability after LPS injection in comparison to wild-type mice $(41.5 \%$ vs. $16 \% ; P<0.05)$. 
a1KO animals had a significant mortality increase after LPS injection (70\% vs. $10 \% ; P<0.05)$. LPS markedly induced the production of proinflammatory cytokines (TNFa, IL-1 1 , IL-6) that were significantly higher in the a1 KO animals. More importantly, LPS treatment leads to an increased left ventricular mass in the a1KO mice within 24 hours, suggesting the onset of edema. Finally LPS-induced vascular hyperpermeability was greatly reduced after AMPK activation by acadesine $(13.2 \%$ vs. $40 \% ; P<0.05)$.

Conclusion AMPK importantly regulates cardiac vascular permeability and could control the sepsis-induced cardiomyopathy. AMPK could represent a new pharmacological target of sepsis.

Reference

1. Gustot T: Curr Opin Crit Care 2011, 17:153-159.

P18

Reduced expression of PPAR- $\beta / \delta$ limits the potential beneficial effects of GW0742 during septic shock in atherosclerotic swine H Bracht', F Simon', J Matallo', M Gröger', O McCook', A Seifritz',

M Georgieff', E Calzia', P Radermacher', A Kapoor², C Thiemermann²

'University Clinic Ulm, Germany; ${ }^{2}$ William Harvey Research Institute, London, UK Critical Care 2012, 16(Suppl 1):P18 (doi: 10.1186/cc10625)

Introduction The PPAR- $\beta / \delta$ agonist GW0742 was shown to attenuate cardiac dysfunction in murine septic shock [1] and renal ischemia/ reperfusion injury in diabetic rats [2]. Since these data originate from unresuscitated models, we investigated the effects of GW0742 during long-term, resuscitated porcine septic shock. In order to assess the role of pre-existing cardiovascular morbidity we used animals with familial hypercholesteremia $(11.1(7.4 ; 12.3)$ vs. $1.4(1.3 ; 1.5) \mathrm{mmol} / \mathrm{l}$ in a healthy strain; $P<0.001)$ and consecutive, diet-induced ubiquitous atherosclerosis resulting in coronary artery disease [3], reduced glomerular filtration rate (76 $(60 ; 83)$ vs. $103(79 ; 120) \mathrm{ml} /$ minute in healthy swine; $P=0.004)$ and presence of chronic histological kidney injury.

Methods Anesthetized and instrumented animals randomly received vehicle $(n=9)$ or GW0742 $(n=10 ; 0.03 \mathrm{mg} / \mathrm{kg})$ at $6,12,18$ hours after induction of fecal peritonitis [4]. Hydroxyethyl starch and noradrenaline were infused to maintain normotensive, hyperdynamic hemodynamics. Creatinine clearance was measured from 0 to 12 hours and from 12 to 24 hours of sepsis, respectively. Data are median (quartiles).

Results GW0742 did not affect the noradrenaline infusion rate required to achieve target hemodynamics $(0.57(0.30 ; 3.83)$ vs. $0.56(0.41 ; 0.91)$ $\mu \mathrm{g} / \mathrm{kg} / \mathrm{minute} ; P=0.775$ ) nor the fall in creatinine clearance (GW0742: from $129(114 ; 140)$ to $78(55 ; 95) \mathrm{ml} /$ minute, $P=0.002$; vehicle: from $130(91 ; 142)$ to $41(31 ; 84) \mathrm{ml} /$ minute, $P=0.004 ; P=0.967$ and $P=0.191$ between groups). Immune histochemistry analysis of kidney biopsies in sham-operated swine showed markedly reduced tissue expression of the PPAR- $\beta / \delta$ receptor in atherosclerotic swine $(281(277 ; 404)$ vs. 57 $(53 ; 77) \times 10^{3}$ densitometric units in healthy swine; $\left.P=0.008\right)$.

Conclusion Even early post-treatment with the PPAR- $\beta / \delta$ agonist GW0742 did not beneficially influence acute kidney injury during longterm, resuscitated fecal peritonitis-induced septic shock in swine with pre-existing impairment of kidney function and histological damage. The lacking beneficial effect of GW0742 was most likely due to the reduced expression of the PPAR- $\beta / \delta$ receptor.

Acknowledgements Supported by the Else-Kröner-Fresenius-Stiftung. References

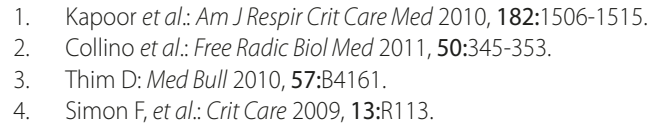

P19

Effects of hexafluoro-2-propanol on inflammatory and hemodynamic responses in a rat model of endotoxic shock M Urner, IK Herrmann, M Hasler, C Booy, B Beck-Schimmer University Hospital Zurich, Institute of Anesthesiology, Zurich, Switzerland Critical Care 2012, 16(Suppl 1):P19 (doi: 10.1186/cc10626)

Introduction Sepsis with multiple organ failure remains a leading cause of hospital morbidity and mortality on ICUs imparting tremendous financial costs. Recently, the primary metabolite of sevoflurane, hexafluoro-2-propanol (HFIP), has been found to exert immunomodulatory properties attenuating inflammatory response to lipopolysaccharides (LPS) in vitro [1]. We investigated whether HFIP attenuates plasma and tissue inflammatory mediator expression in a rat model of endotoxic shock.

Methods Thirty-two male Wistar rats were anesthetized, tracheotomized, and mechanically ventilated. The animals were randomly assigned to one of the following groups: (I) LPS group $(n=8)$, which received intravenous Escherichia coli endotoxin (1 mg/kg); (II) LPS/ HFIP group $(n=8)$, which was treated identically to the LPS group with the additional administration of HFIP ( $67 \mu \mathrm{g} / \mathrm{kg}$ over 30 minutes) after LPS injection. Control groups received Ringer's lactate instead of LPS. General anesthesia was maintained with propofol. All animals received additional $30 \mathrm{ml} / \mathrm{kg}$ Ringer's lactate after injection of LPS over a time period of 1 hour. Arterial blood gases were measured every hour. Animals were euthanized 6 hours after endotoxin injection. The concentrations of monocyte chemoattractant protein-1, key player in the recruitment of monocytes during endotoxemia, was analyzed in bronchoalveolar lavage fluid and in plasma. Linear regression was used to evaluate influence of HFIP on inflammatory mediator expression.

Results Plasma MCP-1 protein levels assessed 6 hours after LPS injection were increased by $+5,192 \mathrm{ng} / \mathrm{ml}$ compared to baseline $\left(R^{2}=0.661\right.$, $P<0.001)$. This increase in MCP-1 protein was attenuated by $-48 \%$ in the LPS/HFIP group $\left(+2,706 \mathrm{ng} / \mathrm{ml}\right.$ to baseline, $\left.R^{2}=0.661 ; P=0.004\right)$. Similar results were found in BALF, in which HFIP decreased the LPSinduced raise in MCP-1 protein concentration by $-62 \%$ (difference of $54 \mathrm{ng} / \mathrm{ml}, P=0.034)$. LPS-stimulated animals had a $+12 \%$ higher mean arterial blood pressure after 6 hours when treated with HFIP $(78 \mathrm{mmHg}$ vs. $\left.67 \mathrm{mmHg}, R^{2}=0.684, P=0.035\right)$. No significant differences in lactate levels were observed. HFIP attenuated base deficit in LPS-stimulated animals by $1 \mathrm{mmol} / \mathrm{l}\left(R^{2}=0.522, P=0.034\right)$.

Conclusion Hexafluoro-2-propanol attenuated LPS-induced inflammatory mediator secretion, the decrease in mean arterial blood pressure, and base deficit. These results suggest that hexafluoro-2-propanol may partly inhibit inflammatory response, hypotension and the development of metabolic acidosis during endotoxic shock.

Reference

1. Urner et al:: Am J Respir Cell Mol Biol 2011, 45:617-624.

\section{P20}

Effects of noradrenaline and lipopolysaccharide exposure on mitochondrial respiration in alveolar macrophages

M Gröger, M Widman, J Matallo, P Radermacher, M Georgieff

University of UIm, Germany

Critical Care 2012, 16(Suppl 1):P20 (doi: 10.1186/cc10627)

Introduction Mitochondrial respiratory capacity of immune cells seems to be impaired in septic patients [1]. On the other hand, the effects of catecholamines on mitochondrial function are still controversial [2] and may confound the genuine mitochondrial response to the septic event. In order to test if catecholamine therapy may influence the impairment of mitochondrial function in immune cells during sepsis, we measured mitochondrial respiration in cultured murine alveolar macrophages (AMJ2-C11) after 24 hours of incubation with noradrenaline and lipopolysaccharide (LPS).

Methods Three states of mitochondrial respiratory activity were quantified in terms of $\mathrm{O}_{2}$-flux $\left(\mathrm{JO}_{2}\right)$ in intact cells at $37^{\circ} \mathrm{C}$ by means of an $\mathrm{O}_{2} \mathrm{~K}$ (Oroboros ${ }^{\circledast}$ Instruments Corp., Innsbruck, Austria) according to a previously published protocol [3] yielding routine respiration ( $R$ ) as the standard respiratory level of the cells without any intervention, proton leak compensation (L) after blocking ATP synthesis by $2.5 \mu \mathrm{M}$ oligomycine, and maximum capacity of the electron transport system (E) after uncoupling by $1 \mu \mathrm{M}$ FCCP. The cells were studied after five different exposure conditions: control (C), $15 \mu \mathrm{mol} / \mathrm{ml}$ noradrenaline (high NoA), $5 \mathrm{nmol} / \mathrm{ml}$ noradrenaline (medium NoA), LPS, and LPS + high NoA. All data are presented in $\mathrm{pmol} /\left(\mathrm{s}^{*}\right.$ million cells) as medians and 25 to $75 \%$ quartiles. Statistical significance was tested by means of the Kruskal-Wallis one-way ANOVA followed by Dunn's method. 
Results After exposure with high but not with medium NoA we observed a statistically significant decrease in maximum mitochondrial respiratory capacity (E-state, C $133(118 ; 148)$ vs. high NoA 111 (106; 113), and medium NoA 129 (123; 140), P<0.05 C vs. high NoA). Both LPS and LPS + high NoA did not affect E-state respiration (LPS: 152 (136; 179), and LPS + NoA $129(125 ; 137))$, but increased routine (R) respiration when compared to control $(C 45(40 ; 55)$ vs. LPS $66(51 ; 72)$ and LPS + NoA $65(55 ; 68), P<0.05$; high NoA 41 (37; 47), and medium NoA $52(51 ; 57)$, NS).

Conclusion High but not moderate doses of noradrenaline reduced mitochondrial respiration in alveolar macrophages in vitro. Surprisingly, LPS increased routine respiration regardless of simultaneous noradrenaline exposure.

References

1. Japiassú AM, et al:: Crit Care Med 2011, 39:1056-1063.

2. Porta F, et al.: Inflammation 2009, 32:315-321.

3. Renner K, et al:: Biochim Biophys Acta 2003, 1642:115-123.

P21

Effects of the anti-diabetic imeglimin in hyperglycemic mice with septic shock

F Wagner', J Vogt', U Wachter', S Weber', B Stahl', M Groeger', O McCook', M Georgieff', P Fouqueray², T Kuhn², E Calzia', P Radermacher',

E Fontaine $^{3}$, K Wagner ${ }^{1}$

'University Medical School Ulm, Anesthesia, Ulm, Germany, ${ }^{2}$ Poxel, Lyon,

France, ${ }^{3}$ Université Joseph Fourier, LBFA, Grenoble, France

Critical Care 2012, 16(Suppl 1):P21 (doi: 10.1186/cc10628)

Introduction Shock-related hyperglycemia impairs mitochondrial function and integrity [1], ultimately leading to apoptosis and organ failure $[1,2]$. Imeglimin is a new anti-diabetic drug with antihyperglycemic and anti-apoptotic properties [3]. Therefore we investigated its effects in hyperglycemic mice with septic shock.

Methods Immediately after cecal ligation and puncture, mice randomly received s.c. vehicle $(n=9)$ or imeglimin $(n=10 ; 100 \mu \mathrm{g} / \mathrm{g})$. Fifteen hours later animals were anesthetized, mechanically ventilated and instrumented for a consecutive 6-hour observation period. After a second imeglimin bolus, colloid fluid resuscitation and continuous i.v. noradrenaline were titrated to maintain normotensive and hyperdynamic hemodynamics. Then $2 \mathrm{mg} / \mathrm{g} / \mathrm{hour}$ glucose was infused to induce hyperglycemia. Glucose oxidation and gluconeogenesis were derived from blood ${ }^{13} \mathrm{C}_{6}$-glucose and mixed expiratory ${ }^{13} \mathrm{CO}_{2} /{ }^{12} \mathrm{CO}_{2}$ isotope enrichment during continuous isotope infusion. Liver mitochondrial activity was assessed using high-resolution respirometry $[4,5]$, Bax, HO-1 and NF-KB expression by immunoblotting and EMSA. All data are median (quartiles).

Results Imeglimin decreased blood glucose levels $(165(153 ; 180)$ vs. $192(184 ; 221) \mathrm{mg} / \mathrm{dl}, P=0.007)$ by increasing whole body glucose oxidation $(55(52 ; 57)$ vs. $51(49 ; 55) \%$ of infused isotope, $P=0.085)$, which coincided with partial restoration of gluconeogenesis $(0.38(0.34 ; 0.41)$ vs. $0.31(0.27 ; 0.33) \mathrm{mg} / \mathrm{g} /$ hour, $P=0.032)$, liver mitochondrial activity (oxidative phosphorylation $\left(136(134 ; 160)\right.$ vs. $116(97 ; 122)$ pmol $\mathrm{O}_{2} /$ second/mg tissue, $P=0.003)$; maximal oxidative capacity (166 (154; $174)$ vs. $147(130 ; 159) \mathrm{pmol} O_{2} /$ second $/ \mathrm{mg}$ tissue, $\left.P=0.064\right)$. Imeglimin increased liver HO-1, reduced liver Bax expression and attenuated NF$\mathrm{KB}$ activation (all $P<0.001$ ).

Conclusion Imeglimin improved whole body glucose utilization and gluconeogenesis, a well-established marker of liver metabolic capacity $[4,5]$, and attenuated organ injury, at least in part due to inhibition of the mitochondrial apoptosis pathway.

Acknowledgements In memoriam of Xavier Leverve who initiated this project; supported by an unrestricted grant from Poxel.

References

1. Vanhorebeek I, et al.: Crit Care Med 2009, 37:1355-1364.

2. Devos P, et al:: Curr Opin Clin Nutr Metab Care 2006, 9:131-139.

3. Fouqueray, et al.: J Diabetes Metab 2011, 2:4

4. Albuszies G, et al:: Intensive Care Med 2007, 33:1094-1101.

5. Baumgart K, et al.: Crit Care Med 2010, 38:588-595.
P22

Adrenomedullin blockade improves catecholamine responsiveness and kidney function in resuscitated murine septic shock

K Wagner', U Wachter ${ }^{1}$, J Vogt', S Weber ${ }^{1}$, M Groeger ${ }^{1}$, O McCook M Georgieff', A Bergmann², H Luettgen², E Calzia', P Radermacher', FWagner

'University Medical School Ulm, Germany; ${ }^{2}$ AdrenoMed AG, Henningsdorf, Germany

Critical Care 2012, 16(Suppl 1):P22 (doi: 10.1186/cc10629)

Introduction The effects of adrenomedullin in circulatory shock states are controversially discussed: while its exogenous supplementation improved organ function and survival [1] in experimental models due to maintenance of hyperdynamic hemodynamics [2] in otherwise hypodynamic conditions, high blood levels were associated with increased mortality in patients with septic shock [3], most likely as a result of excessive vasodilatation [4] and/or impaired systolic heart function [5].

Methods Immediately after cecal ligation and puncture to induce peritonitis, mice randomly received vehicle $(n=11)$ or the adrenomedullin antibody HAM1101 ( $n=9 ; 2 \mu \mathrm{g} / \mathrm{g}$ to achieve antibody concentrations $>4 \mathrm{ng} / \mathrm{ml}$ ). Fifteen hours later animals were anesthetized, mechanically ventilated and instrumented for a consecutive 6-hour observation period. Colloid fluid resuscitation and continuous i.v. noradrenaline were titrated to maintain normotensive (mean blood pressure $>60 \mathrm{mmHg}$ ) and hyperdynamic hemodynamics. Creatinine blood levels and clearance were assessed as surrogate for glomerular filtration $[6,7]$. All data are median (quartiles).

Results Adrenomedullin antagonism decreased the noradrenaline requirements needed to achieve target hemodynamics $(0.009(0.009$; $0.012)$ vs. $0.02(0.015 ; 0.044) \mu \mathrm{g} / \mathrm{g} / \mathrm{hour}, P<0.001)$, increased total diuresis $(2.6(2.3 ; 3.9)$ vs. $0.6(0.5 ; 2.7) \mathrm{ml}, P=0.028)$ resulting in improved fluid balance $(0.18(0.14 ; 0.2)$ vs. $0.26(0.19 ; 0.27), P=0.011)$ and kidney function (creatinine levels at the end of the experiment: $1.3(1.2 ; 1.5)$ vs. $2.0(1.5 ; 2.9) \mu \mathrm{g} / \mathrm{ml}, P=0.006$; creatinine clearance: $400(316 ; 509) \mathrm{vs}$. $197(110 ; 301) \mu \mathrm{l} /$ minute, $P=0.006)$.

Conclusion In resuscitated murine septic shock, early modulation of excess adrenomedullin activity via antibody HAM1101 improves cardiovascular catecholamine responsiveness, ultimately associated with attenuation of acute kidney injury.

Acknowledgements Supported by an unrestricted grant from AdrenoMed AG.

References

1. Wu R, et al:: Mol Med 2009, 15:28-33.

2. Ertmer C, et al.: Br J Anaesth 2007, 99:830-836

3. Guignant C, et al:: Intensive Care Med 2009, 35:1859-1867.

4. Mazzocchi G, et al:: Life Sci 2000, 66:1445-1450.

5. Hyvelin JM, et al.: J Card Surg 2002, 17:328-335.

6. Wagner F, et al: Shock 2011, 35:396-402.

7. Wagner F, et al.: I Trauma 2011. [Epub ahead of print]

P23

Activated protein C, severe sepsis and 28-day mortality

M De La Torre-Prados, A García-de la Torre, M Nieto-González, I Lucena-González, R Escobar-Conesa, A García-Alcántara,

A Enguix-Armada

Hospital Virgen de la Victoria, Málaga, Spain

Critical Care 2012, 16(Suppl 1):P23 (doi: 10.1186/cc10630)

Introduction Protein C (PC) deficiency is prevalent in severe sepsis, studies showing that more than $80 \%$ of patients with severe sepsis have a baseline PC level below the lower limit of normal [1,2]. The aim of the study was to relate the anticoagulation activity evaluated by PC, with clinical parameters and 28-day mortality.

Methods A cohort study of 150 patients $>18$ years with severe sepsis according to the Surviving Sepsis Campaign, in an ICU of a university hospital. Demographic, clinical parameters and coagulation markers during the first 24 hours were studied. PC activity was analysed using a haemostasis laboratory analyser (BCS ${ }^{\circledR}$ XP; Siemens). Descriptive and 
comparative statistical analysis was performed using SPSS version 15.0 (SPSS Inc., Chicago, IL, USA).

Results We analyzed 150 consecutive episodes of severe sepsis $(16 \%)$ or septic shock (84\%) admitted to the ICU. The median age was 64 years old (interquartile range, 48.7 to 71 ); male: $60 \%$. The beginning of severe sepsis took place in the emergency area in $46 \%$ of cases. The main sources of infection were respiratory tract $38 \%$ and intraabdomen $45 \% ; 70.7 \%$ had medical pathology. The 28 -day mortality was $22.7 \%$. The profile of death patients were men $(64.7 \%, n=22)$, with significantly higher average age (63 vs. 57 years; $P=0.049)$, as well as clinical severity scores, APACHE II $(29.8$ vs. $24.1 ; P<0.001)$ and SOFA (12.1 vs. $8.9 ; P<0.001)$ and major dysfunction organs (4.6 vs. 3.6; $P<0,001)$; we observed significantly major consumption of $P C$ (55.2 vs. $70.1, P=0.011$ ). Lower levels of $P C$ were found in surgery septic shock patients, neurological focus or catheter-related infection and Gramnegative pathogens from blood cultures. The ROC analysis showed superior risk prediction of SOFA score for 28-day mortality, AUC 0.81 (95\% Cl: 0.73 to 0.88 , sensitivity: $73.5 \%$; specificity: $76.7 \%, P=0.001$ ), that improves by combining with PC, AUC 0.83 ( $95 \% \mathrm{Cl}: 0.75$ to 0.90 , sensitivity: $77 \%$; specificity: $83 \%, P=0.001$ ).

Conclusion This cohort study showed an improvement in the survival in septic patients under a lower consumption of PC. Low levels of PC are associated with more severity in Sepsis, dysfunction organ and poor outcome.

References

1. Brunkhorst $F$, et al:. Protein $C$ concentrations correlate with organ dysfunction and predict outcome independent of the presence of sepsis. Anesthesiology 2007, 107:15-23.

2. Yan $S B$, et al.: Low levels of protein $C$ are associated with poor outcome in severe sepsis. Chest 2001, 120:915-922.

P24

Soluble usokinase plasminogen activator receptor as a useful biomarker to define advent of sepsis in patients with multiple injuries

M Patrani', M Paraschos', M Georgitsi², E Giamarellos-Bourboulis², K Mandragos'

'Korgialeneion Benakeion Hospital, Athens, Greece; ' University of Athens, Medical School, Athens, Greece

Critical Care 2012, 16(Suppl 1):P24 (doi: 10.1186/cc10631)

Introduction Soluble usokinase plasminogen activator receptor (suPAR) has been considered a useful biomarker to define prognosis in patients with sepsis [1]. The present study aimed to define the kinetics of suPAR during the physical course of patients with multiple injuries. Methods A total of 62 patients were enrolled. All patients were bearing: multiple injuries necessitating ICU admission with an injury severity score (ISS) more than 8 ; and systemic inflammatory response syndrome. Patients with infections upon ICU admission were excluded from the study. Peripheral venous blood was sampled within the first 24 hours after ICU admission. Blood sampling was repeated within the first 24 hours upon advent of sepsis. suPAR was measured in serum by an enzyme immnunoassay.

Results Mean ISS of patients was 14.6. Median suPAR upon ICU admission was $3.74 \mathrm{ng} / \mathrm{ml}$ (range: 1.57 to $16.77 \mathrm{ng} / \mathrm{ml}$ ). No correlation was found between ISS and suPAR. Sepsis was presented in 27 patients. Median suPAR upon sepsis diagnosis was $7.05 \mathrm{ng} / \mathrm{ml}$ (range: 2.18 to $32.51 \mathrm{ng} / \mathrm{ml})(P<0.0001$ compared with ICU admission). This change corresponded to median increase of $57.81 \%$.

Conclusion The presented findings show that measurement of serum suPAR may help diagnosis of sepsis presenting in patients with multiple injuries.

Reference

1. Savva A, et al:: I Infect 2011, 63:344-350.
P25

Role of mannose-binding lectin on pneumococcal infections

J Solé Violán', I García-Laorden', F Rodríguez de Castro', A Payeras²,

J Ferrer Agüero' ${ }^{1}$, M Briones ${ }^{3}$, L Borderías ${ }^{4}$, J Aspa ${ }^{5}$, J Blanquer ${ }^{3}$, O Rajas ${ }^{5}$,

M García-Bello', J Noda', J Rello', C Rodríguez Gallego'

'Hospital Dr Negrín, Las Palmas de Gran Canaria, Spain; ${ }^{2 H o s p i t a l ~ S o n ~ L l a t z e r, ~}$

Palma de Mallorca, Spain; ${ }^{3}$ Hospital Clínico y Universitario, Valencia, Spain;

${ }^{4}$ Hospital San Jorge, Huesca, Spain; ${ }^{5}$ Hospital de La Princesa, Madrid, Spain;

${ }^{6}$ Hospital Universitario Vall d'Hebró, Barcelona, Spain

Critical Care 2012, 16(Suppl 1):P25 (doi: 10.1186/cc10632)

Introduction The role of mannose-binding lectin (MBL) deficiency (MBL2 XA/O + O/O genotypes) in host defences remains controversial. The surfactant proteins (SP)-A1, SP-A2 and SP-D, and other collectins whose genes are located near MBL2, are part of the first-line lung defence against infection. We analyzed the role of MBL on susceptibility to pneumococcal infection and the existence of linkage disequilibrium (LD) among the four genes.

Methods We studied 348 patients with pneumococcal communityacquired pneumonia (P-CAP) and 1,591 controls. A meta-analysis of MBL2 genotypes in susceptibility to P-CAP and to invasive pneumococcal disease (IPD) was also performed. The extent of LD of MBL2 with SFTPA1, SFTPA2 and SFTPD was analyzed.

Results MBL2 genotypes did not associate with either P-CAP or bacteraemic P-CAP in the case-control study. The MBL-deficient O/O genotype was significantly associated with higher risk of IPD in a metaanalysis, whereas the other MBL-deficient genotype (XA/O) showed a trend towards a protective role. We evidenced the existence of LD between MBL2 and SPs genes.

Conclusion The data do not support a role of MBL deficiency on susceptibility to P-CAP or to IPD. LD among MBL2 and SP genes must be considered in studies on the role of MBL in infectious diseases.

P26

Role of serum biomarkers in the diagnosis of infection in patients undergoing extracorporeal membrane oxygenation

M Pieri', T Greco', AM Scandroglio', M De Bonis', G Maj', L Fumagalli',

A Zangrillo', F Pappalardo'

'Istituto Scientifico San Raffaele, Milan, Italy; 'Istituto Scientifico San Raffaele

Turro, Milan, Italy

Critical Care 2012, 16(Suppl 1):P26 (doi: 10.1186/cc10633)

Introduction Although rates and causal organisms of infections occurring in patients on extracorporeal membrane oxygenation (ECMO) have already been described [1], diagnosis of infection itself is challenging in clinical practice. In addition, a significant heterogeneity in infection surveillance practice patterns among ELSO centers has recently been reported [2]. The aim of the study was to analyze the role of C-reactive protein (CRP) and procalcitonin (PCT) in the diagnosis of bacterial and fungal infection in critically ill patients requiring $\mathrm{ECMO}$, and to assess the difference between venovenous (VV) and venoarterial (VA) ECMO setting.

Methods A case-control study on 27 patients. We analyzed serum values of PCT and CRP according to the presence of infection.

Results Forty-eight percent of patients had infection. Gram-negative bacteria were the predominant pathogens (54\%), and Candida was the most frequent isolated microorganism overall (15\%). PCT had an AUC of $0.681(P=0.0062)$, for the diagnosis of infection in patients on VA ECMO, but failed to discriminate infection in the VV ECMO group $(P=0.14)$. The AUC of CRP was $0.707(P \leq 0.001)$ in all ECMO patients. In patients receiving VA ECMO, PCT had good accuracy with $1.89 \mathrm{ng} / \mathrm{ml}$ as the cut-off ( $\mathrm{SE}=87.8 \%, \mathrm{SP}=50 \%$ ) and CRP as well with $97.70 \mathrm{mg} / \mathrm{l}$ as the cut-off ( $\mathrm{SE}=85.3 \%, \mathrm{SP}=41.6 \%$ ). $\mathrm{PCT}$ and CRP tests in parallel had $\mathrm{SE}=87.2 \%$, and $\mathrm{SP}=25.9 \%$. Four variables were identified as statistically significant predictors of infection: PCT and CRP tests in parallel $(\mathrm{OR}=1.184 ; P=0.0008)$, age $(\mathrm{OR}=0.980 ; P \leq 0.001)$, presence of infection before ECMO implantation (OR $=1.782 ; P \leq 0.001)$, and the duration of $\mathrm{ECMO}$ support $(\mathrm{OR}=1.056 ; P \leq 0.001)$.

Conclusion Both traditional and emerging inflammatory biomarkers can help in the diagnosis of infection in patients receiving ECMO. Indeed, we demonstrated for the first time that PCT is a reliable infection marker in patients undergoing VA ECMO. We suggest routine 
concomitant PCT and CRP assay with definite cut-off values as a new test to identify infection in patients undergoing VA ECMO.

\section{References}

1. Bizzarro MJ, et al.: Pediatr Crit Care Med 2011, 12:277-281.

2. Kao LS, et al.: ASAIO J 2011, 57:231-238

P27

Correlation of VAP diagnosis with parameters of critically ill patients in a general ICU

DK Matthaiou, A loannidis, G Gounti, D Lathyris, A Vathis, S Vasiliagkou,

K Kontopoulou, K Mandraveli, E Antoniadou

'G. Gennimatas' General Hospital, Thessaloniki, Greece

Critical Care 2012, 16(Suppl 1):P27 (doi: 10.1186/cc10634)

Introduction We aimed to describe various parameters of critically ill patients who developed VAP and correlate them with its outcome.

Methods Twenty-three VAP cases out of 338 ICU patients were studied retrospectively. Data regarding age, sex, etiology, scores (APACHE II, SOFA, CPIS), CRP, miniBAL cultures, comorbidities, antibiotic exposure, duration of mechanical ventilation, length of ICU and total stay, VAP and patient outcome were recorded. Chi-square and Mann-Whitney $U$ tests were used for statistical analyses.

Results VAP incidence was 23/338 (6.8\%). Fourteen of $23(60.9 \%)$ were males, and $9 / 23(39.1 \%)$ were surgical patients. Their age was $63.5 \pm 16.6$ years. APACHE II was $20.5 \pm 6.7$, initial SOFA was $8.8 \pm 3.7$, SOFA at VAP was $9.4 \pm 3.1$, CPIS 2 days before VAP was $4.6 \pm 2$, CPIS the day before VAP was $6 \pm 1.2$, and CPIS at VAP was $7.6 \pm 1.3$. Length of stay was $25.5 \pm 13.1$ days, ICU stay was $24.8 \pm 13.4$ days, and duration of mechanical ventilation was $22.5 \pm 12.1$ days. Previous antibiotic exposure included: linezolid 10/23 (43.5\%), vancomycin $2 / 23$ (8.7\%), antipseudomonadic penicillins $14 / 23$ (60.9\%), $\beta$-lactams $\pm \beta$-lactamase inhibitor $7 / 23(30.4 \%)$, quinolones $14 / 23(60.9 \%)$, aminoglycosides $6 / 23(26.1 \%)$, antifungals $4 / 23(17.4 \%)$, carbapenems $1 / 23(4.3 \%)$, tigecycline $3 / 23(13 \%)$, and colistin $8 / 23(34.8 \%)$. Antibiotic therapy after the positive miniBAL was modified according to antibiograms. The isolated microorganisms in miniBAL were A. baumannii 10/23 (43.5\%), P. aeruginosa 5/23 (21.7\%), K. pneumoniae $4 / 23$ (17.4\%), Candida spp. $2 / 23(8.7 \%)$, and other $4 / 23(17.4 \%)$; one infection was polymicrobial. In $20 / 23$ cases $(87 \%)$ VAP was of late onset ( $>4$ days) (9.7 \pm 6.8 days). VAP was improved in $17 / 23$ cases $(73.9 \%)$, but $15 / 23$ patients $(65.2 \%)$ died. High overall mortality may be attributed to grave condition. Most patients were admitted to the ICU hours after they were admitted to the hospital. Increased SOFA scores during admission (12 \pm 2 vs. $7.7 \pm 3.4, P=0.009)$ and on the day of VAP diagnosis (11.5 \pm 2.1 vs. $8.6 \pm 3, P=0.016)$ were associated with VAP deterioration. Increased CPIS on the last 2 days before VAP was also associated with worse VAP outcomes $(6.2 \pm 1.7$ vs. $4.1 \pm 1.8$ and $6.8 \pm 1.2$ vs. $5.7 \pm 1.1, P=0.03$ and $P=0.03$, respectively)

Conclusion Our findings support the prognostic value of SOFA score. CPIS values of 6 , although not diagnostic, may need increased alertness on behalf of the clinician.

References

1. Vincent JL, et al.: Intensive Care Med 1996, 22:707-710.

2. Papazian L, et al:: Am J Respir Crit Care Med 1995, 152:1982-1991.

P28

Usefulness of daily monitoring of procalcitonin and C-reactive protein in the early diagnosis of infection after elective colonic surgery

J Rebanda, P Povoa

Hospital São Francisco Xavier, CHLO, Lisbon, Portugal

Critical Care 2012, 16(Suppl 1):P28 (doi: 10.1186/cc10635)

Introduction The diagnosis of infectious complications after elective colonic surgery is frequently misleading, delaying its resolution. Recently several biomarkers, namely procalcitonin (PCT), have been described as more specific in infection diagnosis.

Methods We conducted a prospective observational study segregating patients submitted to elective colonic surgery. Patients were assessed before surgery, and then from the day of surgery until discharge or the 12th day. C-reactive protein (CRP) and PCT were measured daily. We compared infected and noninfected patients.

Results A total of 50 patients were included during a 12-month period (age $70.5 \pm 9.4$ years, $50 \%$ male). The 21 patients (42\%) that subsequently developed infection (16 surgical wound infections) had age, Charlson comorbidity score, primary diagnosis, surgical procedure, intestinal preparation and antibiotic prophylaxis similar to those who had an uneventful recovery. Infection was less frequent in men $(28 \%$ vs. $72 \%, P=0.042$ ). Moreover PCT and CRP before surgery were equally low in patients with or without postoperative infection $(0.10 \pm 0.06 \mathrm{vs}$. $0.07 \pm 0.04 \mathrm{ng} / \mathrm{ml} ; 1.81 \pm 2.83$ vs. $0.72 \pm 1.12 \mathrm{mg} / \mathrm{dl}$, respectively). After surgery, both PCT and CRP increased markedly: PCT increased around $10 \times$ the basal level and peaked at 24 to 48 hours; CRP increased more than $15 \times$ and peaked at 48 hours. Infection was diagnosed a median of 7 days after surgery. The CRP time-course from the day of surgery onwards was significantly different in infected and noninfected patients $(P=0.001)$. In opposition, the PCT time-course was almost parallel in both groups $(P=0.866)$. To assess the diagnostic performance of each biomarker, we performed multiple comparisons between infected and noninfected patients between day 5 and day 9 . The CRP concentration was significantly different $(P<0.01$, Bonferroni correction) on days 6,7 and 8 . The area under the ROC curve of CRP of days 6,7 and 8 were 0.74 , 0.73 and 0.75 , respectively. A CRP concentration $>5.0 \mathrm{mg} / \mathrm{dl}$ at day 6 was predictive of infection with a sensitivity of $85 \%$ and a specificity of $62 \%$ (positive likelihood ratio 2.2 , negative likelihood ratio 0.2 ).

Conclusion After a major elective surgical insult both CRP and PCT serum levels increased independently of the presence of infection. The CRP time-course showed to be useful in the early detection of an infectious complication whereas PCT was unhelpful.

\section{P29}

Procalcitonin as a predictive marker for PCR test and blood culture results in suspected invasive candidemia

A Cortegiani, SM Raineri, F Montalto, MT Strano, A Giarratano

University Hospital Policlinico P. Giaccone, Palermo, Italy

Critical Care 2012, 16(Suppl 1):P29 (doi: 10.1186/cc10636)

Introduction Procalcitonin (PTC) seems to have potential to predict the result of blood culture $(\mathrm{BC})$ supporting the diagnosis of invasive candidemia. Although blood culture is still the gold standard, PCR assays are able to quickly and reliably detect fungi in blood in suspected invasive candidemia. Our aim is to verify the potential of PTC values to predict the result of PCR assay in suspected invasive candidemia.

Methods We retrospectively analyzed 78 patients with suspected invasive candidemia from whom we obtained PCT value, BC and PCR assay. All tests have been obtained on the day in which patients reached a Candida score $\geq 4$. We calculated PTC mean values according to $B C$ and $P C R$ results and compared data using the Mann-Whitney $U$ test. We performed the ROC analysis to test the diagnostic performance of PTC with regards to $B C$ and PCR result.

Results PCR tests and BC were both negative in 48 patients and the PTC mean value in this group was $21.5 \mathrm{ng} / \mathrm{ml}$ while 19 patients were PCR-positive and BC-positive with a PTC mean value of $2.07 \mathrm{ng} / \mathrm{ml}$. The difference between these PCT mean values was significant $(P=0.0001)$. In eight cases $B C$ were negative whereas $P C R$ tests were positive with the PCT mean level in this group being $1.82 \mathrm{ng} / \mathrm{ml}$. No patient resulted PCR-negative and BC-positive. According to PCR results only, there was a significant difference between PTC mean values in positives and negatives $(P=0.0001)$. The ROC analysis showed that the best PTC cutoff value for prediction of $B C$ result was 4.57 with AUC of 0.91 ( $\mathrm{Cl} 0.83$ to 0.96 , sensitivity $99 \%$, specificity $80.39 \%$ ). Concerning the PCR result, the calculated cut-off was 4.31 with AUC of 0.96 (Cl 0.948 to 1 , sensitivity $96.6 \%$, specificity $97.9 \%$; positive predictive value $94.51 \%$; negative predictive value $97.83 \%)$.

Conclusion According to our data, PTC seems to be characterized by a remarkable diagnostic performance and predictive value for both BC and PCR assay in suspected invasive candidemia. PCT could be considered as the first step of the diagnostic process for suspected invasive candidemia in order to spare as much time as possible before starting a pre-emptive antifungal therapy. This may lead to less useless therapies in negative patients and quicker and more reliable start of 
treatment in positive patients while waiting for the $\mathrm{BC}$ and antibiogram results.

\section{Reference}

1. Charles PE, Castro C, Ruiz-Santana S, et al.: Serum procalcitonin levels in critically ill patients colonized with Candida spp: new clues for the early recognition of invasive candidiasis? Intensive Care Med 2009, 35:2146-2150.

P30

Would procalcitonin measurement aid antimicrobial stewardship in a UK district general hospital mixed adult critical care population? J Clayton, J White, L Wilson, M Leonard, J Cuniffe

Wirral University Teaching Hospital NHS Foundation Trust, Wirral, UK

Critical Care 2012, 16(Suppl 1):P30 (doi: 10.1186/cc10637)

Introduction We sought to establish what impact knowledge of procalcitonin (PCT) levels could have on antimicrobial prescribing and stewardship within our 18-bed mixed critical care unit. Assicot and colleagues demonstrated that PCT levels are raised during sepsis and can correlate with the severity [1]. The PCT level peaks after 6 to 12 hours and has a half-life of approximately 25 to 36 hours in critically ill patients [2], declining with adequate treatment. A recent multicentre trial demonstrated reduced duration of antibiotic therapy by using PCT-guided treatment strategy; however, only $10 \%$ of the cohort was surgical patients and therefore this finding cannot be extrapolated to a general critical care population [3].

Methods The question was posed: would knowledge of PCT levels have altered real-time clinical management of patients on established antimicrobial therapy? Over a 2-month period patients were treated in a conventional manner based on clinical findings and standard investigations. Plasma samples from days 0 (respective to antimicrobial therapy) 1, 3, 5 and 7 were analysed for PCT. Nonparametric statistical analysis of PCT levels was available for a retrospective multidisciplinary team review of case notes. This was performed within the context of a local service review and the chair of the local ethics committee gave approval for analysis of plasma samples and case-note review Results Twenty-seven patients were identified. Antimicrobial cessation was deemed possible in seven of these cases at day 5. Nonescalation of treatment was supported in six further cases. In one case treatment had been escalated and PCT supported this decision. This would have resulted in 19 fewer days of antibiotic therapy.

Conclusion Our experience suggests the availability of the PCT response between days 0 and 5 would have been a useful adjunct in monitoring treatment of sepsis on our unit and would have facilitated timely de-escalation and hence exposure to antimicrobial therapy. We hypothesise such a reduction could help to prevent antimicrobial resistance, lead to decreased pharmacy and consumable costs and reduce the incidence of adverse antimicrobial-related events.

References

1. Assicot M, et al:: Lancet 1993, 341:515-518.

2. Meisner M, et al: Intensive Care Med 2000, 26:1193-1200.

3. Bouadma L, et al:: Lancet. 2010, 375:463-474.

\section{P31}

Procalcitonin has a poor prognosis value in critically ill patients with candidemia

PE Charles, R Bruyère, H Roche, JP Quenot, S Prin, A Pavon, F Dalle

University Hospital, Dijon, France

Critical Care 2012, 16(Suppl 1):P31 (doi: 10.1186/cc10638)

Introduction Candidemia is an infrequent but serious infection in the critically ill patients. Although effective antifungal drugs are available, mortality rates remain high so far. Procalcitonin (PCT) repeated measurements have proven useful for assessing the prognosis and the antimicrobial treatment responsiveness in the patients with systemic bacterial infection. Little is known about it in the setting of candidemia. The PCT predictive value regarding the outcome of such patients was therefore addressed.

Methods A retrospective single-centre observational study. All patients with ICU-acquired pure candidemia between 2005 and 2011 were included. Baseline characteristics and both clinical and biological follow-up data including PCT measurement were collected. The SOFA score was calculated daily during the first week of antifungal treatment. Survivors at discharge from the ICU were compared to nonsurvivors by univariate followed by a Cox regression analysis.

Results Fifty patients were included among whom 28 (56\%) died in the ICU. Candida albicans was the most common isolated yeast $(58 \%)$, regardless of the outcome. Nonsurvivors were elder and had a greater SAPS II score value on admission than survivors (55.8 \pm 21.7 vs. $42.5 \pm 14.9$ points, $P=0.01$ ). The time elapsed between the ICU admission and the onset of invasive candidiasis was significantly longer in the nonsurvivors than in the survivors ( $8.3 \pm 12.8$ vs. $1.2 \pm 2.8$ days, $P=0.01$ ). At the onset of candidemia, the nonsurvivors were more severely ill as assessed through SOFA score calculation (10.4 \pm 4.4 vs. $7.8 \pm 3.9$ points, $P=0.04$ ). Antifungal treatment was given within the first 24 hours following the onset of candidemia in $60 \%$ of the whole patients and was always appropriate, regardless of the survival. During therapy, the SOFA score remained greater in the nonsurvivors than in the survivors. In contrast, PCT failed to differentiate the survivors from the nonsurvivors the day antifungals were started $(8.7 \pm 13.1$ vs. $4.5 \pm 4.1 \mathrm{ng} / \mathrm{ml}, P=0.21$ ), as well as the following days. The SAPS $\mathrm{II}$, the SOFA score and the time elapsed between ICU admission and candidemia onset were the sole independent predictors of death in our study population.

Conclusion The late-onset candidemia are more likely to be associated with death than earlier episodes. Unresolved organ failure as assessed through SOFA score despite effective antifungal treatment was associated with death, while PCT failed to predict the outcome.

References

1. Charles PE, et al:: Intensive Care Med 2006, 32:1577-1583.

2. Charles PE, et al.: Intensive Care Med 2009, 35:2146-2150.

3. Martini A, et al.: J Infect 2010, 60:425-430.

\section{P32}

Assessment of the usefulness of presepsin (soluble CD14 subtype) in septic patients

T Nishida', H Ishikura', A Murai', Y Irie'1, R Yuge' ${ }^{1}$ T Kamitani', S Endo ${ }^{2}$

'Fukuoka University Hospital, Fukuoka City, Japan; 'Iwate Medical University, Iwate, Japan

Critical Care 2012, 16(Suppl 1):P32 (doi: 10.1186/cc10639)

Introduction Sepsis is a life-threatening condition that is characterized by a whole-body inflammatory state. The early diagnosis and treatments of sepsis will improve the outcome of the patients. The aims of this study were to investigate the most useful biomarkers which are serum levels of soluble CD14 subtype (sCD14-ST) named presepsin, procalcitonin (PCT), IL-6, and C-reactive protein (CRP) as markers for early diagnosis of sepsis.

Methods A single-center, prospective, observational study. Patients who had one or more systemic inflammatory response syndrome (SIRS) criteria were included in this study. The blood samples for measuring the markers were collected and the severity of sepsis was evaluated at the time of admission and every other day for a week. Eighty-four patients were enrolled for this prospective study from June 2010 to June 2011.

Results Eighteen were SIRS and 42 were sepsis at the time of registration. In the receiver operating characteristics (ROC) analysis, the area under the curve (AUC) to distinguish sepsis was the highest for presepsin (0.92) followed by IL-6 (0.89), PCT (0.88), and CRP (0.83). The ROC analysis showed that at a cut-off value $647 \mathrm{pg} / \mathrm{ml}$, presepsin may be able to discriminate between patients with and without sepsis with a sensitivity and a specificity of $92.9 \%$ and $83.3 \%$ respectively with $95 \%$ confidence intervals of 0.929 ( 0.805 to 0.985$)$. And the presepsin values were significantly higher in the patients with the more severe septic condition (for example, sepsis, severe sepsis, septic shock). In addition, a significant correlation was found between the SOFA scores and the presepsin values $\left(r^{2}=0.258 ; P<0.01\right)$. But there was only weak correlation between APACHE II scores and the presepsin values $\left(r^{2}=0.053\right)$.

Conclusion In this study, presepsin is the most valuable predictor about sepsis compared with PCT, IL-6, and CRP. Moreover, these results suggest that presepsin values can serve as a parameter that closely 
reflects the pathology. So we strongly suggest that presepsin will be not only a very useful new biomarker for a diagnosis of the sepsis, but also useful for monitoring the severity of the disease in the near future. Reference

1. Endo S, Yaegashi Y, Sato N, et al:: Comparative study of soluble CD14 and soluble CD14-subtype in sepsis. Med Postgrad 2006, 44:381-385.

P33

Circulating cell-free DNA levels measured by a novel simple fluorescent assay are predictive for outcome of severe sepsis A Douvdevani, A Avriel, M Paryente Wiessman, V Novack, Y Almog Soroka University Medical Center and Ben-Gurion University of the Negev, Beer-Sheva, Israel

Critical Care 2012, 16(Suppl 1):P33 (doi: 10.1186/cc10640)

Introduction Circulating cell-free DNA (CFD) was found to be a predictor of outcome in severe sepsis and septic shock [1]. The standard CFD assays are work-intensive and not practical for routine clinical laboratory use. We have recently developed a new simple, fast and reliable assay for CFD measurement. The aim was to evaluate the association between admission levels of CFD and severe sepsis outcome in patients hospitalized in intensive care utilizing the new assay.

Methods Seventy-six patients diagnosed with severe sepsis hospitalized in the ICU were enrolled in the study. Serum CFD levels were measured upon admission and after 72 hours using the SYBR-Gold rapid direct fluorescent assay [2]. Primary outcome was 28-day mortality. Logistic regression analysis of CFD quintiles adjusted for baseline comorbidities and severity of the disease was utilized.

Results Out of those diagnosed with severe sepsis, 28 (36.8\%) have died either during hospitalization or within 28 days of admission to the ICU. Decedents had higher APACHE II score on admission (median 24.5 vs. $17.5, P=0.140$ ). Similarly their admission CFD levels were higher than in survivors (median 3,712 vs. $1,974, P=0.001$ ). Spearman's correlation analysis showed significant correlation between APACHE II score and CFD level on admission $(\rho=0.315, P=0.007)$. ROC curve for APACHE II score and CFD level on admission for prediction of 28-day mortality showed area under the curve of $0.59,95 \% \mathrm{Cl} 0.44$ to $0.74(P=0.208)$, for APACHE II score; and area under the curve of $0.73,95 \% \mathrm{Cl} 0.60$ to $0.86(P=0.001)$, for CFD level on admission. The study group was divided into quintiles by CFD levels of admission. The 28-day mortality rate was $12.5 \%$ in the CFD lowest quintile and $60.9 \%$ in the highest quintile. Logistic regression analysis showed that adjusted for age, sex and APACHE II score CFD divided into quintiles was significantly associated with death at 28 days, $\mathrm{OR}=1.83$ per quintile $(95 \% \mathrm{Cl} 1.12$ to $2.98, P=0.015$ ).

Conclusion By using a simple fluorometric assay, we were able to measure CFD levels in severe septic patients. CFD levels were found to be an independent predictors for 28-day mortality. We believe that CFD is an objective, reliable and integrative prognostic marker that will allow fast evaluation of intensive care patients and predicting mortality. References

1. Saukkonen K, et al:: Clin Chem 2008, 54:1000-1007.

2. Goldshtein H, et al.: Ann Clin Biochem 2009, 46:488-494.

P34

Clinical usefulness of measuring endotoxin activity on ICU

admission

A Murai, H Ishikura, T Nishida, Y Irie, T Kamitani, R Yuge, T Kitamura,

TUmemura

Fukuoka University Hospital, Fukuoka City, Japan

Critical Care 2012, 16(Suppl 1):P34 (doi: 10.1186/cc10641)

Introduction According to the Surviving Sepsis Campaign, diagnosis of sepsis and infection is urgent, therefore rapid diagnostic tools play a major role in the management of septic patients. The endotoxin activity (EA) assay (EAA) is one of those tools based on the ability of antigen-antibody complexes to prime neutrophils for an augmented respiratory burst response [1]. EAA has been used widely in patients who had suspected infection in the emergency room and ICU, but the clinical usefulness of measuring EAA in the diagnosis of sepsis in critically ill patients is not yet clear.

Methods We performed an observational cohort study in critically ill patients in the ICU of a tertiary care hospital. We investigated the correlation between EA levels and blood concentration of endotoxin measured by the chromogenic limulus amoebocyte lysate (LAL) assay, causative microorganism identified in laboratory culture, procalcitonin (PCT), soluble CD14 subtype (named presepsin), IL-6, antithrombin, protein C, thrombomodulin, lactate, disseminated intravascular coagulation scores in both the Japanese Ministry of Health and Welfare and the Japanese Association for Acute Medicine, and severity of illness at ICU admission.

Results We enrolled 49 subjects. There was no significant correlation between EA levels and endotoxin concentration measured by LAL assay. There were no significant difference in the EA levels of the Gramnegative infection patients and the others. The diagnostic value of EA levels was investigated using ROC curve analysis. For the diagnosis of sepsis, area under the curve of EA levels, PCT, presepsin, IL-6 and CRP were calculated as $0.76,0.83,0.89,0.88$ and 0.72 , respectively. Both the EA levels and ICU mortalities of the patients who met the criteria for severe sepsis were significantly higher than those of the patients who did not have sepsis $(0.44 \pm 0.21$ vs. $0.22 \pm 0.17, P=0.0004$; EA levels, $33 \%$ vs. $5 \%, P=0.022$; ICU mortalities). There was a positive relationship between EA levels and thrombomodulin $(r=0.30, P=0.049)$, EA levels and lactate $(r=0.31, P=0.028)$, and EA levels and SOFA score $(r=0.34, P=0.02)$. There was a negative relationship between EA levels and platelet counts $(r=-0.34, P=0.018)$, EA levels and antithrombin $(r=-0.41, P=0.004)$, and EA levels and protein $C(r=-0.38, P=0.010)$. Conclusion EA levels in the patients on ICU admission correlated with disease severity. Moreover, we strongly suggested that EAA may have the potential to assess organ dysfunction with sepsis, especially coagulopathy.

Reference

1. Marshall JC, et al.: J Infect Dis 2004, 190:527-534.

\section{P35}

Prognostic value of serum galactomannan in mixed ICU patients: a retrospective observational study

STeering, A Verreth, W Verlinden, J Jacobs, S Pilate, M Peetermans,

A Verrijcken, NVan Regenmortel, I De laet, K Schoonheydt, H Dits,

$M$ Van De Vyvere, M Malbrain

ZNA Stuivenberg, Antwerp, Belgium

Critical Care 2012, 16(Suppl 1):P35 (doi: 10.1186/cc10642)

Introduction Little is known about galactomannan (GM) testing in mixed ICU patients that are often not neutropenic. The aim of this study was to look for the incidence and outcome of invasive aspergillosis (IA) in critically ill patients, to validate previous reported GM thresholds and to evaluate the prognostic value of $\mathrm{GM}$.

Methods A retrospective study of $474 \mathrm{GM}$ samples in 160 patients from 1 January 2003 to 1 February 2004. GM tests were ordered because of clinical suspicion of IA or on a regular basis in immune compromised patients. The number of samples per patient was $3 \pm 2.6$. Similarly to the EORTC criteria we defined 'proven IA' as those patients with positive tissue specimen, 'probable $I \mathrm{~A}^{\prime}$ as those with positive cultures, and 'possible IA' as those treated with antifungals (high clinical index of suspicion). The number of positive samples (GM $>0.5 \mathrm{ng} / \mathrm{ml}$ ) was 230 (48.5\%). Patient characteristics: M/F ratio $1 / 1$, age $64.5 \pm 15.9$, SAPS $45.5 \pm 16.8$, APACHE II $19.3 \pm 8$, SOFA $5.8 \pm 3.5$, mean days on ventilation $12.9 \pm 8.7$, mean CRP $10.4 \pm 11.2 \mathrm{mg} / \mathrm{dl}$.

Results In our study population $5 \%$ had proven IA, 5\% probable IA, $17.5 \%$ possible IA and $72.5 \%$ had no IA. We could not identify a GM threshold for IA. The best threshold was GM $>1.1$ for identifying patients with IA (proven + probable + possible) with a specificity of $70.7 \%$ and negative predictive value of $76.6 \%$. The ICU mortality was $41.9 \%$ and the hospital mortality was $58.1 \%$. Patients who died in the ICU had higher APACHE, SAPS and SOFA scores $(P<0.0001)$, and had a significant increase in GM during their stay $(0.27 \pm 1.26$ vs. $-0.43 \pm 1.7$, $P=0.004)$. We observed higher mean GM values in nonsurvivors but 
this was not statistically significant. Patients who died in the hospital also showed a significant increase in GM during their stay $(0.11 \pm 1.55$ vs. $-0.48 \pm 1.51, P=0.017$ ). There was a trend towards higher $\mathrm{GM}$ values in patients treated with piperacillin/tazobactam $(n=34)$ but this was not statistically significant. Neutropenic patients $(n=31)$ showed an increase in $\mathrm{GM}$ during their stay $(0.32 \pm 1.3$ vs. $-0.43 \pm 1.7, P=0.07)$. Patients on total parenteral nutrition $(n=125)$ had higher maximal GM levels $(1.55 \pm 1.94$ vs. $0.88 \pm 1.25, P=0.058)$. Patients that were mechanically ventilated had significantly higher mean $(P=0.038)$ and maximal $(P=0.007) \mathrm{GM}$ levels. The presence of IA was associated with $100 \%$ hospital mortality.

Conclusion The current GM threshold of $0.5 \mathrm{ng} / \mathrm{ml}$ does not allow one to discriminate between patients with and without IA. A threshold of $1.1 \mathrm{ng} / \mathrm{ml}$ had the best specificity and negative predictive value for IA. There seems to be a correlation between GM levels and total parenteral nutrition due to interference with the ELISA test.

\section{P36}

Analysis of $(1 \rightarrow 3) \beta$-D-glucan as a diagnostic adjunct for invasive

fungal infections in the ICU setting

N Yamada, K Shirai, T Doi, K Kumada, M Nakano, S Yoshida, I Toyoda,

SOgura

Gifu University Hospital, Gifu, Japan

Critical Care 2012, 16(Suppl 1):P36 (doi: 10.1186/cc10643)

Introduction Since invasive fungal infections are associated with high morbidity and increased mortality in the ICU, early diagnosis and treatment are essential. This study assesses the performance of an assay of serum ( $1 \rightarrow 3$ )-D-glucan (BDG) concentration in patients admitted to the ICU.

Methods Patients admitted to our advanced critical care center from April 2007 to March 2011 with measurements of BDG were enrolled in this retrospective study. BDG was measured when invasive fungal infection was suspected based on the Japanese guidelines for diagnosis and treatment of invasive fungal infections. BDG levels were measured using the WAKO method. A BDG level greater than $11 \mathrm{pg} / \mathrm{ml}$ was considered to be positive. No gray zone was considered.

Results Of the 872 patients enrolled in this study, there were 580 males and 292 females. The mean age was 60.7 years (range: 48 to 87 ). The mortality rate was $16.3 \%$. We make a clinical diagnosis of invasive fungal infections according to Japanese guidelines for diagnosis and treatment of invasive fungal infections. The sensitivity of the BDG assay was $71.9 \%$ and the specificity was $91.0 \%$. There were significant differences in sensitivity, specificity, and optimal cut-off points among patients with different clinical conditions (that is, trauma, burn, postoperative, and medical conditions).The area under the summary receiver operating characteristic curve was 0.82 , but there were also differences across clinical categories.

Conclusion The BDG profile in ICU patients is similar to that of other inpatients. It can be useful in clinical practice if implemented in the proper setting and interpreted after consideration of the patient's clinical status.

References

1. Committee for Guideline for Management of Deep-seated Mycoses 2007 : Guideline for Management of Deep-seated Mycoses 2007. Tokyo: Kyowa kikaku; 2007.

2. Drosos EK, et al: $\beta$-D-glucan assay for the diagnosis of invasive fungal infections: a meta analysis. Clin Infect Dis 2011, 52:750-770.

P37

Impaired heart rate variability predicts clinical deterioration and progressive organ failure in emergency department sepsis patients R Arnold, G Green, A Bravi, S Hollenberg, A Seely

Cooper University Hospital, Camden, NJ, USA

Critical Care 2012, 16(Suppl 1):P37 (doi: 10.1186/cc10644)

Introduction Emergency department (ED) sepsis patients without overt shock have a high incidence of clinical deterioration after admission. Heart rate variability (HRV) is decreased in severe sepsis. The objective was to determine the ability of a panel of HRV indices to identify physiologically stable ED sepsis patients who will develop worsening organ failure. We hypothesized that patients meeting the outcome of progressive organ failure will have decreased HRV on initial presentation.

Methods We performed a prospective observational study of adult ED patients admitted to the hospital for infection and treated with i.v. antibiotics. Patients in overt shock (vasopressor requirement or mechanical ventilation) at enrollment or with the inability to provide written informed consent were excluded. A panel of HRV indices was assessed over a 2-hour ED period using CIMVA (continuous individualized multiorgan variability analysis) software including standard deviation (SD), LF/HF ratio, Poincare SD, sample entropy, wavelet AUC, detrended fluctuation analysis (DFA), correlation dimension, and the Lyapunov exponent. Patients were followed to assess the occurrence of the primary outcome of increased organ failure (SOFA score increase greater than 1 point at 24 hours), mechanical ventilation, vasopressor use, or in-hospital mortality.

Results We enrolled 105 ED sepsis patients. Twenty patients were removed due to nonsinus cardiac rhythm or poor data quality of the telemetry signal. Complete HRV assessment was performed on 81 subjects with 17 patients removed who developed shock in the ED. The primary outcome was met in $44 \%(28 / 64)$ of the cohort. On HRV assessment, outcome patients had a lower LF/HF ratio (1.47 vs. 3.11, $P=0.009)$ and DFA (0.65 vs. $0.94, P=0.04)$ compared with stable patients with no differences in other HRV indices. The overall mortality rate was $15 \%$. Compared to stable patients, outcome patients had no difference in age, initial heart rate, systolic blood pressure, or serum lactate with similar initial SOFA scores that were higher at 24 hours $(1.0$ vs. 3.0), a higher ICU transfer rate ( 62 vs. $20 \%, P<0.001)$ and increased ICU length of stay.

Conclusion While standard physiologic parameters in the ED were unable to differentiate sepsis patients who developed increased organ failure, a decreased LF/HF ratio and DFA, measurements of variability representing physiologic reserve, was associated with impending deterioration. The ability of decreased HRV to predict clinical outcomes in a high-risk yet physiologically identical population at presentation supports the need for continued studies into the predictive role of HRV assessment in the ED to supplement clinical decision-making in sepsis patients.

\section{P38}

Severe community-acquired pneumonia: risk factors for in-hospital mortality

JM Pereira', JA Paiva1', JP Baptista², F Froes ${ }^{3}$, J Gonçalves-Pereira ${ }^{4}$ 'Centro Hospitalar S. João, Porto, Portugal; ${ }^{2}$ Hospitais Universidade Coimbra, Portugal; ${ }^{3}$ Hospital Pulido Valente - CHLN, Lisbon, Portugal; ${ }^{4}$ Hospital S. Francisco Xavier, Lisbon, Portugal

Critical Care 2012, 16(Suppl 1):P38 (doi: 10.1186/cc10645)

Introduction Severe community-acquired pneumonia (SCAP) is an important cause of hospital mortality. The goal of this study was to identify variables associated with increased risk of in-hospital mortality at ICU admission.

Methods A prospective, multicentre, observational cohort study of all patients with SCAP consecutively admitted to 15 Portuguese ICUs during a 12-month period. Demographic characteristics, co-morbidities, general severity scores (SAPS II, SAPS3, total SOFA), microbiological data and initial empirical antibiotherapy were recorded. Logistic regression analysis was performed to identify predictors of in-hospital mortality. Results A total of $505(14 \%)$ of the 3,572 enrolled patients had SCAP, mostly male (66\%) with a median age 58 (29 to 82 ). Median general severity scores were: SAPS II 44 (21 to 80$)$, SAPS3 65 (41 to 98) and total SOFA 8 (3 to 17). Comorbidities were present in $74 \%$ of the patients and the most frequent were: diabetes mellitus (22\%), chronic respiratory failure (18\%) and alcoholism (15\%). Median Charlson's comorbidity index was 4 (0 to 13 ). At ICU admission, $44 \%$ of SCAP patients had septic shock. Thirty-seven per cent of the cases were microbiologically documented (St. pneumoniae - 24\%; influenza A 
(H1N1) virus - 20\%; Enterobacteriaceae - 18\%) and 12\% had secondary bacteremia. Antibiotics were administered in the first 3 hours after hospital admission in $71 \%$ of the patients and $76 \%$ of them received combination therapy. Antibiotherapy was appropriate in $80 \%$ with a median duration of 8 days. Median ICU and hospital lengths of stay were 10 and 19 days respectively. Median ICU and hospital mortalities were $25 \%$ and $34 \%$ respectively. Variables independently associated with hospital mortality were: SAPS II score (OR 1.06; $95 \% \mathrm{Cl} 1.037$ to 1.086), severe sepsis (OR $3.61 ; 95 \% \mathrm{Cl} 1.334$ to 9.791), septic shock (OR 4.25; $95 \% \mathrm{Cl} 1.61$ to 11.194 ), inappropriate antibiotherapy (OR 5.06; $95 \% \mathrm{Cl} 1.766$ to 14.516 ) and the use of a macrolide (OR $0.40 ; 95 \% \mathrm{Cl}$ 0.203 to 0.809 ).

Conclusion Disease severity evaluated by SAPS II and sepsis staging score and inappropriate initial antibiotherapy were independent risk factors for in-hospital mortality. The use of a macrolide was independently associated with a reduced risk of death.

\section{P39}

Systemic corticosteroids for community-acquired pneumonia in

adults

RJ Pugh, N Roy

Glan Clwyd Hospital, Rhyl, UK

Critical Care 2012, 16(Suppl 1):P39 (doi: 10.1186/cc10646)

Introduction We aimed to evaluate evidence from randomised controlled trials (RCTs) investigating the effect of systemic corticosteroids in adults with community-acquired pneumonia (CAP). Observational data suggest that corticosteroids may decrease mortality in severe CAP [1], and several large RCTs have been published since the recent Cochrane review [2].

Methods A systematic review of the literature: Cochrane Central Register for Controlled Clinical Trials, MEDLINE, EMBASE and SCOPUS, and reference lists of original studies and reviews. Data were collated and analysed using Review Manager v5.1.

Results A total of 254 RCTs were identified. Seven met inclusion criteria, totalling 806 patients. Studies varied in methodology, participants, interventions, and outcome measures. Where metaanalysis was possible, data are presented in Table 1 (outcomes: hospital mortality, 30-day mortality, hospital length of stay, superinfection, hyperglycaemia). Excepting hyperglycaemia, effect estimates were not statistically significant. Two small studies ( $n=46$ and $n=30$ ) concentrated on severe CAP (using ATS and BTS criteria); one study found a statistically significant reduction in mortality, lengths of stay and duration of mechanical ventilation in the steroid group, but similar improvements in the other study, and in a large subgroup of patients with severe CAP in another study $(n=93)$ were not found. Significant reductions in inflammatory markers in the week following initiation of steroid treatment were found in six studies.

Table 1 (abstract P39). Meta-analysis of clinical outcomes

\begin{tabular}{lccc}
\hline Outcome & Number of studies & Population & Effect \\
\hline Hospital mortality & 5 & 537 & OR 0.65 \\
30-day mortality & 3 & 562 & OR 0.90 \\
Hospital LOS & 2 & 244 & MD -1.52 \\
Superinfection & 3 & 563 & OR 1.24 \\
Hyperglycaemia & 2 & 517 & OR 2.69* \\
\hline
\end{tabular}

LOS, length of stay. ${ }^{*} P<0.0001$.

Conclusion Systemic corticosteroid administration as adjunctive treatment for CAP does not appear to improve relevant clinical outcomes, regardless of severity, and is associated with significantly increased incidence of hyperglycaemia.

References

1. Garcia-Vidal et al: Eur Respir J 2007, 30:951-956.

2. Chen et al: Cochrane Database of Systematic Reviews 2011, 3.
P40

Characteristics of leptospirosis patients admitted to a tropical university hospital during the 2000 to 2010 period

H Mehdaoui, E Caffiot, R Theodose, R Valentino, D Resiere, C Chabartier, $\mathrm{M}$ Jonas

Fort de France University Hospital, Fort De France, Martinique

Critical Care 2012, 16(Suppl 1):P40 (doi: 10.1186/cc10647)

Introduction Leptospirosis is an endemic disease in the intertropical area. Most of the patients present with mild to moderate clinical forms, but leptospirosis may lead to multiple organ failure and death.

Methods We retrospectively analyzed the characteristics of 113 patients with leptospirosis admitted to our emergency department.

Results PCR and/or immunological investigations confirmed the diagnosis for 88 patients. We compared the periods before and after PCR diagnosis implementation (2006), and determined the pattern of the most severe forms. Thirty-two patients were admitted to our ICU. Eight of the ICU patients died including four with confirmed diagnosis. Nineteen patients were diagnosed before 2006, and 69 during the period to 2010. Patients were less frequently admitted to the ICU during the second period ( $29 \%$ vs. $63 \%, P=0.013)$. ICU patients had a higher heart rate $(111 \pm 28$ vs. $93 \pm 21, P=0.001)$, and had more frequently jaundice ( $94 \%$ vs. $64 \%, P=0.002)$ and oliguria ( $81 \%$ vs. $23 \%, P<0.001)$. Glycemia ( $8.7 \pm 3.3$ vs. $7.1 \pm 3.4, P=0.04)$, creatinin $(530 \pm 299$ vs. $142 \pm 113, P<0.0001)$, bilirubin $(423 \pm 251$ vs. $69 \pm 103, P<0.0001), C R P$ $(325 \pm 135$ vs. $210 \pm 127, P<0.0001)$, and WBCC ( $21.7 \pm 9.5$ vs. $9.7 \pm 5.3$, $P<0.0001)$ were higher and protidemia ( $58 \pm 15$ vs. $68 \pm 13, P=0.002$ ), hematocrit $(24 \pm 6$ vs. $34 \pm 6, P<0.0001)$, and $P / F$ ratio $(271 \pm 127$ vs. $352 \pm 84, P=0.036)$ were lower in the ICU group. Troponin was increased more frequently in the ICU group ( $44 \%$ vs. $9 \%, P=0.0003$ ) and ECG anomalies (78\% vs. 52\%, $P=0.02$ ) were more frequent. Among the 22 early cardiac echographies performed in the ICU, 11 patients had LVEF $<50 \%$.

Conclusion The use of PCR dramatically improved the diagnosis of mild to moderate forms of leptospirosis and led to an apparent increase its incidence. Severe forms were less easy to assess as they occur later and we should have a more aggressive policy to improve the immunological diagnosis which was sometimes neglected since the implementation of PCR diagnosis. Severe forms have a more pronounced inflammatory syndrome and diffuse organ failure. Aggressive fluid loading as recommended in septic states may have worsened hemodynamic and respiratory conditions in the ICU group. This is suggested by the hemodilution pattern found in this group. The association of renal, myocardial and respiratory failures in leptospirosis should lead to a careful monitoring of fluid loading and myocardial status.

\section{P41}

Prognostic impact of imported and newly-isolated

methicillin-resistant Staphylococcus aureus in the ICU

S Ohshimo, K Ota, T Tamura, Y Kida, J Itai, K Suzuki, K Kanao, Y Torikoshi,

K Koyama, T Otani, T Sadamori, K Une, R Tsumura, Y Iwasaki, N Hirohashi,

KTanigawa

Hiroshima University, Hiroshima, Japan

Critical Care 2012, 16(Suppl 1):P41 (doi: 10.1186/cc10648)

Introduction Methicillin-resistant Staphylococcus aureus (MRSA) is a leading pathogen of hospital-acquired pneumonia. The difference in outcome between patients with imported and newly-isolated MRSA in the ICU has not been well investigated. The aim of our study was to explore the incidence, risk factors and outcome in patients with imported and newly-isolated MRSA.

Methods Patients admitted to the ICU in our university between April 2009 and May 2010 were prospectively studied. Nasal swabs were collected from all patients on admission and subsequently collected weekly during the ICU stay. When patients were intubated, intratracheal aspirates were concurrently collected. The correlations of positive culture of MRSA with clinical variables were analysed.

Results A total of 1,270 consecutive patients were enrolled. Median follow-up period was 404 (187 to 609) days. There were 803 males and 
467 females. Median age was 63 ( 1 to 97). Of these, imported MRSA was found in $124(10 \%)$ patients, and newly-isolated MRSA in $57(4 \%)$ patients. The incidence of imported MRSA was associated with the comorbidity of cardiovascular disease or malignancy and long hospital stay before admission to the ICU, whereas the incidence of newlyisolated MRSA was associated with the positive culture in intratracheal aspirates or blood/intravenous catheter, the comorbidity of shock, pneumonia or trauma, increased number of isolated sites, higher APACHE II score, prolonged ICU stay and higher mortality during the ICU stay. Although no statistical significance was found in total patients, the subset analysis of the male patients demonstrated that the outcome of newly-isolated patients was significantly poor compared with those of imported MRSA $(P=0.005)$. Multivariate analysis revealed that new isolation of MRSA in the ICU $(P=0.03$; hazard ratio (HR), 2.62), negative culture of MRSA in nasal swab $(P=0.02 ; \mathrm{HR}, 4.18), \geq 2$ isolated sites $(P=0.01 ; \mathrm{HR}, 4.59)$ and comorbidity of ARDS $(P=0.002 ; \mathrm{HR}, 4.63)$ were the independent poor prognostic factors.

Conclusion The new isolation of MRSA during the ICU stay was associated with poor outcome compared with the imported MRSA. Clinicians should be aware of the high-risk group of MRSA infection. Strict hand hygiene plus a careful assessment of the patient, applying aggressive procedures such as patient isolation, staff cohorting, and active surveillance cultures should be indicated.

\section{P42}

Necrotizing pneumonia due to methicillin-sensitive Staphylococcus aureus secreting Panton-Valentine leukocidin: a review of case reports

L Kreienbuehl', E Charbonney², P Eggimann ${ }^{3}$

'HUG, Geneva, Switzerland; 'Li Ka Shing Knowledge Institute, St Michael's

Hospital, Toronto, Canada: ${ }^{3} \mathrm{CHUV}$, Lausanne, Switzerland

Critical Care 2012, 16(Suppl 1):P42 (doi: 10.1186/cc10649)

Introduction Community-acquired necrotizing pneumonia caused by Panton-Valentine leukocidin (PVL)-secreting Staphylococcus aureus is a highly lethal infection, which mainly affects healthy children and young adults $[1,2]$. This study focuses on necrotizing pneumonia due to methicillin-sensitive $S$. aureus strains, with the purpose to determine factors associated with outcome.

Methods We performed a systematic review of case reports on PVL-secreting MSSA necrotizing pneumonia and analyzed factors associated with outcome.

Results A total of 32 patient descriptions were retained for analysis. Septic shock, influenza-like prodrome and the absence of a previous skin and soft tissue infection were associated with fatal outcome. In multivariate analysis, influenza-like prodrome (OR 7.44; $95 \% \mathrm{Cl}$ : 1.24 to $44.76 ; P=0.028$ ) and absence of previous skin and soft tissue infection (OR $0.09 ; 95 \% \mathrm{Cl}: 0.010$ to $0.86 ; P=0.036$ ) remained significant predictors of death. See Table 1.

Table 1 (abstract P42). Univariate analysis of mortality risk factors

\begin{tabular}{|c|c|c|c|c|}
\hline & $\begin{array}{c}\text { Died } \\
(n=13)\end{array}$ & $\begin{array}{c}\text { Survived } \\
(n=19)\end{array}$ & $\begin{array}{c}\text { Univariate } \\
\text { analysis } \\
\text { OR }(95 \% \mathrm{Cl})\end{array}$ & $P$ value \\
\hline Flu-like prodrome & 9/12 (75\%) & $4 / 16(25 \%)$ & 9.00 (1.60 to 50.7 ) & 0.020 \\
\hline SSTI & $1 / 13(8 \%)$ & 9/19 (47\%) & 0.09 (0.01 to 0.86 ) & 0.024 \\
\hline Septic shock & $11 / 11$ & $7 / 15$ (47\%) & 26.0 (1.30 to 522$)$ & 0.007 \\
\hline Leukocytopenia & 9/11 (82\%) & $8 / 17$ (47\%) & 5.06 (0.83 to 30.8$)$ & 0.115 \\
\hline
\end{tabular}

Conclusion Influenza-like prodrome may be predictive of adverse outcome and previous skin and soft tissue infection may be associated with improved prognosis.

References

1. Gillet $Y$, et al: Lancet 2002, 359:753-759.

2. Gillet Y, et al:: Clin Infect Dis 2007, 45:315-321.
P43

Predicting methicillin-resistant Staphylococcus aureus in critically ill patients with pneumonia presenting to the hospital

AF Shorr', DE Myers², DB Huang 3 , BH Nathanson ${ }^{4}$, MF Emmons ${ }^{5}$

'Washington Hospital Centre, Washington, DC, USA; ${ }^{2}$ Pfizer, Inc., Collegeville,

PA, USA; ${ }^{3} V A$ NJ Healthcare System, East Orange, NJ, USA; ${ }^{4}$ OptiStatim, LLC,

Longmeadow, MA, USA; ${ }^{5}$ Cerner LifeSciences, Beverly Hills, CA, USA

Critical Care 2012, 16(Suppl 1):P43 (doi: 10.1186/cc10650)

Introduction Methicillin-resistant Staphylococcus aureus (MRSA) represents an important pathogen in those presenting to the hospital with pneumonia and requiring ICU admission. However, empiric treatment against MRSA in those admitted to the ICU with severe nonnosocomial pneumonia could lead to overuse of anti-MRSA therapy. To address this concern, we sought to develop a simple clinical score for identifying ICU patients presenting to the hospital with pneumonia unlikely to be caused by MRSA.

Methods We retrospectively identified patients admitted to the ICU with community-acquired pneumonia (CAP) or healthcare-associated pneumonia (HCAP) between April 2007 and March 2009 at 62 hospitals in the USA. The diagnosis of pneumonia was based on ICD-9 codes. We only included patients with laboratory evidence of bacterial infection (for example, positive sputum, blood, pleural cultures or urinary antigen testing). We determined, via logistic regression, variables independently associated with the presence of MRSA (two-thirds of cohort) and developed a risk score based on this. We then internally validated (one-third of cohort) the score.

Results The cohort included 957 patients (mean age $65.8 \pm 16.4$ years, $50.2 \%$ male, $43.7 \%$ HCAP). MRSA was identified in $20.1 \%$. The risk score assigned points as follows: 1 point - age $<30$ or $>79$ years, recent immunosuppression other than corticosteroids, shock; 2 points - admission from a skilled nursing facility, history of diabetes without coronary artery disease (CAD) or heart failure without CAD. The prevalence of MRSA increased with escalating score $(P<0.001)$. We collapsed the score into three strata based on risk for MRSA (score of 0 to 1 (low), 2 to 4 (moderate), $\geq 5$ (high)). The respective MRSA rates by strata equaled $15.2 \%, 24.7 \%$, and $31.9 \%,(P<0.001)$. A score $\leq 1$ as a screening test to exclude MRSA performed poorly (sensitivity $58.3 \%$, specificity of $53.3 \%$ ).

Conclusion The prevalence of MRSA in patients with CAP or HCAP requiring ICU care was high. A score to assess the risk for MRSA in these patients performed poorly but requires external validation. Given the high risk of MRSA in this setting along with the limited discriminatory power of our risk score, empiric therapy for MRSA in these patients seems appropriate.

P44

Predictors of multidrug-resistant Acinetobacter baumannii infections: a retrospective analysis in surgical ICU patients

A Camkiran, A Kundakci, C Araz, A Pirat, P Zeyneloglu, H Arslan, G Arslan Baskent University, Ankara, Turkey

Critical Care 2012, 16(Suppl 1):P44 (doi: 10.1186/cc10651)

Introduction Multidrug-resistant Acinetobacter baumannii (MRAB) is an important cause of hospital-acquired infection and leads to an increasing morbidity and mortality in ICUs. The aim of this study was to investigate the predictors of MRAB infection in surgical ICU patients. Methods The charts of the patients who were admitted to the ICU between January 2008 and August 2010 were reviewed to identify patients with MRAB infection. Recorded data were as follows: age, sex, medical history, underlying surgical pathology, APACHE II score on ICU admission, days in hospital before ICU, presence of invasive procedures (intubation, tracheostomy, arterial, central venous lines, urinary and nasogastric catheters, enteral or parenteral nutrition and renal replacement therapy), days in the ICU and white blood cell (WBC) count on infection day, infection site, complications (such as organ/ system failure), length of stay (LOS) in the ICU and hospital, and final outcome.

Results During the study period 25 patients with MRAB infection were identified. When compared with their matched control group $(n=25)$, 
patients with MRAB infection had a significantly higher mean APACHE II score $(P<0.001)$ and more frequently had an open wound $(P=0.002)$ or required mechanical ventilation $(P=0.005)$, arterial catheterization $(P=0.006)$, and central venous catheterization $(P=0.004)$. Multivariate logistic regression revealed that APACHE II score $(\mathrm{OR}, 1.19 ; 95 \% \mathrm{Cl}$, 1.005 to $1.315 ; P=0.043)$ and open wound $(\mathrm{OR}, 0.45 ; 95 \% \mathrm{Cl}, 0.003$ to $0.587 ; P=0.18$ ) were predictors of MRAB infection in these patients. Compared to their controls, patients with MRAB infection had a longer LOS in the ICU $(36.44 \pm 30.44$ days vs. $7.80 \pm 8.13$ days, $P<0.001)$ and hospital $(55.12 \pm 40.81$ days vs. $19.04 \pm 13.44$ days, $P<0.001)$. In-hospital mortality rates for patients with MRAB infection and their controls were $56 \%$ and $32 \%$, respectively $(P=0.154)$.

Conclusion Our results indicate that higher APACHE II scores and presence of an open wound are predictors of MRAB in ICU surgical patients. Patients with MRAB infection tended to have a higher mortality and had a longer LOS in the ICU and hospital than their controls.

P45

Risk factors for bronchial acquisition of resistant Gram-negative bacteria in critically ill patients and outcome

I Papakonstantinou', E Perivolioti', CVrettou'2, I Baraboutis', E Magira², E Balioti', D Panopoulou', T Pitsolis', C Routsi ${ }^{2}$, S Nanas²

'Evaggelismos Hospital, Athens, Greece; ${ }^{2}$ National and Kapodistrian University of Athens, Greece

Critical Care 2012, 16(Suppl 1):P45 (doi: 10.1186/cc10652)

Introduction It has been advocated that resistant Gram-negative bacteria (RGNB) colonization of ICU patients is to some extent a result of increased use of antibiotics. The aim of our study was to investigate, in adjustment with patients' characteristics, the impact of colonization status and antibiotic use during ICU stay on the impending acquisition of RGNB in the bronchial tree of newly intubated patients and to estimate the outcome.

Methods Bronchial and pharyngeal surveillance cultures were obtained up to day 7 (d7) of ICU admission. RGNB considered for analysis on $\mathrm{d} 7$ were $A$. baumannii (RAB) and K. pneumoniae (RKP). Polymicrobial colonization with $\geq 2$ RGNB (PMC) was also evaluated. To assess dependence between different explanatory variables, multivariable logistic regression was used. Variables included in the model were: SOFA score, department prior to ICU admission, medical cause of admission, emergency surgery, CRF, prior aminoglycosides and tigecycline use during ICU stay and concurrent RAB or RKP pharyngeal colonization, respectively. To estimate outcome (death), variables included in multivariate model were: APACHE, SOFA score, department prior to ICU admission, medical cause of admission, emergency surgery, CRF and d7 RAB, RKP.

Results Ninety-five eligible patients with bronchial colonization data on d7 were included for further analysis. In the case of RAB in multivariate model $\left(R^{2}=0.538\right)$, pharyngeal $\mathrm{d} 7 \mathrm{RAB}$ was the only predictor of $\mathrm{d} 7$ RAB bronchial colonization (OR $0.042,95 \% \mathrm{Cl} 0.012$ to $0.148, P<0.001$ ). In the case of RKP in multivariate model $\left(R^{2}=0.648\right)$, pharyngeal d7 RKP (OR $0.037,95 \% \mathrm{Cl} 0.004$ to $0.031, P=0.004$ ), aminoglycoside use (OR $0.094,95 \% \mathrm{Cl} 0.015$ to $0.573, P=0.01)$ and SOFA score (OR $1.66,95 \%$ Cl 1.07 to $2.58, P=0.023)$ characterized bronchial d7 RKP colonization. Multivariate model for PMC $\left(R^{2}=0.49\right)$ revealed only d7 pharyngeal PMC as predictor of bronchial PMC (OR $0.12,95 \%$ Cl 0.026 to 0.50 , $P=0.004$ ). Department prior to ICU, medical cause of admission, CRF, and emergency surgery were not found to influence RGNB bronchial colonization. Outcome death increased with APACHE score (OR 0.84, $95 \% \mathrm{Cl} 0.76$ to $0.94, P=0.002$ ) and bronchial d7 RKP colonization (OR $9.14,95 \% \mathrm{Cl} 1.3$ to $64.4, P=0.026$ ).

Conclusion Of the parameters included in our model, concurrent $\mathrm{d} 7$ pharyngeal RAB and RKP, respectively, resulted eventually in bronchial colonization with the same pathogens. Of the overall antibiotics used only aminoglycosides had significant correlation only for RKP colonization.
P46

Improved antibiotic stewardship resulting from a multifaceted strategy implemented after an outbreak of multiresistant

Acinetobacter baumannii in a university ICU

M Beach, M Cohen, V Grover, J Ho, N Soni, B Azadian, S Singh

Chelsea \& Westminster Hospital, London, UK

Critical Care 2012, 16(Suppl 1):P46 (doi: 10.1186/cc10653)

Introduction A 12-bed ICU experienced an outbreak of multiresistant Acinetobacter baumannii (MRA) from October 2009 to May 2010. A multifaceted strategy involving segregation, enhanced infection control procedures, and microbiological surveillance was implemented. We evaluated its impact on antibiotic stewardship.

Methods A retrospective review of patient notes and results using AcuBase ${ }^{\circledR}$ was conducted: 90 consecutive patients before the outbreak (January to June 2008) and 91 thereafter (October 2010 to May 2011). Data included patient profiles, admission criteria, ICU survival, antimicrobials used, antibiotic days, number of patients on antibiotics, prescribing cost and the demographic of microbes isolated.

Results Following the outbreak, enhanced infection control measures were implemented alongside the Matching Michigan protocols. Daily operational critical care and elective planning meetings and a staff education programme were undertaken. ICU mortality (31 (14\%) vs. 43 (16\%)) was unchanged. Microbiological isolates were overall similar, with a reduction in coagulase-negative Staphylococcus and Klebsiella and an increase in Enterobacter. The use of cefuroxime (3.2 vs. 2.3 antibiotic days/ patient) and quinolones ( 6 vs. 2 ) decreased. There was a reduction in average antibiotic days per patient episode (5.1 vs. 4.2) $(P=0.0291)$ and the prescribing cost savings were $£ 13,558$ (47\%). See Figure 1.

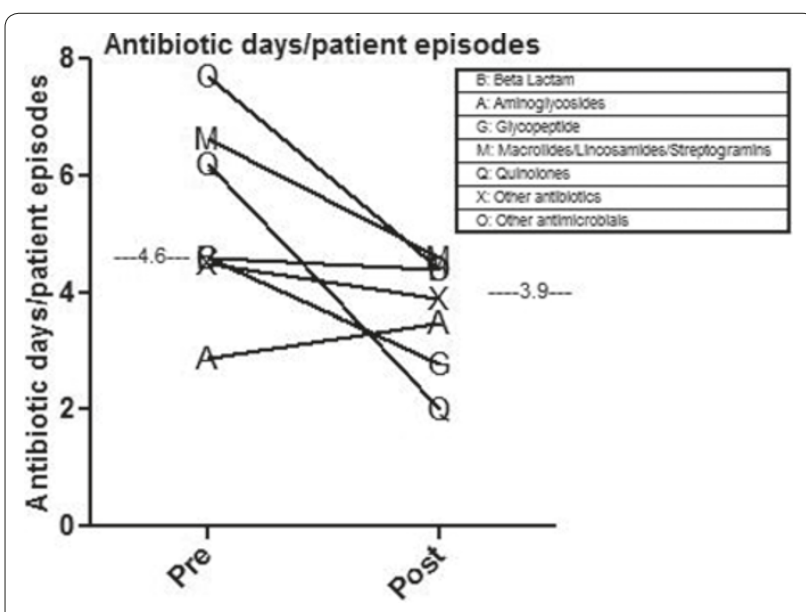

Figure 1 (abstract P46).

Conclusion The need for a complex strategy to manage and eradicate an MRA outbreak in the ICU led to a clinically significant decrease in antibiotic use and prescribing cost saving following eradication of MRA. Improved antibiotic stewardship is achievable through better infection control strategies.

Acknowledgements Thanks to Chelsea and Westminster Healthcare Charity, and ICU and microbiology staff.

Reference

1. Kollef M, et al: Ann Internal Med 2001, 134:298-314.

\section{P47}

Empirical antifungal treatment in the critically ill patients: how does it impact on the outcome?

R Bruyère, C Vigneron, J Quenot, M Hamet, F Dalle, S Prin, PE Charles University Hospital, Dijon, France

Critical Care 2012, 16(Suppl 1):P47 (doi: 10.1186/cc10654)

Introduction Given the high mortality attributable to invasive candidiasis (IC) and the lack of reliable diagnosis tool, antifungals 
are often started in high-risk patients with severe sepsis despite the absence of proven disease. According to current guidelines, echinocandins are the drugs of choice in this setting. However, the level of evidence supporting this statement is low.

Methods A retrospective single-centre observational study including every patient with highly suspected but unproven IC (that is, Candida score $>3$, multifocal Candida sp. colonization, unresolved sepsis despite $>2$-day broad-spectrum antibiotics, negative blood culture) who received at least two doses of one echinocandin between 2008 and 2011. Patients with proven IC (that is, candidemia) over the same period were used as controls. These two groups of patients were compared regarding baseline characteristics and both clinical and biological follow-up data while receiving antifungal therapy. The clinical response to antifungal therapy was assessed through the SOFA score daily decrease from day 0 to day 3 in both groups and compared by repeated-measures ANOVA. Then, independent predictors of death in the ICU were determined by Cox regression analysis.

Results Fifty-one patients were included (30 with suspected IC and 21 with proven IC). At the onset of antifungal therapy, the Candida score was greater in the patients with suspected IC than in those with proven infection $(3.7 \pm 0.7$ vs. $3.0 \pm 0.8, P=0.001)$ since multifocal colonization was more frequent in the former. In addition, the patients with suspected but unproven IC looked more seriously ill according to the SOFA score $(8.3 \pm 3.0$ vs. $6.6 \pm 3.5, P=0.07)$. This mainly resulted from a greater level of hypotension as assessed through the SOFA score ( $2.8 \pm 1.5$ vs. $1.2 \pm 1.5$ points, $P=0.0006)$. Obviously, the clinical response to antifungal therapy was significantly more consistent in the patients with unproven IC than in those with proven infection $(P=0.032)$. In addition, there was a trend toward an improved survival in the former patients ( 53 vs. $47 \%, P=0.42$ ). The only independent protective factor was echinocandin therapy duration ( $\mathrm{HR}=0.84(95 \% \mathrm{Cl} 0.75$ to 0.94$)$, $P=0.0034)$.

Conclusion A significant clinical improvement is achieved in patients with suspected but not proven IC receiving empirical antifungal therapy with an echinocandin. In contrast, the patients with proven IC are less responsive to therapy and are more likely to die in the ICU. Our data support the use of an echinocandin as empirical therapy in very high-risk patients.

References

1. Pappas PG, et al:: Clin Infect Dis 2009, 48:503-535.

2. Leon C, et al:: Crit Care Med 2009, 37:1624-1633.

P48

Relationship between polyclonal immunoglobulin therapy and colonization by Candida spp.

G Serafini, I Cavazzuti, C Venturelli, M Girardis

University Hospital, Modena, Italy

Critical Care 2012, 16(Suppl 1):P48 (doi: 10.1186/cc10655)

Introduction Low IgA levels in blood serum and in saliva have been associated with an increased risk for Candida colonization and infection $[1,2]$. In this retrospective cohort study, we aimed to evaluate the effects of an intravenous immunoglobulin preparation containing polyclonal IgG, IgM and IgA (IgGAM) on the prevention of Candida spp. colonization in patients with septic shock.

Methods In this study we analyzed 69 patients with septic shock and without Candida spp. colonization before shock appearance admitted to the ICU of a university hospital from January 2008 to November 2011. All of the patients were treated in according to the Surviving Sepsis Campaign guidelines. In addition to standard therapy, 44 (64\%) patients received IgGAM therapy (Pentaglobin ${ }^{\otimes} 38 \mathrm{~g} / \mathrm{lg} \mathrm{lg}, 6 \mathrm{~g} / \mathrm{l} \lg \mathrm{M}$, and $6 \mathrm{~g} / \lg \mathrm{lg}$ ) within 24 hours from the diagnosis of septic shock at the dose of $250 \mathrm{mg} / \mathrm{kg} /$ day for 3 days. The colonization by Candida spp. was evaluated by analyzing the results of the microbiological surveillance cultures (two times per week) of pharyngeal swab, tracheal aspirate, urine and surgical drains between 48 hours and 21 days after the diagnosis of septic shock.

Results In the IgGAM group, 11 patients (25\%) developed Candida spp. colonization compared to nine patients $(36 \%)$ of the control group. The Candida colonization index was similar in the two groups: $0.42 \pm 0.16$ in the lgGAM group and 0.45 in the control group.
Conclusion The above data show a slight reduction of Candida spp. colonization in septic shock patients treated with IgGAM therapy. Further studies are needed to confirm this finding.

References

1. Gonçalves e Silva CR, Melo KE, Leão MV, Ruis R, Jorge AO: Relationship between Candida in vaginal and oral mucosae and salivary IgA. Rev Bras Ginecol Obstet 2008, 30:300-305.

2. Bai XD, Liu XH, Tong QY: Intestinal colonization with Candida albicans and mucosal immunity. World J Gastroenterol 2004, 10:2124-2126.

\section{P49}

Predictive and prognostic factors of septic shock of nosocomial origin

JP Quenot', A Pavon', C Binquet', F Kara², O Martinet', F Ganster³,

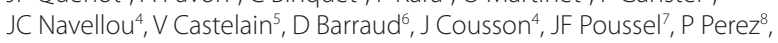
K Kuteifan', A Noirot ${ }^{2}$

'University Hospital Bocage, Dijon, France; ${ }^{2}$ Centre Hospitalier, Haguenau, France; ${ }^{3}$ Nouvel Hopital Civil, Strasbourg, France; ${ }^{4}$ University Hospital, Besancon, France; ${ }^{5}$ Hopital Hautepierre, Strasbourg, France; ${ }^{6} \mathrm{Hopital}$ Central, Nancy, France; ${ }^{7}$ Regional Hospital, Metz-Thionville, France; ${ }^{8}$ Hopital Brabois, Nancy, France; ${ }^{9} \mathrm{CHG}$, Mulhouse, France

Critical Care 2012, 16(Suppl 1):P49 (doi: 10.1186/cc10656)

Introduction The incidence of septic shock in intensive care in France is around 8 to $10 \%$, with in-hospital mortality ranging from 55 to $60 \%$ [1]. Mortality increases by $10 \%$ when the infection causing septic shock is acquired in-hospital or in the ICU [1]. We aimed to determine predictive and prognostic factors for septic shock caused by a nosocomial infection (NI).

Methods Subgroup analysis of a prospective, multicentre, observational study performed between November 2009 and March 2011 in 14 ICUs from 10 university and community (nonacademic) hospitals in the northeast of France. This study was supported by the Collège Interrégional des Réanimateurs du Nord-Est. Patients were included if they were aged $>18$ years and had septic shock plus at least one criterion of hypoperfusion. Infection was classed as nosocomial if acquired in-hospital more than $\mathbf{4 8}$ hours after admission. Data control and statistical analysis were performed by the CIC-EC of Dijon University Hospital (INSERM Unit CIE1).

Results In total, 1,147 patients were included in the cohort, of whom $409(35.6 \%)$ presented a NI (345/409 (84\%) acquired in-hospital and $64 / 409(16 \%)$ acquired in the ICU). The factors significantly associated with NI (in-hospital or in-ICU) were: immunodepression, a Knaus score C to D, SAPS II score, and SOFA score. Other variables such as age, sex, type of admission and type of infection were not significantly related to the nosocomial origin of infection. In-hospital mortality for communityacquired versus Nls was $40.8 \%$ vs. $53.5 \%$ respectively $(P<0.01)$, and $46.9 \%$ vs. $62 \%$ respectively at 28 days $(P<0.01)$.

Conclusion Mortality of patients with septic shock of nosocomial origin is particularly high. Scores evaluating gravity of disease are also higher in patients with $\mathrm{NI}$ versus those with community-acquired infection. This could be explained by delayed presentation or difficulties with management, but also by immunodepression and a poor state of prior health. It is likely that appropriate measures, particularly aimed at prevention, could help to reduce mortality in patients with septic shock caused by NI.

Reference

1. Annane D: Am J Respir Crit Care Med 2003, 168:165.

\section{P50}

Catheter-related bloodstream infection: factors affecting incidence K Boner1, M McGovern², J Bourke', C Walshe ${ }^{3}$, D Phelan'

'Mater Misericordiae University Hospital, Dublin, Ireland; ${ }^{2}$ Harvard University,

Cambridge, MA, USA; ${ }^{3}$ Beaumont Hospital, Dublin, Ireland

Critical Care 2012, 16(Suppl 1):P50 (doi: 10.1186/cc10657)

Introduction Catheter-related bloodstream infection (CRBSI), its associated morbidity, mortality and expense are the most important adverse effect of central venous catheters (CVCs) [1]. The objective of this study of a population in whom the rate of CRBSI fell significantly 
over 12 years [2] was to evaluate the influence of both patient and CVC factors on CRBSI rates in patients receiving total parenteral nutrition (TPN) in this time.

Methods Set in a 525-bed university hospital providing acute and tertiary services. A prospective database was established in 1997, recording data on all patients with CVCs inserted for TPN administration. This database was examined up to 2009 to ascertain the effects of patient and CVC factors on CRBSI.

Results During the 12-year study period, 2,573 CVCs were inserted into 1,343 patients and 15,385 CVC days were accumulated. Overall, $13.8 \%$ of patients developed CRBSI throughout the study. In terms of patient factors affecting CRBSI rates, CRBSI was increased in patients with longer duration of TPN administration (where each additional day was associated with a relative risk ratio of $1.02, P<0.01$ ), increased numbers of CVCs inserted (where each additional line was associated with a relative risk ratio of $1.21, P<0.01$ ), and use of lipid formulation of TPN ( 58.9 vs. $49 \%$ use was associated with a relative risk ratio of $1.56, P<0.01)$. Overall $8.6 \%$ of CVCs inserted became infected. Hospital location of CVC insertion was an important risk factor for CRBSI. The most common site for insertion was the ICU (almost $40 \%$ of CVCs); however, compared to ICU insertion, insertion in the HDU was associated with an increased risk of CRBSI (a relative risk ratio of $1.75, P<0.01$ ), as was insertion in the operating theatre for ward patients (a relative risk ratio of $2.08, P<0.01$ ). CVC maintenance at ward level was associated with increased CRBSI rates, with a relative risk ratio of $2.06(P<0.01)$.

Conclusion CRBSI occurs commonly in TPN populations, but there are very limited published data as regards incidence or factors affecting incidence in this population. This large study of TPN patients provides prospective analysis of both patient and CVC factors influencing the development of CRBSI for the first time.

References

1. O'Grady NP, Alexander M, Dellinger EP, et al.; Healthcare Infection Control Practices Advisory Committee: Guidelines for the prevention of intravascular catheter-related infections. Infect Control Hosp Epidemiol 2002, 23:759-769.

2. Walshe CM, Boner K, Bourke J, et al:: Catheter related blood stream infection (CRBSI) in TPN patients. Benefit of an educational programme using multimodal CRBSI expression. Clin Govern Int J 2010, 15:292-301.

P51

Prognostic factors of septic shock

JP Quenot', A Pavon', C Binquet'2, F Kara 3 , O Martinet ${ }^{4}$, F Ganster ${ }^{4}$,

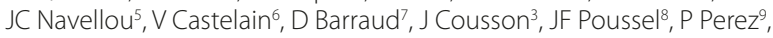
K Kuteifan ${ }^{3}$

'University Hospital Bocage, Dijon, France; ${ }^{2}$ CIC EC, Dijon, France; ${ }^{3}$ Centre Hospitalier, Haguenau, France; ${ }^{*}$ Nouvel Hopital Civil, Strasbourg, France; ${ }^{5}$ University Hospital, Besancon, France; ${ }^{6}$ Hopital Hautepierre, Strasbourg,

France; ${ }^{7}$ Hopital Central, Nancy, France; ${ }^{8}$ Regional Hospitalier, Metz-Thionville, France: ${ }^{\circ H}$ Hopital Brabois, Nancy, France

Critical Care 2012, 16(Suppl 1):P51 (doi: 10.1186/cc10658)

Introduction The incidence of septic shock in intensive care (ICU) in France is around 8 to $10 \%$, with in-hospital mortality ranging from 55 to $60 \%$ [1]. The identification of prognostic factors is essential to guarantee optimal management.

Methods A prospective, multicentre, observational study was performed between November 2009 and March 2011 in 14 ICUs from 10 university and community (nonacademic) hospitals in the northeast of France. This study was supported by the Collège Interrégional des Réanimateurs du Nord-Est. Patients were included if they were aged $>18$ years and had septic shock plus at least one criterion of hypoperfusion. Data control and statistical analysis was performed by the CIC-EC of Dijon University Hospital (INSERM Unit CIE1). Univariate and multivariate logistic regression analysis was used to identify predictors of mortality at 28 days.

Results In total, out of 7,833 patients admitted to intensive care during the study period, $1,147(14.6 \%)$ had septic shock. Factors significantly associated with mortality at 28 days by logistic regression were: age $>70$ (OR $1.98,95 \% \mathrm{Cl} 1.5$ to $2.6, P<0.01$ ); transfer (OR $1.42,95 \% \mathrm{Cl} 1.04$ to $1.95, P=0.02$ ); immunodepression (OR $1.91,95 \% \mathrm{Cl} 1.41$ to 2.57 ,
$P<0.01$ ): Knaus score C-D (OR 2.16, 95\% Cl 1.64 to $2.84, P<0.01$ ): SOFA score (OR for an increase of 1 point $1.32,95 \% \mathrm{Cl} 1.26$ to $1.38, P<0.01$ ); and infection acquired in the ICU (OR $1.86,95 \% \mathrm{Cl} 1.03$ to $3.37, P=0.03)$. Protective factors were surgical admission (OR $0.61,95 \% \mathrm{Cl} 0.41$ to $0.89, P=0.01)$ and urinary tract infection (OR $0.55,95 \% \mathrm{Cl} 0.37$ to 0.82 , $P<0.01)$.

Conclusion Our findings are coherent with the literature. Multivariate analysis found nonmodifiable risk factors such as age, but also modifiable risk factors that warrant further investigation, such as infections acquired in-hospital or in the ICU. Future clinical studies in septic shock should take these findings into account when selecting patients.

Reference

1. Annane D: Am J Respir Crit Care Med 2003, 168:165.

P52

Severe sepsis in the United Sates: a 5-year analysis

J Knittel, S Quraishi

Massachusetts General Hospital, Boston, MA, USA

Critical Care 2012, 16(Suppl 1):P52 (doi: 10.1186/cc10659)

Introduction We describe patient-level healthcare data related to severe sepsis over a 5-year period (2004 to 2008) in the United States. Methods We queried the largest all-payer inpatient care database in the United States to identify cases of hospital admissions between 2004 and 2008 with a primary diagnosis of severe sepsis (ICD 9: 995.92). This retrospective analysis was performed with data from the Healthcare Cost and Utilization Project National Inpatient Sample (NIS) repository. Data related to length of stay, in-hospital mortality, and hospital charge was extracted. The 2004 and 2008 data for these variables were compared and further analyzed by age and sex in SPSS v.19 (IBM Corporation, Amonk, NY, USA). Results are reported with \pm standard error where applicable, and $P<0.05$ represented statistical significance. Results Our query of the NIS data revealed a similar number of hospital admissions with a primary diagnosis of severe sepsis in 2004 versus 2008. Sex (male vs. female) and age group composition (18 to 44 vs. 45 to 64 vs. 65 to 85 vs. $85+$ ) within these cohorts were similar. No significant change in overall length of stay or in-hospital mortality rate was appreciated. However, a significant increase in overall cost was appreciated $(\$ 67,670 \pm 5,742$ vs. $\$ 100,973 \pm 10,525 ; P=0.006)$, which outpaced healthcare-specific and general inflation during this period. Sex did not influence length of stay or in-hospital mortality rate. Cost of care was higher for males versus females (2004: $\$ 78,361 \pm 8,982$ vs. $\$ 57,040 \pm 5,959 ; P=0.048$ and 2008 : $\$ 111,298 \pm 13,835$ vs. $\$ 90,730 \pm 11,380 ; P<0.001)$. Age had a significant influence on inhospital mortality in 2004 and in 2008, with the highest percentage of in-hospital deaths in the $85+$ category. Age also had a significant influence on cost/day. Whereas in 2004 patients in the 85+ category represented the age subset with the lowest cost/day, in 2008 this age group witnessed a threefold increase in daily costs $(P<0.001)$ and represented the highest cost/day subset.

Conclusion Our data suggest that despite significant increases in healthcare costs attributable to severe sepsis, survival and length of stay has not improved significantly between 2004 and 2008. Dramatic increases in cost are particularly notable in males versus females and in patients who are 85 years old and over. Policies to control healthcare costs in the United States should focus on the root causes that lead to such significant increases in cost without appreciable societal returns on investment.

\section{P53}

District hospital experience of organ support requirements for H1N1-associated pneumonia

A Krige, S Chukkambotla

East Lancashire Hospitals NHS Trust, Blackburn, UK

Critical Care 2012, 16(Suppl 1):P53 (doi: 10.1186/cc10660)

Introduction The objective of our study was to describe the disease pattern, outcomes and organ support required in treating H1N1associated pneumonia in a single-centre, district hospital ICU. 
Methods All of the patients with confirmed $\mathrm{H} 1 \mathrm{~N} 1$ infection admitted to our ICU during the months of December 2010 and January 2011 were studied. The outcome measures were incidence, severity and support for organ dysfunction, length of stay in ICU and mortality.

Results During the study period 27 patients were admitted. The mean age was 46.6 years (SD 13.6) with 20 (74\%) patients being female, of whom two were pregnant. The mean APACHE scores were similar between survivors and nonsurvivors, 14.1 and 13.7 respectively. Twenty patients $(74 \%)$ required invasive mechanical ventilation with median duration of 9 days (range 2 to 54 days). Advanced techniques like prone position ventilation and high-frequency oscillatory ventilation were required in $20 \%$ and $10 \%$ of these patients respectively. Two patients were referred for ECMO. Ventilator-associated pneumonia (VAP) ensued in $25 \%$ of invasively ventilated patients resulting in an increase in ventilator days (median) from 9 to 19 and ICU stay (median) from 15 to 23 days. Four (15\%) required advanced cardiovascular support, 14 (52\%) developed acute kidney injury (AKI) of which nine (33\%) patients required renal replacement therapy. The ICU mortality was $11.1 \%$ and hospital mortality was $14.8 \%$. The cohort who developed AKI had $21 \%$ mortality. The median ICU stay (range) was 15 days ( 2 to 68 days).

Conclusion $\mathrm{H} 1 \mathrm{~N} 1$ pneumonia was associated with significant morbidity and mortality requiring advanced multiorgan support in the majority of patients. Although the incidence of organ dysfunction in our cohort mirrored that found in the Swift study [1], in keeping with advances in management of $\mathrm{H} 1 \mathrm{~N} 1$-associated critical illness the mortality was lower in the current study.

Reference

1. Rowan KM, et al:: The Swine Flu Triage (SwiFT) study: development and ongoing refinement of a triage tool to provide regular information to guide immediate policy and practice for the use of critical care services during the H1N1 swine influenza pandemic. Health Technol Assess 2010, 14:335-492.

P54

Compliance with the sepsis resuscitation bundle in patients with severe sepsis and septic shock admitted to Scottish ICUs

JA Davidson', K Dunne ${ }^{2}$

'Victoria Infirmary, Glasgow, UK; ${ }^{2}$ Forth Valley Royal Hospital, Larbert, UK Critical Care 2012, 16(Suppl 1):P54 (doi: 10.1186/cc10661)

Introduction Severe sepsis is the second leading cause for admission to critical care and in spite of advanced care remains associated with a high mortality. When implemented the sepsis resuscitation bundle has been associated with a $20 \%$ reduction in mortality and is therefore recommended as standard care for all patients with severe sepsis [1].

Methods All new admissions to seven west of Scotland ICUs were screened during a 12-week period for evidence of severe sepsis or septic shock. Those meeting the criteria were then assessed for sepsis bundle compliance. The Institute for Healthcare Improvement sepsis resuscitation bundle was taken as standard of care. This has a 6-hour time frame and includes measurement of serum lactate, blood cultures taken prior to antibiotics, antibiotics administered within 3 hours, fluids of $20 \mathrm{ml} / \mathrm{kg}$ if hypotensive or hyperlatataemia and use of early goal-directed therapy in the event of persistent hypotension/ hyperlatataemia in spite of fluid resuscitation.

Results Of the 652 patients screened, 115 met the definition of severe sepsis or septic shock (17.6\%). We collected full data from 108 patients, of which 69 patients $(63.8 \%$ ) had severe sepsis and 39 patients (36.1\%) had septic shock. Full bundle compliance was 5.6\%. Early ICU admission (within 6 hours) was associated with improved compliance with measured lactate $(87.3 \%$ vs. $60.4 \%, P<0.01)$, and where indicated, vasopressor use $(94.4 \%$ vs. $61.3 \%, P<0.01)$, CVP measurement $(77.5 \%$ vs. $44.4 \%, P<0.01)$, and $\mathrm{ScvO}_{2}$ measurement $(25.6 \%$ vs. $2.8 \%, P<0.01)$. ICU mortality was $12 / 64$ patients (18.8\%) with severe sepsis and $18 / 38$ patients $(47.4 \%)$ for those with septic shock. Full bundle compliance and mortality was not different for those reaching ICU early compared with those who were admitted after 6 hours.

Conclusion At present the sepsis resuscitation bundle is not uniformly implemented. Although compliance with early goal-directed therapy and lactate measurement is better in those reaching ICU early, there is still a large proportion of patients not receiving aspects of the bundle in spite of being in a critical care environment.

Reference

1. Dellinger RP, Levy MM, et al:: Surviving Sepsis Campaign: international guidelines for management of severe sepsis and septic shock. Intensive Care Med 2007, 34:17-60.

P55

Improving early administration of antibiotics: a 'Plan Do Study Act' approach

A Revill1', N Wennicke², J Tipping ${ }^{2}$, R Matull²

'Derriford Hospital, Plymouth, UK; '2Taunton Musgrove Park Hospital, Taunton, UK

Critical Care 2012, 16(Suppl 1):P55 (doi: 10.1186/cc10662)

Introduction Delayed administration of antibiotics is associated with an increased mortality in severe sepsis. The Surviving Sepsis Campaign advocates administering antibiotics to severely septic patients within 1 hour. Predicting the patients that will become severely septic is difficult, and therefore we have introduced a pathway via a unique care bundle to identify and treat all patients with suspected sepsis, prior to significant organ dysfunction, and maintain a 1-hour target.

Methods In September 2009, we introduced an audit proforma and management tool into the medical admissions unit of our hospital. This was accompanied by an extensive education programme of all medical and nursing staff. The proforma consists of two parts, a recognition and intervention section. The process is triggered when the patient satisfies two of the SIRS criteria and has symptoms consistent with an infection. All six management processes, including antibiotic administration, must then be completed within 1 hour of the trigger time. By using the 'Plan Do Study Act' cycle, we refined the proforma and streamlined the process and introduced it into emergency department and the surgical admissions unit. A dedicated multidisciplinary team was assigned to review and improve performance every 2 weeks by amending the form and processes.

Results Over a 24-month period we have a database with 1,571 patients. The results demonstrate that the median time to antibiotic administration is consistently near our target of 1 hour for all septic patients included in this pathway. Through continued refinement and staged introduction the proforma and the process has demonstrated consistency from medical to surgical wards; introduction in new areas has rapidly improved results. See Figure 1 overleaf.

Conclusion Our pathway has undergone a successful and dynamic development process guided by a multidisciplinary team. Compared with the usual audit process this has allowed rapid changes and improvements to take place and be tested. Further analysis of our database is ongoing, determining our impact on length of stay, mortality and intensive care admissions with a matched cohort.

P56

Source-directed antimicrobials: a shot in the dark?

L Richardson', GB McNeill', S Gupta

'University Hospital Southampton NHS Foundation Trust, Southampton, UK: ${ }^{2}$ Sheffield Teaching Hospitals NHS Foundation Trust, Sheffield, UK

Critical Care 2012, 16(Suppl 1):P56 (doi: 10.1186/cc10663)

Introduction The Surviving Sepsis Campaign advocates giving early empirical antibiotics directed against all likely pathogens [1]. The failure to instigate antimicrobials against a later confirmed pathogen impacts negatively on mortality [2]. Many hospitals advise sourcedirected therapy from the beginning. Our project aims to elicit the proportion where the source of sepsis is initially predicted incorrectly thereby putting patients at risk.

Methods A prospective cohort study was performed in two UK teaching hospitals of patients presenting with sepsis to critical care between May 2010 and March 2011. Hospital computer systems and patient notes were used to extract the initial suspected source of sepsis, and later verified with true microbiology data. Overall mortality was measured and compared between correctly and incorrectly suspected source of sepsis patients. 


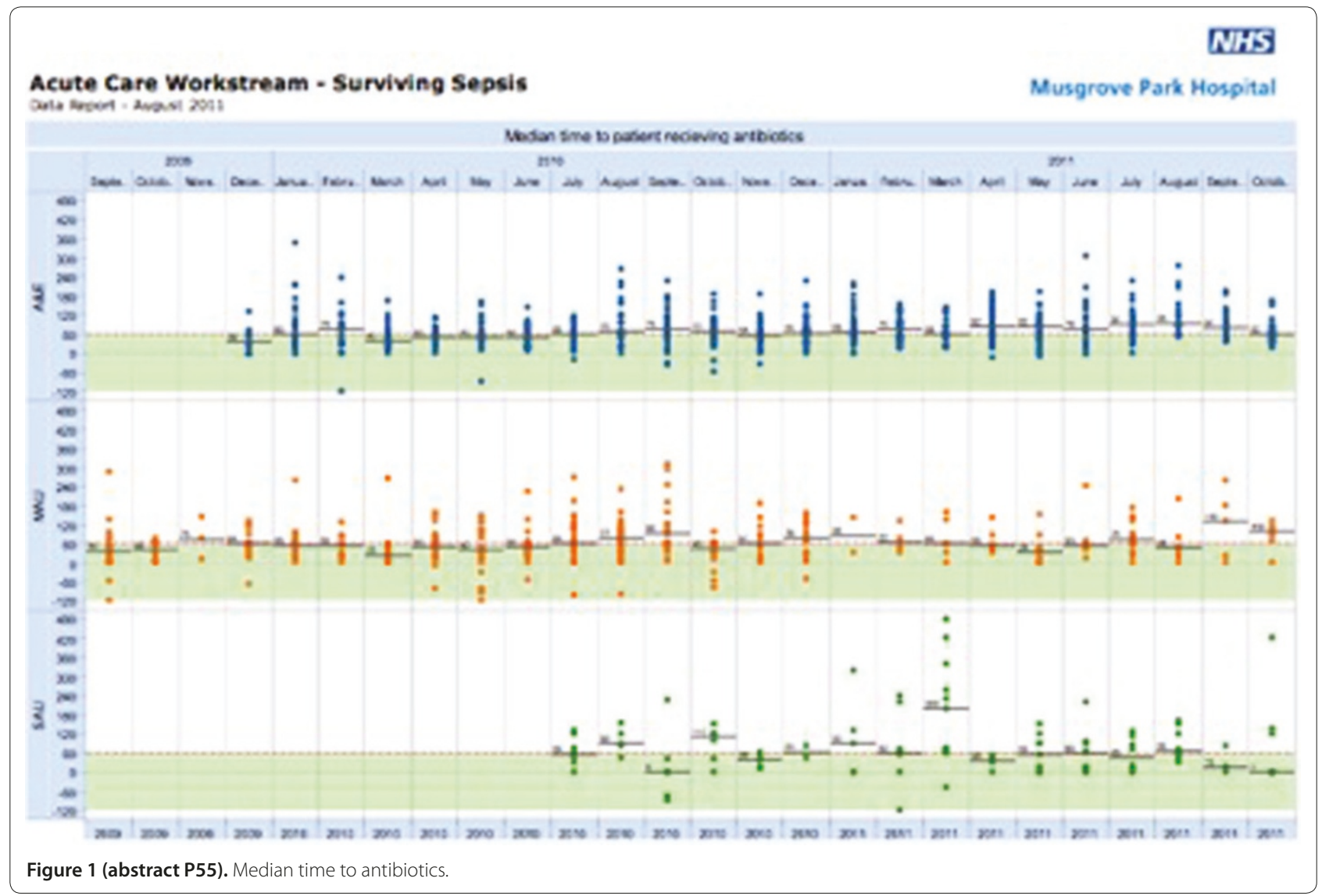

Results Of the 128 patients, the source of sepsis was wrongly identified in 30\% (38/128) (Southampton 28\% 15/53, Sheffield 31\% 23/75 respectively) (Figure 1). The most common source was the bowel, which was initially suspected as a respiratory source in most cases. Interestingly, the mortality was higher in the correctly identified group (13\%, 16/128 vs. $5 \%, 7 / 128)$. This probably reflects the severity of illness where the diagnosis is sometimes more obvious.

Conclusion Good antimicrobial governance requires early administration of narrow-spectrum antibiotics as best guess source-directed therapy from the outset, because de-escalation is often not practical. Our data reveal that in $30 \%$ of cases we incorrectly guess the source.

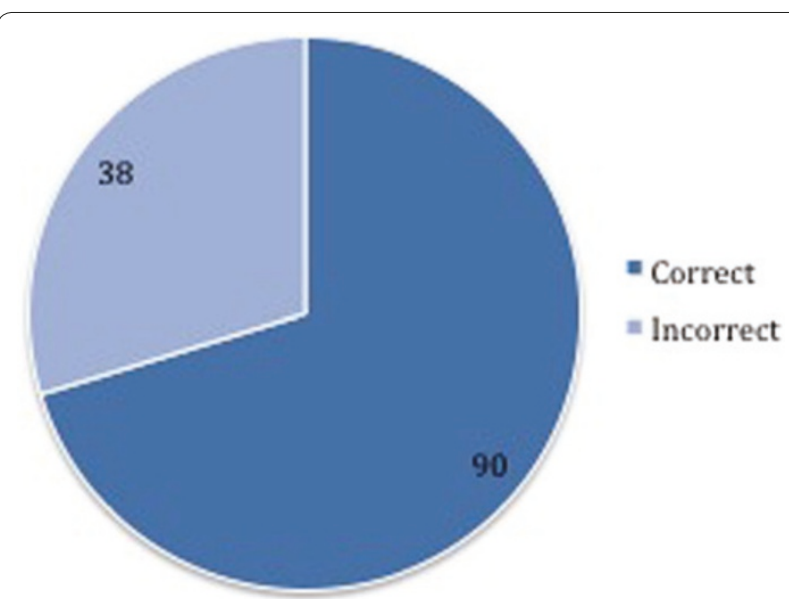

Figure 1 (abstract P56). Proportion of correctly and incorrectly identified sources.
We advise that in patients with severe sepsis or septic shock first-line antibiotics should remain broad spectrum with rigorous follow up to de-escalate as early as possible.

References

1. Dellinger RP, et al:: Surviving Sepsis Campaign: international guidelines for management of severe sepsis and septic shock. Crit Care Med 2008, 36:296-327.

2. Kumar A, et al:: Initiation of inappropriate antimicrobial therapy results in fivefold reduction of survival in human septic shock. Chest 2009, 136:1237-1248.

P57

Relation between temperature in the initial 24 hours in patients with severe sepsis or septic shock with mortality and length of stay in the ICU

R Sanga, S Zanotti, C Schorr, B Milcareck, K Hunter, P Dellinger, J Parrilo Cooper University Hospital, Camden, NJ, USA

Critical Care 2012, 16(Suppl 1):P57 (doi: 10.1186/cc10664)

Introduction Fever is a common event (ranges from 25 to $70 \%$ ) in patients admitted to the ICU. The usual clinical approach in most units is to treat the fever either with medications (acetaminophen, nonsteroid anti-inflammatory drugs) or external measures, like cooling blankets. No studies assessed clinical evidence for these interventions. There is otherwise evidence that fever may be beneficial, inducing heat shock proteins and decreasing NK-KB activation. Also treating fever can mask an important clinical sign and avoid early treatment in patients with severe sepsis.

Methods We did a case-control study using two available databases and collected 750 patients with the diagnosis of severe sepsis and septic shock. We collected age, sex, days on mechanical ventilation, APACHE II score, vasopressor use and correlated with the presence of hyperthermia $\left(>101.3^{\circ} \mathrm{F}\right)$, hypothermia $\left(<96.8^{\circ} \mathrm{F}\right)$ and normothermia 
in the initial 24 hours. We used a mean of the available temperature data. Then we used logistic regression (univariate and multivariate) to compare these temperatures with mortality and length of stay in the ICU.

Results Compared to patients with normal temperature the hyperthermic patients had a lower mortality $(22.58 \%$ vs. $39.1 \%)$ in the univariate analysis $(P<0.01)$. The patients with hypothermia had a mortality of $32.67 \%$ (NS). Length of stay was not significantly different between the groups. In the multivariate logistic regression the factors that were associated independently with mortality were age, APACHE II score, use of vasopressors, mechanical ventilation and temperature. Patients with $T>101.3^{\circ} \mathrm{F}$ were $59 \%$ less likely to die when compared with patients with normal temperature.

Conclusion The results of this study highlight the importance of investigating the real effects of fever in severe sepsis or septic shock. Is it necessary to treat when they are not causing harm to the patients? Are we delaying diagnosis of severe sepsis because of the lack of this important clinical sign? The next step should be a prospective trial of treatment versus no treatment of fever in the ICU.

\section{References}

1. Marik P: Fever in the ICU. Chest 2000, 117:885-869.

2. Levy M: Clinical review of fever in intensive care unit patients. Crit Care 2003, 7:221-225.

3. Dellinger P: Surviving Sepsis Campaign Guidelines. Crit Care Med 2008, 36:296-327

P58

Temperature management for patients without brain injury in Australia and New Zealand ICUs: a point prevalence study NE Hammond, M Saxena, P Young, C Taylor, I Seppelt, P Glass, J Myburgh George Institute for Global Health, Sydney, Australia Critical Care 2012, 16(Suppl 1):P58 (doi: 10.1186/cc10665)

Introduction Our primary aim was to determine the frequency of use of pharmacological and physical cooling strategies in ICU patients in current Australian and New Zealand (ANZ) practice. These patients had sepsis and inflammation but did not have neurological injury or recent surgery. We also aimed to establish current indications for use of antipyretics in these patients, as well as information on the prevalence of fever and the methods to measure temperature.

Methods This point prevalence study was conducted on 17 November and 15 December 2010 in 38 ICUs in ANZ. We identified a cohort of patients with sepsis and inflammation without neurological injury or recent surgery.

Results Of 506 patients surveyed on the point prevalence days, 311 were identified to have sepsis in the absence of neurological injury or recent surgery. These patients had peak temperature of $37.3^{\circ} \mathrm{C}$ (SD $\left.0.8^{\circ} \mathrm{C}\right) . \ln 32.2 \%(n=100 / 311)$ the peak temperature was above

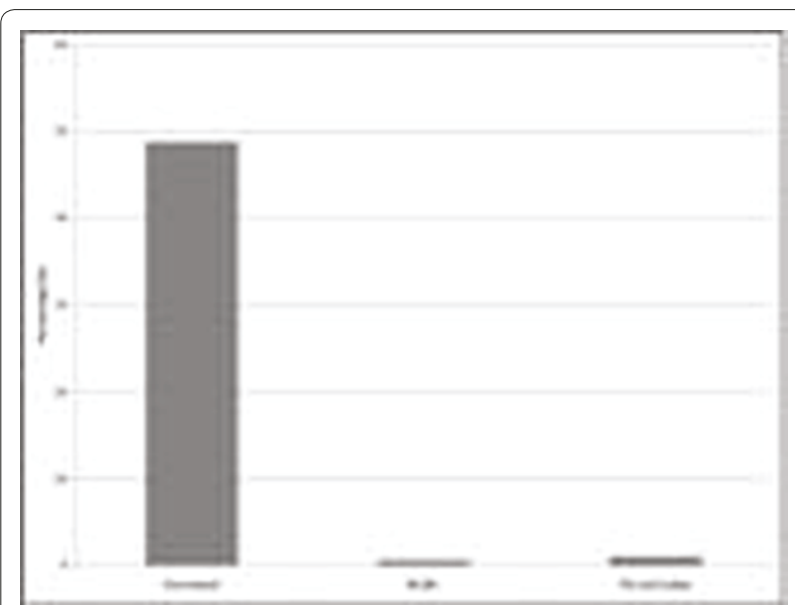

Figure 1 (abstract P58). Type of antipyretic and physical cooling used on the study day $(n=311)$.

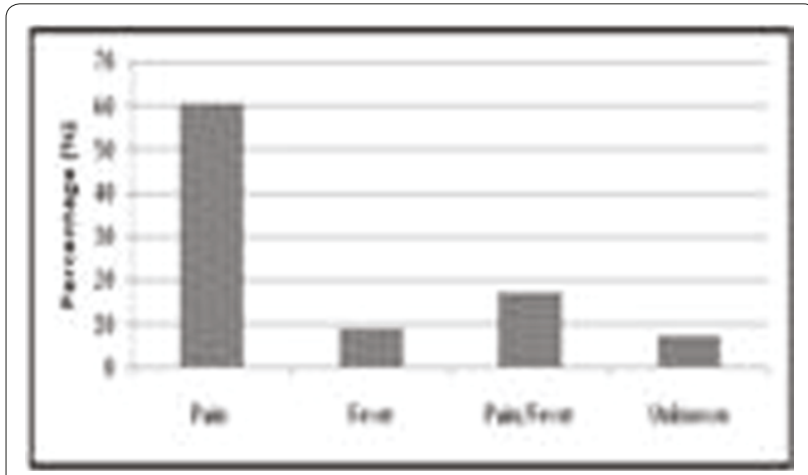

Figure 2 (abstract P58). Indication for paracetamol administration $(n=152)$.

$38^{\circ} \mathrm{C}$. Paracetamol was used in $152 / 311$ (48.8\%), nonsteroidal antiinflammatory drugs (NSAIDS) in 2/311 (0.6\%) and physical cooling in $3 / 311(1.0 \%)$ (Figure 1). Paracetamol was administered for pain in $92 / 152(60.5 \%)$ for both pain and fever in $26 / 152(17.1 \%)$; and for fever alone in 14/152 (10\%) (Figure 2). For the 40 patients who received paracetamol for an indication of fever, the peak recorded temperature was $38.3^{\circ} \mathrm{C}\left(\mathrm{SD} 0.8^{\circ} \mathrm{C}\right)$. The peak temperature for patients receiving physical cooling was $39.2^{\circ} \mathrm{C}\left(\mathrm{SD} 0.9^{\circ} \mathrm{C}\right)$. Temperature measurement were mainly noncore $(n=251 / 311)$ with axillary $(37 \% ; n=116 / 311)$ and tympanic $(35 \% ; n=110 / 311)$ the most common sites.

Conclusion Pharmacological antipyretics are used regularly for pain management rather than fever management, with paracetamol the most common antipyretic therapy. The use of NSAIDS and physical cooling was rare. Noncore temperature measurements were common.

\section{P59}

Impact of antifungal treatment in ICU patients with Candida colonization: analysis of the EPIC II study population

D Kett', G Dimopoulus², E Azoulay³, P Echeverria', C De La Cuesta', U Vincent ${ }^{4}$

University of Miami Miller School of Medicine, Miami, FL, USA; ${ }^{1}$ University Hospital ATTIKO Medical School, University of Athens, Greece; ${ }^{3}$ St-Louis Hospital and Paris VII University, Paris, France; ${ }^{4}$ Erasme University Hospital, Université Libre de Bruxelles, Belgium

Critical Care 2012, 16(Suppl 1):P59 (doi: 10.1186/cc10666)

Introduction We wished to evaluate the impact of receiving antifungal therapy in ICU patients with Candida colonization.

Methods EPIC II recruited 1,265 ICUs in 76 countries. Patient characteristics were collected on the study day. Outcome data were assessed at ICU and hospital discharge. Patients colonized with Candida spp. were classified as having received antifungal treatment or not ( $P P<0.05$ compared between groups). Numerical values are reported as mean \pm SD and length of stay (LOS) data as median (IQR).

Results A total of 13,796 adult patients were in participating ICUs on the study day. Of these, 371 were classified as colonized. Differences

Table 1 (abstract P59). Patients with Candida colonization: characteristics and outcomes

\begin{tabular}{lcc}
\hline & Therapy $(\boldsymbol{n}=\mathbf{1 8 4})$ & No therapy $(\boldsymbol{n}=\mathbf{1 7 5})$ \\
\hline SAPS II & $39 \pm 15$ & $41 \pm 18$ \\
SOFA & $7.6 \pm 4.1$ & $7.4 \pm 4.4$ \\
MV & $76 \%$ & $63 \%$ \\
Pressor & $36 \%$ & $32 \%$ \\
ICU mortality & $35 \%$ & $22 \%$ \\
Hospital mortality & $41 \%$ & $28 \%$ \\
\hline
\end{tabular}


in patient characteristics and outcomes are reported (Table 1). Baseline characteristics were similar in colonized patients treated with antifungal therapy compared to those that were untreated. Only a modest difference in the length of stay in the ICU prior to study day (25 $(14,40)$ vs. $21(8,43))$ and utilization of mechanical ventilatory support (76\% vs. $63 \%$ ) was noted in the treated compared to the untreated patients with Candida colonization $(P<0.05)$. Despite the relatively similar baseline characteristics and equivalent severity of illness scores, treated patients had an increased ICU (35.3 vs. $22.3 \%)$ and hospital (41.0 vs. $27.7 \%)$ mortality $(P<0.05)$.

Conclusion As colonized patients receiving antifungal treatment had significantly higher mortality, our data do not support the routine use of antifungal therapy in ICU patients based solely on colonization.

Reference

1. Vincent JL, et al:. JAMA 2009, 302:2323-2329.

P60

Pharmacokinetics of micafungin in patients with severe burn

injuries

J Sasaki', S Kishino², N Aikawa'1, S Hori'

'Keio University School of Medicine, Tokyo, Japan; 'Meiji Pharmaceutical

University, Tokyo, Japan

Critical Care 2012, 16(Suppl 1):P60 (doi: 10.1186/cc10667)

Introduction Micafungin (MCFG), an echinocandin antifungal agent, exhibits more potent antifungal activity against a broad spectrum of clinically important Candida and Aspergillus species [1]. Few studies have reported the pharmacokinetics (PK) of antifungal agents in patients with burn injuries. A purpose of this study is to characterize the PK of MCFG in severe burn patients.

Methods Eight severe burn patients within 14 days after injuries (M:F $=5: 3,19$ to 85 years old, 35 to $85 \%$ total body surface area) were treated with MCFG (200 to $300 \mathrm{mg}, 3.45$ to $4.49 \mathrm{mg} / \mathrm{kg}$ ) once daily by intravenous infusion over 1 hour. The MCFG concentrations in the plasma at the end of the initial administration of MCFG (P1), just before the second dosing (T1), at the end of the fourth dosing (P4), and just before the fifth dosing (T4) were determined, and were compared with the reported values in health volunteers [2]. MCFG concentrations in the burn eschar at $\mathrm{T} 1$ and $\mathrm{T} 4$ were also measured.

Results The plasma concentrations of MCFG per dose normalized with body weight (C/D) at P1, T1, P4, and T4 were 1.37 to $6.28,0.51$ to 1.38 , 3.20 to 6.46 , and 0.65 to $2.18(\mu \mathrm{g} / \mathrm{ml}) /(\mathrm{mg} / \mathrm{kg})$, respectively, indicating marked interindividual differences. These values were comparable with or slightly lower than the reported values in healthy volunteers (P1: 5.7, T1: 1.3, T4: 2.1). The MCFG concentrations in the burn eschar of three patients at T1 and T4 were $<0.1$ to 3.98 and 1.10 to $14.81 \mu \mathrm{g} /$ $\mathrm{ml}$, respectively. Most of MCFG concentrations in the plasma and burn eschar were higher than the reported MIC $C_{90}$ of MCFG against clinically important Candida and Aspergillus species. There was no correlation between the laboratory parameters of liver/kidney function and the plasma C/D of MCFG.

Conclusion The plasma concentrations of MCFG in patients with severe burn injuries were comparable with or slightly lower than the reported values in healthy volunteers. In addition, MCFG seems to be capable of penetrating burn eschar.

References

1. Aikawa N, et al.: I Infect Chemother 2009, 15:219-227.

2. Azuma J, et al:. Jpn J Chemother 2002, 50(Suppl 1):155-184.

P61

Bacteremia affects the mortality of septic patients with high serum procalcitonin level in the ICU

M Kamochi, K Nagata, Y Isa, S Nihei, N Harayama, K Aibara, T Sata

University Hospital of Occupational and Environmental Health, Kitakyushu, Japan

Critical Care 2012, 16(Suppl 1):P61 (doi: 10.1186/cc10668)

Introduction It is still controversial whether bacteremia affects the severity and the mortality of septic shock. Recent diagnostic criteria of septic shock do not include the presence of bacteremia, because rapid diagnosis and immediate treatment are necessary to improve the survival of septic patients. However, the presence of bacteremia seems to relate to the severity and mortality of septic shock patients in the ICU.

Methods The patients clinically suspected with sepsis were tested for serum procalcitonin level using a procalcitonin kit (BRAHMUS PCT Kit). The PCT test was performed 334 times from March 2008 to August 2010. Sixty-three adult patients showed high PCT level $(>10 \mathrm{ng} / \mathrm{ml})$. Thirty of $62(48 \%)$ patients showed bacteremia. Sixteen of these bacteremic patients were Gram-negative bacteremia and 14 patients were Grampositive bacteremia. The hemodynamic parameter, APACHE II score, SOFA score, serum lactate, some other laboratory data and mortality rate were compared between the patients with bacteremia and those without bacteremia. Statistical analyses were performed by chi-square test and Mann-Whitney $\mathrm{U}$ test.

Results The bacteremic patients with high serum PCT level showed significant higher APACHE II score, SOFA score and serum lactate concentration than nonbacteremic patients. The mortality rate of bacteremic patients was significantly higher than that of nonbacteremic patients $(66 \%$ vs. $28.1 \%, P<0.01)$. There were no differences in the severity and the mortality between Gram-negative and Gram-positive bacteremia.

Conclusion The presence of bacteremia relates to the severity and the mortality of septic patients with high serum PCT in the ICU.

\section{P62}

Cases of tetanus after the Japan crisis 2011

K Morino', M Kobayashi', Y Yamada ${ }^{3}$, S Yamanouchi', Y Tsujimoto',

KTakeda', S Sato', S Kimura', N Mita', M Sato', S Kushimoto ${ }^{4}$, S Endo ${ }^{3}$

'Yamagata Prefectural Medical Center for Emergency, Yamagata, Japan;

2Ishinomaki Red Cross Hospital, Ishinomaki, Japan; 3'Wate Medical University, Morioka, Japan; ${ }^{4}$ Tohoku University, Sendai, Japan

Critical Care 2012, 16(Suppl 1):P62 (doi: 10.1186/cc10669)

Introduction Tetanus is an infectious disease caused by tetanus neurotoxin produced by Clostridium tetani [1]. This bacterium resides in the soil extensively and about 100 people contract this disease annually in Japan. Tetanus is prevented by vaccines. A 1968 law required universal DPT vaccination against diphtheria, pertussis and tetanus in Japan. The survival rate with intensive care has reached more than $90 \%$ in recent years. Tetanus is said to have been found to increase in natural disasters [2]. So we will describe cases in the aftermath of the 2011 Tohoku earthquake and tsunami.

Methods We researched the case reports in a national database and a hospital database which could access patients' exact data. We made and analysed these case profiles.

Results We had nine tetanus cases in this crisis. This number was high compared with previous data. All patients lived in the Pacific coast of Tohoku districts and suffered from the tsunami. Geographically, seven patients were in Miyagi prefecture, and Iwate Prefecture had two cases. Of the nine cases, we could examine seven cases in detail. Mean age was 67 years, two were male cases and five women were injured on the day. Time to onset of symptoms such as trismus was an average of 12 days. The average was 3 days from symptom onset to medical consultation. All seven cases had some wounds, including minimal. Three had obvious wound infection. All patients had tetanus vaccine and tetanus immunoglobulin during their therapy but the time of injection was inconsistent because of the chaotic state. Four people were supported by mechanical ventilation with sedation and three out of four had tracheostomy. Three out of four with mechanical ventilation were treated with intravenous magnesium therapy to reduce spasticity. The average mechanical ventilation period was 23 days. We have no intravenous metronidazole preparation in Japan. No one had a reliable history of tetanus vaccine. No deaths were reported.

Conclusion We reported nine tetanus patients and investigated seven cases in detail. Older people had developed an unknown vaccination history. So we should have more opportunity to give vaccinations to older people and be careful with tetanus in disasters.

References

1. Farrar JJ, et al.: J Neurol Neurosurg Psychiatry 2000, 69:292-301

2. Aceh Epidemiology Group: Glob Public Health 2006, 1:173-177. 
P63

Does the day of the week predict the presence of microbiologically confirmed ventilator-associated pneumonia?

C Linssen, H Van Dessel, WVan Mook

Maastricht University Medical Centre, Maastricht, the Netherlands

Critical Care 2012, 16(Suppl 1):P63 (doi: 10.1186/cc10670)

Introduction At our hospital, ventilator-associated pneumonia (VAP) is diagnosed by microbiological and cytological analysis of bronchoalveolar lavage fluid (BALF). Opening hours of the in-house microbiological laboratory are between 8:00 am and 5:00 pm. During off-hours a laboratory technician is on call for urgent samples including BALF. The total laboratory work-up of the BALF takes 2 hours. The aim of the present study was to detect patterns in the submission time of BALF samples.

Methods During a 60-month period (January 2006 to December 2010), the day and hour of submission of all consecutive BALF samples obtained from patients suspected of VAP were recorded. VAP was microbiologically confirmed if quantitative cultures were $\geq 10^{4} \mathrm{cfu} / \mathrm{ml}$ and/or presence of $\geq 2 \%$ infected cells.

Results A total of 376 BALF samples were included. On weekdays, on average a total of $59.8 \pm 11.4$ were submitted, compared to 34 and 43 samples on Saturdays and Sundays. For more than one-half (203, $54 \%$ ) of the samples, the on-duty laboratory technician was required: $86(23 \%)$ samples arrived within 1 hour before closing time, and an additional 117 (31\%) were submitted thereafter. VAP was diagnosed in 149 (39.6\%) samples, of which $79(53 \%)$ after closing hours. BALF samples were obtained more frequently on Thursdays and Fridays (51 and 47 samples respectively) compared to Mondays and Tuesdays (64 and 76 samples). Interestingly, VAP was confirmed proportionally more frequently on Mondays and Tuesdays (26/51 (51\%) and 23/47 (49\%)) compared to Thursdays and Fridays (20/64 (31\%) and 26/76 (34\%)).

Conclusion The high number of BALFs processed after laboratory opening hours is of concern because of the suboptimal working conditions (fatigue, lack of supervision and experience). Technicians' time spent on these samples puts a strain on the laboratory in terms of costs and absence of the technicians because of legal recuperation. A higher number of confirmed episodes of VAP early in the week compared to just before the beginning of the weekend, combined with a larger number of BALF samples obtained on Thursdays and Fridays, may suggest that clinicians want to exclude VAP before the weekend resulting in a lower threshold for requesting a BALF.

P64

Assessing perforation of acute appendicitis using the delta neutrophil index reflecting the peripheral immature granulocyte count

NG Rhee, S Chung

Yonsei University College of Medicine, Seoul, South Korea

Critical Care 2012, 16(Suppl 1):P64 (doi: 10.1186/cc10671)

Introduction The delta neutrophil index corresponds to the calculated immature granulocyte counts and the severity of sepsis. This study investigated the diagnostic value of the delta neutrophil index as a preoperative laboratory marker for appendiceal perforation in patients with acute appendicitis.
Methods This study was a retrospective analysis of patients confirmed as appendicitis pathologically from November 2009 to September 2010 at two hospitals. The delta neutrophil index was automatically calculated as a subset of routine complete blood count test. The diagnostic performance of the delta neutrophil index for perforated appendicitis was evaluated.

Results During the study period, 308 patients were enrolled. Among them, 32 patients $(10.4 \%)$ were confirmed as perforated appendicitis. The delta neutrophil index was significantly higher in the perforated group than the nonperforated group $(4.8 \pm 7.1 \%$ vs. $2.0 \pm 2.0 \%$, $P<0.05)$. The sensitivity and specificity of the delta neutrophil index for predicting perforated appendicitis was $25.0 \%$ and $96.7 \%$ respectively at a cutoff level of $5 \%$ with an area under the curve of 0.78 on the ROC curve.

Conclusion This study suggested that the delta neutrophil index is associated with perforated appendicitis. However, the sensitivity was not high enough to use as a clinical guidance.

References

1. Nahm CH, Choi JW, Lee J: Delta neutrophil index in automated immature granulocyte counts for assessing disease severity of patients with sepsis. Ann Clin Lab Sci 2008, 38:241-246.

2. Andersson RE: Meta-analysis of the clinical and laboratory diagnosis of appendicitis. Br J Surg 2004, 91:28-37.

3. Oliak D, Yamini D, Udani VM, et al:: Can perforated appendicitis be diagnosed preoperatively based on admission factors? I Gastrointest Surg 2000, 4:470-474.

4. Ansari-Lari MA, Kickler TS, Borowitz MJ: Immature granulocyte measurement using the Sysmex XE-2100. Relationship to infection and sepsis. Am J Clin Pathol 2003, 120:795-799.

P65

Effects of a multifaceted quality improvement intervention in reducing mortality and bloodstream infection in ICUs: insights from the QUALITI initiative

AB Cavalcanti, JC Othero, JC Mouro, KN Silva, ES Victor, AA Kodama, O Berwanger, B Weber, LH Mota

Research Institute - Hospital do Coração, São Paulo, Brazil

Critical Care 2012, 16(Suppl 1):P65 (doi: 10.1186/cc10672)

Introduction Our objective was to evaluate if the implementation of a multifaceted intervention program for quality improvement in ICUs of nonacademic hospitals would decrease mortality and the catheterrelated bloodstream infection rate (CRBSI).

Methods A clinical practice improvement program involving 17 Brazilian ICUs of nonacademic public hospitals located far from major economic centers under coordination of a not-for-profit private hospital with the support of Brazilian Ministry of Health. We implemented the following interventions: (1) hospital visits to assess the facilities, human resources and processes; (2) workshop with hospital directors and ICU coordinators to elaborate improvement proposals based on the initial visit findings; (3) multidisciplinary videoconference lectures every 3 weeks about critical care medicine assistance and quality issues; (4) website containing project educational material, videoconference recordings, and an evidence-based practice course; (5) subscription of an electronic clinical information resource for all participating hospitals (UpToDate ${ }^{\oplus}$ ); (6) 3-day workshop to share the coordinating institution quality improvement practices with directors and ICU coordinators

Table 1 (abstract P65)

\begin{tabular}{|c|c|c|c|c|c|c|}
\hline \multirow[b]{2}{*}{ Outcome } & \multicolumn{4}{|c|}{ Month } & \multirow[b]{2}{*}{ Odds ratio $(95 \% \mathrm{Cl})$} & \multirow[b]{2}{*}{$P$ value } \\
\hline & June & July & August & September & & \\
\hline ICU death, $n(\%)$ & $100(36.0 \%)$ & $122(38.5 \%)$ & $137(34.9 \%)$ & $82(24.9 \%)$ & $0.83(0.75 ; 0.93)$ & 0.001 \\
\hline \multirow[t]{2}{*}{ In-hospital death, $n(\%)$} & $108(38.6 \%)$ & $125(43.0 \%)$ & $151(38.5 \%)$ & $96(29.2 \%)$ & $0.85(0.76 ; 0.94)$ & 0.002 \\
\hline & & & & \multicolumn{3}{|c|}{ Mean difference $(95 \% \mathrm{Cl})$} \\
\hline SMR, mean (SD) & $1.25(0.37)$ & $1.63(0.95)$ & $1.31(0.62)$ & $0.89(0.51)$ & $-0.15(-0.28 ;-0.01)$ & 0.04 \\
\hline CRBSI, mean (SD) & $11.8(19.7)$ & $4.3(11.9)$ & $4.4(5.4)$ & $3.3(6.0)$ & $-2.11(-4.68 ; 0.45)$ & 0.10 \\
\hline
\end{tabular}


from the 17 participant institutions; (7) 3-day nursing visits from the coordinating hospital to perform advice on care practice; (8) basic life support courses, 56 vacancies per hospital, and fundamentals of critical care support, 30 vacancies; and (9) implementation of a web-based system to collect ICU and hospital mortality, SAPS3, standardizedmortality ratio (SMR) and CRBSI after June 2011. We assessed variation of SMR and CRBSI on time using weighted linear regression, and variation of mortality on time using generalized-estimating equations. Results The results are presented in Table 1.

Conclusion A multifaceted intervention program applied to a network of ICUs in nonacademic public hospitals reduced mortality.

\section{P66}

Ceftazidime dosage regimen recommendations in burn patients based on a Monolix population pharmacokinetic study

S Ruiz', JM Conil', B Georges'1, F Ravat², T Seguin'1, P Letocart', O Fourcade1, S Saivin $^{3}$

${ }^{1}$ Hôpital Ranqueil CHU Toulouse, France; ${ }^{2}$ Centre hospitalier St Joseph et St Luc, Lyon, France; ${ }^{3}$ Institut Fédératif de Biologie CHU Toulouse, France Critical Care 2012, 16(Suppl 1):P66 (doi: 10.1186/cc10673)

Introduction The aim of our present work was to predict in burn patients the best adapted ceftazidime dosage regimen to obtain a serum target of 40 to $100 \mathrm{mg} / \mathrm{l}$ taking into account the influence of patients' characteristics on ceftazidime pharmacokinetics (PK).

Methods A Monolix population PK model was developed and validated in 70 burn patients with Pseudomonas aeruginosa infection. Monte Carlo simulations $(n=1,000)$ were performed to explore the appropriateness of different dosage regimens in burn patients. Target concentrations to achieve were defined as a 40 to $100 \mathrm{mg} / \mathrm{l} \mathrm{steady-}$ state concentration interval. The recommended dosage was chosen as the minimum dose providing the maximum of patients in this interval. Results A two-compartment model described ceftazidime disposition. Serum creatinine and age were identified as covariates of ceftazidime clearance. Age also influences the volume of distribution. The simulations showed that the common dosage regimens of $6 \mathrm{~g} /$ day did not allow achieving the desired target interval. This was achieved with continuous administration dosage regimens varying between 8 and $16 \mathrm{~g} /$ day in the youngest patients. Whatever the dosage regimen, the age and the serum creatinine, the mean highest percentage of patients reaching the 40 to $100 \mathrm{mg} / \mathrm{l}$ target interval was $76.43 \pm 2.13 \%$ (range: 65.1 to $80.1 \%$ ) (Table 1 ).

Table 1 (abstract P66). Recommended ceftazidime dosage regimen

\begin{tabular}{lcccccccc}
\hline \multicolumn{10}{c}{ Target $=\mathbf{4 0} \mathrm{mg} / \mathbf{l}$} \\
\hline $\begin{array}{l}\text { Creatinine } \\
(\boldsymbol{\mu m m o l} / \mathrm{l})\end{array}$ & $\mathbf{2 0}$ & $\mathbf{3 0}$ & $\mathbf{4 0}$ & $\mathbf{5 0}$ & $\mathbf{6 0}$ & $\mathbf{7 0}$ & $\mathbf{8 0}$ & $\mathbf{9 0}$ \\
\hline 30 & $16 \mathrm{~g}$ & $16 \mathrm{~g}$ & $16 \mathrm{~g}$ & $16 \mathrm{~g}$ & $14 \mathrm{~g}$ & $12 \mathrm{~g}$ & $12 \mathrm{~g}$ & $10 \mathrm{~g}$ \\
40 & $16 \mathrm{~g}$ & $16 \mathrm{~g}$ & $16 \mathrm{~g}$ & $14 \mathrm{~g}$ & $12 \mathrm{~g}$ & $12 \mathrm{~g}$ & $10 \mathrm{~g}$ & $10 \mathrm{~g}$ \\
50 & $16 \mathrm{~g}$ & $16 \mathrm{~g}$ & $16 \mathrm{~g}$ & $12 \mathrm{~g}$ & $12 \mathrm{~g}$ & $10 \mathrm{~g}$ & $10 \mathrm{~g}$ & $8 \mathrm{~g}$ \\
60 & $16 \mathrm{~g}$ & $16 \mathrm{~g}$ & $14 \mathrm{~g}$ & $12 \mathrm{~g}$ & $12 \mathrm{~g}$ & $10 \mathrm{~g}$ & $8 \mathrm{~g}$ & $8 \mathrm{~g}$ \\
70 & $16 \mathrm{~g}$ & $14 \mathrm{~g}$ & $12 \mathrm{~g}$ & $12 \mathrm{~g}$ & $10 \mathrm{~g}$ & $10 \mathrm{~g}$ & $8 \mathrm{~g}$ & $8 \mathrm{~g}$ \\
80 & $14 \mathrm{~g}$ & $14 \mathrm{~g}$ & $12 \mathrm{~g}$ & $10 \mathrm{~g}$ & $10 \mathrm{~g}$ & $8 \mathrm{~g}$ & $8 \mathrm{~g}$ & $8 \mathrm{~g}$ \\
90 & $14 \mathrm{~g}$ & $14 \mathrm{~g}$ & $12 \mathrm{~g}$ & $10 \mathrm{~g}$ & $10 \mathrm{~g}$ & $8 \mathrm{~g}$ & $8 \mathrm{~g}$ & $6 \mathrm{~g}$ \\
100 & $14 \mathrm{~g}$ & $12 \mathrm{~g}$ & $10 \mathrm{~g}$ & $10 \mathrm{~g}$ & $8 \mathrm{~g}$ & $8 \mathrm{~g}$ & $8 \mathrm{~g}$ & $6 \mathrm{~g}$ \\
120 & $12 \mathrm{~g}$ & $10 \mathrm{~g}$ & $10 \mathrm{~g}$ & $8 \mathrm{~g}$ & $8 \mathrm{~g}$ & $6 \mathrm{~g}$ & $6 \mathrm{~g}$ & $6 \mathrm{~g}$ \\
140 & $10 \mathrm{~g}$ & $10 \mathrm{~g}$ & $8 \mathrm{~g}$ & $8 \mathrm{~g}$ & $6 \mathrm{~g}$ & $6 \mathrm{~g}$ & $6 \mathrm{~g}$ & $4 \mathrm{~g}$ \\
160 & $10 \mathrm{~g}$ & $8 \mathrm{~g}$ & $8 \mathrm{~g}$ & $6 \mathrm{~g}$ & $6 \mathrm{~g}$ & $6 \mathrm{~g}$ & $4 \mathrm{~g}$ & $4 \mathrm{~g}$ \\
\hline
\end{tabular}

Recommended ceftazidime dosage regimen after a $2 \mathrm{~g}$ loading dose required to reach a steady-state concentration between 40 and $100 \mathrm{mg} / \mathrm{l}$ in the highest percentage of typical burn patients in function of serum creatinine and age.

Conclusion This study highlights the peculiarities of ceftazidime pharmacokinetics in burn patients with high interindividual variability.
Age and serum creatinine significantly influence the ceftazidime disposition. These covariates must be used to propose the first doses of ceftazidime. The required dosage regimens are higher than in other ICU patients and doses between 4 and $16 \mathrm{~g} /$ day are proposed.

\section{P67}

Continuous versus intermittent vancomycin in children after cardiac surgery with delayed sternal closure

P Skrak, L Hlinkova, L Kovacikova

National Institute of Cardiovascular Diseases, Bratislava, Slovakia

Critical Care 2012, 16(Suppl 1):P67 (doi: 10.1186/cc10674)

Introduction Delayed sternal closure (DSC) is a technique used in patients with hemodynamic instability, lung dysfunction, edema or prolonged bleeding after cardiac surgery. This group of patients has significant morbidity and mortality with fluid overload and changes in renal function. Adequate antibiotic coverage is of great importance and vancomycin is used as a part of antibiotic prophylaxis in our department. The objective of our study was to compare the efficacy and efficiency of intermittent and continuous vancomycin in pediatric cardiac patients with DSC.

Methods In a retrospective study we compared three groups of patients: patients with intermittent vancomycin (Intermittent group, $n=27$ ) with target trough level of 5 to $10 \mathrm{mg} / \mathrm{l}$, patients with continuous vancomycin (CV1 group, $n=24$ ) with target trough level of 20 to $25 \mathrm{mg} / \mathrm{l}$ and patients with continuous vancomycin (CV2 group, $n=20$ ) with target trough level of 15 to $20 \mathrm{mg} / \mathrm{l}$. The demographic data, total and average vancomycin doses, target level achievement and side effects were analyzed.

Results There was no difference in age, weight, surgical complexity and mortality between the groups. The average vancomycin daily dose $(\mathrm{mg} / \mathrm{kg})$ was the same in the Intermittent and CV2 groups, the dose was twofold higher in CV1 group $(P<0.001)$ (Table 1$)$. The CV2 group has less trough samples per day of treatment than the CV1 and intermittent groups $(P=0.015)$. Target levels were reached in $42.4 \%$, $30.9 \%$, and $42.9 \%$ samples in Intermittent, CV1 and CV2 groups, respectively $(P<0.001)$. Below target were $9.8 \%, 38.5 \%$ and $16.7 \%$ samples in Intermittent, CV1 and CV2 groups, respectively. There was no deep sternal infection in any patient. There was similar incidence of peritoneal dialysis in all three groups. No case of renal insufficiency was directly related to increased trough vancomycin level.

Table 1 (abstract P67)

\begin{tabular}{lccc}
\hline & Intermittent group & CV1 group & CV2 group \\
\hline TLC $(n)$ & 272 & 250 & 233 \\
TLC per day $(n)$ & $1(0.6$ to 2.3$)$ & $0.92(0.5$ to 1.5$)$ & $0.81(0.62$ to 1.8$)$ \\
Daily dose & $15(7.6$ to 45$)$ & $26.2(7.6$ to 54.3$)$ & $15.7(5.9$ to 37.3$)$ \\
\hline
\end{tabular}

$\mathrm{CV}$, continuous vancomycin; TLC, trough level count.

Conclusion In children after cardiac surgery with DSC both intermittent vancomycin with trough level of 5 to $10 \mathrm{mg} / \mathrm{l}$ and continuous vancomycin with trough level of 15 to $20 \mathrm{mg} / \mathrm{l}$ were comparable with regard to administered dose and target values achievement. There was significantly higher daily dose and trough sample count below target values in patients with continuous vancomycin and target of 20 to $25 \mathrm{mg} / \mathrm{l}$.

P68

Elimination of linezolid in patients undergoing low-flow continuous venovenous haemodiafiltration

T Ide, N Hori, Y Ikeda, KTakeda, S Nishi

Hyogo College of Medicine, Nishinomiya City, Japan

Critical Care 2012, 16(Suppl 1):P68 (doi: 10.1186/cc10675)

Introduction It has been reported that linezolid (LZD) is highly removed in patients undergoing high-flow continuous venovenous haemofiltration (CVVH: blood flow and filtration rates were $186 \pm 15$ and $40 \pm 8 \mathrm{ml} /$ minute) compared with patients with normal renal 
function (NRF). It is generally considered that no adjustment of LZD dosage is needed in subjects undergoing CVVH. In Japan, continuous venovenous haemodiafiltration (CVVHDF) has preferentially been administered under low flow rate. Investigating the effects of flow rate on LZD removal during continuous renal replacement therapy is essential to regulate therapeutic dosages. We aimed to investigate the pharmacokinetics of LZD in CVVHDF patients in this setting.

Methods LZD $(600 \mathrm{mg})$ was administered intravenously every 12 hours in ICU patients on CVVHDF and NRF patients (creatinine clearance $50 \mathrm{ml} /$ minute). Blood and filtrate samples were collected at $0,1,1.5$, 2,3 and 5 hours after infusion from both groups. The elimination halflife $\left(T_{-1 / 2}\right)$, maximum concentration, concentration time curve (AUC), volume distribution $\left(\mathrm{V}_{\mathrm{d}}\right)$, clearance $(\mathrm{CL})$ and sieving coefficient $(\mathrm{Sc})$ were evaluated. Patient characteristics and CVVHDF parameters including the filter type, dialysate and filtration flow rates were recorded.

Results Fourteen CVVHDF patients and nine NRF patients were included into the study. CVVHDF was performed using polysulfone and triacetate membranes. Mean blood, dialysate and filtration flow rates were $79.3 \pm 2.7 \mathrm{ml} /$ minute, $8.7 \pm 5.1 \mathrm{ml} /$ minute and $5.5 \pm 2.5$ $\mathrm{ml} /$ minute, respectively. Sc was $0.86 \pm 0.03$. $\mathrm{T}_{-1 / 2}$ data ( $8.78 \pm 3.74 \mathrm{vs}$. $5.54 \pm 3.27$ hours, $P=0.05$ ) were significantly longer in the CVVHDF compared with the NRF group, AUC data $(247.9 \pm 107.8$ vs. $136.0 \pm 84.9$ $\mathrm{g}$ hour $/ \mathrm{ml}, P=0.02$ ) were significantly higher and $\mathrm{CL}(2.94 \pm 1.38 \mathrm{vs}$. $5.92 \pm 2.97 \mathrm{l} /$ hour, $P=0.004)$ and $V_{d}(31.0 \pm 3.8$ vs. $35.8 \pm 3.3 \mathrm{I}, P=0.01)$ data were significantly lower. LZD clearance was not correlated with the type of membrane used (polysulfone vs. triacetate: $2.8 \pm 1.5$ vs. $3.6 \pm 1.2 \mathrm{l} /$ hour, $P=0.39$ ).

Conclusion Clearance of LZD in patients undergoing CVVHDF was significantly lower than in patients with normal renal function. Pharmacokinetic data from CVVHDF patients demonstrated that flow rates significantly influenced the efficiency of LZD removal. The maintenance dose of LZD may need to be reduced in patients undergoing CVVHDF under reduced flow conditions.

Reference

1. Mever B, et al.: J Antimicrob Chemother 2005, 56:172-179.

P69

A post-authorisation survey to analyse the perioperative teicoplanin plasma concentrations in adult patients with chronic bone sepsis, who received loading doses of $12 \mathrm{mg} / \mathrm{kg}$ 12-hourly for 48 hours followed by $12 \mathrm{mg} / \mathrm{kg}$ once daily

AJ Brink', G Richards², C Lautenbach'1, N Rapeport 1 , V Schillack ${ }^{3}$, J Roberts', J Lipman ${ }^{4}$

'Milpark Hospital, Johannesburg, South Africa; ${ }^{2}$ University of Witwatersrand,

Johannesburg, South Africa; ${ }^{3}$ Ampath National Referral Laboratory, Pretoria,

South Africa; ${ }^{4}$ University of Queensland, Brisbane, Australia

Critical Care 2012, 16(Suppl 1):P69 (doi: 10.1186/cc10676)

Introduction To rapidly achieve teicoplanin trough $\left(\mathrm{C}_{\text {min }}\right)$ concentrations $\geq 20 \mathrm{mg} / \mathrm{l}$ suggested for sternal sepsis, loading doses higher than $6 \mathrm{mg} /$ kg 12-hourly might be warranted [1].

Methods Patients ( $n=10$ ) with deep-seated Gram-positive infections were enrolled perioperatively. During the first 4 days of therapy teicoplanin loading doses of $12 \mathrm{mg} / \mathrm{kg} 12$-hourly were administered for 48 hours and $12 \mathrm{mg} / \mathrm{kg}$ once daily thereafter. Surgical debridement was performed on D3. Samples were collected 15 minutes before and 30 minutes and 120 minutes after each teicoplanin administration. Total and unbound teicoplanin levels were determined using HPLC.

Results All patients had hypoalbuminemia (mean $20.2 \mathrm{~g} / \mathrm{l}$ ). The SS PK parameters of teicoplanin are described in Table 1. On D3 the median total and free $C_{\min }$ were 14.66 (8.93 to 19.66) and 3.09 (0.0 to 6.4) $\mathrm{mg} / \mathrm{l}$, respectively. In a multivariate logistic regression model, total teicoplanin concentrations $(P=0.174)$ and serum creatinine concentration $(P=0.034)$ did not impact significantly on free teicoplanin levels whereas, in contrast, albumin concentration did (OR $0.120,95 \% \mathrm{Cl} 0.078$ to $0.161, P<0.001$ ).

Conclusion The levels achieved on D3 in this study are similar to those achieved by Mimoz and colleagues using the same dosing schedule in ICU patients with VAP [2]. Only hypoalbuminemia impacted on the free levels of teicoplanin in this setting. High teicoplanin loading doses of $12 \mathrm{mg} / \mathrm{kg} 12$-hourly should probably be extended beyond 48 hours, before major elective surgery for chronic bone sepsis.
Table 1 (abstract P69). Steady-state pharmacokinetic parameters of teicoplanin

\begin{tabular}{lcc}
\hline & Total & Free \\
\hline$C_{\max }$ & 20.1 & 2.6 \\
$C_{\min }$ & 6.7 & 2.3 \\
$\mathrm{AUC}$ & 137.9 & 28.6 \\
$\mathrm{CL}$ & 7.0 & 33.5 \\
$\mathrm{Vz}$ & 174.1 & 196.6 \\
\hline
\end{tabular}

\section{References}

1. Brink et al.: Int J Antimicrob Agents 2008, 32:455-458.

2. Mimoz et al.: Intensive Care Med 2006, 32:775-779.

P70

Pharmacokinetics of inhaled colistin in critically ill patients with ventilator-associated tracheobronchitis

Z Athanassa' ${ }^{1}$, M Fousteri², S Markantonis², P Myrianthefs³ ${ }^{3}$ E Boutzouka3 ETsigou ${ }^{3}$, A Tsakris 4 , G Baltopoulos ${ }^{3}$

'Hygeia Hospital, Marousi, Greece; ${ }^{2}$ Faculty of Pharmacy, University of Athens, Greece; ${ }^{3}$ Faculty of Nursing, University of Athens, Greece; ${ }^{4}$ Faculty of Medicine, University of Athens, Greece

Critical Care 2012, 16(Suppl 1):P70 (doi: 10.1186/cc10677)

Introduction Although inhaled colistin is frequently used in ventilatorassociated pneumonia (VAP), data regarding its pharmacokinetic properties are scarce [1-3]. The aim of this study was to describe colistin pharmacokinetics in critically ill patients after administration of a single dose of 1 million units of colistimethate sodium (CMS) via nebulization. Methods Patients with ventilator-associated tracheobronchitis dye to polymyxin-only susceptible Gram-negative bacteria were included in the study; patients receiving intravenous and/or nebulized colistin were excluded. CMS was administered at a dose of 1 million units every 8 hours for 7 days, via a vibrating-mesh nebulizer. Mini bronchoalveolar lavage was collected before and at 1, 4 and 8 hours post nebulization, while blood samples were collected before and at $0.16,0.5,1,2,4$, and 8 hours post nebulization. Colistin concentrations in epithelial lining fluid (ELF) and plasma were determined by high-performance liquid chromatography.

Results Our study population included five patients (three female) with mean age 60.6 years. Median (range) colistin concentrations in ELF were 6.9 (6.2 to 13.9), 3.7 (2.7 to 11.6$)$ and 2.1 (1.2 to 8.7$) \mathrm{g} / \mathrm{ml}$ at 1,4 , and 8 hours, respectively, after nebulization. Colistin concentrations in serum were substantially lower than those observed in ELF with peak median (range) values 1.56 (1.19 to 2$) \mathrm{g} / \mathrm{ml}$. The estimated colistin mean half-life was 3.4 hours.

Conclusion Administration of 1 million units of inhaled CMS resulted in high colistin concentrations in the ELF; moreover, concentrations were maintained for up to 8 hours in the majority of patients. This finding might support the use of inhaled CMS for the treatment of patients with VAP due to multidrug-resistant Gram-negative bacteria. Moreover, the low serum concentrations and the short half-life suggest that administration of inhaled colistin may be associated with less systemic toxicity.

References

1. Ratjen F, et al:. I Antimicrob Chemother 2006, 57:306-311.

2. Marchand S, et al:: Antimicrob Agents Chemother 2010, 54:3702-3707.

3. Lu Q, et al:: Intensive Care Med 2010, 36:1147-1155.

P71

Efficacy of inhaled tobramycin in severe nosocomial pneumonia A Kuzovlev', S Polovnikov², V Stec ${ }^{2}$, V Varvarin 2

'V.A. Negovsky Scientific Research Institute of General Reanimatology RAMS, Moscow, Russia; ${ }^{2}$ N.N. Burdenko Main Clinical Military Hospital, Moscow, Russia Critical Care 2012, 16(Suppl 1):P71 (doi: 10.1186/cc10678)

Introduction Nosocomial pneumonia (NP) is one of the most prevalent complications in ICUs. The efficacy of inhaled antibiotics in treatment of NP was shown in several research works. The aim of this study was 
to estimate the efficacy of inhaled tobramycin (IT) as an adjunct to systemic antibiotics in the treatment of severe NP.

Methods Twenty ICU patients with NP were enrolled in the study (all male, $49 \pm 7.3$ years old); primary reason for ICU stay - intraabdominal infections $(60 \%)$, mediastinitis $(10 \%)$, others $(30 \%)$. Diagnosis of NP was made according to standard clinical and CPIS criteria. Associations of multiresistant Gram-negative bacteria were detected in bronchoalveolar lavage (BAL) of all patients. Eighty percent of bacteria were sensitive to tobramycin. Patients were randomized into two groups - 'IT' (group 1, $n=10$ ) + systemic antibiotics (carbapenems, aminoglycosides, protected penicillins); 'no IT' (group $2, n=10$ ), only systemic antibiotics, same as in group 1. Groups were comparable in APACHE II and CPIS scores. IT (Bramitob) was administered $300 \mathrm{mg}$ BID via nebulizer.

Results Duration of IT use in group 1 was $7.5 \pm 2.5$ days. There were no statistically reliable differences between groups detected due to the small number of patients enrolled. But it was clinically detected that treatment with IT in group1 was associated with a decrease of SIRS signs and CPIS scores and an increase of oxygenation index in $70 \%$ of patients. Positive dynamics in chest X-ray and computed tomography was detected in two patients of group 1 (20\%; no dynamics in group 2). The titre of microbes in BAL decreased (100\%) and their sensitivity to other groups of antibiotics, which they were previously resistant to, increased (40\%) in group 1 patients after IT administration. Efficacy of IT in patients with a registered resistance of microbes to tobramycin can be explained by a high local concentrations of tobramycin in lungs. The mortality in groups was similar $(40 \%$ and $40 \%)$ and not related to a progression of NP. Two patients of group 1 (20\%) presented with hearing loss and tinnitus which revealed 3 months after the last IT administration. There were no cases of bronchospasm or renal insufficiency in group 1.

Conclusion Administration of IT as an adjunct to systemic antibiotics is efficient and safe in treatment of severe nosocomial pneumonias caused by multiresistant Gram-negative bacteria. Profound randomized clinical trials on IT are required.

Reference

1. Polovnikov SG, Kuzovlev AN, lliychev AN: Case report of a successful treatment of severe nosocomial pneumonia with inhaled tobramycin. Pulmonologia 2011, 2:109-112.

P72

Comparison of a bronchoscopic microsample probe with bronchoalveolar lavage to measure cytokine levels in critically ill patients

V Grover, LE Christie, P Charles, P Kelleher, P Shah, S Singh

Chelsea and Westminster Hospital, London, UK

Critical Care 2012, 16(Suppl 1):P72 (doi: 10.1186/cc10679)

Introduction The use of bronchoalveolar lavage (BAL) to investigate inflammatory lung disease in the critically ill may not be tolerated in hypoxic patients. Furthermore, soluble protein analysis of BAL fluid suffers from inaccuracies related to saline dilution. The bronchoscopic microsample (BMS) probe allows absolute cytokine levels in epithelial lining fluid (ELF) to be measured directly without lavage [1]. We compared cytokine levels from ELF obtained by the BMS probe with those from BAL, to verify its utility in critical illness.

Methods We recruited 45 patients into five groups in whom BMS and BAL were conducted sequentially: two ventilated with ALI/ARDS, six with burns inhalational injury (five ventilated), 15 with COPD, 18 with interstitial lung disease and four healthy patients. The BMS probe was bronchoscopically inserted to the subsegmental level in order to contact the mucosa for 5 to 7 seconds, collecting approximately $20 \mu \mathrm{l}$ ELF [1]. BAL was performed with $150 \mathrm{ml}$ of $0.9 \%$ saline, discarding the first $20 \mathrm{ml}$ (bronchiolar fraction). We assayed IL-1, IL-6, IL-8, TNFa and G-CSF. Comparisons between paired cytokine ELF concentrations in BMS and BAL were analysed using the nonparametric Wilcoxon's test and Spearman's correlation coefficient.

Results The critically ill patients were aged 18 to 84 years (APACHE II 12 to 21). One patient had ARDS due to urinary tract infection and another related to pneumonia. No adverse incidents noted were noted. Overall, cytokine levels were all higher in the BMS group than
BAL $(P<0.0001)$, consistent with ELF dilution by saline lavage. The ratio of BMS-derived cytokine to BAL for each patient group did not differ significantly. Spearman coefficients $(r)$ for IL-1, IL-6, IL-8, TNFa and G-CSF were $0.38,0.52,0.25,0.38$ and 0.40 . All correlations were significant $(P<0.01)$ except for IL-8 $(P=0.05)$. Both sampling methods demonstrated a gradation of cytokine level, with burns and ALI/ARDS having significantly higher levels than patients with stable chronic lung disease or healthy controls.

Conclusion The BMS probe was well tolerated and provided cytokine data comparable to that obtained by BAL in acute and chronic respiratory diseases. The BMS probe may have utility as a biomarker sampling modality in patients where clinicians have concerns over conventional BAL.

Acknowledgements The BMS probes were provided by Olympus (Tokyo, Japan).

Reference

1. Ishizaka A, et al.: Crit Care Med 2001, 29:896-898.

\section{P73}

Clinical and epidemiological risk factors for ventilator-associated pneumonia in a cohort of critically ill patients

G De Pascale, MA Pennisi, V Raggi, E Piervincenzi, V Bernini, A Occhionero, P De Santis, A Moccaldo, S Cicconi, R Maviglia, M Tumbarello, M Antonelli Sacro Cuore Catholic University, Rome, Italy

Critical Care 2012, 16(Suppl 1):P73 (doi: 10.1186/cc10680)

Introduction Ventilator-associated pneumonia (VAP) represents a major infectious complication in the ICU. The aim of this study is to identify risk factors for VAP acquisition.

Methods All patients admitted to the 18-bed ICU of our university hospital between 1 October 2009 and 31 December 2010 were enrolled on the day of VAP diagnosis. Controls were selected by our computerized database. Statistical analyses were performed using the StatalCI I program.

Results Over the study period, among 902 admissions, 100VAP occurred. The rate of multidrug resistance (MDR) was $23 \%$. Development of VAP was associated with a significantly longer duration of ICU stay (24 days (17 to 30 ) vs. 7 days (5 to 9); $P<0.001$ ) and mechanical ventilation ( 19 days ( 13 to 20 ) vs. 4 days ( 3 to 6$) ; P<0.001$ ). Overall ICU mortality was higher in the VAP population $(41 \%$ vs. $29 \% ; P=0.09)$. Comparing patients affected by VAP with controls (100 matched patients), the former group was significantly more likely to be male $(P<0.001)$ and to be immunosuppressed $(P=0.004)$. In addition, VAP development was associated with higher rate of central venous catheter placements $(P<0.001)$, higher mean SOFA score value $(P<0.001)$ and previous exposure to antimicrobials $(P=0.004)$. Successful use of noninvasive ventilation, and trauma admission appeared as protective factors $(P<0.001)$. Table 1 shows independent risk factors associated with VAP acquisition in multivariate analysis.

Table 1 (abstract P73)

\begin{tabular}{lcc}
\hline & $P$ value & OR (95\% Cl) \\
\hline NIV success & 0.005 & $0.1(0.01$ to 0.4$)$ \\
SOFA score* & 0.01 & $1.2(1$ to 1.3$)$ \\
Male gender & $<0.001$ & $14(5$ to 39.4$)$ \\
Immunosuppressive status & 0.001 & $4(1.7$ to 9.6$)$ \\
\hline
\end{tabular}

*Mean value.

Conclusion VAP occurrence seems to be associated with increased morbidity and ICU mortality. NIV use, avoiding endotracheal intubation and invasive mechanical ventilation, has appeared to be effective in reducing the rate of VAP episodes, particularly in high-risk patients (severe immunosuppressed). The application of behavioural intervention bundles might represent the suitable preventive measure in settings where high rates of MDR pathogens limit the extensive use of pharmacological ones.

Reference

1. Vincent JL, et al:: Drugs 2010, 70:1927-1944. 
P74

Use of a ventilator-associated pneumonia (VAP) bundle to decrease the VAP rate in Syria

R Alsadat ${ }^{1}$, M Mazloum², A Alshamaa³ , A Dakkak², H Al-Bardan', M Eltayeb², A Marie $^{2}$, F Esber', O Naes ${ }^{3}$, M Shama ${ }^{5}$, I Betelmal', M Kherallah ${ }^{6}$

'Al-Mouassat Hospital, Damascus, Syria; ${ }^{2}$ General Assembly of Damascus

Hospital, Damascus, Syria; ${ }^{3} \mathrm{Al}$-Bassel Heart Institute, Damascus, Syria; ${ }^{4}$ lbn

Alnafees Hospital, Damascus, Syria; ${ }^{5}$ World Health Organization, Damascus,

Syria; ${ }^{6}$ King Faisal Specialist Hospital and Research Center, Riyadh, Saudi

Arabia

Critical Care 2012, 16(Suppl 1):P74 (doi: 10.1186/cc10681)

Introduction Implementation of a ventilator-associated pneumonia (VAP) bundle as a performance improvement project in the critical care units for all mechanically ventilated patients aiming to decrease the VAP rates over the study period at four major teaching hospitals in Damascus.

Methods CDC criteria were used to define VAP. VAP rates were calculated based on occurrences per 1,000 ventilator days, VAP rates were monitored on a monthly basis throughout the project period. VAP bundle elements included elevation of the head of the bed to between 30 and $45^{\circ}$, daily sedation vacation, daily assessment of readiness to wean, peptic ulcer disease prophylaxis and deep venous thrombosis prophylaxis if not contraindicated. Each hospital formed a task force with a team leader, one or two physicians and one or two nurses. Education took place at an initial conference and a follow-up meeting for the implementation process and frequent staff education session in individual units. Compliance with the VAP bundle was considered based on the implementation of all elements of the bundle. Statistical Control Chart (SPC) was used to monitor the compliance with the individual bundle elements as well the bundle as a whole.

Results VAP bundle compliance rates were steadily increasing from 33 to $80 \%$ in Hospital 1, from 33 to $86 \%$ in Hospital 2 and from 83 to $100 \%$ in Hospital 3 during the study period. The VAP bundle was not applied in Hospital 4 and therefore no data were available. This correlated with a decrease in VAP rates from 30 to 6.4 per 1,000 ventilator days in Hospital 1 , from 12 to 4.9 per 1,000 ventilator days in Hospital 3, whereas the VAP rate failed to decrease in Hospital 2 (despite better compliance) and it remained high around 33 per 1,000 ventilator days in Hospital 4 where the VAP bundle was not implemented or monitored.

Conclusion The VAP bundle is known to be an effective way to decrease VAP but has performed differently in different hospitals in our study. Prevention of VAP requires concerted efforts on the part of hospital administration, physicians, and ICU personnel. The program must be evidence-based, maintained, and accepted by ICU personnel. Monitoring and collection of data should be strict and objective. Continued education and feedback are crucial to maintain a low VAP rate. Other factors of healthcare infection prevention should also be taken into consideration.

P75

A strategy for prevention and control of catheter-related bloodstream infection of ICU patients in China (Prevent CRBSI): a prospective, multicenter, controlled study

GCai, J Yan

Zhejiang Hospital, Hangzhou, China

Critical Care 2012, 16(Suppl 1):P75 (doi: 10.1186/cc10682)

Introduction Catheter-related bloodstream infection (CRBSI) continues to be a key issue in ICUs despite recent improvements in the clinical technique, standardization of the CVC insertion protocol and hand hygiene. The impact of catheter maintenance on CRBSI rates in China needs to be further investigated. The objective of study is to evaluate a bundle of interventions for reducing CRBSI in ICUs. The bundle includes new technology (BD Q-Syte ${ }^{\mathrm{TM}}$ and BD Posiflush ${ }^{\mathrm{TM}}$ ) in addition to updated standards of practice for catheter maintenance.

Methods This is a prospective, multicenter, controlled study. Patients receiving CVCs in the ICUs were eligible for inclusion. The study was performed in seven general and teaching hospitals from June 2010 to June 2011 in China. The clinicians conducted their original catheter maintenance standards in the baseline period (Phase 1). The bundle was introduced in Phase 2. CRBSI was determined according to US CDC diagnostic criteria. The rates of CRBSI before and after the introduction of the bundle of interventions were compared.

Results A total of 619 patients were enrolled in the study. During the baseline period (Phase 1), 238 patients with 2,456 catheter days were assessed, 30 patients developed a CRBSI. The CRBSI rate during this period was 12.2 per 1,000 catheter days. All nurses and principle doctors in the seven ICUs received training on the standard of care for catheter maintenance along with the introduction of Q-Syte ${ }^{\mathrm{TM}}$ and Posiflush $^{\mathrm{TM}}$. In Phase 2, following introduction of the interventions, 12 of 381 patients developed a CRBSI. Total catheter days during this period were 3,562. The CRBSI rate decreased to 3.4 per 1,000 catheter days. This was significantly lower than during the baseline period (Wilcoxon nonparametric test, $u=4.36, P=0.0003$ ). Additional analyses demonstrated that patients were at higher risk for developing a CRBSI if associated with: a prolonged catheter dwell time, a higher number of insertion attempts, a blood infusion or an increased frequency of catheter connector changes. We also found that the patients who developed a CRBSI had prolonged hospital stay and significantly added to the cost of treatment.

Conclusion Introduction of Q-Syte ${ }^{T M}$ and Posiflush ${ }^{T M}$ and improved standards of practice for catheter maintenance can significantly decrease CRBSI in the ICU.

\section{Reference}

1. O'Grady NP, Alexander M, Burns LA, et al.: Guidelines for the Prevention of Intravascular Catheter-Related Infections 2011 [www.cdc.gov/hicpac/bsi/ bsi-guidelines-2011.html]

P76

Wash your hands: simple measures save lives

S Macedo, GV Bispo, LA Ferreira, TO Cavalcanti, PF Rosa, C Paiva,

DR De Melo, LG Rezende

São Jose do Avai Hospital, Itaperuna, Brazil

Critical Care 2012, 16(Suppl 1):P76 (doi: 10.1186/cc10683)

Introduction Sepsis is a challenge for the intensive therapy unit, being the principal cause of death during hospitalization.

Methods We realized a longitudinal and individuated intervention authorized by the HSJA ethics committee applying the campaign 'Simple Measures Save Lives' in which 105 educational adhesives served as a guide for washing hands and flags for high-contaminated locations. A decontamination routine of monitors, control panels, fans and infusion bombs was established at each 12 hours; and continued education for the health team was intensified during the intervention. Was separated two groups, patient enrollments in periods of 45 days before and after the intervention, with more than 24 hours of hospitalization: group $A$ with 18 patients and group $B$ with 15 patients. Results The hospital infection incidence decreased by $40 \%$ and VAP by $39.6 \%$. Urine culture was positive in $33.3 \%$ of those patients $(n=5)$ in group $\mathrm{A}$ and in $16.7 \%(n=1)$ in group B (a $50.1 \%$ decrease). The cultures of catheter tip were positive in $68.8 \%(n=22)$ of catheters in group $A$, which used 32 catheters in total, and none in group B, which used 13 catheters. The sepsis incidence decreased by $39.6 \%$. Septic shock was detected in $16.6 \%(n=3)$ of patients in group A. There was a drop of the costs between groups (R4,479.28, 10.5\%). The cost of campaign material was $\mathrm{R} \$ 50.00$.

Conclusion This intervention was a simple form to decrease the related number of infections in the neurovascular ICU, having spent irrelevant values when compared with treatment of these clinical tables.

References

1. Zanon F, Caovilla JJ, Michel RS, et al:: Sepsis in the intensive care unit: etiologies, prognostic factors and mortality. Rev Bras Terapia Intensiva 2008, 20:128-134.

2. Silva E, Pedro MA, Sogaya ACB, et al.: Brazilian Sepsis Epidemiological Study. Crit Care 2004, 8:R251-R260.

3. Chesley R: Getting to zero: an emerging policy framework for the elimination of hospital-associated infections. Infect Control Hosp Epidemiol 2008, 30:71-73. 
P77

Comparison of hand hygiene in single-room versus open-plan ICUs

I Gork, S Benenson, M Brezis, CL Sprung, PD Levin

Hadassah Hebrew University Medical Center, Jerusalem, Israel

Critical Care 2012, 16(Suppl 1):P77 (doi: 10.1186/cc10684)

Introduction In a previous study we showed that cross-contamination with resistant bacteria occurred less frequently in a single-room (SR) ICU when compared to an open-plan (OP) ICU. We attempted to identify whether this was mediated by a change in human behavior; that is, whether hand hygiene $(\mathrm{HH})$ practices were similar in the OP versus SR ICU. Methods The SR ICU comprises eight single-patient rooms. The OP ICU includes four beds in a common area. Covert HH observations were made of physicians and nurses in both ICU areas. Defined $\mathrm{HH}$ opportunities occurred before and after contact with the patient or their environment. Each observation session lasted 20 minutes. Compliance was defined as use of alcohol hand rub or chlorhexidine wash. Qualitative records were made of tasks preceding missed $\mathrm{HH}$ opportunities (patient contact, computer use, obtaining additional supplies or other).

Results Observations sessions were completed on 34 and 35 occasions in the SR and OP ICUs respectively including 277 and $418 \mathrm{HH}$ opportunities. The number of staff observed per session was $2.6 \pm 0.7$ in the SR ICU versus $2.1 \pm 0.5$ in the OP ICU $(P=0.01)$. There were fewer HH opportunities per session in the SR ICU $(8.4 \pm 3.3$ vs. OP ICU $11.9 \pm 5.2$, $P<0.001)$. $\mathrm{HH}$ compliance before patient contact was higher in the $\mathrm{SR}$ ICU than the OP ICU ( $1.8 \pm 1.4$ vs. $0.8 \pm 1.1$ episodes/session, $P=0.001)$, but similar after patient contact $(2.6 \pm 1.4$ vs. $2.2 \pm 1.5$ episodes/ session, $P=0.29$ ). Causes of missed $\mathrm{HH}$ opportunities were recorded on 98 and 140 occasions in the SR and OP ICUs. Comparing the SR to OP ICU: patient contact accounted for $21 / 98$ (21\%) versus $50 / 140$ (36\%, $P=0.02$ ) missed $\mathrm{HH}$ opportunities respectively; use of the bedside computer $1 / 98(1 \%)$ versus $14 / 140(10 \%, P=0.005)$; additional supplies (drugs, cleaning, dressing, and so forth) $9 / 98$ (9\%) versus 20/140 (14\%, $P=0.24)$; and other $4 / 98(6 \%)$ versus $15 / 140(10 \%, P=0.06)$.

Conclusion There were more $\mathrm{HH}$ opportunities in the OP ICU and $\mathrm{HH}$ compliance there was lower. The main difference in compliance occurred before patient care, with compliance after patient care being similar. This may reflect ease of access from patient to patient in the OP ICU where turning around brings you easily from one patient to the next. In the SR ICU movement from patient to patient requires exiting one room and entering another with a clear end to patient care in one room and a beginning in the next. Patient contact and use of the bedside computer accounted for the majority of missed $\mathrm{HH}$ opportunities and present possibilities for interventions to improve $\mathrm{HH}$ compliance.

\section{P78}

Compliance for decontamination of bedside computer keyboards

on an ICU

A Disney, K Sim

St Helens \& Knowsley NHS Trust, Prescot, UK

Critical Care 2012, 16(Suppl 1):P78 (doi: 10.1186/cc10685)

Introduction An audit to assess the compliance of decontamination of bedside computer keyboards by medical staff on an ICU. The topic was used for audit as previously identified by the hospital infection control department as the worst-performing area on 'clean trace' scoring, and had been a regular topic of discussion between members of the medical team. Bedside keyboards automatically emit an alarm sound and small flashing LED light if cleaning is required prior to use. Discussion and hypothesis on reasons for rates of compliance follow this, including social/human factors that are barriers to compliance with infection control measures.

Methods Observations and recording of medical staff and their compliance of cleaning bedside keyboards when required during a morning consultant-led ward round over a 10-day period. Further observations were taken at two other points in time during each day over the same time period. Observations were conducted by the author. Medical staff were informed at the beginning of the audit that this behaviour was being observed and staff were reminded of the principles of infection control, specifically maintaining clean IT equipment. A standard of $100 \%$ compliance for cleaning the keyboard appropriately when required was set for audit purposes.

Results Total number of patient consultations during morning ward round $n=99$; total number of occasions a keyboard used $n=40$; total number of times a keyboard used and keyboard required cleaning $n=37$; keyboard used and cleaned when required prior to use $n=5$. In total, a rate of compliance at $14 \%$ for cleaning keyboards when appropriate. Further observations over the same time period showed that keyboards were indicating they required cleaning on $96 \%$ of occasions observed.

Conclusion The rate of compliance for cleaning bedside keyboards was $14 \%$, below the standard set of $100 \%$. This may be due to several factors, including lack of education and resistance to changes in behaviour which have previously been described in various models of human behaviour such as the theory for planned behaviour. This may reflect compliance with other areas of infection control and have a detrimental effect on patient safety and health. This may also become a significant issue in the future if compliance rates remain low as more IT and touchscreen equipment is employed in ICUs. The ICU has adjusted the induction programme for new medical staff to include education on infection control measures with bedside IT equipment.

\section{P79}

\section{Reduced air contamination in an ICU environment with a portable} air purification system

J Papaparaskevas ${ }^{1}$, V Papas², M Pratikaki², A Tsakris' ${ }^{1}$ C Routsi ${ }^{2}$

'Medical School, University of Athens, Greece; 'Evangelismos Hospital, Athens, Greece

Critical Care 2012, 16(Suppl 1):P79 (doi: 10.1186/cc10686)

Introduction Indoor air contamination has been implicated in hospitalacquiring infections, especially in immunocompromised patients. This implies that, along with other preventing measures, maintenance of good air quality in critical areas in hospitals is helpful to reduce the incidence of these infections. The objectives of this study were to evaluate the quality of an ICU air environment regarding total and fungal flora and the ability of a mobile air purification system (Hegoa; ANEMO, Oullins, France). This device uses UVc technology (photocatalysis) to destroy a wide range of microorganisms, including fungi.

Methods Air samples were obtained before and after the Hegoa air purification system was started in seven ICU rooms, including a total of 10 beds, during a 24-hour period and at 3-hour intervals. From each room and time point, $200 \mathrm{I}$ air samples were collected using a calibrated biocollector (Air Ideal; bioMerieux, Marcy L'Etoile, France). Cultures were performed on Triptycase Soy Agar and Sabouraud chloramphenicol agar plates, for the total and the fungal flora, respectively. Plates were incubated at $36^{\circ} \mathrm{C}$ and room temperature for a period of 7 days.

Results A total of 112 air samples from sampling sites in the ICU rooms were collected during the 24-hour study period. Before starting the air purification unit, total flora ranged from 175 to $70 \mathrm{cfu} / \mathrm{m}^{3}$ and fungal flora from 30 to $35 \mathrm{cfu} / \mathrm{m}^{3}$. Total flora values were continuously decreasing and at 24 hours after air purification onset were significantly reduced to 30 to $50 \mathrm{cfu} / \mathrm{m}^{3}$ ( $72 \%$ reduction). Similarly, environment fungal levels were continuously decreased and at 24 hours after the start were undetectable.

Conclusion The Hegoa mobile air purification system shown a rapid lowering of contaminates with eventual elimination of fungal flora. Therefore, that equipment may provide an efficient method of reducing air contamination into the ICU. Whether equipping ICU rooms with such devices could protect immunocompromised patients admitted to the ICU against fungal and microbial risk has to be examined.

P80

Effectiveness of an innovative system for the bio-decontamination

of the ICU

A De Nicola, MJ Sucre

San Leonardo Hospital, Castellammare di Stabia, Italy

Critical Care 2012, 16(Suppl 1):P80 (doi: 10.1186/cc10687)

Introduction The ICU contains a large quantity of sensitive electrical equipment which must not be affected by any bio-decontamination 
process. To disinfect the ICU environment we have used the original device, Medisize $99.99^{\circledR}$, which releases a synergistic formulation of hydrogen peroxide with silver ions. The machine launches a dry cloud of 0.5 to $2 \mathrm{~m}$ particles which penetrates everywhere, without humidity or corrosive activity.

Methods The study has been conducted in the ICU area, just after the patients have been discharged, before and after the use of the Medisize $99.99^{\circledR}$ device. The overall number of samples taken has been 54 on three different days. The sampling has been taken with Petri contact plates and incubated at $35^{\circ} \mathrm{C}$ for 48 hours, counting afterwards the CFU/plate.

Results We found the annulment of the contamination at all sites tested after sanitation (Table 1).

Table 1 (abstract P80). CFU of sample sites before and after sanitation

\begin{tabular}{lcc}
\hline Sample sites & Before UFC/cm & After UFC/cm \\
\hline Mattress & $10^{4}$ & $<0.5$ \\
Vital parameters monitor & 5,000 & $<0.5$ \\
Wall & 2,000 & $<0.5$ \\
Bed rail & $<0.5$ & $<0.5$ \\
Bed remote control & $<0.5$ & $<0.5$ \\
Ventilator screen & 5,000 & $<0.5$ \\
Ventilator chassis & $10^{4}$ & $<0.5$ \\
Infusion pump & $<0.5$ & $<0.5$ \\
\hline
\end{tabular}

Conclusion The destruction of the bacteria has practically taken place in all the points tested. The system has resulted to be compatible with the electronic equipment and a few minutes after the end of the procedure it is possible to use the area. The catalytic action of the silver atoms produces the tyndallisation of the surfaces, increasing the effectiveness of the sanitizer. Eight minutes after the end of the treatment, $98 \%$ of the $\mathrm{OH}^{-}$radicals have been destroyed and $95 \%$ of the dry cloud has been deposited, inhibiting the possibility of regeneration of any resistant microorganism.

P81

Massive hemoptysis in a respiratory ICU: causes, interventions and outcomes - Indian study

D Talwar, J Chudiwal, R Jain, S Kumar

Metro Center for Respiratory Diseases, Noida, India

Critical Care 2012, 16(Suppl 1):P81 (doi: 10.1186/cc10688)

Introduction Massive hemoptysis carries high mortality and morbidity, requiring multidisciplinary management. In India, tuberculosis is a very common cause of severe hemoptysis and is being treated in tuberculosis hospitals where such an approach is not available. We evaluated the profile of patients admitted with massive hemoptysis in a well-equipped Indian tertiary-care respiratory center.

Methods Retrospective analysis of 376 patients admitted with hemoptysis to the respiratory ICU of the Metro Center for Respiratory Diseases, India was done. We identified 90 patients with massive hemoptysis (>600 ml in 24 hours) between 2005 and 2011 and the results were analyzed. As per our protocol all patients had active medical management and those suitable for surgery underwent elective or emergent surgery. Unsuitable candidates underwent bronchial artery embolisation (BAE) or bronchoscopic interventions (BI) and if suitable were taken for surgery later.

Results The mean age of patients was $49.5 \pm 16.53$ years with $73.33 \%$ $(n=66)$ being male. Mortality in male patients was significantly higher than females (64.7 vs. $35.3 \%, P=0.02)$. The mean length of stay in hospital was $10.44 \pm 6.9$ days and significantly less $(7.06 \pm 4.8, P=0.01)$ in the mortality group. Massive hemoptysis was due to tuberculosis (active and old) in 61\%, pneumonia in $25.5 \%$, bronchiectasis in $21.1 \%$, aspergillus-releated disease in $11.1 \%$. Lung cancer in $6.6 \%$ cases but this carried highest mortality. The bleeding site was identified on
Table 1 (abstract P81). Types of management versus mortality in massive hemoptysis

\begin{tabular}{lccc}
\hline Management & Total & Mortality & Percentage \\
\hline Medical & 6 & 5 & 83.3 \\
Bl & 35 & 7 & 20 \\
BAE & 19 & 1 & 5.2 \\
Surgery & 30 & 4 & 13.3 \\
Multiple & 21 & 2 & 9.5 \\
\hline
\end{tabular}

CT chest in $65.5 \%$ and in $64.4 \%$ by fiber optic bronchoscopy (FOB). However, combined FOB and CT scan could localize bleeding in $87.8 \%$. See Table 1.

Conclusion All-cause mortality in massive hemoptysis at our center was $18.8 \%$. Lung cancer, necrotizing pneumonia and bronchiectasis carried significantly higher mortality. BAE showed low mortality but required multiple interventions in nearly two-thirds of cases. Hence, surgery remains the intervention of choice in massive hemoptysis at our setup with acceptable mortality and outcome.

P82

\author{
Capnography use in Scottish ICUs \\ CWallace, S Cole, B McGuire \\ Ninewells Hospital, Dundee, UK \\ Critical Care 2012, 16(Suppl 1):P82 (doi: 10.1186/cc10689)
}

Introduction Almost 20\% of adverse airway events reported to the Royal College of Anaesthetists 4th National Audit Project (NAP4) occurred in the ICU [1]. NAP4 commented that the failure to use capnography probably contributed to $77 \%$ of the ICU airway mortality. NAP4 subsequently made a number of recommendations pertaining to capnography use. We designed a survey to describe practice with regards to these.

Methods A survey was sent to an intensivist at each of the 23 adult ICUs in Scotland.

Results There was a $100 \%$ response rate. Nineteen (83\%) units used capnography for all tracheal intubations on the unit, two (9\%) in over three-quarters, one (4\%) in under one-half and one (4\%) unit reported never using it. For tracheal intubations prior to unit admission, the corresponding usage was three (13\%) always, seven $(30 \%)$ in over three-quarters, seven (34\%) in over one-half and six (26\%) in less than one-half of all intubations. Continuous capnography monitoring was in use on $54 \%$ of the intubated patients and $63 \%$ of the ventilatordependent patients. Twelve (52\%) units reported using capnography in all the intubated and ventilated patients. Of the units not using continuous capnography routinely, two (18\%) had no equipment for continuous monitoring.

Conclusion UK Intensive Care Society (ICS) guidelines make strong recommendations for the use of capnography in all critically ill patients during intubation [2]. We show a reassuring compliance with those guidelines during tracheal intubations performed on ICUs. Compliance was much poorer with the guidelines for those intubations performed outside units. An AAGBI safety statement recommended that continuous capnography should be used in all patients with intubated tracheas, regardless of location [3]. This was not echoed in the 2009 ICS guidelines (although in the light of NAP4, these have been updated to support this). Despite the majority of units in Scotland having facilities to monitor patients using capnography, just over one-half were doing so routinely. Capnography monitoring will surely increase in the advent of NAP4 and because of the change to the ICS guidelines.

References

1. CookTM, et al:: Br J Anaesth 2011, 106:617-631 and 632-642.

2. Thomas AN, et al:: Standards for Capnography in Critical Care. London: Intensive Care Society Standards and Guidelines; 2009.

3. The Association of Anaesthetists of Great Britain and Ireland: Safety Statement on Capnography Outside The Operating Theatre. London: AAGBl; 2009 [http:// www.aagbi.org/sites/default/files/AAGBI\%20SAFETY\%20STATEMENT_0] 
P83

Digitalized acoustic monitoring of lung congestion

S Lev', L Wolloch², I Kagan', M Grienv', P Singer

'Rabin Medical Center, Petah Tikva, Israel; ' 2 Deep Breeze Ltd, Or-Akiva, Israel

Critical Care 2012, 16(Suppl 1):P83 (doi: 10.1186/cc10690)

Introduction Changes in lung water are known to change breath sound acoustics [1]. Using two pig models, we observed that continuous elevation of lung sound amplitude may indicate an increase in total lung water content [2]. Here we report three cases of ventilated patients in whom continuous acoustic monitoring was done during extravascular lung water (EVLW) measurements.

Methods We retrospectively analyzed cases in which EVLWi (PiCCO) and other clinical parameters were measured, during continuous acoustic monitoring (VRI), using eight small sensors adhered to the anterior chest. A transmission factor (TF) was calculated, using the sound transfer function between different sensors. The TF changes in correspondence to changes in tissue density [1]. The difference in TF was calculated between recordings when pulmonary edema was observed $(>7 \mathrm{ml} / \mathrm{kg}$ threshold accompanied with an increase of $2 \mathrm{ml} / \mathrm{kg}$ in the EVLWi) and when absent. Statistical analysis was made using a $t$ test.

Results A total of 336 continuous acoustic recordings in three patients (acoustic monitoring was applied together with EVLWi measurements) were analyzed (146 recordings when lung edema was present; 190 with no edema). In all patients, the acoustic profile corresponded to changes in the clinical picture. In two of the cases, changes in acoustic profile were similar to the ones in the EVLWi and other clinical parameters (Figure 1). In one case, where there was stability in lung sound acoustics, EVLWi and other clinical parameters were also stable. Significant differences existed between recordings with edema $(-3.61 \pm 0.39)$ and without edema $(-5.71 \pm 0.15)(P<0.001)$.

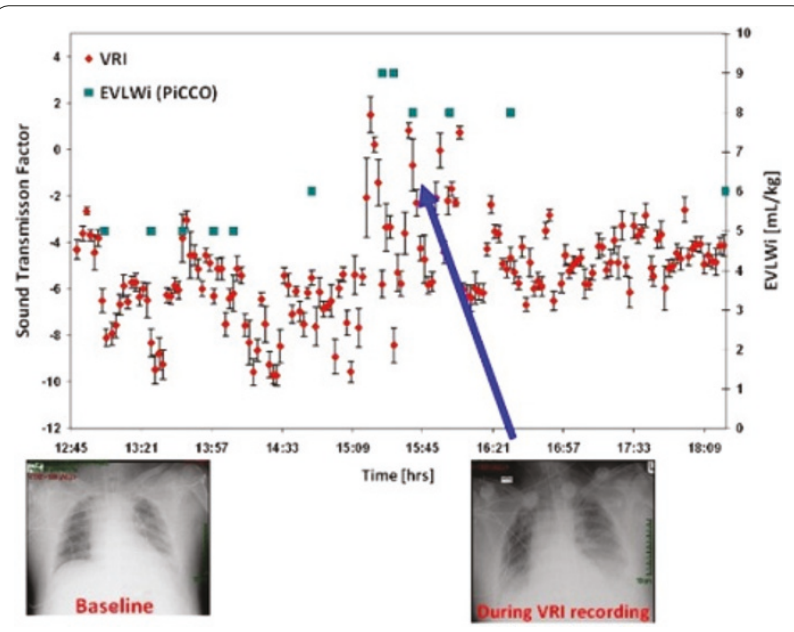

Figure 1 (abstract P83). VRI (average \pm SE) versus PiCCO and CXR.

Conclusion Changes in lung water tend to result in changes in the sound TF, due to changes in the tissue's density. These preliminary results indicate that monitoring lung sounds has the potential to monitor changes in lung water.

References

1. Donnerberg: Br J Dis Chest 1980, 74:23

2. Lev: Crit Care 2011, 15:P174.

P84

Usefulness of electrical activity of the diaphragm to detect intrinsic positive end-expiratory pressure during pressure support ventilation S Arrigoni, T Mauri, G Bellani, A Pradella, M Turella, V Sala, E Rezoagli, A Pesenti University of Milano-Bicocca, Monza, Italy

Critical Care 2012, 16(Suppl 1):P84 (doi: 10.1186/cc10691)

Introduction Intrinsic positive end-expiratory pressure (PEEPi) may add a substantial workload on respiratory muscles of patients undergoing pressure support ventilation (PSV). This can be reduced with the application of an external positive end-expiratory pressure (PEEPe) [1]. However, an accurate measurement of PEEPi during PSV is challenging [2]. The aim of the present study is to investigate if the use of the electrical activity of diaphragm (EAdi) may yield the detection of PEEPi in patients undergoing PSV. We reasoned that if PEEPi was present the inspiratory airflow would start after EAdi had reached a given value (EAdi-threshold) necessary to generate the muscle pressure overcoming PEEPi.

Methods Ten patients with a clinical suspicion of PEEPi undergoing PSV were enrolled. Exclusion criteria were: age $<18$ years, hemodynamic instability, fever and $\mathrm{PaO}_{2} / \mathrm{FiO}_{2}<100 \mathrm{mmHg}$. All patients were tested during PSV for seven steps of 3 minutes each with increasing PEEPe (2, $4,6,8,10,12,14 \mathrm{cmH}_{2} \mathrm{O}$ ). At the end of each step, PEEPi was estimated with an end-expiratory occlusion maneuver. During the study, we continuously recorded airway pressure, flow, volume and EAdi waveforms for off-line analysis. Data were analysed by linear regression and $t$ test. $P<0.05$ was considered statistically significant.

Results If PEEPi is present, EAdi-threshold is supposed to gradually decrease together with the raise of PEEPe; thus we divided patients into five responders for whom EAdi-threshold was significantly correlated with PEEPe, as opposed to five nonresponders. In the group of responders we observed significant correlations between the reduction of PEEPi and the increase of PEEPe $\left(r^{2}=0.86, P<0.01\right)$, and between EAdi-threshold and PEEPi at different PEEPe levels $\left(r^{2}=0.96\right.$, $P<0.001)$. In the same group, respiratory rate (RR) decreased $\left(r^{2}=0.76\right.$, $P=0.01)$, tidal volume increased $\left(r^{2}=0.71, P=0.02\right)$ and the peak of EAdi decreased $\left(r^{2}=0.94, P<0.001\right)$ at increasing levels of PEEPe. On the contrary, in the nonresponder group the increase of PEEPe was associated only with an increase of $\operatorname{RR}\left(r^{2}=0.75, P=0.01\right)$.

Conclusion In five of 10 patients with clinical suspicion of PEEPi, when the PEEPe was increased we observed a decrease of EAdi-threshold, associated with improved respiratory mechanics, suggesting that EAdithreshold could be a useful indicator for the presence of PEEPi.

References

1. Mancebo J, et al:: Anesthesiology 2000, 93:81-90.

2. Marini JJ: Am J Respir Crit Care Med 2011, 184:756-762.

P85

Adequate lung sliding identification is not influenced by the level of academic or ultrasound training

E Piette' ${ }^{1}$, R Daoust ${ }^{1}$, J Lambert ${ }^{2}$, A Denault ${ }^{3}$

'Hôpital du Sacré-Coeur de Montréal, Canada: 'Université de Montréal,

Canada: ${ }^{3}$ Institut de Cardiologie de Montréal, Canada

Critical Care 2012, 16(Suppl 1):P85 (doi: 10.1186/cc10692)

Introduction Rapid confirmation of the adequacy of endotracheal intubation is critical in the field of emergency medicine (EM). Methods confirming endotracheal tube (ET) position should have accuracy near $100 \%$. Studies confirming ET position using lung sliding (LS) identification were done by physicians with extensive ultrasound (US) training using sometimes lengthy examination. These conditions are not easily reproduced in the emergency department. Our primary objective was to compare the accuracy of EM physicians with different levels of academic and US training to correctly identify presence or absence of LS on random short sequences of lung US. Our secondary objective was to determine if results were better when participants had the choice to abstain themselves in uncertain cases.

Methods We recorded in the operating room 280 short lung US sequences (one respiratory cycle), of present and absent LS of intubated patients and randomly presented them to two groups of EM physicians. Accuracy was calculated for different academic and US training: none, basic Focused Assessment with Sonography in Trauma (FAST), FAST and advanced cardiac US, fellowship in EM US. We compared them using an ANOVA test. Only participants in the second group where instructed to abstain from answering in uncertain cases and accuracy was compared to the first group using a Student's $t$ test. The project was approved by the research and ethics committees.

Results Two medical students, 42 EM residents and 31 EM attendings participated. No difference in accuracy was shown between the subgroups of academic training with mean accuracies of $66.3 \%$ (medical 
students), $70.9 \%$ (residents) and $69.0 \%$ (attendings) $(P=0.361)$. No difference was shown between the subgroups of US training with means of $63.9 \%$ (no formation), 70.2\% (FAST), 70.9\% (FAST + advanced cardiac US), and $74.2 \%$ (fellowship) ( $P=0.119$ ). Accuracy was significantly better when participants could abstain from answering in uncertain cases with means of $67.5 \%(95 \% \mathrm{Cl}: 65.7$ to 69.4$)$ in the first group and $73.1 \%(95 \% \mathrm{Cl}: 70.7$ to 75.5$)$ in the second $(P<0.001)$. Conclusion Correct LS identification on short lung US sequences is not influenced by the level of academic or US training. Accuracy is better when the possibility to abstain oneself from answering is given. LS identification using one respiratory US sequences should be used with caution to confirm adequacy of endotracheal intubation.

P86

Lung ultrasound can differentiate Pneumocystis jiroveci versus other etiologies among critically ill AIDS patients with pneumonia

A Japiassu, F Bozza

IPEC-FIOCRUZ, Rio de Janeiro, Brazil

Critical Care 2012, 16(Suppl 1):P86 (doi: 10.1186/cc10693)

Introduction Lung ultrasound (US) can be applied as a point-of-care approach for diagnosis of pneumonia in AIDS patients. We compare US examinations of Pneumocystis jiroveci versus other etiologies of pneumonia in critically ill patients.

Methods Every HIV/AIDS patients admitted to the ICU with pneumonia was included. The first US examination was performed until 72 hours after admission. Pneumonia was defined by clinical examination, laboratorial parameters and chest X-rays. Etiologic agents were defined according to appropriate cultures and serology. US was applied to four fields (apex, lateral middle third, anterior basal and posterior basal regions) for each hemithorax, with $2.5 \mathrm{MHz}$ curved transducer Three pneumonia patterns were defined: interstitial pneumonia, bronchopneumonia and pneumonia with consolidation. The presence of B lines, peripheral microabscesses (bronchopneumonia), consolidations and pleural effusions were compared between the Pneumocystis pneumonia group (PCP) versus other etiologies.

Results We included 21 patients (age (median) 38 years; male 71\%). Most (80\%) patients were admitted because of acute respiratory insufficiency by pneumonia. Seventeen (81\%) had CD4 cell counts lower than $200 / \mathrm{mm}^{3}$. The SAPS 2 score was 47 points and the SOFA score on day 1 of admission was 6 points. Hospital mortality was $43 \%$. All radiographic pneumonia images were viewed on lung US examinations. Possible and probable pneumonia by $P$. jiroveci was diagnosed in six patients; all of these patients presented diffuse thin and/or gross B lines on both lungs. Bacterial $(n=7)$, mycobacterial (tuberculosis $(n=6)$ and Mycobacterium kansasii $(n=1)$ ), and fungal (Aspergillus sp. $(n=1)$ ) were diagnosed in other patients. Peripheral microabscesses were viewed on one patient with PCP and four patients with other etiologies $(P=N S)$; pleural effusions were present on US of seven patients with diverse etiologies (no PCP patient had pleural effusions; $P=0.06$ ); no pneumothorax was diagnosed in the study. Consolidation was present in one patient with PCP and 11 patients with bacterial, mycobacterial and fungal pneumonia $(P=0.05)$. There was a high degree of symmetry on lung US examinations of PCP patients, while there was always differences between the right and left hemithorax among other etiologic pneumonia $(P<0.001)$.

Conclusion We suggest that high-degree symmetric and diffuse $B$ lines, without pleural effusions, are compatible with $P$. jiroveci as the etiology of recent diagnosed pneumonia in critically ill AIDS patients.

\section{P87}

Difference in accuracy of lung sliding identification between the right and left hemithorax

R Daoust ${ }^{1}$, E Piette $^{1}$, J Lambert ${ }^{2}$, A Denault ${ }^{3}$

'Hôpital du Sacré-Coeur de Montréal, Canada: ${ }^{2}$ Université de Montréal,

Canada: ${ }^{3}$ nstitut de Cardiologie de Montréal, Canada

Critical Care 2012, 16(Suppl 1):P87 (doi: 10.1186/cc10694)

Introduction The field of lung ultrasound (US) in critical care is in rapid expansion. Lung sliding (LS) identification has been used in emergency medicine (EM) to diagnose pneumothorax as well as to evaluate the adequacy of endotracheal intubation. Presence of the Lung Pulse artefact (back and forth pleural motion induced by the heartbeat) as well as the underlying heart may affect correct identification of LS in the left hemithorax, but this has never been studied. Our main objective was to evaluate the rate of correct identification (accuracy) of the presence or absence of LS in the right and left hemithorax.

Methods A total of 280 short lung US sequences (one respiratory cycle), recorded in the operating room, of presence and absence of LS in intubated patients were randomly presented to two groups of physicians (in total: two medical students, $42 \mathrm{EM}$ residents and $31 \mathrm{EM}$ attendings). Sequences were divided equally between the right and left hemithorax. Each participant's knowledge of the Lung Pulse artefact was noted. Only the second group was instructed not to answer in case of uncertainty. A Kolmogorov-Smirnov test showed the rate of correct LS identification did not follow a normal distribution. Median rates are reported with interquartile range (IQR) and compared using a MannWhitney test.

Results Knowledge of Lung Pulse was higher in the second group ( $55 \%$ vs. $21 \%, P<0.05$ ). Globally, median accuracy of identification of LS presence or absence was $74.0 \%$ (IQR: 48.0 to 90.0 ) in the first group and 83.7\% (IQR: 53.3 to 96.2$)$ in the second $(P=0.006)$. For the first group, median accuracy was $80.0 \%$ (IQR: 57.0 to 95.0 ) in the right hemithorax and $67.0 \%$ (IQR: 43.0 to 83.0$)$ in the left $(P<0.001)$. For the second group, median accuracy was $88.7 \%$ (IQR: 63.1 to 96.9$)$ in the right hemithorax and $76.3 \%$ (IQR: 42.9 to 90.9$)$ in the left $(P<0.001)$.

Conclusion Accuracy of identification of LS presence or absence is higher in the right hemithorax. Our study is the first to report this finding. Presence of the Lung Pulse artefact, as well as the underlying heart, probably explains the worse accuracy found in the left hemithorax. Caution should be taken in using LS identification as a diagnostic tool in the left hemithorax and knowledge of the Lung Pulse artefact should be emphasized in chest US curriculum.

P88

Trans-thoracic echo evaluation before and during noninvasive ventilation

L Vetrugno, M Costa, C Centonze, N Langiano, M Rojatti, G Della Rocca University Hospital of Udine, Italy

Critical Care 2012, 16(Suppl 1):P88 (doi: 10.1186/cc10695)

Introduction Over the last decade noninvasive ventilation (NIV) gained the dignity of first-line intervention for acute lung injury (ALI) and acute respiratory distress syndrome (ARDS) in the ICU. Its great interest is based on a lower complications rate compared with traditional invasive ventilation. However, the NIV application, although less invasive, cannot ignore its hemodynamic effect over the patient. This study evaluates the NIV effects on the left ventricle in terms of systolic and diastolic function through trans-thoracic echocardiography (TTE). We also try to obtain a preload value index equivalent of flow time corrected (FTC).

Methods Thirteen patients admitted to our ICU with ALI/ARDS underwent TTE before and during NIV. NIV was set as a 1 hour cycle with 5 to $7 \mathrm{cmH}_{2} \mathrm{O}$ of PEEP and 5 to $7 \mathrm{cmH}_{2} \mathrm{O}$ of pressure support ventilation. During NIV for a better patient compliance a continuous i.v. infusion of remifentanil was used (range 0.03 to $0.05 \mu \mathrm{g} / \mathrm{kg} / \mathrm{minute}$ ). At baseline (TO = before NIV) and after 30 minutes of NIV (T1), the following data were recorded: respiratory - $\mathrm{RR}, \mathrm{SaO}_{2} \%, \mathrm{PaO}_{2^{\prime}} \mathrm{PaCO}_{2}, \mathrm{pH}, \mathrm{BE}$, and $\mathrm{HCO}_{3}^{-}$; and cardiac - heart rate (HR), arterial blood pressure (systolic, diastolic and media), diastolic and systolic volume (EDV, ESV), ejection fraction $(\mathrm{EF})$, stroke volume (SV), velocity time integral (VTI), FTc, E wave, deceleration time (Dt), A wave, ventricular flow propagation velocity (Vp).

Results From T0 to T1 the following changes with Wilcoxon matched pairs test were statistically significant $\left(P<0.05^{*}\right)$. $\mathrm{PaO}_{2}\left(94\right.$ to $\left.123 \mathrm{mmHg}^{*}\right), \mathrm{SaO}_{2}$ ( 87 to $97 \% *$ ) and $\mathrm{PaO}_{2} / \mathrm{FiO}_{2}, \mathrm{RR}$ ( 37 to $28 /$ minute*). At TO, EF was $>55 \%$ in seven patients and $<55 \%$ in six patients. In the group with $\mathrm{EF}<55 \%$ (T0) the EF increased at T1 (42 to 52\%*). Dt significantly increased from T0 to T1 (182 to $198 \mathrm{~cm} /$ second $^{*}$ ). No significant changes were observed in VTI, E/A ratio, Vp, and E/Vp ratio, from $\mathrm{T} 0$ to $\mathrm{T} 1$.

Conclusion Our study suggests that NIV improves cardiac function in patients with reduced EF, positioning the patients to a more favorable 
point of the Frank Starling curve. In these patients we also showed an increase in FTc that seems to be affected by either preload or afterload reduction.

References

1. Antonelli M, Pennisi MA, Montini L: Clinical review: Noninvasive ventilation in the clinical setting - experience from the past 10 years. Crit Care 2005, 9:98-103.

2. Shekerdemian L, Bohn D: Cardiovascular effects of mechanical ventilation. Arch Dis Child 1999, 80:475-480.

P89

Listen to $\mathrm{PaO} / \mathrm{FiO}_{2}$ ratios: they tell us about length of stay

$\checkmark$ Inal, B Comert, L Yamanel

GATA, Ankara, Turkey

Critical Care 2012, 16(Suppl 1):P89 (doi: 10.1186/cc10696)

Introduction Classification of respiratory distress has been dependent on $\mathrm{PaO}_{2} / \mathrm{FiO}_{2}$; that is, $<300$ acute lung injury (ALI) and $<200$ acute respiratory distress syndrome (ARDS). In this study, $\mathrm{PaO}_{2} / \mathrm{FiO}_{2}$ was analyzed for predicting ICU patients' length of stay (LOS).

Methods Data of 273 patients admitted to the ICU with RI were retrospectively analyzed for LOS in the ICU. Patients admitted to the emergency department (ED) with Rl, documented arterial blood gas analysis (ABGA), and hospitalized in the ICU were eligible for this study within 4 years. The first $\mathrm{ABGA}$ in $\mathrm{ED} \mathrm{PaO} / \mathrm{FiO}_{2}$ were taken for predicting ICU LOS. Patients' comorbid diseases, APACHE II/Glasgow scores, non/ invasive mechanical ventilation supports were not included in the analysis. Patients were classified into three groups as: (1) $>300$ not having $\mathrm{RI},(2)<300 \mathrm{ALI},(3)<200 \mathrm{ARDS}$; they were then compared for predicting ICU LOS, and also receiver operating curve (ROC) analysis and area under curve (AUC) were calculated.

Results Analysis showed statistical significance of $P<0.01$ for all groups pointing out that $\mathrm{ED} \mathrm{ABGAPaO} / \mathrm{FiO}_{2}$ levels negatively affected patients' LOS in the ICU. ROC analysis of $\mathrm{PaO}_{2} / \mathrm{FiO}_{2}$ for LOS showed significant AUC: 0.917 levels, which was predicted as a powerful indicator. Patients' data are presented in Table 1.
Table 1 (abstract P89). Patient data

\begin{tabular}{lcccc}
\hline$(n)$ & $\begin{array}{c}\text { Age } \\
\text { (years) }\end{array}$ & $\begin{array}{c}>300(n) \\
\text { LOS (days) }\end{array}$ & $\begin{array}{c}\text { ALI }(\boldsymbol{n}) \\
\text { LOS (days) }\end{array}$ & $\begin{array}{c}\text { ARDS }(\boldsymbol{n}) \\
\text { LOS (days) }\end{array}$ \\
\hline Male 165 & $65 \pm 8.2$ & $478 \pm 2.1$ & $6512 \pm 3.4$ & $5316 \pm 4.2$ \\
Female 108 & $69 \pm 7.6$ & $337 \pm 2.9$ & $4311 \pm 3.7$ & $3217 \pm 3.8$ \\
\hline
\end{tabular}

Conclusion We concluded that the $\mathrm{PaO}_{2} / \mathrm{FiO}_{2}$ ratio was a powerful indicator for predicting ICU LOS in patients with RI. In addition there was no need to classify patients according to $\mathrm{PaO}_{2} / \mathrm{FiO}_{2}$ to predict LOS; any decreased ratio meant a longer LOS. However, this study was weak in power; it had a small sample, did not include comorbid conditions, did not account for accepted scoring systems, and did not include daily $A B G A$ for prediction. On the other hand, these results are promising for future observations that ABGA taken in the ED would be a supplemental tool for the physician's approach in the ICU.

\section{P90}

Worst Oxygenation Index during the first 24 hours of ventilation predicts mortality

RJ Jackson, TH Gould, MJ Thomas

Bristol Royal Infirmary, Bristol, UK

Critical Care 2012, 16(Suppl 1):P90 (doi: 10.1186/cc10697)

Introduction The ratio of $\mathrm{PaO}_{2}$ to $\mathrm{FiO}_{2}(\mathrm{P} / \mathrm{F}$ ratio) is often used to classify patients with hypoxic respiratory failure, and is recommended in guidelines from a UK expert group [1] but does not take airway pressures into account. A study found that adjusting for PEEP did not affect the predictive ability of the $P / F$ ratio [2]; however, the mean airway pressure (MAP) may be a better indicator of lung recruitment. The Oxygenation Index $\left.\left(\mathrm{Ol}=\left(\mathrm{FiO}_{2} \times \mathrm{MAP}\right) / \mathrm{PaO}_{2}\right)\right)$ includes an adjustment for MAP.

Methods We retrospectively assessed a computerised record (from 2008 to 2010) of ventilator parameters and identified the highest OI for all ventilated patients from a general adult university teaching hospital ICU, during the first 24 hours of ventilation. Patients were grouped according to highest $\mathrm{Ol}$, and mortality was calculated for subgroups.

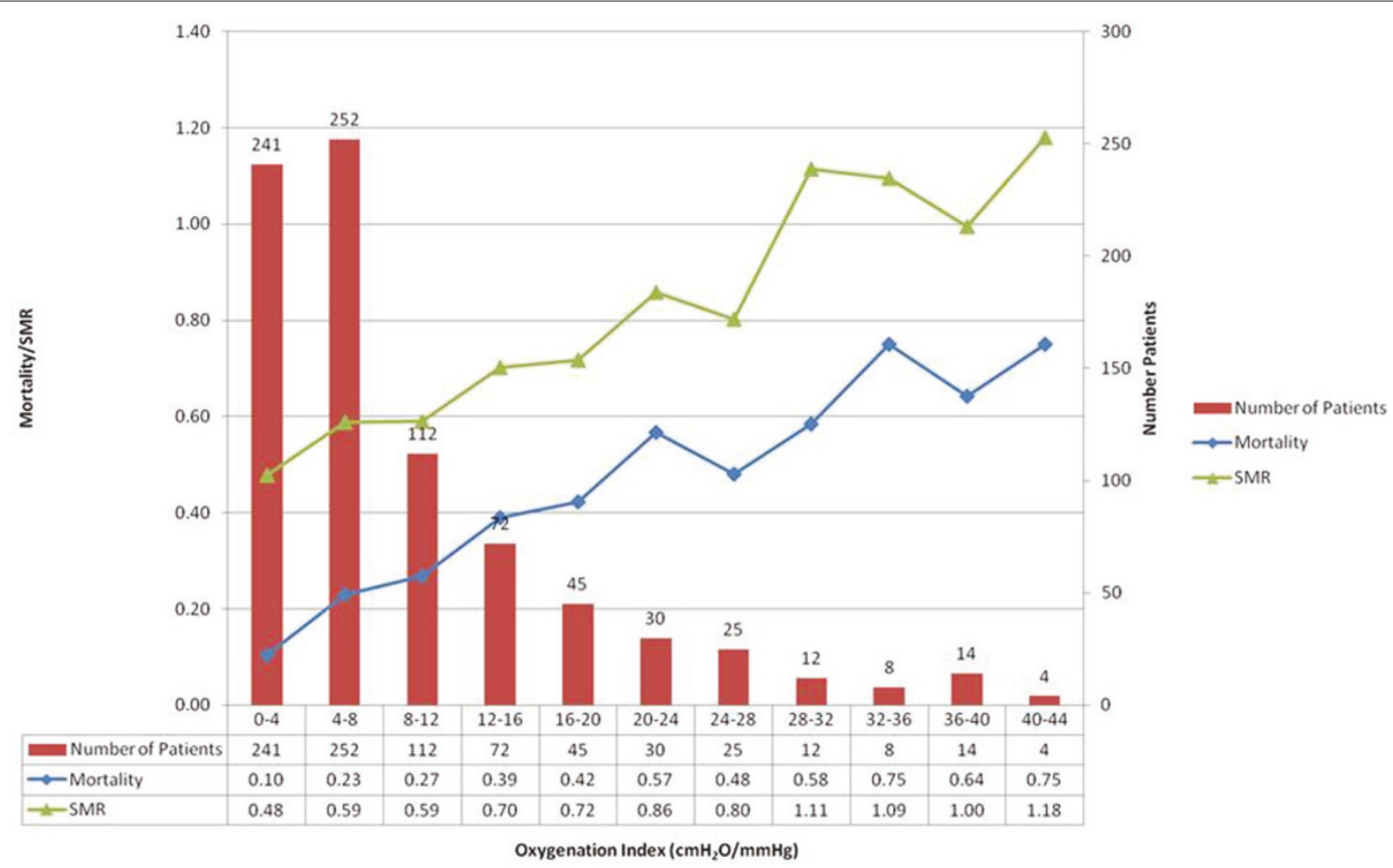

Figure 1 (abstract P90). Mortality and number of patients by Oxygenation Index. 
Results Data were available for 815 patients (see Figure 1). Increasing Ol was associated with increasing mortality $(P<0.0001$ chi-squared test for trend). Each step increase in OI was associated with approximately a $6 \%$ absolute increase in mortality. The Ol was also associated with increasing Standardised Mortality Ratio (ICNARC model).

Conclusion The highest Ol occurring in the first 24 hours of ventilation is an independent predictor of mortality. Collection of OI data may allow better prediction of outcome than P/F ratio data alone.

\section{References}

1. Winter B, et al:: Management of Severe Refractory Hypoxia in Critical Care in the UK in 2010 Report from UK Expert Group [http://www.ics.ac.uk] latest_news/management_of_severe_respiratory_failure_in_critical_care_]

2. Britos $M$, Smoot E, Liu KD, et al: The value of positive end-expiratory pressure and $\mathrm{FiO}_{2}$ criteria in the definition of the acute respiratory distress syndrome. Crit Care Med 2011, 39:2025-2030.

P91

The Oxygenation Index compared with the P/F ratio in ALI/ARDS

M Van Haperen', PH Van der Voort², RJ Bosman²

${ }^{\prime} A M C$, Amsterdam, the Netherlands; ${ }^{2} \mathrm{O} / \mathrm{vg}$, Amsterdam, the Netherlands

Critical Care 2012, 16(Suppl 1):P91 (doi: 10.1186/cc10698)

Introduction The usual way to describe the severity of pulmonary dysfunction in ventilated ICU patients is by using the $\mathrm{PaO}_{2} / \mathrm{FiO}_{2}$ ratio (PF). The PF may be adjusted by the ventilator pressure settings in order to reduce inspiratory oxygen fraction but the PF does not take the mean airway pressure (MAP) into account. In contrast, the Oxygenation Index $(\mathrm{OI})$ is defined as the reciprocal of PF times MAP: $\mathrm{OI}=\left(\mathrm{FiO}_{2} \times\right.$ mean airway pressure) / $\mathrm{PaO}_{2}$. As such, the $\mathrm{Ol}$ is a better representative of oxygenation dysfunction. The objective was to study the correlation between and the impact of the MAP on the PF and OI.

Methods We performed a retrospective analysis of 27 consecutive mechanically ventilated patients admitted to our ICU with bilateral interstitial/alveolar lung disease, defined as ALI or ARDS. The data of these patients were collected during a time period of maximum 30 consecutive days. Demographic data were recorded and the PF, OI and MAP were assessed daily at 6:00 am during the first 30 days of admission. OI $>8.1$ is usually regarded as ARDS and $>5.3$ as ALI [1].

Results We included 27 patients, 25 were male, the mean APACHE II score was 22, the median length of stay on the ICU 11 days and the ICU mortality was $11 / 16$ (69\%). The mean PF was 165 (SD 83), the mean OI was 8.2 (SD 5) and the mean MAP was $16 \mathrm{cmH}_{2} \mathrm{O}$ (SD 5). The 27 patients resulted in 364 measurements. Of these measurements 158 had OI $>8.1$, of which 157 had PF $<200$ and a mean MAP of $19.3 \mathrm{cmH}_{2} \mathrm{O}$. In one patient PF was $>200$ while Ol was $>8.1$ with MAP $18 \mathrm{cmH}_{2} \mathrm{O}$. Of the 100 measurements with OI 5.3 to $8.1,14$ had PF 200 to 300 and 85 had $\mathrm{PF}<200$. The MAP in these measurements was 17,64 and $24 \mathrm{cmH}_{2} \mathrm{O}$ respectively. Figure 1 shows the nonlinear relation between $\mathrm{Ol}$ and PF. Conclusion In patients with ARDS, OI $>8.1$ is usually in agreement with $\mathrm{PF}<200$. However, patients with ALI based on OI 5.3 to 8.1 frequently had $P F<200$. More studies are needed to determine the optimal level of OI for the diagnosis of ALI/ARDS.

\section{Reference}

1. Pediatr Crit Care Med 2010, 11:12-17.

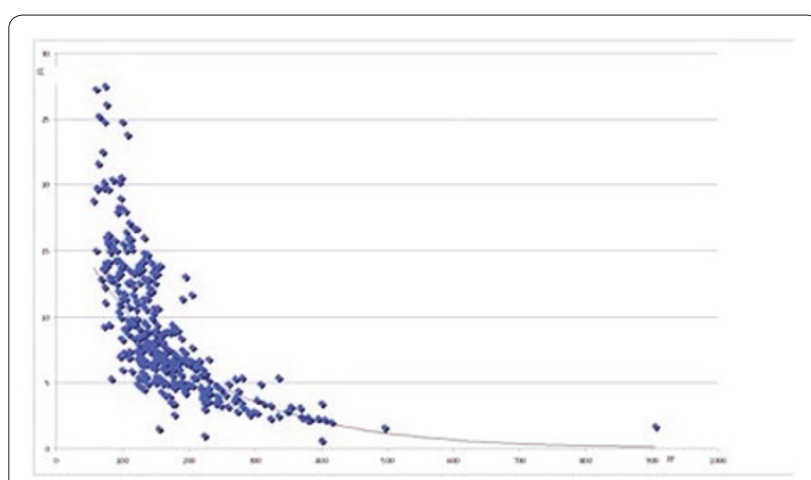

Figure 1 (abstract P91). Oxygenation Index versus P/F ratio.
P92

Do actual tidal volumes differ from prescribed tidal volumes?

R Kleijn, B Kalkman, N Verburg, H Oudijk, B Van Vondelen, M Luttmer,

I Slagers, M Ruijters, I Meynaar

Reinier de Graaf Groep, Delft, the Netherlands

Critical Care 2012, 16(Suppl 1):P92 (doi: 10.1186/cc10699)

Introduction Studies have shown that the selection of incorrect tidal volume can cause ventilator-induced lung injury and increased mortality [1]. This study was done to determine if the actual tidal volume $(\mathrm{aVt})$ differs from the prescribed tidal volume $(\mathrm{pVt})$ based on predicted body weight (PBW).

Methods The ICU is a 10-bed intensivist-led unit in a 500-bed teaching hospital. All consecutive patients receiving invasive mechanical ventilation in June 2011 were included. Patients with noninvasive ventilation or with continuous positive airway pressure only were excluded. The ICU has a mechanical ventilation protocol that prescribes tidal volume to be between 6 and $8 \mathrm{ml} / \mathrm{kg}$ PBW. A table with prescribed tidal volumes based on PBW is available at the bedside throughout the ICU. All patients were ventilated with Drager Evita XL ventilators on pressure support (ASB) or pressure control mode (BIPAP). During the study period we compared the aVt with the $\mathrm{pVt}$ each day at $0,6,10,14$, 18 and 22 hours for all patients.

Results Seventeen patients with mean age of 70.2 years (SD 14.1) and median APACHE IV expected mortality of 31\% (IQR 14 to 70 ), 10 admitted for medical reasons and seven for surgical reasons, and ventilated for 4 days median (IQR 3 to 6) fulfilled inclusion criteria and were included in the study. Results of tidal volume measurements are shown in Table 1.

Table 1 (abstract P92). Tidal volume measurements

\begin{tabular}{lc}
\hline Total number of aVt measurements & 286 \\
Number of aVt measurements per patient (IQR) & $12(4$ to 20$)$ \\
aVt $<6 \mathrm{ml} / \mathrm{kg}$ PBW & $25(9 \%)$ \\
aVt 6 to $8 \mathrm{ml} / \mathrm{kg} \mathrm{PBW}$ & $156(58 \%)$ \\
aVt 8 to $10 \mathrm{ml} / \mathrm{kg}$ PBW & $82(29 \%)$ \\
aVt $>10 \mathrm{ml} / \mathrm{kg}$ PBW & $23(8 \%)$ \\
Mean aVt per kg PBW (SD) & $7.85(1.23)$ \\
\hline
\end{tabular}

$\mathrm{aVt}$, actual tidal volume; PBW, predicted body weight.

Conclusion In this small single-centre study, the mean aVt is between 6 and $8 \mathrm{ml} / \mathrm{kg}$ PBW as prescribed, but only $58 \%$ of measured tidal volumes are indeed between 6 and $8 \mathrm{ml} / \mathrm{kg}$ PBW.

Reference

1. Ventilation with lower tidal volumes as compared with traditional tidal volumes for acute lung injury and the acute respiratory distress syndrome. The Acute Respiratory Distress Syndrome Network. N Engl J Med 2000, 342:1301-1308.

P93

Intratracheal administration of siRNA targeting FAS reduces ischemia-reperfusion-induced lung injury

L Del Sorbo, G Muraca, A Costamagna, G Rotondo, L Laudari, F Civiletti, E Tonoli, E Martin, V Fanelli, V Ranier

University of Turin, Italy

Critical Care 2012, 16(Suppl 1):P93 (doi: 10.1186/cc10700)

Introduction Ischemia-reperfusion injury is one of the main causes of primary graft dysfunction after lung transplantation. Fas-mediated apoptosis plays a major role in the pathogenesis of ischemiareperfusion injury. Exogenous administration of small interfering RNA (siRNA) is an effective strategy to specifically silence the expression of proteins through blocking the translation of mRNA. The aim of this study was to investigate in an ex vivo mouse model of lung ventilation and perfusion whether a specific siRNA targeting Fas is able to reduce ischemia-reperfusion injury. 
Methods $\mathrm{C} 57 \mathrm{BL} / 6$ male mice were randomized to intratracheally receive a specific sequence of siRNA targeting FAS (siRNA-FAS) or a scrambled siRNA 48 hours before undergoing 6 hours of cold ischemic time $\left(4^{\circ} \mathrm{C}\right)$ followed by 2 hours of ex vivo ventilation (peak inspiratory pressure $=7 \mathrm{cmH}_{2} \mathrm{O}, \mathrm{PEEP}=2 \mathrm{cmH}_{2} \mathrm{O}$, respiratory rate $=100$ breaths/minute, $\mathrm{FiO}_{2}=100 \%$ ) and reperfusion ( $4 \%$ bovine serum albumin RPMI medium with $10 \%$ fresh blood at $1 \mathrm{ml} /$ minute flow rate) in a predisposed humidified chamber at $37^{\circ} \mathrm{C}$. At the end of the experiment, lung elastance, assessed through tidal volume, and total protein concentration in the bronchoalveolar lavage (BAL) fluid were measured. A separate set of lungs were analysed by western blot before undergoing cold ischemia to assess the expression of FAS protein.

Results The intratracheal administration of siRNA-FAS reduced the expression of FAS in the lung by $44 \%$ (siRNA-FAS $0.90 \pm 0.11$ vs. scrambled siRNA $1.61 \pm 0.18 \mathrm{AU}$ ). Lung elastance and BAL total protein concentration were significantly reduced in the siRNA-FAS group as compared to control in lungs exposed to 6 hours of cold ischemia followed by 2 hours of reperfusion. See Table 1.

Table 1 (abstract P93)

\begin{tabular}{lcc}
\hline & siRNA-FAS & siRNA scrambled \\
\hline Elastance $\left(\mathrm{cmH}_{2} \mathrm{O} / \mathrm{ml}\right)$ & $11.34 \pm 0.24^{*}$ & $13.75 \pm 0.99$ \\
BAL proteins $(\mu \mathrm{g} / \mathrm{ml})$ & $529.1 \pm 64.8^{*}$ & $928.5 \pm 138.2$
\end{tabular}

Data are mean \pm SE. Comparison between groups was performed with the Student's $t$ test. ${ }^{*} P<0.05$.

Conclusion The intratracheal administration of siRNA targeting FAS prevents the increase of the alveolar membrane permeability during ischemia-reperfusion injury.

Acknowledgements Funded by PRIN and Regione Piemonte.

P94

Acute lung injury in mice associates with p44/42 and c-Jun $\mathrm{N}$-terminal kinase activation and requires the function of TNFa receptor I

N Maniatis', A Sfika', I Nikitopoulou', A Vassiliou', C Magkou', M Kardara', A Armaganidis', C Roussos'1, G Kollias², S Orfanos', A Kotanidou

'University of Athens, Greece; 'Al. Fleming' Biomedical Sciences Research Center, Vari, Greece

Critical Care 2012, 16(Suppl 1):P94 (doi: 10.1186/cc10701)

Introduction Aspiration of hydrochloric acid-containing gastric juice leads to acute lung injury and hypoxemic respiratory failure due to an exuberant inflammatory response associated with pulmonary edema from increased endothelial and epithelial permeability. The aim of this study was to determine the role and signaling mechanisms of TNFa in experimental acute lung injury from hydrochloric acid aspiration using a combination of genetic animal models and pharmacologic inhibition strategies.

Methods Subjects were male and female C57BI/6 mice, wild-type, TNFa knockout, TNFa receptor I knockout $(n=135)$. Hydrochloric acid was instilled intratracheally to mice, followed by respiratory system elastance measurement, bronchoalveolar lavage and lung tissue harvesting 24 hours post injection. The TNFa inhibitor etanercept was administered as pretreatment to a subset of mice prior to hydrochloric acid exposure.

Results Hydrochloric acid instillation induced an inflammatory response in the lungs of wild-type mice, evidenced as increased bronchoalveolar lavage total cells, neutrophils and total protein, histologic lung injury score and respiratory system elastance, while TNFa receptor I mRNA levels were maintained. These alterations could be prevented by pretreatment with etanercept or genetic deletion of the $55 \mathrm{kDa}$ TNFa receptor l, but not by deletion of the TNFa gene. Hydrochloric acid induced a sixfold increase in apoptotic, caspase3-positive cells in lung sections from wild-type mice, which was abrogated in mice lacking TNFa receptor I. In immunoblotting and immunohistochemistry studies, hydrochloric acid stimulated signaling via p44/42 and c-Jun N-Terminal kinase, which was blocked in TNFa receptor I knockout mice.
Conclusion Acute lung injury induced by intratracheal hydrochloric acid instillation requires the function of TNFa receptor I and associates with activation of downstream proinflammatory signaling pathways p44/42 and c-Jun N-Terminal kinase.

P95

Retrieval of patients with severe respiratory failure on venovenous extracorporeal membrane oxygenation: an intensivist-led model A Burrell', V Pellegrino', D Pilcher', S Bernard', M Kennedy²

'The Alfred Hospital, Melbourne, Australia; ${ }^{2}$ Adult Retrieval Victoria, Melbourne, Australia

Critical Care 2012, 16(Suppl 1):P95 (doi: 10.1186/cc10702)

Introduction Patients with severe respiratory failure may require venovenous extracorporeal membrane oxygenation (vv-ECMO). However, this treatment is only available in specialized centres. Previous reports of vv-ECMO cannula insertion and retrieval have included large teams of surgeons, perfusionists, physicians, retrieval doctors, paramedics and nurses. We hypothesized that an intensivist-led model for rapid response to a referring hospital, the insertion of vv-ECMO cannulae and subsequent retrieval would be safe and feasible.

Methods The Alfred Hospital ICU is the specialist centre for ECMO services for the states of Victoria and Tasmania in Australia. The intensivists in our ICU are trained to insert ECMO cannulae using a percutaneous femoral approach and manage the ECMO circuit during transport. A new ECMO retrieval service was set up in 2008 to allow the cannulation and retrieval of patients from other referring hospitals. The retrieval team comprises two intensivists to insert femoral cannulae and manage the ECMO circuit, a third physician to manage the ventilator and infusion pumps and a paramedic to manage the logistics of the patient transfer. We reviewed all consecutive patients from 2008 to 2011 with severe respiratory failure who received vv-ECMO and were retrieved to our specialist center.

Results There were 23 patients from 2008 to 2011. All cannulations were successfully performed percutaneously at the referring hospital by the intensivists. The underlying condition was $\mathrm{H} 1 \mathrm{~N} 1$ in 11 patients, bacterial pneumonia in six, acute lung injury in four, metastatic seminoma in one and multiple lung abscesses in one. The average age was 36 years (range 17 to 60 years). Males were $61 \%$. Transport was by fixed-wing aircraft in $35 \%$ and road ambulance in $65 \%$. The retrieval distance averaged $76 \mathrm{~km}$ (range 7 to $1,770 \mathrm{~km}$ ). During transport, there were two transient pump failures requiring hand cranking and one monitor failure. These resulted in no adverse clinical effects. The average ICU length of stay was 14 days. Overall survival to hospital discharge was $17 / 23$ (74\%).

Conclusion An intensivist-led model of vv-ECMO cannulation and retrieval appears to be a safe and effective model for vv-ECMO retrieval. This model may lead to a more rapid and cost-effective response and is the subject of further study.

P96

A new miniaturized extracorporeal membrane oxygenator with integrated rotary blood pump (Ilias): first results in a porcine model of lung injury

K Pilarczyk', J Heckmann'1, K Lyskawa' ', A Strauß², U Aschenbrenner², H Jakob ${ }^{1}$, M Kamler ${ }^{1}$, N Pizanis ${ }^{1}$

'West German Heart Centre Essen, University Hospital, Essen, Germany; 2iliasmedical GmbH, Bochum, Germany

Critical Care 2012, 16(Suppl 1):P96 (doi: 10.1186/cc10703)

Introduction Extracorporeal membrane oxygenation (ECMO) is used for most severe acute respiratory distress syndrome cases in specialized centres. However, critically ill patients fulfilling ECMO criteria are often not suitable for transportation and currently available ECMO systems are not designed for emergency use or interhospital transfer. Therefore, a new miniaturized ECMO (Ilias; Figure 1) with only $5 \mathrm{~kg}$ weight was developed to reduce filling volume and simplify management.

Methods Acute lung injury was induced with repeated pulmonary saline infusion in 13 pigs until the Horowitz Index was $<100 \mathrm{mmHg}$. Pigs were assigned to the following three groups: group $1(n=3)$, 


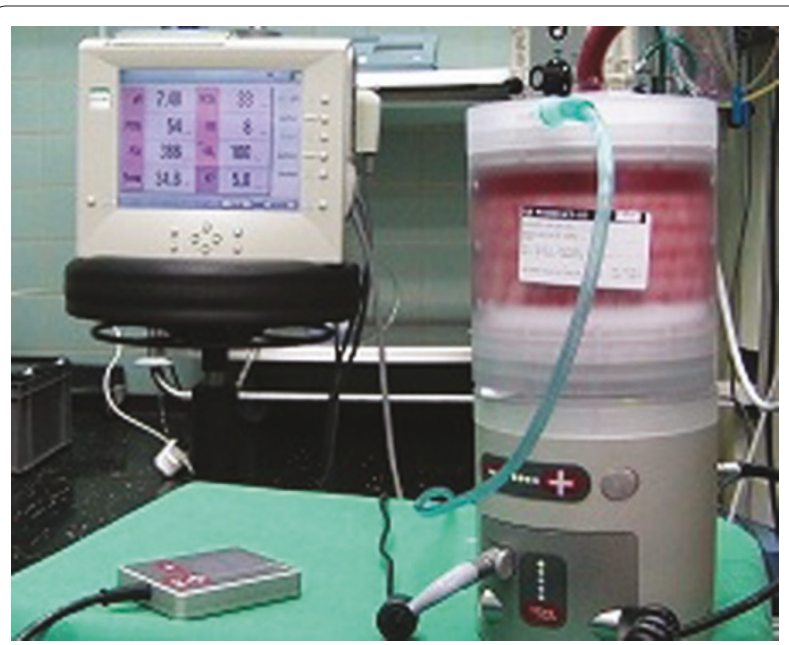

Figure 1 (abstract P96). The llias prototype.

control group, conventional ventilation; group $2(n=5)$, standard venovenous ECMO (Maquet); group $3(n=5)$, Ilias group. Gas exchange, hemodynamics, hemolysis, and coagulation activation were examined over a period of 8 hours.

Results No device failed during the observation period. Oxygenation increased significantly in both ECMO groups compared to baseline and to control ( $\mathrm{paO}_{2}$ from $79 \pm 8$ before llias to $340 \pm 108 \mathrm{mmHg}$ and from $61 \pm 12 \mathrm{mmHg}$ to $309 \pm 59 \mathrm{mmHg}$ in the standard ECMO group). The $\mathrm{CO}_{2}$ elimination by the llias reduced arterial $\mathrm{paCO}$ from $134 \pm 25 \mathrm{mmHg}$ at baseline to $53 \pm 7 \mathrm{mmHg}$. Hemodynamic instability, significant activation of the plasmatic coagulation or platelet consumption was not observed. However, hemolyses were significantly higher in the llias group compared to the Maquet group.

Conclusion The llias prototype provided excellent gas exchange with hemodynamic stability comparable to a standard ECMO system. Further development and design modifications (optimized rotation speed and surface coating of rotor) are already done and another experiment is projected to reduce hemolysis for clinical application.

\section{P97}

ECMO in nonintubated patients as a bridge to lung transplant: our experience

M Chierichetti, A Santini, F Pagan, S Crotti, A Lissoni, L Gattinoni Fondazione IRCCS Ca' Granda Ospedale Maggiore Policlinico Milano, Milan, Italy

Critical Care 2012, 16(Suppl 1):P97 (doi: 10.1186/cc10704)

Introduction Extracorporeal membrane oxygenation (ECMO) has now been used by an expanding number of centres for bridging to lung transplant (LTX) in patients with advanced cardiac and respiratory failure [1]. ECMO has been used for bridging to LTx almost exclusively in patients receiving mechanical ventilation. In order to avoid the drawbacks and complications associated with intubation and prolonged mechanical ventilation we hypothesized that the use of venovenous ECMO (VV-ECMO) in awake and spontaneously breathing patients might be an option for respiratory support in those patients who are severely deteriorating while waiting for lung transplant.

Methods We performed a retrospective analysis of seven patients (three female, mean age $31.7 \pm 12.1$ years) who underwent lung transplant while on ECMO support between May 2009 and October 2011 and who had not been ventilated for more than 24 hours before the LTx. All patients were fully awake and they kept on receiving noninvasive ventilation for a variable amount of time per day after ECMO support was started, according to clinical evaluation. Mean blood gas values before ECMO support was started were: $\mathrm{pH} 7.26 \pm 0.13, \mathrm{PaCO}_{2}$ $81.7 \pm 31.6, \mathrm{PaO}_{2} 151.4 \pm 164.2, \mathrm{PEEP} 9 \pm 4, \mathrm{FiO}_{2} 83 \pm 20$, mean time on ECMO before LTX $11.7 \pm 17.7$ days.
Results All patients survived successfully until the transplant. All patients underwent BLTX on VV-ECMO support, three were converted to VA during transplant and then back to VV at the end of the procedure. One patient died after BLTX due to hemorrhagic complications. Mean ECMO support was BloodFlow $3.1 \pm 0.8 \mathrm{l} /$ minute, GasFlow $4.7 \pm 2.5 \mathrm{l} /$ minute, no one needed mechanical ventilation before BLTx. After lung transplant five patients remained intubated and they were ventilated for $13.9 \pm 16.4$ days. Mean duration of ECMO support after LTx was $4.7 \pm 5.4$ days. Mean ICU LOS after LTx was $18 \pm 17.9$ days. Among this population three patients developed hemorrhagic complication, two primary graft dysfunction, two neuromuscular dysfunction, while only one chronic renal failure.

Conclusion Our experience shows that bridge to lung transplant with VV-ECMO in awake and spontaneously breathing patients is not only feasible but also successful. Survival to BLTx in our center was $100 \%$, while survival after BLTx is comparable to that of patients who were not on ECMO support.

\section{Reference}

1. Bermudez CA, et al:: Extracorporeal membrane oxygenation as a bridge to lung transplant: midterm outcomes. Ann Thorac Surg 2011, 92:1226-1232.

P98

Extracorporeal life support in major trauma: case series from a tertiary referral trauma

R Spina, S Biondi, A Circelli, G Cianchi, S Degl'Innocenti, M Bonacchi, A Peris

Careggi Teaching Hospital, Florence, Italy

Critical Care 2012, 16(Suppl 1):P98 (doi: 10.1186/cc10705)

Introduction Major trauma (MT) is a leading cause of death and disability worldwide. Immediate fatal complications are bleeding shock followed in the post-emergency phase by severe head and spine injury and post-traumatic respiratory failure. Venoarterial (va) or venovenous (vv) extracorporeal life support (ECLS) could represent a valid option to face these life-threatening trauma complications. Moreover, ECLS can potentially be employed to expand the pool of donors in patients with brain death diagnosis after trauma event.

Methods Patient data were collected from January 2009 to November 2011. A multidisciplinary algorithm-based protocol was written by our ECLS Team. The va-ECLS indications were bleeding shock, with potential controllable bleeding sites, not responding to massive fluid and blood resuscitation and to vasopressor support and cardiac arrest not responding after 10 minutes of cardiopulmonary resuscitation (CPR). vv-ECLS criteria establishment were severe hypoxia and/or hypercarbia due to acute lung failure not responding to conventional ventilatory strategies. The ECLS device is composed of a centrifugal pump and a hollow fiber membrane oxygenator (Quadrox-D; Maquet, Germany). Coagulation status was controlled by the activated partial thromboplastin time every 4 hours and thromboelastography.

Results A total of 19 trauma patients, 15 males and four females, underwent the ECLS technique. All of the following data are expressed as median and 25th and 75th percentiles are enclosed in parenthesis. Median age was 48 (31.8 to 63.8) years. Injury severity score was 59 (41.3 to 73.8 ). Thirteen patients had polytrauma with brain injury. Fourteen patients received va ECLS and five patients vv ECLS. Indications to ECLS placement were: cardiac arrest in nine patients, severe bleeding shock in five patients and acute respiratory failure in five patients. In four patients, ECLS was placed in the shock room, two patients received ECLS in the operating room during damage control surgery and 13 patients in the ICU. Timing to ECLS from the trauma event was within 6 hours for six patients, between 6 and 24 hours for five patients and over 24 hours for eight patients. Sixteen patients were admitted to the ICU. Five patients were discharged from the ICU. Brain death diagnosis as a consequence of traumatic injury was performed in six patients. In four of these patients organ donation was possible.

Conclusion ECLS in a multimodal approach in MT represents a challenging opportunity to face potential fatal complications. Moreover ECLS applied to selected trauma patients could expand the potential donor pool. 
P99

Two years' experience with bicaval dual lumen cannula for venovenous extracorporeal membrane oxygenation in adult refractory acute respiratory distress syndrome

J Reeb, PE Falcoz, J Pottecher, X Delabranche, N Santelmo, A Steib, M Hasselmann, G Massard

Hôpitaux universitaires de Strasbourg, France

Critical Care 2012, 16(Suppl 1):P99 (doi: 10.1186/cc10706)

Introduction Venovenous extracorporeal membrane oxygenation (ECMO) is a respiratory support increasingly used in adult refractory acute respiratory distress syndrome (ARDS). Technological advances such as bicaval dual lumen cannula (DLC) allow one to decrease drawbacks associated with this cardiopulmonary bypass technique and to implement it in the ICU setting. We report our 2 years' experience of using DLC for ECMO in adult refractory ARDS.

Methods A prospective single-center study between November 2009 and November 2011 including all medical and surgical adult patients receiving ECMO for refractory ARDS. All ECMOs were performed with DLC implanted percutaneously in the right internal jugular vein. Variables under study were: arterial blood gases, duration of ECMO support, activated cephalin time (TCA) values, number of blood products transfused, and patient's outcome. Statistical test: Student's $t$ test.

Results Twenty-five ECMOs were performed in 24 patients (16 men and eight women). Mean age of patients was 52.2 years \pm 17.5 . All these patients had severe ARDS despite optimal medical therapy. At DLC implantation, mean $\mathrm{pH}, \mathrm{PaCO}_{2^{\prime}}, \mathrm{PaO}_{2^{\prime}}$ and $\mathrm{PaO}_{2} / \mathrm{FiO}_{2}$ ratio were $7.25 \pm 0.11,60.5 \pm 17.5 \mathrm{mmHg}, 58.9 \pm 13.6 \mathrm{mmHg}$, and $61 \pm 14$ respectively. Mean duration of respiratory support with ECMO was $9.5 \pm 4.8$ days and mean blood flow was $3.3 \pm 0.6 \mathrm{l} /$ minute. During ECMO, arterial blood gases were significantly improved $(P<0.05)$ : mean $\mathrm{PaCO}_{2}$, and $\mathrm{PaO}_{2}$ were $39.9 \pm 4.8 \mathrm{mmHg}$, and $92.7 \pm 21.1 \mathrm{mmHg}$ respectively. Concerning haemostasis and provision of blood products, mean TCA and mean pellets of red blood cells transfused were $53.6 \pm 1.2$ seconds and $10.7 \pm 7$ respectively. Eleven patients $(46 \%)$ died under ECMO. Causes of death with ECMO support were: five multiorgan failures, two septic shocks, two withdrawal of care, one hemodynamic shock, and one refractory hypoxemia. ECMO withdrawal was possible in 13 patients (twice in one patient) with $\mathrm{PaO}_{2} / \mathrm{FiO}_{2}$ ratio $258 \pm 24$ at withdrawal. Removal of the endotracheal tube was performed for eight patients (33\%), $18.3 \pm 6.2$ days after DLC implantation. Eight patients (33\%) were discharged alive from the ICU, $18.3 \pm 6.3$ days after DLC implantation. These eight patients were discharged alive from hospital. No adverse event related to the DLC was observed.

Conclusion ECMO with a bicaval DLC is feasible in the ICU. It improves significantly haemostasis parameters in patients suffering from refractory ARDS. DLC also decreases drawbacks associated with the ECMO respiratory support.

\section{P100}

Resolution of organ functional scores to predict outcomes in severe acute respiratory distress syndrome patients receiving extracorporeal membrane oxygenation

L Chiu, Y Chen, H Hu, C Huang, F Tsai, K Kao

Chang Gung Memorial Hospital, Taoyuan, Taiwan

Critical Care 2012, 16(Suppl 1):P100 (doi: 10.1186/cc10707)

Introduction Extracorporeal membrane oxygenation (ECMO) may be used as an alternative therapy for severe acute respiratory distress syndrome (ARDS) patients who have failed conventional mechanical ventilation. We undertook a study to investigate the determinants of mortality and the sequential evolution of organ failures in ECMO-treated ARDS patients.

Methods This was a prospective observational study of severe ARDS patients who received venovenous ECMO in the ICU of Chang Gung Memorial Hospital between March 2006 and December 2010. We included data on all 38 consecutive patients who receive venovenous ECMO. Retrospective data included the following: demographics, primary diagnosis for ARDS, ventilator setting before ECMO, oxygenation, durations of ECMO, SOFA scores and outcome.

Results A total of 38 severe ARDS patients receiving ECMO were eligible. The causes of ARDS in these 38 patients were pneumonia in 21 patients, trauma in 10 patients, sepsis in three patients, pulmonary hemorrhage in two patients and others in two patients. The overall hospital mortality rate was 39\% (15/38). Compared with the nonsurvivors group, the survivors group was younger $(33.3 \pm 15.1$ vs. $52.2 \pm 18.1$ years old, $P=0.001)$ and had lower APACHE II scores $(18.7 \pm 6.3$ vs. $26.7 \pm 6.6, P=0.001)$ and SOFA scores $(10.4 \pm 2.7$ vs. $12.7 \pm 2.4, P=0.014)$. Furthermore, the survivors group had early significant resolution in the sequential SOFA scores compared with the nonsurvivors group.

Conclusion Survivors had early improvements in SOFA scores after ECMO for severe ARDS patients. SOFA score evolution may be used for evaluating the effect of ECMO.

\section{P101}

Corticosteroids for critically ill patients: an international survey of intensivists

F Lamontagne ${ }^{1}$, N Adhikari², D Cook ${ }^{3}$, KK Koo $^{4}$, F Lauzier ${ }^{5}$, KE Burns²,

I Douglas 6 , AF Turgeon ${ }^{5}$, H Quiroz', Y Poulin', K Choong ${ }^{3}$, N Ferguson ${ }^{2}$,

ME Kho ${ }^{7}$, M Duffett $^{3}$, C Chant ${ }^{2}$, O Lesur ${ }^{1}$, MO Meade $^{3}$

'Université de Sherbrooke, Canada; ${ }^{2}$ University of Toronto, Canada: ${ }^{3}$ McMaster University, Hamilton, Canada; ${ }^{4}$ University of Western Ontario, London, Canada; Université Laval, Québec, Canada; ${ }^{6}$ Denver Health Medical Center, Denver, CO, USA; ${ }^{7}$ Johns Hopkins University, Baltimore, MD, USA

Critical Care 2012, 16(Suppl 1):P101 (doi: 10.1186/cc10708)

Introduction We surveyed intensivists to evaluate their stated use of systemic steroids in the ICU. The efficacy of steroids in septic shock and ARDS remains uncertain and clinicians' perceptions of competing indications and contraindications may jeopardize future randomized controlled trials (RCT). Knowledge of current practice will inform the design of future RCTs addressing the efficacy of systemic steroids in septic shock and ARDS.

Methods We designed and conducted a self-administered survey of intensivists practicing in academic settings with expertise in ARDS clinical research. We generated questionnaire items in focus group sessions with content experts and refined them through a standardized process of clinical sensibility, pilot and intra-rater reliability testing. Respondents used a four-point scale to grade how frequently they would administer systemic steroids in a 14 different clinical situations and reported their opinions of 16 near absolute indications or contraindications for systemic steroids. Local research staff distributed the survey to all intensivists practicing in the 11 centres (Canada and USA) with most patients enrolled in the OSCILLATE trial.

Results In total, 103 of 125 potential respondents returned completed surveys (response rate $82 \%$ ). A majority of respondents 'almost always' prescribe systemic steroids in the setting of recent systemic steroid use and low blood pressure (93\%), significant bronchospasm in a mechanically ventilated patient $(93 \%)$ and vasopressor refractory septic shock (52\%). A majority of respondents would 'almost never' prescribe steroids in severe community-acquired pneumonia (81\%), ALI (76\%) and ARDS (65\%). One-half (50\%) would 'almost never' prescribe steroids for severe ARDS (50\%). The near absolute indications selected by a majority of respondents were 'known adrenal insufficiency' (99\%) and 'suspicion of cryptogenic organizing pneumonia' (89\%). The only near absolute contraindication selected by a majority of respondents was 'systemic fungal infection' (52\%).

Conclusion Certain clinical conditions may prompt intensivists to almost always prescribe systemic steroids and reduce equipoise for future placebo-controlled trials. Moreover, this survey shows that in selected academic centres a majority of intensivists do not prescribe corticosteroids for pneumonia, ALI and ARDS.

\section{P102}

Effects of salbutamol on airway characteristics in mechanically ventilated adults without COPD

J Van Rosmalen, QL Habes, I Havinga, FVan Beers, A Van Hees,

D Ramnarain, JA Van Oers

St Elisabeth Hospital, Tilburg, the Netherlands

Critical Care 2012, 16(Suppl 1):P102 (doi: 10.1186/cc10709)

Introduction In our ICU, salbutamol inhalation to prevent bronchospasm is standard care in mechanically ventilated (MV) patients. In MV patients without COPD the effect of salbutamol remains unclear. 
Table 1 (abstract P102)

\begin{tabular}{|c|c|c|c|c|c|c|c|c|c|}
\hline & $\mathrm{T}-1$ & T0 & $\mathrm{T}+1$ & $\mathrm{~T}+15$ & $\mathrm{~T}+30$ & $\mathrm{~T}+60$ & $\mathrm{~T}+90$ & $T+240$ & \\
\hline $\mathrm{R}_{\text {ins }}\left(\mathrm{cm} \mathrm{H}_{2} \mathrm{O} / \mathrm{l} /\right.$ second $)$ & $14(6)$ & $\begin{array}{c}\text { Salbutamol } \\
\text { administration }\end{array}$ & $14(4)$ & $13(5)$ & $13(5)$ & $12(4)$ & $12(5)$ & $12(4)$ & NS \\
\hline $\mathrm{R}_{\text {exp }}\left(\mathrm{cm} \mathrm{H}_{2} \mathrm{O} / \mathrm{l} /\right.$ second $)$ & $17(7)$ & & $17(6)$ & $16(6)$ & $17(8)$ & $18(10)$ & $18(8)$ & $17(7)$ & NS \\
\hline $\mathrm{C}_{\text {dyn }}\left(\mathrm{ml} / \mathrm{cm} \mathrm{H}_{2} \mathrm{O}\right)$ & $44(12)$ & & $42(11)$ & $42(11)$ & $37(10)$ & $36(9)$ & $38(9)$ & $38(8)$ & NS \\
\hline $\mathrm{C}_{\text {stat }}\left(\mathrm{ml} / \mathrm{cm} \mathrm{H}_{2} \mathrm{O}\right)$ & $49(13)$ & & $49(12)$ & $47(13)$ & $43(11)$ & $44(12)$ & $45(12)$ & $43(12)$ & NS \\
\hline Elastance $\left(\mathrm{cm} \mathrm{H}_{2} \mathrm{O} / \mathrm{l}\right)$ & $19(3)$ & & $20(4)$ & $21(4)$ & $22(4)$ & $23(4)$ & $21(4)$ & $18(8)$ & NS \\
\hline Heart rate (beats/minute) & $81(22)$ & & $84(24)$ & $85(23)$ & $87(22)$ & $85(22)$ & $82(18)$ & $84(20)$ & NS \\
\hline $\mathrm{SpO}_{2}(\%)$ & $98(1)$ & & $98(2)$ & $98(2)$ & $98(1)$ & $98(2)$ & $98(2)$ & $97(2)$ & NS \\
\hline $\mathrm{EtCO}_{2}(\mathrm{kPa})$ & $4.7(0.7)$ & & $4.7(0.7)$ & $4.7(0.7)$ & $4.7(0.7)$ & $4.7(0.7)$ & $4.7(0.8)$ & $4.7(0.7)$ & NS \\
\hline
\end{tabular}

Therefore we examined the effect of inhaled salbutamol on resistance and compliance in MV patients without COPD.

Methods In this prospective study, we enrolled 11 critically ill MV patients without COPD. These intubated patients were on volumecontrolled ventilation $(6 \mathrm{ml} / \mathrm{kg} / \mathrm{PBW})$. Exclusion criteria were the use of $\beta$-blockers, propofol or neuromuscular blockers. They received five puffs of salbutamol (100 $\mathrm{\mu g} /$ puff) delivered by metered dose inhaler via the adapter on the Y-piece. Ventilator settings and body position were unchanged during the study. Before and after salbutamol administration vital signs were recorded and lung mechanics were measured using the ventilator (Servo- $\mathrm{i}^{\oplus}$ or Hamilton-G5 ${ }^{\oplus}$ ) at $-1,+1$, $+15,+30,+60,+90$ and +240 minutes. Values after administration of salbutamol (T0) were compared to those before administration. Results are presented as mean \pm SD. Data were evaluated by paired $t$ test and $P<0.05$ was taken as statistically significant.

Results The study group consisted of seven men and four women, mean age 53 years. Underlying causes for ventilation were diverse. The median time spent on the ventilator before inclusion was 36 hours (6 to 151). After salbutamol administration inspiratory resistance and dynamic compliance decreased, but not significantly. Expiratory resistance, dynamic compliance, elastance, $\mathrm{SpO}_{2}$ and $\mathrm{EtCO}_{2}$ did not change (Table 1).

Conclusion There was no significant effect of salbutamol inhalation on airway characteristics and vital signs in non-COPD patients on MV. Therefore standard salbutamol inhalation in MV patients without COPD can be aborted.

Reference

1. Malliotakis $P$, et al:: Influence of respiratory efforts on $\beta_{2}$-agonist induced bronchodilation in mechanically ventilated COPD patients: a prospective clinical study. Respir Med 2007, 101:300-307.

\section{P103}

Respiratory system elastance monitoring during PEEP titration YS Chiew', JG Chase1', GM Shaw ${ }^{2}$, T Desaive ${ }^{3}$

'University of Canterbury, Christchurch, New Zealand; ${ }^{2}$ Christchurch Hospital, Christchurch, New Zealand; ${ }^{3}$ University of Liege, Belgium

Critical Care 2012, 16(Suppl 1):P103 (doi: 10.1186/cc10710)

Introduction PEEP selection during mechanical ventilation (MV) for patients with acute lung injury (ALI) and acute respiratory distress syndrome (ARDS) remains a challenge for clinicians. Clinicians often rely on experience and intuition in setting $\mathrm{MV}$, resulting in a more variable treatment and outcome. We hypothesise that monitoring patient-specific respiratory system elastance (Ers) during PEEP change may provide an insight into the patient's condition.

Methods Thirteen patients with ALI/ARDS underwent a step-wise PEEP increase $\left(5 \mathrm{cmH}_{2} \mathrm{O}\right)$ recruitment manoeuvre (RM) until peak airway pressure reaches $45 \mathrm{cmH}_{2} \mathrm{O}$. Airway pressure and flow profile were recorded using a pneumotachometer. The change of patient's respiratory system elastance (Ers $=1 /$ compliance) and the end of expiratory lung volume (EELV) during RM were estimated and studied. The trials were approved by New Zealand South Island Regional Ethics Committee.

Results The median (IQR) Ers over all patients was $34.0 \mathrm{cmH}_{2} \mathrm{O} / \mathrm{l}$ (26.1 to 51.0 ), reflecting the heterogeneity of the patients and their response to PEEP. This outcome supports the idea that MV/ PEEP should be individualised. During RM, patients' Ers decreased with PEEP increase until a specific minimum and increase at higher PEEP. The decreased of Ers suggest alveolar recruitment whereas an increase of Ers at higher PEEP shows potential overinflation. An example is shown in Figure 1 a. A clear inflection/minimum Ers can be found in Figure 1a, indicating a

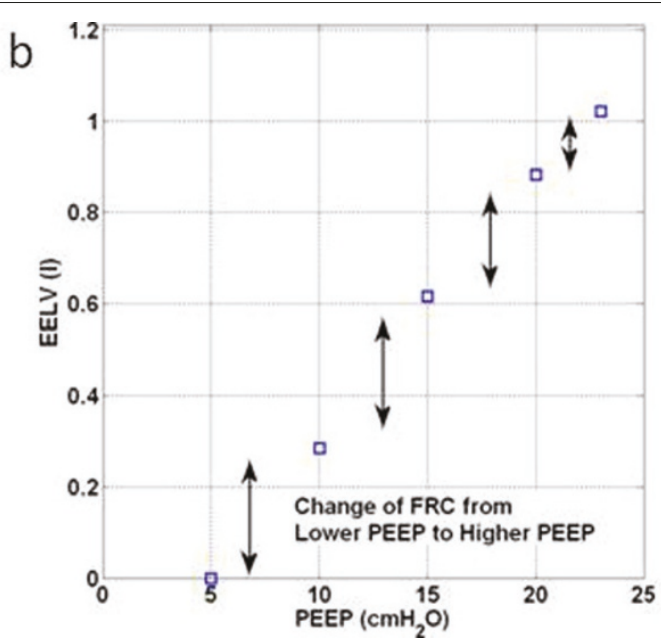

Figure 1 (abstract P103). Ers and EELV change with PEEP increase. 
potential method to optimise PEEP selection for a particular patient. Figure $1 \mathrm{~b}$ shows the change of patient's EELV with PEEP increase. As PEEP increases, the potentially recruitable collapsed lung decreases.

Conclusion The change of patient-specific Ers and EELV during minimally invasive PEEP titration provides an insight into the patient's lung condition, and thus could potentially be used as a method to individualise MV treatment and, in particular, PEEP selection.

\section{P104}

Accuracy of the pressure-volume curve method compared to quantitative lung $\mathrm{CT}$ scan to assess the recruitable lung in patients with acute respiratory failure

D Chiumello', A Marino ${ }^{2}$, I Cigada 2 , F Menga², M Brioni², IR Piva ${ }^{2}$

'Fondazione IRCCS Ca' Granda - Ospedale Maggiore Policlinico, Milan, Italy; ¿Università degli Studi di Milano, Milan, Italy

Critical Care 2012, 16(Suppl 1):P104 (doi: 10.1186/cc10711)

Introduction In patients with acute lung injury the knowledge of recruitable lung is useful for a physiological PEEP setting. The quantitative lung CT scan analysis remains the reference method [1]. However, it is time consuming and often it is not applicable in clinical management. The PV curve at two PEEP levels has been proposed as an alternative method [2]. The aim of this study was to evaluate the accuracy of these two methods in predicting the lung recruitability. Methods Sedated and paralyzed patients underwent a PV curve using the low-flow method and whole-lung CT scan at 5 and $15 \mathrm{cmH}_{2} \mathrm{O}$ of PEEP. The lung recruitability was defined as the decrease in the not aerated tissue by the quantitative lung $\mathrm{CT}$ analysis and as the difference between the lung volume computed on the two PV curves for an airway pressure of $20 \mathrm{cmH}_{2} \mathrm{O}$.

Results Ten patients (mean age $65.4 \pm 10.4$ years, body mass index $24.0 \pm 6.8 \mathrm{~kg} / \mathrm{m}^{2}, \mathrm{PaO}_{2} / \mathrm{FiO}_{2} 181 \pm 37$ ) were enrolled. The mean recruitable lung was $3.9 \pm 6.3 \%$ of the total lung weight and $218 \pm 266$ $\mathrm{ml}$ for the quantitative lung CT scan and PV curve. The linear regression between the two methods (Figure 1) was not significant $(P=0.338$ and $R^{2}=0.115$ )

Conclusion The recruitable lung computed as the difference in not aerated tissue was not related to the difference in volume estimated by the PV curve. The role of the PV curve to estimate the lung recruitability remains to be elucidated.

\section{References}

1. Gattinoni L, et al.: Lung recruitment in patients with the acute respiratory distress syndrome. N Engl J Med 2006, 354:1775-1786.

2. Ranieri VM, et al:: Volume-pressure curve of the respiratory system predicts effects of PEEP in ARDS: 'occlusion' versus 'constant flow' technique. Am J Respir Crit Care Med 1994, 149:19-27.

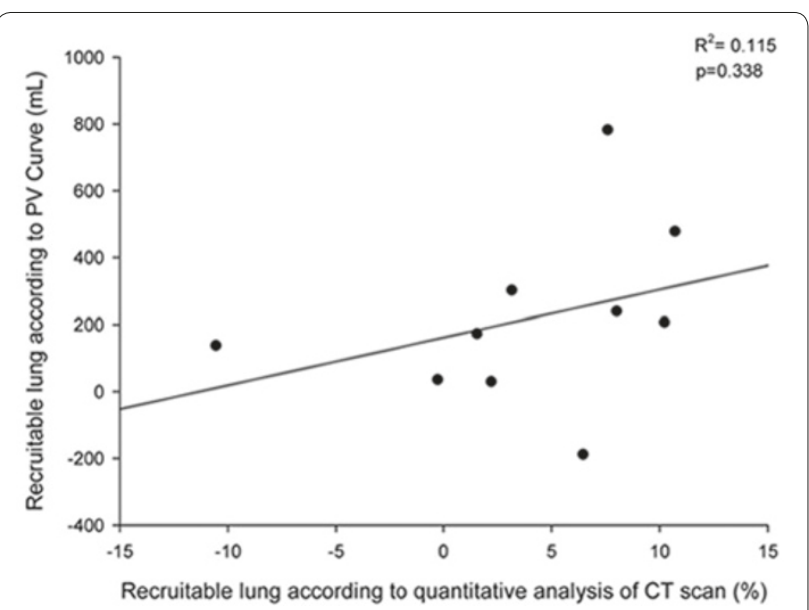

Figure 1 (abstract P104). Linear regression between quantitative CT scan analysis and PV curve method.
P105

Flow-controlled expiration discloses PEEP-dependent dynamic hysteresis of the pressure-volume loop

S Schumann', L Vimlati², M Lichtwarck-Aschoff2', J Guttmann'

'University Medical Center Freiburg, Germany; ${ }^{2}$ Uppsala University, Uppsala, Sweden

Critical Care 2012, 16(Suppl 1):P105 (doi: 10.1186/cc10712)

Introduction Hysteresis of the pressure-volume loop is a measure for the additional energy that is required during inspiration to recruit and inflate additional alveoli. The hysteresis area is usually constructed using data from a low-flow inflation/deflation maneuver; that is, from a quasi-static situation that the lung never sees during ongoing ventilation. However, during the dynamic conditions of mechanical ventilation the hysteresis area is biased by resistive pressure portions. Therefore we uncoupled flow and volume by linearizing expiratory flow (flow-controlled expiration). This enabled calculation of compliance separately for inspiration and expiration. We hypothesized that the volume-dependent intratidal compliance profiles differ between inspiration and expiration, describing a dynamic hysteresis behavior. Methods In five Swedish Landrace Hybrid pigs weighing $26 \pm 2 \mathrm{~kg}$ the lungs were ventilated in the volume-controlled mode. PEEP was set to $0,6,12$ and $15 \mathrm{cmH}_{2} \mathrm{O}$. The flow-controlled expiration was realized by a computer-controlled expiratory resistance which was adjusted in a fashion that expiratory flow was strongly limited in the beginning and continuously facilitated towards the end of expiration. Using the gliding-SLICE method [1], intratidal inspiratory and expiratory compliance profiles were calculated from inspiration data only and from expiration data only, respectively. The dynamic hysteresis area was calculated as the area within the dynamic tracheal pressurevolume loop. The relative hysteresis area was calculated as the quotient of hysteresis area divided by the rectangular area which is limited by the minima and maxima of pressure and volume of the respective pressure-volume loop [2].

Results Intratidal compliance profiles of inspiration and expiration differed strongly in mean value and slope at low PEEP. With increasing PEEP the inspiratory compliance profile approximated closer to the expiratory compliance profile. This was accompanied by a decreased relative hysteresis area by $26 \%$.

Conclusion Flow-controlled expiration allows for calculation of respiratory system compliance separately for inspiration and expiration. This compliance displays the hysteresis behavior of the respiratory system during uninterrupted ventilation. Such analysis, which is similar to the time-honored quasi-static hysteresis area analysis, could be helpful for finding an optimal PEEP.

References

1. Schumann et al:: Anesthesiology 2011, 114:1111-1117.

2. Bachofen H, Hildebrandt J: J Appl Physiol 1971, 30:493-497.

P106

Feasibility of early spontaneous breathing in acute respiratory

distress syndrome

S Mortaza, A Mercat

CHU Angers, France

Critical Care 2012, 16(Suppl 1):P106 (doi: 10.1186/cc10713)

Introduction Airway pressure release ventilation (APRV) is as a pressure preset mode that allows unrestricted spontaneous breathing throughout the entire ventilator cycle. In ARDS, this mode may decrease the need for sedation, increase alveolar recruitment and improve hemodynamic tolerance. The aim of this study is to assess the feasibility of a protocol combining APRV settings and sedation adaptation in order to obtain levels of spontaneous breathing between 10 and $50 \%$ of total minute ventilation.

Methods We designed a monocentric study including 10 patients with early ARDS. We used a Dräger Evita XL ventilator. We initially used the volume-assist control (VAC) mode to set the PEEP, tidal volume and respiratory rate according to the increased recruitment strategy of the ExPress trial [1]. These settings were used to adjust the ventilator's parameters in APRV mode: low pressure at the same level as the PEEP applied in VAC, high pressure $\left(<32 \mathrm{cmH}_{2} \mathrm{O}\right)$ set to reach a tidal volume 


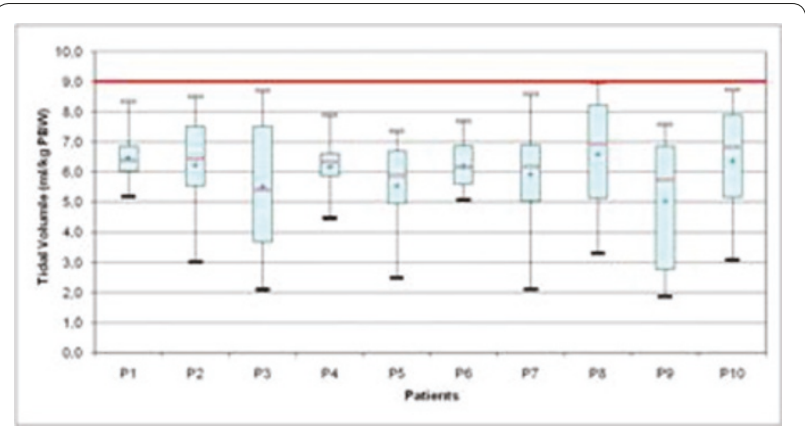

Figure 1 (abstract P106). Tidal volume distribution ( $\mathrm{ml} / \mathrm{kg} \mathrm{PBW)} \mathrm{for}$ 5 days for each patient.

of $6 \mathrm{ml} / \mathrm{kg}$ of predicted body weight (PBW), T high between 0.8 and 1 second, T low set to reach the same respiratory rate as in VAC. Patients were initially paralyzed. Then sedation was adapted to keep RASS $\geq-4$ and a level of spontaneous breathing between 10 and $50 \%$ of total minute ventilation. A computer was continuously connected to the ventilator for 5 days in order to record respiratory variables.

Results At inclusion, baseline characteristics of the patients were the following: five men and five women, 59 years old on average (25 to 85), SAPS II score of 42 (20 to 71), $\mathrm{PO}_{2} / \mathrm{FiO}_{2}$ ratio of 107 (74 to 175) and respiratory system compliance (Cst,rs) of $25 \mathrm{ml} / \mathrm{cmH}_{2} \mathrm{O}$ (18 to 36). We did not observe any pneumothorax. Eight of patients had RASS $\geq-4$ during the 5 days of enrollment. From day 2, the level of spontaneous breathing ranged between 10 and $50 \%$ of total minute ventilation in eight patients. The tidal volume (spontaneous and mechanical) measured for 5 days for each patient was mainly distributed around $6 \mathrm{ml} / \mathrm{kg}$ PBW (Figure 1). The mean $\mathrm{PO}_{2} / \mathrm{FiO}_{2}$ ratio increased from $107 \pm 29$ at enrollment to $173 \pm 91$ at day $5(P<0.05)$.

Conclusion APRV settings and the protocol for sedation used in this trial allowed to reach sufficient spontaneous breathing for the majority of the patients without any major complication and with a tidal volume below $8 \mathrm{ml} / \mathrm{kg}$ PBW. For a few patients, spontaneous breathing seems to be hard to obtain, especially within the first 2 days.

\section{Reference}

1. Mercat A, Richard JC, Vielle B, et al.; Expiratory Pressure (Express) Study Group: Positive end-expiratory pressure setting in adults with acute lung injury and acute respiratory distress syndrome: a randomized controlled trial. JAMA 2008, 299:646-655.

P107

A device for ventilation-analogue mechanostimulation in vitro K Gamerdinger, M Schneider, E Smudde, J Guttmann, S Schumann University Medical Center Freiburg, Germany Critical Care 2012, 16(Suppl 1):P107 (doi: 10.1186/cc10714)

Introduction The mechanical stress-strain characteristics of most living tissues is nonlinear and frequency dependent. During spontaneous breathing the mechanical strain on the pulmonary tissue is akin to a sinusoidal profile. In contrast, during mechanical ventilation the stimulation profile of the lung tissue differs considerably from a sinusoidal pattern. While all in vitro experiments aiming at dynamic stimulation typically use sinusoidal patterns, we here describe the establishment of a new device affording a ventilation-analogue stimulation pattern, allowing a better imitation of the situation in vivo. The new device includes a linear motor connected to four piston pumps and it allows the identical stimulation of four probes at the same time. Here we show how we stressed four test samples with sinusoidal, rectangular and ventilation-analogue mechanostimulation and how we analyzed them for frequency contents by means of a fastFourier transform.

Methods Silicone membranes serving as substitutes for biological tissue samples were placed inside a bioreactor [1] either in single-membrane or in double-membrane configuration. Cyclic mechanostimulation at repetition rates ranging from $15 /$ minute to 2,000 /minute at amplitude volumes of 0.5 up to $2.8 \mathrm{ml}$, corresponding to a surface increase of $5 \%$ up to $100 \%$, were used. The system was driven with sinusoidal, rectangular and ventilation-analogue profiles simulating the ventilatory pattern which is associated with the volume-controlled ventilation.

Results The drive system allowed us to vary the amplitude from 0 up to $100 \%$ surface increase. At amplitudes of 0.5 and $1.0 \mathrm{ml}$ we were able to apply a frequency range from 0 up to 2,000/minute, and at an amplitude of $2.0 \mathrm{ml}$ a frequency range from 0 up to 800 sinusoidal deflections per minute. We were able to apply the rectangular and the ventilationanalogue volume patterns to the probes. Close inspection of the pressure curves revealed that rapid volume increases were followed by peaks with subsequent relaxation decays when rectangular or ventilation-analogue stimulation patterns were applied. The frequency spectra of the pressure variation revealed side frequencies of up to $10 \mathrm{~Hz}$ for the rectangular mechanostimulation profile.

Conclusion With our new mechanostimulation system we are able to configure the frequency content of the applied strain profile and furthermore to identify the frequency content of the resulting stress on the tissue.

Reference

1. Schumann S, et al.: J Biomed Mater Res B Appl Biomater 2008, 86:483-492.

\section{P108}

Hemodynamics effects of recruitment maneuver

D Protsenko, R Magomedov, O Ignatenko, Al Yaroshetskiy, B Gelfand

Russian National Research Medical University, Moscow, Russia

Critical Care 2012, 16(Suppl 1):P108 (doi: 10.1186/cc10715)

Introduction Acute respiratory distress syndrome (ARDS) is a frequent complication in critically ill patients. Recruitment maneuver (RM) is a rescue procedure which improves oxygenation [1-3]. However, it is not clear whether improving oxygen delivery $\left(\mathrm{DO}_{2}\right)$ exists after $\mathrm{RM}$. The aim of this study was to evaluate the effects of RM on hemodynamics and $\mathrm{DO}_{2}$.

Methods A prospective, randomized trial in ARDS patients (AECC criteria). The protocol was approved by the local ethics committee. Fifty-seven patients with extrapulmonary ARDS were randomized into three groups: group $\mathrm{A}(n=17)-40 \times 40 \mathrm{RM}\left(\mathrm{CPAP} 40 \mathrm{cmH}_{2} \mathrm{O}\right.$ for 40 seconds), group B ( $n=17)$ - PCV RM (PIP 40 to $50 \mathrm{cmH}_{2} \mathrm{O}$, PEEP 18 to $20 \mathrm{cmH}_{2} \mathrm{O}$ for 120 seconds), and group $\mathrm{C}(n=17)$ - stepwise PCV RM. Gas exchange and systemic hemodynamics by aortal blood flow (transesophageal Doppler; ARROW, USA) were measured before, after, 30 and 120 minutes after RM.

Results In all groups we observed rapid increasing of $\mathrm{paO}_{2}(\mathrm{mmHg})$ from $65.9 \pm 24.9 ; 77.2 \pm 14.0 ; 87.0 \pm 16.7$ to $110.3 \pm 38.7 ; 124.5 \pm 45.5$; $115.2 \pm 32.6(P<0.0001)$ after RM. We also observed significant improvement of oxygenation 120 minutes after RM $(95.6 \pm 25.6$; $99.3 \pm 25.3 ; 108.1 \pm 26.8$ ). There was no statistical difference between groups. Contrarily, $\mathrm{DO}_{2}\left(\mathrm{ml} / \mathrm{minute} / \mathrm{m}^{2}\right)$ after RM statistically significantly decreased from $709.5 \pm 297.5 ; 804.9 \pm 217.3 ; 811.7 \pm 638.3$ to $569.8 \pm 211.9 ; 675.5 \pm 244.7 ; 661.7 \pm 421.3(P=0.053)$ and lasted more than 2 hours. The reason for this alteration was decreasing of cardiac output (CO) from $5.3 \pm 2.5 \mathrm{l} /$ minute to $3.6 \pm 1.7 \mathrm{l} /$ minute $(P<0.0001)$ after RM. We hypothesized that the main reason for decreasing $\mathrm{CO}$ is rapid increasing of intrathoracic pressure during RM.

Conclusion Three different RMs increase oxygenation and decrease CO equally. But RM does not improve oxygen delivery due to decreasing CO.

References

1. Marini JJ, Amato MB: Lung recruitment during ARDS. In Acute Lung Injury. Edited by Marini JJ, Evans TW. Berlin: Springer; 1998:236-257.

2. Odenstedt $\mathrm{H}$, Lindgren $\mathrm{S}$, et al.: Slow moderate pressure recruitment maneuver minimizes negative circulatory and lung mechanic side effects: evaluation of recruitment maneuvers using electric impedance tomography. Intensive Care Med 2005, 31:1706-1714.

3. Medoff $\mathrm{BD}$, Harris RS, Kesselman $\mathrm{H}$, et al:: Use of recruitment maneuvers and high-positive end-expiratory pressure in a patient with acute respiratory distress syndrome. Crit Care Med 2000, 28:1210-1216. 
P109

Differential pulmonary and circulatory effects of preventive lung protective ventilation in an experimental postoperative sepsis model

J Sperber ${ }^{1}$, M Lipcsey², A Larsson², A Larsson², J Sjölin², M Castegren ${ }^{1}$ Centre for Clinical Research Sörmland, Eskilstuna, Sweden; ${ }^{2}$ Uppsala University, Uppsala, Sweden

Critical Care 2012, 16(Suppl 1):P109 (doi: 10.1186/cc10716)

Introduction It has been proposed that low tidal volume (VT) ventilation combined with higher PEEP should be used in patients with risk of developing postoperative lung injury instead of the commonly used VT of $10 \mathrm{ml} / \mathrm{kg}$ with lower PEEP [1]. Such a ventilatory mode would in theory reduce postoperative lung and organ dysfunction. However, this hypothesis has neither been tested clinically nor experimentally. Therefore we developed an experimental endotoxemic postoperative sepsis model to evaluate the effect of different modes of ventilation. Methods Twenty-five healthy pigs were randomized to three ventilation groups: I: PEEP $10 \mathrm{cmH}_{2} \mathrm{O}$, VT $6 \mathrm{ml} / \mathrm{kg}$; II: PEEP $5 \mathrm{cmH}_{2} \mathrm{O}$, VT $10 \mathrm{ml} / \mathrm{kg}$, changed to PEEP $10 \mathrm{cmH}_{2} \mathrm{O}, \mathrm{VT} 6 \mathrm{ml} / \mathrm{kg}$ at the end of laparotomy; III: PEEP $5 \mathrm{cmH}$ O, VT $10 \mathrm{ml} / \mathrm{kg}$. For all groups the plateau pressure was kept below $28 \mathrm{cmH}_{2} \mathrm{O}$, normocapnia was reached by respiratory rate and $\mathrm{FiO}_{2}$ was adjusted to reach $\mathrm{PaO}_{2}>12 \mathrm{kPa}$. Laparotomy for 2 hours was performed to simulate a surgical procedure and then a continuous endotoxin infusion was started at $0.25 \mu \mathrm{g} / \mathrm{kg} / \mathrm{hour}$ for 5 hours. Differences between groups were analyzed with ANOVA for repeated measures.

Results The groups were equal before and at the end of laparotomy. During the endotoxin infusion, $\mathrm{PaO}_{2} / \mathrm{FiO}_{2}$ was higher in groups I and II than in group III, whereas in pulmonary compliance or functional residual capacity no differences were found. In contrast, group I showed greater negative changes than group III in the circulatory variables; that is, arterial blood pressure, cardiac index, oxygen delivery and oxygen consumption. In all measured variables, group II showed an intermediate response to groups I and III, but no significant differences were found between groups I and II. Groups I and II had slightly higher mean airway pressure at the end of the experiment than group III. However, this does not explain the circulatory differences since they occurred early in the course, temporally different from the continuous slow increase of the airway pressures $(P \leq 0.01$ ANOVA group by time interaction).

Conclusion Low VT ventilation combined with higher PEEP in healthy animals exposed to laparotomy and subsequent experimental postoperative sepsis leads to a less prominent pulmonary dysfunction but to a more hypodynamic circulatory state compared to animals ventilated with a medium-high VT and lower PEEP.

Reference

1. Schultz et al:: Anesthesiology 2007, 106:1226-1231

P110

High NT-proBNP level is correlated with high PEEP, low PH and low $\mathrm{PaO}_{2} / \mathrm{FiO}_{2}$ in ARDS

Y Nassar, D Monsef, S Abdelshafy, G Hamed

Cairo University, Giza, Egypt

Critical Care 2012, 16(Suppl 1):P110 (doi: 10.1186/cc10717)

Introduction Cardiac injury may occur in ARDS patients with structurally normal hearts and may be correlated with respiratory parameters [1]. We aimed at observing NT-proBNP, troponin I and troponin T relations with different respiratory parameters in ARDS.

Methods Inclusion criteria were any adult patient diagnosed to have ARDS according to the criteria of the American-European Consensus Conference in 1994. Exclusion criteria were any structural heart disease by echo, pulmonary embolism, atrial fibrillation, renal insufficiency, and age $<18$. All patients benefited from a lung protective ventilation strategy. Plasma NT-proBNP, troponin I and troponin T were measured on day 0 and on day 2 and day 7 of ARDS diagnosis. $\mathrm{PH}, \mathrm{PaCO}, \mathrm{PaO}$, $\mathrm{P}(\mathrm{A}-\mathrm{a}) \mathrm{O}_{2}$ (alveolar-arterial gradient), $\mathrm{PaO}_{2} / \mathrm{FiO}_{2}$ ratio, a/A ratio, $\mathrm{PEEP}, \mathrm{PIP}$ (peak airway pressure), $P_{\text {mean }}, P_{\text {plat }}$ (plateau pressure) and $C_{\text {eff }}$ (effective compliance), and $\mathrm{R}_{\mathrm{aw}}$ (airway resistance) were monitored daily.
Results The study comprised 20 patients with mean age of $58.9 \pm 20.69$ years, 11 men versus nine women $(P>0.05)$. NT-proBNP was negatively correlated with $\mathrm{PH}$ on day $2(P=0.008, r=-0.53)$ and day 7 with $(P=0.02, r=-0.50)$. NT-proBNP was positively correlated with PEEP on day $2(P=0.05, r=0.46)$ and day $7(P=0.035, r=0.48)$. NT-proBNP was negatively correlated with the $\mathrm{PaO}_{2} / \mathrm{FiO}_{2}$ ratio on day $7(P=0.0035$, $r=0.60)$. However, there was no significant correlation between NTproBNP and other respiratory indices including $\mathrm{PaCO}, \mathrm{HCO}_{3}, \mathrm{PaO}_{2}$, $\mathrm{SaO}_{2^{\prime}}, \mathrm{FiO}_{2}, \mathrm{PAO}_{2}, \mathrm{P}(\mathrm{A}-\mathrm{a}) \mathrm{O}_{2}$ and a/A ratio $(P>0.05)$. Neither troponin I nor troponin $T$ showed any significant correlation with any respiratory indices $\mathrm{PH}, \mathrm{PEEP}, \mathrm{PaO}_{2} / \mathrm{FiO}_{2}, \mathrm{PaCO}_{2}, \mathrm{HCO} 3, \mathrm{PaO}_{2}, \mathrm{SaO}_{2}, \mathrm{FiO}_{2}, \mathrm{PAO}_{2}, \mathrm{P}(\mathrm{A}-\mathrm{a})$ $\mathrm{O}_{2}$ and a/A ratio on any day $(P>0.05)$. None of the cardiac markers NTproBNP, troponin I or troponin T showed any significant correlation with the lung mechanics parameters $C_{\text {dyn }}, \mathrm{R}_{\text {aw' }} C_{\text {eff' }}$ PIP, $P_{\text {plat' }}$ and $\mathrm{P}_{\text {mean }}(P>0.05)$. Conclusion High NT-proBNP level was correlated with high PEEP, low $\mathrm{PH}$ and low $\mathrm{PaO}_{2} / \mathrm{FiO}_{2}$ ratio while troponin $\mathrm{T}$ and troponin I did not show significant correlations with respiratory parameters in ARDS patients with structurally normal hearts.

Reference

1. Mitaka C, et al:: Increased plasma concentrations of BNP in patients with ALI. J Crit Care 1997, 12:66-71.

P111

Early application of high-frequency oscillatory ventilation in H1N1 influenza-related severe ARDS is associated with better outcome

S Jog, M Patel, D Patel

Deenanath Mangeshkar Hospital and Research Centre, Pune, India

Critical Care 2012, 16(Suppl 1):P111 (doi: 10.1186/cc10718)

Introduction High-frequency oscillatory ventilation (HFOV) is a promising rescue modality for refractory hypoxia and was used extensively in H1N1 influenza-related ARDS in 2009 and 2010. The aim of this study was to find predictors of successful outcome of HFOV in H1N1 influenza-related severe ARDS [1].

Methods Patients with H1N1 influenza-related severe ARDS by the new Berlin definition (applied retrospectively) receiving volumecontrolled ventilation (VCV) as per the ARDSnet protocol with $\mathrm{PO}_{2} / \mathrm{FiO}_{2}$ $\leq 100$ at $\mathrm{PEEP} \geq 12 \mathrm{cmH}_{2} \mathrm{O}$ and $\mathrm{FiO}_{2} \geq 0.7$ were connected to $\mathrm{HFOV}$ as a rescue therapy for refractory hypoxia. All patients were followed until discharge from the hospital (survivors) or death (nonsurvivors).

Results About 80 parameters were evaluated as outcome predictors of HFOV like demographics, comorbidities, clinical features, laboratory parameters, X-rays, ventilatory and blood gas parameters and therapyrelated complications. Previously collected data of 19 patients were analysed applying the new Berlin definition. Demographic, clinical, comorbidity, laboratory and radiological parameters were comparable in survivors and nonsurvivors. Table 1 shows comparison of survivors and nonsurvivors with respect mainly to ventilatory and gas exchange parameters before application of HFOV. Duration of conventional mechanical ventilation before HFOV, $1.4 \pm 0.69$ versus $3.66 \pm 3.53$ days $(P=0.03)$, was the only discriminating parameter between survivors and nonsurvivors.

Table 1 (abstract P111). Comparison of survivors and nonsurvivors

\begin{tabular}{lccc}
\hline Variable & Survivors & Nonsurvivors & $P$ value \\
\hline APACHE & $13.3 \pm 1.7$ & $13.2 \pm 2.2$ & 0.14 \\
Time VCV & $1.4 \pm 0.69$ & $3.66 \pm 3.5$ & 0.03 \\
PIP & $35.6 \pm 7.1$ & $35.2 \pm 5.1$ & 0.44 \\
PEEP & $13.4 \pm 2.0$ & $13.4 \pm 2.8$ & 0.48 \\
P/F & $82.6 \pm 31$ & $68.8 \pm 34$ & 0.37 \\
OI & $36.08 \pm 24$ & $25.32 \pm 7$ & 0.1 \\
\hline
\end{tabular}

Conclusion In H1N1 influenza-related severe ARDS, early application of HFOV is a significant predictor of successful outcome.

Reference

1. European Society of Intensive Care Medicine: Conference Proceedings of 24th Annual Conference of ESICM, Berlin. 
P112

Outcomes of early delivery in pregnant patients with acute respiratory distress syndrome

$\mathrm{CY}$ Hung, $\mathrm{HC} \mathrm{Hu}$, CH Chang, CC Huang, KC Kao

Chang Gung Memorial Hospital, Taoyuan, Taiwan

Critical Care 2012, 16(Suppl 1):P112 (doi: 10.1186/cc10719)

Introduction Critical illnesses in pregnancy account for 0.11 to $0.89 \%$ of deliveries resulting in ICU admissions. The high rate of perinatal asphyxia in infants and high mortality rate in gravid patients supported a strategy of early delivery during the third trimester. The mortality rate of acute respiratory distress syndrome (ARDS) is high and varied from 15 to $72 \%$ among the studies. The present study reports the outcomes of early delivery within 48 hours after ICU admission in pregnant patients with ARDS.

Methods A total of 23 pregnant patients with gestational age more than 20 weeks admitted to the ICU was recorded from January 2009 to November 2012. Emergent delivery was performed within 48 hours after ICU admission. The collected data included etiologies of ICU admission, patients' characteristics and ventilator setting, infant and maternal clinical outcomes.

Results The gestational age more than 25 weeks was in 21 patients and between 20 and 25 weeks was in two patients. The mean age of these patients were $31 \pm 5.7$. The leading causes of ICU admission were obstetric emergency (26\%), cardiovascular disease $(26 \%)$ and infectious disease (26\%) in these 23 patients. A total of 19 patients were respiratory failure and ARDS was diagnosed in nine of 19 patients. Of these nine ARDS patients, tidal volume (mean: $385 \pm 31 \mathrm{ml}$ ), $\mathrm{PaO}_{2} / \mathrm{FiO}_{2}$ ratio (mean: $116 \pm 47$ ), positive end-expiratory pressure (PEEP) (mean: $13 \pm 1.4 \mathrm{mmHg}$ ), peak airway pressure (mean: $34 \pm 9.1 \mathrm{mmHg}$ ) and $\mathrm{FiO}_{2}$ (mean: $93 \pm 7 \%$ ). The intra-uterine fetal death ratio was $33 \%(3 / 9)$ and the Apgar score of the other six living births $(6 / 9)$ was $7.8 \pm 0.7$. The hospital mortality rate of these ARDS patients was only $11 \%(1 / 9)$.

Conclusion For pregnant ARDS patients, intensivists had a challenge for fetal and maternal life-threatening distress. In our study, early delivery combined with a lung-protective ventilation strategy may provide significantly better fetal and maternal outcomes.

References

1. Oram MP, et al:: Severe acute respiratory distress syndrome in pregnancy. Caesarean section in the second trimester to improve maternal ventilation. Anaesth Intensive Care 2007, 35:975-978.

2. Cole DE, et al:: Acute respiratory distress syndrome in pregnancy. Crit Care Med 2005, 33:S269-S278.

3. Bandi VD, et al:: Acute lung injury and acute respiratory distress syndrome in pregnancy. Crit Care Clin 2004, 20:577-607.

P113

Mechanical ventilation demographics between 1999 and 2009

DS Sulemanji, E Burns, R Kacmarek

Massachusetts General Hospital, Boston, MA, USA

Critical Care 2012, 16(Suppl 1):P113 (doi: 10.1186/cc10720)

Introduction Efforts at many levels are being directed at decreasing the economic burden of mechanical ventilation (MV), its related complications and their consequences. Our aim was to determine the length of MV, reintubation rates and use of noninvasive ventilation (NIV), over a 10-year period.

Methods Data were retrospectively collected using the Respiratory Care Services' Database. The number of invasive and noninvasive MV services, their sequence if both were used for a given patient, the duration of the services, and reintubation episodes for years 1999 to 2009 were extracted. Four ICUs were included; surgical, medical, neuro and burn ICUs. If a patient was reintubated within 48 hours of extubation, the case was regarded as a single episode of MV and the duration was calculated accordingly. For NIV, if restarted within 48 hours, it was counted as a single episode as well.

Results A total of 19,734 IV and 2,472 NIV episodes were identified during this period. The number of MV episodes increased from 1,660 in 1999 to 2,182 in 2009 with an increasing NIV/IV ratio (from 0.05 to 0.17 ). In the medical and surgical ICUs, median IV days decreased from 4 to 3 and 3 to 2 days respectively. Overall, $76 \%$ of IV episodes lasted $<7$ days, $14 \%$ between 8 and 14 days and $10 \%>15$ days. The number of $<7$ day IV episodes increased by $8 \%$ and $>15$ day episodes decreased by $7 \%$ from 1999 to 2009 . The overall reintubation rate was $13.8 \%$. Less than 48 -hour reintubation dropped from $14.4 \%$ in 1999 to 6.7 in 2009 while more than 48-hour reintubation remained similar (5.6\% in 1999 and $6.2 \%$ in 2009). NIV use significantly increased over this time - almost quadrupled (from 78 in 1999 to 315 in 2009). The most prominent increase was noted in the surgical and burn ICUs where, in 1999, NIV use was minimal (burn: 0 , SICU: 6 and 16 and 131 in 2009). Medical and neuro ICUs doubled their use. A total $52.7 \%$ of NIV applications were associated with IV within 48 hours of NIV therapy. The sequence of IV-NIV revealed similar patterns through the years, overall, $58.4 \%$ of NIV application followed extubation, $24.2 \%$ preceded intubation and $17 \%$ was in between.

Conclusion We found that the duration of MV decreased, reintubations within 48 hours decreased and the use of NIV increased over this 10year period. The analysis of outcomes from our data has yet to be completed, but it would not be premature to speculate these results are related to the incorporation of SBT protocols and awaking trials, lesser use of neuromuscular blocking agents as well as extensive application of lung-protective ventilation strategies.

P114

Mechanical ventilation in intensive and critical care units of Russia: RuVent national epidemiologic study

DN Protsenko', AI Yaroshetskiy', SG Suvorov², AU Lekmanov², BR Gelfand

${ }^{1}$ Russian National Research Medical University, Moscow, Russia; ${ }^{2}$ Moscow

Research Institute of Pediatrics and Child Surgery, Moscow, Russia

Critical Care 2012, 16(Suppl 1):P114 (doi: 10.1186/cc10721)

Introduction Experimental data have shown that mechanical ventilation can amplify or possibly trigger lung injury $[1,2]$. The biggest up-to-date clinical trial by the ARDS Network demonstrates reduction of mortality in ARDS patients with a protective lung strategy [3]. But we can see some gaps between international recommendations and real clinical practice $[4,5]$.

Methods The multicenter clinical trial included 470 patients from 101 centers (ICUs) in Russia. Inclusion criteria were all patients without age restrictions ventilated for more than 12 hours for any reason from 14 to 18 February 2011. Recruitment of centers and data collection were made online.

Results Total mortality was $35.1 \%$, mortality in ARDS was $44.9 \%$. Prevalence of ARDS was $18.7 \%$. Leading causes for initiation of respiratory support were pathology of the central nervous system (severe TBI 13.3\%, stroke $15.7 \%$, craniocephal tumors $5 \%$ ), sepsis $(8.3 \%)$, communityacquired pneumonia $(8.8 \%)$ and ARDS (10.5\%). Controlled modes of mechanical ventilation were predominant in our study (A/C 20.2\%, SIMV $45.1 \%$, BIPAP $12.6 \%$ ), other modes includes pressure support ventilation, ASB and PAV. Prevalence of noninvasive respiratory support was only $1.1 \%$. Mean tidal volume calculated by ideal body weight was $8.13(6.84$ to 9.35 ) for boys and men and 9.1 (7.6 to 10.9) for girls and women. Mean PEEP was 5 ( 4 to 8 ) in the whole study and 6 (5 to 9) for ARDS patients.

Conclusion Results of the RuVent study are comparable with international epidemiologic multicenter studies. Further investigations are needed for evaluation of the situation in ICUs which are a long distance from big medical centers.

References

1. Dreyfuss D, Saumon G: Am J Respir Crit Care Med 1998, 157:294-323.

2. Ignatenko O, Protsenko D, Yaroshetskiy A, Gelfand B: Crit Care 2010, 14(Suppl 1):P200

3. ARDS Network: N Engl J Med 2000, 342:1301-1308

4. Esteban A, et al:. JAMA 2002, 287:345-355.

5. Esteban A, et al:: Am J Respir Crit Care Med 2008, 177:170-177.

P115

Use of a fully closed-loop ventilation mode in long-term ventilated ICU patients: a prospective study

J Arnal', A Garnero', M Wysocki², D Demory', G Corno', A Berric',

S Donati', L Ducros', J Durand-Gasselin'

${ }^{1}$ Hôpital Ste Musse, Toulon, France; ${ }^{2}$ Hamilton Medical, Bonaduz, Switzerland Critical Care 2012, 16(Suppl 1):P115 (doi: 10.1186/cc10722)

Introduction IntelliVent-ASV ${ }^{\circledR}$ is a closed-loop ventilation mode that automatically adjusts ventilation and oxygenation settings in passive 
and active breathing patients. The minute volume is adjusted according to end-tidal $\mathrm{CO}_{2}\left(\mathrm{ETCO}_{2}\right)$ information in passive breathing patients (and respiratory rate in active breathing patients), and oxygenation is adjusted according to $\mathrm{SpO}_{2}$ information. This study reports the ventilation and oxygenation delivered by IntelliVent-ASV ${ }^{\circledR}$ in long-term ventilated ICU patients.

Methods This prospective, observational study included 100 patients invasively ventilated using IntelliVent-ASV ${ }^{\circledR}$ from admission to weaning or death. The rate and reason for stopping automation were recorded. Settings automatically selected, delivered ventilation, respiratory mechanics and arterial blood gas results were collected once a day. Patients were categorized in different lung conditions: normal lung, ALI/ARDS, COPD. Analysis of variance compared the ventilation-days for each type of lung condition for active and passive breathing patients. Results Patients (age 73 (64 to 79) years; SAPS II 56 (48 to 69)) were ventilated using Intellivent-ASV ${ }^{\otimes}$ to weaning or death (31\%) for a median duration of 3.0 (2.0 to 7.0) days without any safety issue. The ventilation controller was deactivated in two patients because of high $\mathrm{PaCO}_{2}-\mathrm{ETCO}_{2}$ gradient. Oxygenation controller was deactivated in seven patients for 1 day because of a poor $\mathrm{SpO}_{2}$ signal. In passive and active ventilation-days, minute volume, $\mathrm{VT} / \mathrm{PBW}$, respiratory rate, $\mathrm{FiO}_{2^{\prime}}$ and PEEP were statistically different based on lung condition. In passive ALI/ARDS ventilation-days, VT/PBW was significantly lower (7.5 (6.9 to 7.9) $\mathrm{ml} / \mathrm{kg}$ ) than passive normal lung $(8.1(7.3$ to 8.9$) \mathrm{ml} / \mathrm{kg} ; P<0.05)$ and passive COPD patients (9.9 (8.3 to 11.1$) \mathrm{ml} / \mathrm{kg} ; P<0.05)$. In passive ALI/ARDS ventilation-days, $\mathrm{FiO}_{2}$ and PEEP were statistically higher than passive normal lung (35 (33 to 47 )\% vs. 30 (30 to 31 )\% and 11 (8 to 13) $\mathrm{CmH}_{2} \mathrm{O}$ vs. 5 (5 to 6$) \mathrm{CmH}_{2} \mathrm{O}$, respectively; $P<0.05$ ). In active normal lung ventilation-days, VT/PBW was not different (8.4 (7.8 to 9.1$) \mathrm{ml} / \mathrm{kg}$ ) than in active ALI/ARDS (8.1 (7.5 to 9.3) ml/kg), and in active COPD (9.3 (8.6 to $11.6) \mathrm{ml} / \mathrm{kg}$ ). In active ALI/ARDS and COPD ventilation-days, PEEP was significantly higher than active normal lung (8 (5 to 10$) \mathrm{CmH}_{2} \mathrm{O}, 7$ (5 to 10) $\mathrm{CmH}_{2} \mathrm{O}$, and 5 ( 5 to 5$) \mathrm{cm} \mathrm{H}_{2} \mathrm{O}$, respectively; $P<0.05$ ).

Conclusion IntelliVent-ASV ${ }^{\circledR}$ can be used safely in long-term ventilated ICU patients and selects automatically different ventilation and oxygenation settings according to the lung condition, especially for passive breathing patients.

P116

Effects of low and high tidal volume and pentoxifylline on intestinal blood flow and leukocyte-endothelial interactions in mechanically ventilated rats

N Nakagawa', P Aikawa', HZ Zhang'2, C Correia', R Pazzeti',

CValente Barbas'1', T Mauad', E Silva', P Sannomiya'

'Faculdade de Medicina da Universidade de São Paulo, Brazil; ${ }^{2}$ University of

Toronto and Saint Michael Hospital, Toronto, Canada

Critical Care 2012, 16(Suppl 1):P116 (doi: 10.1186/cc10723)

Introduction The combination of high positive end-expiratory pressure (PEEP) and low tidal volume (VT) decreases some risks of mechanical ventilation, including pulmonary overdistention, damage due to cyclic opening and closing of the alveoli, and inflammatory responses that can lead to multiple-organ dysfunction. We hypothesized that high VT and high PEEP induce mesenteric microcirculatory disturbances and that those disturbances would be attenuated by pentoxifylline, which is anti-inflammatory.

Methods We anesthetized (isoflurane 1.5\%), tracheostomized, and mechanically ventilated 57 male Wistar rats with PEEP of $10 \mathrm{cmH}_{2} \mathrm{O}$ and $\mathrm{FIO}_{2}$ of 0.21 for 2 hours. One group received low VT $(7 \mathrm{ml} / \mathrm{kg})$, another group received high VT $(10 \mathrm{ml} / \mathrm{kg})$, and a third group received high VT (25 mg/kg) plus pentoxifylline. We measured mean arterial pressure, respiratory mechanics, mesenteric blood flow, and leukocyteendothelial interactions.

Results The mean arterial pressure was similar among the groups at baseline (108 mmHg (IQR 94 to $118 \mathrm{mmHg})$ ) and after 2 hours of mechanical ventilation (104 mmHg (IQR 90 to $114 \mathrm{mmHg})$ ). Mesenteric blood flow was also similar between the groups: low VT $15.1 \mathrm{ml} /$ minute (IQR 12.4 to $17.7 \mathrm{ml} /$ minute), high VT $11.3 \mathrm{ml} /$ minute (IQR 8.6 to $13.8 \mathrm{ml} /$ minute), high-VT/pentoxifylline $12.4 \mathrm{ml} /$ minute $(10.8$ to $13.7 \mathrm{ml} /$ minute). Peak airway pressure was lower $(P=0.03)$ in the lowVT group (10.4 $\mathrm{cmH}_{2} \mathrm{O}$ (IQR 10.2 to $10.4 \mathrm{cmH}_{2} \mathrm{O}$ )) than in the high-VT group (12.6 $\mathrm{cmH}_{2} \mathrm{O}$ (10.2 to $14.9 \mathrm{cmH}_{2} \mathrm{O}$ )) or the high-VT/pentoxifylline group (12.7 $\mathrm{cmH}_{2} \mathrm{O}$ (10.7 to $\left.16.0 \mathrm{cmH}_{2} \mathrm{O}\right)$ ). There were fewer adherent leukocytes $(P=0.005)$ and fewer migrated leukocytes $(P=0.002)$ in the low-VT group ( 5 cells $/ 100 \mu \mathrm{m}$ length (IQR 4 to 7 cells $/ 100 \mu \mathrm{m}$ length) and 1 cell/5,000 $\mu^{2}$ (IQR 1 to 2 cells $/ 5,000 \mu \mathrm{m}^{2}$ ), respectively) and the high-VT/pentoxifylline group ( 5 cells/100 $\mu \mathrm{m}$ length (IQR 3 to 10 cells/100 $\mu \mathrm{m}$ length) and 1 cell/5,000 $\mathrm{mm}^{2}$ (IQR 1 to 3 cells $/ 5,000 \mu \mathrm{m}^{2}$ ), respectively) than in the high-VT group ( 14 cells $/ 100 \mu \mathrm{m}$ length (IQR 11 to 16 cells $/ 100 \mu \mathrm{m}$ length) and 9 cells $/ 5,000 \mu \mathrm{m}^{2}$ (IQR 8 to 12 cells/5,000 $\left.\mu \mathrm{m}^{2}\right)$, respectively).

Conclusion Low VT with high PEEP was lung-protective, and early pentoxifylline reduced the inflammatory response to high VT with high PEEP (and presumed lung overdistention) during mechanical ventilation.

\section{P117}

A method for continuous noninvasive assessment of respiratory mechanics during spontaneous breathing

K Lopez-Navas'1, S Brandt², H Gehring², M Strutz', U Wenkebach'

'Fachhochschule Lübeck, Germany; '2Universitätsklinikum Schleswig-Holstein, Lübeck, Germany

Critical Care 2012, 16(Suppl 1):P117 (doi: 10.1186/cc10724)

Introduction The proper assessment of patient's work of breathing (WOB) is the key to a better or even automatic setting of ventilation parameters. We introduce the Occlusion+Delta method $(\mathrm{O}+\mathrm{D})$ to continuously determine resistance (R) and compliance (C), allowing one to assess noninvasively the inspiratory force.

Methods The O+D method uses a short expiratory occlusion producing immediate changes in airway pressure (Paw), flow $\left(\mathrm{V}^{\prime}\right)$ and volume (V) but not in transdiaphragmatic pressure (Pdi). The differences between an occluded and an undisturbed cycle are related by V'R + $\mathrm{V} / \mathrm{C}=$ Paw + Pdi. If both cycles are similar Pdi can be neglected, making its measurement unnecessary. Then $\mathrm{R}$ and $\mathrm{C}$ are derived from linear regression (MLR) and used to make a reconstruction of Pdi (rPdi). As control, $\mathrm{R}$ and $\mathrm{C}$ were calculated by MLR using the objectively measured (with balloon catheters) Pdi. The inspiratory pressure time product (PTPinsp) of measured Pdi (APdi) and reconstructed Pdi (ArPdi) were compared as expression of WOB.

Results After validation with simulations, we used data from two healthy adults breathing at several levels of WOB. The occlusions caused the expected signals reproducing Pdi as desired with $R$ and $C$ values typical for healthy men (Table 1). Measured and assessed PTPinsp correlated well $\left(R^{2}=0.93\right.$ and 0.89$)$ and had small mean differences (mean $\pm 2 \mathrm{SD}=1.78 \pm 3.81$ and $0.27 \pm 4.80 \mathrm{cmH}_{2} \mathrm{O}$. second) (Figure 1).

Table 1 (abstract P117)

\begin{tabular}{lcc}
\hline & Male 1 & Male 2 \\
\hline R estimated & $3.7 \pm 0.7$ & $3.2 \pm 0.7$ \\
R measured & $5.2 \pm 1.9$ & $2.9 \pm 1.2$ \\
Cestimated & $97.7 \pm 20.6$ & $85.4 \pm 18.7$ \\
C measured & $100.5 \pm 21.9$ & $76.5 \pm 18.7$
\end{tabular}

Mean $\pm \mathrm{SD}$ of $\mathrm{R}$ in $\mathrm{cmH}_{2} \mathrm{O} / \mathrm{l} /$ second and $\mathrm{C}$ in $\mathrm{ml} / \mathrm{mbar}$ (measured $=\mathrm{MLR}$ estimated $=\mathrm{O}+\mathrm{D})$.
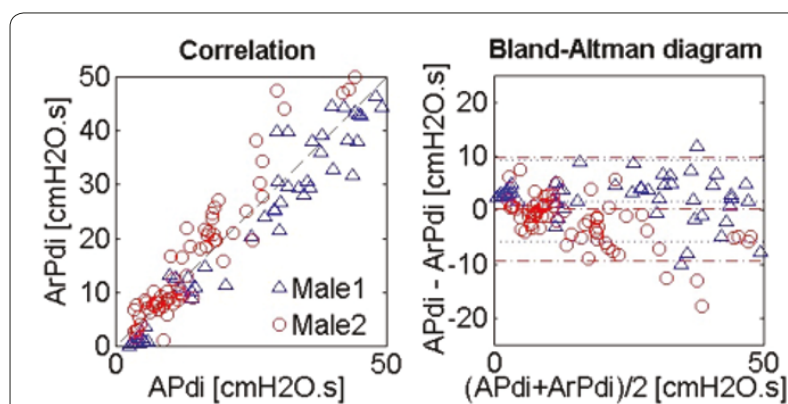

Figure 1 (abstract P117). PTPinsp from measured Pdi (APdi) versus PTPinsp from reconstruction (ArPdi). 
Conclusion Our first results demonstrate a great potential in the proposed method. A study with 30 volunteers is being carried out and results will be presented in 2012.

P118

Influence of catheter diameter, endotracheal tube diameter, suction pressure, and PEEP on the tracheal pressure and lung volume during endotracheal suctioning using a lung model KJ Snijders', PE Spronk', TW Fiks', H Boon', TTen Kleij', AA Becht', FH De Jongh ${ }^{2}$

'Gelre Ziekenhuizen, Apeldoorn, the Netherlands; '2Academic Medical Center, Amsterdam, the Netherlands

Critical Care 2012, 16(Suppl 1):P1 18 (doi: 10.1186/cc10725)

Introduction Endotracheal suctioning (ETS) is frequently performed in the ICU for clearing bronchial secretions in intubated and ventilated patients. However, research shows that subatmospheric pressures in the trachea and decreases in lung volume are measured during ETS when unfavourable parameters are chosen, causing complications (for example, atelectasis) $[1,2]$. The aim of this study was to investigate the influence of the parameters: area ratio 'catheter/endotracheal tube', suction pressure, type of ventilation, and positive end-expiratory pressure (PEEP) on tracheal pressure and lung volume during ETS. Methods A lung model (two intersurgical balloons of 2 litres each and an artificial trachea with a $25 \mathrm{~mm}$ internal diameter) for spontaneous breathing and pressure-controlled ventilation (PCV) was designed. Spontaneous breathing was simulated by varying the pressure inside the chamber in which the balloons were mounted by an electronically controlled syringe. During PCV, a Servo 300 ventilator was added. An open suction system (VBM, $5 \mathrm{~mm}$ suction gap) was used. After insertion of the catheter, suction (pressures ranged from 20 to $65 \mathrm{kPa}$ ) was applied for 15 seconds during withdrawal, as used in clinical practice. During spontaneous breathing the parameters pressure and area ratio (79\%, 58\%, 34\%, 25\%, 13\%) were varied, while during PCV the PEEP was varied too. Each setting was repeated three times and the mean results were used for analysis.

Results For spontaneous breathing $(n=45)$ the mean tracheal pressure and lung volume decreased strongly when the area ratio and/or suction pressure increased (for example, mean tracheal pressure $-13 \pm 1.3 \mathrm{cmH}_{2} \mathrm{O}$ compared to atmospheric pressure, and lung volume $-524 \pm 37 \mathrm{ml}$ using $20 \mathrm{kPa}$ suction pressure and area ratio 0.58 ), the first having the greater influence. Similar results $(n=84)$ were found for PCV (for example, $22 \pm 0.35 \mathrm{cmH}_{2} \mathrm{O}$ and $-536 \pm 137 \mathrm{ml}$, using $20 \mathrm{kPa}$ suction pressure, area ratio 0.58 and PEEP $20 \mathrm{cmH}_{2} \mathrm{O}$ ).

Conclusion During endotracheal suctioning the area ratio (between the catheter and the endotracheal tube) and the applied suction pressure should be minimal to avoid high pressure and lung volume losses.

References

1. Wood CJ, et al:. Intensive Crit Care Nurs 1998, 14:124-136.

2. Pedersen CM, et al:. Intensive Crit Care Nurs 2009, 25:21-30.
P119

Risk factors of mortality in severe cutaneous adverse reactions patients with pulmonary involvement

H Hu, K Kao, C Huang, C Yang, C Hung

Chang Gung Memorial Hospital, Taoyuan, Taiwan

Critical Care 2012, 16(Suppl 1):P119 (doi: 10.1186/cc10726)

Introduction Severe cutaneous adverse reactions, such as StevensJohnson syndrome/toxic epidermal necrolysis (SJS/TEN) and drug reaction with eosinophilia and systemic symptoms (DRESS) are uncommon but potentially critical ills. Pulmonary involvements in these severe cutaneous reactions patients are rare but are life-threatening complications. Therefore, we conducted a study to investigate the outcomes and risk factors of patients with severe cutaneous reactions with pulmonary complications.

Methods This is a retrospective study conducted in a tertiary teaching hospital in Taiwan. Between September 2002 and June 2011, 23 consecutive patients admitted to our hospital under the diagnosis of severe cutaneous adverse reactions with pulmonary involvements were enrolled. The collected demographic data included gender, age and comorbidity. Laboratory data and possible offending etiology also were collected by reviewing the medical records.

Results A total of 21 severe cutaneous adverse reactions patients were eligible. In these 21 patients, $16(76.2 \%)$ patients were SJS/TEN and five (23.8\%) patients were DRESS. Allopurinol was the most common culprit medicine $(n=9)$. There were $11(52.4 \%)$ patients progressing to respiratory failure with mechanical ventilation. Among these 11 patients, one was upper airway obstruction, two patients were pneumonia, three patients were acute respiratory distress syndrome and the other five patients were acute pulmonary edema. The overall hospital mortality rate was $47.6 \%(11 / 21)$. The survivors group was younger $(51.5 \pm 25.4$ years vs. $70 \pm 10.7$ years, $P=0.046$ ) and had less chronic kidney disease ( $9 \%$ vs. $60 \%, P=0.021$ ) compared with the nonsurvivors group.

Conclusion Severe cutaneous adverse reaction with lung involvement may contribute a high mortality rate. Older age and comorbidity of chronic kidney disease were the risk factors of mortality in severe cutaneous adverse reactions patients.

\section{P120}

During spontaneous breathing cardiac output lacks major effect on pulmonary shunting in porcine lungs with partial collapse

L Vimlati, A Larsson, G Hedenstierna, M Lichtwarck-Aschoff Uppsala University, Uppsala, Sweden

Critical Care 2012, 16(Suppl 1):P120 (doi: 10.1186/cc10727)

Introduction Spontaneous breathing (SB) improves oxygenation compared to mechanical ventilation (MV), and does so even without recruiting atelectasis $[1,2]$. Since it cannot be excluded that cardiac output (CO) impacts on pulmonary shunt, we investigated whether pulmonary shunt correlates with $\mathrm{CO}$ in a porcine model of lung collapse.

Methods $\ln 12$ anaesthetized and relaxed supine piglets, lung collapse was induced by negative pressure application to the endotracheal tube during MV. Six animals resumed SB after 15 minutes; the other six
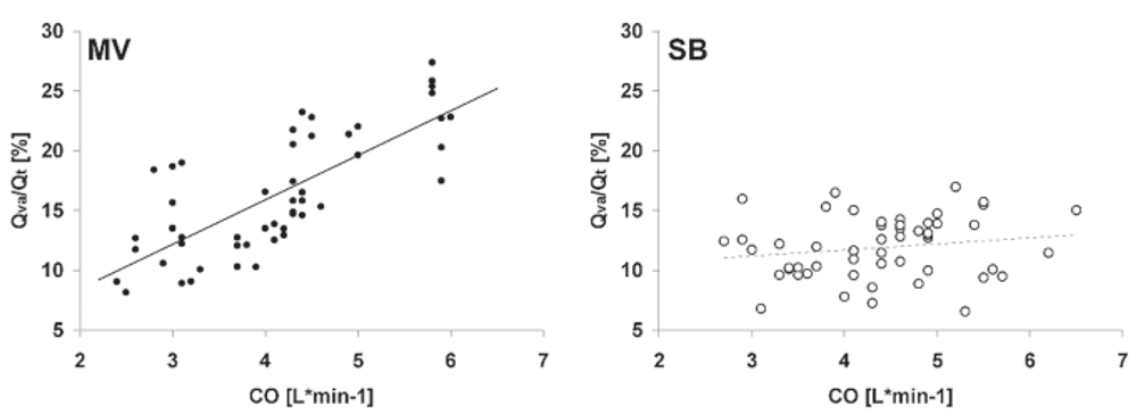

Figure 1 (abstract P120). Venous admixture (Qva/Qt) plotted against CO (pooled data for each group). Solid circles, mechanical ventilation (MV); open circles, spontaneous breathing (SB). 
were kept on $\mathrm{MV}$ at a respiratory rate and tidal volume corresponding to SB. All animals were followed over 120 minutes, and repeated measurements were converted to the area under curve and analysed by Mann-Whitney test and linear regression.

Results $\mathrm{PaO}_{2} / \mathrm{FiO}_{2}$ was higher and venous admixture (Qva/Qt) was lower in the SB group. Hemodynamics was stable and CO was similar in both groups. Qva/Qt correlated with $\mathrm{CO}(r=0.83, P=0.04)$ in the MV group, but not in the SB group $(r=0.08, P=0.88)$ (Figure 1).

Conclusion $\mathrm{SB}$ achieves higher $\mathrm{PaO}_{2} / \mathrm{FiO}_{2}$ and lower Qva/Qt compared to MV. During SB, Qva/Qt seems to be unaffected by CO. This lung collapse model has stable hemodynamics and gas exchange for at least 2 hours irrespective of the mode of ventilation.

\section{References}

1. Carvalho AR, Spieth PM, Pelosi P, et al:: Pressure support ventilation and biphasic positive airway pressure improve oxygenation by redistribution of pulmonary blood flow. Anesth Analg 2009, 109:856-865.

2. Vimlati L, Kawati R, Hedenstierna G, Larsson A, Lichtwarck-Aschoff M: Spontaneous breathing improves shunt fraction and oxygenation in comparison with controlled ventilation at a similar amount of lung collapse. Anesth Analg 2011, 113:1089-1095.

P121

Oxygenation correlates with lung aeration during unsupported spontaneous breathing in porcine lung collapse model

L Vimlati, A Larsson, G Hedenstierna, M Lichtwarck-Aschoff Uppsala University, Uppsala, Sweden

Critical Care 2012, 16(Suppl 1):P121 (doi: 10.1186/cc10728)

Introduction We investigated whether oxygenation correlates with lung aeration during unsupported spontaneous breathing (SB) and mechanical ventilation (MV) in a porcine lung collapse model.

Methods In 14 anesthetized supine piglets, lung collapse was induced by negative pressure application (NPA) to the endotracheal tube. Eight animals resumed SB 5 minutes after NPA, six animals were kept on MV at a respiratory rate and tidal volume corresponding to $\mathrm{SB}$. Thoracic CTs and arterial blood gases were taken 2.5 and 30 minutes after NPA. Spearman rank correlation was used for testing; values are given as mean $(95 \% \mathrm{Cl})$.

Results Thirty minutes after NPA the amount of lung tissue in collapsed regions was similar in both groups (MV: $40 \%$ (36 to 44), SB: 35\% (26 to 43); $P=0.22$ ). Resuming $\mathrm{SB}, \mathrm{PaO}_{2} / \mathrm{FiO}_{2}$ improved significantly more with less amount of collapsed lung tissue 2.5 minutes after NPA $(r=-0.87$, $P=0.033$ ). During SB a significant negative correlation between $\mathrm{PaO}_{2} /$ $\mathrm{FiO}_{2}$ and the amount of collapsed lung tissue $(r=-0.76, P=0.038)$ was observed; no such correlation could be seen during MV ( $r=-0.3$, $P=0.2$ ) (Figure 1).

Conclusion In porcine lung collapse $\mathrm{PaO}_{2} / \mathrm{FiO}_{2}$ correlates with lung aeration during unsupported $\mathrm{SB}$, but not during $\mathrm{MV}$ at a similar breathing pattern. The less lung collapse the animals have, the more $\mathrm{PaO}_{2} / \mathrm{FiO}_{2}$ improves resuming $\mathrm{SB}$.

Reference

1. Cressoni M, Caironi P, Polli F, et al:: Anatomical and functional intrapulmonary shunt in acute respiratory distress syndrome. Crit Care Med 2008, 36:669-675
P122

Safety and effect of intermittent intrapulmonary percussive ventilation on oxygen saturation and hemodynamic functions

I Blum, R Janssen-Dean, A Van Overdijk, B Speelberg

St Anna Hospital Geldrop, the Netherlands

Critical Care 2012, 16(Suppl 1):P122 (doi: 10.1186/cc10729)

Introduction Intrapulmonary percussive ventilation (IPV) is a ventilatory technique which is used to clear endobronchial secretions in patients. IPV uses a Phasitron, which delivers rapid, high-flow, minibursts of air mixed with oxygen to the patients. We investigated the safety of IPV on hemodynamic values and the effect of IPV on oxygen saturation and respiratory rate.

Methods From April until August 2011 we investigated 42 consecutive patients admitted to our eight-bed adult general ICU with respiratory failure. Variables such as heart rate, mean arterial pressure, respiratory rate, and oxygen saturation were measured and compared at three different time points: before starting IPV therapy, directly after and 15 minutes later. All patients received IPV using a Bird Intrapulmonary Percussionator Ventilator Model IPV-2C for a period of 20 minutes consisting of two cycles of 10 minutes. After the first 10 minutes of IPV therapy in combination with chest compressions the frequency rate was reduced in order to suction the mobilized secretions. This cycle was then repeated. Statistical analysis was done with SPSS version 17 . Student's $t$ test was used to compare values before therapy with directly after and after 15 minutes of therapy. $P<0.05$ was considered significant.

Results Neither heart rate, mean arterial pressure nor respiratory rate showed any significant change after IPV. Oxygen saturation improved immediately after IPV and was also present after 15 minutes. See Table 1.

Table 1 (abstract P122). Values before, after and 15 minutes after therapy

\begin{tabular}{|c|c|c|c|c|}
\hline & $\begin{array}{l}\text { Heart } \\
\text { rate }\end{array}$ & $\begin{array}{l}\text { Mean } \\
\text { arterial pressure }\end{array}$ & $\begin{array}{l}\text { Respiratory } \\
\text { rate }\end{array}$ & $\begin{array}{l}\text { Oxygen } \\
\text { saturation }\end{array}$ \\
\hline Before & $84.5 \pm 18.2$ & $86.6 \pm 19.0$ & $24.7 \pm 5.6$ & $93.9 \pm 3.0$ \\
\hline After & $86.0 \pm 17.6$ & $87.4 \pm 21.0$ & $24.1 \pm 6.7$ & $95.8 \pm 2.8^{*}$ \\
\hline After 15 minutes & $83.1 \pm 16.7$ & $85.4 \pm 18.9$ & $23.4 \pm 6.0$ & $95.5 \pm 2.8^{*}$ \\
\hline
\end{tabular}

${ }^{*} P<0.01$.

Conclusion We demonstrated that IPV is a safe therapy, and oxygen saturation improved after therapy with IPV.

P123

Impact of an open lung approach on hemodynamic parameters

after cardiac surgery

A Leme, F Galas, M Volpe, J Fukushima, J Almeida, R lanotti, L Hajjar,

M Amato

Heart Institute, São Paulo, Brazil

Critical Care 2012, 16(Suppl 1):P123 (doi: 10.1186/cc10730)

Introduction Lung recruitment maneuver (RM) has been associated with an increase of arterial oxygen saturation and improvement of
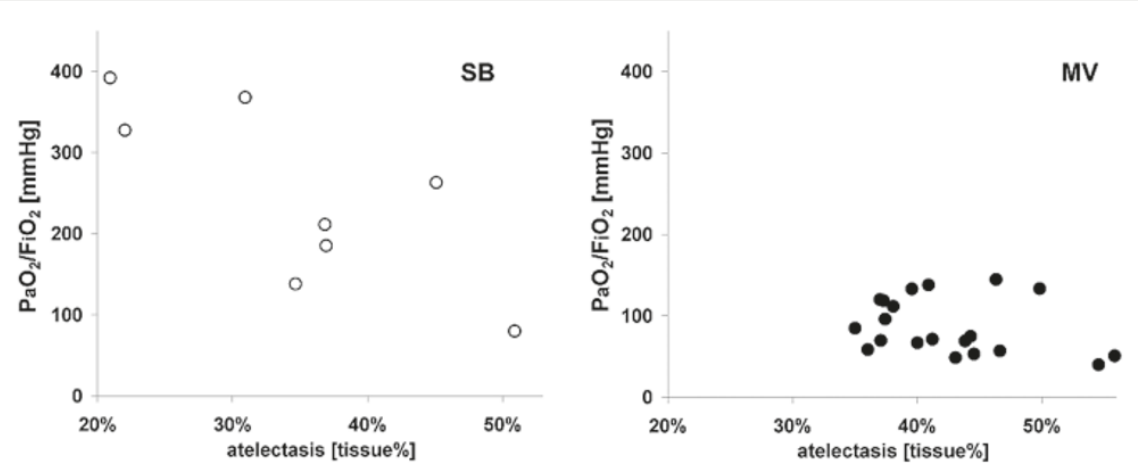

Figure 1 (abstract P121). $\mathrm{PaO}_{2} / \mathrm{FiO}_{2}$ plotted against the proportion of atelectatic lung tissue. Open circles, SB; solid circles, MV. 
respiratory parameters. Nevertheless, adverse hemodynamic effects can occur due to the RM technique. The aim of this study is to evaluate the effect of the RM on hemodynamic parameters in the immediate postoperative period after cardiac surgery.

Methods A total of 120 patients with $\mathrm{PaO}_{2} / \mathrm{FiO}_{2}$ ratio $<250$ was randomized to a conventional strategy of mechanical ventilation or open lung strategy. The open lung strategy was performed using RM with an inspiratory pressure amplitude of $15 \mathrm{cmH}_{2} \mathrm{O}$ and PEEP of 30 $\mathrm{CmH}_{2} \mathrm{O}$ three times during 1 minute and setting PEEP after RM at 13 $\mathrm{cmH}_{2} \mathrm{O}$. The conventional strategy was done using PEEP $=8 \mathrm{cmH}_{2} \mathrm{O}$ and RM with CPAP $=20 \mathrm{cmH}_{2} \mathrm{O}$ three times during 30 seconds and setting PEEP after RM at $8 \mathrm{cmH}_{2}^{2} \mathrm{O}$. The heart rate, systolic, diastolic and mean arterial blood pressures were recorded before, immediately and 5 minutes after RM. Respiratory mechanics and blood gas analysis were recorded before and after RM.

Results The open lung group presented a higher variability on blood pressure immediately after RM compared to the conventional group. There were no differences in baseline blood pressure or 5 minutes after $\mathrm{RM}$ and heart rate between groups. The open lung group presented higher lung compliance ( $60 \pm 17$ vs. $48 \pm 13 \mathrm{ml} / \mathrm{cmH}_{2} \mathrm{O}$ ) and $\mathrm{PaO}_{2} / \mathrm{FiO}_{2}$ ( $431 \pm 124$ vs. $229 \pm 68$ ) ratio compared to the conventional group.

Conclusion An open lung approach after cardiac surgery improves lung compliance and the $\mathrm{PaO}_{2} / \mathrm{FiO}_{2}$ ratio with minimum hemodynamic detrimental effect.

Reference

1. Amato MB, Barbas CS, Medeiros DM, et al.: Effect of a protective-ventilation strategy on mortality in the acute respiratory distress syndrome. NEng/J Med 1998, 338:347-354.

P124

A protective-ventilation strategy reduces pulmonary complications after cardiac surgery

F Galas', A Leme', J Almeida', M Volpe², R lanotti', J Fukushima', L Hajjar', $\mathrm{M} \mathrm{Amato}^{3}$

'Heart Institute, São Paulo, Brazil;' 2 Federal University of Triângulo Mineiro

Minas Gerais, Uberaba, Brazil; ${ }^{3}$ Hospital das Clinicas, São Paulo, Brazil

Critical Care 2012, 16(Suppl 1):P124 (doi: 10.1186/cc10731)

Introduction Cardiac surgical procedures are associated with a high incidence of postoperative complications, increasing costs and mortality. The aim of this study is to evaluate the effect of a strategy of protective ventilation on pulmonary complications after cardiac surgery.

Methods We prospectively evaluated 120 patients immediately after cardiac surgery, presenting hypoxemia and $\mathrm{PaO}_{2} / \mathrm{FiO}_{2}<250$. Patients were randomized to protective or conventional ventilation strategy. Protective strategy: PEEP $=13 \mathrm{cmH}_{2} \mathrm{O}$, recruitment maneuver (RM) with inspiratory pressure amplitude of $15 \mathrm{cmH}_{2} \mathrm{O}$ and PEEP of $30 \mathrm{cmH}_{2} \mathrm{O}$. Conventional strategy: PEEP $=8 \mathrm{cmH}_{2} \mathrm{O}$ and $\mathrm{RM}$ with CPAP $=20 \mathrm{cmH}_{2}^{2} \mathrm{O}$. Both patients were ventilated in pressure controlled at $6 \mathrm{ml} / \mathrm{kg}$. Pulmonary mechanic and oxygenation parameters were collected at baseline, 15, 240 and 255 minutes after the start of treatment. Occurrence of respiratory complications was assessed in the first 5 days according to the severity score 1 to 4 .

Results The protective group compared to the conventional group had better lung compliance ( $60 \pm 17$ vs. $48 \pm 13 \mathrm{ml} / \mathrm{cmH}_{2} \mathrm{O}, P<0.001$ ) and higher $\mathrm{PaO}_{2} / \mathrm{FiO}_{2}(431 \pm 124$ vs. $229 \pm 68, P<0.001)$ at 15 minutes after the start. Also, the protective group had a lower incidence of complications after 5 days of follow-up (grade $2=47 \%$ vs. $55 \%$, grade $3=9 \%$ vs. $13 \%$, grade $4=0 \%$ vs. $3 \%, P=0.045$ ).

Conclusion A protective-ventilation strategy after cardiac surgery reduces hypoxemia, increases lung compliances and results in less respiratory complications without adverse effects.

Reference

1. Amato MB, Barbas CS, Medeiros DM, et al.: Effect of a protective-ventilation strategy on mortality in the acute respiratory distress syndrome. NEng/ J Med 1998, 338:347-354.
P125

A rule for predicting the new equilibrated carbon dioxide partial

pressure after changes in the ventilation frequency

S Buehler, M Jensen, S Lozano, S Schumann, J Guttmann

Uniklinik Freiburg, Germany

Critical Care 2012, 16(Suppl 1):P125 (doi: 10.1186/cc10732)

Introduction In mechanical ventilation the arterial carbon dioxide partial pressure $\left(\mathrm{PCO}_{2}\right)$ is one of the key parameters to control the ventilation frequency. Qualitatively, the effect of changes in the ventilation frequency on the arterial $\mathrm{PCO}_{2}$ level is well known. However, little is known about the time it takes for the $\mathrm{PCO}_{2}$ value to reach a new equilibrium after a change in the ventilation frequency (the period of latency), nor in what way the transition between two states of equilibrium takes place.

Methods We carried out a clinical study on patients without any history of lung disease or intracranial surgery in order to determine these relations. We collected data for the arterial $\mathrm{PCO}_{2}$ from blood gas analyses at discrete points in time as well as continuous end-tidal $\mathrm{CO}_{2}$ $\left(\right.$ et $\left.\mathrm{PCO}_{2}\right)$ and transcutaneous $\mathrm{CO}_{2}\left(\mathrm{PtcCO}_{2}\right)$ data and checked for the accuracy of the latter two. Least-squares fitting and a statistical analysis were carried out.

Results We determined a general rule to estimate the period of latency after a change in the ventilation frequency. Furthermore, we specified the relation between a change in the ventilation frequency and the change in the $\mathrm{PCO}_{2}$ level. Last, the transition between two $\mathrm{PCO}_{2}$ levels was found to follow an exponential law and the fitting resulted in a formula for the prediction of the new $\mathrm{PCO}_{2}$ level. The new equilibrium can be predicted with high confidence in all cases after only 3 to 4 minutes using four data points while the period of latency lasts much longer, usually between 10 and 20 minutes.

Conclusion The general rule for the period of latency allows an estimation of the amount of time it takes for the $\mathrm{PCO}_{2}$ value to stabilise again after a disturbance. A quantitative knowledge of the transition between two $\mathrm{PCO}_{2}$ equilibria allows for the prediction of the new $\mathrm{PCO}_{2}$ level long before the period of latency is over. Thus, with our relation between the change in ventilation frequency and the change in $\mathrm{PCO}_{2}$ at hand, an optimal $\mathrm{PCO}_{2}$ level can be aimed for at bedside in the shortest time span possible.

P126

Patient-ventilator asynchrony during conventional or automated pressure support ventilation in difficult-to-wean patients

MM Bitondo ${ }^{1}$, HM Aguirre-Bermeo ${ }^{2}$, A Moccaldo', P De Santis' ${ }^{1}$ V Bernini', A Tersali', S Italiano', DL Grieco', FA Idone', J Grandjean², F Roche-Campo², M Antonelli', J Mancebo Cortes', SM Maggiore ${ }^{1}$

'Catholic University of the Sacred Heart, Roma, Italy; '2 San Pau University Hospital, Barcelona, Spain

Critical Care 2012, 16(Suppl 1):P126 (doi: 10.1186/cc10733)

Introduction Patient-ventilator asynchrony, defined as a mismatch between patient's inspiratory time and the ventilator insufflation time, occurs in nearly $25 \%$ of intubated patients. High asynchrony rates are associated with higher incidence of weaning failure and tracheostomy, and prolonged mechanical ventilation. The aim of this study was to compare the asynchrony rate during conventional pressure support ventilation (PSV) and automated PSV (SmartCare; Draeger) in difficultto-wean patients.

Methods A prospective, crossover study in difficult-to-wean patients (patients who required up to three spontaneous breathing trials (SBTs) or as long as 7 days to achieve successful weaning). Patients were ventilated with an Evita XL ventilator for two consecutive 3-hour periods applied in random order: with conventional PSV managed by the attending physicians; and with PSV managed by SmartCare. The periods were administered in the afternoon (3:00 to 9:00 pm) and in the night $(12: 00 \mathrm{pm}$ to $6: 00 \mathrm{am})$. In both periods, the starting PS level with either conventional or automated PSV was the basal level before enrolment. During every period, airway pressure, flow and volume signals were continuously recorded on a PC connected to the ventilator using dedicated software (VentView). These signals were analyzed offline by two clinicians. The asynchrony index was defined as 
the number of asynchronies (wasted efforts, double cycles, premature cycling off) divided by the total respiratory rate (ventilator cycles + asynchrony events), multiplied by 100 .

Results Sixteen patients were enrolled (age $64 \pm 11$ years; SAPS II $66 \pm 14$; COPD 25\%; days of mechanical ventilation before enrollment $9 \pm 4$, number of SBTs $3 \pm 1$ ). The asynchrony index was lower with Smartcare $(10 \%$ vs. $14 \%, P=0.01)$, but not different between afternoon and night. Mean PS level (11 vs. $12 \mathrm{cmH}_{2} \mathrm{O}$ ) was not different between conventional and automated PSV, although the coefficient of variability of PS level was greater with Smartcare $(20 \%$ vs. $0 \%, P<0.01)$. No differences were observed in $\mathrm{PaCO}_{2}$ ( 36 vs. 36 mm Hg), $\mathrm{PaO}_{2}$ (106 vs. 102 $\mathrm{mmHg}$ ), total respiratory rate (22 vs. 23$)$, and P0.1 (1.4 vs. $1.6 \mathrm{cmH}_{2} \mathrm{O}$ ) between conventional PSV and Smartcare.

Conclusion As compared with conventional PSV, Smartcare may reduce asynchronies in difficult-to-wean patients, possibly because of greater variability of the PS level. This needs to be further confirmed.

P127

High levels of B-type natriuretic peptide predict weaning failure from mechanical ventilation in adult patients after cardiac surgery

L Hajjar', T Lara', J Almeida', J Fukushima', C Barbas', A Rodrigues',

E Nozawa', JL Vincent ${ }^{2}$, F Jatene', J Auler Jr', F Galas' ${ }^{1}$

${ }^{1}$ Heart Institute, São Paulo, Brazil;' 'Erasme Hospital, Universitélibre de Bruxelles, Belgium

Critical Care 2012, 16(Suppl 1):P127 (doi: 10.1186/cc10734)

Introduction Failure to wean from mechanical ventilation is related to worse outcomes after cardiac surgery. The aim of the study was to evaluate B-type natriuretic peptide (BNP) as a predictor factor of failure to wean from mechanical ventilation after cardiac surgery.

Methods We conducted a prospective and observational cohort study of 101 patients that underwent on-pump coronary artery bypass grafting. BNP was measured postoperatively after ICU admission and at the end of a spontaneous breathing test (SBT). Demographic data, hemodynamic and respiratory parameters, fluid balance, need for vasopressor or inotropic support, lengths of ICU and hospital stay were recorded. Weaning failure was considered as either the inability to sustain spontaneous breathing after 60 minutes or the need for reintubation within 48 hours.

Results BNP levels were significantly higher both at ICU admission and in the end of breathing test in patients with weaning failure than in the other patients. A BNP concentration of $299 \mathrm{ng} / \mathrm{l}$ at the end of the SBT identified weaning failure with $92 \%$ sensitivity and $87 \%$ specificity, resulting in an area under the curve value of 0.91 ( $95 \% \mathrm{Cl}(0.86$ to 0.97$)$,

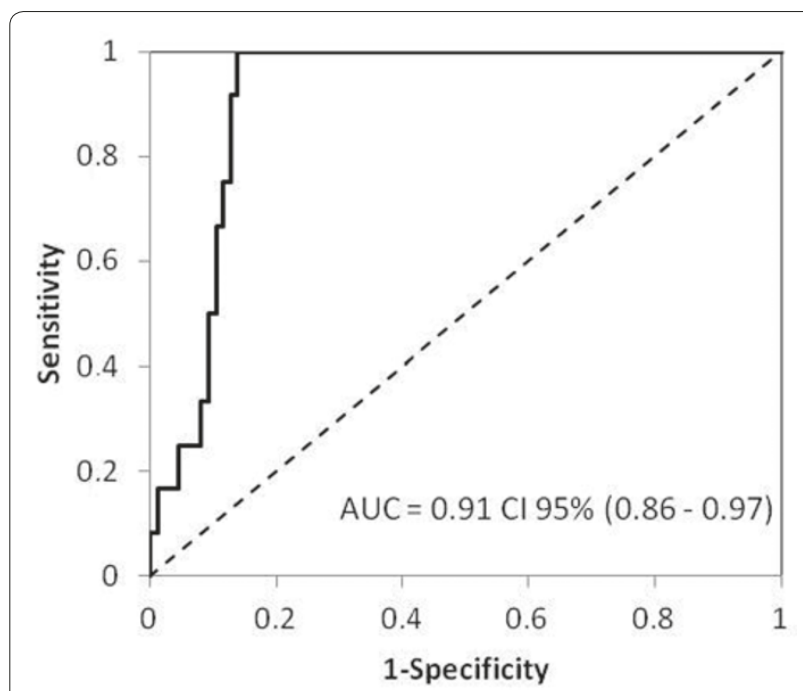

Figure 1 (abstract P127). Area under receiving operating characteristic curve for BNP-2 (at the end of spontaneous breathing test) to predict weaning failure.
$P<0.001$ ) (Figure 1). In a multivariate model, BNP level at the end of SBT was the only predictor of weaning failure from mechanical ventilation. Conclusion BNP was an independent predictor factor of failure to wean from mechanical ventilation after cardiac surgery, which suggests that optimization of the ventricular function must be a goal prior to liberation from mechanical ventilation.

\section{P128}

Case-control study of failed extubation

J Krinsley, P Reddy, A labal

Stamford Hospital, Stamford, CT, USA

Critical Care 2012, 16(Suppl 1):P128 (doi: 10.1186/cc10735)

Introduction Failed extubation (FE), defined as reintubation within 48 hours of planned extubation (PE), is common. The literature suggests that FE complicates 10 to $20 \%$ of PE. The consequences of FE have not been well described, nor have its risk factors.

Methods We performed a retrospective study of prospectively collected data involving 2,012 consecutive patients undergoing mechanical ventilation (MV) in a 16-bed university-affiliated hospital between 1 October 2005 and 31 August 2011. Eighty-five patients with FE were matched 1:3 with successfully extubated patients (SE) using diagnostic category, age, Acute Physiology Score (APS) and duration of ventilation (DOV) before PE as matching criteria.

Results Patients undergoing MV included 1,209 (60.1\%) with SE; 224 (11.1\%) died during ventilation (without prior FE); 206 (10.2\%) were extubated to withdraw support; 180 (8.9\%) were transferred from the ICU while ventilated; 81 (4.0\%) were liberated from MV after tracheostomy; 85 (6.6\%) failed PE. APS scores were higher (53 (42 to 69 ) vs. 43 (32 to 60), $P<0.0001$ ) and DOV before PE longer (1.8 (0.8 to 4.4) vs. $0.9(0.4$ to 2.6$), P=0.0001)$ in $\mathrm{FE}$ than in $\mathrm{SE}$. There was $100 \%$ concordance of diagnostic category and no statistically significant differences between the groups in regards to age, APS and DOV before PE. Table 1 illustrates the results of the case-control analysis. In addition, FE had more days in the hospital after ICU discharge than did SE: 11 (4 to 24) versus 5 ( 2 to 9 ), $P<0.0001$.

Table 1 (abstract P128). Case-control analysis of failed extubation: key outcomes

\begin{tabular}{lccc}
\hline & FE & SE & $P$ value \\
\hline ICU LOS & $11.8(7.7$ to 17.5$)$ & $3.8(2.1$ to 7.5$)$ & $<0.0001$ \\
VAP (\%) & 7.1 & 0.8 & 0.0043 \\
Mortality (\%) & 23.5 & 10.2 & 0.0052 \\
\hline
\end{tabular}

Conclusion FE is associated with increased ICU and hospital LOS, increased risk of VAP and increased mortality. Efforts to prospectively identify patients at risk for FE may reduce its incidence and improve outcomes.

P129

Out-of-bed extubation: changing paradigms

F Dexheimer Neto, R Cremonese, J Maccari, F Carlin, C Rodrigues, A Raupp, P Vesz, C Leaes, J De Andrade

Hospital Ernesto Dornelles, Porto Alegre, Brazil

Critical Care 2012, 16(Suppl 1):P129 (doi: 10.1186/cc10736)

Introduction The position of the patient at the time of extubation is an important topic as several studies have shown that early mobilization is beneficial for the critically ill patient and, generally, it occurs simultaneously with the weaning from mechanical ventilation (MV). Extubations are currently performed with the patient in a supine position (SP) with the head elevated, and there are no data available concerning the safety of removing the endotracheal tube of a patient seated in an armchair (SA). The aim of this study was to evaluate the safety of proceeding extubations in SA patients compared with those in a SP. Methods A retrospective cohort study of a clinical and surgical 23-bed ICU, in a private hospital in Brazil - Hospital Ernesto Dornelles (Porto Alegre, RS, Brazil). Extubation success was the primary outcome defined as tolerating removal of the endotracheal tube for at least 
48 hours. All statistical analysis were done using SPSS version 16 and the differences between the groups were assessed using Student's $t$ test and the chi-square test.

Results Ninety-one patients were included in the analysis - from December 2010 to June 2011. Mean ( \pm SD) age of the population was $71 \pm 12$ years, mean APACHE II score was $21 \pm 7.6$, mean duration of MV was $2.6 \pm 2$ days and mean number of spontaneous breathing trials was $1.3 \pm 0.6$. Extubation was performed in $33 \mathrm{SA}$ patients $(36 \%)$ and 58 SP patients (64\%), with a similar success rate of $82 \%$ and $85 \%$, respectively $(P>0.05)$. Furthermore, no significant differences between these groups were found in terms of APACHE II score, time of MV and postextubation distress or complications.

Conclusion The outcomes of proceeding extubation in patients seated in armchairs are similar to those extubated in supine position with the head elevated. This new practice can be considered safe and allow extubations to be performed simultaneously with early mobilization.

P130

Prediction of post-extubation failure by portable ICU ultrasound

Y Sutherasan, P Theerawit, T Hongpanat, C Kiatboonsri, S Kiatboonsri

Ramathibodi Hospital, Mahidol University, Bangkok, Thailand

Critical Care 2012, 16(Suppl 1):P130 (doi: 10.1186/cc10737)

Introduction Stridor and vocal cord oedema are common in ICU patients. Currently, the cuff leak volume test is a standard technique to assess these complications [1,2]; however, wide variations in terms of its sensitivity and specificity have been demonstrated in many studies. Recently, ultrasound is a promising noninvasive method widely used in ICU patients and allows visualization of the vocal cords and larynx [3]. Thus, we would like to determine the diagnostic accuracy of portable ultrasound for detection of these post-extubation complications.

Methods We conducted a prospective, observational study from December 2010 to September 2011 using portable critical care ultrasound to examine air-column width differences of vocal cords before and after deflation of a endotracheal cuff balloon. All patients also underwent cuff leak volume tests and vocal cord examination by direct video laryngoscopy.

Results We enrolled 101 patients with planned extubation. The overall prevalence of post-extubation stridor and/or vocal cord oedema was $17 \%$. Age, gender, duration of intubation and BMI were not different between patients with and without post-extubation complications. The average sizes of endotracheal tubes were similar in both groups (No. 7.5). The mean difference of increasing of air-column width in patients without complications was considerably higher than those with complications $(1.9 \mathrm{~mm}$ vs. $1.1 \mathrm{~mm} ; P<0.001)$. The sensitivity and specificity at air-column width differences $\geq 1.6 \mathrm{~mm}$ were 0.706 and 0.702 respectively. The positive predictive value and negative predictive value were 0.324 and 0.922 . The area under the ROC curve of tracheal ultrasound was 0.823 ( $95 \% \mathrm{Cl}: 0.698$ to 0.947$)$ and that of the cuff leak volume test was 0.840 ( $95 \% \mathrm{Cl}: 0.715$ to 0.964 ).

Conclusion Portable ICU ultrasound visualising air-column width differences between pre and post deflation cuff balloon is a promising objective tool which aids in prediction of successful extubation.

\section{References}

1. De Bast Y, De Backer D, Moraine JJ, et al.: The cuff leak test to predict failure of tracheal extubation for laryngeal edema. Intensive Care Med 2002, 28:1267-1272.

2. Chung YH, Chao TY, Chiu CT, et al:: The cuff-leak test is a simple tool to verify severe laryngeal edema in patients undergoing long-term mechanical ventilation. Crit Care Med 2006, 34:409.

3. Ding LW, Wang HC, Wu HD, et al:: Laryngeal ultrasound: a useful method in predicting post-extubation stridor. A pilot study. Eur Respir J 2006, 27:384.

P131

Intermittent aspiration of pharyngeal secretion for re-intubation prevention

T Nakamura, O Nishida, J Shibata, N Kuriyama, Y Hara, M Yumoto

Fujita Health University School of Medicine, Toyoake, Japan

Critical Care 2012, 16(Suppl 1):P131 (doi: 10.1186/cc10738)

Introduction The inability of extubated patients to clear oropharyngeal secretion increases the risk of re-intubation. To eliminate excessive

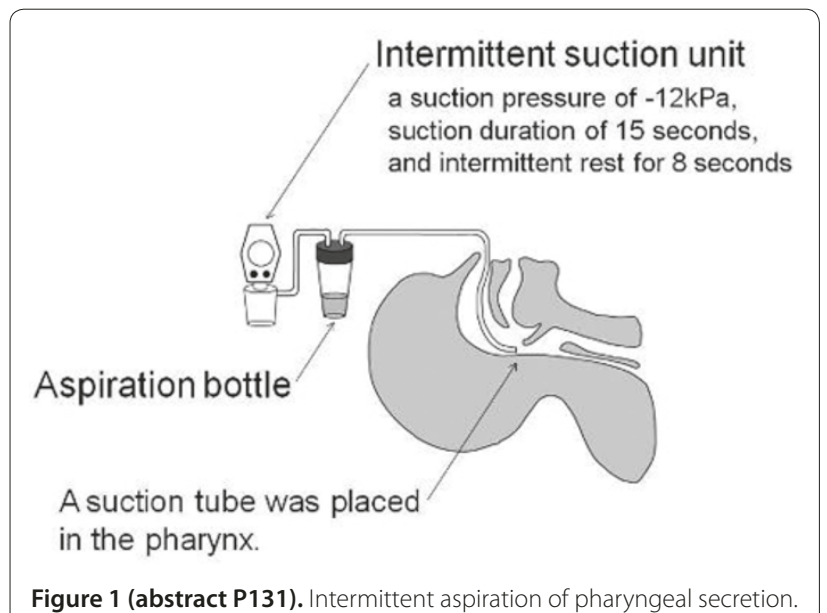

oropharyngeal secretion, we devised a suctioning method: intermittent aspiration of pharyngeal secretion (IAPS). IAPS is a simple, low-cost technique utilizing an intermittent suction unit and a common suction tube (Figure 1), which may reduce the risk of re-intubation on extubated patients requiring supraglottic airway management.

Methods A retrospective study was performed on 24 patients who received IAPS after extubation from June 2009 to May 2011. A suction tube was placed in the pharynx after extubation. The same suction unit used in intermittent subglottic secretion drainage was applied. IAPS is effective for patients with large amounts of oropharyngeal secretion (A), patients with poor laryngopharyngeal function (B), and patients unable to expel viscous sputum (C). Efficacy of IAPS in each of these patient groups was studied.

Results The average age was $64.3 \pm 17.8$ years, APACHE II score $21.0 \pm 7.7$, and SOFA score $8.4 \pm 3.1$. Six patients were diagnosed with $A$, three with $B$, two with $C$, and others had multiple diagnoses. Combinations with NPPV or cricothyroidotomy were also successful. Of the patients who required re-intubation, four were re-intubated for reasons other than aspiration. Two had possibly aspirated. Among patients receiving IAPS, the rate of re-intubation due to oropharyngeal aspiration was $8.3 \%$. No major complication was observed.

Conclusion IAPS is a potential method for supraglottic airway management after extubation that may reduce the re-intubation risk. IAPS is a simple method requiring common instruments. Combined effects of IAPS with NPPV or cricothyroidotomy can modify airway management. IAPS is a temporary method in which the exact timing for re-intubation should not be missed. To successfully apply IAPS and reduce aspiration, the suctioning method, duration of application and position of the suctioning tube should be further optimized.

\section{P132}

Efficacy of biphasic cuirass ventilation in the critical care department

TYamashita', Y Taniwaki², H Takayama², Y Sakamoto'

'Saga University, Saga, Japan; ${ }^{1}$ National Hospital Organization Nagasaki Medical Center, Omura, Japan

Critical Care 2012, 16(Suppl 1):P132 (doi: 10.1186/cc10739)

Introduction Biphasic cuirass ventilation (BCV) assists ventilation by applying intermittent or continuous negative pressure to the thorax. $B C V$ has been reported to improve lung function in various respiratory failures. However, to determine the therapeutic effect of $B C V$ is difficult, because it is too difficult to include animal experiments. Therefore it is important to compile amounts of clinical cases for discussion. We have tried to find a way of developing BCV in critical care.

Methods This is a retrospective, nonrandomized study. Before and after $\mathrm{BCV}$, we compared $\mathrm{pO}_{2}, \mathrm{pCO}_{2}$, tidal volume, $\mathrm{P} / \mathrm{F}$ ratio, respiratory index, $\mathrm{A}-\mathrm{aDO}_{2^{\prime}}$ shunt ratio, dead space ventilation rate, and chest $\mathrm{X}$-ray. We also performed a questionnaire study about BCV which focused on physicians and nurses working in the ICU. 
Results From April 2008 to May 2010, BCV was performed by applying RTX (Medivent Ltd, London, UK) for 18 patients admitted to the ICU, National Hospital Organization Nagasaki Medical Center. All of them had acute respiratory failure, and 15 of them were intubated and mechanically ventilated. Thirteen were men, and the mean age was 68 years ( 1 to 82 years). One case could not continue the treatment due to discomfort of wearing the cuirass. We used the control mode (negative pressure $-21 \mathrm{cmH}_{2} \mathrm{O}$, positive pressure $+7 \mathrm{cmH}_{2} \mathrm{O}$, I:E ratio 1:1). It improved the tidal volume, $\mathrm{P} / \mathrm{F}$ ratio, shunt ratio in all cases during BCV $(P<0.05)$. Skin damage caused by the cuirass was observed in one case. According to the questionnaire survey, they had some problems about the durability of the urethane of the cuirass, too close to a thin body or deformation. Some of them had no confidence because of unfamiliarity with the machine.

Conclusion We conclude that BCV is also useful for respiratory care in the ICU. Further confirmation is needed regarding problems such as the criteria to start and terminate BCV.

\section{References}

1. Chari S, King J, Rajesh PB, Stuart-Smith K: Resolution of left lower lobe collapse postesophagectomy using the Medivent RTX respirator, a novel noninvasive respiratory support system. J Cardiothorac Vasc Anesth 2004, 18:482-485.

2. Dolmage TE, De Rosie JA, Avendano MA, Goldstein RS: Effect of external chest wall oscillation on gas exchange in healthy subjects. Chest 1995, 107:433-439.

3. Ciesla ND: Chest physical therapy for patients in the intensive care unit. Phys Ther 1996, 76:609-625.

4. Rocker GM, Mckenzie MG, Williams B, Logan PM: Noninvasive positive pressure ventilation:successful outcome in patients with acute lung injury/ARDS. Chest 1999, 115:173-177.

5. Hill NS: Clinical applications of body ventilators. Chest 1986, 90:897.

P133

Differences in neurophysiologic effects between CPAP and a novel high-flow therapy system

NTiffin, S Connelly

Special Care Technologies, Banbury, UK

Critical Care 2012, 16(Suppl 1):P133 (doi: 10.1186/cc10740)

Introduction CPAP therapy for respiratory insufficiency is an established and accepted mode of therapy; however, patient compliance remains an issue. Recent studies have shown that high-flow therapy (HFT), which uses high flows of warmed and humidified air $/ \mathrm{O}_{2}$ mixtures through a nasal cannula, can also be effective in treating respiratory insufficiency. Although a nasal cannula is commonly preferred over a CPAP mask, patient comfort with HFT and CPAP has not been measured empirically. We sought to examine the autonomic neurophysiologic responses as a measure of comfort between these therapies.

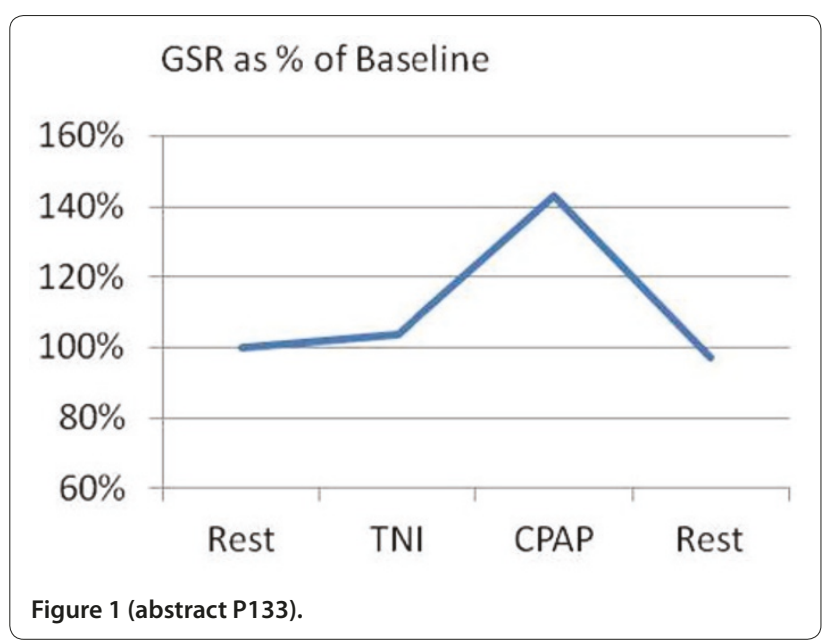

Methods We used the Sensewear Armband (Bodymedia Inc., USA) to measure the Galvanic Skin Response (GSR) in 11 healthy volunteers (36 to 53 years). The 60 -second averages of each test condition were made after 20 minutes of stabilization. Test conditions were pre and post baseline (no intervention), $10 \mathrm{cmH}_{2} \mathrm{O}$ CPAP (Resmed, Sydney, Australia) and 15 LPM HFT (TNI, Würzburg, Germany) both in room air. Repeated ANOVA with $P<0.05$.

Results There were no statistically significant differences in GSR between pre and post baselines. CPAP produced an increase in GSR compared to both baselines (45\%; $P<0.05)$ and to HFT $(41 \%$; $P<0.05)$. HFT produced no significant change in GSR compared to baseline. See Figure 1.

Conclusion GSR is a measurement of the sympathetic component of the autonomic nervous system. It is commonly referred to as the 'Fight or Flight' response, and when elevated indicates a state of psychological or physiological stress. Our data suggest that CPAP produces an increase in the GSR compared to rest, whilst TNI therapy produces no change in GSR compared to rest. This increased stress may lead to lower patient compliance when using CPAP therapy compared to TNI therapy, which has very high patient compliance rates.

P134

Management of acute bronchospasm respiratory distress with CPAP ventilation associated with nebulization in the prehospital emergency setting

J Cuny, C Berteloot, P Goldstein, E Wiel

CHRU de Lille, France

Critical Care 2012, 16(Suppl 1):P134 (doi: 10.1186/cc10741)

Introduction In emergency medicine, noninvasive ventilation (NIV) has grown up for COPD and acute pulmonary edema through the use of continuous positive airway pressure (CPAP). Recently, several studies have reported the use of NIV coupled with nebulized bronchodilators to optimize the management of acute asthma patients in emergency departments and ICUs. This has indicated an improvement in gas exchanges, decreased lung resistances and decreased work of breathing. The purpose of this study is to assess prehospital practices in CPAP for these patients, to target patients for its use, and to compare clinical data before and after achievement of CPAP with nebulization.

Methods We have conducted a retrospective, descriptive and observational study, by collecting all files (EMA, Dispatching Center) for each patient receiving CPAP associated with nebulization, for pulmonary bronchospasm (excluding acute pulmonary edema), and supported by the emergency medical service. Several data were analyzed: age, sex, history, severity signs, cardiac and respiratory rate, blood pressure, pulse oxymetry, need for intubation, nebulization of $\beta_{2}$-agonists, anticholinergics, intravenous corticosteroids, and arterial blood gases.

Results Over an 18-month period, 21 patients were enrolled: 38\% for severe asthma, and $62 \%$ for COPD exacerbation. Regarding the history: $67 \%$ were under long-term corticosteroid, $48 \%$ smokers, $29 \%$ received antibiotics, and all of them presented a clinical bronchospasm, and severity criteria for respiratory distress. Sixty percent of patients were hypoxic $\left(\mathrm{SpO}_{2}<92 \%\right)$. All patients received salbutamol inhalation, associated with inhaled anticholinergic agent in $71.4 \%$. Intravenous glucocorticoid drug was dispensed in $71.4 \%$ and intravenous salbutamol in $23.8 \%$. None of the asthma patients was intubated, five COPD patients (24.8\%) were intubated. Twelve patients were admitted to the ICU (one with asthma and 11 with COPD). Comparison of clinical parameters between prehospital care and the emergency room shows a significant difference $(P<0.05)$ for respiratory rate $(35.9 \pm 7.48$ vs. $24.95 \pm 8.25)$ and pulse oxymetry ( $81.8 \pm 15.8$ vs. $96.4 \pm 3.54)$.

Conclusion NIV through CPAP associated with nebulizations appears to provide benefit by reducing respiratory work (decreased respiratory rate) and improving alveolar ventilation (increased $\mathrm{SpO}_{2}$ ) in patients with asthma. However, in COPD patients, no improvement of symptoms has been observed. 
P135

Difference between continuous positive airway pressure via mask therapy plus chest physiotherapy (CPT) and incentive spirometry therapy plus CPT to treat or prevent acute atelectasis after cardiac surgery

F ALMutairi', S Fallows', W Abukhudair', B Islam²

'University of Chester, Manchester, UK; ${ }^{2}$ King Fahd Armed Forces Hospital, Jeddah, Saudi Arabia

Critical Care 2012, 16(Suppl 1):P135 (doi: 10.1186/cc10742)

Introduction All types of therapy such as an incentive spirometry (IS) or continuous positive airway pressure (CPAP) have a valuable role to play in the prevention or the treatment of acute atelectasis. However, the type of therapy that should be used is not yet completely clear. This study aims to clarify the difference in effectiveness between CPAP therapy plus chest physiotherapy (CPT) and IS therapy plus CPT to treat or prevent acute atelectasis.

Methods Seventy-two patients who fit the inclusive criteria (smoker, hemodynamically stable, normal lung and above 50 years old) participated in this study. The participants were divided randomly into two groups: the control group used IS 15 times per hour plus CPT 4 hours for 3 days, and the trial group used CPAP via mask therapy for half an hour every 2 hours plus CPT 4 hours. The inspiratory capacity (IC) in liters was used to compare the two groups of therapy and it was measured by incentive spirometer after the operation as baseline test, after 12 hours, 24 hours, 48 hours and post therapy. At the same time, $\mathrm{RR}, \mathrm{HR}$ and $\mathrm{SpO}_{2}$ were measured for both groups. Failure was defined as a need for advanced therapy.

Results Thirty-six patients participated in each group (57 male and 15 female). IC was increased significantly in the CPAP group $(P=0.005)$ and $\mathrm{SpO}_{2}$ was decreased significantly in the control group $(P=0.037)$. There were no significant differences in RR and HR. See Figure 1.

Conclusion Adding chest physiotherapy to CPAP via mask therapy had better outcomes to treat or prevent acute postoperative atelectasis.

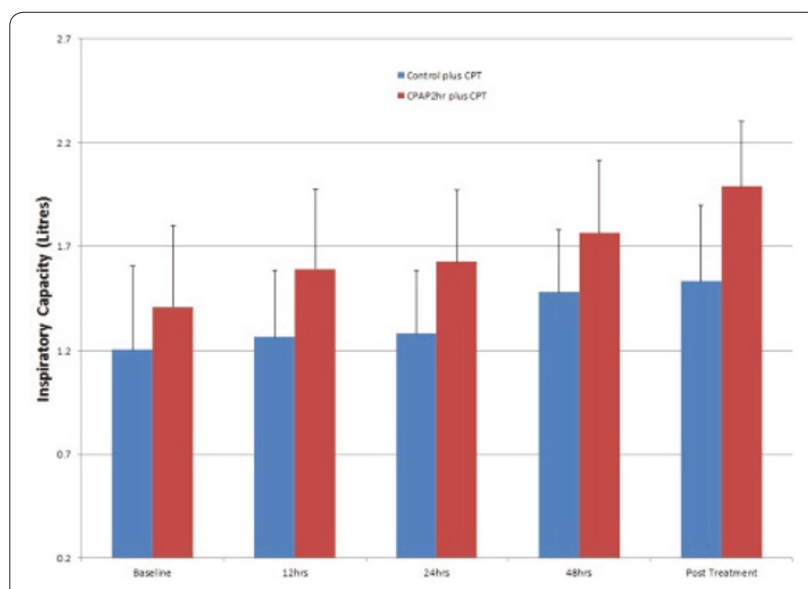

Figure 1 (abstract P135). Effect of adding CPT to CPAP via mask therapy to treat acute atelectasis.

P136

Nasal high-flow oxygen in patients with hypoxic respiratory failure: effect on functional and subjective respiratory parameters compared to conventional oxygen therapy and noninvasive ventilation

R Riessen, N Schwabbauer, B Berg, G Blumenstock, M Haap, J Hetzel University Hospital Tübingen, Germany

Critical Care 2012, 16(Suppl 1):P136 (doi: 10.1186/cc10743)

Introduction This study compared a nasal high-flow oxygen therapy $\left(\mathrm{NHFO}_{2}\right.$ ) with conventional oxygen therapy via a Venturi mask (VM) or noninvasive ventilation (NIV) in patients with hypoxic respiratory failure. Study endpoints were functional respiratory parameters, dyspnea, patient comfort and a global rating by the patients.
Methods We included 14 patients with hypoxic respiratory failure ( $\mathrm{paO}_{2}<55 \mathrm{mmHg}$ under room air). Exclusion criteria were ventilatory failure, hemodynamic instability, cardiogenic pulmonary edema, NIV contraindications and inability to cooperate. Patients were treated in a randomized order for 30 minutes each with $\mathrm{NHFO}_{2}$ (Optiflow ${ }^{\oplus}$; Fisher-Paykel), VM or NIV, using a $\mathrm{FiO}_{2}$ of 0.6. Every treatment phase was preceded by a 15 -minute baseline phase in which the patients received oxygen via a standard nasal prong $\left(\mathrm{SaO}_{2}\right.$ goal $\left.>88 \%\right)$. At the end of each treatment phase vital signs and blood gases were measured and patients rated their dyspnea and their general comfort on a 10-point scale. Finally, patients were ask for a global rating of all three devices ranging from 1 (very good) to 6 (failed) and could choose one device for further treatment.

Results The $\mathrm{paO}_{2}$ was highest under NIV with $129 \pm 38 \mathrm{mmHg}$, followed by $\mathrm{NHFO}_{2}(101 \pm 34 \mathrm{mmHg}, P<0.01 \mathrm{vs}$. NIV) and VM $(85 \pm 21 \mathrm{mmHg}$, $P<0.001$ vs. NIV, $P<0.01$ vs. NHFO $2^{\prime}$ ANOVA). All other vital and blood gas parameters did not show significant differences. Dyspnea rating on a 10-point Borg scale was significantly better under $\mathrm{NHFO}_{2}(2.9 \pm 2.1)$ and VM $(3.3 \pm 2.3)$ compared to NIV $(5.0 \pm 3.3)\left(P<0.05\right.$, vs. $\mathrm{NHFO}_{2}$ or VM). Comfort rating showed similar results: $\mathrm{NHFO}_{2} 2.7 \pm 1.8$; VM $3.1 \pm 2.8$; NIV $5.4 \pm 3.1(P<0.05$, NIV vs. NHFO or VM). In the final global rating using German school grades from 1 to $6 \mathrm{NHFO}_{2}$ also received the best rating $(2.3 \pm 1.4)$, followed by VM $(3.2 \pm 1.7, P=\mathrm{NS}$ vs. NHFO $)$ and NIV $\left(4.5 \pm 1.7, P<0.01\right.$ vs. NHFO ${ }_{2}$ and $P<0.05$ vs. VM). For further treatment 10 patients chose $\mathrm{NHFO}_{2}$, three VM and one NIV.

Conclusion $\mathrm{NHFO}_{2}$ is a promising new device for oxygen supply in respiratory failure, offering better oxygenation than the VM and better patient comfort and tolerance than NIV.

\section{P137}

Short-term effect of humidified high nasal flow oxygen in critically ill patients

FVan Beers, A Van Hees, J Van Rosmalen, D Ramnarain

St Elisabeth Hospital Tilburg, the Netherlands

Critical Care 2012, 16(Suppl 1):P137 (doi: 10.1186/cc10744)

Introduction Recently, humidified high-flow nasal cannula oxygen (HFNC) has gained popularity in treating patients with acute respiratory insufficiency. Studies have shown that HFNC generates a low level of positive airway pressure, reduction of airway resistance and flushes nasopharyngeal dead space leading to less work of breathing. However, in which type of patient HFNC could be of benefit, the short-term as well as long-term effects, tolerance and outcome are unknown. We used HFNC in a variety of patients. We evaluated the short-term effect of HFNC. Methods We retrospectively studied respiratory, oxygen-derived and hemodynamic parameters before and 1 hour after start of HFNC in 50 patients during the past 12 months. All patients were treated in a mixed medical, surgical, neurological ICU of a teaching hospital. The HFNC used consisted of an air-oxygen blender with adjustable $\mathrm{FiO}_{2}(0.21$ to 1.0 ), delivering a modifiable gas flow up to $60 \mathrm{l} /$ minute (Optiflow; Fisher \& Paykel, Auckland, New Zealand).

Results Fifty patients were included, 29 men and 21 women, mean age $65 \pm 14$, mean APACHE II score on admission $19 \pm 5$.9. The mean duration of HFNC was $22 \pm 21$ hours. Indications for HFNC could be divided into five categories: (1) no acceptance of noninvasive positive pressure ventilation (NPPV) $(n=8)$, (2) weaning from NPPV, (3) hypoxia $(n=14)$, (4) respiratory distress/discomfort $(n=9)$, and (5) other $(n=5)$. Despite the use of HFNC, in 15 patients intubation was unavoidable; group $1, n=8$, group $3, n=6$, group $4, n=1$. Oxygen saturation increased from $91 \pm 7.2$ to $97.5 \pm 1.7(P \leq 0.05)$. $\mathrm{PaO} / \mathrm{FiO}_{2}$ ratio increased from $140 \pm 79.1$ to $169.8 \pm 68(P \leq 0.05)$. $\mathrm{PCO}_{2}$ decreased from $6.5 \pm 3.0$ to $6.2 \pm 2.9 \mathrm{mmHg}(P \leq 0.05)$. No significant differences were seen in heart rate, blood pressure and respiratory rate. Ten patients died, in eight patients of which the policy was not to reanimate and not to be intubated due to extensive comorbidity. Two patients died during treatment in the ICU due to underlying disease.

Conclusion We used HFNC therapy for a variety of indications. In $70 \%$ of our study population HFNC was successful. Oxygen-derived parameters significantly increased after 1 hour of HFNC. HFNC was successful and well tolerated in patients weaning from NPPV. After noncompliance of NPPV in $42 \%$ of patients in our population, intubation could be avoided with the use of HFNC. 
P138

Good response on high nasal oxygen flow reduces the need for intubation in adult respiratory failure

L Van Wagenberg, IM Hoekstra, GC Admiraal, M Slabbekoorn

Medisch Centrum Haaglanden, Den Haag, the Netherlands

Critical Care 2012, 16(Suppl 1):P138 (doi: 10.1186/cc10745)

Introduction High nasal flow (HNF) therapy has proven its efficiency in acute respiratory failure when compared to conservative oxygen therapy [1]. This study was performed to find a responding and nonresponding group on HNF therapy in adults with hypoxic respiratory insufficiency measured by oxygenation and work of breathing.

Methods A prospective observational study during a 6-month period in patients $\geq 18$ years with acute hypoxic respiratory failure when conservative oxygen therapy ( $15 \mathrm{l} /$ minute) failed. Arterial blood gas analysis was done before HNF therapy and after 1 hour on flow $50 \mathrm{l} /$ minute with $\mathrm{FiO}_{2}$ 1.0. Breaths per minute and saturation were noted. When patients remained respiratory insufficient they were intubated. Results A total of 20 patients was included. Mean age $63.95 \pm 3$ years and APACHE II score $23 \pm 7$. Mean $\mathrm{PaO}_{2} / \mathrm{FiO}_{2}$ (P/F) ratio on admission was $77.7 \pm 4.2$. A total of seven out of 20 patients $(35 \%)$ needed endotracheal intubation. After 1 hour of $\mathrm{HNF}$ therapy $\mathrm{PaO}_{2}$ and saturation measured in arterial blood gas significantly increased from respectively $8.9 \pm 0.3$ $\mathrm{kPa}$ to $16.1 \pm 2.4 \mathrm{kPa}(P=0.023)$ and from $91.8 \pm 1.2 \%$ to $96.5 \pm 0.8 \%$ $(P=0.001)$. Work of breathing, measured by the frequency of breathing, significantly decreased from $35 \pm 3$ times a minute to $22 \pm 2$ times a minute. The group that was in need of endotracheal intubation showed a less prominent response to 1-hour HNF therapy, expressed in $\mathrm{PaO}_{2}$ $(13.2 \pm 2.6 \mathrm{kPa}$ vs. $16.1 \pm 3.4 \mathrm{kPa}, P=0.548)$, saturation $(94.4 \pm 1.6 \%$ vs. $96.5 \pm 0.8 \%, P=0.228)$ and breathing frequency $(25 \pm 2.4 /$ minute vs. $22 \pm 2$ /minute, $P=0.357$ ). The duration of HNF therapy was $26.1 \pm 6.3$ hours in the nonintubated group and $15.1 \pm 9.8$ hours for those who were intubated $(P=0.345)$.

Conclusion All included patients did have a reduced $\mathrm{P} / \mathrm{F}$ ratio and are therefore to be considered severely respiratory compromised. $\mathrm{PaO}_{2}$ and saturation increased with the use of HNF therapy, while work of breathing decreased. These changes were less prominent in the nonresponding group (Figure 1). The nonresponders, except one, were intubated within 15 hours after the start of HNF therapy.

Reference

1. Roca et al.: Respir Care 2010, 55:408-413.

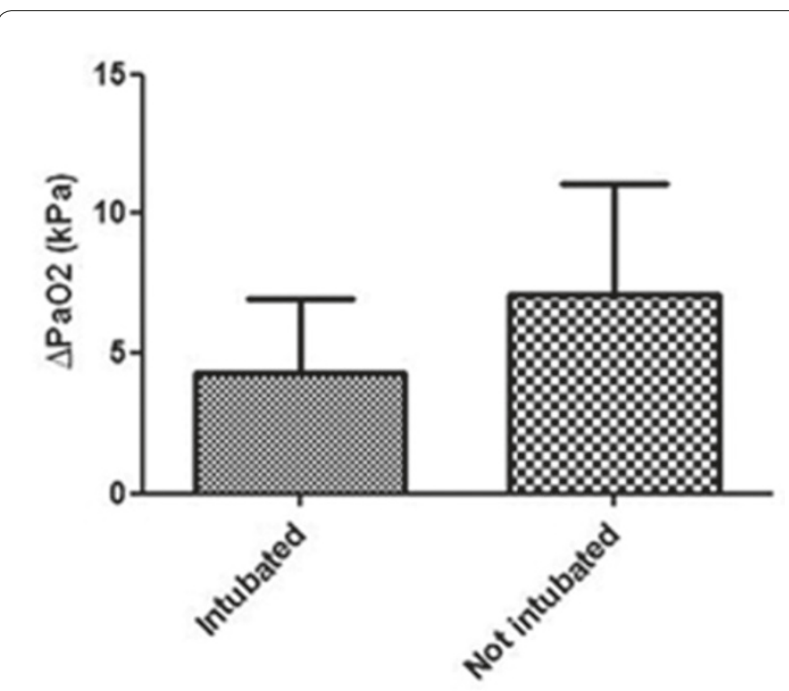

Figure 1 (abstract P138). $\mathrm{PaO}_{2}$ after 1-hour HNF therapy.
P139

An audit of airway complications in a district general hospital ICU

JW Chan, KJ Turner, R Lloyd, R Howard-Griffin

Ipswich Hospital NHS Trust, Ipswich, UK

Critical Care 2012, 16(Suppl 1):P139 (doi: 10.1186/cc10746)

Introduction The 4th National Audit Project of The Royal College of Anaesthetists and The Difficult Airway Society (NAP4) highlighted the increased incidence of airway-related complications in an ICU setting [1]. The aim of this audit was to establish our local ICU airway intubation complication rate as well as our compliance with the NAP4 recommendation that continuous capnography should be used on all intubated patients.

Methods All intubated patients who were admitted to the Ipswich Hospital ICU between April and December 2010 were identified and data relating to basic demographics, airway management and the use of capnography were collected. An airway was classed as difficult if there were two or more attempts at intubation, a bougie was used, or it was Cormack-Lehane grade III/IV. Complications arising from airway intubation were also noted.

Results A total of 139 intubations on 118 patients were identified. Fifty-eight (42\%) intubations occurred on the ICU, 41 (29\%) in the emergency department (ED) and five (4\%) on the ward; $29(21 \%)$ intubations occurred in theatre for surgery and six (4\%) out of hospital. Of the 104 intubations on the ICU, ED or ward, nine (9\%) were classed as difficult and there were 21 (20\%) documented complications (hypoxia, hypotension, oesophageal intubation, cardiac arrest and aspiration). Complication rates were similar across junior trainees, senior trainees and consultants. Only $27 \%$ of all intubated patients received continuous capnography.

Conclusion Our findings are consistent with the NAP4 view that airway management outside the controlled confines of a theatre setting has the potential to be more difficult. Steps should be taken to minimise the risk associated with this procedure, including a thorough airway assessment, use of continuous capnography and the presence of suitably trained operators and assistants. The finding that complications occurred at a similar rate regardless of the seniority could be explained by more senior staff intubating the most unwell patients.

\section{Reference}

1. CookTM, et al:: Br J Anaesth 2011, 106:632-642.

P140

Multidisciplinary care for patients with tracheostomy shortened time to decannulation

A Van Hees, F Van Beers, JVan Rosmalen, D Ramnarain,

WVan den Wildenberg

St Elisabeth Hospital, Tilburg, the Netherlands

Critical Care 2012, 16(Suppl 1):P140 (doi: 10.1186/cc10747)

Introduction Care for cannulated patients in our hospital is not uniform and clear, leading to an unnecessarily long period of cannulation and even unsafe situations. Therefore our hospital formed a specialized multidisciplinary cannula team (SMCT) consisting of an intensivist, three ventilation practitioners and a registered nurse. The aim of this team was to shorten the period of cannulation and to guarantee uniform care and safety around cannulated patients in our ICU and on the ward.

Methods The study was conducted in the mixed medical and neurosurgical ICU of a teaching hospital with a duration of 22 months. Two groups of patients were studied. Group one received PDT before introduction of a SMCT $(n=49)$ and group two received PDT $(n=27)$ after introduction of a SMCT. During treatment in the ICU and after discharge, all patients were followed by the cannula team. This team eventually made the decision to decannulate the patients. We evaluated the results of a multidisciplinary cannula team (SMCT) during a follow-up period of 22 months.

Results For patient data see Table 1. Seventy-six patients were included in this study. The study showed a reduction in time to decannulation after a mechanical ventilation period of 8.4 days. However clinically relevant, this was not statistically significant. 
Table 1 (abstract P140). Results of comparative analysis between patients before and after the cannula team

\begin{tabular}{lcc}
\hline & $\begin{array}{c}\text { Pre-cannula } \\
\text { team }(\boldsymbol{n}=\mathbf{4 9})\end{array}$ & $\begin{array}{c}\text { Post-cannula } \\
\text { team }(\boldsymbol{n}=\mathbf{2 7})\end{array}$ \\
\hline Age (years) & $52.2 \pm 16.3$ & $56.4 \pm 16.8$ \\
APACHE II score & $20.9 \pm 5.2$ & $21.4 \pm 5.7$ \\
Intubated days before tracheostomy & $12.8 \pm 7.9$ & $9.9 \pm 7.3$ \\
Length of stay in ICU & $34.1 \pm 4.7$ & $36.6 \pm 28.9$ \\
Cannulation days & $22 \pm 15.4$ & $20 \pm 0.7$ \\
Mechanical ventilation after tracheostomy & $9.3 \pm 7.4$ & $9.1 \pm 9$ \\
Tracheostomy after mechanical ventilation & $18.2 \pm 27.7$ & $9.8 \pm 9.5$ \\
\hline
\end{tabular}

Conclusion With the introduction of a SMCT a clinically relevant reduction of cannulation period could be achieved. The group was small and probably underpowered to show a statistically significant reduction in the cannulation period.

P141

Influence of percutaneous tracheostomy on gas exchange in mechanically ventilated patients

A Pradella', G Bellani', S Abd El Aziz El Sayed Deab', T Mauri', E Rezoagli',

S Arrigoni', F Leone', G Citerioº, A Pesenti'

'University of Milano-Bicocca, Monza, Italy; ${ }^{2}$ San Gerardo Hospital, Monza, Italy

Critical Care 2012, 16(Suppl 1):P141 (doi: 10.1186/cc10748)

Introduction The influence of percutaneous tracheostomy on patients' ventilator-dependency and clinical outcomes has been deeply investigated [1]. However, except for immediate intraprocedural variations [2], tracheostomy's impact on gas exchange has scarcely been explored. The aim of the present study is to investigate the persisting effects of percutaneous tracheostomy on pulmonary function in a group of ICU-admitted patients.

Methods Clinical records of 107 patients from San Gerardo Hospital General and neurosurgical ICUs that underwent a percutaneous tracheostomy were retrospectively revised to compare ventilator settings, gas exchange and hemodynamic parameters on the day before and on the day after the procedure. For each parameter we averaged the values of three different recordings during the day. A pre-established subgroup analysis on the hypoxemic $\left(\mathrm{PaO}_{2} / \mathrm{FiO}_{2}<300\right.$ $\mathrm{mmHg}$ ) patients $(n=38)$ was performed. Analyses were performed by paired $t$ test and linear regression; a level of $P<0.05$ was considered statistically significant.

Results Among all analyzed patients, we found, after tracheostomy, a marginal decrease in $\mathrm{PaCO}_{2}(43 \pm 9$ vs. $42 \pm 8 \mathrm{mmHg}$, before vs. after $P<0.01)$ and increase in $\mathrm{pH}(7.43 \pm 0.04$ vs. $7.44 \pm 0.03 \mathrm{mmHg}$, before vs. after $P<0.01$ ), with no variation in $\mathrm{PaO}_{2} / \mathrm{FiO}_{2}$. Considering the subgroup of hypoxemic patients, despite unchanged ventilator parameters, after the tracheostomy a higher $\mathrm{PaO}_{2} / \mathrm{FiO}_{2}(222 \pm 60 \mathrm{mmHg}$ vs. $256 \pm 84$ $\mathrm{mmHg}$, before vs. after $P<0.01)$ and a lower $\mathrm{PaCO}_{2}(46 \pm 11$ vs. $43 \pm 9$ $\mathrm{mmHg}$, before vs. after $P<0.01$ ) were found. For hypoxemic patients, a positive correlation was found between $\mathrm{PaCO}_{2}$ on the day before tracheostomy and the decrease of $\mathrm{PaCO}_{2}\left(r^{2}=0.29 ; P<0.01\right)$. Moreover, taking in account the subgroup of hypoxemic patients under pressure support ventilation $(n=28)$, the $\mathrm{PaCO}_{2}$ decrease was loosely but significantly correlated with the pressure support level on the day before the procedure $\left(r^{2}=0.25 ; P<0.01\right)$.

Conclusion In a relatively large cohort of mechanically ventilated patients, percutaneous tracheostomy seems to increase the carbon dioxide elimination. This effect was even more pronounced in the subgroup of hypoxic patients, in whom also oxygenation improved.

References

1. Terragni PP, et al:: JAMA 2010, 303:1483-1489.

2. Benini A, et al:: Intensive Care Med 2002, 28:726-730.
P142

Bronchoscope-guided percutaneous dilatational tracheostomy performed by an experienced intensivist: a 26-month experience at a tertiary care center in United Arab Emirates

M Rahman, R Ammar, D Abdullah, F Chedid, S Abuhasna

Tawam Hospital, Al Ain, United Arab Emirates

Critical Care 2012, 16(Suppl 1):P142 (doi: 10.1186/cc10749)

Introduction Bedside percutaneous dilatational tracheostomy (PDT) is a safe procedure with an acute complication rate of 10 to $15 \%$. Our hypothesis was that having an experienced person performing or supervising the procedure results in extremely low complications with PDT. We formed a tracheostomy team which always included at least a consultant or specialist experienced (at least 25 procedures) in performing the procedure.

Methods A retrospective chart review of all patients who had PDT in a multidisciplinary adult medical surgical ICU during November 2008 to December 2010. The patients' demographics, indications for intubation and PDT, early and late complications, date weaned off the ventilator, date of decannulation, discharge from ICU and hospital, and outcome of these patients in the hospital were noted.

Results Out of a total of 2,364 admission 57 patients underwent PDT, all with bronchoscopic guidance by an intensivist experienced in PDT (>25 procedures); there were 45 (78.9\%) males and 12 (21\%) females with the median age of 42 (range 18 to 90 ) years. The most common admission diagnosis was cardiac arrest $n=14$ (24\%) followed by severe head injury $n=13(23 \%)$ and cerebrovascular accident $n=8(14 \%)$. The commonest indication for tracheostomy was airway protection $n=40$ (73\%) followed by prolonged mechanical ventilation $n=25$ (45\%). The median duration of intubation before PDT was 11 days (IQ 8 to 18). The median time elapsed between tracheostomy and weaning of ventilator was 1 day (IQ 1 to 3). However, the median time to decannulation was 37 day (IQ 10 to 136). Acute complication of paratracheal insertion occurred in $n=1(1.8 \%)$ patient. No deaths were reported related to the procedure. However, $n=13(22.8 \%)$ patients died during the hospital stay. No procedure was converted to surgical tracheostomy. The median duration between tracheostomy and discharge from ICU was 12 days (IQ 5 to 21). Chronic complication of subglottic stenosis occurred in $n=1(1.8 \%)$ patient.

Conclusion PDT is an extremely safe procedure when performed by an experienced intensivist under bronchoscopic guidance. Our low complication rate is due to careful screening and selection of patients and being performed or supervised by an experienced intensivist under direct vision.

P143

Risk factors for poor outcome in patients with osmotic demyelination syndrome

MA Peters', JG Van der Hoeven², C Hoedemaekers²

'Canisius Wilhemina Hospital, Nijmegen, the Netherlands; ${ }^{2}$ Radboud

University Nijmegen Medical Centre, Nijmegen, the Netherlands

Critical Care 2012, 16(Suppl 1):P143 (doi: 10.1186/cc10750)

Introduction The osmotic demyelination syndrome (ODS) is a devastating complication of rapid correction of hyponatremia. The objective of this study was to identify prognostic factors that determine outcome in patients with ODS.

Methods We performed a literature search using MEDLINE and Embase. Case reports or case series were eligible for this study in cases of: (1) hyponatremia defined as a serum sodium $\leq 130 \mathrm{mEq} / \mathrm{l}$ on hospital admission or thereafter, but preceding the clinical signs of ODS; (2) a clear diagnosis of ODS, confirmed by MRI scanning or pathology; and (3) a description of patient outcome. We defined a favourable outcome as a Glasgow Outcome Score $>3$ or a Modified Rankin Scale $<4$.

Results A total of 120 manuscripts were identified describing 125 cases: $86 / 125(69 \%)$ had a favourable outcome. Mean age in the favourable outcome group was $44.7 \pm 14.4$ years versus $52.3 \pm 13.6$ years in the poor outcome group $(P=0.006)$. The ODS was exclusively pontine in $44 / 125(35 \%)$, extrapontine in $34 / 125$ (37\%) and combined pontine and extrapontine in $47 / 125(37 \%)$ of cases. The anatomical localisation of the lesion was not associated with outcome $(P=0.64)$. Forty-two 
out of 125 (34\%) of cases were associated with alcohol abuse, 14/125 (11\%) with malnutrition, 26/125 (21\%) with use of diuretics and 9/125 (7\%) with use of psychoactive medication; none of these characteristics were significantly related to outcome. The sodium concentration on admission was $107.3 \pm 9.6$ in the patients with a favourable outcome versus $108.4 \pm 9.4$ in the patients with a poor outcome $(P=0.54)$. The speed of sodium correction was $1.12 \pm 1.6 \mathrm{mmol} /$ hour versus $1.16 \pm 0.9$ $\mathrm{mmol} /$ hour respectively in the favourable and poor outcome cases $(P=0.19)$. The highest sodium concentration after correction was significantly higher in the patients with a poor outcome (139.0 \pm 9.3 vs. $134.0 \pm 7.3, P=0.003$ ). Serum osmolality, and concentrations of potassium, chloride, creatinin and glucose were comparable between the outcome groups. The development of tetraparesis (55/125 (44\%), $P=0.02)$ or a decreased level of consciousness (58/125 (46\%), $P<0.001)$ were associated with a poor outcome. In contrast, mutism or dysarthria $(82 / 125(66 \%), P=0.002)$, tremors $(29 / 125(23 \%), P=0.001)$ or ataxia $(58 / 125(46 \%), P<0.001)$ were associated with a favourable outcome. Conclusion The highest serum sodium concentration during sodium correction rather than the speed of sodium correction or severity of the hyponatremia is a determinant of outcome in patients with ODS. The development of tetraparesis and decreased consciousness are associated with a poor outcome in these patients.

\section{P144}

Is inappropriate secretion of anti-diuretic hormone (SIADH) the cause of hyponatremia in Legionella pneumonia?

P Schuetz, S Haubitz, B Mueller, for the ProHOSP Study Group

Medical University Clinic, Kantonsspital Aarau, Switzerland

Critical Care 2012, 16(Suppl 1):P144 (doi: 10.1186/cc10751)

Introduction Medical textbooks list Legionella as a differential diagnosis for the syndrome of inadequate anti-diuretic hormone (ADH) secretion (SIADH), but empirical evidence supporting this association is largely lacking. Partly this is explained by the analytical challenges of $\mathrm{ADH}$ measurement. With the recent availability of an immunoassay that measures the more stable ADH precursor peptide (CT-ProVasopressin), we sought to investigate whether increased ADH levels would explain hyponatremia found in Legionella patients.

Methods We measured CT-ProVasopressin and sodium levels in a prospective cohort of 925 pneumonia patients from a previous multicenter study with 31 patients having positive antigen tests for Legionella pneumophilia. We calculated Spearman rank correlations and multivariate regression models.

Results Legionella patients had higher rates of hyponatremia (sodium $<130 \mathrm{mmol} / \mathrm{l})(43 \%$ vs. $8 \%, P<0.01)$, but similar median CTProVasopressin levels (pmol/I) (20 (12 to 26$)$ vs. 26 (13 to 53$), P=0.89$ ) compared to pneumonia of other etiology. In Legionella patients, high CT-ProVasopressin was not associated with low sodium levels, but showed a positive correlation with sodium levels $(r=0.42, P<0.05)$. Independent of pneumonia etiology, CT-ProVasopressin were significantly correlated with the pneumonia severity index $(r=0.56$, $P<0.05)$ and showed an association with risk for ICU admission (odds ratio per decile, $95 \% \mathrm{Cl})(1.4,1.2$ to 1.6$)$ and 30 -day mortality $(1.3,1.2$ 1.4).

Conclusion We found no evidence that increased ADH secretion would explain low sodium levels in Legionella patients, or other pneumonia patients, challenging the common believe of Legionella causing $\mathrm{SIADH}$. Rather, $\mathrm{ADH}$ precursors were upregulated as a response to severe disease. Future studies continuing to explore the cause of sodium disturbance in Legionella are warranted.

\section{P145}

Fluctuations in serum sodium level are associated with an increased risk of death in surgical ICU patients

Y Sakr, S Rother, AM Ferreira, C Ewald, P Dünich, K Reinhart

Friedrich Schiller University Hospital, Jena, Germany

Critical Care 2012, 16(Suppl 1):P145 (doi: 10.1186/cc10752)

Introduction Dysnatremia may have an impact on outcomes in critically ill patients, but this has not been widely investigated in surgical ICU patients. We investigated the epidemiology of dysnatremia in a large cohort of surgical ICU patients and evaluated the possible influence of the time of acquisition of dysnatremia and fluctuations in serum sodium concentrations on hospital mortality in these patients.

Methods All patients admitted to the ICU between January 2004 and January 2009 were included retrospectively in this study. Hyponatremia was defined as a serum sodium concentration $(\mathrm{sNa})<135 \mathrm{mmol} / \mathrm{l}$ and hypernatremia as a sNa $>145 \mathrm{mmol} / \mathrm{l}$. Hyponatremia was defined as a sNa less than $135 \mathrm{mmol} / \mathrm{l}$ and hypernatremia as a sNa greater than 145 $\mathrm{mmol} / \mathrm{l}$. Patients were classified according to the onset of dysnatremia into those who had abnormal sodium concentrations in the initial blood sample, analyzed within 2 hours of admission to the ICU, or those acquiring dysnatremia thereafter. We performed a logistic regression multivariate analysis with hospital outcome as the dependent factor to investigate the possible influence of dysnatremia on hospital outcome. Results Of the 10,923 surgical ICU patients included in the study, 1,215 (11.2\%) had hyponatremia and 277 (2.5\%) hypernatremia at admission to the ICU. Among patients with normonatremia at admission to the ICU $(n=9,431)$, the incidence of ICU-acquired dysnatremia was $31.3 \%$. Dysnatremia present at ICU admission (OR $=2.53 ; 95 \% \mathrm{Cl}: 2.06$ to $3.12, P<0.001)$ and ICU-acquired dysnatremia (OR $=2.06 ; 95 \% \mathrm{Cl}: 1.71$ to $2.48, P<0.001)$ were independently associated with an increased risk of in-hospital death. Dysnatremia at ICU admission $(O R=1.23$; 95\% Cl: 1.01 to 1.50 ) was associated with a higher risk of in-hospital death, compared to ICU-acquired dysnatremia. Fluctuation in serum sodium concentration was also independently associated with an increased risk of in-hospital mortality; both in patients who remained normonatremic ( $>6 \mathrm{mmol} / \mathrm{l} / \mathrm{ICU}$ stay) and those with dysnatremia ( $>12 \mathrm{mmol} / \mathrm{l} / 24$ hours or $>12 \mathrm{mmol} / \mathrm{l} / \mathrm{ICU}$ stay).

Conclusion Dysnatremia was common in surgical ICU patients and was independently associated with an increased risk of in-hospital death in these patients. Dysnatremia at ICU admission was associated with a higher risk of death compared to ICU-acquired dysnatremia. Fluctuations in serum sodium concentrations were independently associated with an increased risk of in-hospital death, even in patients who remained normonatremic during the ICU stay.

P146

Impact of ketogenesis and strong ion difference on acid-base in our CICU

T Clark, B McGrath, P Murphy, M Jayarajah

Derriford Hospital, Plymouth, UK

Critical Care 2012, 16(Suppl 1):P146 (doi: 10.1186/cc10753)

Introduction Persistence of a mild metabolic acidosis or base deficit was occasionally observed in our otherwise well patients post cardiac surgery, sometimes delaying discharge. We hypothesised that this metabolic abnormality may be due to either ketogenesis caused by a combination of starvation and the surgical stress response, or strong ion imbalances following fluid administration. The administration of large volumes of chloride-rich fluids (as may occur during cardiac surgery to prime the cardiopulmonary bypass circuit or resuscitate the patient) is known to induce hyperchloraemic metabolic acidosis [1]. Using simplifications of the original Fencl-Stewart's equations, it is possible to partition the base deficit into its constituent parts, subsequently determining the relative contribution of chloride, albumin and unmeasured anions to acidosis [2,3]. Ketone production may contribute significantly to the unmeasured anion component.

Methods A prospective cohort analysis. Fifty postoperative cardiac patients were recruited. For each we measured urinary ketones three times per day for the first 48 hours of their $\mathrm{CICU}$ admission. Arterial blood gas $(A B G)$ data were recorded in conjunction each time. For each blood gas we partitioned the base deficit into its constituent components using previously published equations [1-3].

Results A total of 231 ABGs were analysed. Urinary ketones were checked along with 181 of the ABGs. A total of 14 ketonuria checks were positive (8\%) in 11 patients (22\%). In nine ABGs ketonuria was associated with a significant base deficit, whilst in three it was also associated with a metabolic acidosis. The average starvation time was 39 hours (SD 11 hours). In 121 (52\%) ABGs the chloride component of the base deficit (BECl) was below -2. In 104 (45\%) ABGs the BECl 
contributed to greater than $75 \%$ of the BETOTAL, whilst in $74(32 \%)$ of these the $\mathrm{BECl}$ was greater than the BETOTAL. In $18 \mathrm{ABGs}$ a BECl of less than -2 caused a metabolic acidosis.

Conclusion Our observation of persistent metabolic abnormalities in otherwise well postoperative cardiac patients may be due to iatrogenic strong ion imbalances caused by hyperchloraemic solutions. Ketogenesis was not a significant contributing factor. The impact of relative hyperchloraemia on $\mathrm{pH}$ was buffered by other counteracting metabolic factors (for example, hypoalbuminaemia), as in 74 ABGs the $\mathrm{BECl}$ was greater than the BETOTAL.

References

1. Taylor et al:: Intensive Care Med 2006, 32:295-301.

2. Story et al:: Br J Anaesth 2004, 92:54-60.

3. O'Dell et al: Crit Care 2005, 9:R464-R470,

P147

Buffer therapy in metabolic acidosis after surgery-associated hemorrhage in pediatric oncology

N Matinyan, A Saltanov, I Letyagin, O Obukhova

N.N. Blokhin Russian Cancer Research Center, Moscow, Russia

Critical Care 2012, 16(Suppl 1):P147 (doi: 10.1186/cc10754)

Introduction Surgery in pediatric oncology is usually massive and traumatic and often leads to acute blood loss, which can result in metabolic acidosis. To treat acidosis, sodium bicarbonate is often used; however, its application has some side effects. In this situation trishydroxymethyl aminomethane (THAM) seems to be more effective. The objective of this study was to evaluate the effect of THAM for treating metabolic acidosis after surgery-associated hemorrhage in pediatric oncology.

Methods The observational study included 50 children aged 12 months to 16 years (among them 27 boys) with metabolic acidosis after surgeryassociated hemorrhage: $40 \%$ patients lost $58 \pm 8.5 \%$ of total blood volume, $26 \%$ lost $150 \pm 9.5 \%$ of total blood volume. Patients received $3.66 \%$ THAM infusion. The dose of THAM infusion was calculated as the dose administered $(\mathrm{ml})$ :negative standard $\mathrm{BE}(\mathrm{mmol} / \mathrm{l}) \times \mathrm{kg}$ body weight, and did not increase $1.5 \mathrm{ml} / \mathrm{kg}$ body weight every 24 hours. The following were analyzed: $\mathrm{Na}^{+}, \mathrm{K}^{+}$, ionized calcium, lactate, $\mathrm{pH}, \mathrm{pCO}_{2}$, $\mathrm{HCO}_{3}$ and $\mathrm{BE}$ of arterial blood, before therapy, and after receiving a onehalf dose and a full dose of THAM. The significance of differences was assessed by Student's $t$ test, Mann-Whitney coefficient and chi-square test; $P<0.05$ was considered statistically valid.

Results There were no differences in the concentrations of electrolytes and lactate. At the stages of the research the following significant dynamics have been noted: $\mathrm{pH}(7.27 \pm 0.01 ; 7.31 \pm 0.01 ; 7.35 \pm 0.01$; $P<0,01), \mathrm{HCO}_{3}(18.59 \pm 0.26 ; 19.5 \pm 0.3 ; 21.2 \pm 0.41 \mathrm{mmol} / \mathrm{l} ; P<0.01)$ and $\mathrm{BE}(-8.34 \pm 0.3 ;-6.58 \pm 0.37 ;-4.47 \pm 0.45 \mathrm{mmol} / \mathrm{l} ; P<0.01)$. PaCO tension did not change significantly $(38.9 \pm 0.83 ; 37.3 \pm 0.94 ; 37.5 \pm 0.95$ $\mathrm{mmHg} ; P>0.05$ ).

Conclusion THAM infusion resulted in metabolic acidosis correction without the development of hypernatremia and increase of $\mathrm{CO}_{2}$ tension. However, the small number of observations does not allow one to assess accurately the clinical effect of THAM for these patients.

\section{P148}

An unusual cause of high anion gap metabolic acidosis:

\section{pyroglutamic acidosis}

RJ Wardell, LA Burrows, K Myall, A Marsh

Frenchay Hospital, Bristol, UK

Critical Care 2012, 16(Suppl 1):P148 (doi: 10.1186/cc10755)

Introduction Metabolic acidosis is a common acid-base disturbance in intensive care. A high anion gap indicates the presence of endogenous acids, which in critically ill patients are most commonly ketones, lactate and those accumulated in renal failure. However, excluding these causes means more rare forms of acid must be considered, including pyroglutamic acidosis. Pyroglutamic acidosis is caused by the accumulation of 5-oxoproline [1] due to the depletion of glutathione. This leads to loss of negative feedback and therefore the build-up of Y-glutamyl cysteine, which is converted to 5-oxoproline.
Methods During a 12-month period, three patients on our ICU with unexplained high anion gap metabolic acidosis had their urine screened for organic acids.

Results All had chronic methicillin-sensitive Staphylococcus aureus infections treated with long-term paracetamol and flucloxacillin. All cases presented to intensive care with reduced level of consciousness after several weeks of treatment. In each case, common causes of high anion gap metabolic acidosis were excluded and urine specimens contained grossly elevated levels of pyroglutamic acid. Flucloxacillin and paracetamol were stopped and $\mathrm{N}$-acetylcysteine commenced, which led to resolution of the metabolic acidosis within 48 hours. All three patients made full recoveries. The first case has been previously described [2].

Conclusion Pyroglutamic acidosis is an uncommon condition, but should be considered in a high anion gap metabolic acidosis of unknown cause. The incidence in critical care may be more prevalent due to lack of screening currently. It is associated with sepsis, hepatic and renal dysfunction [3], and in patients who are receiving drugs such as paracetamol and flucloxacillin. If known precipitants are stopped, the condition can be rapidly reversed with full patient recovery.

\section{References}

1. Croal BL, et al: Clin Chem 1998, 44:336-340.

3. Peter J, et al:: Med J Aust 2006, 185:223-225.

\section{P149}

Metabolic acid-base disturbances in patients in the emergency

department

EM Antonogiannaki, E Lilitsis, D Georgopoulos

University Hospital of Heraklion, Greece

Critical Care 2012, 16(Suppl 1):P149 (doi: 10.1186/cc10756)

Introduction The aim of the present study is to determine in unselected patients that visit the emergency department whether the physicochemical approach improves the ability to diagnose acidbase disorders compared with the two commonly used diagnostic approaches; one relying on the plasma bicarbonate concentration $\left(\mathrm{HCO}_{3}^{-}\right)$and anion gap (AG), and the other on the base excess (BE).

Methods A prospective observational study took place in the emergency department at a university hospital during the period of March to September 2011. Three hundred and sixty-five patients were included. Arterial and venous samples were drawn for blood gases and a serum biochemical panel, respectively. The decision to collect arterial samples was made by the attending physician in the emergency department who was not involved in the study.

Results All patients were admitted to the hospital, while 103 of them (28\%) were transferred directly to the ICU. Hypoalbuminemia (serum albumin $\leq 35 \mathrm{~g} / \mathrm{ll}$ ) was observed in 191 patients (52\%). The BE and $\mathrm{HCO}_{3}{ }^{-}$ were normal in $35 \%$ and $38 \%$ of the total patients, respectively. The corresponding values in patients admitted to the ICU were $41 \%$ and $28 \%$. In a significant proportion of patients in whom $\mathrm{BE}$ and/or $\mathrm{HCO}_{3}^{-}$ were normal the physicochemical approach detected the presence of acidifying and/or alkalinizing disturbances. Hypoalbuminemia (metabolic alkalosis) was identified in $45 \%$ of patients with normal $\mathrm{HCO}_{3}{ }^{-}$and $48 \%$ of patients with normal BE. Strong ion difference (SID) acidosis (SID $\leq 36 \mathrm{mEq} / \mathrm{l}$ ) was observed in $49 \%$ and $44 \%$ of patients with normal $\mathrm{HCO}_{3}^{-}$and $\mathrm{BE}$, respectively. A high unmeasured strong ion concentration ( $\left[\mathrm{XA}^{-}\right] \geq 8 \mathrm{mEq} / \mathrm{l}$, metabolic acidosis) was observed in $48 \%$ of patients with normal $\mathrm{HCO}_{3}{ }^{-}$and in $52 \%$ of patients with normal $\mathrm{BE}$. Patients in whom hidden acidosis of high unmeasured strong anion type was observed were identified by the common diagnostic approach only using the $A G$ adjusted for hypoalbuminemia $\left(A G_{\text {adj }}\right.$ $\geq 13 \mathrm{mEq} / \mathrm{l}$ ). Patients who were admitted to clinical wards with acidosis, other than hyperchloremic, remained significantly more days in the hospital than those without the disturbance.

Conclusion Hypoalbuminemia is a common finding in patients in the emergency department and complicates the interpretation of acid-base data using the common diagnostic approaches. A physicochemical approach may better identify metabolic disturbances in this population. 
P150

Aberrant bone metabolism in critical illness

I Vanhees, L Solie, SJ Roberts, A Wauters, J Gunst, F Luyten,

S Van Cromphaut, G Van den Berghe, HC Owen

Katholieke Universiteit Leuven, Belgium

Critical Care 2012, 16(Suppl 1):P150 (doi: 10.1186/cc10757)

Introduction Critically ill patients present with distinct alterations in bone metabolism. We have previously reported a decrease in bone formation markers and a dramatic increase in bone resorption markers. In a rabbit model of critical illness, we observed significantly lower bone mineral content in the trabeculae of critically ill rabbits compared to healthy controls. This suggested uncoupling between bone formation and degradation during critical illness, and could increase risk of fracture during rehabilitation or impaired healing of bone fractures. In this study, we investigated the effect of critical illness on bone metabolism at the tissue and cellular level.

Methods Circulating CD14/CD11b osteoclast precursors in peripheral blood samples of critically ill patients and healthy controls were measured by flow cytometry. Peripheral blood mononuclear cells (PBMCs) were isolated and differentiated towards osteoclasts in vitro in $10 \%$ healthy (HS) or patient serum (PS) for 14 days. When analyzing bone formation, human periosteal-derived cells (hPDCs) were cultured in vitro in $10 \% \mathrm{HS}$ or PS, and analyzed for osteoblast differentiation after 14 days. Bone formation was studied using serum-treated hPDCs implanted onto $\mathrm{NuOss}^{\mathrm{TM}}$ calcium phosphate scaffolds in a murine in vivo model.

Results Circulating mononuclear precursors were increased in patients compared to healthy controls $(99.1 \%$ vs. $83.9 \% ; P<0.05)$. Patient PBMCs differentiated into mature actively resorbing osteoclasts in the presence or absence of osteoclastogenic factors (3.2-fold increase vs. healthy cells; $P<0.01)$ and when cultured in PS this spontaneous osteoclast formation was increased further $(2.3$-fold; $P<0.05)$. There were no differences in the osteogenic differentiation of hPDCs treated with PS, but there was a twofold $(P<0.01)$ decrease in vascular endothelial growth factor receptor 1 expression. Scaffolds with patient serum-treated hPDCs displayed decreased vascularization and increased osteoclast activity leading to a $28.9 \%(P<0.001)$ decrease in bone formation.

Conclusion Circulating mononuclear precursors from critically ill patients seem prone to form osteoclasts both in the presence of osteoclastogenic factors and spontaneously. The murine in vivo model confirmed an increase in osteoclastic resorption and a decreased vascularization, leading to decreased bone formation in patient scaffolds. These findings will help to unravel the mechanisms behind bone loss during critical illness.

\section{P151}

Low serum 25-hydroxyvitamin D levels and acute kidney injury in the critically ill

AB Braun', AA Litonjua', T Moromizato', FK Gibbons², E Giovannucci³ KB Christopher

'Brigham and Women's Hospital, Boston, MA, USA;'2Massachusetts General Hospital, Boston, MA, USA; ${ }^{3}$ Harvard School of Public Health, Boston, MA, USA Critical Care 2012, 16(Suppl 1):P151 (doi: 10.1186/cc10758)

Introduction Given the importance of inflammation in acute kidney injury and the relationship between vitamin $D$ and inflammation, we sought to elucidate the effect of vitamin $D$ status on acute kidney injury. We hypothesized that deficiency in 25-hydroxyvitamin D $(25(\mathrm{OH}) \mathrm{D})$ prior to hospital admission would be associated with acute kidney injury in the critically ill.

Methods We performed an observational study of patients treated in medical and surgical ICUs in two teaching hospitals in Boston, Massachusetts between 1998 and 2009. We studied 2,075 patients, age $\geq 18$ years, in whom serum $25(\mathrm{OH}) \mathrm{D}$ was measured prior to hospitalization. The exposure of interest was pre-admission serum $25(\mathrm{OH}) \mathrm{D}$ and categorized $a$ priori as deficiency $(25(\mathrm{OH}) \mathrm{D} \leq 15 \mathrm{ng} /$ $\mathrm{ml})$, insufficiency $(25(\mathrm{OH}) \mathrm{D} 15$ to $30 \mathrm{ng} / \mathrm{ml})$ or sufficiency $(25(\mathrm{OH}) \mathrm{D}$ $\geq 30 \mathrm{ng} / \mathrm{ml}$ ). The primary outcome was acute kidney injury defined as meeting RIFLE Injury or Failure criteria in the 7 days prior to critical care initiation and the 7 days following critical care initiation. We applied the serum creatinine criteria to determine the maximum RIFLE class.
Pre-admission baseline creatinine was available on all subjects. Logistic regression examined the RIFLE criteria outcome. Adjusted odds ratios (ORs) were estimated by multivariable logistic regression models. Estimates were adjusted for age, gender, race (white, nonwhite), DeyoCharlson index, sepsis and patient type (surgical vs. medical).

Results Pre-admission 25(OH)D deficiency is predictive for acute kidney injury. Patients with 25(OH)D deficiency have an OR for acute kidney injury of $1.73(95 \% \mathrm{Cl}, 1.30$ to $2.30 ; P<0.0001)$ relative to patients with $25(\mathrm{OH}) \mathrm{D}$ sufficiency. The $25(\mathrm{OH}) \mathrm{D}$ deficiency remains a significant predictor of acute kidney injury following multivariable adjustment (adjusted OR 1.50; $95 \% \mathrm{Cl}, 1.42$ to $2.24 ; P<0.0001)$. Patients with $25(\mathrm{OH})$ D insufficiency have an OR for acute kidney injury of $1.49(95 \% \mathrm{Cl}, 1.15$ to $1.94 ; P=0.003)$ and an adjusted $\mathrm{OR}$ of $1.23(95 \% \mathrm{Cl}, 1.12$ to 1.72 ; $P=0.003)$ relative to patients with $25(\mathrm{OH}) \mathrm{D}$ sufficiency. The vitamin $\mathrm{D}$-acute kidney injury association is independent of the time between 25(OH)D draw and hospital admission.

Conclusion Deficiency of 25(OH)D prior to hospital admission is a significant predictor of acute kidney injury in a critically ill patient population.

P152

Concentration of major vitamins in critically ill patients

H Hayami, K Mizutani, M Shiota, N Nakayasu, T Masubuchi, M Idei, T Gotoh Yokohama City University Hospital, Yokohama, Japan

Critical Care 2012, 16(Suppl 1):P152 (doi: 10.1186/cc10759)

Introduction Commercially available vitamin solutions have been improved during the last decade, but there have been a few recent reports on deficiency occurring in the ICU setting. In general, daily delivery of a comprehensive modern vitamin regimen will suffice and TPN solution which contains vitamins and trace elements is now widely used because of the view of convenience and infection control. But the dose of vitamins is determined by the American Medical Association (AMA) recommendation which is based on requirement by healthy subjects and it is not clear whether the dose is applicable for critically ill patients. We measured the concentration of major vitamins in 19 critically ill patients who stayed in the ICU and analyzed those treated for more than 3 weeks.

Methods Of 19 patients, 10 were treated for more than 3 weeks under artificial ventilation; seven of which received renal replacement therapy (RRT). Early enteral nutrition was established in six patients who were assessed to have normal intestinal function. For those patients who were diagnosed to have malfunction in intestine, nutrition was supplied via peripheral route for 1 week, and led to total parenteral nutrition after 1 week. Multivitamin product (Vit B1 3 mg, Vit B6 4 mg, Vit C $100 \mathrm{mg}$, Folate $400 \mathrm{mg}$, and so on) was administered from day 0 . We measured the concentration of those vitamins every 7 days.

Results Concentrations of Vit B1, Vit 12, and Folate were $37 \pm 16 \mathrm{pg} /$ $\mathrm{ml}, 1,068 \pm 1,702 \mathrm{pg} / \mathrm{ml}$, and $9.9 \pm 14.4 \mathrm{ng} / \mathrm{ml}$ on day 7 , and there was tendency of increasing to normal range subsequently. On the other hand, the concentration of Vit C was low $(2.5 \pm 2.4 \mathrm{~g} / \mathrm{ml}$ : median 1.75) on day 7 , and it remained low through 3 weeks (median $2.0 \mathrm{~g} / \mathrm{ml}$ on day 21). Especially, the concentration of Vit $C$ was extremely low in seven patients who received RRT (median $1.0 \mathrm{~g} / \mathrm{ml}$ ) on day 7 compared with those without RRT (median $2.6 \mathrm{~g} / \mathrm{ml}, P=0.05$ ). We administered a high dose of Vit C (ascorbic acid 1,000 mg/day) for three patients in this group but restoration to normal range was seen in only two patients. Conclusion In critically ill patients, especially those who received RRT, the concentration of water-soluble vitamins such as Vit $\mathrm{C}$ remained low even when they received the AMA recommended dose. Measurement of vitamins and additional administration will be needed as necessary depending on the disease condition.

\section{P153}

No association between vitamin $D$ deficiency at admission and outcome in a medical ICU

M Claus, L Schmitz, A Roman, E Stevens, P Dechamps

Hôpital Saint Pierre, Brussels, Belgium

Critical Care 2012, 16(Suppl 1):P153 (doi: 10.1186/cc10760)

Introduction Vitamin D deficiency is associated with chronic illness and an excess in morbidity and mortality in the general population. Studies have found an even higher prevalence in ICU patients. 
The aim of our study was to evaluate the relationship between $25-\mathrm{OH}$ vitamin $D$ deficiency at admission and the outcome in a medical ICU. Methods A prospective observational study in a 10-bed medical ICU at an inner-city hospital in Brussels. Patients with an expected stay in ICU $>48$ hours were included.

Results Vitamin D deficiency was defined as a serum $25-\mathrm{OH}$ vitamin $\mathrm{D}$ concentration $<20 \mathrm{ng} / \mathrm{ml}$. The study was conducted between February and August 2011. A total of 105 patients were included. Dosages were performed on day $3(2,4)$ (median, interquartiles). The number of patients with $25-\mathrm{OH}$ vitamin $\mathrm{D}<10 \mathrm{ng} / \mathrm{ml}$, between 10 and $20 \mathrm{ng} / \mathrm{ml}$, between 20 and $30 \mathrm{ng} / \mathrm{ml}$ and $>30 \mathrm{ng} / \mathrm{ml}$ was respectively $56,26,14$ and 9 . No differences were seen between deficient and nondeficient patients if we compare SAPS III ( $58 \pm 13$ vs. $60 \pm 15)$, predicted mortality ( $34 \pm 21 \%$ vs. $40 \pm 25 \%$ ), intra-ICU mortality ( 8.5 vs. $8.7 \%$ ), intrahospital mortality (19.5 vs. $21.7 \%)$, mean length of stay in the ICU (10 days \pm 8 ), and median SOFA score during the first 5 days $(5,4,4,3,3$ vs. 4, 4, 3, $3,4)$. A higher (but nonsignificant) prevalence of sepsis was found at admission in deficient patients ( $42 / 82$ patients vs. $8 / 23$ patients). Eleven deficient patients were treated with oral vitamin $D(25,000$ units/day) for 5 days. After treatment, $25-\mathrm{OH}$ vitamin D was above $20 \mathrm{ng} / \mathrm{ml}$ in seven patients $(31 \pm 14 \mathrm{ng} / \mathrm{ml})$. If we adjust groups for vitamin $D$ post treatment, no differences were found if we compare deficient versus nondeficient patients for intra-ICU mortality $(9.3 \%$ vs. $6.6 \%)$ and intrahospital mortality ( $14.6 \%$ vs. $23.3 \%)$.

Conclusion Our study confirmed the high prevalence of vitamin D deficiency in ICU patients but not the association with an excess of mortality.

Reference

1. Lee P, et al:: N Engl J Med 2009, 360:1912-1914.

P154

Plasma levels of Coenzyme Q10 are reduced in critically ill patients as compared to healthy volunteers and correlate with age

A Coppadoro', L Berra², A Kumar², M Yamada², R Pinciroli², E Bittner²,

U Schmidt ${ }^{2}$, M Kaneki $^{2}$

'University of Milan-Bicocca, Monza, Italy; ${ }^{2}$ Massachusetts General Hospital,

Boston, MA, USA

Critical Care 2012, 16(Suppl 1):P154 (doi: 10.1186/cc10761)

Introduction The purpose of this study is to investigate Coenzyme Q10 (Q10) levels in critically ill patients as compared to healthy volunteers. Q10 is an essential cofactor for the electron transport chain reactions necessary for the aerobic cellular respiration. Q10 insufficiency, therefore, leads to mitochondrial dysfunction. It also acts as an antioxidant. Oxidative state is prominent in critically ill patients, favoring the production of oxygen-free radicals. A recent study showed reduced Q10 levels in septic shock patients [1].

Methods We recruited 18 healthy volunteers and 36 critically ill patients in the surgical ICU of the Massachusetts General Hospital. Ethical committee approval and written informed consent were obtained. At the moment of blood sampling, height, weight, and age as well as clinical data were collected. Plasma total Q10 concentrations were measured by high-performance liquid chromatography. The Assessment of Daily Living (ADL) score was obtained after discharge. Results Patients' age and gender did not differ as compared to healthy volunteers $(P=$ NS). Plasma Q10 levels were lower in critically ill patients as compared to healthy volunteers $(0.81 \pm 0.22$ vs. $0.50 \pm 0.36 \mu \mathrm{g} / \mathrm{ml}$, $P<0.001)$. In critically ill patients, plasma Q10 levels inversely correlated with age $(R=0.40, P=0.015)$. Lower levels of plasma Q10 $(<0.4 \mu \mathrm{g} /$ $\mathrm{ml}$, median) were associated with lower ADL score after discharge $(P=0.005)$. In our patient population, plasma Q10 levels were not related to $\mathrm{PaO} / \mathrm{FiO}_{2}$, septic shock, SAPS 2 at ICU admission, SOFA score or mortality (all $P=\mathrm{NS}$ ).

Conclusion Plasma Q10 levels are reduced in critically ill patients, suggesting reduced antioxidant capacity. Older patients seem to be more prone to exhibit low Q10 levels. Oral supplementation might be considered for those patients.

Reference

1. Donnino MW, et al: Coenzyme Q10 levels are low and may be associated with the inflammatory cascade in septic shock. Crit Care 2011, 15:R189.
P155

Reduced cortisol metabolism drives hypercortisolism in critical illness

E Boonen ${ }^{1}$, H Vervenne1, P Meersseman², L Mortier ${ }^{3}$, YM Vanwijngaerden'1, I Spriet², L Langouche', I Vanhorebeek', G Van den Berghe'

${ }^{1}$ KU Leuven, Belgium; ${ }^{2}$ University Hospitals, Leuven, Belgium; ${ }^{3}$ Virga Jesse

Hospital, Hasselt, Belgium

Critical Care 2012, 16(Suppl 1):P155 (doi: 10.1186/cc10762)

Introduction Critical illness is hallmarked by elevated cortisol levels, reflecting the severity of illness. Paradoxically, previous studies reported suppressed $\mathrm{ACTH}$, implicating another mechanism driving elevated cortisol during critical illness. We hypothesized that cortisol metabolism is reduced in critical illness, in part via elevated bile acids, which may explain the paradoxical ACTH-cortisol dissociation by negative feedback inhibition.

Methods In a first clinical study $(n=59)$, we determined the time course of ACTH and cortisol levels during the first week in the ICU. In a second study $(n=28)$, we calculated the plasma half-life of exogenous cortisol in critically ill patients. In a third clinical study $(n=51)$, urinary cortisol metabolites were quantified to estimate the activity of cortisol metabolizing enzymes. In a fourth study $(n=64)$, we quantified the major cortisol metabolizing enzymes in the liver and adipose tissue in relation to circulating cortisol and bile acids. We performed every study in a similar, heterogeneous ICU population, in comparison with a healthy control group matched for age, gender and BMI.

Results In the presence of elevated total cortisol, ACTH remained much lower in patients than in healthy controls $(P<0.001)$, confirming the ACTH-cortisol dissociation during critical illness. Cortisol half-life was substantially prolonged in patients compared to controls. Based on urinary metabolites, the activity of 5 a-reductase and $5 \beta$-reductase was significantly lower in patients than controls $(P<0.0001)$. Furthermore, the calculated activity of 11-hydroxysteroid dehydrogenase type 2 was reduced $(P<0.0001)$. In the liver, gene and protein expression of 5 a-reductase and $5 \beta$-reductase was reduced $(P<0.0001)$ and correlated inversely with circulating cortisol. Moreover, the enzyme expression correlated inversely with circulating levels of conjugated bile acids, which were markedly elevated in patients [1] and which have been shown capable of suppressing expression and activity of cortisol metabolizing enzymes [2].

Conclusion Reduced expression and activity of cortisol metabolizing enzymes, possibly driven by elevated bile acids, contributes to the hypercortisolism in the critically ill, which explains the increased cortisol plasma half-life and feedback-inhibited ACTH release. Reduced cortisol metabolism could inferentially suppress the cortisol response to an ACTH stimulation test, thereby reducing its diagnostic value for adrenal failure.

References

1. Vanwijngaerden et al: Hepatology 2011, 54:1741-1752.

2. McNeilly et al:. I Hepatol 2010, 52:705-711.

P156

Effect of low-dose hydrocortisone on the expression of glucocorticoid receptor alpha of the septic kidney in rats and its protective effect on kidney injury

DWWu, HP Guo

Qilu Hospital of Shandong University, Jinan, Shandong, China Critical Care 2012, 16(Suppl 1):P156 (doi: 10.1186/cc10763)

Introduction Inflammation out of control caused by sepsis can eventually lead to multiple organ dysfunction, of which the kidney is one of the most common injured organs. Sepsis-induced acute kidney injury (SI-AKI) can obviously increase the mortality of sepsis. At present, there are controversial views about the impact of exogenous glucocorticoid to SI-AKI on kidney pathological changes and glucocorticoid receptor (GR) expression. So, we want to investigate whether low-dose glucocorticoid has a protective effect on SI-AKI and what is the mechanism.

Methods Healthy Wistar male rats were randomly divided into a sham group, SI-AKI group and SI-AKI hydrocortisone group (HC group). The 
SI-AKI model was reproduced using the cecum ligation and puncture method. Pathological changes of the kidney were detected by $\mathrm{H}$ \& $\mathrm{E}$ staining. The expression of GRa and NF-KB in the kidney was detected by immunohistochemistry. The levels of IL-1, IL-6, TNFa and IL-10 in the plasma were detected by ELISA.

Results The survival rate of the AKI group and HC group showed no statistical difference $(P>0.05)$. H \& E stain showed renal tubular epithelial cells swelling and falling off, and the tubular brush border disappeared and vacuolated in the AKI group. Pathological changes of the renal tubular could be alleviated after hydrocortisone treatment. Compared with the AKI group, immunohistochemistry showed that GRa expression was increased and NF-KB expression was decreased in the HC group $(P<0.01)$. The level of TNFa, IL- 6 , and IL-1 were reduced and the level of IL-10 was increased in the $\mathrm{HC}$ group compared with the AKI group $(P<0.01)$.

Conclusion Low-dose hydrocortisone can inhibit the NF-KB activity, possibly in part by increasing the expression of GRa in renal sepsis rats. Accordingly, it could reduce the production of inflammatory factors participating in sepsis, effectively inhibit the inflammation and extenuate the sepsis-induced renal pathological changes.

References

1. Robter W, et al: NEngl J Med 2004, 351:159-169.

2. Rittirsch D, et al:: Nat Protoc 2009, 4:31-36.

3. Leelahavanichkul A, et al:. Am J Physiol Renal Physiol 2008, 295:1825-1895.

P157

Hydrocortisone increases the risk of dysglycemia in critically ill

patients

RT Van Hooijdonk, JM Binnekade, RE Harmsen, MJ Schultz

Academic Medical Center, Amsterdam, the Netherlands

Critical Care 2012, 16(Suppl 1):P157 (doi: 10.1186/cc10764)

Introduction Hyperglycemia and hypoglycemia are independently associated with mortality and morbidity of critically ill patients $[1,2]$. Critically ill patients frequently receive hydrocortisone for refractory shock. While hydrocortisone infusion is associated with hyperglycemia [3], the effect of hydrocortisone on the incidence of hypoglycemia is uncertain. We hypothesized hydrocortisone infusion to increase the risk of hyperglycemia and hypoglycemia in critically ill patients.

Methods Blood glucose measurements $(n=73,400)$ of patients admitted to the ICU from January 2007 to December $2009(n=2,167)$ were analyzed. Logistic regression was used to analyze the effect of hydrocortisone infusion on mild (blood glucose level $\geq 150 \mathrm{mg} / \mathrm{dl}$ ) and severe hyperglycemia ( $\geq 180 \mathrm{mg} / \mathrm{dl}$ ) and mild hypoglycemia ( $\leq 70 \mathrm{mg} /$ dl) separately. To adjust for severity of disease, patients were stratified in APACHE II score groups $(<15 ; 15$ to $24 ;>24)$.

Results Hydrocortisone infusion was independently associated with mild hypoglycemia (APACHE II score $<15$, OR $2.40,95 \% \mathrm{Cl} 2.01$ to 2.85; APACHE II score 15 to 24 , OR $1.53,95 \%$ Cl 1.44 to 1.62 ; APACHE II score $>24$, OR $1.10,95 \% \mathrm{Cl} 1.05$ to 1.15 ) and severe hyperglycemia in all APACHE II groups (APACHE II score $<15$, OR 3.26, 95\% Cl 2.59 to 4.10; APACHE II score 15 to 24 OR $1.45,95 \% \mathrm{Cl} 1.33$ to 1.68 ; and APACHE II score $>24$ OR $1.09,95 \% \mathrm{Cl} 1.02$ to 1.17$)$. Hydrocortisone infusion was independently associated with mild hypoglycemia in patients with APACHE II score 15 to 24 (OR $1.74,95 \% \mathrm{Cl} 1.42$ to 2.13 ) and $>24$ (OR $1.64,95 \% \mathrm{Cl} 1.42$ to 1.90 ), but not in patients with APACHE II score $<15$ (OR $1.83,95 \% \mathrm{Cl} 0.94$ to 3.55 ).

Conclusion Hydrocortisone increases the risk of dysglycemia in critically ill patients. Whether these dysglycemic effects diminish the beneficial effects of hydrocortisone treatment should be investigated in future studies.

References

1. Krinsley JS, et al:: Crit Care Med 2007, 35:2262-2267.

2. Bagshaw SM, et al.: Crit Care Med 2009, 37:463-470.

3. Annane D, et al:. JAMA 2009, 301:2362-2375.
P158

Nutritional status of patients occupying ICUs in the state of Rio de Janeiro

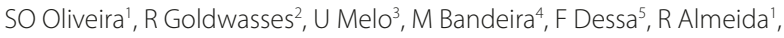
I Kouh ${ }^{6}$

'Albert Schweitzer State Hospital, Rio de Janeiro, Brazil; ${ }^{2}$ Healh Provider

State Government, Rio de Janeiro, Brazil; ${ }^{3}$ Alberto Torres State Hospital, São Gonçalo, Brazil; ${ }^{4}$ Real Cordis Hospital, Rio de Janeiro, Brazil; ${ }^{5}$ Bangu Hospital, Rio de Janeiro, Brazil; ${ }^{6}$ UFRJ University Hospital, Rio de Janeiro, Brazil Critical Care 2012, 16(Suppl 1):P158 (doi: 10.1186/cc10765)

Introduction Nutritional status and anemia influence the clinical course of hospitalized patients. Anemia appears in the first days of hospitalization and can sustain itself and grow worse over time and is caused by a number of factors such as dilution secondary to fluid replacement, hemolysis, abnormalities in iron metabolism, blood loss in the gastrointestinal tract and also due to decreased production of erythropoietin, a consequence of decreased erythropoiesis caused by the presence of inflammatory cytokines.

Methods A cross-sectional study on 30 November, patients $>18$ years. Evaluated characteristics of all patients admitted with age, sex, APACHE II score, mean length of stay, cause of hospitalization in mechanical ventilation, organ failure, sedation and analgesia, coma and underuse of vasoactive drugs.

Results The study included 247 patients hospitalized in ICUs, mean age 63 years and $60 \%$ (148 patients) were male. Sepsis was the most frequent cause of hospitalization at $57 \%$ and the average hospital stay was 16 days. The rate of albumin and mean hemoglobin level were respectively 2.1 and $9.5 \mathrm{~g} / \mathrm{dl}$. For those patients hospitalized over 10 days were observed average levels of 1.5 and $8.9 \mathrm{~g} / \mathrm{dl}$. For mechanical ventilation in patients with septic shock the results were 1.4 and $7.9 \mathrm{~g} / \mathrm{dl}$ with a mean hospital stay of 14 days. The postoperative group was the highest level observed at 2.6 and $10.4 \mathrm{~g} / \mathrm{dl}$ and mean total time of hospitalization of 5 days. The worst results based on diagnosis were respectively pulmonary septic shock, ischemic hemispheric brain stroke and cardiogenic shock. All patients with length of stay over 11 days resulted in a clinically malnourished state.

Conclusion The age, length of stay and diagnosis associated with the level of organ dysfunction are key factors to progress to the state of malnutrition. The multidisciplinary team has an ongoing role in controlling the supply of proteins and calories with essential nutrients in order to improve the provision, preventing complications and adverse outcomes.

\section{Reference}

1. Heymsfield SB, Baumgartner RN, Pan FS: Nutritional assessment of malnutrition by anthropometric methods. Treaty of Modern Nutrition in Health and Disease. Edited by Shils M, Olson JA, Shike M, Ross C. New York: Manole; 2003.

P159

Investigating diarrhoea on the ICU: a retrospective study

N Tirlapur, M Kelsey, H Montgomery

Whittington Hospital, London, UK

Critical Care 2012, 16(Suppl 1):P159 (doi: 10.1186/cc10766)

Introduction Diarrhoea is common in ICU patients, with a reported prevalence of 15 to $38 \%$ [1]. Many factors may cause diarrhoea, including Clostridium difficile, drugs (for example, laxatives, antibiotics), faecal impaction with overflow and enteral feeds. Diarrhoea increases nursing workload, impacts on patient dignity, increases costs and exacerbates morbidity through dermal injury, impaired enteral uptake and subsequent fluid imbalance. We aimed to identify prevalence, yield of stool investigations and clinical impact of diarrhoea on our ICU.

Methods A retrospective observational study of all ICU patients treated in a 15-bed district general hospital from 1 January 2010 to 31 December 2010 was performed. ICU patients from whom stool samples had been sent for microbiological analysis (including microscopy and C. difficile toxin (CDT)) were assumed to have suffered diarrhoea. Stool sample results were compiled with patient demographics, ICU length of stay (LOS) and mortality data. 
Results Of 782 patients (mean age \pm 2 SD $62.1 \pm 37.1$, 52.3\% female) treated on our ICU, 334 stool samples were sent from 133 (17.0\%) patients. Two samples $(0.6 \%)$ yielded abnormal results: one out of $131(0.8 \%)$ patients with CDT samples sent and one out of $108(0.9 \%)$ patients with stool microscopy samples sent had a positive sample. The prevalence of $C$. difficile (1/782) and other organisms (1/782) was $0.1 \%$ and $0.1 \%$ respectively. In terms of diagnostic yields, positive findings were found in one out of $191(0.5 \%)$ CDT samples and one out of $141(0.7 \%)$ stool microscopy samples (for Candida). When compared to patients without diarrhoea, sufferers were older $(64.1 \pm 33.2$ vs. $61.7 \pm 37.8$ years, $P=0.16)$ with greater female preponderance $(55.6 \%$ vs. $51.6 \%, P=0.40)$. Sufferers experienced longer ICU LOS $(16.3 \pm 45.6$ vs. $4.6 \pm 19.4$ days, $P<0.0001)$ and greater ICU mortality $(19.5 \%$ vs. $12.6 \%, P=0.035)$ during the study period.

Conclusion Diarrhoea was common on our ICU, its prevalence $(17 \%)$ being consistent with established literature. It was associated with statistically increased ICU LOS and mortality, although any direction of causality remains to be established. A low stool investigation yield and low prevalence of $C$. difficile suggests that other noninfective causes of diarrhoea need excluding. Further research is required to establish the prevalence and pathogenesis of diarrhoea on UK ICUs, in order to develop evidence-based management plans for reducing its incidence, and its clinical and financial impact.

Reference

1. Wiesen P, et al:: Curr Opin Crit Care 2006, 12:149-154

P160

Preliminary report of surface electrogastrography in critically ill septic patients after resuscitation

C Mancilla, R Galvez, G Landskron, E Tobar, A Madrid

Hospital Clinico Universidad de Chile, Santiago, Chile

Critical Care 2012, 16(Suppl 1):P160 (doi: 10.1186/cc10767)

Introduction Impaired gastrointestinal motility is common in critically ill patients. Multiple conditions such as shock with diminished splanchnic perfusion, surgery, fluid overload, intra-abdominal hypertension, and drugs are responsible for this phenomena. Assessing gastric motility in this setting is complex. Surface electrogastrography (sEGG) is a recent noninvasive technique that determines basal and postprandial gastric motility [1]. Our aim is to study basal gastric motility in critically ill septic patients in the post-resuscitative phase, by sEGG.

Methods Eligible patients were those admitted to the ICU with diagnosis of septic shock as stated by the Sepsis Conference 2001 [2]. At the moment of the study the patients were in the post-resuscitative phase, defined as normal clinical and laboratory perfusion parameters. sEGG is a noninvasive technique that uses skin abdominal electrodes to record myoelectrical stomach activity. The basal slow wave originates in the proximal stomach and propagates to the antrum with a frequency of approximately 3 cycles per minute (cpm). Basal activity below $2.4 \mathrm{cpm}$ is defined as bradygastria and above $3.7 \mathrm{cpm}$ as tachygastria [1]. Data were correlated with severity scores, lactate levels, and doses of sedatives. The study was approved by the Ethics Committee of the Hospital Clínico Universidad de Chile.

Results We recruited 16 patients ( 10 females). Mean age 62 years (50 to 76) $(P=0.8)$. APACHE II score 25 (19 to 28$)(P=0.4)$ and SOFA score 9 (7 to 11$)(P=0.29)$. Lactate at admission $3.8 \mathrm{mmol} / \mathrm{l}(1.2$ to 6.5$)(P=0.72)$. Fentanyl total dose $172.7 \mu \mathrm{g} / \mathrm{kg}$ (59 to 256.6$)(P=0.91)$ and midazolan total dose $3.4 \mathrm{mg} / \mathrm{kg}$ (0.1 to 3.1$)(P=0.07)$. We obtained a reliable register in all the patients and found six patients with bradigastria, three with tachygastria and nine with normal motility. In this small sample size study there was a trend to bradygastria in relation to high total doses of midazolam.

Conclusion sEGG is a feasible technique in critically ill septic patients. In the post-resuscitative phase $43.8 \%$ of patients present normal gastric motility, and 37\% showed bradygastria. Future research is warranted in order to find risk factors of gastrointestinal dismotility.

References

1. Chang F-Y: J Gastroenterol Hepatol 2005, 20:502-516.

2. Levy MM, et al.: Crit Care Med 2003, 31:1250-1256.
P161

Frequency, determinants and impact of feed intolerance amongst the critically ill

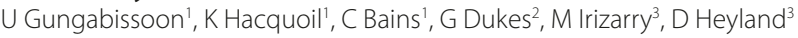
'GlaxoSmithKline, Stockley Park, UK; 'laxoSmithKline, Research Triangle Park, NC, USA; ${ }^{3}$ CERU, Queen's University, Kingston, Canada

Critical Care 2012, 16(Suppl 1):P161 (doi: 10.1186/cc10768)

Introduction Provision of early and adequate enteral nutrition (EN) to critically ill patients is associated with improved clinical outcomes; however, 50 to $60 \%$ of prescribed EN is received. We aimed to characterise the incidence and determinants of intolerance and assess its influence on nutritional and clinical outcomes using the 2009 Critical Care Nutrition Survey (CCNS).

Methods The CCNS survey is a prospective observational cohort study of nutrition practices from over $150 \mathrm{ICU}$ around the world. Included patients were those that remained in ICU for $\geq 72$ hours and were mechanically ventilated $\leq 48$ hours of admission to ICU. We collected pertinent baseline and outcome data that included nutritional adequacy, ventilator-free days, 60-day mortality and ICU stay. Intolerance was defined as interruption of EN due to gastrointestinal (GI) reasons (high gastric residuals, increased abdominal girth/ abdominal distension, vomiting/emesis, diarrhoea or subjective discomfort). In the analysis of intolerance we included each potential effect into a logistic regression analysis to determine its significance. Results Data from 1,888 ICU patients receiving EN were analysed. The incidence of intolerance was $30.5 \%$, and occurred after a median 3 days from EN initiation. Factors associated with intolerance were: diagnosis category $(P=0.0009)(\mathrm{Gl}$, cardiovascular and sepsis categories with the highest risk), pre-emptive motility agent use $(P=0.0125)$, non$\mathrm{Gl}$ interruptions to feed $(P=0.0086)$ and global region $(P=0.0006)$. Intolerance was associated with poor nutritional adequacy, increased mortality, longer ventilator dependence and increased length of ICU stay $(P<0.05)$ (Table 1). Poorer clinical outcomes were seen with increasing number of days of intolerance.

Table 1 (abstract P161). Nutritional adequacy and clinical endpoints in tolerant and intolerant EN patients

\begin{tabular}{lcccccc}
\hline & $\begin{array}{c}\% \\
\text { Calorific } \\
\text { adequacy }\end{array}$ & $\begin{array}{c}\text { Protein } \\
\text { adequacy }\end{array}$ & $\begin{array}{c}\text { Ventilator- } \\
\text { free } \\
\text { days }\end{array}$ & $\begin{array}{c}\text { 60-day } \\
\text { mortality }\end{array}$ & $\begin{array}{c}\text { ICU stay } \\
\text { (days) }\end{array}$ & $\begin{array}{c}\text { Tische to } \\
\text { alive }\end{array}$ \\
\hline Tolerant & 64.3 & 63.7 & 11.2 & 26.2 & 11.3 & 25.2 \\
Intolerant & 55.5 & 55.6 & 2.5 & 30.8 & 14.4 & 31.1 \\
\hline
\end{tabular}

Conclusion Intolerance is common amongst the EN ICU population and is associated with poor nutritional and clinical outcomes.

\section{P162}

Gastric emptying assessment in critically ill patients with feed intolerance; comparison of ${ }^{13} \mathrm{C}$ octanoic acid, paracetamol and 3-O-methylglucose absorption tests

M Chapman', R Fraser ${ }^{1}$, N Nguyen'1, A Deane ${ }^{1}$, LS Vasist ${ }^{2}$, K Hacquoil $^{2}$, M Barton ${ }^{2}$, GE Dukes ${ }^{2}$

${ }^{1}$ University of Adelaide, Australia; ${ }^{2}$ GlaxoSmithKline, Research Triangle Park, NC, USA

Critical Care 2012, 16(Suppl 1):P162 (doi: 10.1186/cc10769)

Introduction Delayed gastric emptying (GE) occurs frequently in critically ill patients and may result in impaired small intestinal delivery of drugs and nutrients. Use of direct methods of GE assessment (scintigraphy) in the ICU for clinical monitoring or research is challenging. Indirect methods that utilize substances which rely on effective GE and rapid absorption from the small intestine offer a feasible estimate of GE. Three substances with these characteristics are ${ }^{13} \mathrm{C}$-octanoic acid $\left({ }^{13} \mathrm{C}\right)$, paracetamol (PA) and 3-O-methylglucose (OMG). We have previously shown significant correlation to scintigraphy for ${ }^{13} \mathrm{C}(r=0.63)$ and OMG absorption ( $r=-0.77$ to -0.87 ). The current study examined the relationship between three indirect methods of GE assessment: ${ }^{13} \mathrm{C}$, PA, OMG. 
Methods GE was concurrently assessed in mechanically ventilated patients $(n=33)$ with enteral feeding intolerance (gastric residual volume $>200 \mathrm{ml}$ ) on two occasions 24 hours apart. A test meal of 100 $\mathrm{ml}$ Ensure with $100 \mu \mathrm{l}{ }^{13} \mathrm{C}, 1,000 \mathrm{mg} \mathrm{PA}, 3 \mathrm{~g}$ OMG was infused into the stomach over 5 minutes. Breath samples for ${ }^{13} \mathrm{C}$ and plasma samples for PA, OMG determination were collected over the subsequent 4-hour period. Bivariate Pearson correlations were calculated between the following parameters; ${ }^{13} \mathrm{C}$ (gastric emptying coefficient (GEC)), PA (concentration at 60 minutes $\left.\left(C_{60}\right), A \cup C_{0-60 \text { min }}\right)$, OMG $\left(\left(C_{60}\right), A \cup C_{0-60 \text { min }}\right)$. See Table 1.

Table 1 (abstract P162). Correlations between GE parameters

\begin{tabular}{lcl}
\hline${ }^{13} \mathrm{CGEC}$ & OMG AUC $_{60}$ & $0.675^{*}$ \\
${ }^{13} \mathrm{CGEC}$ & OMG C $_{60}$ & $0.758^{*}$ \\
${ }^{13} \mathrm{CGEC}$ & PA AUC $_{60}$ & $0.678^{*}$ \\
${ }^{13} \mathrm{CGEC}$ & $\mathrm{PAC}_{60}$ & $0.590^{*}$ \\
$\mathrm{PAAUC}_{60}$ & $\mathrm{OMG} \mathrm{AUC}_{60}$ & $0.534^{*}$ \\
$\mathrm{PAC}_{60}$ & OMGC $_{60}$ & $0.553^{*}$ \\
\hline
\end{tabular}

${ }^{*} P<0.0001$.

Results Observed GE rates for all parameters were across the spectrum from fast to delayed (for example, with $r$ range $=0.534$ to 0.758 , $P<0.0001)$

Conclusion These three practical indirect methods of GE assessment may provide similar estimates of GE in critically ill patients.

P163

Early enteral nutrition in the critically ill: a single-centre study

A Gupta, E Rupert, R Sharma, D Mishra

Rabindranath Tagore Hospital, Kolkata, India

Critical Care 2012, 16(Suppl 1):P163 (doi: 10.1186/cc10770)

Introduction Early enteral nutrition in critically ill patients has been established as a valuable addition to improve overall outcome and mortality. The recommendation is to initiate feeding within 24 to 48 hours of admission and to meet the calorie goal within the next 48 to 72 hours. The purpose of this study was to find out whether these guidelines are followed in the ICU as per the new protocol.

Methods This is a prospective observational study done in a 32-bed mixed medical and surgical ICU over the period from March 2011 to August 2011. Consecutively, 575 patients admitted to this ICU were followed up. Nineteen patients were excluded from the study where enteral nutrition could not be commenced within 48 hours due to various reasons. The remaining 556 patients' data were analyzed. Data were collected by interviewing the doctors and nurses as well as reviewing medical notes and all ICU charts.

Results In the 556 study patients, early enteral feeding was started in 379 patients $(68.16 \%)$. Out of 379 patients, $100 \%$ calorie requirements were met only in 43 patients (7.73\%). For the remaining study patients, more than $40 \%, 50 \%$ and $60 \%$ calorie goals were achieved in 115 (30.34\%), 128 (33.77) and 93 (24.53\%) patients respectively.

Conclusion The initiation of early enteral feeding is still far off for a significant proportion of the ICU population despite evidence-based definite recommendations to improve ICU outcome. The calorie goal achievements were also very suboptimal. This important but still neglected nutritional therapy must be carefully looked at and implemented in all ICUs.

References

1. McClave SA, Martindale RG, Vanek VW, et al:: Guidelines for the provision and assessment of nutrition support therapy in the adult critically ill patient: Society of Critical Care Medicine (SCCM) and Americal Society for Parenteral and Enteral Nutrition (A.S.P.E.N.). JPEN J Parenter Enteral Nutr 2009, 33:277-316

2. Kreyman $\mathrm{KG}$, Berger MM, Deutz NE, et al:: ESPEN guidelines on enteral nutrition: intensive care. Clin Nutr 2006, 25:210-223.
P164

Preliminary experience with ketone-targeted treatment of diabetic ketoacidosis

F Riccio, T Drake, S Mathieu, J Cranshaw

Royal Bournemouth Hospital, Bournemouth, UK

Critical Care 2012, 16(Suppl 1):P164 (doi: 10.1186/cc10771)

Introduction In May 2011 the Joint British Diabetes Societies (JBDS) published new guidance for managing adult diabetic ketoacidosis. We developed a JBDS-based protocol that measured and treated capillary ketonaemia (not blood glucose) hourly with i.v. $0.1 \mathrm{IU}$ insulin/kg/hour increased by $1 \mathrm{IU} /$ hour if the ketone reduction was $<0.5 \mathrm{mM} /$ hour. The final target was capillary ketonaemia $<0.3 \mathrm{mM}$. To allow this insulin rate and avoid hypoglycaemia, $125 \mathrm{ml} /$ hour of 10 or $20 \%$ dextrose was started when blood glucose was $<14 \mathrm{mM}(250 \mathrm{mg} / \mathrm{dl})$. As the effects of this new protocol were unknown, all patients were managed in our high-dependency unit (HDU). We report our experience of the new protocol compared to our old 'sliding scale' insulin titration to blood glucose protocol.

Methods We prospectively gathered results of the new protocol over 3 months and performed a chart review of the same results from patients admitted in the previous year managed on wards and the HDU. Results are expressed as median (range).

Results Patients on the new protocol $(n=7)$ cleared ketones to $<0.3 \mathrm{mM}$ in 8 (7 to 20) hours. The insulin rate needed was 10 (6 to 17) IU/hour. Potassium during treatment was 4 (3.2 to 5.2$) \mathrm{mM}$ and required 35 (12 to 60 ) $\mathrm{mM} /$ hour to maintain the target 3.5 to $5 \mathrm{mM}$. No episodes of blood glucose $<4 \mathrm{mM}$ were recorded. Time to reach glucose $<14 \mathrm{mM}$ was 7 ( 1 to 15 ) hours with a fall rate of 3.7 ( 2.9 to 6.8 ) $\mathrm{mM} /$ hour. Patients on the old protocol $(n=39)$ were treated for 15 (5 to 20) hours with 3 (0.5 to 6) IU/hour. Potassium during treatment was 3.5 (2.8 to 5.5) mM and required 9 ( 6 to 16$) \mathrm{mM} /$ hour to maintain the target 3.5 to $5 \mathrm{mM}$. Time to reach glucose $<14 \mathrm{mM}$ was 3.5 (1 to 13 ) hours with a fall rate of $4.2(0.3$ to 13$) \mathrm{mM} /$ hour. A total 0.02 results per hour $<4 \mathrm{mM}$ were recorded in the old protocol.

Conclusion The median insulin infusion rate in an individual patient and the range required to suppress capillary ketonaemia in all patients with diabetic ketoacidosis using this protocol was more than three times that in the old protocol and the amount of i.v. potassium required to maintain near-normal blood potassium during treatment was four times more. There was a slower correction of initial blood glucose. Blood glucose and potassium maintenance during treatment with this protocol would appear to require high-intensity nursing care to maintain patient safety.

Reference

1. Joint British Societies Guideline for the management of diabetic

ketoacidosis. Diabet Med 2011, 28:508-515.

P165

Impact of blood glucose on blood lactate levels in a medical ICU:

a retrospective cohort study

G Adelsmayr, R Brunner, U Holzinger

Medical University Vienna, Austria

Critical Care 2012, 16(Suppl 1):P165 (doi: 10.1186/cc10772)

Introduction Although blood lactate and glucose both represent important markers in the intensive care setting, they have been considered quite independently. Especially, the ideal glucose target range has been the topic of recent studies with conflicting results [1,2]. Blood lactate is an acknowledged predictor of outcome in critically ill patients [3]. The aim of this study was to establish a possible correlation between elevated blood glucose and lactate levels in intensive care patients.

Methods Blood gas data of 1,170 patients, admitted to the medical ICU of the Department of Medicine III, Medical University Vienna, between the years 2001 and 2009, were analysed retrospectively. The association of circulating blood glucose levels with corresponding lactate levels was investigated using a linear regression model. The impact of different blood glucose intervals $(<80,80$ to 120,120 to 160 , 160 to $200,>200 \mathrm{mg} / \mathrm{dl}$ ) on blood lactate levels was analysed using ANOVA. The influence of blood glucose variability, expressed as the 
blood glucose standard deviation, on mean lactate concentrations for the period of ICU stay was analysed using a linear regression model. To adjust for the severity of illness, a multivariate regression analysis was conducted including SAPS II and APACHE II scores.

Results Blood glucose and lactate presented a U-shaped curve with a minimum blood lactate $(1.5 \mathrm{mmol} / \mathrm{l})$ between 80 and $120 \mathrm{mg} / \mathrm{dl}$ blood glucose. ANOVA and linear regression demonstrated a significant influence of blood glucose and blood glucose variability on blood lactate $(P=0.0001)$. The identification of this relation was supported by the result of a multivariate regression analysis, adjusting for severity of illness $(P=0.0001)$.

Conclusion The results demonstrate an influence of blood glucose and blood glucose variability on blood lactate, independent of severity of illness, in a medical ICU patient population.

References

1. van den Berghe $G$, et al:. Intensive insulin therapy in the critically ill patients. N Engl J Med 2001, 345:1359-1367.

2. Finfer $\mathrm{S}$, et al:. Intensive versus conventional glucose control in critically ill patients. N Engl J Med 2009, 360:1283-1297.

3. Khosravani $\mathrm{H}$, et al.: Occurrence and adverse effect on outcome of hyperlactatemia in the critically ill. Crit Care 2009, 13:R90.

P166

Relationship between glycemic Lability Index, infections and outcome in critically ill patients

A Donati, L Botticelli, R Castagnani, V Gabbanelli, E Damiani, R Domizi, P Pelaia

Università Politecnica delle Marche, Ancona, Italy

Critical Care 2012, 16(Suppl 1):P166 (doi: 10.1186/cc10773)

Introduction Hyperglycemia and glucose variability are important factors associated with morbidity and mortality in critically ill patients $[1,2]$. Our objective was to determine the association between the glucose Lability Index (LI), infections and outcome in critical illness.

Methods We performed a retrospective study in 2,943 adult patients admitted to our ICU from 2004 until 2010. Glucose variability was calculated for all subjects as the LI [3] during the hospital stay on capillary, arterial and venous blood. The ROC curve was performed to verify discrimination of the LI towards mortality and ICU infections.

Results There were 709 infections and 447 deaths. There was a significant interaction between the $\mathrm{LI}$ and infections in patients. The LI had a great ability to predict hospital mortality (area under the curve $=0.62,95 \% \mathrm{Cl}=0.59$ to $0.65, P<0.5$; Figure 1) but moreover infections (area under the curve $=0.80,95 \% \mathrm{Cl}=0.78$ to $0.82, P<0.5$; Figure 2).

Conclusion Glucose variability has ability to predict outcome but moreover infections in patients in the ICU, because it is a predictor of clinical outcomes in patients with hyperglycemia and diabetes.

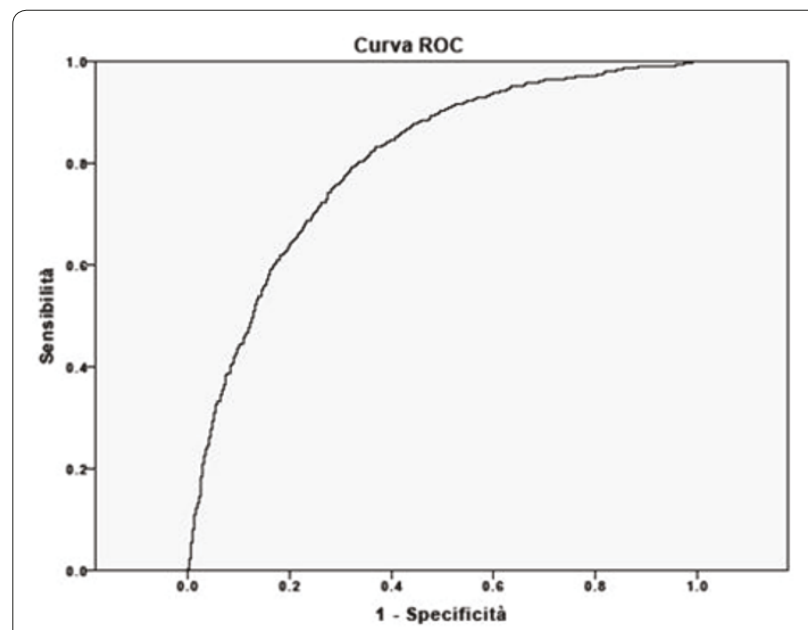

Figure 1 (abstract P166).

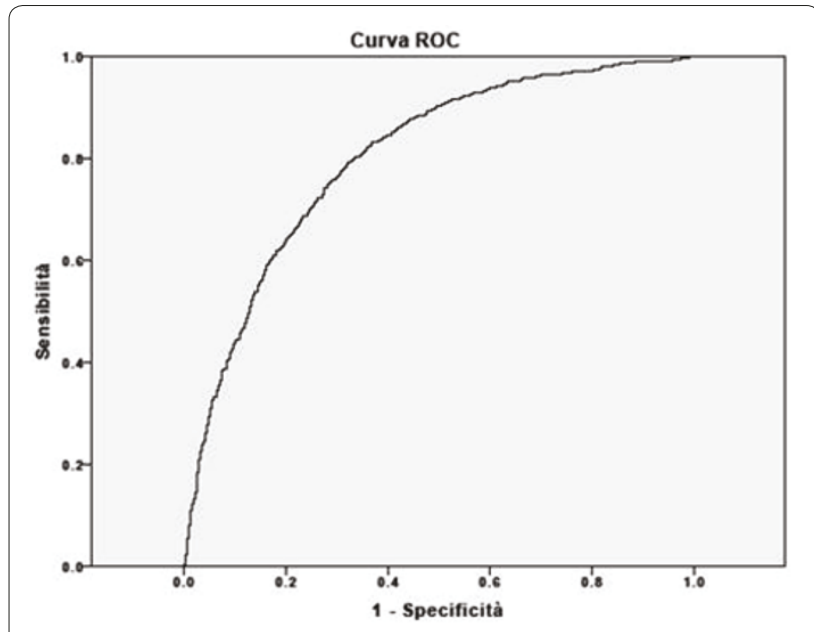

Figure 2 (abstract P166).

Strategies to reduce glucose variability should be studied to improve the outcomes in ICU patients.

References

1. N Eng/ J Med 2001, 345:1359

2. Crit Care Med 2008, 36:2316.

3. Diabetes 2004, 53:955.

P167

Variability of insulin sensitivity during the first 4 days of critical

illness

C Pretty', A Le Compte1, JG Chase1, G Shaw², JC Preiser³, S Penning 4 ,

T Desaive ${ }^{4}$

'University of Canterbury, Christchurch, New Zealand; '2Christchurch Hospital, Christchurch, New Zealand; ${ }^{3}$ Hospital Erasme, Free University of Brussels,

Belgium; ${ }^{4}$ University of Liege, Belgium

Critical Care 2012, 16(Suppl 1):P167 (doi: 10.1186/cc10774)

Introduction Safe, effective tight glycaemic control (TGC) can improve outcomes in critical care patients, but is difficult to achieve consistently. Insulin sensitivity defines the metabolic balance between insulin concentration and insulin-mediated glucose disposal. Hence, variability of insulin sensitivity can cause variable glycaemia. This study investigates the evolution of insulin sensitivity level and variability in patients receiving TGC during the first 4 days of their ICU stay.

Methods A retrospective analysis of patient data ( $n=164$ patients) from the SPRINT TGC study in the Christchurch Hospital ICU [1]. All patients commenced TGC within 12 hours of ICU admission and spent at least 24 hours on the SPRINT protocol. Model-based insulin sensitivity (SI) was identified using a validated glucose-insulin system model developed for critical care patients. SI was identified every hour for each patient using clinical data and the model. Level and hour-tohour percentage changes in SI were assessed on cohort and per-patient bases. Level and variability of SI were compared over time on 24-hour and 6-hour timescales for the first 4 days of the ICU stay.

Results Cohort and per-patient median SI levels increased by $34 \%$ and $33 \%(P<0.001)$ between days 1 and 2 of the ICU stay. Concomitantly, cohort and per-patient SI variability reduced by $32 \%$ and $36 \%(P<0.001)$. For $72 \%$ of the cohort, median SI on day 2 was higher than day 1 . The day 1 and 2 results were the only clear, statistically significant trends across both analyses. Analysis of the first 24 hours using 6 -hour blocks of SI data showed that most of the improvement in insulin sensitivity level and variability seen between days 1 and 2 occurred during the first 12 to 18 hours of day 1 . This rapid improvement was probably due to the decline of counterregulatory hormones as the acute phase of critical illness progressed.

Conclusion ICU patients have significantly lower and more variable insulin sensitivity on day 1 than later in their ICU stay and particularly during the first 12 hours. Clinically, these results suggest that while using 
TGC protocols with patients during their first few days of ICU stay, extra care should be afforded. Increased measurement frequency, higher target glycaemic bands, conservative insulin dosing and modulation of carbohydrate nutrition should be considered to safely minimize outcome glycaemic variability and reduce the risk of hypoglycaemia. Reference

1. Chase JG, et al:: Crit Care 2008, 12:R49.

P168

Endogenous insulin secretion in critically ill patients

C Pretty', A Le Compte1, J Lin', G Shaw², JG Chase'

'University of Canterbury, Christchurch, New Zealand; ${ }^{2}$ Christchurch Hospital,

Christchurch, New Zealand

Critical Care 2012, 16(Suppl 1):P168 (doi: 10.1186/cc10775)

Introduction Glucose-insulin system models can be used for improved glycemic control of critically ill patients. A key component of glucoseinsulin models is pancreatic insulin secretion. There are limited data in the literature quantifying insulin secretion in critically ill patients at physiologic levels. This study presents a model of pancreatic insulin secretion in critically ill patients based on data from a critically ill population.

Methods Samples were collected from 19 patients enrolled in a prospective clinical trial studying sepsis at the Christchurch Hospital ICU. Fifteen of the patients had confirmed sepsis and three were diagnosed type 2 diabetics. All patients were on the SPRINT glycaemic control protocol [1]. Each patient had arterial blood samples assayed for insulin and C-peptide. Two sets of four samples were taken from each patient, with each set collected over 60 minutes. Blood glucose (BG) data were collected with a bedside glucometer. C-peptide data were deconvolved using the model and population parameter values of van Cauter and colleagues [2] to determine pancreatic insulin secretion rates (ISRs). Data from Kjems and colleagues investigating the potentiating effects of glucagon-like peptide-1 on insulin secretion [3] suggested a maximum secretion rate of $16 \mathrm{U} /$ hour. A minimum rate of $1 \mathrm{U} /$ hour was also adopted.

Results The best model for insulin secretion was based on blood glucose concentration alone. There was clear separation of secretion levels between normal glucose tolerant (NGT) and impaired glucose tolerant (IGT) patients. Hence, ISR was modeled as a constrained linear function of BG (in $\mathrm{mmol} / \mathrm{l}$ ) for NGT and IGT patients separately with $R^{2}=0.61$ and 0.69 respectively. NGT: ISR $=893 \times \mathrm{BG}-2,996$ ( $\mathrm{mU} /$ hour). IGT: ISR $=296 \times B G-1,644$ (mU/hour). The glucose coefficients of 893 and $296 \mathrm{mU} . \mathrm{I} / \mathrm{mmol}$.hour were comparable to data published in a number of other studies for healthy and diabetic subjects.

Conclusion This work presents a simple model for pancreatic insulin secretion in critically ill patients based on clinical data. The model is a function of blood glucose level and glucose tolerance status and compares well with published data for healthy and diabetic subjects. This model can be incorporated into glucose-insulin system models and could potentially improve model-based glycaemic control.

\section{References}

1. Chase JG, et al.: Crit Care 2008, 12:R49.

2. Van Cauter E, et al:: Diabetes 1992, 41:368-377.

3. Kjems L, et al:: Diabetes 2003, 52:380-386.

P169

Impact of the type of glucose monitoring on the assessment of glycemic variability in critical care patients

P Kalfon, M Chilles

CHChartres, France

Critical Care 2012, 16(Suppl 1):P169 (doi: 10.1186/cc10776)

Introduction While minimizing glycemic variability (GV) during glucose control in critical care patients could become a new therapeutic goal, it is important to have a reliable assessment of GV. The aim of the study is to compare a real continuous glucose monitoring (CGM) system in comparison with a semi-continuous glucose monitoring (sCGM) system, with respect to the reliability of a marker of GV.
Methods We used the Eirus ${ }^{\mathrm{TM}}$ system (DipylonMedical, Solna, Sweden) based on microdialysis for CGM in three mechanically ventilated patients necessitating continuous intravenous insulin. The CGM system consists of a dedicated triple-lumen central venous catheter, a disposable sensor on a reusable sensor holder outside the patient, and a monitor producing a new measurement each minute. Calibrations were performed every 8 hours using arterial blood samples and a blood gas analyzer (Radiometer SAS, Neuilly-Plaisance, France). The attending nurses also performed intermittent blood glucose measurements with a point-of-care glucometer in order to set the insulin rate according to our glucose control method. GV was assessed by the variability index defined as the mean of the absolute value of the first derivative of the glucose signal during CGM. In order to simulate sCGM, we extracted CGM values every 15 minutes for calculating the corresponding variability index as the mean of the absolute value of the variation rate between two consecutive measurements.

Results The variability indexes were respectively $1.97,1.65,1.55 \mathrm{mmol} / \mathrm{l} /$ hour for CGMS, and 1.07, 0.65, $0.83 \mathrm{mmol} / \mathrm{l} /$ hour for sCGMS.

Conclusion SCGM in comparison with CGM may considerably underestimate a marker of GV during glucose control in critical care patients. Reference

1. Mackenzie et al: The metrics of glycaemic control in critical care. Intensive Care Med 2011, 37:435-443.

P170

Evaluation of a continuous blood glucose monitoring system using a central venous catheter with an integrated microdialysis function

F Möller, J Liska, A Öwall, A Franco-Cereceda

Karolinska Institutet, Solna, Sweden

Critical Care 2012, 16(Suppl 1):P170 (doi: 10.1186/cc10777)

Introduction Glycemic control in critically ill patients has been debated over the last decade. An accurate glucose monitoring system is essential to understand and study this concern. We have evaluated the accuracy and technical feasibility of a continuous glucose monitoring system using intravascular microdialysis.

Methods Thirty patients undergoing cardiac surgery were monitored using a triple-lumen central venous catheter (Eirus TLC ${ }^{\oplus}$; Dipylon Medical $A B$, Sweden) with an integrated microdialysis membrane. The catheter was placed with the tip in the superior vena cava, and functions both as a central venous catheter, enabling blood sampling and administration of medication, while simultaneously measuring

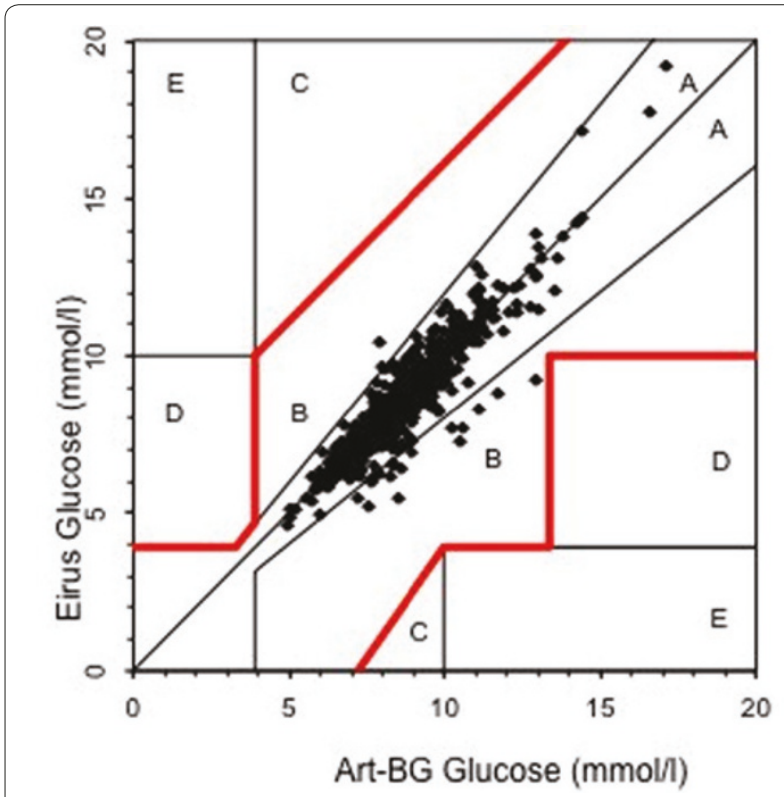

Figure 1 (abstract P170). Clarke error grid analysis. 


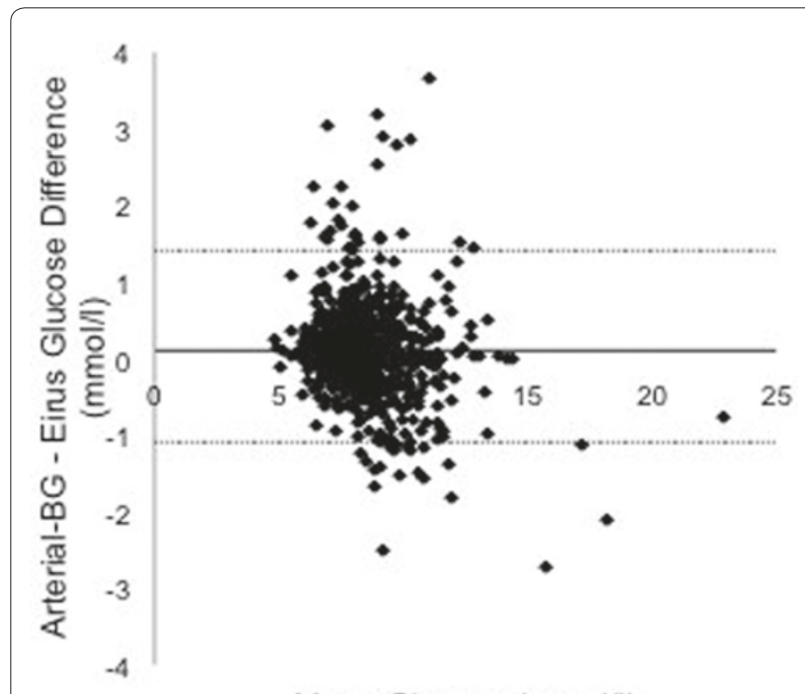

Mean Glucose (mmol/l)

Figure 2 (abstract P170). Bland-Altman analysis.

glucose. The patients were monitored for up to 48 hours postoperatively in the ICU. As reference, arterial blood samples were taken every hour. Results Data were available from all 30 patients. A total of 725 paired (arterial blood gas-microdialysis) samples were obtained. Glucose correlation coefficient was 0.87 . Using Clarke error grid analysis, $100 \%$ of the paired samples were in region $A B$ and $97.4 \%$ in region $A$ (Figure 1). Mean glucose level was $8.6 \mathrm{mmol} / \mathrm{l}$, bias $-1.3 \%$ and mean absolute relative difference was $4.8 \%$. A total $97.5 \%$ of the paired samples were correct according to ISO criteria. Bland-Altman analysis showed bias \pm limits of agreement $-0.11 \pm 1.3 \mathrm{mmol} / \mathrm{l}$ (Figure 2).

Conclusion Central venous microdialysis is an accurate and reliable method for continuous blood glucose monitoring in critically patients.

P171

Variable and maximum blood glucose levels during the first week after ICU admission are related to the severity of the patients

M Hoshino', Y Haraguchi', K Oda', S Kajiwara'

'Shisei Hospital, Saitama, Japan; ${ }^{2}$ Geriatric Health Services Facility,

Kokubunnji-shi, Japan

Critical Care 2012, 16(Suppl 1):P171 (doi: 10.1186/cc10778)

Introduction Significance of blood glucose (BG) control in ICU patients, especially in terms of mortality reduction, has been recognized in recent years. However, the relationship between BG profile and the severity, as well as BG target, is not clearly elucidated. A preliminary study was performed in order to clarify the relationship between BG profile and the severity of ICU patients.

Methods Forty-seven patients were studied. The following parameters were calculated during first week after ICU admission. (1) Mean and maximum value of SOFA scores (SOFAm and SOFAmax, respectively). (2) Mean, standard deviation, maximum, minimum, and difference of BG levels (BGm, BGsd, BGmax, BGmin, BGd(BGmax-BGmin), respectively). BG levels were measured basically every 6 hours with capillary blood. (3) Correlation coefficients $(r)$ between the abovementioned SOFA scores and BG parameters were calculated using two-dimensional and linear regression analysis $\left(r_{t}, r_{1}\right.$, respectively).

Results (1) $r_{t}$ and $r_{1}\left(r_{t} / r_{1}\right)$ between SOFAmax and BG parameters: BGsd (0.48/0.36), BGmax (0.47/0.33), BGd (0.47/0.35), BGm (0.30/0.22), BGmin (0.21/0.36). (2) $\left(r_{t} / r_{1}\right)$ between SOFAm and BG parameters: BGsd (0.45/0.33), BGmax (0.45/0.28), BGd (0.45/0.30), BGm (0.28/0.17), BGmin $(0.17 / 0.29)$.

Conclusion (1) Variable and maximum BG levels during the first week (BGsd, BGd, BGmax), rather than mean and minimum BG levels (BGm, BGmin), were related to the severity. (2) Suppression of the higher variable and maximum BG levels was considered to link to better outcome. (3) On the other hand, part of the severe patients seemed to have the lowest of those variable and maximum BG levels, judging from two-dimensional or parabolic correlations between those BG parameters and SOFA scores.

P172

Pilot trial of STAR in the medical ICU

LM Fisk', AJ Le Compte', GM Shaw', S Penning ${ }^{3}$, T Desaive', JG Chase ${ }^{1}$

'University of Canterbury, Christchurch, New Zealand; 'Christchurch Hospital, Christchurch, New Zealand; '3niversité de Liege, Belgium

Critical Care 2012, 16(Suppl 1):P172 (doi: 10.1186/cc10779)

Introduction Medical ICU patients often develop stress-induced hyperglycemia. Regulating blood glucose (BG) levels in these patients using insulin can be difficult due to varying patient conditions and therapy, leading to increased risk of hypoglycaemia. This abstract describes a pilot trial of STAR, a computerised risk-management accurate glycemic control (AGC) protocol.

Methods Thirteen hyperglycaemic patients (BG >145 mg/dl) were consented from Christchurch Hospital ICU. The BG target range was 80 to $145 \mathrm{mg} / \mathrm{dl}$ or 108 to $162 \mathrm{mg} / \mathrm{dl}$ (chosen clinically). Model-based insulin sensitivity was calculated for every measurement and its variability for the next 1 to 3 hours forecast using stochastic models. These data and model were used to calculate new insulin/nutrition interventions for the next 1 to 3 hours, limiting risk of BG $<80 \mathrm{mg} / \mathrm{dl}$ to a maximum of $5 \%$. Nursing staff selected the BG measurement interval to manage workload. Insulin was delivered as boluses ( $\max 6 \mathrm{U} / \mathrm{hour}$; $\max$ increase $+2 \mathrm{U} /$ intervention), with infusions up to $3 \mathrm{U} /$ hour for highly resistant patients. Nutrition was 30 to $100 \%$ of clinical goal feed (max change $\pm 30 \%$ per intervention) and constant rates were used when desired clinically. Limiting insulin/nutrition changes prevents over-response to erroneous BG measurements and results were resampled hourly for consistency. Approval was granted by the Upper South A Regional Ethics Committee (Christchurch, New Zealand).

Results Median BG was $109 \mathrm{mg} / \mathrm{dl}$ for 80 to $145 \mathrm{mg} / \mathrm{dl}$ target patients and $145 \mathrm{mg} / \mathrm{dl}$ for 108 to $162 \mathrm{mg} / \mathrm{dl}$ target patients. In total, $85.6 \%$ of time was in the specified target band, with $1.18 \%$ of $B G<72 \mathrm{mg} / \mathrm{dl}$ and $2.41 \%$ BG $<80 \mathrm{mg} / \mathrm{dl}$. BG measurement frequency was 13.3 measures/ day. Per-patient median carbohydrate intake was $10.7 \mathrm{~g} /$ hour (IQR: 4.0 to $11.9 \mathrm{~g} /$ hour) and median insulin usage was $2.5 \mathrm{U} /$ hour (IQR: 1.75 to $3.5 \mathrm{U} /$ hour). Requirements varied considerably by patient. Observed response to insulin varied by a factor of $14 \times$ between patients. Accurate control was maintained over a range of metabolic conditions, and STAR adapted safely to therapies including high-dose steroids, long-acting insulin (Glargine) and changing insulin response.

Conclusion STAR provided AGC in a clinical setting. Tight and accurate control was able to be extended to patients with a range of metabolic requirements, and the risk-management approach proved capable of balancing clinical workload and risks presented by patient variability.

P173

Glucometer accuracy and implications for clinical studies

AJ Le Compte', CG Pretty', GM Shaw', JG Chase'

'University of Canterbury, Christchurch, New Zealand; ${ }^{2}$ Christchurch Hospital, Christchurch, New Zealand

Critical Care 2012, 16(Suppl 1):P173 (doi: 10.1186/cc10780)

Introduction Elucidating links between glycemic control and clinical outcome requires reliable discrimination between groups with different target blood glucose (BG) cut-offs. Point-of-care glucometers are commonly used, but lower accuracy means BG errors will impact classification and thus outcome analyses. This study reanalyses a BG control trial with an error model of a typical glucometer to assess the impact of sensor errors on interpretation of trial results.

Methods BG profiles from 301 patients (stay $>24$ hours) from the SPRINT trial with BG measurements $(n=25,000)$ using the Arkray SuperGlucocard II GT-1630. A model of sensor bias and variance (CV 2.7 to $3.5 \%$, regression: $y=3.92+0.97 x$ ) was used to estimate possible 'true' BG profiles from measured BG and repeated 100 times for each 
patient. The defined cut-off for 'good' control for a patient was $\geq 70 \%$ of BG in 72 to $126 \mathrm{mg} / \mathrm{dl}$ (cTIB $\geq 0.7$ ), and 'poor' as $<70 \%$ (cTIB $<0.7$ ), based on original observed clinical BG. The number of true BG profiles that resulted in misclassification between 'good' and 'poor' control for a patient was recorded over all Monte-Carlo runs. The maximum change in true and observed BG mean and standard deviation were used to evaluate potential worst-case scenarios.

Results Good control was clinically measured in $76 \%$ of patients $(24 \%$ with cTIB $<0.7$ ). Of these, $83 \%$ of 'good' and $64 \%$ of 'poor' control would never be misclassified over all 100 runs due to sensor error. A total of $91 \%$ (good) and $87.5 \%$ (poor) could be misclassified $10 \%$ of the time. Patients with CTIB near 0.7 were more likely to be misclassified when accounting for glucometer error. Hence, a deadband around the cut-off would reduce this misclassification. If 'good' cut-off was CTIB $\geq 0.5$ (95\% of clinical patients) then $100 \%$ correct classification was $97 \%$ for good control patients, but fell to $40 \%$ of poor control patients. The median largest difference in observed and true mean BG across patients was $-54 \mathrm{mg} / \mathrm{dl}$ (90th percentile: $-21 \mathrm{mg} / \mathrm{dl}$ ) and the standard deviation was $3.2 \mathrm{mg} / \mathrm{dl}$ (90th percentile: $1.8 \mathrm{mg} / \mathrm{dl}$ ).

Conclusion Glucometers can distinguish between patients that received good and poor BG control but risk of misclassification rises for patients nearer cut-offs. Reliable classification to associate with outcomes relies on the control protocol and cut-off choice to achieve sufficient separation between groups so that device errors do not result in significant misclassification confounding the results. A deadband around cut-off values to eliminate patients at high risk of misclassification may be required.

\section{P174}

Initial experience with continuous intra-arterial fluorescent glucose monitoring in patients in the ICU following cardiac surgery

S Bird', L Macken', O Flower', E Yarad', F Bass', N Hammond', D LaCour², P Strasma², S Finfer

'Royal North Shore Hospital, St Leonards, NSW, Australia; ${ }^{2}$ GluMetrics, Inc., Irvine, CA, USA

Critical Care 2012, 16(Suppl 1):P174 (doi: 10.1186/cc10781)

Introduction Continuous glucose monitoring (CGM) in ICUs has the potential to improve patient safety and outcomes. The GluCath Intravascular CGM System uses a novel quenched chemical fluorescence sensing mechanism to measure glucose concentration (BG) in venous or arterial blood. This is the first report of its use in cardiac surgery patients.

Methods This ongoing clinical study is evaluating the system deployed via a standard $20 \mathrm{G}$ radial artery catheter inserted for routine care in 20 patients undergoing cardiac surgery. Data are presented from five run-in patients. Outcome measures are qualitative (ease-of-use, workflow fit) and quantitative (accuracy vs. reference analyzer). Sensors were inserted shortly after ICU admission with placement confirmed by ultrasound and in vivo calibration 30 minutes later. Clinical staff managed blood glucose according to usual protocols. Glucose values were recorded each minute for 24 hours; hourly reference samples from the same arterial catheter were analyzed on a Radiometer $A B L$ Blood Gas Analyzer.

Results The sensor was successfully deployed in all five patients and did not interfere with clinical care, blood pressure monitoring or sampling. One patient suffered a cardiopulmonary arrest; the sensor functioned successfully during resuscitation and urgent return to the operating room. One hundred and twenty reference samples ranging from 5.9 to $13.4 \mathrm{mmol} / \mathrm{l}$ were collected; $107 / 120$ (89.2\%) of GluCath measurements met ISO 15197 criteria (within $\pm 20 \%$ of reference when BG $>4.2 \mathrm{mmol} /$; Figure 1). In Subject 1 the sensor was inadvertently retracted into the arterial catheter during the study, leading to measurement error from arterial flush solution contamination. In a sensitivity analysis excluding this patient, 89/95 (93.7\%) of measurements met ISO 15197 with a mean absolute relative difference of $9.4 \%$.

Conclusion The GluCath System measured glucose concentration continuously in a cardiac surgery ICU without compromising arterial line function or patient care. In all patients the sensor operated without interruption for 24 hours following a single in vivo calibration.

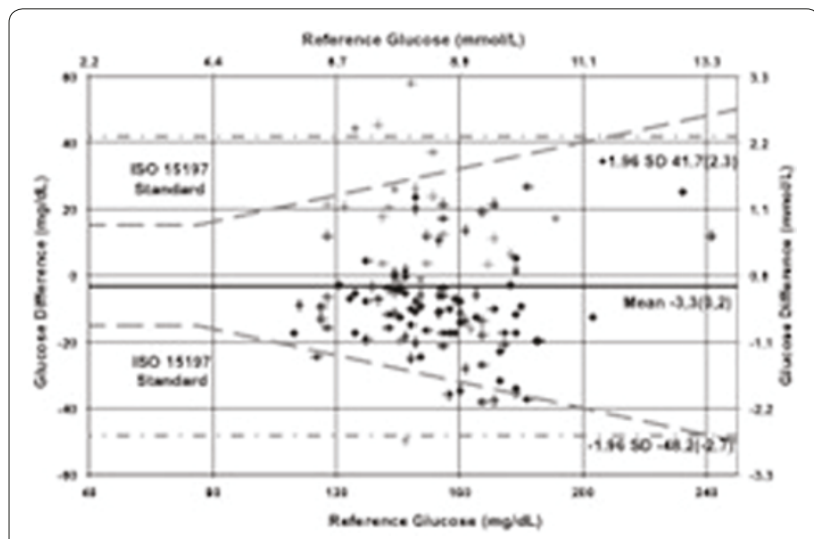

Figure 1 (abstract P174). ISO-modified Bland-Altman plot.

P175

Preliminary ICU experience of a novel intravascular blood glucose sensor

KP Mulavisala', PB Gopal², B Crane 3 , A Mackenzie ${ }^{3}$

'Axon Anaesthesia Associates Care Hospital Nampally, Hyderabad, India;

${ }^{2}$ Apollo Hospitals, Hyderabad, India; ${ }^{3}$ Glysure, Abingdon, UK

Critical Care 2012, 16(Suppl 1):P175 (doi: 10.1186/cc10782)

Introduction A need for continuous blood glucose monitoring has always been expressed by critical care practitioners. The results from several iterations of a novel optical fluorescence-based intravascular blood glucose sensor were examined for correlation with an accepted laboratory assay. Ever since Van Den Berghe's group demonstrated reductions in hospital mortality and morbidity from the application of tight glycaemic control [1], many groups have attempted to replicate those results with limited success. Practitioners have speculated upon the reasons behind this observation, and have cited manpower implications and incidence of hypoglycaemic episodes as contributing factors [2]. Investigators have speculated that a continuous blood glucose sensor might contribute towards safe effective glycaemic control [3].

Methods A series of postoperative and direct admission ICU patients had an optical fluorescence-based intravascular glucose sensor (GlySure Ltd, Abingdon, UK) placed into the left internal jugular vein on admission to the ICU. The sensor remained in situ throughout the ICU stay. Periodic blood samples and simultaneous real-time values of blood glucose measured by the sensor were recorded. The results were correlated with the results of blood sample analysed by a Yellow Springs Instrument glucose analyser. The sensor, which has a heparin coating on its surface, required no further heparinisation; a 'keep vein open' rate of normal saline infusion was maintained throughout the period of operation.

Results Sixteen patients received the current configuration blood glucose sensor; during their combined length of stay, 296 paired values were obtained for correlation purposes. A total $99.6 \%$ of these values fall within the $A+B$ areas of the Clarke error grid. All sensors continued to function throughout the length of stay, maximum 92 hours, and were withdrawn immediately prior to discharge from the ICU.

Conclusion The pre-production intravascular blood glucose sensors successfully track blood glucose values, with improved insight into blood glucose variability in ICU patients.

\section{References}

1. Van den Berghe $G$, et al:: Intensive insulin therapy in critically ill patients. NEngl J Med 2001, 345:1359-1367.

2. Aragon D, et al:: Evaluation of nurse work effort and perception about blood glucose testing in TGC. Am J Crit Care 2006, 15:370-377.

3. Krinsley J, Preiser JC: Moving beyond TGC to safe effective glycemic control (SEGC). Crit Care 2008, 12:149 
P176

Does tight glycemic control positively impact on patient mortality? S Penning ${ }^{1}$, AJ Le Compte ${ }^{2}$, M Signal2 ${ }^{2}$, P Massionn ${ }^{3}$, JC Preiser ${ }^{4}$, GM Shaw ${ }^{5}$ T Desaive', JG Chase 2

'Université de Liege, Belgium; '2University of Canterbury, Christchurch, New Zealand; ${ }^{3} \mathrm{CHU}$ de Liège, Belgium; ${ }^{4}$ Erasme University Hospital, Brussels,

Belgium; ${ }^{5}$ Christchurch Hospital, Christchurch, New Zealand

Critical Care 2012, 16(Suppl 1):P176 (doi: 10.1186/cc10783)

Introduction High and variable blood glucose (BG) levels have been associated with increased mortality. Tight glycemic control (TGC) aims at reducing BG levels to improve patient outcome and mortality. This research evaluates the impact of TGC on mortality.

Methods This study used glycemic data from 1,488 patients of two cohorts: Glucontrol $(n=704)$ and SPRINT $(n=784)$. TGC glycemic outcome is measured by cumulative time in the 4 to $7 \mathrm{mmol} / \mathrm{l}$ band (cTIB), defined daily for each patient. Each day, patients were divided into two groups: CTIB $<70 \%$ and CTIB $\geq 70 \%$. For each group, odds of living $(\mathrm{OL}=$ \#lived $/$ \#died) was calculated.

Results OL for CTIB $\geq 70 \%$ patients tends to increase over time while OL for CTIB $<70 \%$ patients decreases (Figure 1). On Day 1, OL for CTIB $<70 \%$ patients and $\mathrm{CTIB} \geq 70 \%$ patients are similar $(\mathrm{OL}=5.1$ and $\mathrm{OL}=5.5$ respectively). The difference between the two groups increases over the ICU stay. On Day $10, \mathrm{OL}=2.8$ and $\mathrm{OL}=10.5$ for $\mathrm{CTIB}<70 \%$ and $\mathrm{CTIB}$ $\geq 70 \%$ patients respectively. These results suggest that survival rate is higher when CTIB $\geq 70 \%$ and thus when BG levels are tightly controlled around normoglycemia. The longer patients'ICU stay, the lower survival rate they have when $\mathrm{CTIB}<70 \%$.

Conclusion Results show that, irrespective of TGC protocols, high CTIB and thus normoglycemia are associated with higher odds of living. This suggests that TGC positively influences patient outcome.

P177

Glycaemic control in ICUs in large English hospitals: a follow-up telephone survey

CR Bullock, A Pang, A Routledge, I Mackenzie

Queen Elizabeth Hospital Birmingham, UK

Critical Care 2012, 16(Suppl 1):P177 (doi: 10.1186/cc10784)

Introduction Following van den Berghe's landmark paper in 2001 (Leuven study) [1], the critical care community became very interested in 'tight' glycaemic control [2]; however, recent negative studies have dampened this interest [3]. In view of more recent analyses, which offer possible explanations for equivocal results [4], it is possible there will be renewed interest in glycaemic control. The purpose of this survey is to assess the utilisation of tight glycaemic control protocols in a sample of ICUs in England, as a reflection of current UK intensive care practice. Methods We identified 171 large acute hospital trusts, of which 87 were randomly selected. Of these, 85 had ICUs, which were contacted by telephone. The senior nurse in charge at the time was asked whether their ICU used a protocol for the management of blood glucose, and what were the upper and lower target limits.

Results A blood glucose protocol was used in $87.1 \%$ of ICUs surveyed. Of these, the median lower limit of allowed blood glucose concentration was $4.0 \mathrm{mmol} / \mathrm{l}$ (range 3.0 to 7.0 ), with an upper limit of $8.0 \mathrm{mmol} / \mathrm{I}$ (range 6.0 to 12.0 ). Only $22 \mathrm{ICUs}(25.9 \%)$ had a target range similar to the Leuven study. A further 34 ICUs used a lower limit similar to the Leuven study, of 4.0 to $4.5 \mathrm{mmol} / \mathrm{l}$, but had a higher upper limit. This is reflective of the general opinion from the nurses contacted, that a tight protocol is difficult to achieve, can result in hypoglycaemia, and has been recently relaxed in many departments.

Conclusion Our data suggest that glycaemic control has, to a very large extent, been accepted as a standard of care in the UK, although in most ICUs this does not constitute tight glycaemic control. The full benefit of tight glycaemic control, achieved by minimisation of mean glucose, glucose variability and episodes of hypoglycaemia, will not be achieved until robust techniques for continuous, or semi-continuous, blood glucose measurement are available.

\section{References}

1. Van den Berghe G, et al:: N Engl J Med 2001, 345:1359-1367.

2. Mackenzie et al: Intensive Care Med 2005, 31:1136.

3. NICE-SUGAR Study Investigators: N Engl J Med 2009, 360:1283.

4. Mackenzie et al.: Intensive Care Med 2011, 37:435-443.

\section{P178}

Model-based regulation of glucose in critical care

SP Gawel', G Clermont², RS Parker

'University of Pittsburgh, PA, USA; 'University of Pittsburgh Medical Center,

Pittsburgh, PA, USA

Critical Care 2012, 16(Suppl 1):P178 (doi: 10.1186/cc10785)

Introduction Glucose control in critical care has been shown to improve patient outcome, yet tight glucose control has led to increased

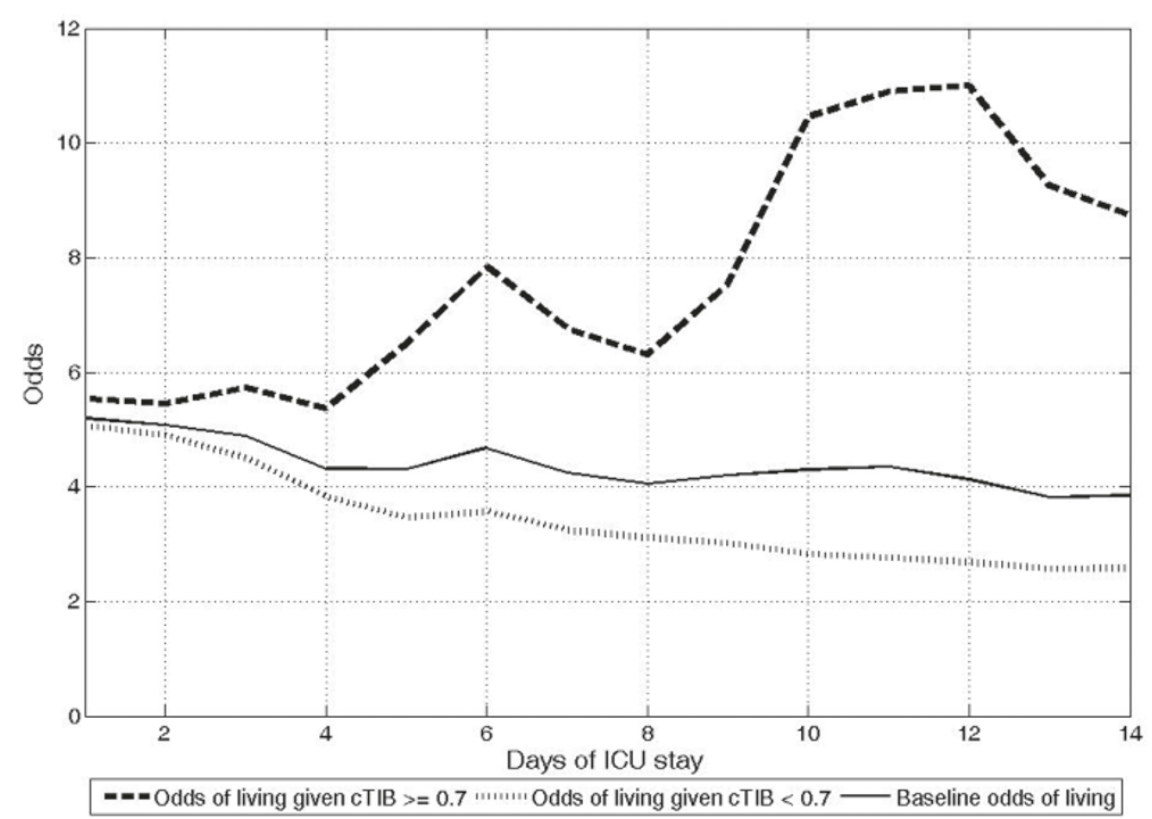

Figure 1 (abstract P176). Whole-cohort odds of living over ICU stay. 


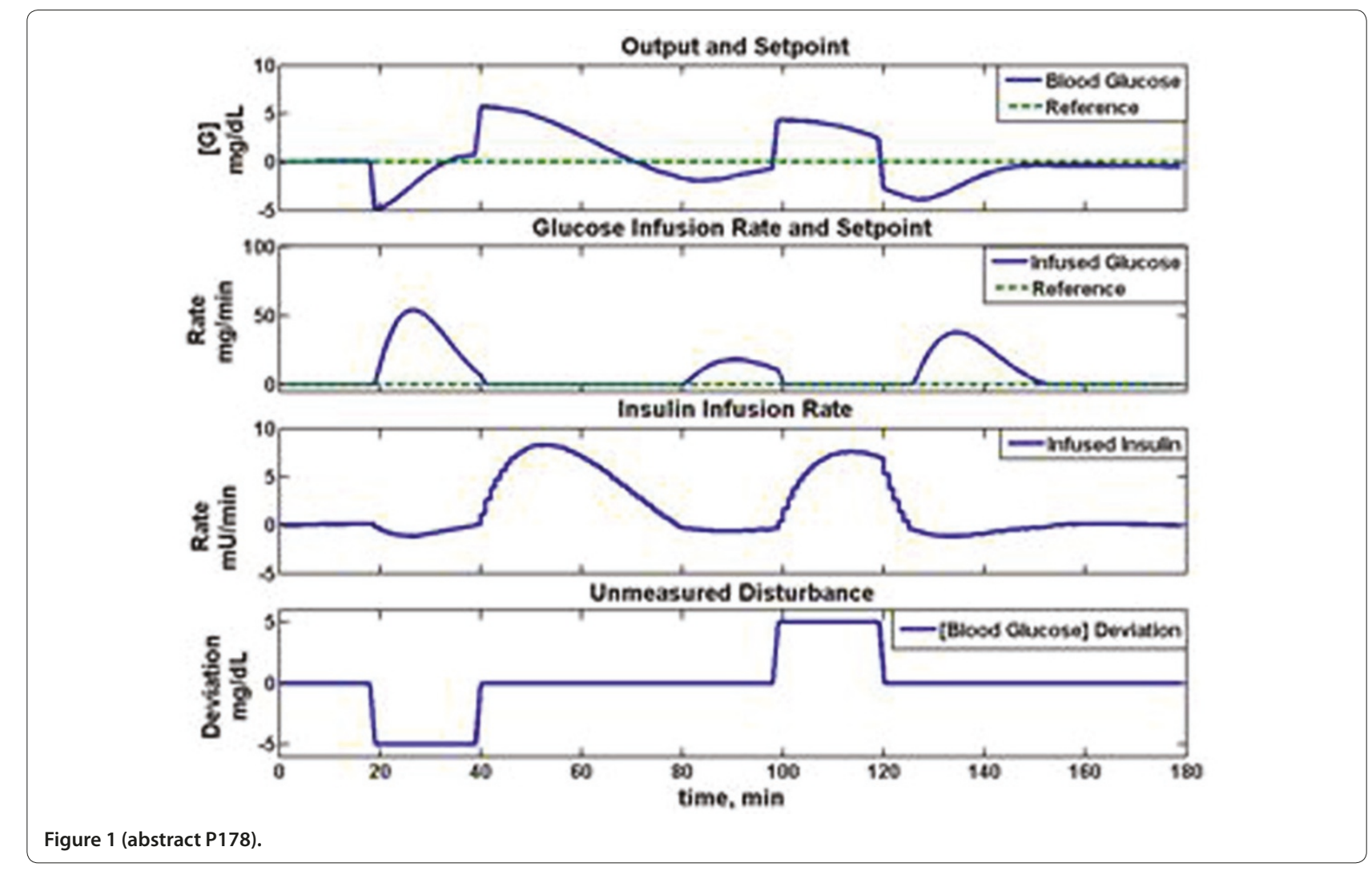

hypoglycemia in the clinic. We employed a systems engineering approach to assist clinicians in maintaining blood glucose within a desired target range while avoiding hypoglycemia in the critically ill. The long-term vision is a decision support system that provides recommended insulin and glucose administrations leading to patientspecific achievement of tight glucose control without hypoglycemia. Methods To achieve these goals, we employ a model predictive control (MPC) algorithmic platform using two control inputs: insulin for glucose control and glucose for hypoglycemia. The MPC controller is designed based on a nonlinear dynamic model of glucose-insulin-fatty acid interactions [1]. A moving horizon estimation (MHE) technique is used to alter the tissue sensitivity to insulin based on deviations between measurements and model predictions of glucose concentration as a mechanism for tailoring the controller model to individual patient dynamics.

Results The response of the MPC controller to measured deviations in glucose is shown in Figure 1. For glucose measurements below target, glucose is administered, while insulin administration is used to lower blood glucose from an elevated state to a desired target. The model parameter pG2, representing patient insulin sensitivity (insulin action on glucose uptake), was used by the MHE algorithm to tailor the model response to simulated patient dynamics. In response to PG2 changes in the simulated patient, MHE provided a $93 \%$ improvement in glucose reference tracking performance.

Conclusion The algorithm achieves tight glucose control in response to multiple measured and unmeasured disturbances. Furthermore, the MHE scheme updates patient parameters in real time in response to changing patient dynamics. The adaptive MPC algorithm is currently being validated using a retrospective cohort of critically ill patients at the University of Pittsburgh Medical Center.

Acknowledgements Funded by NIH-R21-DK092813.

Reference

1. Roy et al: Diab Tech Ther 2006, 8:617-626.
P179

Perioperative glycemic control with a computerized algorithm versus conventional glycemic control

M Punke', S Bruhn', M Goepfert', S Kluge' ', H Reichenspurner², A Goetz', D Reuter ${ }^{1}$

University Medical Center Hamburg-Eppendorf, Hamburg, Germany;

2 University Heart Center, Hamburg, Germany

Critical Care 2012, 16(Suppl 1):P179 (doi: 10.1186/cc10786)

Introduction In critically ill patients, both hypoglycemia and hyperglycemia seem to influence outcome. Since hypoglycemia can lead to organ dysfunction, hyperglycemia seems to boost surgical site infections (SSI) [1]. It was shown that intensive insulin therapy (IIT) reduced mortality in critically ill patients [2]. Unfortunately several studies could not reproduce the effects $[3,4]$. In particular, IIT bears the risk of accidental hypoglycemia which could even have a negative effect on patient outcome $[3,4]$. In cardiac surgery, the use of blood cardioplegia for cardiopulmonary bypass frequently leads to high blood glucose levels during surgery. In particular, a computerbased algorithm that guides the insulin therapy might be beneficial. We hypothesized that in patients undergoing major cardiac surgery with cardiopulmonary bypass and blood cardioplegia, the use of a computer-based algorithm for the application of insulin will lead to a tighter adherence of normoglycemia. Our primary study end-point was the duration, in which the patients fulfilled the predefined target range of 80 to $150 \mathrm{mg} / \mathrm{dl}$ blood glucose. Patients with conventional blood glucose therapy served as controls.

Methods Seventy-five patients were enrolled and randomized into three groups. Start of therapy was determined as the beginning of cardiopulmonary bypass. Group 1: therapy with computer-based blood glucose control (TGC System; Braun, Melsungen, Germany) and measurement of blood glucose every 30 minutes. Group 2: same therapy as group 1 and measurement of blood glucose every 15 minutes. Group 3: conventional therapy using a fixed insulin dosing scheme. End of therapy was defined as discharge from the ICU. 
Statistical analysis was performed with using ANOVA and the LPS post hoc test. Data shown are mean \pm standard deviation, $n=$ number of patients.

Results There were no statistical differences between the groups regarding age, height, weight, premedical history or intraoperative amount of glucose administration during cardioplegia (33 $\pm 15 \mathrm{~g}$ ). Blood glucose levels in groups 1 and 2 stayed significantly longer in the target interval compared with group 3 (75 $\pm 19 \%$ vs. $72 \pm 19 \%$; vs. $50 \pm 34 \%, P<0.01, n=25$, respectively). There was no significant difference between the groups regarding ICU or hospital stay and SSI rates.

Conclusion Early computer-based insulin therapy allows one to better warrant normoglycemia in patients undergoing major cardiac surgery with the use of blood cardioplegia.

References

1. Ann Intern Med 2007, 146:233-243

2. NEngl J Med 2001, 345:1359-1367.

3. NEngl J Med 2006, 354:449-461.

4. NEngl J Med 2009, 360:1283-1297.

\section{P180}

Efficacy of the novel heart attack centre extension pathway: a pilot study

D Perera', B Hoonjan', K Krishnathasan', M Selvanyagam', H Neugebauer ${ }^{2}$ 'Barts and The London School of Medicine and Dentistry, London, UK;

${ }^{2} Q u e e n s$ Hospital, Barking Havering and Redbridge NHS Trust, London, UK Critical Care 2012, 16(Suppl 1):P180 (doi: 10.1186/cc10787)

Introduction The Barts and the London Heart Attack Centre Extension (HACX) programme was introduced to provide a direct pathway for high-risk non-ST elevation myocardial infarction (NSTEMI) patients from the A\&E of a district general hospital to a tertiary intervention centre. As a result, patients have earlier access to angiography and subsequent treatment, including percutaneous coronary intervention $(\mathrm{PCl})$, coronary artery bypass grafting (CABG) or nonsurgical interventions. There is no research on the effectiveness of this novel HACX programme.

Methods Over 3 months, 33 patients transferred via the HACX pathway and 37 patients transferred via the conventional interhospital transfer pathway (IHT) were followed up. All patients with acute coronary syndrome symptoms, relevant ECG changes (ST segment depression in two or more contiguous leads $>1 \mathrm{~mm}$, pathological T-wave inversion in V1 to V4, a GRACE score $>88$ and troponin I levels $>0.1 \mathrm{ng} / \mathrm{ml}$ ) were discussed with the cardiology team at the interventional centre prior to immediate transfer. We assessed patient suitability for angiography, post-angiography procedures, and 3-month mortality outcomes. Data were obtained from the hospital's PAS computer system.

Results The average time for patients to have an angiography via the IHT pathway was 5.5 days. Of the 33 patients (mean age $61 \pm 15.2$ SD) transferred via HACX, 30 patients (91\%) were appropriately identified for an angiogram. Seventeen patients $(52 \%)$ required $\mathrm{PCl}$, five patients $(15 \%)$ required CABG, four patients $(12 \%)$ required nonsurgical intervention, and four patients (12\%) required no treatment. Controls included 37 patients (mean age $71 \pm 12.6 \mathrm{SD}$ ) of whom 17 patients (46\%) required $\mathrm{PCl}$, six patients (16\%) required $\mathrm{CABG}$, eight patients (22\%) required nonsurgical intervention and six patients $(16 \%)$ required no treatment. At 3-month follow-up, 32 patients (97\%) in the HACX cohort and 36 patients (97\%) in the IHT cohort were alive.

Conclusion HACX is an effective pathway that accurately identifies and rapidly transfers appropriate NSTEMI patients requiring early coronary revascularisation. However, there was no additional mortality benefit at 3-month follow-up compared to the conventional IHT pathway. Further studies with larger patient cohorts and longer follow-up periods are required to substantiate the benefits of the HACX programme in order to consider whether this service could be implemented nationwide, or whether this is a service that does not need to exist at all.
P181

Integral assistance process implantation for ST-elevated acute coronary syndrome

JC Rodriguez-Yañez, M Celaya-Lopez, MJ Huertos-Ranchal, I Diaz-Torres, C Navarro-Ramirez, J Gomez-Ramos

Hospital Universitario Puerto Real, Spain

Critical Care 2012, 16(Suppl 1):P181 (doi: 10.1186/cc10788)

Introduction The objective was to evaluate the implantation of assistance process implantation (PAI) for ST-elevated acute coronary syndrome (SCASTE) in our sanitary district. When we refer to PAl, we mean protocolysed assistance guidelines developed and published by Andalucia sanitary authorities that include recommendations to direct the assistance from the beginning of the process until patient discharge from the hospital.

Methods All ICU patients from HUPR diagnosed with SCASTE within the first 24 hours from January 2005 to December 2010 were included in this study and registered in the ARIAM-Andalucia Project. This database gathers the whole PAI from preadmission (PH), ER, ICU, hemodynamics laboratory and cardiology ward to discharge. Within these 6 years three main interventions were carried out: fibrinolysis protocol $\mathrm{PH}$ with ER and critical care unit EMS involving the ICU, continuous update of protocols based on AHA clinical guidelines, and 24-hour availability of the hemodynamic laboratory for primary coronary intervention (P-ICP available since 1 February 2007). Revascularization indexes are analyzed and grouped in 2-year periods (A, B, C), the time justified as necessary for modification after the intervention, attention times and $\mathrm{PH}$ action. The latter was measured by a score (aspirin, nitroglycerine, ECG, vein access, intravenous treatment and monitoring during transport) up to 6 points. A correct intervention must obtain at least 4 points. Statistical processing was by the R-UCA pack from R-Commander.

Results $A$ total of 590 patients were included in this study: $188(A)$, 227 (B) and 175 (C). All groups were similar in mean age, gender, IAM location and origin. A statistically significant increase was found in the revascularization and $\mathrm{PHA}$ attention between periods $\mathrm{A}$ versus $\mathrm{C}$ and B versus $C$ with $P<0.0001$ and $\mathrm{Cl}(0.15$ to 0.42$) /(0.17$ to 0.45$)$ and $(0.2$ to 0.6$) /(0.11$ to 0.39$)$. No statistically significant difference was found among groups A versus B. No significant difference was observed in attention times.

Conclusion Coordination of the SCASTE attention, constant analysis by continuous registry of different action levels (ARIAM-Andalucia registry), clinical guideline updates and adjustment to resources and environment, in this case a rural setting, meaning quality and a continuous improvement circle, reduce variability and lead undoubtedly to better assistance for our patients.

P182

Prognostic value of Killip classification in terms of health-related quality of life

A loannidis', D Tsounis', A Pechlevanis², M Paraskelidou²

'HOU, Kalamaria, Greece; 'HT'Agios Pavlos', Kalamaria, Greece Critical Care 2012, 16(Suppl 1):P182 (doi: 10.1186/cc10789)

Introduction The aim of the study was to evaluate the prognostic value of Killip classification in terms of health-related quality of life (HRQoL). Methods The sample consisted of 112 patients treated for myocardial infarction (MI), as onset manifestation of coronary artery disease (CAD), during 2008/09 in a prefectural hospital in northern Greece. At 1-year follow-up visit, HRQoL was measured using a generic and a diseasespecific instrument. The $15 \mathrm{D}$ consists of a visual analogue scale (VAS) and a total score. The scoring algorithm of the MacNew generates a global score, and three separate domains scores: emotional, physical and social.

Results Patients were grouped into the four Killip classes according to the degree of pulmonary congestion at admission (Table 1). Mean HRQoL for each group differed in the expected manner: the higher the class, the lower the HRQoL. Statistical significant differences were observed in VAS of the 15D and the emotional and social domain scores of the MacNew. Accordingly, the majority of patients with no signs of pulmonary congestion at admission were classified in NYHA functional class I at 1-year follow-up visit. No difference was observed in the type 
Table 1 (abstract P182). Patient characteristics according to Killip classification

\begin{tabular}{|c|c|c|c|c|c|}
\hline & Class I & Class II & Class III & Class IV & $P$ value \\
\hline$n(\%)$ & $52(46.4)$ & $40(35.7)$ & $14(12.5)$ & $6(5.4)$ & NA \\
\hline \multicolumn{6}{|l|}{ HRQoL (mean) } \\
\hline \multicolumn{6}{|l|}{$15 D$} \\
\hline VAS & 78.9 & 76.9 & 72.9 & 70.8 & 0.025 \\
\hline Total & 0.844 & 0.842 & 0.823 & 0.803 & 0.478 \\
\hline \multicolumn{6}{|l|}{ MacNew } \\
\hline Emotional & 5.60 & 5.61 & 5.38 & 5.05 & 0.030 \\
\hline Physical & 5.49 & 5.26 & 5.25 & 5.05 & 0.144 \\
\hline Social & 5.72 & 5.47 & 5.30 & 5.14 & 0.014 \\
\hline Global & 5.52 & 5.44 & 5.27 & 4.98 & 0.056 \\
\hline Age (years) & 61.8 & 66.0 & 70.2 & 61.2 & 0.013 \\
\hline \multicolumn{6}{|l|}{ Gender (n, \%) } \\
\hline Male (85) & $40(47.1)$ & $31(36.5)$ & $12(14.1)$ & $2(2.4)$ & 0.080 \\
\hline Female (27) & $12(44.4)$ & $9(33.3)$ & $2(7.4)$ & $4(14.8)$ & \\
\hline BMI & 28.7 & 29.1 & 30.0 & 34.8 & 0.001 \\
\hline Systolic BP & 127.5 & 122.5 & 115.7 & 140.0 & 0.000 \\
\hline Diastolic BP & 75.8 & 73.6 & 70.7 & 76.7 & 0.173 \\
\hline Heart rate & 64.9 & 68.7 & 72.3 & 71.7 & 0.030 \\
\hline CRP & 3.6 & 4.9 & 6.0 & 3.8 & 0.087 \\
\hline NT-proBNP & 741 & 1645 & 2193 & 1674 & 0.000 \\
\hline LVEF & 61.9 & 55.2 & 49.0 & 45.0 & 0.000 \\
\hline \multicolumn{6}{|l|}{ Ml type $(n, \%)$} \\
\hline STEMI (48) & $24(50.0)$ & $18(37.5)$ & $4(8.3)$ & $2(4.2)$ & 0.638 \\
\hline NSTEMI (64) & $28(43.8)$ & $22(55.0)$ & $10(15.6)$ & $4(6.3)$ & \\
\hline \multicolumn{6}{|c|}{ Affected arteries ( $n, \%)$} \\
\hline $1(38)$ & $19(50.0)$ & $15(39.5)$ & $4(10.5)$ & $0(0.0)$ & 0.005 \\
\hline $2(30)$ & $17(56.7)$ & $9(30.0)$ & $4(13.3)$ & $0(0.0)$ & \\
\hline $3(44)$ & $16(36.4)$ & $16(36.4)$ & $6(13.6)$ & $6(13.6)$ & \\
\hline \multicolumn{6}{|c|}{ Revascularization technique ( $n, \%)$} \\
\hline None (14) & $7(50.0)$ & $3(21.4)$ & $4(28.6)$ & $0(0.0)$ & 0.002 \\
\hline $\mathrm{PCl}(63)$ & $35(55.6)$ & $22(34.9)$ & $6(9.5)$ & $0(0.0)$ & \\
\hline CABG (35) & $10(28.6)$ & $15(42.9)$ & $4(11.4)$ & $6(17.1)$ & \\
\hline \multicolumn{6}{|l|}{ NYHA } \\
\hline I (73) & $42(57.5)$ & $21(28.8)$ & $8(11.0)$ & $2(2.7)$ & 0.013 \\
\hline ॥ (37) & $8(21.6)$ & $19(51.4)$ & $6(16.2)$ & $4(10.8)$ & \\
\hline III (2) & $2(100.0)$ & $0(0.0)$ & $0(0.0)$ & $0(0.0)$ & \\
\hline Rho & 0.28 & & & & 0.001 \\
\hline
\end{tabular}

of $\mathrm{MI}$, although patients in higher Killip classes had more affected arteries and were treated more often with CABG than PCI. Additionally, Killip class I patients were favored in a number of parameters including age, systolic blood pressure, heart rate, BMI, NT-proBNP level and LVEF. Conclusion Patients with $\mathrm{MI}$ as an onset manifestation of CAD present with varied degrees of pulmonary congestion. The prognostic value of the Killip classification is highlighted in terms of the extent of CAD (number of affected vessels and revascularization technique), of NYHA class and, last but not least, of HRQoL.
P183

Modes of arrival, door to balloon time and its impact on morbidity and mortality for ST elevation myocardial infarct

YC Chia

Tan Tock Seng Hospital, Singapore

Critical Care 2012, 16(Suppl 1):P183 (doi: 10.1186/cc10790)

Introduction Timely reperfusion of the occluded coronary artery is crucial in reducing the amount of myocardial damage in patients with ST elevation myocardial infarct (STEMI). This study aims to examine the common presenting symptoms of patients with STEMI, their modes of arrival at the emergency department (ED) and its impact on door-toballoon (D2B) time and in-hospital morbidity and mortality.

Methods In this retrospective study, the medical records of 619 patients with an admitting diagnosis of STEMI from Tan Tock Seng Hospital, Emergency Department between 1 January 2009 and 31 December 2009 were reviewed. We extracted data from the electronic records of the emergency case notes and inpatient discharge summaries.

Results Among 619 patients, 363 (58.6\%) arrived by emergency medical services (EMS) and $256(41.4 \%)$ by self-transport. Three hundred and thirty $(53.3 \%)$ patients underwent emergency angiography, of which $313(94.9 \%)$ were treated with percutaneous coronary intervention $(\mathrm{PCl})$, eight $(2.4 \%)$ with coronary artery bypass grafting (CABG) and nine $(2.7 \%)$ were conservatively managed. The D2B time was significantly shorter in patients who arrived by EMS ( 60 vs. 82 minutes; $P<0.001$ ). There was no difference in D2B time between patients who arrived in the day (06:00 to 17:59 hours) or at night (18:00 to 05:59 hours). Chest pain, shortness of breath and diaphoresis were the three commonest presenting symptoms in patients with STEMI regardless of their mode of arrival. Previous myocardial infarction, $\mathrm{PCl}$ or CABG did not influence the mode of transport. Patients who arrived by EMS had a higher incidence of cardiogenic shock $(20.7 \%$ vs. $11.7 \% ; P=0.020)$ and were significantly older (63 vs. 59 years; $P=0.004$ ) than patients who arrived by self-transport. Patients who arrived by EMS had a higher in-hospital mortality rate $(12.1 \%$ vs. $5.1 \% ; P=0.003)$ and a longer mean length of stay compared to those who arrived by self-transport ( 6 vs. 4 days; $P=0.004$ ).

Conclusion In our study population, patients with STEMI who used EMS tend to be older and arrived in cardiogenic shock. They therefore had a higher incidence of in-hospital mortality and morbidity although their D2B time was shorter compared to those who arrived by self-transport.

P184

Next-generation, fast and accurate point-of-care test for NT-proBNP based on Magnotech technology

B Inçaurgarat ${ }^{1}$, J Nieuwenhuis ${ }^{2}$

'bioMérieux, Marcy L'Etoile, France; ${ }^{2}$ Philips, Eindhoven, the Netherlands Critical Care 2012, 16(Suppl 1):P184 (doi: 10.1186/cc10791)

Introduction In the emergency care setting, where time is of the essence, there is a need for fast and reliable information on NT-proBNP levels for diagnosis and management of acute dyspnea. Rapid NTproBNP testing near the patient has the potential to streamline the process of care, but only if it is robust, fast and accurate enough to operate safely at the point of care (POC). Here we report on a novel NT-proBNP POC test which can be entirely carried out in a handheld device. This test has the potential to be rapid ( $<8$ minutes), easy to use (fingerprick sampling), and with good accuracy compared to state-ofthe-art automated laboratory assays.

Methods This new NT-proBNP POC test is based on Magnotech technology. A one-step sandwich immunoassay is performed in a compact plastic disposable cartridge with on-board dry reagents and magnetic nanoparticles. After a short incubation step the amount of bound nanoparticles, proportional to the concentration of NT-proBNP in the sample, is detected optically [1]. The precision of the assay was determined for plasma samples with NT-proBNP levels at clinically relevant values of $125 \mathrm{ng} / \mathrm{l}$ and $411 \mathrm{ng} / \mathrm{l}$ (10 replicates). Assay accuracy was determined by measuring 104 patient samples (lithium heparin plasma, NT-proBNP levels from 20 to $5,000 \mathrm{ng} / \mathrm{l}$ ) on both the handheld device and the bioMérieux VIDAS laboratory system, and comparing results by Passing and Bablok regression analysis. 
Results Assay precision was characterised by CV levels of less than $10 \%$. NT-proBNP results correlated well with VIDAS $(r=0.89)$, with a corresponding slope of the regression line of $1.12(95 \% \mathrm{Cl} 1.01$ to 1.22$)$ and an intercept of 64.04 (95\% Cl -73.50 to 109.83). In the current format under development, the NT-proBNP assay time with plasma samples is only 5 minutes. We are in the process of adding a filter that will allow measurements from whole blood directly. Flow experiments show that the filling time of the cartridge with whole blood is less than 30 seconds, resulting in a total assay time of less than 6 minutes, and a time-to-result of less than 8 minutes.

Conclusion In its current implementation the Magnotech-based NTproBNP assay shows promising performance for rapid, reliable NTproBNP testing at the POC in emergency settings. Development work is presently focused on the integration of a blood filter into the cartridge, to allow fingerprick tests.

Reference

1. Bruls DM, et al:: Lab Chip 2009, 9:3504-3510.

P185

Right ventricular apical versus septal pacing: impact on left ventricular synchrony and function

I Atteia, A Alazab, K Hussein, N Abeed, H Nagi

Cairo University, Cairo, Egypt

Critical Care 2012, 16(Suppl 1):P185 (doi: 10.1186/cc10792)

Introduction Right ventricular apical pacing alters the LV activation resulting in an adverse effect on LV function and synchrony. On the contrary, RV septal pacing results in narrower QRS and may be more physiological with less deleterious long-term effect on LV echocardiographic and hemodynamic parameters.

Methods Forty patients indicated for permanent DDD pacing were studied. All patients were subjected to transthoracic echocardiography calculating LVESD, LVEDD, EF\% and CO together with tissue Doppler imaging (TDI) to detect LV dyssynchrony. Patients were randomly classified into two groups, group I having RV apical pacing and group II having RV septal pacing. The acute threshold, R-wave sensing and fluoroscopic time were measured in all patients and compared in both groups. Both groups were followed-up over a period of 6 months.

Results QRS durations were significantly narrower in group II patients $(148 \pm 6.9$ vs. $162 \pm 6 \mathrm{~ms}, P=0.001)$. Electrical parameters at the time of implantation were satisfactory for all patients (acute stimulation threshold was $0.5 \pm 0.18 \mathrm{~V}$; $\mathrm{R}$ wave sensing was $11 \pm 1.6 \mathrm{mV}$ and ventricular impedance was $630 \pm 90 \mathrm{Ohm})$. No single patient needed ventricular lead repositioning. The acute pacing threshold, R-wave sensing, ventricular impedance and fluoroscopic time did not change significantly in both groups. During follow-up, it was found that in group II patients with RV septal pacing there was significantly lower LVESD $(3.0 \pm 0.6$ vs. $3.4 \pm 0.6 \mathrm{~cm}, P=0.004)$, significantly higher LVEF\% $(69 \pm 13$ vs. $61 \pm 8, P=0.01)$, significantly higher CO $(4.9 \pm 0.3$ vs. $4.5 \pm 0.6 \mathrm{I})$, and significantly lower septal to lateral wall delay in LV using TDI (72 \pm 5 vs. $83 \pm 6 \mathrm{~ms}, P=0.001)$ if compared to group I patients with RV apical pacing.

Conclusion Long-term RV septal pacing is feasible, and reliable with less adverse effects on LV synchrony and function when compared to $\mathrm{RV}$ apical pacing.

References

1. Kutarski A, Ruciniski P, Sodolski T, Trojnar M: Factors influencing differences of RVA and RVOT pacing hemodynamic effects. Europace 2005, 7:288 doi:10.1016/j.eupc.2005.02.104.

2. Hafez M, Small GR, Hannah A, et al:. Impact of temporary right ventricular pacing from different sites on echocardiographic indices of cardiac function. Europace 2011. doi: 10.1093/europace/eur 207.

P186

Consecutive case series of Takotsubo cardiomyopathy: a disease potentially triggered by the Great East Japan Earthquake T Suzuki, S Sakai, T Abe

Mito Kyodo General Hospital, University of Tsukuba, Mito City, Japan Critical Care 2012, 16(Suppl 1):P186 (doi: 10.1186/cc10793)

Introduction Takotsubo cardiomyopathy (TC) is a rare disease that mimics ST elevated myocardial infarction (STEMI). TC is known to involve psychic or physical stressors such as a devastating disaster, but those clinical features have been not fully investigated. As Ibaraki prefecture suffered from the Great East Japan Earthquake, we tried to clarify the characteristics of TC and investigate whether the Great East Japan Earthquake increased the occurrence of TC or not.

Methods Eleven consecutive patients with TC (five men, six women) were enrolled between October 2009 and October 2011 in this study. Patients were diagnosed by echocardiography, left ventriculography, or nuclear scintigraphy. Absence of significant coronary stenosis was confirmed by coronary angiography or coronary computedtomography angiography. Clinical characteristics (age, season, coronary risk factors, the condition that preceded onset as possible triggering factors and so on), laboratory data (troponin $\mathrm{T}$, creatinine kinase, and so on) and data of electrocardiography (ECG) were obtained from reviewing medical records.

Results The number of cases of TC after the earthquake was five for 7 months and that of before is six for 17 months. The occurrence rate of TC seemed to increase after the earthquake. Reviewing all of our cases, $45.5 \%(n=5 / 11)$ of patients have TC in the autumn, $72.7 \%(n=8 / 11)$ of patients suffered from a physical stressor, and $27.3 \%(n=3 / 11)$ of patients a psychic stressor. No obvious stressor was found in only one patient. The patients complained of chest pain or dyspnea ( $54.5 \%$ each). The rate of coronary risk factors were; family history, $10 \%(n=1 / 10)$; smoking, $60 \%(n=6 / 11)$; diabetes, $57.1 \%(n=4 / 7)$; hypertension, $63.6 \%$ $(n=7 / 11)$; dyslipidemia, 44.4\% ( $n=4 / 9)$; and obesity, $22.2 \%(n=2 / 9)$. Laboratory data showed that elevated troponin $\mathrm{T}$ was observed in $60 \%(n=6 / 10)$, high CK and CK-MB were $45.5 \%(n=5 / 11)$ and $100 \%$ $(n=9 / 9)$, respectively. ECG findings of all of the patients; ST elevation was observed in precordial leads of V2 to V4 $(27.3 \%, 54.5 \%$ and $27.3 \%$, respectively) and ST depression was in V5 (36.4\%). Reversed $r$ progression was observed in $18.2 \%$, poor $r$ progression was $27.3 \%$, abnormal Q was $18.2 \%$, long QT interval was $72.7 \%$ and negative T was $63.6 \%$ of TC patients.

Conclusion Although TC seems to mimic anterior STEMI, limb leads did not tend to show ST change in ECG in our cases. The Great East Japan Earthquake could increase patients with TC until the tremendous damage caused by the disaster will be over.

\section{P187}

Stress cardiomyopathy after live donor liver transplantation: incidence, risk factors and mortality

S Gupta, D Govil, S Bhatnagar, S Patel, S Srinivasan, P Pandey, M Sodhi, J KN, P Singh, S Saigal, A Soin, V Vohra, Y Mehta

Medanta - The Medicity, Gurgaon, India

Critical Care 2012, 16(Suppl 1):P187 (doi: 10.1186/cc10794)

Introduction The incidence of cardiac complications in the post live donor liver transplantation (LDLT) period has been reported to be nearly $70 \%$ [1]. Stress cardiomyopathy (SC) is a severe complication which has varied presentation and has grave prognosis if not diagnosed and managed aggressively.

Methods Data for 250 LDLTs (June 2010 to July 2011) were collected to assess incidence, risk factors and mortality due to SC. Diagnostic criteria [2] for SC were taken as: global hypokinesia or new ST segment elevation or T-wave inversion in absence of coronary artery disease (CAD) or pheochromocytoma. Etiologies of chronic liver disease and preoperative cardiac status along with intraoperative vasopressor use and dosages were noted.

Results Out of 250 patients five patients had preoperative CAD and were excluded. Seven patients (incidence $2.8 \%$ ) were diagnosed to have SC. Five out of seven (71.4\%) patients were ethanolic and vasopressor requirement was high in all these patients (Figure 1). Echocardiography revealed global hypokinesia with left ventricular ejection fraction between 10 and $25 \%$. They were managed with inotropic support and four patients required an intraaortic balloon pump (IABP). Two patients succumbed to cardiogenic shock on the second day (mortality 28.5\%). IABP was weaned between 7 and 9 days. Patients had normal cardiac status at the time of discharge around the fourth week post liver transplant.

Conclusion Our incidence was $3 \%$. SC generally presents on the second to third postoperative day and usually recovers by the second 


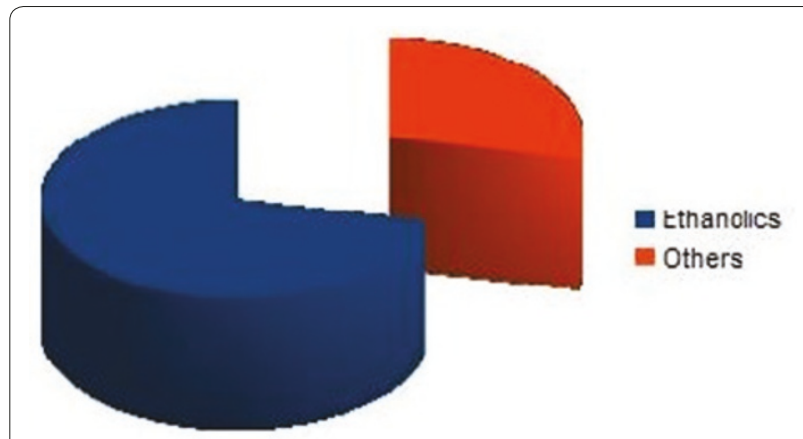

Figure 1 (abstract P187).

week. Ethanolics and patients who require high vasopressor support intraoperatively are more prone to develop SC.

References

1. Therapondos G, et al.: Cardiac morbidity and mortality related to orthotopic liver transplantation. Liver Transp/ 2004, 10:1441-1453.

2. Bybee KA, et al.: Systematic review: Transient left ventricular apical ballooning: a syndrome that mimics ST-segment elevation myocardial infarction. Ann Intern Med 2004, 141:858-865.

\section{P188}

Short-term hemodynamic effects of nebivolol in acute decompensated heart failure: a randomized clinical trial R Puig, M Ochiai, J Cardoso, KVieira, E Brancalhao, M Lima, A Pereira Barretto

Hospital Auxiliar de Cotoxo - InCor - HCFMUSP, São Paulo, Brazil

Critical Care 2012, 16(Suppl 1):P188 (doi: 10.1186/cc10795)

Introduction Acute decompensation heart failure in patients in use of $\beta$-blocker has become frequent and maintenance of this drug remains controversial, mainly in low cardiac output. Nitric-oxide-dependent vasodilation of nebivolol could be useful in this situation.

Methods We evaluated hospitalized patients with acute decompensated heart failure, NYHA IV, EF $<0.45$, in use of dobutamine and carvedilol. Intervention: patients were randomly assigned to carvedilol maintenance or exchange to nebivolol according to Table 1. Hemodynamic parameters were compared using a noninvasive model flow technique (Nexfin ${ }^{\oplus}$; BMEYE), 24 hours before, 6 and 24 hours after the randomization. $P<0.05$ was significant.

Table 1 (abstract P188)

\begin{tabular}{cc}
\hline Carvedilol & Nebivolol \\
\hline $6.25 \mathrm{mg} / \mathrm{bid}$ & $2.5 \mathrm{mg} / \mathrm{qd}$ \\
$12.5 \mathrm{mg} / \mathrm{bid}$ & $5.0 \mathrm{mg} / \mathrm{qd}$ \\
$25.0 \mathrm{mg} / \mathrm{bid}$ & $10.0 \mathrm{mg} / \mathrm{qd}$ \\
\hline
\end{tabular}

Results We selected 30 patients, $75 \%$ men, age $56.0(S D=13.0)$ years, ejection fraction $23.4(\mathrm{SD}=7.2) \%$, ischemic myocardiopathy present in $16.7 \%$, Chagas disease in $40 \%$ and $43.3 \%$ of patients were nonischemic/ non-Chagas. Baseline indexed systemic vascular resistance was 2,255.9 $(\mathrm{SD}=792.4)$ dynes.second $/ \mathrm{cm}^{5} / \mathrm{m}^{2}$, and cardiac index was $2.7(\mathrm{SD}=0.6)$ $\mathrm{l} /$ minute/m. In the nebivolol group $(n=15)$ the indexed systemic vascular resistance reduced $0.6 \%$ and in the carvedilol group $(n=15)$ it reduced $5.0 \%$ in 24 hours (mean difference $4.4 \%$; $95 \% \mathrm{Cl}:-12.6$ to $21.4 \% ; P=0.513)$. The cardiac index maintained unchanged $(P=0.274)$. Comparing patients that received a high dose of nebivolol ( 5 to $10 \mathrm{mg} /$ day) to those with a low dose ( $<5 \mathrm{mg} /$ day) or carvedilol, we observed a tendency to superiority of high dose in reduction of systemic vascular resistance, although not statistically significant (Figure 1).

Conclusion Short-term nebivolol use in decompensated heart failure was hemodynamically safe. Further studies should be done to clarify this matter.

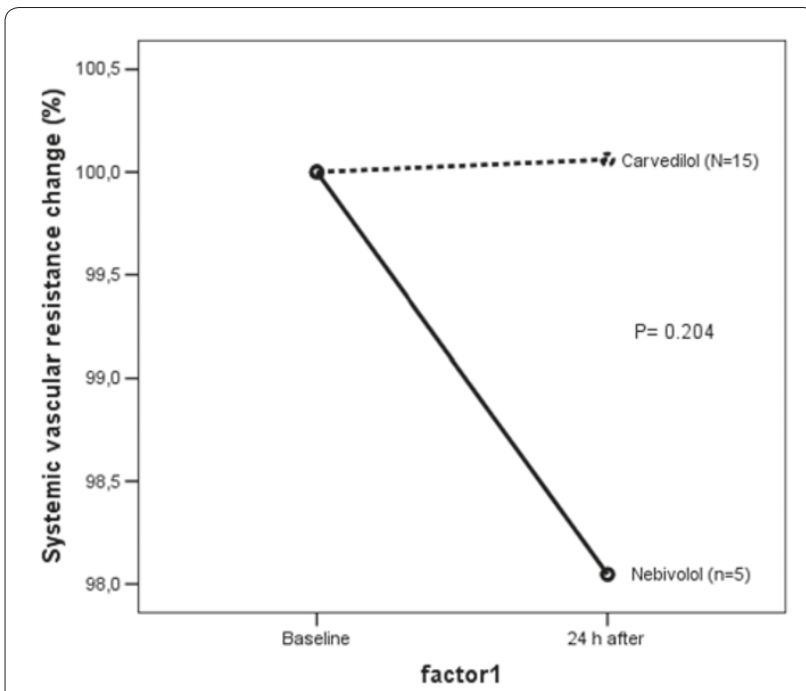

Figure 1 (abstract P188). Systemic vascular resistance change: nebivolol high $\times$ nebivolol low or carvedilol.

P189

Patients with infective endocarditis patients in the ICU: how are they? P Fernandez Ugidos', R Gomez Lopez', P Vidal Cortes',

AV Aller Fernandez², JM Lopez Perez

${ }^{1}$ Complejo Hospitalario Universitario Ourense, Spain; ${ }^{2}$ Complejo Hospitalario Universitario A Coruña, Spain

Critical Care 2012, 16(Suppl 1):P189 (doi: 10.1186/cc10796)

Introduction The objective was to analyze clinical characteristics of patients with infective endocarditis (IE) requiring surgery when the disease is diagnosed.

Methods A retrospective study of all patients, during 5 years in a tertiary hospital in Spain, which required admission to the ICU with the diagnosis of IE (Duke criteria modified) and required surgery at the same time. We compiled demographics, clinical characteristics and complications. Data were analyzed with SPSS 17.

Results We had 73 patients, $79 \%$ male, mean age 65 . Forty-five percent had previous heart disease. Eighty-four percent presented with fever, $56.5 \%$ general syndrome, $56.2 \%$ heart failure, $19.2 \%$ pain, and $7 \%$ coma. The duration of the clinic before diagnosis was mainly between 7 and 30 days (32\%), followed by more than 30 days (27\%). Less than 3 days duration represented $13 \%$. Blood cultures were positive in $82 \%$. The most common agent was Streptococcus (39\%), followed by Staphylococcus aureus MS (16\%), SCN (12\%), Enterococcus (12.3\%), S. aureus MR (1.4\%), Escherichia coli (1.4\%), Pseudomonas Aeruginosa (1.4\%), Aspergillus (1.4\%), and polymicrobial (1.4\%). Twelve percent were negative cultures. The valve more frequently affected was aortic. In all cases TTE was carried out for diagnosis. In 69 cases TEE was performed. The principal echo findings were: vegetation (42\%), new insufficiencies (26\%), and also stenosis, perivalvular abscess and normal echo. Fifty-eight percent of patients had no distal emboli. Other localizations: splenic (11\%), hepatic $(2.7 \%)$, bones $(2.7 \%)$, brain $(4 \%)$, lung $(5 \%)$ and more than one (11\%). Forty-one percent of patients required ICU admission before surgery with an average stay of 5.6 days. A total of $31.5 \%$ suffered multiorgan failure. Antibiotics were given 17 days before surgery. In $6.8 \%$ it was not possible to give them preoperatively. Eighty-two percent of patients took combination therapy ( $19 \%$ four). Cephalosporins, aminoglucosids and vancomycin were the most used. Two patients died before surgery. Thirty-five percent of the interventions were urgent. In $16.4 \%$, reoperation was necessary, mainly for bleeding, followed by prosthetic dysfunction, recurrent IE, mediastinitis and pseudoaneurysm repair. A total $56 \%$ of patients presented postoperative shock. MV was needed during 5 days (range 0 to 53). Acute renal failure post surgery was present in $58 \%$. Other complications were secondary infection, ventricular dysfunction, 
atrioventricular block, stroke, perioperative $\mathrm{MI}$, and liver failure. The ICU stay was 33 days (median 6). The hospital mortality was $31.5 \%$. Conclusion IE has high morbi-mortality. The subgroup of patients requiring early surgery presents the most severe disease. This corresponds with our patients: one-third of cases need urgent surgery, $56 \%$ have shock, about $60 \%$ ARF, and mortality reaches $30 \%$.

\section{P190}

Malperfusion and branch compromise in acute type $\mathrm{A}$ aortic syndrome

R Gomez Lopez'1, P Fernandez Ugidos' 1 P Vidal Cortes' ${ }^{1}$ J Lopez Perez²,

J Priego Sanz'1, M Bouza Vieiro², A Aller Fernandez², L Seoane Quiroga²,

S Fojon Polanco ${ }^{2}$

${ }^{1}$ Complexo Hospitalario Universitario de Ourense, Spain; ${ }^{2}$ Complexo

Hospitalario Universitario de A Coruña, Spain

Critical Care 2012, 16(Suppl 1):P190 (doi: 10.1186/cc10797)

Introduction Malperfusion is a factor associated with higher risk of death and complications in patients with acute type A aortic syndrome (AAAS). Our objective is to determine the incidence and characteristics of this disease in our population and to verify the relevance in morbidity and in-hospital mortality.

Methods A historical cohort study that includes all patients with AAAS admitted to the ICU after surgical management in a single institution from January 2000 to July 2010. Anatomical, clinical, biochemical, electrocardiographic and echocardiographic signs of ischemia were considered. The events of interest were death or major complication (neurological damage, multiorgan failure (MOF), acute lung injury (ALI), postoperative hemorrhage) during hospitalization.

Results A total of 65 patients were identified (24.6\% women, $61.86 \pm 12$ years old, APACHE II score $12.9 \pm 7.2$, EuroSCORE 7.4 \pm 2.6 ). Thirty-three (50.8\%) presented branch compromise, affecting coronary arteries in 12 patients (18.4\%) (symptomatic (S) seven (10.5\%), asymptomatic (A) five (7.7\%)), nine (13.8\%) carotid (S five (7.7\%), A four (6.1\%)), 28 (43\%) brachiocephalic or subclavian (S 17 (26.1\%), A 11 (16.9\%)), 15 (22.8\%) mesenteric (S seven (10.5\%), A eight (12.3\%)), $13(20 \%)$ renal (S nine (13.8\%), A four (6.1\%)), and 31 (47.7\%) iliac (S $16(24.6 \%)$, A 15 (23\%)). Twenty-eight (43.1\%) showed clinical ischemia of at least one system and $54(83 \%)$ clinical signs of global hypoperfusion. Comparing patients with and without data of hypoperfusion, differences in incidence of death $(45.5 \%$ vs. $18.8 \%, P=0.03)$, neurological complication $(35.7 \%$ vs. $10.8 \%, P=0.03)$, MOF ( $16.6 \%$ vs. $25 \% . P=0.07)$ and ALI ( $21.3 \%$ vs. $29.6 \%$, $P=0.09)$ were found.

Conclusion More than $80 \%$ of the patients with AAAS suffered malperfusion in our series. They had a higher risk of death and neurological complication during hospitalization.

\section{P191}

Prognostic value of the echocardiographic-derived calcium index in coronary artery disease

J Jimenez, J Iribarren, J Lacalzada, A De La Rosa, R Juárez, A Barragán,

J Bonilla, G Blanco, R Pérez, M Brouard, I Laynez

Hospital Universitario de Canarias, La Laguna, Spain

Critical Care 2012, 16(Suppl 1):P191 (doi: 10.1186/cc10798)

Introduction Calcification of different cardiac structures is associated with atherosclerotic risk factors. The aim of this study is to determine whether the echocardiography-derived calcium index (ECI) assessed by transthoracic echocardiography (TTE) predicts cardiovascular events, besides determining the coronary artery calcium score (CACS), the presence of obstructive coronary artery disease (CAD) and the composition of plaques, all of which determined by multidetector computed tomography (MDCT).

Methods We carried out a prospective study of 82 consecutive patients, with an intermediate likelihood for $C A D$, who were evaluated by noninvasive coronariography by MDCT. ECI was blindly assessed by TTE. A 36-month follow-up was conducted to detect cardiovascular events. Results The area under the ROC curve (AUC) of the Agatston score scale as a predictor of significant obstruction identified by MDCT was 0.80 ( $95 \% \mathrm{Cl}: 0.68$ to 0.91 ); $P<0.001$. The optimal cut-off was 239 .
Agatston score $\geq 239$ has a sensitivity (Se) of $60.6 \%$ (95\% Cl: 0.42 to 0.77 ), specificity (Sp) of $97.8 \%$ ( $95 \% \mathrm{Cl}: 0.88$ to 0.99 ), positive predictive value (PPV) of $95.2 \%$ and negative predictive value (NPV) of $77.2 \%$. The AUC of ECI to predict an optimal cut-off value for Agatston score was 0.90 ( $95 \% \mathrm{Cl}: 0.83$ to 0.96$) ; P<0.001$. $\mathrm{ECl} \geq 7$ had a Se of $59.1 \%(95 \% \mathrm{Cl}$ : 0.36 to 0.79 ), a Sp of $93.3 \%$ ( $95 \% \mathrm{Cl}: 0.83$ to 0.98 ), PPV of $76.5 \%$ and NPV of $86.2 \%$. There was a significant linear trend of $\mathrm{ECl}$, and $\mathrm{ECl} \geq 7$ has in MDCT a greater presence of both severe calcified wall and obstructive $C A D$, number of affected vessels, and mixed/calcified plaques (all $P<0.001)$. There were 23 coronary ischemic events. The AUC of ECl as a predictor of adverse cardiac events post MDCT was 0.92 (95\% Cl: 0.852 to 0.987 ); $P<0.001$. $\mathrm{ECl} \geq 7$ had a Se of $77.3 \%$ (95\% Cl: 54.6 to 92.2$)$, a Sp of $90 \%$ (95\% Cl: 79.5 to 96.2 ), PPV of $73.9 \%$ and NPV of $91.5 \%$. The Kaplan-Meier survival analyses show a statistically significant difference between patients with VCSI $\geq 7$ or not regarding an ischemic event $\left(X^{2}: 52, P<0.001\right)$. This accumulation of risk occurs mainly in the first 2 years after the determination of $\mathrm{ECl}$.

Conclusion $\mathrm{ECl} \geq 7$ determines a poor CAD prognosis of coronary ischemic events. Furthermore, $\mathrm{ECl} \geq 7$ may serve as a marker of the content of wall calcium, obstruction level and composition of the plaques. $\mathrm{ECl}$ seems to provide prognostic information as well as providing information about the characteristics of the plaque of atheroma.

\section{P192}

Echocardiography in the ICU: an audit of 3 years practice

A Hall, J Walker, I Welters

Royal Liverpool Hospital, Liverpool, UK

Critical Care 2012, 16(Suppl 1):P192 (doi: 10.1186/cc10799)

Introduction Assessment of the haemodynamically unstable patient is a core part of ICU management and relies predominantly on a combination of clinical skill and measurement of physiological variables. Echocardiography in the ICU has become increasingly popular as a tool for assessment of cardiac output, fluid status and ventricular function. Traditionally transoesophageal echo (TOE) has been favoured due to the belief that it gave superior images [1]. Transthoracic echo (TTE) is not often performed as it relies on 24-hour availability of trained personnel, availability of equipment and good patient windows [1]. There was also a perceived lack of benefit; however, recent studies have shown good or adequate images in over 85 to $90 \%$ of patients resulting in a change of management in $48 \%$ [1].

Methods Data were collected prospectively in all patients undergoing echocardiography in a teaching hospital ICU from January 2008 to January 2011. The main focus of our investigation was to ascertain the clinical questions to be answered and the outcome of echo on management.

Results A total of 238 echoes were performed on 216 patients with an average age of 59.75 years (TTE: 198, TOE: 19, and both: 14). The most commonly asked questions were on filling status and contractility (40\%) and left ventricular function (33\%). Ninety percent of clinical questions asked were answered fully (74\%) or partially (16\%) by echo. Sixty-one percent of echoes resulted in a change of management ( $5 \%$ of which were to continue with increased confidence). TTE performed by operators with basic training resulted in a $54 \%$ change in management. Changes included more filling (39\%) and changes in inotropes or diuretics.

Conclusion Echocardiography in the ICU patient relies on numerous factors including skill and equipment availability and patient windows [1]. Our results confirm that there is a role for echo in these patients, important in a population where assessment of cardiac output and filling status is notoriously difficult. Our results also show that TTE performed by ICU physicians with basic training provides very useful information for the management of patients. This makes the focused courses on echocardiography very important $[2,3]$. Limitations of the study: an unknown amount of missing data and a likelihood of patient and operator bias as to which patients had echocardiography. In conclusion, echocardiography is a useful tool in the management of the haemodynamically unstable patients.

References

1. Orme R, et al.: Br J Anaesth 2009, 102:340-344.

2. Vieillard-Baron et al: Intensive Care Med 2008, 34:243-249.

3. Jensen et al: Eur J Anaesth 2004, 21:700-707. 
P193

Left ventriculum diastolic dysfunction in pediatric septic shock

M Georgiyants, V Korsunov

Kharkov Medical Academy Post-Graduate Education, Kharkov, Ukraine

Critical Care 2012, 16(Suppl 1):P193 (doi: 10.1186/cc10800)

Introduction One of the causes of septic mortality is a low cardiac output secondary to preload failure. Same patients demonstrate preload failure after aggressive volume replacement [1].

Methods Ultrasound impulse-wave Doppler evaluation of transmitral flow:VmaxE, VmaxA, ejection time E,A; DT E wave, IVRT of LV. Ultrasound evaluation of end-diastolic and end-systolic LV volume, stroke volume (LVEDV, LVESV, SV) on Teichholz L. EDLVP $=1.06+15.15 \times V T$ T peakA/ VTI peakE. Coronary perfusion pressure $(C P P)=$ EDLVP - diastolic BP. We evaluate these parameters in 34 patients (age $28.1 \pm 8.0$ months) with septic shock (SS) diagnosed according to Consensus 2002. Control (C) -44 healthy children (age $40.7 \pm 8.5$ months). Statistical analyses with $t$ criteria.

Results The increase of VmaxA and decrease of VmaxE in patients of SS are demonstrated. IVRT and DT are less than in control group. We evaluated a decrease in E/A proportion. EDLVP in patients was more, and CPP lower, than in controls. See Table 1.

Table 1 (abstract P193). Diastolic function in pediatric septic shock

\begin{tabular}{lccc}
\hline Value & SS & C & $P$ value \\
\hline VmaxA & 81 & 65 & $<0.05$ \\
VmaxE & 99 & 108 & $>0.05$ \\
ETA & 80 & 102 & 0.01 \\
ETE & 102 & 149 & 0.001 \\
DTE & 51 & 93 & 0.001 \\
IVRT & 48 & 87 & 0.01 \\
E/A & 1.3 & 1.7 & 0.01 \\
EDLVP & 23 & 9.8 & $<0.001$ \\
CPP & 22 & 50 & 0.001 \\
EDLVV & 46 & 65 & $<0.001$ \\
SV & 14 & 26 & 0.001 \\
\hline
\end{tabular}

Conclusion Pediatric SS accompanied with LV diastolic dysfunction, which decreases the effectiveness of volume restoration therapy, reduces preload and cardiac output.

Reference

1. Jardin F, et al.: Persistent preload defect in severe sepsis despite fluid loading. A longitudinal echocardiographic study in patients with septic shock. Chest 1999, 116:1354-1359.

\section{P194}

Existence of interference between the heart and respiratory sounds: preliminary report

N Finahari

Brawijaya University, Malang East Java, Indonesia

Critical Care 2012, 16(Suppl 1):P194 (doi: 10.1186/cc10801)

Introduction Heart diseases still persist as one of the first-ranked causes of mortality in the world and Indonesia. Currently, mortality from coronary heart diseases is estimated to reach 53.5 per 100,000 population [1]. Auscultation is a fundamental diagnostic method for heart disease, noninvasive and inexpensive [2], but highly dependent on the expertise and experience of the listener. Improved accuracy of diagnosis is usually then performed through further examination using the electrocardiogram, magnetic resonance imaging and the computed tomography scan. Unfortunately, these tools require very expensive investment costs that are only available in large hospitals [3]. This is the main reason for supporting the development of computerbased auscultation technique tools that are cheaper and are able to

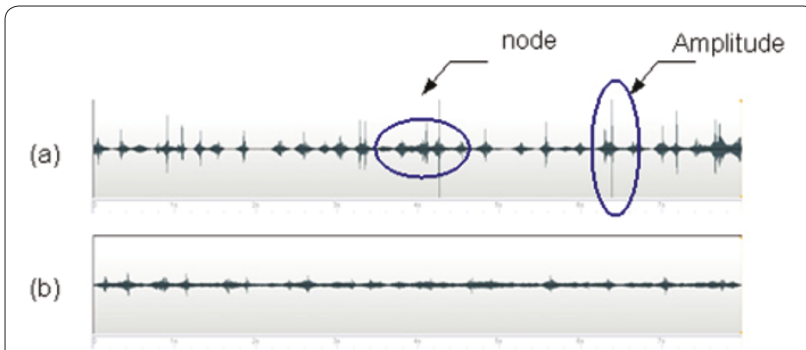

Figure 1 (abstract P194). Characteristics of the data differences between (a) free and (b) hold breathing.

improve the accuracy and reliability of diagnosis on early stages [2]. If the device can be designed as portable, then it can be used by heart disease patients for daily monitoring to avoid or minimize heart attack accidents. To improve the accuracy of heart auscultation analysis, usually the lung sound must be minimized, or vice versa. It is very difficult. This study tried to use heart and lung interference sounds as physiological parameters. So this preliminary research aims to prove that interference does occur between heart and respiratory sounds. This interference sound will be used as an analysis technique to improve the accuracy of a new auscultation device.

Methods This research was conducted on nine randomly chosen volunteers whose heart sounds were recorded in two conditions: 30 seconds free and hold breathing. The heart sound recording process is done electronically using a modified standard stethoscope to generate digital data. Modifications were performed using a mic condenser combined with a voice processing system based on Windows XP. Accuracy of the equipment is ensured by the noise-signal ratio test.

Results Generally, it can be seen (Figure 1) that there are pronounced differences in heart sound data recorded in the conditions of free and hold breathing. This means that the respiration process is likely to affect the heart sounds heard on the chest surface. The differences that appear are in the form of nodes and amplitude. Differences in the form of a node indicate a difference in frequency of sounds and color (timbre), while the amplitude differences may indicate differences in strength and speed of sound propagation. In general, the number of differences in the recording position is close to the number of respiratory cycles so that it is possible these differences are caused by respiratory processes. Conclusion The differences that can be noticed from the graphical visualization of recorded sounds are in the form of nodes and amplitude. These differences that indicate the frequency, sound color, strength and speed of sounds improve the existence of an interference wave between the heart and respiratory sounds. These characteristics will be used to design the new portable auscultation device.

References

1. Persatuan Perawat Nasional Indonesia [http://inna-ppni.orid/html]

2. Javed F, Venkatachalam PA, Fadzil A: J Phys Conf Ser 2006, 34:1098-1105.

3. Stasis A, et al:: A Multiple Decision Tree Architecture for Medical Diagnosis: The

Differentiation of Opening Snap, Second Heart Sound Split and Third Heart

Sound. CMS Springer-Verlag; 2004:254-274.

4. Mrowka R, Cimponeriu L, Patzak A, Rosenblum MG: Am J Physiol Regul Integr Comp Physiol 2003, 285:R1395-R1401

5. Schikowski T, et al:: Respir Res 2007, 8:1-11

6. Toledo E, et al: Med Eng Phys 2002, 24:45-52.

7. Darowski M: Front Med Biol Eng 2000, 10:157-165.

P195

Effects of the intravenous administration of purine nucleosides guanosine or inosine against hemorrhagic shock in pigs

A Schmidt, D Otsuki, DO Souza, J Auler Jr

Faculdade de Medicina da Universidade de São Paulo, Brazil

Critical Care 2012, 16(Suppl 1):P195 (doi: 10.1186/cc10802)

Introduction Hemorrhagic shock leads to the appearance of substances in plasma that depress $\mathrm{Na} / \mathrm{K}$ ATPase activity, an effect that could be related to significant morbidity and mortality. Recently, some findings 
indicated that purine nucleosides such as guanosine, inosine or adenosine might prolong survival in shocked rats, an effect potentially related to the stimulation of $\mathrm{Na} / \mathrm{K}$ ATPase activity. This study aimed to evaluate the effects of intravenous administration of guanosine or inosine combined with lactate Ringer solution (LR) on hemodynamic and oxygenation parameters and survival in an experimental model of hemorrhagic shock (HS).

Methods HS was induced in 24 pigs ( 25 to $30 \mathrm{~kg}$ ) by blood removal for 20 minutes to target a mean arterial pressure (MAP) of $40 \mathrm{mmHg}$, which was maintained for 60 minutes with additional blood removal or retransfusion. Animals were treated with LR alone (three times the volume of blood withdrawn) or associated to $1 \mathrm{mmol} / \mathrm{l}$ guanosine or $1 \mathrm{mmol} / \mathrm{l}$ inosine. Hemodynamic and oxygenation parameters were evaluated at baseline, after $\mathrm{HS}$, immediately after fluid resuscitation, and 30,60, 120, 240 and 360 minutes after fluid resuscitation. Primary outcome was post-shock survival. Statistical analysis of parametric data was performed with one-way ANOVA for repeated measures followed by Student-Newman-Keuls. Kruskal-Wallis followed by the Dunn test was used for analysis of nonparametric data. The post-shock survival was evaluated by the Kaplan-Meier curve.

Results The hemodynamic and oxygenation parameters were not significantly different among pigs treated with RL alone or in combination with guanosine or inosine. No effects on post-shock survival were observed in any group.

Conclusion The actual preliminary results did not demonstrate any additional improvement induced by guanosine or inosine on the hemodynamic and oxygenation parameters or on the postshock survival during HS. These findings need to be confirmed in a larger group of animals and further investigation with cellular and biochemical analysis may help to elucidate the effects of guanosine and inosine during $\mathrm{HS}$.

Acknowledgments Supported by FAPESP and CNPq.

References

1. Darlington DN, Gann DS:. J Trauma 2005, 58:1055-1060.

2. Schmidt AP, et al.: Pharmacol Ther 2007, 116:401-416.

\section{P196}

Norepinephrine versus angiotensin II in septic shock: effects on isolated kidney, heart and liver mitochondrial respiration

$\checkmark$ Jeger, M Vuda, T Correa, J Takala, S Djafarzadeh, SM Jakob Inselspital University Hospital Bern, Switzerland

Critical Care 2012, 16(Suppl 1):P196 (doi: 10.1186/cc10803)

Introduction Mitochondrial dysfunction has been proposed to influence organ function and outcome in sepsis. Both vasopressor agents norepinephrine and angiotensin II can interfere with mitochondrial function. The aim of this study was to compare mitochondrial respiration after exposure of septic animals to either of these two drugs.

Methods In 16 anesthetized pigs, evolving septic shock after 12 hours of fecal peritonitis was randomly treated with either norepinephrine $(0.8 \pm 0.6 \mu \mathrm{g} / \mathrm{kg} /$ minute; mean \pm SD) or angiotensin II $(0.31 \pm 0.37 \mu \mathrm{g} /$ $\mathrm{kg} /$ minute; $n=8$, each) and fluids for 48 hours. Organs were harvested at the end of the experiment, and mitochondria isolated by tissue homogenization and differential centrifugation. Mitochondrial oxygen consumption $\left(\mathrm{VO}_{2}\right)$ was measured by high-resolution respirometry (Oroboros Instruments, Innsbruck, Austria). Groups were compared using Mann-Whitney $U$ test. In addition, mitochondrial respiration was also compared to a similarly instrumented control group without fecal peritonitis ( $n=8$; Kruskal-Wallis test).

Results Achieved blood pressure levels and cardiac output were not different between the two septic groups, and both groups received the same amount of fluids (norepinephrine: $1.6 \pm 0.5 \mathrm{ml} / \mathrm{kg} / \mathrm{hour}$, angiotensin II: $1.3 \pm 0.8 \mathrm{ml} / \mathrm{kg} / \mathrm{hour} ; P=\mathrm{NS}$ ). Compared to controls, mitochondrial $\mathrm{VO}_{2}$ was not different in septic animals. The only difference between the two septic groups was higher renal Complex I, State 4 respiration in norepinephrine-treated (median (range): 309 (164 to 415$) \mathrm{pmol} /\left(\right.$ second $\left.{ }^{*} \mathrm{mg}\right)$ ) versus angiotensin-II-treated animals (210 (89 to 273 ) $\mathrm{pmol} /\left(\right.$ second $\left.{ }^{*} \mathrm{mg}\right) ; P=0.05$ ).

Conclusion We found no significant effects of septic shock treated with either angiotensin II or norepinephrine and fluids on mitochondrial function, under similar hemodynamic conditions. Hepatic, renal and myocardial respiration of the measured mitochondrial complexes did not significantly differ between the two treatment groups, except for renal Complex I, State 4 respiration.

P197

Goal-directed hemodynamic resuscitation in high-risk patients undergoing cardiac surgery: a randomized controlled trial preliminary data (GRICCS STUDY)

E Osawa', A Rhodes'2, J Fukushima', J Almeida', F Jatene', R Nakamura', M Sundin', J Auler Jr', R Kalil Filho', F Galas', L Hajjar

'Heart Institute, São Paulo, Brazil;'2Charing Cross Hospital, Imperial College

NHS Trust, London, UK

Critical Care 2012, 16(Suppl 1):P197 (doi: 10.1186/cc10804)

Introduction Low cardiac output is a frequent clinical circumstance after cardiac surgery and results in higher morbidity and mortality rates. Goal-directed therapy (GDT) is a validated design that has been proved to reduce the number of perioperative outcomes. We investigated the results of a cardiac index optimization protocol through the use of the LiDCO rapid device.

Methods A prospective study that randomized 34 high-risk patients (EuroSCORE higher than 6 or LVEF lower than $45 \%$ ) to a GDT protocol or a conventional hemodynamic therapy. Patients from the GDT group were resuscitated to a cardiac index higher than $3 \mathrm{l} /$ minute $/ \mathrm{m}^{2}$ through the implementation of a three-step approach: (1) fluid challenge of $250 \mathrm{ml}$ aliquots, (2) dobutamine infusion up to a dose of $20 \mu \mathrm{g} / \mathrm{kg} / \mathrm{minute}$, and (3) blood transfusion to reach a hematocrit higher than $28 \%$. The control group was managed according to institutional protocol. Categorical variables were compared using Fisher's exact test and categorical variables were compared using the Mann-Whitney U test.

Results Sixteen patients from the GDT group were compared with 18 patients from the control group. There was a tendency towards reduction in ICU stay in patients from GDT group in relation to the control group ( 7 days vs. 6 days, $P=0.18$ ). Comparison of the primary endpoint variable (composite of death or major postoperative complications within 30 days after surgery or before discharge) between groups showed a reduced complication rate in the GDT group $(52.2 \%$ vs. $45.6 \%, P=0.12)$, mainly attributed to worse acute renal failure RIFLE criteria in the control group.

Conclusion Goal-directed hemodynamic resuscitation with the use of a minimally invasive device seems to be a promising perioperative strategy aimed at reducing the rates of worse outcomes and the ICU stay after cardiac surgery.

P198

Economic evaluation of early-goal directed therapy for high-risk surgical patients

C Ebm, M Cecconi, H Aya, M Geisen, A Rhodes, M Grounds

St George's Healthcare Trust, London, UK

Critical Care 2012, 16(Suppl 1):P198 (doi: 10.1186/cc10805)

Introduction Early goal-directed therapy (EGDT) has been shown to reduce postoperative morbidity and length of hospital stay. Our objective was to analyse the cost-effectiveness of early goal-directed proactive therapy versus standard reactive care in patients at high risk of developing postoperative complications.

Methods Patient-level outcome data used were based on a previous randomised, controlled trial. A Markov decision model was constructed to analyse costs and outcomes associated with the use of EGDT. Outcomes assessed were postoperative complications, mortality, quality-adjusted life expectancy (QALY) and incremental costs/QALY. Results The main analysis, based on 28-day survival data of 122 patients, revealed an incremental cost-effectiveness ratio of EGDT of $£ 280.15$ per patient. Additional costs of $£ 525.43$ per patient associated with EGDT were mainly due to costs related to monitor acquisition and staffing (two additional nurses). These costs were balanced by savings due to the significant reduction in length of stay in the hospital and in the ICU and lower complication rates in the GDT arm (mean expenditures/patient $£ 4,511.25$ vs. $£ 5,218.75$ ). This outcome was 
Table 1 (abstract P198)

\begin{tabular}{lccc}
\hline Outcome & Unit & GDT & Standard \\
\hline Ward stay & (days) & 11 (7 to 15) & 14 (11 to 27) \\
Incr. costs & $(£)$ & 525.43 & - \\
Inc. effect & $($ QALY $)$ & 1.88 & - \\
ICER & $(£ /$ QALY $)$ & 280.15 & - \\
\hline
\end{tabular}

ICER, incremental cost-effectiveness ratio.

robust to variations in treatment effect (probability of morbidity and mortality) and sensitive to implementation costs of EGDT. See Table 1. Conclusion The implementation of EGDT appears clinical and costeffective. Additional implementation costs will be offset by savings due to a marked decrease in complication rates and hospital length of stay. We conclude that GDT provides significant benefits with respect to both clinical and financial outcomes.

Reference

1. Pearse R, Dawson D, Fawcett J, Rhodes A: Early goal directed therapy after major surgery reduces complications and duration of hospital stay. A randomized, controlled trial. Crit Care 2005, 9:R687-R693.

P199

What matters during a hypotensive episode: fluids, vasopressors, or both?

J Lee ${ }^{1}$, R Kothari², JA Ladapo 3 , DJ Scott ${ }^{1}$, LA Celi

'Massachusetts Institute of Technology, Cambridge, MA, USA; ${ }^{2}$ Mount Sinai School of Medicine, New York City, NY, USA; ${ }^{3}$ New York University School of Medicine, New York City, NY, USA

Critical Care 2012, 16(Suppl 1):P199 (doi: 10.1186/cc10806)

Introduction The objective of this retrospective study was to investigate the relationships between fluid and vasopressor interventions and patient outcomes. In intensive care, it is imperative to resolve hypotensive episodes (HEs) in a timely manner in order to minimize end-organ damage. The current clinical practice is first to attempt fluid resuscitation and then to follow with vasopressor therapy if fluid resuscitation is unsuccessful. However, the effects of fluid and vasopressor interventions on patient outcomes have not been clearly established.

Methods Hypotension was defined as MAP below $60 \mathrm{mmHg}$. The primary outcome was in-hospital mortality. Secondary outcomes included ICU LOS, HE duration, Hypotension Severity Index (HSI) (MAP curve area below $60 \mathrm{mmHg}$ during the $\mathrm{HE}$ ), and rise in serum creatinine. The patient cohort included patients in the MIMIC-II database [1] who experienced a single HE. Multivariate logistic regression and propensity score analysis were employed. Sensitivity analyses were conducted in subpopulations stratified by treatment type and diagnosis.

Results A total of 3,163 patients in MIMIC-II met the inclusion criteria. The multivariate regression results showed that fluid resuscitation was significantly associated with shorter ICU LOS (OR $=0.71, P=0.007)$ and greater $\mathrm{HSI}(\mathrm{OR}=1.26, P=0.04)$. Vasopressor administration significantly decreased HE duration $(\mathrm{OR}=0.29, P<0.001)$ and $\mathrm{HSI}(\mathrm{OR}=0.72$, $P=0.002)$ but was correlated with increased in-hospital mortality risk $(\mathrm{OR}=2.86, P<0.001)$ (even after propensity adjustment; $\mathrm{OR}=2.44$, $P<0.001)$, prolonged ICU LOS (OR $=1.29, P=0.04)$, and rise in serum creatinine (OR $=1.44, P=0.002)$. Sensitivity analyses in treatmentspecific and diagnosis-specific subpopulations corroborated the relationship between vasopressors and increased in-hospital mortality. Conclusion Regarding the relationship between vasopressor therapy and in-hospital mortality, similar findings have been reported in previous studies analyzing sepsis [2], cardiac surgery [3], and heart failure [4]. We speculate that benefits of vasopressor use may be restricted to subsets of patients with specific conditions. This study illustrates the utility of electronic medical records in research when randomized controlled trials are difficult to conduct.

\section{References}

1. Saeed M, et al.: Crit Care Med 2011, 39:952-960.

2. Dunser M, et al.: Crit Care 2009, 13:R181.

3. Shahin J, et al:: Crit Care 2011, 15:R162.

4. Thackraya S, et al:: Eur J Heart Fail 2002, 4:515-529.
P200

Elevated central venous pressure in septic patients is associated with impairment of microcirculatory blood flow

N Vellinga' ${ }^{1}$ C Ince ${ }^{2}$, EC Boerma

'Medisch Centrum Leeuwarden, the Netherlands; ${ }^{2}$ Erasmus Medical Center, Rotterdam, the Netherlands

Critical Care 2012, 16(Suppl 1):P200 (doi: 10.1186/cc10807)

Introduction The microcirculation plays a pivotal role in oxygen delivery to the tissue. Microcirculatory alterations have been observed to occur independently of the major inflow variable for microcirculation: mean arterial pressure. According to physiological theory, the microcirculation is considered to be a low-pressure compartment. Maximum optimal central venous pressure (CVP) according to Surviving Sepsis Campaign (SSC) guidelines is 12 to $15 \mathrm{mmHg}$ in mechanically ventilated patients. We hypothesized that a CVP $>12 \mathrm{mmHg}$ would hamper microcirculatory perfusion but not diffusion, by acting as outflow obstruction.

Methods We retrospectively analyzed combined measurements of CVP and sidestream dark-field derived sublingual microcirculatory variables in patients with severe sepsis or septic shock. Measurements were made $0,0.5,2,12$ and 24 hours after resuscitation in accordance with SSC guidelines. Differences in small vessel microvascular flow index (MFI) and total vessel density (TVD) between two groups (CVP $\leq 12 \mathrm{mmHg}$ and CVP $>12 \mathrm{mmHg}$ ) were analyzed with a Mann-Whitney $U$ test.

Results A total of 345 measurements in 70 patients (APACHE II 21 (6.5) (mean (SD))) were included. MFI in patients with CVP $>12 \mathrm{mmHg}$ was significantly lower than in CVP $\leq 12 \mathrm{mmHg}$ (1.83 (0.92 to 2.75) vs. 2.25 (1.35 to 2.90 ) (median (IQR)), $P=0.032$ ), whereas TVD in both groups did not differ significantly (14 (12.84 to 15.75$)$ vs. 14.3 (13 to 15.8$) \mathrm{mm} /$ $\left.\mathrm{mm}^{2}, P=0.38\right)$. See also Figure 1.

Conclusion In septic patients with CVP >12 mmHg after resuscitation, microcirculatory flow was significantly lower as compared to patients with CVP $\leq 12 \mathrm{mmHg}$, whereas capillary density did not differ between groups.

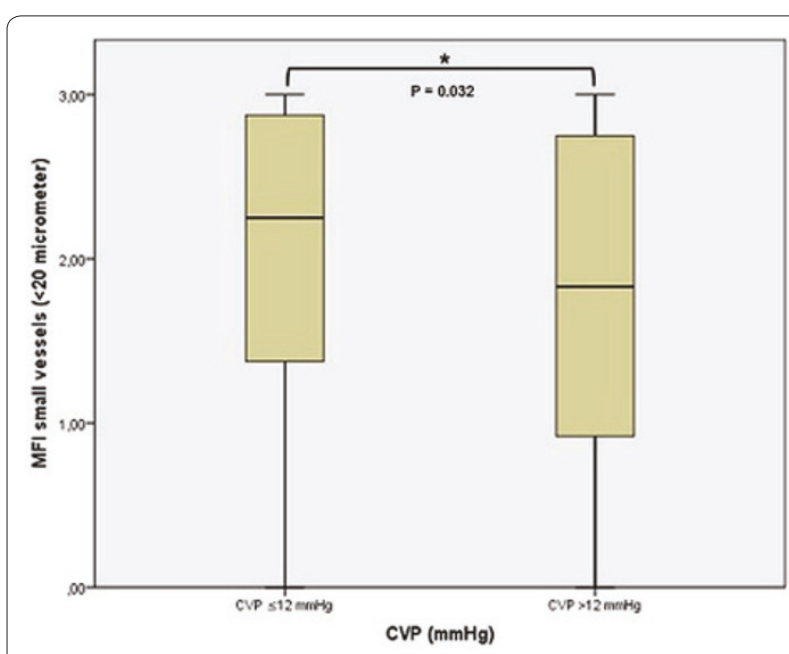

Figure 1 (abstract P200). Boxplots of microvascular flow index (MFI) in patients with a central venous pressure (CVP) $\leq 12 \mathrm{mmHg}$ or $>12 \mathrm{mmHg}$.

\section{P201}

Human protein $C$ concentrate to restore physiological values in adult septic shock patients: effects on microcirculation A Morelli ${ }^{1}$, A Donati $^{2}$, A Di Russo' , F D'lppolito', C Raffone', A D'Egidio', MR Lombrano ${ }^{2}$, S Tondi ${ }^{2}$, E Damiani ${ }^{2}$, V Cecchini', A Orecchioni' ${ }^{1}$ P Pietropaoli 'University La Sapienza, Rome, Italy; ${ }^{2}$ Marche Polytechnique University, Ancona, Italy

Critical Care 2012, 16(Suppl 1):P201 (doi: 10.1186/cc10808)

Introduction We investigated whether human protein $C(P C)$ concentrate to restore physiological values in adult septic shock patients can influence microcirculatory blood flow. 
Methods We enrolled 36 septic shock patients with plasma protein $C$ activity $<60 \%$. Patients were randomly allocated to be treated with either a continuous infusion of PC concentrate at $3 \mathrm{UI} / \mathrm{kg} / \mathrm{hour}$ for 72 hours to reach plasma protein C activity between 70 and $120 \%$ or a standard treatment (control; each $n=18$ ). In both groups, NE was titrated to achieve a MAP between 65 and $75 \mathrm{mmHg}$. Data from right heart catheterization and sidestream dark-field imaging were obtained at baseline and after 24, 48 and 72 hours.

Results For the same MAP and cardiac output, no significant differences were found between groups in terms of microvascular flow index of the small vessels (MFIs) and perfused vessel density (PVD). Results are summarized in Table 1.

Table 1 (abstract P201). Microcirculatory variables

\begin{tabular}{lcccc}
\hline & Baseline & $\mathbf{2 4}$ hours & $\mathbf{4 8}$ hours & $\mathbf{7 2}$ hours \\
\hline MFIs & & & & \\
Treated & $2.8(2.6 ; 3)$ & $3(2.7 ; 3)$ & $2.9(2.8 ; 3)$ & $3(2.9 ; 3)$ \\
Controls & $2.8(2.1 ; 2.9)$ & $2.8(2.1 ; 2.8)$ & $2.8(2.2 ; 3)$ & $3(2.6 ; 3)$ \\
PVD & & & & \\
Treated & $17.8(16.5 ; 22.2)$ & $19.7(17.4 ; 22.5)$ & $19.7(18.1 ; 23)$ & $19.9(17 ; 22.2)$ \\
Controls & $20.2(17.4 ; 23.5)$ & $18.8(17.6 ; 20.2)$ & $19.4(17.5 ; 20.7)$ & $18.7(17.5 ; 21.2)$ \\
\hline
\end{tabular}

Conclusion The administration of human PC concentrate did not influence microcirculatory blood flow in septic shock patients.

\section{P202}

Heart rate reduction with esmolol in septic shock: effects on microcirculation

A Morelli', A Donati' ${ }^{2}$ A Di Russo' , F D'lppolito', A Carsetti ${ }^{2}$, R Domizi', A D'Egidio', C Raffone', C Scarcella², C Ertmer ${ }^{3}$, S Rehberg ${ }^{3}$, P Pietropaoli', MWestphal ${ }^{3}$

'University La Sapienza, Rome, Italy: ${ }^{2}$ Marche Polytechnique University,

Ancona, Italy; ${ }^{3}$ University Hospital of Muenster, Germany

Critical Care 2012, 16(Suppl 1):P202 (doi: 10.1186/cc10809)

Introduction Preclinical and clinical studies report that $\beta$-blockers may be an interesting option to attenuate the deleterious effects of prolonged catecholamine exposure during septic shock. Nevertheless, there are concerns that $\beta$-blockers may have negative chronotropic and inotropic effects leading to inappropriately low cardiac output. The objective of the present study was therefore to elucidate whether a reduction in heart rate (HR) with esmolol may negatively affect microcirculation in patients with septic shock who remained tachycardic after hemodynamic optimization.

Methods After 36 hours of initial hemodynamic stabilization, 11 septic shock patients with $\mathrm{HR}>95 \mathrm{bpm}$ and requiring norepinephrine (NE) to maintain mean arterial pressure (MAP) between 65 and $75 \mathrm{mmHg}$, despite adequate volume resuscitation, received a continuous esmolol infusion to maintain HR between 94 and $80 \mathrm{bpm}$. NE was titrated to achieve a MAP between 65 and $75 \mathrm{mmHg}$. Data from right heart catheterization and sidestream dark-field imaging were obtained at baseline and after 24 hours.

Results Apart from a statistically significant decrease in $\mathrm{HR}$ and cardiac index $(\mathrm{Cl})(P<0.05)$, stroke volume $(\mathrm{SV})$, microvascular flow index of

Table 1 (abstract P202)

\begin{tabular}{lcc}
\hline & Baseline & 24 hours \\
\hline $\mathrm{HR}$ & $119 \pm 12$ & $85 \pm 9^{*}$ \\
$\mathrm{Cl}$ & $4.4 \pm 1$ & $3.1 \pm 1^{*}$ \\
$\mathrm{SV}$ & $81 \pm 35$ & $80 \pm 23$ \\
$\mathrm{MFIS}$ & $2.6 \pm 0.6$ & $2.8 \pm 0.3$ \\
$\mathrm{NE}$ & $0.7 \pm 0.7$ & $0.5 \pm 0.4$ \\
\hline
\end{tabular}

${ }^{*} P<0.05$. the small vessels (MFIs) and norepinephrine requirements did not vary during the 24-hour observational period. Results are summarized in Table 1.

Conclusion In patients with established septic shock who remained tachycardic after hemodynamic optimization in accordance with the current guidelines, titration of esmolol to reduce the HR to a predefined threshold did not affect microcirculatory blood flow.

\section{P203}

Early course of microcirculatory perfusion in the eye and digestive tract during experimental sepsis

A Pranskunas', R Rasimaviciute', E Milieskaite', A Vitkauskiene',

P Dobozinskas', V Veikutis², Z Dambrauskas', D Vaitkaitis', V Pilvinis ${ }^{1}$

'Lithuanian University of Health Sciences, Kaunas, Lithuania; 'Institute of

Cardiology, Lithuanian University of Health Sciences, Kaunas, Lithuania

Critical Care 2012, 16(Suppl 1):P203 (doi: 10.1186/cc10810)

Introduction Studies show that sublingual mucosa is a reproducible part for small intestine mucosal microcirculatory perfusion in sepsis, when they are not exposed by local factors. However, it is of great interest how sublingual microcirculation can reflect other beds of microcirculation. The aim of the study is to evaluate and compare the microcirculatory perfusion of potentially available parts of the body, such as sublingual mucosa, conjunctiva of the eye, mucosa of jejunum and rectum, at the same time points during experimental sepsis.

Methods Pigs were randomly assigned to sepsis $(n=9)$ and sham $(n=4)$ groups. The sepsis group received a fixed dose of live Escherichia coli infusion over 1 hour. Animals were observed 5 hours after the start of $E$. coli infusion. In addition to systemic hemodynamic assessment, we performed conjunctival, sublingual, jejunal and rectal evaluation of microcirculation using sidestream dark-field videomicroscopy at the same time points: at baseline, 3 and 5 hours after the start of live $E$. coli infusion. Assessment of microcirculatory parameters of convective oxygen transport (microvascular flow index (MFI), proportion of perfused vessels (PPV)) and diffusion distance (perfused vessel density, total vessel density) was done using a semiquantitative method.

Results Infusion of $E$. coli resulted in a hypodynamic state of sepsis despite fluid administration. Significant decreases in MFI and PPV of small vessels were in sublingual, conjunctival, jejunal and rectal lodges 3 and 5 hours after the start of $E$. coli infusion in comparison to baseline variables. Correlation between sublingual and conjunctival $(r=0.80$, $P=0.036)$, sublingual and jejunal $(r=0.94, P=0.005)$, sublingual and rectal $(r=0.79, P=0.03)$ MFI was observed 3 hours after onset of sepsis. There was no correlation in change of MFI and PPV between sublingual mucosa and other evaluated regions. However, the sublingual mucosa exhibited the most pronounced alterations of microcirculatory flow in comparison to conjunctival, jejunal and rectal mucosa microvasculature $(P<0.05)$.

Conclusion Microcirculatory alterations were observed in all investigated lodges, including sublingual, jejunal and rectal mucosa, and conjunctiva of the eye at the same time point during experimental sepsis. There is a clear association between sublingual microcirculation and conjunctival, jejunal or rectal microcirculation in the very early course of an extreme hypodynamic state of sepsis.

P204

Microcirculation and blood transfusion: effects of three different types of concentrated red blood cells - preliminary results

A Donati, E Damiani, R Domizi, C Scorcella, A Carsetti, MR Lombrano, $\checkmark$ Fiori, P Pelaia

Università Politecnica delle Marche, Ancona, Italy

Critical Care 2012, 16(Suppl 1):P204 (doi: 10.1186/cc10811)

Introduction Red blood cell (RBC) transfusions are used to increase oxygen delivery; however, a restrictive transfusion strategy (predefined hemoglobin threshold of $7 \mathrm{~g} / \mathrm{dl}$ ) was demonstrated to be associated with lower mortality and incidence of nosocomial infections than a liberal one $[1,2]$. This may be related to the storage process, which could affect the ability of RBCs to transport and delivery oxygen, or to immunomodulating effects of cytokines from residual leukocytes [2]. 


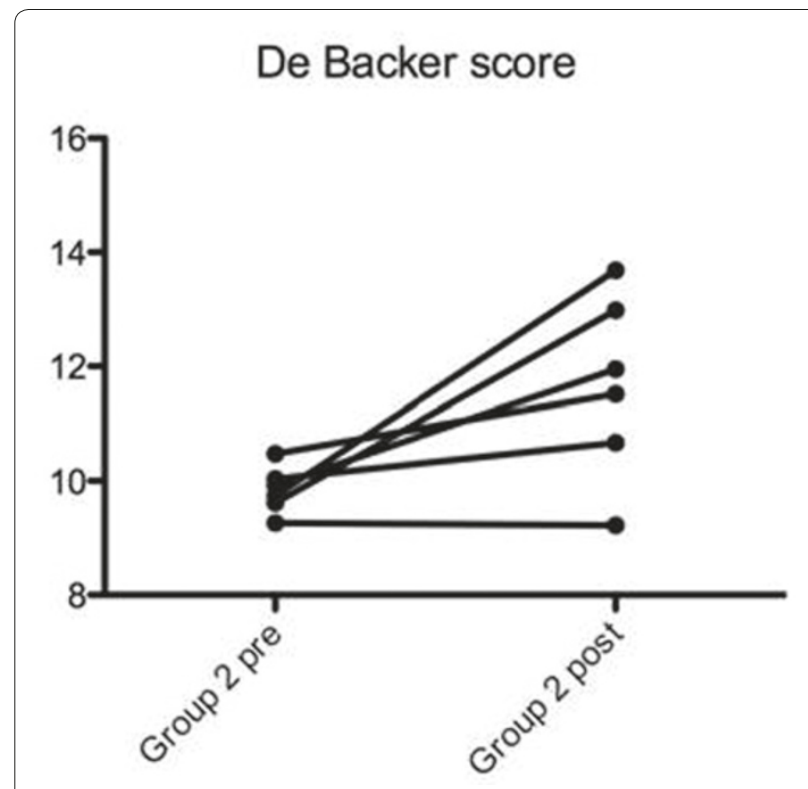

Figure 1 (abstract P204). De Backer score pre and post transfusion in group 2.

The aim of the study is to evaluate the effects, on microcirculation of septic patient, of three types of RBCs.

Methods A controlled randomized prospective study on 45 patients with sepsis, severe sepsis or septic shock requiring RBC transfusion. Patients are randomized into three groups receiving: (1) fresh standard RBCs (storage < 10 days); (2) leukodepleted RBCs; and (3) old standard RBCs (storage $>20$ days) respectively. Before and 1 hour after the transfusion, microcirculation is evaluated using sidestream dark-field imaging [3] and near-infrared spectroscopy with a vascular occlusion test. We also monitor temperature, heart rate, mean blood pressure, hemochrome, blood gases, blood lactates and SOFA score.

Results Preliminary data on 18 patients, six for each group: before and after transfusion, in group 2, but not in groups 1 and 3 , there is a trend to an increase in MFIs $(P=0.09)$, DeBacker score (Figure $1, P<0.05)$, $\operatorname{PPV}(P=0.07)$ and PVD $(P=0.07)$. No relevant differences for other parameters.

Conclusion After transfusion, microcirculation seems to be improved in the leukodepleted RBC group with a significant improvement of De Backer score and a trend to improve the other microcirculatory parameters, while in the other three groups there was not this trend. References

1. Hebert PC: N Engl J Med 1999, 340:409-417.

2. Rosemary L: Blood Transfusion 2010, 8(Suppl 3):S26.

2. De Backer D: Crit Care 2007, 11:R101.

P205

Peripheral perfusion is correlated to metabolic perfusion parameters and microvascular reactivity but not with hepatosplanchnic or microcirculatory flow parameters in hyperdynamic septic shock

G Hernandez', T Regueira', A Bruhn', P Mcnab', EVeas', C Pedreros', A Fuentealba', E Kattan', G Bugedo', M Rovegno', R Castro', C Ince ${ }^{2}$ 'Pontificia Universidad Catolica de Chile, Santiago, Chile; ${ }^{2}$ University of Amsterdam, the Netherlands

Critical Care 2012, 16(Suppl 1):P205 (doi: 10.1186/cc10812)

Introduction Peripheral perfusion assessment is increasingly being recognized as a potential surrogate of global perfusion parameters during septic shock resuscitation. Nevertheless, its correlation with other perfusion parameters is not well established. This study explores the relationship between peripheral perfusion parameters and macrohemodynamic, metabolic, hepatosplanchnic, and microcirculatory-related parameters during hyperdynamic septic shock.

Methods Thirty-nine sets of parallel assessments of hemodynamic or perfusion-related parameters were performed in 13 hyperdynamic (cardiac index $>2.5 \mathrm{l} / \mathrm{minute} / \mathrm{m}^{2}$ ) septic shock patients (age $68 \pm 18$ years, APACHE II score $26 \pm 6$, SOFA score $11 \pm 4$, ICU mortality 2/13) during the first 24 hours of resuscitation. Assessment included: echocardiographic and pulmonary artery catheter-derived parameters; indocyanine green plasma disappearance rate (ICG-PDR, Limon) and gastric tonometry; metabolic parameters (lactate, $\mathrm{SvO}_{2}$ and $\mathrm{p}(\mathrm{v}-\mathrm{a})$ $\mathrm{CO}_{2}$ ); sublingual microcirculatory assessment (SDF); thenar $\mathrm{StO}_{2}$ and vascular occlusion test (VOT) derived parameters (NIRS); peripheral perfusion parameters including capillary refill time and central to toe temperature difference.

Results Peripheral perfusion was normal in 22 sets (56\%) and abnormal in 17 (44\%). A normal peripheral perfusion was associated with lower APACHE II scores $(23.2 \pm 3$ vs. $28.4 \pm 7, P=0.009)$, better metabolic parameters (lactate: $2.3 \pm 0.6 \mathrm{mmol} / \mathrm{l}$ vs. $3.5 \pm 1.1 \mathrm{mmol} / \mathrm{l}, P=0.002$ and $\mathrm{SvO}_{2}: 78.1 \pm 6 \%$ vs. $73.9 \pm 5 \%, P=0.049$ ), and better $\mathrm{StO}_{2}$ recovery slope after VOT ( $3.54 \pm 1.4$ vs. $0.94 \pm 0.5 \% /$ second, $P<0.001$ ) as compared with an abnormal one. No correlation could be demonstrated with macrohemodynamic parameters, hepatosplanchnic perfusion parameters (gastric tonometry and ICG-PDR), or with sublingual microcirculatory parameters.

Conclusion A normal peripheral perfusion is associated with normal metabolic perfusion parameters and less impaired microvascular reactivity. No relation between peripheral perfusion and hepatosplanchnic or sublingual microcirculatory flow could be established in this study.

ClinicalTrials.gov Identifier: NCT01271153

Acknowledgements Supported by grant FONDECYT 1100610 (Chile).

\section{P206}

Hyperoxia affects peripheral tissue microcirculation in patients with pulmonary arterial hypertension

S Dimopoulos, G Tzanis, C Manetos, A Tasoulis, A Mpouchla, ETseliou,

I Vasileiadis, N Diakos, J Terrovitis, S Nanas

University of Athens, Greece

Critical Care 2012, 16(Suppl 1):P206 (doi: 10.1186/cc10813)

Introduction Pulmonary microcirculation abnormalities play a central role in pulmonary arterial hypertension (PAH) pathophysiology. We hypothesized that PAH patients also have systemic muscle microcirculation alterations compared to healthy subjects. The aim of this study was to investigate peripheral muscle microcirculation by near-infrared spectroscopy (NIRS) in PAH patients and to test the effects of hyperoxia into their tissue microcirculation.

Methods Eight PAH patients and eight healthy subjects matched for age, gender and body mass index underwent NIRS evaluation. Tissue $\mathrm{O}_{2}$ saturation $\left(\mathrm{StO}_{2}, \%\right)$, defined as the percentage of hemoglobin saturation in the microvasculature compartments, was measured on the thenar muscle. Subsequently, the 3-minute brachial artery occlusion technique was applied before, during, and after 15 minutes of $100 \%$ of $\mathrm{O}_{2}$-breathing. Main measurements included the oxygen consumption rate (OCR, \%/minute), the reactive hyperemia time (RHT, minutes), and the time needed for $\mathrm{StO}_{2}$ to reach its baseline values after the release of the occlusion.

Results PAH patients had a significantly lower resting $\mathrm{StO}_{2}(65.8 \pm 14.9$ vs. $82.1 \pm 4.0, P=0.01)$, a lower OCR $(35.3 \pm 9.1$ vs. $43.4 \pm 19.7)$ and a higher RHT ( $3.0 \pm 0.6$ vs. $2.0 \pm 0.3, P<0.001)$ compared to controls. Hyperoxic breathing increased $\mathrm{StO}_{2}(65.8 \pm 14.9$ to $71.4 \pm 14.5, P<0.05)$ in PAH patients, while OCR was reduced ( $35.3 \pm 9.1$ to $25.1 \pm 6.6$, $P<0.05)$ and RHT was further increased $(3.0 \pm 0.6$ to $4.2 \pm 0.7, P<0.01)$. Conclusion $\mathrm{PAH}$ patients present a significant impairment of peripheral tissue microcirculation as assessed by the NIRS occlusion technique. Acute hyperoxic breathing affects peripheral microcirculatory function in PAH patients, possibly due to oxidative stress and evoked vasoconstriction. 
P207

Supraclavicular ultrasound-guided subclavian vein cannulation in infants under $5 \mathrm{~kg}$

P Kenderessy

Faculty Children Hospital, Banska Bystrica, Slovakia

Critical Care 2012, 16(Suppl 1):P207 (doi: 10.1186/cc10814)

Introduction Central venous cannulation is at some point difficult in small children and is associated with many complications especially in multiple-attempt cases. Various techniques exist to achieve successful cannulation. Ultrasound (US)-guided techniques are reported to be safe and reduce the rate of complications for internal jugular vein (IJV) cannulation. We describe an US-guided supraclavicular approach to another central vein - the subclavian vein (SCV). The supraclavicular approach to the SCV with anatomical landmarks was described by Yoffa, but physicians are hesitant to use this technique because of the short distance to pleura.

Methods The principle of the US-navigated technique is to find the SCV at the supraclavicular level and to obtain a longitudinal view of the SCV and to allow access to the vein in-plane view (absolute control of the needle). The ultrasound probe $(2.5 \mathrm{~cm}, 6$ to $13 \mathrm{MHz})$ was placed above the clavicle to visualize the IJV and tilted showing the subclavian artery and SCV in longitudinal view. This view permitted an in-plane puncture of the vein avoiding arterial or plural hit.

Results Seventy-eight infant and newborns under $5 \mathrm{~kg}(1.2$ to $5 \mathrm{~kg})$ and $83 \mathrm{SCV}$ cannulations were enrolled in this observational study during a period of 11 months (January 2011 to November 2011). All cannulations were performed by a single anesthesiologist trained for ultrasound in central line cannulation with established eye-hand coordination (5 years experience with peripheral blocks under US). For all cases the SCV was easily and quickly visualized, one case had an extremely narrow SCV. The US window for cannulation was always established for free in-plane placement of the needle. The overall success rate for puncture was $100 \%$ and for cannulation was $98 \%$. In the case with an extremely narrow vein (because of oedema and stricture) the SCV was punctured but it was impossible to pass the catheter in. The success rate of puncture at first attempt was $97 \%$, at second attempt was $100 \%$. A second attempt was necessary in two cases because needle visualization and angle of the needle movement were not considered correct. No complication was reported.

Conclusion A supraclavicular US-guided approach to SCV cannulation is safe and effective possibility for central vein cannulation in small infants. More studies are needed to establish a learning curve for pure paediatric intensivists without experience with US navigation.

\section{P208}

Central venous catheter placement: where is the end of the line?

KTizard, I Welters

Royal Liverpool University Hospital, Liverpool, UK

Critical Care 2012, 16(Suppl 1):P208 (doi: 10.1186/cc10815)

Introduction There is still controversy regarding safe placement of central venous catheters (CVCs) as to where the tip should lie to avoid mechanical complications whilst maintaining effective use $[1,2]$. The carina has previously been suggested as a useful landmark to avoid intracardiac placement and its associated risks, and also that the catheter tip should lie within the superior vena cava parallel to its walls $[1,2]$. However, this has been disputed and there remains no consensus as to optimal tip placement. To gauge our current practice we performed a retrospective review of CVCs placed via the internal jugular or subclavian route in intensive care patients to assess where CVC tips were placed.

Methods We retrospectively reviewed the chest radiographs of 197 consecutive intensive care patients admitted on and before 30 June 2011. A total of 101 patients had evidence of 137 new CVCs. For each new catheter the Picture Archiving \& Communication System was used to record the tip position (after any repositioning) in relation to the carina and the degree of angulation from the vertical.

Results Twenty-five per cent (34/137) of all catheter tips lay $>10 \mathrm{~mm}$ below the carina, therefore potentially increasing the likelihood of intracardiac placement. This was reduced for left-sided catheters (6/37;
$16 \%)$. All right-sided catheters lay at an angle $<30^{\circ}$. However, $38 \%$ (14/37) of left-sided catheters had not crossed the midline, and $59 \%$ $(22 / 37)$ lay at an angle $>30^{\circ}$ to the vertical. Only $11 \%(4 / 37)$ of left-sided catheters had crossed the midline and lay at an angle of $<30^{\circ}$, and all of these lay below the level of the carina. No immediate complications of insertion were identified. See Table 1.

Table 1 (abstract P208). Site of CVC insertion $(n=137)$

\begin{tabular}{lcc}
\hline & Internal jugular & Subclavian \\
\hline Right & 95 & 5 \\
Left & 32 & 5 \\
\hline
\end{tabular}

Conclusion There was a wide variation of catheter tip placements accepted without re-positioning. Left-sided catheter tips are more at risk of less precise (and thus potentially nonoptimal) placement. Our results indicate that a clearer placement strategy is required.

References

1. Stonelake PA, et al:: The carina as a radiological landmark for central venous catheter tip position. Br J Anaesth 2006, 96:335-340.

2. Bodenham A: Reducing major procedural complications from central venous catheterization. Anaesthesia 2011, 66:1-9.

\section{P209}

Power-injectable peripherally inserted central catheters in intensive care patients

MG Annetta, C Marano, A Brutti, D Celentano, M Pittiruti

Catholic University, Rome, Italy

Critical Care 2012, 16(Suppl 1):P209 (doi: 10.1186/cc10816)

Introduction In ICUs, peripherally inserted central catheters (PICCs) may be an alternative option to standard central venous catheters, particularly in patients with coagulation disorders or at high risk for infection. Some limits of PICCs (such as low flow rates) may be overcome by the use of power-injectable catheters.

Methods We have retrospectively reviewed all of the power-injectable PICCs inserted in adult and pediatric patients in the ICU during a 12-month period, focusing on the rate of complications at insertion and during maintenance. All PICCs were inserted by specifically trained nurses, using ultrasound guidance and the microintroducer technique, according to a specific insertion protocol.

Results We have collected 89 power-injectable PICCs $(65$ in adults and 24 in children), 4 to $6 \mathrm{Fr}$, both multiple and single lumen. All insertions were successful. There were no major complications at insertion and no episodes of local infection or catheter-related bloodstream infection. Noninfective complications during management were not clinically relevant. There was one episode of symptomatic thrombosis during the stay in the ICU and one episode after transfer of the patient on a nonintensive ward.

Conclusion Power-injectable PICCs have many advantages in the ICU: they can be used as multipurpose central lines for any type of infusion including high-flow infusion, for hemodynamic monitoring, and for high-pressure injection of contrast media during radiological procedures. Their insertion is successful in $100 \%$ of cases and is not associated with significant risks, even in patients with coagulation disorders. Their maintenance is associated with an extremely low rate of infective and noninfective complications.

\section{P210}

Comparison of internal jugular and subclavian access for central venous catheterization in pediatric cardiac surgery

A Pirat', A Camkiran', P Zeyneloglu', M Ozkan', E Akpek², G Arslan

'Baskent University, Ankara, Turkey; ${ }^{2}$ Acibadem University, Istanbul, Turkey Critical Care 2012, 16(Suppl 1):P210 (doi: 10.1186/cc10817)

Introduction Central venous catheterization (CVC) is an essential component of perioperative care in pediatric cardiac surgery. Traditionally the internal jugular vein (IJV) is used for CVC in cardiac surgery. The aim of this study was to compare IJV and subclavian vein (SV) routes for CVC 
in pediatric cardiac surgery in terms of success rate and mechanical and infectious complications.

Methods After Ethics Committee approval and written informed consent from the parents of the children were obtained, 200 children who were scheduled for cardiac surgery were randomly allocated to IJV $(n=100)$ and SV $(n=100)$ groups.

Results The mean age was 37 months ( $95 \% \mathrm{Cl}, 29$ to 45 months) in group IJV and 35 months ( $95 \% \mathrm{Cl}, 29$ to 42 months) in group SV $(P=0.619)$. The $95 \% \mathrm{Cl}$ for weight in groups IJV and SV were 10.4 to $14.2 \mathrm{~kg}$ and 10.2 to $13.0 \mathrm{~kg}$, respectively $(P=0.595)$. The CVC success rates at first attempt for groups IJV and SV were $67 \%$ and $70 \%$, respectively $(P=0.761)$. An alternative location was required to perform CVC in 90 patients in group IJV and in 92 patients in group SV $(P=0.806)$. The overall frequency of mechanical complications during the catheter insertion and its use was $26 \%$ in group IJV and $28 \%$ in group SV (log-rank test: $P=0.753)$. Significantly more arterial punctures occurred in group IJV than in group SV (14\% vs. $4 \%, P=0.024)$. Catheter tip misplacement was observed more frequently in group SV than group IJV (12\% vs. $1 \%, P=0.003)$. Catheter colonization rates were significantly higher in group IJV than group SV (15\% vs. $5 \%$, log-rank test: $P=0.020)$. There was no difference in bloodstream infection per 1,000 catheter days between group IJV and group SV ( 3.4 vs. 1.4 , respectively: $P=0.319$ ). Conclusion In pediatric cardiac surgery patients, IJV and SV catheters had similar success rates as well as overall mechanical complication rates. Although the catheter colonization rate was significantly higher with IJV than SV, both access routes had similar rates of bloodstream infection.

P211

Ultrasound-guided central venous line placement in critically

ill patients: is chest X-ray needed to assess post-insertion pneumothorax?

O Samir Abdel Gelil Kotb, A Ali Abel Aziz, Y Awad

Sheikh Khalifa Medical City, Abu Dhabi, United Arab Emirates

Critical Care 2012, 16(Suppl 1):P211 (doi: 10.1186/cc10818)

Introduction Critically ill patients, mostly on positive pressure ventilation, are at higher risk of pneumothorax as well as their need for a central venous line (CVL) to optimize fluid status, CVP measurement, and so forth, and where the CVL is not being placed in the best circumstances with the patients being critically ill, unstable and with higher chances of error predisposed by pre-existing lung disease, obesity or whatever the admitting diagnosis. Before CVL placement was a blind technique relying on the anatomical positions identifying the position of major blood vessels and thus post-insertion X-ray was needed to confirm correct placement and to assess for pneumothorax. But with ultrasound (US) being more widely available, and most CVLS placed as US guided, the ultimate question develops: is post-insertion chest X-ray still needed?

Methods A retrospective study of 856 lines placed in 602 patients being evaluated over a period of 11 months. All cases were performed in a controlled ICU environment. Chest X-rays were performed 30 minutes post-insertion in the DO adult ICU unit in a tertiary medical center in Abu Dhabi, UAE. The DO ICU has a capacity of 24 beds with an average admission rate of 55 to 60 patients per month. Records were assessed and evaluated, and data collected and statistically studied.

Results A total of 856 lines performed in 602 patients were evaluated. In 607 US-guided cannulating internal jugular veins with only four cases of malposition, there were no cases of pneumothorax recorded. A total of 161 subclavian veins were cannulated with no US, of which six cases of pneumothorax were reported; two cases needed intercostal tube insertion. Eighty-eight femoral vein cannulations with no US were performed and no complications were recorded.

Conclusion Chest X-ray is not necessary after US-guided CVL placement. Cutting out the chest $\mathrm{X}$-ray procedure post insertion proved to be cost-effective.

References

1. Ultrasound detects central line placement and postprocedure pneumothorax. Emerg Med 2009.

2. Ultrasound-guided central line placement: no X-ray needed. Emerg Med 2011.
P212

Errors in the arterial blood pressure measurement

F Franchi', V De Palo', A Faltoni', S Cecchini', L Cubattoli², P Giomarelli'

'University of Siena, Italy; ${ }^{2}$ Hospital of Siena, Italy

Critical Care 2012, 16(Suppl 1):P212 (doi: 10.1186/cc10819)

Introduction The artefacts affecting arterial wave morphology may compromise recorded values of arterial blood pressure (ABP) and can lead to therapeutic errors. The aim of this study is to evaluate the errors between invasive and noninvasive arterial pressure values, the incidence of artefacts due to an inadequate dynamic response of the transducer-tubing system, and their detection by the ICU staff.

Methods Seventy-five consecutive patients ( 50 male, mean age $55 \pm 18$ ) admitted to the ICU for heterogeneous pathologies were enrolled. Inclusion criteria were: the presence of an intra-arterial catheter (IAC) for invasive blood pressure monitoring, and age $>18$ years. Pregnancy was excluded. At admission and every time the IAC was replaced we acquired invasive systolic, diastolic, and medium arterial pressure values (I-SP, I-DP, I-MP) during hemodynamic stability (variations of mean arterial pressure $<10 \%)$; at the same time, noninvasive systolic and diastolic arterial pressure values (Ni-SP, Ni-DP) were measured with a sphygmomanometer at the same arm of the IAC. Noninvasive medium arterial pressure (Ni-MP) was calculated as follows: (SP + 2DP) / 3. At every time of the study, before ABP value acquisition, medical and nursing staff answered a questionnaire on the reliability of the arterial waveform. The staff could perform the fast flush test if considered appropriate. However, the fast flush test was executed by the main investigator at the end of questionnaire in all patients. Bland-Altman analysis was performed.

Results We compared 130 pairs of Ni-SP, Ni-DP and Ni-MP and I-SP, I-DP and I-MP. The mean bias between Ni-SP and I-SP was $-11 \mathrm{mmHg}$ (limit of agreement (LoA) -43.6 to $21.4 \mathrm{mmHg}$ ). The mean bias between $\mathrm{Ni}-\mathrm{DP}$ and I-DP and between Ni-MP and I-MP was $6.1 \mathrm{mmHg}$ (LoA -15.5 to $27.7 \mathrm{mmHg}$ ) and $0.37 \mathrm{mmHg}$ (LoA -21.0 to $21.7 \mathrm{mmHg}$ ), respectively. We performed the fast flush test 130 times; an inadequate dynamic response of the transducer-tubing system was observed 55 times: in 45 cases the arterial signal was underdumped and in 10 cases was overdumped. The arterial dumping was correctly detected by the medical staff in $95 \%$ of cases, by nursing staff and postgraduates in $35 \%$ of cases.

Conclusion The bias between invasive and noninvasive ABP measure can be relevant and mislead in the therapeutic management. These errors can be avoided by identifying the artefacts that affect arterial signal and so the ICU staff must pay attention to the recognition of arterial dumping in critically ill patients.

\section{Reference}

1. Pickering TG: Principles and techniques of blood pressure measurement.

Cardiol Clin 2002, 20:207-223.

P213

The T-Line TL-200 system for continuous noninvasive blood pressure measurement in medical ICU patients

B Saugel, F Fassio, A Hapfelmeier, AS Meidert, RM Schmid, W Huber Klinikum Rechts der Isar, Technischen Universität München, Munich, Germany Critical Care 2012, 16(Suppl 1):P213 (doi: 10.1186/cc10820)

Introduction The T-Line TL-200 (Tensys Medical Inc., San Diego, CA, USA) is a noninvasive arterial blood pressure (BP) monitoring system allowing continuous beat-to-beat monitoring of systolic arterial pressure (SAP), mean arterial pressure (MAP), and diastolic arterial pressure (DAP). It provides a real-time BP waveform like that obtained using an arterial catheter for BP monitoring. The aim of this study was to compare BP measurements obtained using the T-Line TL-200 system with simultaneous invasive BP measurements using a femoral arterial catheter in unselected critically ill medical patients.

Methods In 28 patients treated in a medical ICU of a German university hospital, BP values were simultaneously obtained using a femoral arterial catheter and the T-Line TL-200 device. All recorded data were included in the final analysis. For comparison of BP measurements, Bland-Altman analysis accounting for repeated measurements was performed. 
Results A total of 76,826 pairs of BP measurements (each consisting of SAP, MAP, and DAP) were analyzed. For MAP, Bland-Altman analysis revealed a mean difference of $+0.47 \mathrm{mmHg}$ ( $95 \%$ limits of agreement: -16.53 to $+17.46 \mathrm{mmHg}$ ). For SAP and DAP, the bias and $95 \%$ limits of agreement were $-9.01 \mathrm{mmHg}(-37.47$ to $+19.45 \mathrm{mmHg})$ and +5.22 $\mathrm{mmHg}(-13.50$ to $+23.94 \mathrm{mmHg})$, respectively.

Conclusion The T-Line TL-200 system allows determination of MAP with a satisfactory agreement when compared to invasive assessment of MAP using a femoral arterial catheter in unselected critically ill medical patients. Higher mean differences and $95 \%$ limits of agreement for SAP and DAP measurements might be explainable by limited comparability of central (femoral) and peripheral (radial) SAP and DAP measurements.

P214

Reliability of radial arterial pressure monitoring after cardiac surgery

C Lavault ${ }^{1}$, MC Fevre ${ }^{2}$, A Hebrard ${ }^{2}$, M Durand ${ }^{2}$, F Grimbert ${ }^{1}$, Y Lavault' 1 , P Albaladejo ${ }^{2}$

'UJF-Grenoble1/CNRS/TIMC-IMAG UMR 5525 (Equipe PRETA), Grenoble, France; ${ }^{2} \mathrm{CH} \cup$ Grenoble, France

Critical Care 2012, 16(Suppl 1):P214 (doi: 10.1186/cc10821)

Introduction Invasive monitoring in critically ill patients allows a continuous measurement of arterial pressure, cardiac output, and the derivation of dynamic predictors of fluid responsiveness. However, the pressure signal may be altered by the dynamic characteristics of the fluid-filled tubing. The aim of the present study was to evaluate the reliability of radial artery blood pressure measurement and derived indexes during the early period after cardiac surgery.

Methods After IRB approval, 30 patients admitted to the ICU after elective cardiac surgery (CABG: 16 , valve surgery: 11 ; combined: 3 ) with a radial artery catheter were included. In the ICU, an independent continuous recording of arterial pressure during at least 18 hours was started via a double-head pressure transducer (Flotrac; Edwards Lifesciences, Irvine, CA, USA) for a retrospective analysis and three fast flushes were performed. First, the whole record was examined for episodes of overdamping (Ov) or attenuation (At). Ov was defined as a decrease in systolic (SAP), an increase diastolic (dAP), and an unchanged mean pressure (mAP). At was defined as a decrease in SAP, $\mathrm{dAP}$ and $\mathrm{mAP}$. Second, three periods of 10 minutes during the first hour were analysed assuming that the dynamic characteristics remained constant. This allowed the correction of the distorted raw signals and the study of the consequences of an underdamped signal on SAP, pulse pressure variation (PPV) and $\mathrm{dP} / \mathrm{dt}$ as an estimate of left ventricular contractility. A paired $t$ test was used for statistical comparison, $P<0.05$ was considered statistically significant.

Results Mean age was $69 \pm 13$ years, 14 patients received noradrenaline, eight patients dobutamine, and nine patients volume expansion. During the whole record, the number of episodes of Ov or At ranged from 0 to 15 with a duration of 0 to 6 hours: 17 patients had at least one episode of Ov and/or At tracing, 10 patients had at least two episodes, eight patients had at least five episodes. Seven episodes lasted more than 20 minutes and three more than 1 hour. During the first hour, SAP was overestimated by $5.0 \pm 1.4 \mathrm{mmHg}(P<0.0001)$ (range: 0.3 to 5.9 ) or by $4.3 \pm 0.9 \%$ (range: 0.4 to $15.9 \%$ ), raw PPV was $9.5 \pm 7.3$ versus $10.0 \pm 7.8$ for the corrected PPV (range from -2.6 to 4.3 ); raw $\mathrm{dP} / \mathrm{dt}$ was overestimated by $134 \pm 47 \mathrm{mmHg} / \mathrm{second}(P<0.0001)$ (range: -13 to $353)$ or by $24 \pm 6 \%$.

Conclusion These results showed that frequent artefacts and distortions induced by the fluid-filled tubing could modify the arterial waveform and could lead to inaccurate therapy [1]. More attention should be paid to the quality of the pressure signal.

Reference

1. Wax DB, et al.: Anesthesiology 2011, 115:973-978.
P215

Right/left ventricular area ratio does not correlate with right ventricular impedance

F Paalvast' ${ }^{1}$ D Reis Miranda ${ }^{1}$, M Knook² ${ }^{2}$ A Rossi ${ }^{1}$, J Van Bommel', D Gommers ${ }^{1}$

${ }^{1}$ Erasmus Medical Centre, Rotterdam, the Netherlands; ${ }^{2}$ Reinier de Graaf

Ziekenhuis, Delft, the Netherlands

Critical Care 2012, 16(Suppl 1):P215 (doi: 10.1186/cc10822)

Introduction The right ventricular/left ventricular (RV/LV) area ratio is often used to titrate airway pressure during mechanical ventilation [1]. This ratio has only been validated for detecting pulmonary embolism [2]. The purpose of this study was to evaluate the relationship between $\mathrm{RV} / \mathrm{LV}$ area ratio and RV impedance in a pig model with a closed pericardium.

Methods Eight anesthetized pigs were instrumented with closed pericardium for the measurement of arterial blood pressure, central venous pressure, $\mathrm{RV}$ and pulmonary pressure. On the main pulmonary artery, an ultrasonic flowprobe (MA14PAX; Transonic) was positioned to obtain pulmonary flow. Distally, a balloon occluder was positioned facilitating gradual constriction of the pulmonary artery. To obtain a stepwise pressure difference increment over the banding we gradually inflated the balloon occluder. Occluder resistance is computed as the systolic right ventricle pressure minus systolic pulmonary pressure divided by cardiac output times 79.9. An ECG-gated CT scan of the heart was performed, 10 minutes after each banding. The RV/LV area ratio was computed by reconstructing the $\mathrm{CT}$ images to a typical echocardiographic four-chamber view and dividing the RV area by the LV area. All measurements were performed in triplicate and averaged. As repeated measurements in eight independent animals were used, a significant relation was sought between RV/LV area ratio and occluder resistance with ANOVA. Correlation was obtained by a Spearson's correlation.

Results Cardiac output dropped from $4.4 \pm 0.8$ to $1.7 \pm 0.9 \mathrm{l} /$ minute. The relations between occluder resistance and RV/LV area ratio during diastole and during systole were not significant $(r=0.48$ resp. $r=0.54$ ). Conclusion The RV/LV area ratio does not correlate with right ventricular impedance in this closed pericardium model.

References

1. Bouferrache K: Acute respiratory distress syndrome, mechanical ventilation, and right ventricular function. Curr Opin Crit Care 2011, 17:30-35.

2. Mansencal N: Comparison of different echocardiographic indexes secondary to right ventricular obstruction in acute pulmonary embolism. Am J Cardiol 2003, 92:116-119.

P216

Techniques to measure cardiac output: minimally invasive method versus thermodilution

A Donati, S Tondi, A Carsetti, R Domizi, C Melia, D Kolgjini, C Munch,

C Scorcella, P Pelaia

Università Politecnica delle Marche, Ancona, Italy

Critical Care 2012, 16(Suppl 1):P216 (doi: 10.1186/cc10823)

Introduction Hemodynamic monitoring is important to manage critically ill patients. The thermodilution pulmonary catheter is considered the gold standard; however, it is invasive and associated with the onset of complications. Our study compared cardiac output (CO) obtained with the MostCare (COMC), which uses the pressure recording analytical method, to CO obtained with a Swan-Ganz (COSG) catheter in hemodynamically unstable patients.

Methods We conducted a prospective clinical study in our cardiosurgical ICU. Sixteen post-cardiosurgical adult patients were enrolled. They had a Swan-Ganz catheter and were mechanically ventilated. The SwanGanz catheter was connected to the monitor Vigilance Edwards ${ }^{\oplus}$, while the MostCare was connected to the patient's artery. For each patient three measurements of $\mathrm{CO}$ have been carried out and the mean was considered for statistical analysis. The correlation coefficient, BlandAltman test and percentage of error were measured.

Results The correlation coefficient between COSG and COMC was 0.824 ( 0.567 to $0.935,95 \% \mathrm{Cl} ; P<0.001)$ The Bland-Altman analysis showed 
a mean difference between the two methods (bias) of $0.22 \pm 0.55 \mathrm{I} /$ minute $/ \mathrm{m}^{2}$ with lower and upper $95 \%$ limits of confidence of -0.87 and $1.30 \mathrm{l} / \mathrm{minute} / \mathrm{m}^{2}$ respectively. The percentage of error was of $25 \%$.

Conclusion This study demonstrated a good correlation between the two methods. MostCare is resulted to be reliable and accurate even in hemodynamically unstable patients. It would be interesting to study the new device before and after having modified the therapy, such as fluid challenge or inotropic therapy or the use of vasopressors.

\section{References}

1. Zangrillo A, et al.: I Cardiothorac Vasc Anesth 2010, 24:265-269.

2. Scolletta S, et al:. Br J Anaesth 2005, 95:159-165.

3. Romano SM, et al: Crit Care Med 2002, 30:1834-1841.

P217

Effects of cardiac output levels on the measurement of transpulmonary thermodilution cardiac output in patients with acute lung injury

K Kao, H Hu, C Hung, C Chang, C Huang

Chang Gung Memorial Hospital, Kwei-Shan,Taoyuan, Taiwan

Critical Care 2012, 16(Suppl 1):P217 (doi: 10.1186/cc10824)

Introduction Transpulmonary thermodilution cardiac output (CO) correlates closely with pulmonary artery (PA) thermodilution CO. Levels of CO may contribute varying amounts of thermal indicator loss and recirculation during thermodilution CO measurement. This study aimed to investigate the effects of $\mathrm{CO}$ levels on the agreement between transpulmonary and PA thermodilution $\mathrm{CO}$ in acute lung injury (ALI) patients.

Methods Twenty-two ALI patients were prospectively enrolled. Paired bolus transpulmonary thermodilution cardiac index (BCltp) and continuous PA thermodilution cardiac index (CClpa) data were recorded at baseline and repeated immediately and at 2, 4, and 6 hours after volume expansion with a $500 \mathrm{ml}$ infusion of $10 \%$ pentastarch (HES 200/0.5).

Results One hundred and ten paired $\mathrm{Cl}$ measurements were recorded and divided into four quartiles from the lowest to the highest CClpa. The mean BCltp was higher than CClpa, and the Bland-Altman analysis revealed a bias of $0.57 \pm 0.75 \mathrm{l} / \mathrm{minute} / \mathrm{m}^{2}$. The limits of agreement (2SD) were +2.07 to $-0.93 \mathrm{l} /$ minute $/ \mathrm{m}^{2}$. BCltp correlated closely with CClpa $(R=0.887)$. CClpa negatively correlated with the difference between BCltp and CClpa $(R=-0.26)$. The bias of quartile 1 with the least CClpa was significantly greater than those of the three other quartiles.

Conclusion In ALI patients, transpulmonary thermodilution is a clinically acceptable and interchangeable alternative to PA thermodilution for $\mathrm{CO}$ measurement. Levels of $\mathrm{CO}$ weakly and negatively correlate with the difference between $\mathrm{BCltp}$ and CClpa. There is greater overestimation of BCItp over CClpa in low than in high CO states.

References

1. Harvey S, et al: Lancet 2005, 366:472-477.

2. Koo KKY, et al:: Crit Care Med 2011, 39:1613-1618.

\section{P218}

Comparison of bioimpedance and oesophageal Doppler cardiac output monitoring during abdominal aortic surgery

HK Jørgensen, J Bisgaard, T Gilsaa

Littlebaelt Hospital Kolding, Denmark

Critical Care 2012, 16(Suppl 1):P218 (doi: 10.1186/cc10825)

Introduction Abdominal aortic surgery is a high-risk procedure. Cardiac output monitoring allowing haemodynamic optimisation may reduce the complication rate. Minimally invasive, continuous techniques are preferable. Cardiac output using oesophageal Doppler has been validated in several studies, showing good agreement with the gold standard. The aim of this study was to assess the degree of correlation and agreement between cardiac output measured by oesophageal Doppler and bioimpedance obtained from an endotracheal tube. Methods Twelve patients scheduled for elective abdominal aortic surgery were included. Patients were intubated with an $\mathrm{ECOM}^{\mathrm{TM}}$ endotracheal tube (ConMed Corporation, NY, USA) which was connected to the $\mathrm{ECOM}^{\mathrm{TM}}$ monitoring system. An oesophageal Doppler

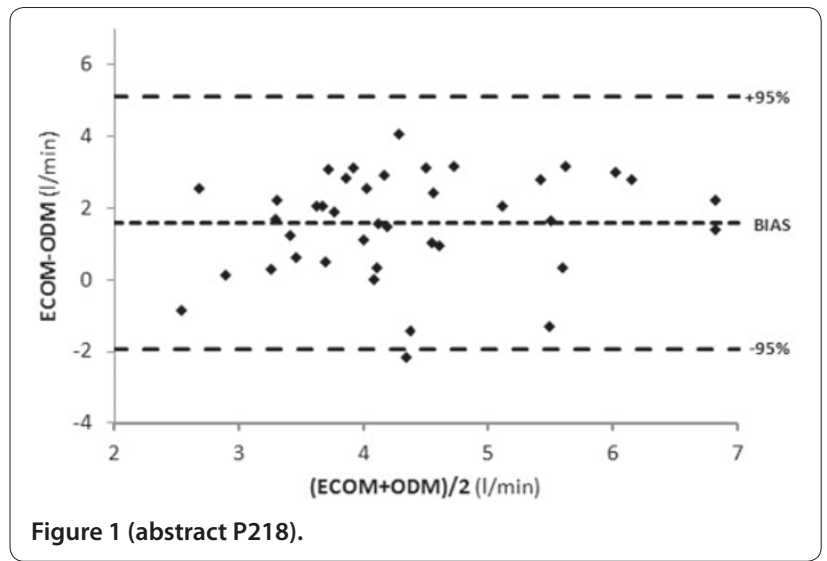

probe (Cardio ${ }^{\mathrm{TM}}$; Deltex Medical, UK) was inserted, connected to the CardioQ-ODM ${ }^{\mathrm{TM}}$ monitoring system and correct positioning verified. Simultaneous determination of cardiac output by ODM and ECOM ${ }^{\mathrm{TM}}$ was performed before and after cross-clamping of the aorta.

Results Cardiac output ranged from 1.4 to $13.1 \mathrm{I} /$ minute. Linear regression is represented by the equation $y=0.30 x+2.2$ and the correlation coefficient $r^{2}=0.15$. The bias was $+1.5 \mathrm{l} /$ minute with $95 \%$ limits of agreement between -2.1 and $5.1 \mathrm{l} /$ minute (Figure 1).

Conclusion Using the CardioQ ${ }^{\mathrm{TM}}$ as a reference, the $\mathrm{ECOM}^{\mathrm{TM}}$ system cannot be recommended as a clinical cardiac output measurement technique in abdominal aortic surgery, due to its poor correlation and wide limits of agreement.

\section{P219}

Cardiac output monitoring in cirrhotic patients: EV1000 versus pulmonary artery catheter - preliminary data

G Costa, T Cecconet, D Baron, G Serena, P Chiarandini, L Pompei, L Vetrugno, G Della Rocca

University of Udine, Italy

Critical Care 2012, 16(Suppl 1):P219 (doi: 10.1186/cc10826)

Introduction The EV1000 platform, a new calibrated device for intermittent and continuous cardiac output monitoring, has recently been introduced into clinical practice [1]. This study aims to assess the level of agreement between intermittent and continuous cardiac output obtained from VolumeView (ICOvv and CCOvv) connected to the EV1000 platform (Edwards Lifesciences, Irvine, CA, USA) and intermittent (ICOvig) and continuous cardiac output (CCOvig) obtained using an advanced pulmonary artery catheter (PAC) connected to the Vigilance System (Edwards Lifesciences) in cirrhotic patients undergoing liver transplantation.

Methods Seven consecutive patients (seven male, zero female), mean age $56.5( \pm 12)$ years, were enrolled into the study. ICO data were

Table 1 (abstract P219). Bias, 2SD and PE for all data pairs and for $\mathrm{CO}$ higher and lower than $8 \mathrm{l} /$ minute

\begin{tabular}{lccc}
\hline & Bias & 2SD & PE (\%) \\
\hline ICO & & & \\
Total & -0.89 & 3.6 & 49 \\
$<8$ & -1.37 & 2.4 & 40 \\
$>8$ & -0.05 & 4.8 & 52 \\
CCO & & & \\
Total & -0.83 & 4.88 & 63 \\
$<8$ & -1.8 & 2.4 & 37 \\
$>8$ & 2.07 & 5.64 & 47 \\
\hline
\end{tabular}


obtained from the two devices after ICU admission (T0) and after 12 (T12) and 24 hours (T24). CCOvig and CCOvv were recorded every hour from T0 up to 48 hours after ICU admission. Agreement and precision between $\mathrm{CO}$ values were evaluated with Bland-Altman analysis. The percentage error (PE) was calculated as $2 \mathrm{SD} /$ mean CO [2].

Results Twenty-one ICO data pairs were compared. Two patients were excluded from CCO data analysis for technical reasons. A total 240 CCO data pairs from five patients were analysed. Data yielded were analysed as total and for $\mathrm{CO}$ values lower and higher than $8 \mathrm{l} /$ minute (Table 1).

Conclusion These data, even if very preliminary, showed low agreement and high PE either for intermittent and continuous $\mathrm{CO}$ obtained from the VolumeView. However, for CO data lower than $8 \mathrm{I} /$ minute the PE was improved.

References

1. Bendjelid et al:: Crit Care 2010, 14:R209

2. Cecconi et al:: Crit Care 2009, 13:201

\section{P220}

Pulse contour cardiac output monitoring is less reliable in critically ill children

JC Verheul, A Nusmeier, J Lemson

Radboud University Nijmegen Medical Centre, Nijmegen, the Netherlands Critical Care 2012, 16(Suppl 1):P220 (doi: 10.1186/cc10827)

Introduction Intermittent cardiac output measurement using the transpulmonary thermodilution (TPTD) method is considered to be the gold standard in young children but a validated continuous cardiac output technique is not available in these patients. We compared the continuous pulse contour cardiac output (PCCO) measurements with the TPTD method in critically ill children.

Methods We compared PCCO, measured with the PiCCO device (Pulsion, Munich, Germany), with TPTD measurements $\left(\mathrm{CO}_{\mathrm{TPTD}}\right)$ using the same device in a general pediatric intensive care (PICU) population. Because PCCO is calibrated with each TPTD measurement $\left(\mathrm{CO}_{\text {TPTD }}\right)$ we compared the mean PCCO value just before a new TPTD measurement was done. We approved only $\mathrm{CO}_{\text {Tpro }}$ measurement consisting of three consecutive TPTD measurements and we checked the thermodilution curve for a temperature difference of at least $0.2^{\circ} \mathrm{C}$ and a normal appearance. Only the intervals between two approved series of TPTD measurements were analysed. We calculated the correlation coefficient and used the Bland-Altman method for analysis.

Results Sixty-one measurements in 10 children were included. Mean age was 24.5 (range 5 to 123) months; mean weight was 11.2 (range 3.8 to 18 ) $\mathrm{kg}$, mean heart rate was $131 /$ minute (range 87 to 193) and the mean blood pressure was 73 (range 49 to 96 ) $\mathrm{mmHg}$. The mean $\mathrm{CO}_{\text {трто }}$ was 2.60 (range 0.66 to 5.64 ) $\mathrm{l} /$ minute, mean cardiac index was 5.16 (range 2.76 to 10.83 ) $\mathrm{l} / \mathrm{minute} / \mathrm{m}^{2}$ and mean duration of the interval was

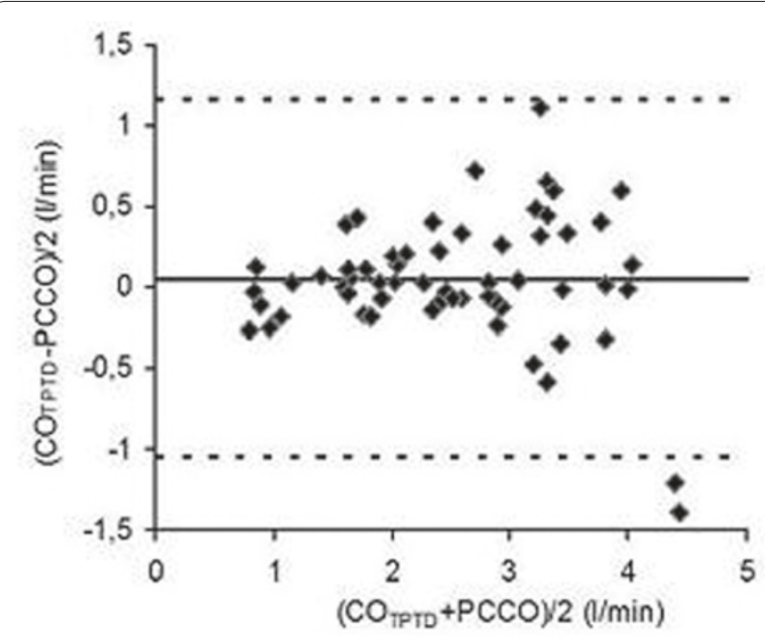

Figure 1 (abstract P220). Bland-Altman analysis of $\mathrm{CO}_{\text {TPTD }}$ and PCCO.
5 hours and 33 minutes (range 14 minutes to 15 hours). The correlation coefficient between the $\mathrm{CO}_{\text {TPTD }}$ and PCCO was $0.85(P<0.0001)$. The Bland-Altman analysis showed a mean bias of $0.06 \mathrm{l} /$ minute (limits of agreement (LoA) $\pm 2.22 \mathrm{l} /$ minute) (Figure 1). The percentage error was $43 \%$. The correlation coefficient between the recalibration interval and the bias between $\mathrm{CO}_{\text {тртD }}$ and PCCO was $-0.26(P=0.05)$. There was no correlation between CO ${ }_{\text {TPTD }}$ and PCCO $(r=0.09(P=0.57))$.

Conclusion The PCCO method cannot replace the transpulmonary thermodilution method in critically ill children.

\section{P221}

Impact of arterial catheter location on the accuracy of cardiac output provided by an endotracheal bioimpedance device F Gennart, S Beckers, C Verborgh, A De baerdemaeker, J Poelaert UZ Brussels, Belgium

Critical Care 2012, 16(Suppl 1):P221 (doi: 10.1186/cc10828)

Introduction With respect to a radial arterial pressure measurement, a more central achievement of this pressure should improve the reliability of endotracheal bioimpedance cardiac output (CO) monitoring. We therefore compared prospectively the impact on accuracy of this device, in comparison with thermodilution (TD) CO.
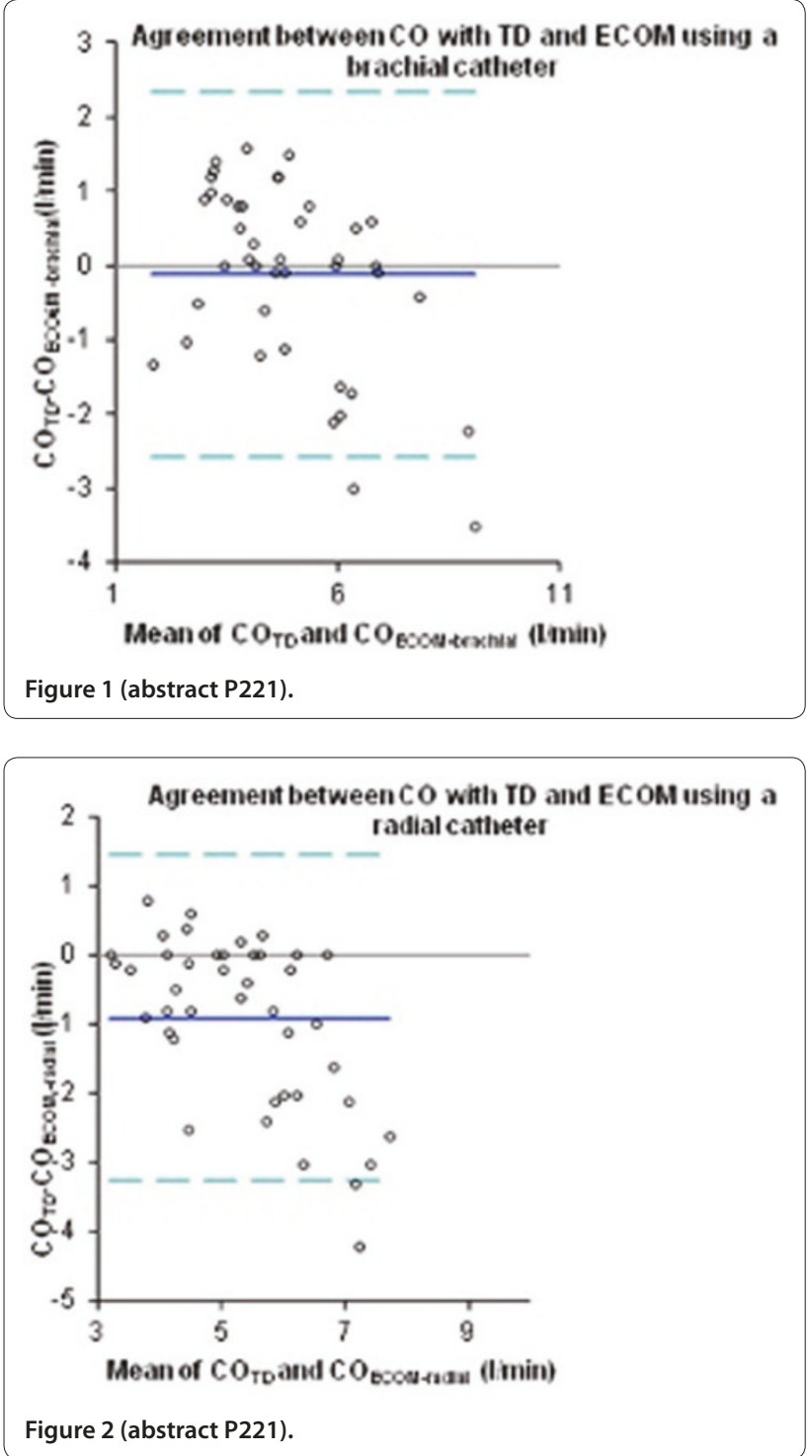
Methods Fourteen patients undergoing cardiac surgery with cardiac output monitoring by TD have been enrolled. A specially designed endotracheal tube (ECOM; ConMed) was placed in conjunction with a catheter located either in the brachial $(18 \mathrm{G})$ or in the radial $(20 \mathrm{G})$ artery in each group of seven patients. Six individual measurements have been carried out in each patient at fixed period, resulting in a total of 42 measurements for each subset. The mean CO by TD was compared with $\mathrm{CO}$ by ECOM for each operative period and assessed for agreement by linear regression, Bland-Altmann analysis and percentage error methods. The measurement error should not exceed $30 \%$ to be considered as valid, according to Critchley and colleagues. Results Mean patient age was 71 years (56 to 89) (13 male, one female). $\mathrm{R}^{2}$ values of $0.47(P<0.01)$ and $0.63(P<0.01)$ in the linear regressions and errors of $41 \%$ and $50 \%$ were found for the radial and brachial catheter data, respectively. See Figures 1 and 2.

Conclusion Accuracy was considerably improved using a brachial artery catheter. Nevertheless, measurement errors between TD and ECOM using either a radial or brachial catheter both exceed $30 \%$. Based on these results and under the current technical conditions, ECOM should not replace TD in CO monitoring for patients undergoing cardiac surgery.

References

1. Critchley et al.: J Clin Monit 1999, 15:85-91.

2. Bland et al.: Lancet 1986, i:307-310.

\section{P222}

Accuracy of the $\mathrm{PiCCO}_{2}$-derived pulse contour cardiac index (Clpc): development and validation of a calibration index in two independent collectives

W Huber, J Koenig, B Saugel, T Schuster, R Schmid, S Mair

Klinikum Rechts der Isar, Technical University of Munich, Germany

Critical Care 2012, 16(Suppl 1):P222 (doi: 10.1186/cc10829)

Introduction After calibration by thermodilution (TD), the PiCCO device is able to assess $\mathrm{CO}$ using pulse contour (PC) analysis. Despite an overall good correlation of Cltd and Clpc in several studies, the manufacturer suggests recalibration by TD after 8 hours. A calibration index derived from PC parameters indicating a certain probability of a relevant bias and triggering the next calibration would be of great practical use. Therefore, it was the aim of our study to prospectively evaluate predictors of the bias Cltd-Clpc exactly 1 hour, 2 hours, 4 hours, 6 hours and 8 hours after the last calibration.

Methods In 28 consecutive patients 56 datasets each including six TDs were recorded. In each triplicate TD measurement, Clpc was recorded immediately before recalibration by TD and compared to Cltd. Results derived from this evaluation collective were validated in an independent second collective of 48 patients with 67 datasets. SPSS 19 software was used.

Results The sample was 19 males, nine females, age $60.2 \pm 11.8$ years; APACHE II score $23.3 \pm 5.4$. The 280 pairs of Clpc and Cltd showed a significant correlation $(P<0.001 ; r=0.907)$. There was no difference between Clpc versus Cltd ( $4.15 \pm 1.46$ vs. $4.09 \pm 1.41 \mathrm{l} /$ minute*$^{*}$ sqm; $P=0.265$ ). Bland-Altman analysis demonstrated a mean bias of

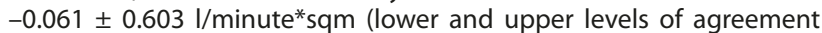
-1.24 and $1.12 \mathrm{l} /$ minute $^{*}$ sqm; percentage error of $28.7 \%$ ). In univariate analyses, the bias $\mathrm{Cltd2}$-Clpc was not correlated to the interval to the last calibration ( $P=0.705 ; r=-0.023)$, but it was correlated to Clpc immediately before recalibration $(r=-0.275 ; P<0.001)$ and to changes from Clpc versus the previous Cltd1 (Delta-Clpc-Cltd1; $r=-0.504$; $P<0.001)$. These findings were confirmed in the validation collective $(P<0.001)$. Multiple regression analysis demonstrated independent association of the bias to Delta-Clpc-Cltd1. This association was best described by bias Cltd2-Clpc $=-0.014-0.372 x+0.145 x^{2}-1.260 x^{3}$ with $x=$ Delta-Clpc-Cltd1. This formula as a potential calibration index provided ROC AUCs of 0.882 and $0.751(P<0.001)$ to predict a bias Cltd2-Clpc $>20 \%$ or $<-20 \%$ in the evaluation collective. This formula was confirmed with ROC AUCs of 0.809 and $0.714(P<0,001)$ to predict a bias Cltd2-Clpc $>20 \%$ or $<-20 \%$ in the independent validation collective.

Conclusion The difference $\mathrm{Clpc}-\mathrm{Cltd} 1$ is an independent predictor of the bias Cltd2-Clpc. A calibration index was developed and validated. It could be a useful decision support to initiate the next TD.
P223

Cardiac output monitoring using the LiDCOplus ${ }^{\mathrm{TM}}$ monitor in abdominal aortic surgery: changes in calibration factor in aortic aneurysm disease versus aortic occlusive disease

HK Jørgensen, J Bisgaard, T Gilsaa

Lillebaelt Hospital, Kolding, Denmark

Critical Care 2012, 16(Suppl 1):P223 (doi: 10.1186/cc10830)

Introduction Monitoring of cardiac output (with subsequent haemodynamic optimisation) may improve outcome after high-risk surgery. The pulmonary artery catheter is still considered the gold standard, but has potential serious complications. Much effort has been put into developing equally good, but less invasive techniques. One of these, the LiDCOplus ${ }^{T M}$ system, uses pulse power analysis to calculate cardiac output and is calibrated by a lithium indicator dilution technique. Since cardiac output is affected by the compliance of the aorta, the LiDCO calculates a calibration factor (CF) each time it is calibrated. The purpose of this study was to investigate whether insertion of aortic prosthetic material would affect aortic compliance and thereby the CF. It was hypothesised that the change in CF would be larger in patients with aortic occlusive disease (AOD) than in patients with aortic aneurysm disease (AAD), since previous studies have shown that these two groups differ considerably on both haemodynamic capacity and their response to aortic cross-clamping [1].

Methods A prospective study in 51 patients undergoing open elective abdominal aortic surgery - 30 patients with AAD and 21 with AOD. CF values were obtained at baseline, before induction of anaesthesia (T1) and 30 minutes after reperfusion (T2).

Results AAD patients were older ( 70 vs. 65 years, $P<0.05$ ), predominantly males $(80 \%$ vs. $47 \%)$, weighed more $(80 \mathrm{~kg}$ vs. $73 \mathrm{~kg}, P<0.1)$ and preoperative cardiac co-morbidity was more prevalent ( $43 \%$ vs. $14 \%$ ). No difference was found in the use of epidural analgesia, vasopressors, or inotropes between the groups. At T1, CF was significantly higher for $\mathrm{AAD}=0.83$ versus $\mathrm{AOD}=0.68(P=0.01)$. After reperfusion, $\mathrm{T} 2$, there was no significant difference in $C F, A A D=0.86$ versus $A O D=0.81(P=0.53)$. The percentage change in $\mathrm{CF}$ from $\mathrm{T} 1$ to $\mathrm{T} 2$ was significantly larger in AOD than in AAD (20\% vs. $1.3 \%)(P<0.05)$.

Conclusion Operative insertion of an abdominal aortic prosthesis significantly affects the calibration factor in patients with AOD, indicating an increase in aortic compliance and the need for recalibration of the LiDCOplus ${ }^{\mathrm{TM}}$. No significant change was seen in patients with aortic aneurysm disease.

Reference

1. Shteinberg D, et al:. Eur J Vasc Endovasc Surg 2000, 20:462-465.

\section{P224}

Haemodynamic changes during the peri-extubation period using bioreactance flow monitoring

J Thirsk, D Magimairaj, A Douiri, D Hadfield, P Hopkins

King's Health Partners, London, UK

Critical Care 2012, 16(Suppl 1):P224 (doi: 10.1186/cc10831)

Introduction Here we present a prospective, observational study examining the effect of extubation on cardiac index, measured by bioreactance (Nicom Cheetah), in critically ill patients with or without a history of left ventricular impairment [1]. A number of simple interventions are known to improve the process of weaning patients from mechanical ventilation. Despite this progress, the pathophysiology underlying failure to wean remains incompletely understood. In particular, the role of cardiac ventricular dysfunction may be underestimated [2].

Methods Cardiac index was measured by bioreactance monitoring at 30-second to 60-second intervals for 1 hour pre and 1 hour post extubation. Individual data were presented by box plot, showing median and interquartile ranges (Figure 1). Combined results from multiple patients in each test group were analysed by covariance (Stata version 11.2)

Results Group A $(n=5)$ had impaired left ventricular systolic function, documented on formal transthoracic echo, of which three had ejection fractions $<25 \%$. One patient in this group failed extubation due to cardiogenic pulmonary oedema. Group B $(n=6)$ had normal systolic function. Figure 1 shows representative absolute data obtained from 


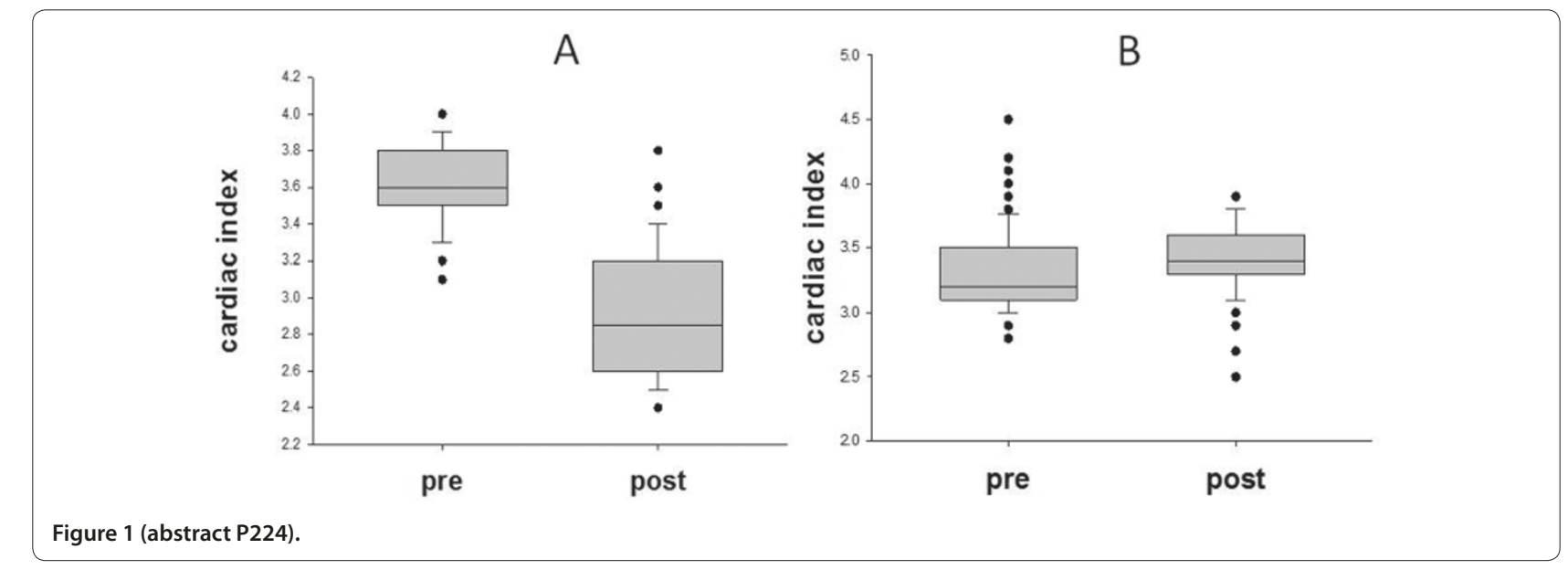

a patient in each group. There was a statistical difference between the two groups $(P=0.02)$. In the impaired LV group, the cardiac index fell from $3.2 \mathrm{l} /$ minute $/ \mathrm{m}^{2}( \pm 0.5)$ to $2.9 \mathrm{l} / \mathrm{minute} / \mathrm{m}^{2}( \pm 2.5)$.

Conclusion In this small observational study we demonstrated a consistent fall in cardiac index post extubation in patients with known cardiac ventricular dysfunction when compared with patients with normal hearts. These data suggest that bioreactance monitoring may be valuable during spontaneous breathing trials and extubation. References

1. Benomar B, Ouattara A, Estagnasie P, Brusset A, Squara P: Fluid responsiveness predicted by non-invasive bioreactance-based passive leg raise test. Intensive care Med 2010, 36:1875-1881.

2. Papanikolaou J, Makris D, Saranteas T, et al: New insights into weaning from mechanical ventilation: left ventricular diastolic dysfunction is a key player. Intensive Care Med 2011, 37:1976-1985.

\section{P225}

Left ventricular stroke volume measurement by impedance cardiography correlates with echocardiography in neonates ME Blohm', J Hartwich', D Obrecht', G Müller', JWeil2, D Singer' 'University Medical Center Hamburg - Eppendorf, Hamburg, Germany; 2University Center for Cardiology and Cardiothoracic Surgery, Hamburg, Germany

Critical Care 2012, 16(Suppl 1):P225 (doi: 10.1186/cc10832)

Introduction The aim of this study was to validate impedance cardiography (electrical velocimetry (EV)) as a continuous noninvasive cardiac output monitoring in neonates and infants. As the reference method, discontinuous transthoracic echocardiography (TTE) was used.

Methods In a prospective single-center observational study, simultaneous left ventricular stroke volume (LVSV) measurements by EV (using an Aesculon ${ }^{\circledast}$ Monitor) and by TTE were compared. LVSV measurement by TTE was based on the aortic valve velocity time integral multiplied by the area of the aortic valve outflow tract. A total of 102 healthy neonates with normal biventricular cardiac morphology (including PDA or patent foramen ovale) were included - further patient details: 43 female, 59 male, median weight $3.32 \mathrm{~kg}$, median length $51 \mathrm{~cm}$, median age 49.24 hours, mean heart rate $133 \pm 22 /$ minute. In total 328 simultaneous LVSV measurements in triplicate irrespective of respiratory cycle were analyzed.

Results Significant correlations $(P<0.05)$ were noted between EV-LVSV and body weight, TTE-LVSV and body weight, EV-LVSV and age, TTE-LVSV and age. A significant inverse correlation was seen between EV-LVSV and heart rate, and TTE-LVSV and heart rate. No significant correlation was found for EV-LVSV and age (if age $\leq 120$ hours). No significant effect was seen for a small persistent foramen ovale $(n=66)$ and a small PDA $(n=26)$ on EV-LVSV and TTE-LVSV in the observed cohort. Bland-Altman analysis of logarithmic data showed a bias of the EV-LVSV measurements in comparison to the TTE-LVSV measurements with smaller LVSVs resulting in lower EV than TTE measurements and higher LVSVs resulting in higher EV than TTE measurements. The bias defined by the difference of the means of the two methods was $9.65 \%$, with a mean percentage error of the individual measurements of $55 \%$. Based on the Bland-Altman analysis, a deduced approximated correction factor between TTE-LVSV and EV-LVSV was TTE-LVSV = EVLVSV $^{0.539} \times 10^{0.335} \approx(\sqrt{ }$ EV - LVSV $) \times 2.2$.

Conclusion Correlation between EV and TTE in LVSV measurement was significant. Bland-Altman analysis showed that - despite a large mean error of the individual measurements of $55 \%$ - the bias between the means of the two methods was only $9.65 \%$. A correction factor between TTE and EV could be deduced.

P226

Validation of less-invasive hemodynamic monitoring with Pulsioflex in critically ill patients

M Peetermans, WVerlinden, J Jacobs, A Verrijcken, S Pilate,

N Van Regenmortel, I De laet, K Schoonheydt, H Dits, ML Malbrain

ZNA Stuivenberg, Antwerp, Belgium

Critical Care 2012, 16(Suppl 1):P226 (doi: 10.1186/cc10833)

Introduction Thermodilution (TD) is a gold standard for cardiac index (CI) measurement. The aim of this study is to compare intermittent bolus TDCI with intermittent automatic calibration $\mathrm{Cl}$ (AutoCl) and continuous $\mathrm{Cl}(\mathrm{CCl})$ obtained by pulse contour analysis with $\mathrm{PiCCO}_{2}$ (PiCCl) and Pulsioflex (PuCCI).

Methods A prospective study in 20 patients (all mechanically ventilated, 14 male). Age 54.4 \pm 16.7, BMI 28.1 \pm 7.3 , SAPS $\|$ 52.9 \pm 13.4 , APACHE $\|$ score $26.7 \pm 7.8$ and SOFA score $10 \pm 3$. All patients underwent PiCCO monitoring via a femoral line whilst the radial line was kept in place during four 8-hour time periods (in the first two periods the Pulsioflex was connected to the radial line, in the last two it was connected to the femoral line). In the first and third 8-hour periods the Pulsioflex was calibrated with the TDCl obtained at baseline, for the second and fourth 8-hour periods the Pulsioflex was calibrated with the AutoCl value. Simultaneous $\mathrm{PiCCl}$ and $\mathrm{PuCCl}$ measurements were obtained every 2 hours while simultaneous TDCI and AutoCl were obtained every 8 hours. The $\mathrm{PiCCl}$ and $\mathrm{PuCCl}$ values were recorded within 5 minutes before TDCI was determined. We also looked at the effects of 22 interventions: passive leg raise $(n=6)$, fluid bolus $(n=5)$, change in vasopressor $(n=9)$ or dobutamine $(n=1)$, increase in sedation $(n=1)$. Statistical analysis was performed using Pearson correlation and Bland-Altman analysis.

Results In total, 305 paired PiCCI-PuCCI and 128 paired AutoCl-TDCI values were obtained. TDCl values ranged from 1.5 to $6.7 \mathrm{l} / \mathrm{minute} / \mathrm{m}^{2}$ (mean $3.9 \pm 1$ ), AutoCl from 2.4 to $6.5(3.8 \pm 0.8)$, PiCCI from 1.5 to 7.1 $(3.8 \pm 1.2)$ and $\mathrm{PuCCl}$ from 2 to $7.6(3.8 \pm 1)$. The Pearson correlation coefficient comparing all and mean $\mathrm{PuCCl}$ and $\mathrm{PiCCl}$ values had an $R^{2}$ of 0.77 and 0.86 respectively; for AutoCl and TDCl, $R^{2}$ was 0.76 . The above $R^{2}$ values were $0.73,0.84$ and 0.71 respectively when the Pulsioflex was connected to a radial line. Changes in AutoCl correlated well with changes in TDCI $\left(R^{2}=0.68\right)$, as did changes in PuCCl versus changes 
in PiCCI $\left(R^{2}=0.53\right)$. PPV obtained from Pulsioflex and PiCCO correlated better than SVV $\left(R^{2}=0.86\right.$ vs. 0.62). Changes in PiCCl and PuCCl induced by an intervention correlated well with each other $\left(R^{2}=0.94\right)$. BlandAltman analysis comparing AutoCl with TDCI revealed a mean bias $\pm 2 \mathrm{SD}$ (LA) of $0.05 \pm 0.94 \mathrm{l} / \mathrm{minute} / \mathrm{m}^{2}$ (with $27.3 \%$ error) while analysis of PuCCl versus PiCCl showed a bias $\pm \mathrm{LA}$ of $0.01 \pm 1.12$ ( $29.1 \%$ error). Conclusion Although TDCI remains a gold standard, the preliminary results of an ongoing prospective study indicate that in unstable critically ill patients $\mathrm{Cl}$ can be reliably monitored with Pulsioflex technology. Moreover, the Pulsioflex was also able to keep track of changes in $\mathrm{Cl}$.

\section{P227}

A preliminary study on the use of noninvasive hemodynamic monitoring with the Nexfin monitor in critically ill patients

M Peetermans, W Verlinden, J Jacobs, A Verrijcken, S Pilate, N Van Regenmortel, I De laet, K Schoonheydt, H Dits, ML Malbrain ZNA Stuivenberg, Antwerp, Belgium

Critical Care 2012, 16(Suppl 1):P227 (doi: 10.1186/cc10834)

Introduction Noninvasive hemodynamic monitoring may become a new tool in the ICU armamentarium. The Nexfin monitor (BMEYE, Amsterdam, the Netherlands) enables continuous noninvasive analysis of the finger blood pressure waveform using an inflatable finger cuff, a technology based on the volume-clamp principle of Penaz in combination with the physical criteria of Wesseling. The aim of the present study was to validate the Nexfin in a mixed population of medical ICU patients and to look for a pattern recognition that may be linked with outcome.

Methods A prospective study in 40 patients admitted to the medical ICU (17 patients mechanically ventilated, M/F ratio $1 / 1)$. Age $63.5 \pm 16.7$, BMI $26.4 \pm 5.4$, APACHE II score $20.8 \pm 9.5$, SAPS II $45.9 \pm 18.9$, SOFA score $7.2 \pm 4.2$. For all patients, simultaneous recording of arterial pressure by radial line $(n=46)$, by PiCCO monitor $(n=15)$ or by NIBP measurement with arm cuff $(n=17)$ was compared with noninvasive hemodynamic parameters obtained with the Nexfin monitor. Statistical analysis was performed with Student's $t$ test, Pearson correlation and Bland-Altman analysis.

Results A total of 69 measurements in 40 patients were performed. In three patients measurement with the Nexfin was not possible. For CO (26 paired measurements), values were $6.4 \pm 2.1 \mathrm{l} /$ minute (range 3.3 to 12). The Pearson correlation coefficient comparing Nexfin-CO with reference $\mathrm{CO}$ showed a good correlation $\left(R^{2}=0.5\right)$. Bland-Altman analysis comparing both $\mathrm{CO}$ techniques revealed a mean bias $\pm 2 \mathrm{SD}$ (LA) of $0.7 \pm 3.9 \mathrm{l} /$ minute $(58.3 \%$ error). The MAP was $84.6 \pm 17.7 \mathrm{mmHg}$ ( 57.5 to 131.5 ) and values obtained with the Nexfin correlated well with the reference method (PiCCO in eight; radial line in 43) with an $R^{2}$ of 0.75 . Bland-Altman analysis comparing both MAP techniques revealed a mean bias \pm 2 SD (LA) of $0.2 \pm 19.7 \mathrm{mmHg}$ (23.3\% error). However, Nexfin-MAP did not correlate well with $\operatorname{NIBP}\left(R^{2}=0.1\right)$. The nine patients that died in the ICU had higher APACHE II $(P=0.07)$, SAPS II $(P=0.07)$ and SOFA $(P=0.01)$ scores and significantly lower MAP $(P=0.028)$ and lower dp/dtmax $(P=0.029)$, a marker for contractility. There were no outcome differences with regard to subgroup analysis in patients with either low or high CO or SVR.

Conclusion The preliminary results of this ongoing prospective trial indicate that in unstable critically ill patients $\mathrm{CO}$ and MAP can be monitored noninvasively with the Nexfin. The exact patient population for this technology has yet to be defined and more patients are probably needed for pattern recognition, although the results indicate that low MAP and dp/dtmax are associated with poor outcome.

P228

Computer-based monitoring of global cardiovascular dynamics during acute pulmonary embolism and septic shock in swine JA Revie1, DJ Stevenson', JG Chase', BC Lambermont ${ }^{2}$, A Ghuysen², P Kolh², GM Shaw ${ }^{3}$, T Desaive ${ }^{2}$

'University of Canterbury, Christchurch, New Zealand; '2University of Liege,

Belgium; ${ }^{3}$ Christchurch Hospital, Christchurch, New Zealand

Critical Care 2012, 16(Suppl 1):P228 (doi: 10.1186/cc10835)

Introduction Acute pulmonary embolism (APE) and septic shock (SS) are highly prevalent dysfunctions in the ICU due to the immunocompromised and immobile state of ICU patients. This research retrospectively tests the ability of a computer-based method to monitor acute hemodynamic changes in pigs. If proven, this method could assist ICU staff by providing a clear physiological, patient-specific picture of cardiovascular status for decision support.

Methods In two porcine studies, APE $(n=5)$ and SS $(n=4)$ were induced using autologous blood clots and endotoxin infusions. Hemodynamic measurements were recorded every 30 minutes for 4 hours $(n=80)$. Subject-specific cardiovascular models were identified from typical ICU measurements obtained from each of these datasets, including aortic and pulmonary artery pressure, stroke volume, heart rate, global enddiastolic volume, and mitral and tricuspid valve closure times. Model outputs and identified parameters were compared to experimentally derived indices, measurements not used in the identification process, and known trends to validate the accuracy of the models.

Results The models accurately predicted maximum ventricular pressures and volumes, not used in the identification process, to mean percentage errors of $7.1 \%$ and $6.7 \%$ (less than measurement error $\sim 10 \%)$. Mean modelled pulmonary vascular resistances (PVR) compared well ( $R^{2}=0.81$ for APE and $R^{2}=0.95$ for SS) to experimentally derived values. Importantly, in the APE study a $91 \%$ rise from baseline in the mean PVR was identified with an $89 \%$ increase seen in the SS pigs. Contrasting behaviour between the two studies was observed for systemic vascular resistance (SVR) with a maximum drop of $40 \%$ from baseline recorded at T120 for SS, indicating a loss of vascular tone as expected, where at the same time in the APE study the average SVR had increased by $13 \%$. An increase in the ratio of right to left ventricle end volume was identified in all nine pigs, indicating right ventricular distension and a leftward shift in the intraventricular septum.

Conclusion These results indicate that subject-specific cardiovascular models are capable of tracking well-known global hemodynamic trends of two common forms of shock in the ICU. The method shows potential and could provide a means for continuous cardiovascular monitoring at little extra cost as no extra measurements or expensive devices are required.

\section{P229}

Homeodynamic complexity: multifractal analysis of physiological instability

A Ercole, SM Bishop, SI Yarham, VU Navapurkar, DK Menon University of Cambridge, UK

Critical Care 2012, 16(Suppl 1):P229 (doi: 10.1186/cc10836)

Introduction Physiological instability is a common clinical problem in the critically ill. Physiological adaptation can be regarded as a dynamic process, with stability being conferred by a number of apparently complex, fluctuating homeokinetic processes [1]. Many natural systems are nonlinear, and seemingly random fluctuations may result as a consequence of their underlying dynamics. Fractal geometry offers a method to characterize the underlying nonlinear state, providing a technique for monitoring complex physiology in real time, which may be of clinical importance.

Methods We employ the wavelet modulus maxima technique to characterize the multifractal properties of physiological time series such as heart rate (HR) and mean arterial pressure (MAP) under conditions of clinical physiological instability. We calculated point estimates for the dominant Hölder exponent $\left(\mathrm{h}_{\mathrm{m}}\right)$ and multifractal spectrum width-athalf-height (WHH). We investigated how these parameters changed with pharmacological interventions such as vasoconstriction.

Results Hypotensive patients showed lower values of $h_{m}$ for MAP, consistent with a more highly fluctuating, antipersistent and complex behavior. Blood pressure support with pharmacological vasoconstriction led to a transient increase in $h_{m}$ for MAP (Figure 1) revealing the appearance of longer-range correlations, but did not affect $h_{m}$ as estimated for HR. On the other hand, supporting the heart rate with atropine had no effect on $h_{m}$ for MAP, but did tend to increase $\mathrm{h}_{\mathrm{m}}$ for HR.

Conclusion We demonstrate increasing signal complexity under physiological challenge consistent with the activation of homeokinetic processes. Differential fractal behavior for HR and MAP suggests that the homeokinetic systems are recruited in a targeted way depending 

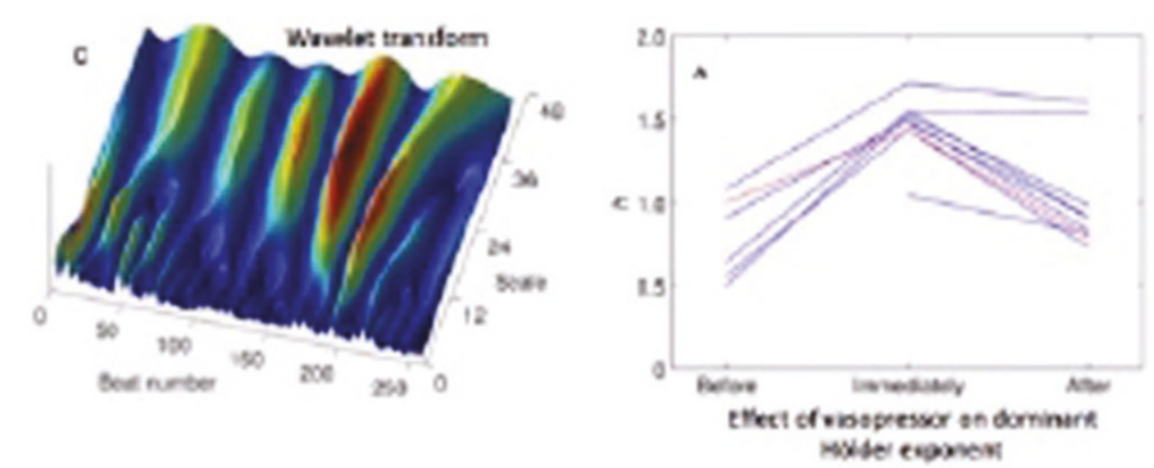

Figure 1 (abstract P229).

on the physiological challenge. Pharmacological restoration of homeostasis leads to system decomplexification suggesting that homeokinetic mechanisms are derecruited as physiology is restored. We suggest fractal geometry provides a method for characterizing physiological instability and measuring the homeokinetic stress response during physiological challenges.

Reference

1. Goldberger AL, Amaral LAN, Hausdorff JM, Ivanov PC, Peng C-K, Stanley HE: Proc Natl Acad Sci U S A 2002, 99:2466-2472.

P230

Accuracy of conventional urinary output monitoring in the ICU

E Bouwhuijsen, A Oude Lansink, MW Nijsten, W Dieperink

University of Groningen, University Medical Center Groningen, the Netherlands

Critical Care 2012, 16(Suppl 1):P230 (doi: 10.1186/cc10837)

Introduction In patients who are treated in the ICU an accurate fluid balance is an important tool to assess their hydration status. In most ICUs, intake of fluid is monitored precisely by sophisticated volumetric infusion and feeding pumps. In contrast to fluid intake, fluid output - especially urine as its most important component - is usually monitored visually by hourly assessment of the amount of fluid lost and urine production. Thus measurement of urinary output is a repetitive procedure 24 times a day which requires handling of the urinary collection system, visual assessment and manual data recording, actions that are easily affected by human errors.

Methods In a bench test we investigated the accuracy and precision of conventional urinary output monitoring, by visual hourly readings and manual data recording, as performed by experienced intensive care nurses with the purpose to provide insight into potential errors in urinary output measurement as well as identifying systematic sources of error. Two different types of ordinary 24-hour urine meters were used. The meters were filled with a predetermined amount (gold standard) of yellow lemonade. Both urine meters were filled with variable but identical volumes for a range of 8 to $325 \mathrm{ml}$, to a total amount of $3,600 \mathrm{ml}$. Hereafter the nursing staff manually recorded the reading of 48 prefilled urine meters.

Results Forty-eight nurses performed 2,285 urine volume measurements in two different types of ordinary urine meters (Bard Urine meter drainage bag; Bard Medical, Covington, Georgia, USA and Rüsh U-bag; Jiangsu, People's Republic of China). The mean measured output for the Bard urine meter was $3,688 \mathrm{ml}, \mathrm{SD} \pm 45$ and for the Rüsh urine meter $3,692 \mathrm{ml}, \mathrm{SD} \pm 55$. The limits of agreement between both types of urine meters were $2.4 \%$ to $2.6 \%$ respectively. Compared with the gold standard, analysis demonstrated deviations of $2.6 \%$ for both types of urine meters.

Conclusion Conventional urinary output measurement with ordinary urine meters constitutes a simple and accurate method for measuring urine volume in the ICU.
P231

Changes in B-type natriuretic peptide and related hemodynamic parameters following a fluid challenge in patients with severe sepsis or septic shock

S Omar, LR Mathivha, A Ali

Wits University, Johannesburg, South Africa

Critical Care 2012, 16(Suppl 1):P231 (doi: 10.1186/cc10838)

Introduction The aim of the study is to describe the hemodynamic changes and relate them to the changes in B-type natriuretic peptide (BNP) following fluid challenge in patients with severe sepsis and septic shock.

Methods This prospective observational study enrolled 30 patients with severe sepsis or septic shock who required a fluid challenge within 48 hours of admission to the ICU. All patients had a basic cardiac echocardiogram (echo) performed, blood for BNP collected and baseline hemodynamic measurements recorded. A $500 \mathrm{ml}$ colloid challenge was administered within 30 minutes. The echo and hemodynamic measurements were repeated at this point. One hour after the fluid challenge the BNP test was repeated.

Results One patient was excluded due to missing data. There were significant increases in mean arterial pressure (MAP), left ventricular dimensions at the end of diastole and systole, stroke volume (SV), cardiac output (CO) and BNP after the fluid challenge, while the heart rate decreased. Impaired cardiac contractility was defined as an ejection fraction (EF) $<50 \%$. The left ventricular end-systolic dimension (LVESd) before $(4 \mathrm{~cm}$ vs. $2.9 \mathrm{~cm})$ and after the fluid challenge $(4.2 \mathrm{~cm}$ vs. $3.29 \mathrm{~cm})$ was significantly greater (statistically and beyond reference intervals) in the $\mathrm{EF}<50 \%$ group compared to the $\mathrm{EF}>50 \%$ group. In the group with $\mathrm{EF}<50$, the median LVEDd 2 and LVESd 2 post fluid challenge increased to values of $5.72 \mathrm{~cm}$ and $4.28 \mathrm{~cm}$ respectively (above the reference thresholds). For the group with $E F>50$, the median post-challenge LVEDd2 and LVESd2 increased significantly $(P=0.01)$ to $5.38 \mathrm{~cm}$ and $3.39 \mathrm{~cm}$ but within the reference thresholds. The BNP increased by $53.6 \%$ in the $\mathrm{EF}<50$ group in contrast to a decrease by $12.7 \%$ in the $\mathrm{EF}>50$ group. The median $\mathrm{EF}$ in the $\mathrm{EF}<50$ and $\mathrm{EF}>50$ groups were significantly different (0.44 vs. 0.66 respectively). Multiple regression analysis found LV dimension at end diastole at baseline was one of four independent predictors of an increase in \%BNP.

Conclusion A significant increase in \%BNP after a fluid challenge (irrespective of initial value) may indicate that cardiac contractility is impaired and the LV dilated, indicating a strategy away from fluid resuscitation and towards inotrope use.

\section{Reference}

1. Pirracchio R, et al:: Impaired plasma BNP clearance in human septic shock. Cri Care Med 2008, 36:2542-2546. 
P232

The Brody effect to detect hypovolemia in clinical practice

R Giraud, N Siegenthaler, DR Morel, K Bendjelid

Hôpitaux Universitaires de Genève, Switzerland

Critical Care 2012, 16(Suppl 1):P232 (doi: 10.1186/cc10839)

Introduction The electrocardiogram (EKG) is a common monitoring method in intensive care medicine. Several studies suggest that changes in EKG morphology may reflect changes in volume status. The Brody effect, a theoretical analysis of left ventricular chamber size influence on QRS-wave amplitude, is the key element of this phenomenon. It is characterized by an increase in QRS-wave amplitude induced by an increase in ventricular preload [1]. This study investigated the influence of changes in intravascular volume status on respiratory variations of QRS-wave amplitude (EKG) compared with respiratory pulse pressure variations (PP).

Methods In 17 pigs, EKG and arterial pressure were recorded. QRSwave amplitude was measured from the Biopac recording ensuring that in all animals EKG electrodes were always at the same location. Maximal QRS amplitude (EKGmax) and minimal QRS amplitude (EKGmin) were determined over one respiratory cycle. EKG was calculated as 100x((EKGmax - EKGmin) / (EKGmax + EKGmin) / 2). EKG and PP were simultaneously recorded. Measurements were performed during normovolaemic conditions, after haemorrhage and following retransfusion with constant tidal volume $(10 \mathrm{ml} / \mathrm{kg})$ and respiration rate $(15 /$ minute).

Results At baseline, PP and EKG were both $<12 \%$. PP were significantly correlated with EKG $\left(r^{2}=0.89, P<0.001\right)$. Volume loss induced by haemorrhage increased significantly PP and EKG. Moreover, during this state, PP were significantly correlated with EKG $\left(r^{2}=0.86, P<0.001\right)$. Retransfusion significantly decreased both PP and EKG, and PP were significantly correlated with EKG $\left(r^{2}=0.90, P<0.001\right)$.

Conclusion Available correlations between PP and EKG at each time of the study were observed, meaning that EKG is a reliable parameter to estimate the changes in intravascular volume status and provide experimental confirmation of the Brody effect [2].

\section{References}

1. Cannesson M, Keller G, Desebbe O, Lehot JJ: Relations between respiratory changes in R-wave amplitude and arterial pulse pressure in mechanically ventilated patients. J Clin Monit Comput 2010, 24:203-207.

2. Brody DA: A theoretical analysis of intracavitary blood mass influence on the heart-lead relationship. Circ Res 1956, 4:731-738.

\section{P233}

Stroke volume variation guided fluid therapy in septic shock with ARDS

S Jog, D Patel, M Patel, S Sable

Deenanath Mangeshkar Hospital and Research Centre, Pune, India

Critical Care 2012, 16(Suppl 1):P233 (doi: 10.1186/cc10840)

Introduction Optimal fluid resuscitation guided by central venous pressure (CVP) in patients having septic shock with ARDS is a perplexed issue having risk of underfilling and worsening of shock versus fluid overload leading to pulmonary edema. Whether stroke volume variation (SVV) (Flotrac-Vigileo system) guided fluid resuscitation has an impact on improvement of shock, oxygenation and mortality were tested in this single-center prospective study $[1,2]$.

Methods Inclusion criteria were: (1) septic shock patients with dose of norepinephrine $\geq 0.1 \mu \mathrm{g} / \mathrm{kg} /$ minute or dopamine $\geq 10 \mu \mathrm{g} / \mathrm{kg} /$ minute; (2) CVP $\geq 12 \mathrm{mmHg}$; (3) $\mathrm{PO}_{2} / \mathrm{FiO}_{2}$ ratio $\leq 200$ with ARDSnet protocol ventilation under deep sedation. Exclusion criteria were atrial/ventricular arrhythmias, spontaneous triggering of inspiration, established renal failure needing continuous renal replacement therapy (CRRT). During the 24-hour study period, SVV was continuously monitored with the third-generation Flotrac-Vigileo system (version 3.02). Intravenous fluids were given in the boluses of 250 to $500 \mathrm{~cm}^{3}$ to keep SVV $<12 \%$ throughout the study period. Vasopressor infusion was titrated to keep MAP $>70 \mathrm{mmHg}$.

Results Thirty-seven patients with severe sepsis-induced multiorgan dysfunction syndrome with average APACHE II score of 24.6 and PEEP of $8.2 \mathrm{~cm}$ were enrolled. SVV guided fluids received during the 24-hour
Table 1 (abstract P233). Hemodynamic variables at 0 and 24 hours

\begin{tabular}{lccc}
\hline Variable & 0 hours & 24 hours & P value \\
\hline CVP & 15.51 & 16.2 & $>0.1$ \\
MAP & 73.6 & 76.4 & $>0.1$ \\
SW\% & 13.61 & 10.8 & $>0.1$ \\
Arterial lactate & $3.9 \pm 2.6$ & $2.3 \pm 1.5$ & $<0.001$ \\
$\mathrm{PO}_{2} / \mathrm{FiO}_{2}$ & $120.6 \pm 42$ & $193.4 \pm 76$ & $<0.001$ \\
\hline
\end{tabular}

study period were $5.1 \pm 2.6$ I. Arterial lactates reduced significantly without worsening of hypoxia. The $\mathrm{PO}_{2} / \mathrm{FiO}_{2}$ ratio increased significantly at 24 hours. Twenty-two out of 37 survived (59.45\%) until hospital discharge. See Table 1.

Conclusion SVV guided fluid therapy in septic shock with ARDS may improve shock by optimizing preload in a targeted way without worsening oxygenation.

References

1. Intensive Care Med 2011, 37:233-240.

2. Crit Care Med 2088, 36:2810-2816.

P234

Assessment fluid responsiveness in septic shock patients: a comparison of automated pulse pressure variation and manually calculated pulse pressure variation

S Panyawatanaporn, B Khwannimit

Prince of Songkla University, Hat Yai, Thailand

Critical Care 2012, 16(Suppl 1):P234 (doi: 10.1186/cc10841)

Introduction Pulse pressure variation (PPV) is an accurate predictor of fluid responsiveness in mechanically ventilated patients. The aim of this study was the assessment and comparison of the ability of automated PPV, when measured by an Intellivue MP 70 monitor, and manually calculated PPV to predict fluid responsiveness in mechanically ventilated septic shock patients.

Methods We conducted a prospective study on 36 septic shock patients. Automated and manually calculated PPV and other hemodynamic data were recorded before and after fluid administration of $500 \mathrm{ml}$ of $6 \%$ hydroxyethyl starch (130/0.4) over 30 minutes. Responders were defined as patients with an increase in their cardiac index $>15 \%$ after fluid loading.

Results The agreement (mean bias \pm SD) between automated and manually calculated PPV was $4.03 \pm 7.37 \%$. The baseline automated PPV correlated with the baseline manually calculated PPV $(r=0.79, P<0.01)$. Twenty-three (63.9\%) patients were classified as fluid responders. Automated PPV and manually calculated PPV were significantly higher in responders than in nonresponders $(16.0 \pm 4.5 \%$ vs. $7.2 \pm 2.0 \%$ and $11.1 \pm 5.6$ vs. $4.6 \pm 2.8 \%$, respectively; $P<0.001$ for both). The area under the receiver operating characteristic curves of automated PPV was significantly greater than the manually calculated PPV (0.982 vs. 0.87 , respectively; $P=0.04$ ). The optimal threshold values for predicting fluid responsiveness were $11 \%$ for automated PPV (sensitivity $91.3 \%$, specificity $92.3 \%$ ) and $13 \%$ for manually calculated PPV (sensitivity $73.9 \%$, specificity $84.6 \%)$.

Conclusion Our results indicate that the automated PPV, obtained by the IntelliVue MP 70 monitor, and manually calculated PPV, showed comparable performance for predicting fluid responsiveness in passively ventilated septic shock patients.

\section{P235}

Applicability of methods for fluid responsiveness prediction in the ICU P Mendes', L Miranda', M Park', L Azevedo', B Rodrigues², E Queiroz², G Schettino², L Taniguchi

'Hospital das Clinicas, São Paulo, Brazil;' ${ }^{2}$ Hospital Sirio Libanes, São Paulo, Brazil Critical Care 2012, 16(Suppl 1):P235 (doi: 10.1186/cc10842)

Introduction Volume expansion is a frequent and widely used therapy in hemodynamically unstable patients but may lead to complications. 
To ensure appropriate indication of fluid administration, evaluation of fluid responsiveness by dynamic parameters is suggested, although it requires specific conditions not always present in ICU patients. The aim of this study was to analyze the applicability of parameters for evaluation of fluid responsiveness in the ICU.

Methods We conducted a prospective observational study in two ICUs. Volume expansions performed in ICU patients at the discretion of the physician in charge were analyzed for the presence of conditions that allowed adequate fluid responsiveness evaluation. The presence of central venous, pulmonary arterial or peripheral arterial catheters, invasive mechanical ventilation and ventilator settings, echocardiography availability, presence of arrhythmias, use of sedation and vasoactive drugs were registered. Percentages of patients who fulfilled conditions for dynamic parameters (such as pulse pressure variation, stroke volume variation and echocardiographic analysis) were recorded.

Results Ninety volume expansions in 68 patients were performed during the study period. Central venous catheter was present $58.9 \%$ of the time. In $41.1 \%$ of the cases patients were in spontaneous ventilation. No patients used a pulmonary artery catheter. An echocardiography machine with an attending physician trained for critical care echocardiography was available in $8.9 \%$. An arterial catheter was available in $21 \%$ of the volume expansions and mechanical ventilation was present in $31.1 \%$ of the cases $(67.3 \%$ of ventilated patients were using controlled mode of ventilation). The association of mechanical ventilation in controlled mode with an arterial catheter in place and no restrictions for performing analysis of dynamic parameters was present in only $7.7 \%$ of patients. Considering all dynamic parameters described here, the use of any method for predicting fluid responsiveness was possible in $15.6 \%$ of the volume expansions performed in our ICU.

Conclusion The use of dynamic parameters for predicting fluid responsiveness in the ICU may have restricted applicability since the necessary conditions are often not present.

\section{P236}

Fluid responsiveness during weaning from mechanical ventilation

M Geisen, UM Schmid, O Dzemali, A Zollinger, CK Hofer

Triemli City Hospital, Zürich, Switzerland

Critical Care 2012, 16(Suppl 1):P236 (doi: 10.1186/cc10843)

Introduction To overcome the limited accuracy of functional hemodynamic parameters such as stroke volume and pulse pressure variation (SVV and PPV) during spontaneous breathing, a passive leg raising (PLR) maneuver has been suggested as a reliable predictor of fluid responsiveness $[1,2]$. The aim of this study was to evaluate fluid responsiveness using SVV, PPV and PLR during the transition from controlled to spontaneous breathing.

Methods Thirty-four patients after off-pump CABG were enrolled. Measurements were performed in the ICU using a PiCCO, system. Fluid $(500 \mathrm{ml})$ was given: (A) during controlled mechanical ventilation, (B) during pressure support ventilation with spontaneous breathing and (C) after extubation. The stroke volume (SV), SVV and PPV as well as the mean arterial pressure and heart rate were assessed. A PLR was performed before fluid administration at all three time points. Fluid response was defined as an increase in SV $>15 \%$. Prediction of fluid responsiveness was tested using ROC analysis.

Results In 34 patients significant hemodynamic changes were observed, with 19 (55.9\%), 22 (64.7\%), and $13(40.6 \%)$ responders at time points $A, B$ and $C$, respectively. Prediction of fluid responsiveness is depicted in Table 1.

Table 1 (abstract P236). Prediction of fluid responsiveness

\begin{tabular}{|c|c|c|c|c|c|c|c|c|c|}
\hline & \multicolumn{3}{|c|}{ A } & \multicolumn{3}{|c|}{ B } & \multicolumn{3}{|c|}{ C } \\
\hline & AUC & $P$ value & TS\% & AUC & $P$ value & TS\% & AUC & $P$ value & TS\% \\
\hline $\mathrm{SW}$ & 0.88 & 0.0001 & 15.5 & 0.70 & 0.056 & 12.5 & 0.56 & 0.604 & 13.5 \\
\hline PPV & 0.83 & 0.001 & 14.5 & 0.69 & 0.063 & 11.0 & 0.48 & 0.863 & 11.5 \\
\hline PLR SV\% & 0.72 & 0.028 & 8.0 & 0.74 & 0.021 & 10.0 & 0.70 & 0.058 & 8.0 \\
\hline
\end{tabular}

TS, threshold.
Conclusion Prediction of a fluid response with SVV/PPV was less reliable in spontaneous breathing. PLR predicted fluid responsiveness, but was less accurate than previously reported.

References

1. Marik et al:: Crit Care Med 2009, 37:2642-2647.

2. Monnet et al.: Crit Care Med 2006, 34:1402-1407.

\section{P237}

Microcirculatory blood flow is related to clinical signs of impaired organ perfusion, and its dynamics to the macrohemodynamic concept of fluid responsiveness

A Pranskunas' ${ }^{1}, \mathrm{M} \mathrm{Koopmans}^{2}$, V Pilvinis ${ }^{3}$, P Koetsier2 ${ }^{2}$ EC Boerma²

'Lithuanian University of Health Sciences, Kaunas, Lithuania; ${ }^{2}$ Medical Centre Leeuwarden, the Netherlands; ${ }^{3}$ Hospital of Lithuanian University of Health

Sciences, Kaunas, Lithuania

Critical Care 2012, 16(Suppl 1):P237 (doi: 10.1186/cc10844)

Introduction Fluid responsiveness is not equal to a clinical need for fluid therapy. The aim of our study was to assess the incidence of microcirculatory flow alterations, according to a predefined arbitrary cut-off value, in patients with clinical signs of impaired organ perfusion. The secondary endpoint was to establish the correlation between the microcirculatory and macrocirculatory response to a fluid challenge.

Methods We performed a prospective, single-centre, observational study. Included were ICU patients $\geq 18$ years with invasive hemodynamic monitoring and clinical signs of impaired organ perfusion, as the principal reason for fluid administration. Fluid challenge was performed by the infusion of $500 \mathrm{ml}$ crystalloid or a balanced colloid (Volulyt ${ }^{\oplus}$ ) solution in 30 minutes. Before and after fluid challenge, systemic hemodynamics and direct in vivo observation of the microcirculation were obtained with sidestream dark-field imaging. Assessment of microcirculatory parameters of convective oxygen transport (microvascular flow index (MFI) and proportion of perfused vessels), and diffusion distance (perfused vessel density and total vessel density) was done using a semiquantitative method.

Results We enrolled 50 patients. MFI $<2.6$ was present in $66 \%$ of the patients. After fluid challenge, signs of impaired organ perfusion reduced from $100 \%$ to $68 \%$ of the patients, $P<0.001$. The incidence of $\mathrm{MFI}<2.6$ decreased to $46 \%$, and was higher in patients with persistent signs of impaired organ perfusion: $56 \%$ versus $25 \%, P=0.04$. Median MFI increased from 2.5 (2.3 to 2.8) at baseline to 2.7 (2.4 to 2.8) after fluid challenge, $P=0.003$, but its change was only significant in fluidresponsive patients.

Conclusion These data demonstrate a relationship between clinical signs of impaired organ perfusion and MFI $<2.6$. Fluid responsiveness did not discriminate between patients with and without clinical signs of impaired organ perfusion or MFI <2.6. However, significant improvement of microvascular alterations and attenuation of clinical signs of impaired organ perfusion was restricted to patients who were fluid responsive. Noninvasive assessment of microvascular perfusion may help to define patients with potential need for fluid therapy, and to evaluate its effect.

\section{P238}

Frank-Starling and Guyton together at bedside during a fluid challenge

H Aya, M Cecconi, M Geisen, C Ebm, M Grounds, N Fletcher, A Rhodes St George's Hospital, London, UK

Critical Care 2012, 16(Suppl 1):P238 (doi: 10.1186/cc10845)

Introduction According to Guyton, the difference between mean systemic filling pressure (Pms) and right atrial pressure (RAP) is the venous pressure gradient (VP). This is proportional to venous return and cardiac output (CO). According to the Frank-Starling law a fluid challenge successfully increases the stroke volume if the preload increases in the ascending part of the curve. The aim of this study was to assess the significance of the analogue of the Pms (Pmsa) measured with the Navigator ${ }^{T M}$ (Applied Physiology, St Leonards, Australia), the central venous pressure (CVP) (as a surrogate of RAP) and the VP during a fluid challenge. 
Methods A prospective observational study was performed in postsurgical patients. Patients were monitored with a central venous catheter, an arterial line, a calibrated LiDCO ${ }^{\mathrm{TM}}$ plus (LiDCO, Cambridge, UK) and the Navigator ${ }^{\mathrm{TM}}$. A $250 \mathrm{ml}$ fluid challenge was used to maximise the stroke volume (SV). Data were recorded before and after the fluid challenge which was given over 5 minutes. A positive response to the fluid challenge was defined as either a stroke volume or $\mathrm{CO}$ increase of $10 \%$ or more.

Results Twenty-five fluid challenges in 14 patients were observed. In seven cases (28\%), the fluid challenge increased SV (and CO) by $\geq 10 \%$ (Table 1). At baseline there were no differences between HR, Pmsa, CVP or $\triangle \mathrm{VP}$ for responders or nonresponders. The responders had greater changes in $\triangle \mathrm{VP}$ in response to the challenge.

Table 1 (abstract P238). Haemodynamic parameters in responders and nonresponders

\begin{tabular}{lccc}
\hline & Nonresponders & Responders & P value \\
\hline MAP & 78.4 & 71.4 & 0.07 \\
Pmsa & 17 & 15 & 0.3 \\
CVP & 9.7 & 9 & 0.7 \\
HR & 89 & 91 & 0.7 \\
$\Delta$ VP & 7.2 & 6 & 0.09 \\
$\Delta$ Pmsa & 19.3 & 22.1 & 0.6 \\
$\Delta$ CVP & 2.9 & 1.6 & 0.1 \\
$\Delta(\Delta V P)$ & 1.1 & 24.8 & $<0.01$ \\
\hline
\end{tabular}

Conclusion Our study demonstrates that the Navigator ${ }^{\mathrm{TM}}$ may be used to monitor the effect of fluid challenges by assessing the change in VP to the challenge.

\section{P239}

Prediction of fluid responsiveness in intensive care (PREFERENCE study): fluid challenge versus passive leg raising in high-risk surgical patients

M Cecconi, F Caliandro, J Mellinghoff, D Dawson, S Ranjan, M Hamilton,

$M$ Grounds, A Rhodes

St Georges Hospital, London, UK

Critical Care 2012, 16(Suppl 1):P239 (doi: 10.1186/cc10846)

Introduction The aim of this study is to evaluate whether the passive leg raising (PLR) maneuver could be used to predict fluid responsiveness in awake postoperative patients admitted to the ICU. PLR has been demonstrated to be a good indicator of fluid responsiveness even in spontaneously breathing patients, but few data are available in the immediate postoperative period. Nexfin is a new cardiac output monitor that measures and tracks stroke volume (SV) by analyzing the arterial pressure pulse contour noninvasively from a finger probe. Methods We enrolled self-ventilating patients admitted to the ICU postoperatively. A PLR maneuver $\left(45^{\circ}\right.$ bed tilt from the 30 to $45^{\circ}$ head up) was performed and followed by a fluid challenge (FC, $250 \mathrm{ml}$ fluid bolus over 5 minutes). Changes in SV during PLR and after administration of FC were monitored with the Nexfin monitor. Receiver operator characteristic (ROC) analysis was performed.

Results Forty-five patients were enrolled. Twenty-three patients responded to the FC with an increase of SV $>5 \%$. Twenty-eight patients (62\%) were excluded from the PLR analysis as a result of haemodynamic instability (difference in heart rate, mean arterial pressure or SV baselines pre PLR and pre FC $>5 \%$ ). Seventeen patients were analyzed. The area under the curve for the ROC analysis was 0.93 ( $\mathrm{SE}=0.06$; $P=0.003$ ) (Figure 1). A SV increase $>1 \%$ during a PLR test predicts a SV increase $>5 \%$ after $\mathrm{FC}$ with a sensitivity of $75 \%$ and a specificity of $78 \%$. Conclusion In $62 \%$ of patients a PLR test could not be performed due to haemodynamic instability. In these patients, FC is the best way to assess fluid responsiveness. During haemodynamic stability PLR shows great sensitivity and specificity to predict fluid responsiveness. The Nexfin monitor can be used to track SV changes both during FC and during a PLR test.

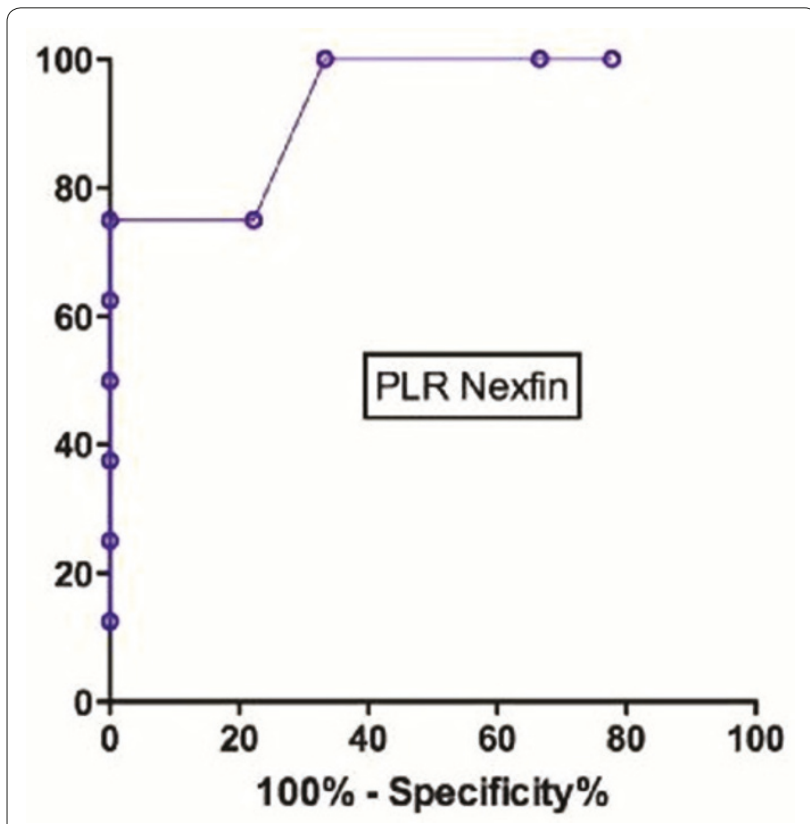

Figure 1 (abstract P239).

P240

Tight control of fluid balance may reduce incidence of intraabdominal hypertension in patients after major abdominal surgery and trauma: a pilot study

P Szturz', J Maca', J Neiser1, J Jahoda', R Kula', JT Tichy²

${ }^{1}$ Faculty Hospital Ostrava, Czech Republic; ${ }^{2}$ Yeovil District Hospital, Yeovil, UK Critical Care 2012, 16(Suppl 1):P240 (doi: 10.1186/cc10847)

Introduction Massive fluid resuscitation followed by hypoperfusion abnormalities in the ICU is a risk factor for development of intraabdominal hypertension (IAH). The aim of our study was to determine what influence would have the control of daily fluid balance on the incidence of IAH in patients after extensive abdominal surgery or after abdominal trauma.

Methods A prospective observational study included a total of 82 adult patients (age: $59 \pm 10$ years, APACHE II score at admission: $18 \pm 11$, predicted mortality according to APACHE II score at admission: $34 \%$, observed in-hospital mortality: 20\%), number of patients with intraabdominal pressure (IAP) above 12 at admission: 23 (28\%), admitted to a single ICU with the diagnosis of abdominal trauma $(n=22)$ or after abdominal surgery $(n=60)$. During first 7 days the fluid intake and balance was monitored and corrected at 6-hour intervals not to exceed $1,500 \mathrm{ml}$ positivity over 24 hours (oncodiuretic therapy was administered - repeated boluses of starch solutions and/or albumin followed by furosemide). IAP was measured from admission twice daily (standardized measurement by instillation of $25 \mathrm{ml}$ normal saline into the bladder). A sustained elevation of the IAP above $12 \mathrm{mmHg}$ in two consecutive measurements was considered as IAH.

Results The incidence of IAH in relation to daily fluid intake and daily fluid balance is shown in Figure 1.

Conclusion The incidence of IAH in patients after abdominal surgery or abdominal trauma may exceed the value of $40 \%$, especially in situations associated with massive fluid resuscitation [1]. We have identified a close relationship of the daily dynamics of changes in IAP and fluid balance. When we maintained the daily fluid balance not to exceed the positivity of $1,500 \mathrm{ml} / 24$ hours, the incidence of IAH in our study dropped from $28 \%$ to less than $20 \%$, despite high daily fluid intake (about 5,000 to $8,000 \mathrm{ml} /$ day). Tight control of the fluid balance seems an effective method to prevent the development of IAH.

Reference

1. Ball ChG, et al:: The secondary abdominal compartment syndrome: not just another post-traumatic complication. Can J Surg 2008, 51:399-405. 


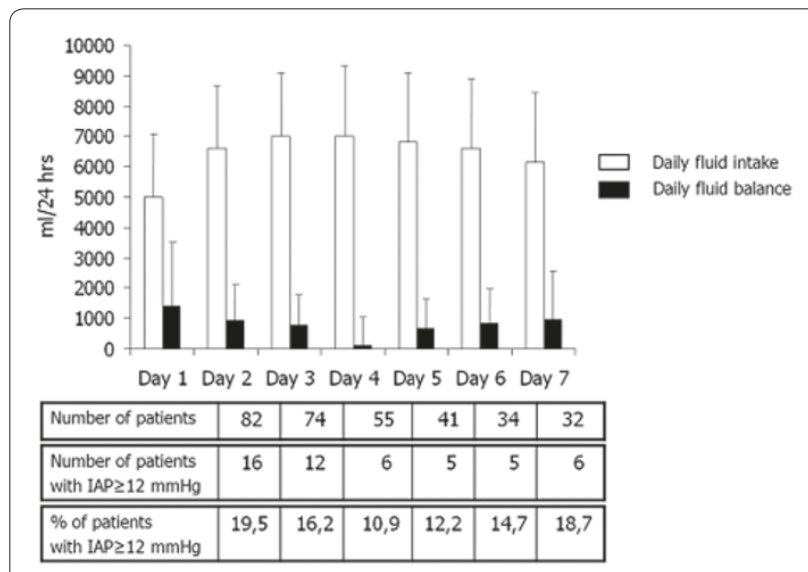

Figure 1 (abstract P240). Fluid intake, fluid balance and incidence of IAH.

P241

Negative fluid balance 48 hours after admission improves survival at $\mathbf{2 8}$ days in critically ill patients

M Cuartero, AJ Betbese, K Nuñez, J Baldira, L Zapata

Hospital De Sant Pau, Barcelona, Spain

Critical Care 2012, 16(Suppl 1):P241 (doi: 10.1186/cc10848)

Introduction Fluid infusion may be lifesaving in critically ill patients, but following initial resuscitation a positive fluid balance is associated with increased mortality. This study aimed to determine whether a negative fluid balance $(\leq-500 \mathrm{ml})$ within the first 48 hours of admission in the ICU is associated with improved survival at 28 days in a heterogeneous cohort of critically ill patients.

Methods We conducted a retrospective study in a 20-bed ICU at a university-affiliated teaching hospital. Patients admitted for acute heart failure, those who required dialysis before admission and those who died within 24 hours after admission were excluded. Demographic data, SAPS II and APACHE II scores were recorded at admission and SOFA, fluid balance, hemodynamic, respiratory and renal variables once per day. Variables were compared between survivors and nonsurvivors and between patients who did and those who did not achieve negative fluid balance by day 2 of admission. Multiple logistic regression was used to identify variables significantly associated with ICU mortality in the univariate analysis. Survival was assessed using Kaplan-Meier analysis.

Results We studied 87 patients: 53 males, mean age $58 \pm 18$ years, SAPS II $39.3 \pm 15.8$, APACHE II score $15.9 \pm 7.5$, SOFA score $5.0 \pm 3.4$, and ICU stay $10.3 \pm 9.8$ days. The main syndrome diagnosis at admission was septic shock $(n=26)$, acute respiratory failure $(n=19)$, trauma $(n=13)$, neurocritical illness $(n=14)$ and others $(n=15)$. Overall mortality in the ICU reached $20.7 \%$ and survival at 28 days was $73.6 \%$. When patients were classified according to 28 -day outcome, we observed statistically significant differences in negative fluid balance at 48 hours $(P<0.001)$, SAPS II $(P<0.001)$, APACHE II score $(P=0.007)$, age $(P=0.046)$ and incidence of acute kidney injury at admission $(P=0.02$; defined as at least Risk in RIFLE criteria), but urinary output, hemodynamic and respiratory parameters did not differ. Multivariate analysis showed that negative fluid balance at 48 hours was independently associated with improved survival: odds ratio $=7.9(P=0.013)$. Kaplan-Meier analysis showed that survival was significantly lower in patients without negative fluid balance at 48 hours $(P=0.015)$.

Conclusion Our findings show that negative fluid balance 48 hours after admission may correlate with better outcome in a heterogeneous population of critically ill patients.

Reference

1. Boyd JH, Forbes J, Nakada T, Walley KR, Russell JA: Fluid resuscitation in septic shock: a positive fluid balance and elevated central venous pressure are associated with increased mortality. Crit Care Med 2011, 39:259-265.
P242

Goal-directed fluid and hemodynamic therapy in major colon surgery with the pressure recording analytical method cardiac output monitor (MostCare ${ }^{\oplus}$-PRAM ${ }^{\circledR}$ ): prospective analysis of 58 patients

JM Alonso-Iñigo, MJ Fas, V Osca, A Nacher, JE Llopis

Hospital Universitario de la Ribera, Alzira, Spain

Critical Care 2012, 16(Suppl 1):P242 (doi: 10.1186/cc10849)

Introduction Optimal fluid therapy in colon surgery is controversial. The aim of this study is to assess the impact on postoperatory complications, fluid administration, and length of hospital stay, of GD fluid and hemodynamic therapy protocol based on PRAM ${ }^{\oplus}$-MostCare ${ }^{\circledast}$ hemodynamic variables.

Methods Patients scheduled for elective colorectal operations were included. The MostCare ${ }^{\oplus}$ was connected to a radial artery catheter for hemodynamic monitoring. Hemodynamic variables including the stroke volume index (SVI), cardiac index $(\mathrm{Cl})$, and pulse pressure variation (PPV) were measured. Fluids and hemodynamic drugs were administered to achieve the primary endpoints: $\mathrm{Cl} 2.5$ to $4.5, \mathrm{PPV}<13 \%$, SVRI $>1,500$, MAP similar to basal values. Hemodynamic data were noted at induction and at the end of surgery. Data of fluids, urine output, postoperative complications and length of stay were assessed. Descriptive statistics were used. A paired-simple $t$ test was used for the analysis of the differences between variables.

Results Data of 58 patients were collected. The mean length of hospital stay was $9 \pm 3$ days. Postoperative complications included suture leak $(3.4 \%)$, surgical site infection $(6.9 \%)$, and heart failure (3.4\%) with no other medical or surgical complications. Total fluids during surgery were $2,775 \pm 934 \mathrm{ml}(1,259 \pm 498 \mathrm{ml}$ crystalloids, 1,302 \pm 622 $\mathrm{ml}$ colloids, $214 \pm 330 \mathrm{ml} \mathrm{RBC}$ ). Fluid infusion and urine output were $10.6 \pm 3.2$ and $1.8 \pm 1.2 \mathrm{ml} / \mathrm{kg}$ hour respectively. Hemodynamic data are shown in Table 1.

Table 1 (abstract P242). Hemodynamic variables

\begin{tabular}{lccc}
\hline & Start SG & End SG & $P$ value \\
\hline $\mathrm{Cl}$ & $2.85 \pm 0.9$ & $3.16 \pm 0.4$ & 0.020 \\
$\mathrm{SVI}$ & $40 \pm 10$ & $41 \pm 8$ & 0.59 \\
$\mathrm{HR}$ & $70 \pm 12$ & $77 \pm 12$ & $<0.001$ \\
$\mathrm{PPV}$ & $7 \pm 5$ & $4 \pm 2$ & 0.001 \\
$\mathrm{MAP}$ & $76 \pm 14$ & $83 \pm 14$ & 0.002 \\
SVRI & $2051 \pm 588$ & $2026 \pm 551$ & 0.74 \\
\hline
\end{tabular}

Conclusion GDT with MostCare ${ }^{\circledR}$ resulted in a low incidence of postoperative complications and provided an optimal hemodynamic management during major colon surgery.

References

1. Gan TJ, et al:: Anesthesiology 2002, 97:820-826.

2. Vincent IL, et al:: Crit Care 2011, 15:229.

P243

Implementation of an optimal fluid management protocol using the PiCCO system delays development of ARDS secondary to severe sepsis

N Saito, T Yagi, Y Hara, H Matsumoto, K Mashiko

Chiba-Hokusoh Hospital, Nippon Medical School, Chiba, Japan

Critical Care 2012, 16(Suppl 1):P243 (doi: 10.1186/cc10850)

Introduction Acute respiratory distress syndrome (ARDS) is often associated with sepsis, and one recommended approach in the fluid management of ARDS is to keep sepses dry. On the other hand, optimal fluid management after the early phase of septic shock remains unknown. An excessive positive fluid balance in patients with septic shock is associated with increased mortality and morbidity. We implemented an optimal fluid management (OFM) protocol using the PiCCO system from April 2009. The purpose of this study was to 
examine the effect of the OFM protocol in comparison with historical controls.

Methods A retrospective study was conducted in a Japanese mixed ICU of a tertiary-care teaching hospital from July 2007 to March 2011. Our protocol includes daily volume assessment using the PiCCO system after ICU admission and a change of fluid therapy after evaluation; for example, additional diuretic use or fluid restriction. We retrospectively analyzed 96 consecutive patients with severe sepsis or septic shock who required mechanical ventilation between July 2007 and December 2010. We divided patients into the OFM protocol group ( $P ; n=49$; April 2009 to December 2010) and the control group ( $C ; n=47$; July 2007 to March 2009) and compared their clinical and laboratory data.

Results Median (IQR) age was 69.5 (55.5 to 78.5) years, and the median APACHE II score and SOFA score were 23.0 (19.0 to 27.0) and 10.0 (7.0 to 12.0), respectively. The proportion of patients with septic shock was $75 \%$. There was no difference in patient characteristics between the two groups. At 28 days, the mortality rate was similar in both groups ( $P$ : $14.3 \% ; C: 17.0 \% ; P=0.78)$. The incidence of ARDS after ICU admission in the $P$ group was significantly lower than that in the $C$ group $(P: 20.4 \% ; C$ : $57.4 \% ; P=0.02)$. In addition, the onset of ARDS in the $P$ group occurred later than that in the $C$ group $(P<0.01)$. Achievement of a negative water balance in the $\mathrm{P}$ group occurred earlier than in the $\mathrm{C}$ group. The incidence of AKI (RIFLE criteria: failure) and another organ failure was similar in both groups. Multivariate regression analysis revealed that the OFM protocol independently suppressed the onset of ARDS (OR $0.17(P=0.001 ; 95 \% \mathrm{Cl}: 0.06$ to 0.51$))$.

Conclusion Implementation of an OFM protocol using the PiCCO system significantly decreased the development of ARDS secondary to severe sepsis with no other complications.

P244

Confrontation of the increase in ELWI rate regarding the septic

polytraumatised patient administering furosemide: is it effective?

P Sarafidou, E Pappa, D Dimitriadou, D Litis, I Pavlou

KAT General Hospital Kifisia, Athens, Greece

Critical Care 2012, 16(Suppl 1):P244 (doi: 10.1186/cc10851)

Introduction The ELWI rate (measurable with the PICCO catheter) conveys the extravascular lung water. The increase of the ELWI rate is a frequent finding in heavily septic patients and it is connected with very high mortality. The purpose of this study is to find out if the administration of furosemide is possible to reduce effectively the ELWI rate and if this decrease can be maintained regardless of the confrontation of the sepsis.

Methods We studied 20 septic poytraumatized patients (mean ISS score 35) with ARDS syndrome and ELWI >10, with good renal function and on medical treatment with levophed. We administered furosemide $10 \mathrm{mg} /$ hour, for 24 hours, while at the same time we confronted the septic source. During all these we noted $\mathrm{PO}_{2} / \mathrm{FiO}_{2}, \mathrm{MAP}, \mathrm{CVP}$ and ELWI every 8 hours. Moreover, we noted the changes in the levophed dose and the total balance of fluid at the end of the 24-hour interval.

Results The ELWI rate was up 12 to 16 before the administration of furosemide. We marked that after the administration of furosemide and at the end of the first 8-hour interval, the ELWI rate decreased about 2 to 3 units but we had to increase the dose of vasoconstriction, until the end of the 24-hour interval the ELWI rate restored to the initial high level and could not manage to decrease the dose of vasoconstriction to have a negative balance of fluids. The improvement of $\mathrm{PO}_{2} / \mathrm{FiO}_{2}$ was insignificant statistically and we confronted operatively the septic source for five to 20 patients at the end of the 24-hour interval.

Conclusion We managed to decrease very little the ELWI rate, only temporarily after the administration of furosamide about a 24-hour interval. The small improvement of the $\mathrm{PO} 2 / \mathrm{FiO}_{2}$ finally leads to a decrease of the ELWI rate but it is not important statistically and on the other side leads to an increase of vasoconstriction. Therefore, this method is not effective. We gain only a short time for the safer surgical treatment if it is needed.
P245

Indexation of extravascular lung water in unselected adult patients with and without mechanical ventilation: a prospective study in 50 patients with 843 transpulmonary thermodilutions

W Huber, B Saugel, D Paradellis, J Hoellthaler, V Phillip, C Schultheiss, PThies, U Mayr, A Herrmann, RM Schmid

Klinikum Rechts der Isar, Technischen Universität München, Munich, Germany Critical Care 2012, 16(Suppl 1):P245 (doi: 10.1186/cc10852)

Introduction Extravascular lung water (EVLW) has been indexed to actual BW (BW-act), termed the EVLW index (ELWI). Since in obese patients indexation to BW-act might inappropriately diminish the indexed ELWI-act, ELWI indexed to predicted BW (ELWI-pred) has been introduced. Indexation of EVLW to height might be superior to ELWIpred/-act. Recent data in a selected collective of ARDS patients suggest that indexation to height might improve the predictive capabilities of ELWI regarding $\mathrm{pO}_{2} / \mathrm{FiO}_{2}$. We aimed to investigate which indexation of EVLW provides the best association of ELWI and $\mathrm{pO}_{2} / \mathrm{FiO}_{2}$ in patients without pulmonary impairment or without ventilation.

Methods In 50 consecutive ICU patients with PiCCO monitoring, 843 triplicate measurements of EVLW and simultaneous blood gas analysis were performed. The endpoint was prediction of $\mathrm{pO}_{2} / \mathrm{FiO}_{2}<200 \mathrm{mmHg}$ and other critical thresholds provided by unindexed EVLW as well as ELWI indexed to ideal BW, adjusted BW, BMI, body surface area, height and total lung capacity.

Results Measurements in patients without pulmonary impairment $463 / 843(54.9 \%)$; acute $188 / 843$ (22.3\%), chronic 106/843 (12.6\%), and both acute and chronic pulmonary disease $86 / 843(10.2 \%)$. Mechanical ventilation in 458/843 (54.3\%) measurements. The largest ROC AUCs regarding $\mathrm{pO}_{2} / \mathrm{FiO}_{2}<200 \mathrm{mmHg}$ were found for ELWl-height (AUC $0.658 ; 95 \% \mathrm{Cl} 0.554$ to 0.735$)$ and $\operatorname{EVLW}(0.655 ; 95 \% \mathrm{Cl} 0.544$ to 0.732$)$, the lowest AUC for ELWI-act $(0.629 ; 95 \% \mathrm{Cl} 0.514$ to 0.742$)$. Similarly ELWI-height and unindexed EVLW provided the largest ROC AUCs regarding $\mathrm{pO}_{2} / \mathrm{FiO}_{2}>300 \mathrm{mmHg}(0.659$ and 0.657$)$, normal $\mathrm{pO}_{2} / \mathrm{FiO}_{2}$ (>381 mmHg; 0.665 and 0.657 ) and acute and/or chronic pulmonary impairment (0.622 and 0.625). All these associations were significant with $P<0.001$. Among patients with pulmonary impairment, first values of ELWI-height and EVLW provided the largest ROC AUCs regarding mortality $(0.815$ and $0.815 ; P=0.016)$ compared to ELWI-act $(0.694$; $P=0.136)$ and APACHE II score $(0.792 ; P=0.025)$.

Conclusion Indexation to $\mathrm{BW}$-act results in reduced predictive capabilities compared to no indexation at all. ELWI-pred performs slightly better than ELWI-act, but our data do not support that ELWI-pred is superior to no indexation at all in adult ICU patients. In this unselected and prospectively evaluated collective, the highest predictive capabilities regarding several predefined thresholds were found for ELWI-height.

P246

How to perform indexing of extravascular lung water data

S Wolf ${ }^{1}$, A Riess², J Landscheidt² , C Lumenta², L Schuerer ${ }^{2}$, P Friederich ${ }^{2}$ ${ }^{1}$ Charite Berlin, Germany; ${ }^{2}$ Klinikum Bogenhausen, Munich, Germany Critical Care 2012, 16(Suppl 1):P246 (doi: 10.1186/cc10853)

Introduction Extravascular lung water (EVLW) is a marker for the severity of acute lung injury. To allow assessment of normal and pathologic states, traditionally EVLW data are either indexed to real or predicted body weight. Surprisingly and despite widespread use, this has so far not been validated in a larger cohort of subjects without cardiopulmonary compromise. The aim of the study was to prospectively evaluate a different ways of indexing EVLW data.

Methods EVLW was measured using single indicator transpulmonary thermodilution at predefined time points in 101 patients requiring elective brain tumor surgery. This database was used to investigate the properties of indexing EVLW data to real and predicted body weight, body surface area and body height.

Results EVLW indexed to predicted body weight was inversely correlated with a patient's body height $(P<0.001)$, while values indexed to real body weight remained inversely dependent on weight $(P<0.001)$. Indexing to estimated body surface area, again based on real or predicted body weight, provided no advantage. In contrast, 
indexing to body height presents an alternative method without dependence on physical properties or gender of a patient, yielding a uniform $95 \%$ confidence interval of normal values from 0.22 to $0.43 \mathrm{l} / \mathrm{m}$. Conclusion Traditional ways of indexing EVLW data do not resolve value dependence on physical properties or gender. Therefore, the currently used definition of a normal range from 3 to $8 \mathrm{ml} / \mathrm{kg}$ seems to be invalid. Our data suggest indexing EVLW to plain body height instead of weight-based methods. As we are not aware of any abnormal hemodynamic profile for brain tumor patients, we propose our findings as a close approximation to normal values. This will require further validation in critically ill patients.

\section{P247}

Near-normal values of extravascular lung water in children

J Lemson', C Cecchetti², A Nusmeier

'Radboud University Nijmegen Medical Centre, Nijmegen, the Netherlands;

2IRCS Bambino Gesù Roma, Rome, Italy

Critical Care 2012, 16(Suppl 1):P247 (doi: 10.1186/cc10854)

Introduction Extravascular lung water (EVLW) reflects the amount of pulmonary edema and can be measured at the bedside using the transpulmonary thermodilution method (TPTD) incorporated in the PiCCO device (Pulsion, Germany). Currently, normal values of EVLW for the use in children are unavailable. This study was designed to collect near-normal values of EVLW in children after recovery from critical illness.

Methods In this prospective observational multicenter study (five sites), pediatric TPTD measurements were collected from children admitted to a pediatric ICU without or after resolution of pulmonary abnormalities. Inclusion criteria were minimal or no respiratory support and stable hemodynamics. We searched typically for the last lung water measurement prior to removal of the PiCCO system. EVLW was indexed using predicted body weight (EVLWI) calculated using height, based upon WHO data.

Results Fifty-five children aged from 0 to 16 years were included. Mean values (range) were: age 6.5 (0.04 to 16) years, weight 25.8 (3.7 to 80) $\mathrm{kg}$, mean arterial blood pressure 79 (48 to 131$) \mathrm{mmHg}, \mathrm{PaO}_{2} / \mathrm{FiO}_{2}$ ratio 388 (171 to 662$) \mathrm{mmHg}$, cardiac index (Cl) 4.5 (2.2 to 6.7$) \mathrm{l} /$ minute $/ \mathrm{m}^{2}$, global end-diastolic volume (GEDVI) 490 (211 to 718 ) $\mathrm{ml} / \mathrm{m}^{2}$, EVLWI 12.7 (4.7 to 34.6$) \mathrm{ml} / \mathrm{kg}$. Figure 1 shows the logarithmic relation between EVLWI and age with an $r^{2}$ of 0.7. There was no significant correlation between GEDVI or $\mathrm{Cl}$ and age.

Conclusion Near-normal values of EVLW in children are strongly correlated with age. Based upon these data, normal values can be constructed for future clinical use.

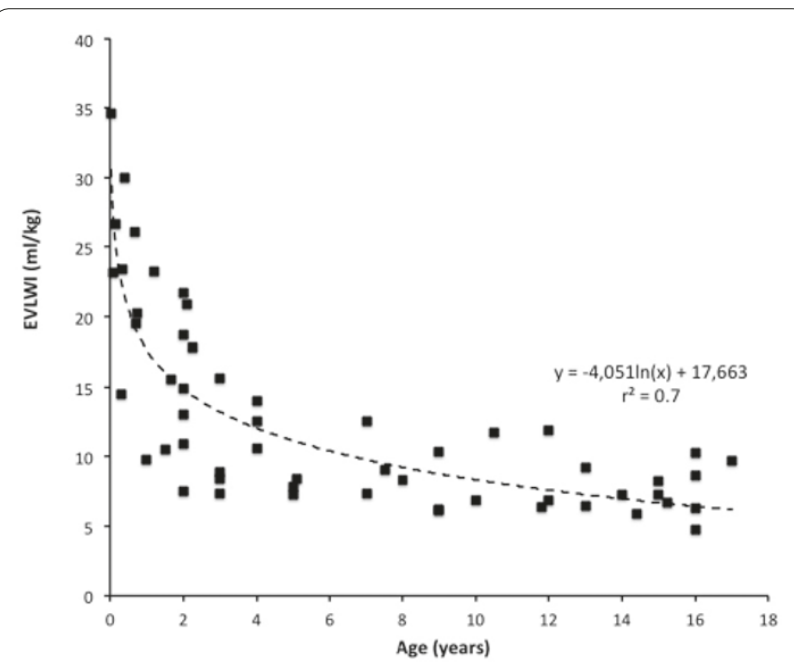

Figure 1 (abstract P247).
P248

Transthoracic ultrasound assessment of B-lines for identifying the increment of extravascular lung water in shock patients requiring fluid resuscitation

PTheerawit, N Tomuan, Y Sutherasan, S Kiatboonsri

Ramathibodi Hospital, Mahidol University, Bangkok, Thailand

Critical Care 2012, 16(Suppl 1):P248 (doi: 10.1186/cc10855)

Introduction Sonographic B-lines are commonly observed in cases of increasing extravascular lung water (EVLW). These findings became prominent when interstitial and alveolar tissues were filled with fluid $[1,2]$. Thus, we hypothesized that the increment of sonographic B-lines would be observed when the EVLW increased after fluid resuscitation in shock patients and be associated with the impaired gas exchange. Methods Transthoracic portable ultrasound before and after fluid resuscitation was performed. Patients with pleural disease were excluded. The B-lines were measured in 23 lung zones. The total numbers of B-lines seen in each patient were counted as the total B-line score (TBS). The primary outcome was to demonstrate the increase of TBS after fluid resuscitation. The secondary outcome was to examine the magnitude of the incremental number of TBS.

Results Twenty patients were enrolled in this study. All patients had septic shock. Six of all had lung involvement. Twelve patients received mechanical ventilation. The mean of net fluid balance was $+2,228 \pm 1,982 \mathrm{ml}$ and the mean of duration between two ultrasound measurements was $31 \pm 13$ hours. The means of TBS at pre and post fluid therapy were $37 \pm 26$ and $64 \pm 29$ respectively $(P<0.0001,95 \% \mathrm{Cl}$ 13.47 to 33.67). This increase was found in all areas of measurement. In particular, the number of B-lines measured at the anterior axillary line area very well correlated to the TBS $(r=0.90, P<0.01)$ and its increment had reverse correlation to the $\mathrm{PaO}_{2} / \mathrm{FiO}_{2}$ ratio $(r=0.704, P<0.05)$. The volume of fluid per one B-line increasing was $119 \pm 134 \mathrm{ml}$. The interobserver reliability between two ultrasound readers was very high $(r=0.92, P<0.01)$. The changing of TBS did not correlated to that of the chest radiologic score for EVLW assessment $(r=0.002, P>0.05)$. There was no linear correlation observed between net fluid balance and total number of increasing B-lines.

Conclusion The number of B-lines definitely increased after fluid resuscitation in shock and correlated to the deterioration of pulmonary gas exchange. These data support the benefit of transthoracic portable ultrasound for assessment of the increment of EVLW in shock patients receiving fluid resuscitation.

References

1. Bouhemad B, Zhang M, Lu Q, et al: Clinical review: Bedside lung ultrasound in critical care practice. Crit Care 2007, 11:205.

2. Soldati G, Copetti R, Sher S: Sonographic interstitial syndrome: the sound of lung water. J Ultrasound Med 2009, 28:163-174.

\section{P249}

Fluid therapy tactics in patients with polytrauma during interhospital transportation

D Skopintsev, S Kravtsov, A Shatalin, V Agadzhanyan

Federal State Budgetary Medical Prophylactic Institution, Scientific Clinical

Center of Miners' Health Protection, Leninsk-Kuznetsky, Russia

Critical Care 2012, 16(Suppl 1):P249 (doi: 10.1186/cc10856)

Introduction The study's aim was to carry out a comparative evaluation of fluid therapy's influence using 130/04 hydroxyethyl (HES) starch and dextrans on hemodynamics values in patients with traumatic shock in polytrauma during interhospital transportation.

Methods Eighty patients with polytrauma were included in the study. Mean age was $35 \pm 1$ years. All patients were divided into two similar groups: experimental (EG) and control (CG). Each group was apportioned by two subgroups depending on the shock severity. Subgroup 1 consisted of patients with degree I shock, subgroup 2 comprised patients with degree II shock. The Algover-Burry index was used to evaluate the shock severity. ISS was applied to determine the injuries' severity. The injuries' severity values of the EG were the following: in subgroup 1, $25 \pm 1$ points; in subgroup $2,46 \pm 2$ points. The values of the CG were $26 \pm 1$ in the first subgroup and $44 \pm 2$ points in the second subgroup. All patients were transported during the first 
24 hours after trauma. The distance was $177 \pm 9 \mathrm{~km}$. The components of the fluid therapy in the CG were crystalloids and dextrans. The latter were not used in degree I shock. Crystalloid infusion was carried out on the basis of $3 \mathrm{ml}$ crystalloids per $1 \mathrm{ml}$ blood loss. The crystalloids and HES 130/04 starch were used in the EG. The dose of HES 130/04 starch comprised 10 to $25 \mathrm{ml} / \mathrm{kg}$ of the body mass and depended on the shock severity state. Statistical analysis was performed using Statistica 6.1. We used the Mann-Whitney criterion.

Results The EG patients with degree I shock had higher hemodynamics parameters (BPsys, BPdias, MAP) and less expressed tachycardia as compared to the CG patients with degree I shock $(P<0.05)$. The EG patients with degree II shock had higher hemodynamics parameters (BPsys, MAP, ESV, Cl, SVR) as compared to the CG patients with degree II shock $(P<0.05)$. The change of the fluid therapy tactics in the EG resulted in the normalization of the HR, SVR and in the increase of the BPsys, MAP, ESV and $\mathrm{Cl}$ in patients of both degrees of shock during transportation. The values of the EG were higher than in the CG during all periods of the transportation $(P<0.05)$.

Conclusion Inclusion of the HES 130/04 starch in the fluid therapy complex of the patients with traumatic shock in polytrauma allows one to normalize hemodynamics values at short notice and to support them adequately during all periods of transportation.

\section{P250}

A prospective, randomized, clinical trial comparing the hemodynamics, efficacy, and safety of $6 \%$ hydroxyethyl starch $130 / 0.4$ compared to albumin in postoperative patients undergoing pancreaticoduodenectomy

SK Hong ', K Kyoung' ${ }^{2}$, Y Kim'1, S Kim

'Ulsan University College of Medicine, Asan Medical Center, Seoul, South

Korea; 'Inje University College of Medicine, Harundae Paik Hospital, Busan, South Korea

Critical Care 2012, 16(Suppl 1):P250 (doi: 10.1186/cc10857)

Introduction Hypovolemia is often present in patients undergoing extensive abdominal surgery. As the first colloid used in the clinical setting, albumin is still widely employed during perioperative periods. We hypothesized that $6 \%$ hydroxyethyl starch (HES) 130/0.4 is equally efficacious and has the added advantages of its low cost and convenience of use. This study's objective is to compare the hemodynamics, efficacy, and safety of HES 130/0.4 compared with that of albumin.

Methods This study was a prospective, randomized, active-controlled study comparing the hemodynamics, efficacy, and safety of HES 130/0.4 to that of albumin in patients undergoing pancreaticoduodenectomy. Eligible adult patients of both sexes were assigned following the surgery into either the HES group or the albumin group at a ratio of 1:1. Crystalloids for hydration and colloid therapy for volume support were administered. The primary endpoint of this study was the hemodynamic evaluation. Secondary endpoints were measurement of the inputoutput, ICU stay, ventilation time, length of hospital stay, time to liquid mealtime and the use of blood products. Safety assessment was carried out by performing physical examination, laboratory examination, and assessment of any adverse events during the study period.

Results A total of 50 patients were randomized to study groups ( 25 each). The volume of the crystalloid was the same in both groups; however, significantly more colloids were infused after 24 hours post surgery in the HES group than in the albumin group, the voluven patient group had lower heart rates, and the difference in the lowest MAP value was $-1.64 \mathrm{mmHg}$ (lower limit of confidence interval, $-8.228 \mathrm{mmHg}$ ) than in the albumin group. Routine hematology and biochemical profiles, including blood coagulation test and renal function assessment, were comparable in the two groups. The mean duration of the ICU stay, ventilation, hospital stay, and tolerance of a liquid meal were similar. The mean cost of the colloid was significantly lower in the HES 130/0.4 group than in the albumin group $(P<0.001)$.

Conclusion This study demonstrated that $6 \%$ HES 130/0.4 may be used as a valuable alternative to $5 \%$ albumin in patients undergoing extensive abdominal surgery, as its low cost is also of value.
P251

Effect of balanced versus unbalanced HES solution on cytokine response in a rat model of peritonitis

M Schläpfer', M Urner', S Voigtsberger', R Schimmer², B Beck-Schimmer ${ }^{1}$

'University Hospital Zurich, Switzerland; 'University of Zurich, Switzerland

Critical Care 2012, 16(Suppl 1):P251 (doi: 10.1186/cc10858)

Introduction Sepsis with multiple organ failure remains a leading cause of death in ICUs. Acute renal failure is a common complication of severe sepsis and septic shock. The effect of hydroxyethyl starch (HES) on the kidney as well as on liver tissue remains controversial and has never been tested in detail. We investigated in a model of fecal peritonitis the influence of fluid resuscitation with HES $6 \%$ in unbalanced versus balanced solutions on inflammatory mediator expression in renal and hepatic tissue.

Methods Cecal ligation and puncture was performed in anesthetized Wistar rats (CLP group). Sham group animals were treated in the same manner but without CLP. One hour after this procedure, Ringer lactate (RL) was intravenously infused to all animals at a volume of $30 \mathrm{ml} / \mathrm{kg}$. Two hours after initiation of injury rats received RL (control, $75 \mathrm{ml} / \mathrm{kg}$ ), unbalanced HES 130/0.42 (HES, $25 \mathrm{ml} / \mathrm{kg}$ ) or balanced HES 130/0.42 (Tetraspan, $25 \mathrm{ml} / \mathrm{kg}$ ). Animals were euthanized 4 hours after induction of peritonitis. Monocyte chemotactic protein-1, intercellular adhesion molecule-1, and TNFa mRNA expression were assessed in the kidneys and liver. Linear regression was used to evaluate influence of the different fluid resuscitation procedures on inflammatory mediator expression.

Results CLP had a significant effect on production of inflammatory mediators in the kidneys $(P \leq 0.03)$ and liver $(P \leq 0.02)$. While HES did not alter expression of inflammatory mediators compared to $R L$, fluid resuscitation with Tetraspan provoked a burst in inflammatory mediator expression, which was at least threefold higher in the kidneys $(P<0.001)$ and eightfold in the liver $(P=0.001)$ compared to the $\mathrm{RL}$ group.

Conclusion While unbalanced HES did not show a proinflammatory effect on renal and hepatic tissue in early sepsis, the balanced HES solution upregulated inflammatory mediators. Further studies have to be performed to elucidate this phenomenon in detail and to assess the functional implication of these results.

P252

Evaluation of effectiveness and safety of hydroxyethyl starch (HES $130 \mathrm{kDa} / 0.4$ ) in burn resuscitation

A Mokline, I Rahmani, L Gharsallah, H Oueslati, B Gasri, I Harzallah,

A Ksontini, A Messadi

Burn and Trauma Center, Tunis, Tunisia

Critical Care 2012, 16(Suppl 1):P252 (doi: 10.1186/cc10859)

Introduction Excessive fluid resuscitation of large burn injuries has been associated with adverse outcomes including worsening of burn oedema, conversion of superficial into deep burns, and compartment syndromes. So, there have been efforts recently to address these concerns, particularly with the use of physiologically balanced fluids. Starches, as effective plasma expanders, may limit resuscitation requirements and burn oedema. This study aims to evaluate clinical results of HES in early burn resuscitation of major burn-injured patients. Methods A case-control study conducted in a burn care center in Tunis. Adult burned patients admitted within the first 24 hours post burn, with a burn injury exceeding $30 \%$ of total body surface area, from 1 January to 31 December 2010 were included. Exclusion criteria were pregnancy, history or biochemical evidence of renal impairment on admission (serum creatinine $>130 \mu \mathrm{mol} / \mathrm{l}$ ), history or hematological evidence of disorders of hemostasis. Fluid volume resuscitation was evaluated according to the Parkland formula. HES supplementation was limited to $33 \mathrm{ml} / \mathrm{kg} / 24$ hours. The HES supplementation group was compared with a group of patients from the same center matched in age, sex and severity of burns at baseline.

Results Patients were assigned to two groups: G1 $(n=15)$ : HES supplemented, and G2 ( $n=15)$ : crystalloids only. The mean age was $44 \pm 18$ years old for $G 1$ and $43 \pm 17$ years old for $G 2$. The average TBSA was $51.8 \% \pm 19$ for $\mathrm{G} 1$ versus $43.6 \pm 7$ for $\mathrm{G} 2$. The addition of 
HES $130 \mathrm{kDa} / 0.4$ reduces significantly body weight gain within the first 72 hours after injury: $8 \mathrm{~kg}$ for $\mathrm{G} 1$ versus $13.6 \mathrm{~kg}$ for $\mathrm{G} 2(P=0.002)$, occurrence of $\mathrm{ALI}$ ( $35 \%$ for $\mathrm{G} 1$ versus $65 \%$ for $\mathrm{G} 2)(P=0.01)$, and length of ICU stay ( 19 days \pm 13 for $G 1$ vs. 30 days \pm 15 for $G 2$ ). There was no evidence of renal dysfunction with the use of HES in burns patients comparative to the crystalloids group.

Conclusion HES supplementation in early burn resuscitation allows, for smaller fluid volume requirement, less tissue oedema. This along with a significantly lower in ALI occurrence and length of ICU stay.

\section{P253}

Normal saline resuscitation worsens lactic acidosis in experimental sepsis

F Zhou, ME Cove, ZY Peng, J Bishop, K Singbartl, JA Kellum

University of Pittsburgh Medical School, Pittsburgh, PA, USA

Critical Care 2012, 16(Suppl 1):P253 (doi: 10.1186/cc10860)

Introduction Infusing large volumes of $0.9 \%$ sodium chloride (saline) causes hyperchloremic acidosis. The clinical relevance of this effect remains contentious and saline is still the most commonly used resuscitation fluid in the US. However, a recent trial showed that saline or albumin in saline increased mortality in children with malarial sepsis, compared to no fluid [1]. Infusion of these fluids may have perpetuated the underlying metabolic acidosis sepsis, causing cardiovascular collapse and death. In this study, we investigated the effect of saline versus a balanced crystalloid (plasmalyte) in a cecal ligation and puncture (CLP) model of sepsis. We hypothesized that saline resuscitation would increase acidosis and worsen hemodynamics, compared to resuscitation using a balanced crystalloid.

Methods Fifty adult male Sprague-Dawley rats were subjected to CLP ( $25 \%$ cecum length, two punctures with a 25 -gauge needle). Eighteen hours later, they were randomly assigned to receive either $30 \mathrm{ml} / \mathrm{kg}$ saline $(n=25)$ or plasmalyte $(n=25)$ over 4 hours. Arterial blood gases, serum creatinine, urea, and lactate were measured at baseline, 18 hours after CLP (before resuscitation), after resuscitation, and 24 hours after resuscitation. Blood pressure and pulse rate were measured during fluid infusion.

Results Saline-treated animals developed significantly higher levels of serum chloride $(111 \mathrm{mmol} / \mathrm{l}$ vs. $102 \mathrm{mmol} / \mathrm{l}, P<0.0001)$ and lower $\mathrm{pH}$ (7.35 v. $7.44, P<0.01$ ) compared to plasmalyte. In addition, lactate was significantly higher after fluid infusion in the saline group $(4.8 \mathrm{mmol} / \mathrm{l}$ vs. $2.5 \mathrm{mmol} / \mathrm{l}, P<0.001)$ compared to plasmalyte, despite being similar before infusion ( 2.61 vs. $2.39, P>0.05)$. However, neither mean arterial blood pressure ( $83 \mathrm{mmHg}$ vs. $91 \mathrm{mmHg}, P>0.10$ ) nor heart rate (310 vs. $299, P>0.10)$ differed between the two groups.

Conclusion Saline infusion worsens lactic acidosis, despite similar blood pressure, when compared to plasmalyte. The mechanisms responsible for this effect are unclear. However, deoxygenated hemoglobin readily binds hydrogen ions, forming $\mathrm{HbH}^{+}$, which is stabilized in the presence of chloride [2]. Consequently, the oxygen affinity for hemoglobin is reduced, which could impair oxygen delivery, perpetuating the lactic acidosis. Further study is needed to better understand the mechanisms of this effect and their clinical relevance.

References

1. Maitland K, et al:: N Engl J Med 2011, 364:2483-2495.

2. Prange HD, et al.: J Appl Physiol 2001, 91:33-38.

\section{P254}

Albumin in early septic shock resuscitation: examination of plasma and urine inflammatory markers

A Fox-Robichaud', C Leger ${ }^{2}$, KD Burns ${ }^{3}$, E Sabri³, B Lo' 1 , P Kubes²,

LA McIntyre ${ }^{3}$

'McMaster University, Hamilton, Canada; '2University of Calgary, Canada;

${ }^{3}$ Ottawa Hospital Research Institute, Ottawa, Canada

Critical Care 2012, 16(Suppl 1):P254 (doi: 10.1186/cc10861)

Introduction A recent meta-analysis has suggested that albumin may be beneficial in sepsis; however, there is no clear biological rationale for the pharmacological use of this negative acute-phase protein. Our objective was to describe the temporal production of plasma and urine biomarkers in a pilot study of early septic shock patients resuscitated with either $5 \%$ albumin or normal saline.

Methods Patients presenting in early septic shock received albumin or saline in a randomized, double-blind pilot study. Blood and urine was collected at enrolment and 6, 12, 24, 72 hours and 7 days later and processed using standard operating procedures. A panel of 27 cytokines, chemokines and growth factors was measured by multiplex technology. Mean values were separated by treatment and analyzed using $\mathrm{R}$ to generate heat maps, by principal component analysis (PCA) and hierarchal clustering. Urinary neutrophil gelatinase-associated lipocalin (NGAL) was measured by ELISA.

Results Twenty-five patients (median age 66 years, median APACHE II score 26) received albumin (median amount $3 \mathrm{I}$ ) and 21 (median age 62 years, median APACHE II score 22) received normal saline (median amount $3.5 \mathrm{I}$ ) as study fluid over 7 days. PCA revealed that $60 \%$ of the variance in the chemokines was accounted for with the first two components. Analyzing the first component using a threshold of greater than 0.5 or -0.5 we saw a clustering of IL-17, IL-12p70, IL-9 and IL-5. Heat map analysis suggests that by 72 hours albumin-resuscitated patients are distinguished by the cluster of IL-17, IL-9 and II-12p70 and VEGF when compared to saline. Hierarchal clustering also separates IL-17, IL-19, IL-12p70 and IL-2 in the albumin-treated patients but not the saline-treated patients at 72 hours. At enrolment, mean urine NGAL levels were greater than 1,000 $\mathrm{ng} / \mathrm{ml}$ (albumin 1,121 $\pm 2,172$ $(n=21)$, saline $1,375 \pm 3,197(n=17))$. Over the next 24 hours there was a marked increase in urine NGAL in the saline-resuscitated patients, peaking at $5,793 \pm 15,948 \mathrm{ng} / \mathrm{ml}$, whereas levels remain blunted over the first 12 hours, peaking at 2,216 $\pm 3,177 \mathrm{ng} / \mathrm{ml}$ at 24 hours in the albumin group.

Conclusion In this cohort of patients treated with albumin or saline in early septic shock, there appeared to be a marked increase in the clustering of early T-cell-mediated immune responses. Also striking was the blunted rise in urine NGAL over time for patients in the albumin fluid group. These results should be considered hypothesis generating and prompt further studies to explore possible biological mechanisms for albumin resuscitation in sepsis.

P255

Study of the correlation between central venous oxygen saturation and venous saturation from the antecubital vein in severe sepsis/ septic shock patients

K Piyavechviratana, W Tangpradubkiet

Phramongkutklao Hospital, Bangkok, Thailand

Critical Care 2012, 16(Suppl 1):P255 (doi: 10.1186/cc10862)

Introduction Early goal-directed therapy has been used for severe sepsis and septic shock in the ICU to achieve a balance between systemic oxygen delivery and oxygen demand before global tissue hypoxia develops and proceeds to multiorgan failure. One of the resuscitation end points includes normalized values for central venous oxygen saturation $\left(\mathrm{ScvO}_{2}\right)$ that needs insertion of a central venous catheterization, which is still impractical in small-to-medium-sized hospitals in Thailand. The purpose of this study was to examine whether the venous oxygen saturation from the antecubital vein has correlation with the central venous oxygen saturation or can be applied instead of the central venous oxygen saturation.

Methods This was an observational study performed during 4 July 2007 to 31 March 2009 in the 10-bed ICU of Pramongkutklao Hospital in severe sepsis or septic shock patients who already had a central venous catheter inserted. Two blood samples were collected and sent to the laboratories for blood gas analysis. We then calculated for the correlation using correlation and linear regression analysis.

Results Of the 44 enrolled patients, 24 were males (54.54\%). Mean age was around $69.86 \pm 16.819$ years. A total of $84.1 \%$ was in septic shock. The most common source of infection was pneumonia (38.6\%). The central $\mathrm{ScvO}_{2}$ and peripheral venous oxygen saturation ranges and means were 46.0 to $93.2 \%, 31.5$ to $99.0 \%$ and $71.66 \pm 10.39 \%$, $71.18 \pm 19.79 \%$ respectively. The correlation between $\mathrm{ScvO}_{2}$ and antecubital venous oxygen saturation significant $P$ value was 0.000 : calculated $\mathrm{ScvO}_{2}=52.386+0.271$ (peripheral), $R^{2}=0.266$. The specificity, sensitivity, positive predictive value and negative predictive value of 


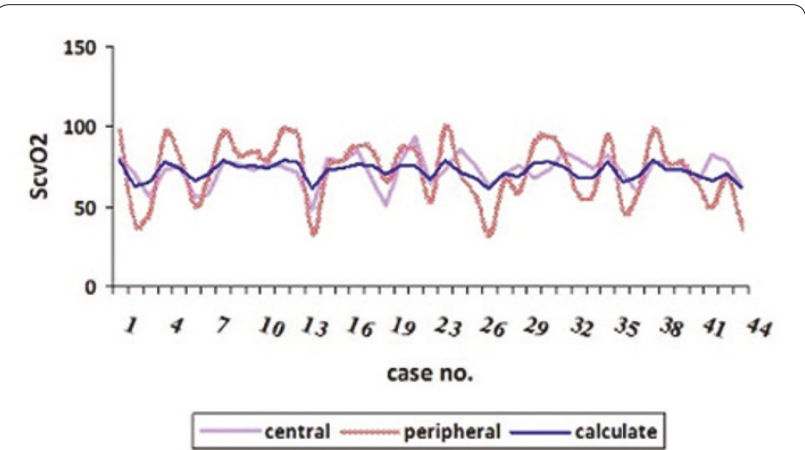

Figure 1 (abstract P255). Central and peripheral oxygen saturation range.

the predicting equation were $69.23 \%, 77.41 \%, 85.71 \%$ and $56.25 \%$ respectively. The accuracy was $75 \%$. See Figure 1.

Conclusion Venous oxygen saturation from the antecubital vein was not the exact value of central venous oxygen saturation but there were significant correlations.

P256

Central venous hyperoxia is related to changes in tissue perfusion and morbi-mortality of patients in shock

G Friedman', A Do Canto², D Araujo ${ }^{2}$

'UFRGS, Porto Alegre, Brazil; '2Complexo Hospitalar Santa Casa, Porto Alegre, Brazil

Critical Care 2012, 16(Suppl 1):P256 (doi: 10.1186/cc10863)

Introduction Mixed or central venous hyperoxia is associated with organ dysfunction and worse mortality. Venous hyperoxia may reflect altered tissue oxygen extraction. We aim to assess the relationship between central venous hyperoxia $\left(\mathrm{ScvO}_{2}\right)$ and markers of tissue perfusion; and to evaluate the relationship between central venous hyperoxia and morbidity.

Methods The setting was a university general ICU with 18 beds. The population was adult patients (age $>18$ years) in circulatory shock. Blood lactate, arterial and central venous blood gases were collected on admission to the study and after 6, 12, 18 and 24 hours of shock. Venous hyperoxia was defined as a $\mathrm{ScvO}_{2} \geq 85 \%$. The severity of the patients was assessed using the APACHE II score on admission to the study. Mortality was evaluated in the ICU and after 28 days.

Results Preliminary data from 40 patients (205 measurements) are presented. Mean blood lactate levels were higher (3.2 vs. $2.3 \mathrm{mmol} / \mathrm{l}$ ), the mean venoarterial $\mathrm{CO}_{2}$ difference was lower $(4.7$ vs. $5.8 \mathrm{mmHg})$, and the mean base deficit was greater (11 vs. $8 \mathrm{mEq} / \mathrm{l})$ for patients with venous hyperoxia at any time. Mean APACHE II score was higher (28 vs. 24) for patients with venous hyperoxia. The ICU mortality was higher $(4 / 5(80 \%)$ vs. $17 / 35(46 \%))$ among patients who already had venous hyperoxia at time 0 . The proportion of death remained the same in the next day among patients that persisted or developed venous hyperoxia in the following 18 hours.

Conclusion These preliminary data suggest that the presence of central venous hyperoxia is associated with persistent changes in perfusion. The presence of venous hyperoxia at both the onset of shock and in the following hours is associated with a worse clinical outcome.

\section{Reference}

1. Pope JV, et al:: Multicenter study of central venous oxygen saturation $\left(\mathrm{ScvO}_{2}\right)$ as a predictor of mortality in patients with sepsis. Ann Emerg Med 2010, 55:40-46.
P257

Curve analysis of tissue oxygen desaturation after a venous occlusion test does not identify the central venous hemoglobin oxygen saturation

G Friedman', C Alan², A Meregalli², A Lima³, J Bakker³

'UFRGS, Porto Alegre, Brazil; ${ }^{2}$ Complexo Hospitalar Santa Casa, Porto Alegre,

Brazil; ${ }^{3}$ University Medical Center Erasmus, Rotterdam, the Netherlands

Critical Care 2012, 16(Suppl 1):P257 (doi: 10.1186/cc10864)

Introduction We aim to compare the time for the equivalence of tissue oxygen saturation $\left(\mathrm{StO}_{2}\right)$ with central venous hemoglobin oxygen saturation $\left(\mathrm{ScvO}_{2}\right)$ measured at depths of 15 and $25 \mathrm{~mm}$.

Methods Twenty-one critically ill patients were included. The $\mathrm{ScvO}_{2}$ was measured by blood gas analysis. Thenar $\mathrm{StO}_{2}$ was continuously monitored (Model 650 InSpectra Tissue Spectrometer; Hutchinson Technology Inc., MN, USA) in $15 \mathrm{~mm}\left(\mathrm{StO}_{2-} 15\right)$ and $25 \mathrm{~mm}\left(\mathrm{StO}_{2} 25\right)$ depths. The venous occlusion was performed using an automatic pneumatic device maintaining inflation pressure $10 \mathrm{mmHg}$ above the diastolic pressure. $\mathrm{A} \mathrm{StO}_{2}$ desaturation curve was plotted to identify the time for equivalence to $\mathrm{ScvO}_{2}$.

Results Age: $59 \pm 17$ years, APACHE II score: $21 \pm 7$, SOFA score: $7 \pm 4$, $\mathrm{ScvO}_{2}: 75 \pm 6 \%$, blood lactate: $1.6 \pm 1.2 \mathrm{mmol} / \mathrm{l}$, capillary refill time: $9.1 \pm 8.1$ seconds, body temperature: $36.7 \pm 1.1^{\circ} \mathrm{C}$. Measurements were performed for up to three consecutive days (total measurements: 43). In four patients the equivalence was not identified. The curve analysis showed that $\mathrm{StO}_{2}$ desaturation time equivalency for $\mathrm{ScvO}_{2}$ was greater for $\mathrm{StO}_{2-} 15$ than for $\mathrm{StO}_{2-} 25$ (88 \pm 54 seconds vs. $79 \pm 56$ seconds, $P<0.01)$. The Pearson correlation index for equivalence times for $\mathrm{StO}_{2-} 15$ and $\mathrm{StO}_{2} 25$ was $0.92(P<0.001)$, but Bland-Altman analysis showed a significant difference between the times (mean difference: $\mathrm{StO}_{2} 25-\mathrm{StO}_{2} 25:-7.9 \pm 37.8$ seconds). An arbitrary time of 80 seconds identifies the $\mathrm{ScvO}_{2}$ in $58 \%$ of cases.

Conclusion The analysis of the $\mathrm{StO}_{2}$ desaturation curve does not adequately identify the hemoglobin central venous oxygen saturation. References

1. Lima A, et al:: The relation of near-infrared spectroscopy with changes in peripheral circulation in critically ill patients. Crit Care Med 2011, 39:1649.

2. Bezemer R, et al:: Assessment of tissue oxygen saturation during a vascular occlusion test using near-infrared spectroscopy: the role of probe spacing and measurement site studied in healthy volunteers. Crit Care 2009, 13(Suppl 5):S4.

3. Lima A, et al.: Low tissue oxygen saturation at the end of early goaldirected therapy is associated with worse outcome in critically ill patients. Crit Care 2009, 13(Suppl 5):S13.

P258

Lactate in burn patients: biomarker of sepsis and mortality

A Mokline, L Gharsallah, A Abdenneji, H Oueslati, I Rahmani, B Gasri,

I Jami, A Ghanem, A Messadi

Burn and Trauma Center, Tunis, Tunisia

Critical Care 2012, 16(Suppl 1):P258 (doi: 10.1186/cc10865)

Introduction In this study, we attempted to assess whether the early plasma lactate $(\mathrm{PL})$ level is a useful biomarker to predict septic complications and outcome in burn patients.

Methods A retrospective study was conducted in the burn care center in Tunis. Patients admitted within 24 hours from the thermal injury, from 1 January 2009 to 30 June 2010, were included. PL was measured early in the first 24 hours and controlled more than twice. For each measurement, $5 \mathrm{ml}$ venous blood was drawn into a heparin-coated syringe. The normal lactate value was defined as $1 \pm 0.5 \mathrm{mmol} / \mathrm{l}$.

Results Over an 18-month period of study, 80 patients were enrolled. There were 60 males and 20 females. The mean age was $40.7 \pm 19.5$ and the average TBSA was $32 \pm 21 \%$. Upon admission, patients with an initial lactate value of more than $2 \mathrm{mmol} / \mathrm{l}$ were $86.7 \%$. Fifty-eight percent of them have a lactate initial value of more than $4 \mathrm{mmol} / \mathrm{l}$. In order to evaluate the potential impact of using early lactate measurements (H24 post burn injury) as predictor biomarker of sepsis in burn patients, a linear discrimination function was performed, by measuring the area under the ROC curve, and found that initial lactate value of more than $4 \mathrm{mmol} / \mathrm{l}$ provides the best sensitivity and specificity: $88 \%$ and $79 \%$ 
respectively. Also, the PL cut-off value for prediction of mortality was $4 \mathrm{mmol} / \mathrm{l}$ with a good sensitivity (86\%) and specificity (92\%). The area under the ROC curve was 0.96 .

Conclusion Lactate appears to be a powerful predictor biomarker of sepsis and mortality in burn patients. A serum lactate of $4 \mathrm{mmol} / \mathrm{l}$ provides the best sensitivity and specificity.

\section{P259}

Can we predict arterial lactate from venous lactate in the emergency department?

A Mikami', S Ohde'2, G Deshpande², T Mochizuki', N Otani', S Ishimatsu

'St Luke's International Hospital, Tokyo, Japan; 'St Luke's Life Science Institute,

Tokyo, Japan

Critical Care 2012, 16(Suppl 1):P259 (doi: 10.1186/cc10866)

Introduction Analysis of arterial blood has an important role in the clinical assessment of critically ill patients. Particularly, measured arterial lactate (a-Lac) provides valuable information on peripheral circulatory failure, although it is invasive and frequent measurement is often impractical. The aim of this study is to clarify the relationship between a-Lac and the more easily accessed venous lactate ( $v$-Lac) and to generate a formula to predict a-Lac using v-Lac and other laboratory data.

Methods A prospective cohort study was conducted from June to November 2011 in the emergency department at a tertiary-level community hospital in Tokyo, Japan. Patients were eligible for entry into the study if an arterial blood gas (ABG) analysis was required for appropriate diagnostic care by the treating physician. Arterial and venous samples were taken within 5 minutes of each other from the ipsilateral radial artery and cephalic vein. Samples were analyzed as soon as possible after collection on the same blood gas analyzer. Univariate linear regression analysis was conducted to generate an equation to calculate a-Lac incorporating only v-Lac. Then, a multivariate forward stepwise logistic regression model ( $P$ value of 0.05 for entry, 0.1 for removal) was used to generate an equation including $\mathrm{v}$-Lac and other potentially relevant variables including age, sex, systolic blood pressure, heart rate, and venous blood parameters $(\mathrm{pH}$, $\mathrm{pO}_{2}, \mathrm{pCO}_{2}$, hemoglobin, creatine kinase, potassium). A Bland-Altman plot was drawn and the two equations were compared for model fitting using R-squared.

Results Seventy-two arterial samples from 72 patients $(61 \%$ male; mean age, 58.2 years) were included in the study. Indications for ABG included respiratory failure (16\%), assessment of shock (21\%), altered mental status (26\%), and others (36\%). An initial regression equation was derived from univariate linear regression analysis: (a-Lac) $=-0.259$ $+(v-L a c) \times 0.996$. Subsequent multivariate forward stepwise logistic regression analysis, incorporating venous lactate and venous $\mathrm{pO}_{2}$ $\left(\mathrm{v}-\mathrm{pO}_{2}\right)$, generated the following equation: $(\mathrm{a}-\mathrm{Lac})=-0.469+(\mathrm{v}-$ $\left.\mathrm{pO}_{2}\right) \times 0.005+(\mathrm{v}$-Lac $) \times 0.997$. Calculated R-squared values by single and multiple regression were 0.94 and 0.96 , respectively.

Conclusion Venous lactate estimates showed a high correlation with arterial values and our data provide two clinically useful equations to calculate a-Lac from v-Lac data. Considering clinical flexibility, Lac $=-0.259+$ VLac $\times 0.996$ might be more useful, while avoiding a time-consuming and invasive procedure.

\section{P260}

Cross-correlation analysis of blood and microdialysis-assessed tissue lactate monitoring: a study in critically ill septic patients I llias'1, P Kopterides², N Nikitas², D Vassiliadi², M Theodorakopoulou², E Papadomichelakis², M Lygnos², A Flevari², M Rizos², F Frantzeskaki², C Diakaki², E Paramythiotou², E Dimitriadou'², S Orfanos², A Armaganidis², I Dimopoulou²

'Elena' Hospital, Athens, Greece; 'Attiko' University Hospital, Haidari, Athens, Greece

Critical Care 2012, 16(Suppl 1):P260 (doi: 10.1186/cc10867)

Introduction In the critical care setting, blood lactate (BL) concentration is measured to assess - albeit indirectly - tissue oxygenation. In addition, serial $B L$ measurements are clinically useful since a drop in $B L$ is a good prognostic finding, whereas persistent BL elevation portends poor outcome. Microdialysis (MD) enables direct monitoring of tissue metabolic changes. This study aimed to describe the dynamics of $\mathrm{MD}$-assessed tissue lactate (TL) vis-à-vis BL in septic patients with and without shock.

Methods We measured BL and thigh adipose tissue TL serially every 4 hours for 6 days in 88 patients with septic shock and 45 patients at various sepsis stages hospitalized in a tertiary-care hospital ICU. Analysis was done with measurement of the area under the curve (AUC) of lactate*hours, cross-approximate entropy (X-ApEn) and cross-correlation. Comparisons of septic shock versus nonseptic shock patients' results were done with $t$ tests and $z$ statistics.

Results BL and TL were higher in septic shock patients compared to nonseptic shock patients (AUCs of $276 \mathrm{vs} .176$ and $355 \mathrm{vs.} 273 \mathrm{mmol} /$ I*hours, respectively; Welch's $t$ test: $P<0.0001$ ). X-ApEn for MDL/BL was lower in septic shock patients compared to those without septic shock (mean \pm SD: $0.79 \pm 0.12$ vs. $1.14 \pm 0.13$, respectively; $t$ test: $P<0.0001$ ). Cross-correlation of TL versus BL was stronger in septic shock patients, with TL leading BL by 4 hours compared to TL versus BL with no lag time ( $r=+0.85, P<0.0001$ and $r=+0.66, P<0.0001$, respectively) than in nonseptic shock patients $(r=+0.58, P=0.0003$ with TL leading BL by 4 hours and $r=+0.66, P<0.0001$ with no lag time; $z$ statistic $=2.41$ and $P=0.016$ for leading $B L$ compared to $z$ statistic $=0.036, P=0.971$ for no lag time).

Conclusion In septic shock patients, tissue lactate levels - measured by $\mathrm{MD}$ - are higher compared to nonseptic shock patients. Furthermore, TL is better correlated with and precedes - within 4 hours - BL in septic shock patients compared to nonseptic shock patients. Further studies are warranted to assess the clinical value of serial TL monitoring.

\section{P261}

Admission lactate and outcome after high-risk surgery

M Geisen, HD Aya, C Ebm, N Arulkumaran, MA Hamilton, M Grounds, A Rhodes, M Cecconi

St George's Hospital NHS Trust, London, UK

Critical Care 2012, 16(Suppl 1):P261 (doi: 10.1186/cc10868)

Introduction The aim of this study was to assess the ability of serum lactate level in patients admitted to the ICU after surgery to predict outcome.

Methods A retrospective, clinical observational study in patients undergoing high-risk surgery admitted to a 17-bed ICU of a large teaching hospital. Data were obtained during haemodynamic optimization using an established GDT protocol in the first 8 hours after admission and included demographic data as well as haemodynamic and laboratory parameters. Outcome data included morbidity (defined as $>1$ complications on the postoperative morbidity survey) and clinical outcome (hospital mortality, length of ICU stay, length of hospital stay, readmission to the $I C U$ ).

Results Sixty-seven patients were included. Lactate clearance (decrease of lactate $>10 \%$ in 2 hours) occurred in 64 patients (96\%). Sixty patients developed at least one surgical complication. There were no significant correlation between lactate levels on admission and development of

Table 1 (abstract P261). Lactate on admission, complications and clinical outcome

\begin{tabular}{|c|c|c|c|}
\hline & $\begin{array}{l}\text { Lactate } \\
<1.7 \mathrm{mmol} \\
(n=46)\end{array}$ & $\begin{array}{l}\text { Lactate } \\
>1.7 \mathrm{mmol} \\
(n=21)\end{array}$ & $P$ value \\
\hline Complications & $41(89 \%)$ & $19(90.5 \%)$ & NS \\
\hline Complications $>1$ & $36(78.3 \%)$ & $16(76.2 \%)$ & NS \\
\hline Total complications per patient & $3(2$ to 7$)$ & $4(2$ to 7$)$ & NS \\
\hline Hospital stay & 14 (8 to 39$)$ & 13 (8 to 24$)$ & NS \\
\hline ICU stay & 1 (1 to 2$)$ & $2(1$ to 10$)$ & 0.045 \\
\hline Readmission to ICU & $3(6.5 \%)$ & $6(28.6 \%)$ & 0.022 \\
\hline Mortality & $2(4.3 \%)$ & $3(14.3 \%)$ & NS \\
\hline
\end{tabular}




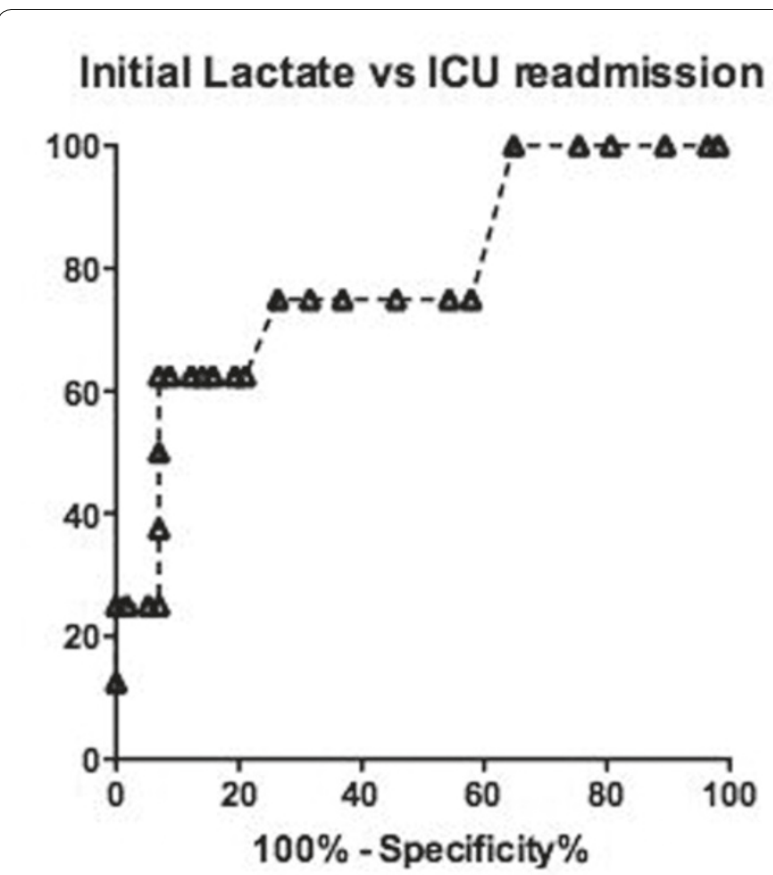

Figure 1 (abstract P261). Prediction of ICU readmission according to initial lactate concentration.

complications and length of hospital stay. Nine patients (13\%) were readmitted to the ICU. A receiving operator characteristic analysis for readmission to the ICU showed an area under the curve of 0.79. A lactate higher than $1.7 \mathrm{mmol} / \mathrm{l}$ on admission had a sensitivity of $75 \%$ and a specificity of $74 \%$ to predict ICU readmission (Figure 1). Patients with a lactate on admission $>1.7 \mathrm{mmol} / \mathrm{l}$ also had a longer length of ICU stay (Table 1).

Conclusion Lactate on admission correlates with length of ICU stay and readmission to the ICU.

References

1. Pearse R, et al:: Crit Care 2005, 9:R687-R693.

2. Jansen TC, et al: Am J Respir Crit Care Med 2010, 182:752-761.

P262

Effects of induced relative hypoxia during the postoperative period of abdominal oncologic surgery, on hemoglobin and reticulocyte levels: a prospective, randomized controlled clinical trial

M Khalife', KWiams'1, M Ben Aziz', M Paesmans'1, C Balestra², M Sosnowski' 'Institut Jules Bordet, Brussels, Belgium; ${ }^{2}$ Divert Alert Network Europe Research Division, Brussels, Belgium

Critical Care 2012, 16(Suppl 1):P262 (doi: 10.1186/cc10869)

Introduction Anemia is a frequent complication in oncologic patients. Erythropoietin (EPO) stimulating agents are known as alternatives to transfusion. However, they expose patients to thrombosis and are expensive. Recently, a new phenomenon, the normobaric oxygen paradox (NOP), has been described. In brief, transient hyperoxia followed by a prolonged return to normoxia acts as an effective trigger for EPO production. The mechanism depends on free oxygen radicals and on reduced glutathione (GSH) availabilities. Also, $\mathrm{N}$-acetylcystein (NAC) is known to regenerate the stock of GSH. Very few clinical trials have investigated this phenomenon [1]. The goal of this study was to test the NOP theory on the evolution of hemoglobin and reticulocytes in patients receiving intermittent oxygen with or without NAC compared to a control group.

Methods This prospective, randomized study included 78 patients (three groups). The first group $(\mathrm{G} 1 ; n=26)$ received $60 \% \mathrm{FiO}_{2}$ for 2

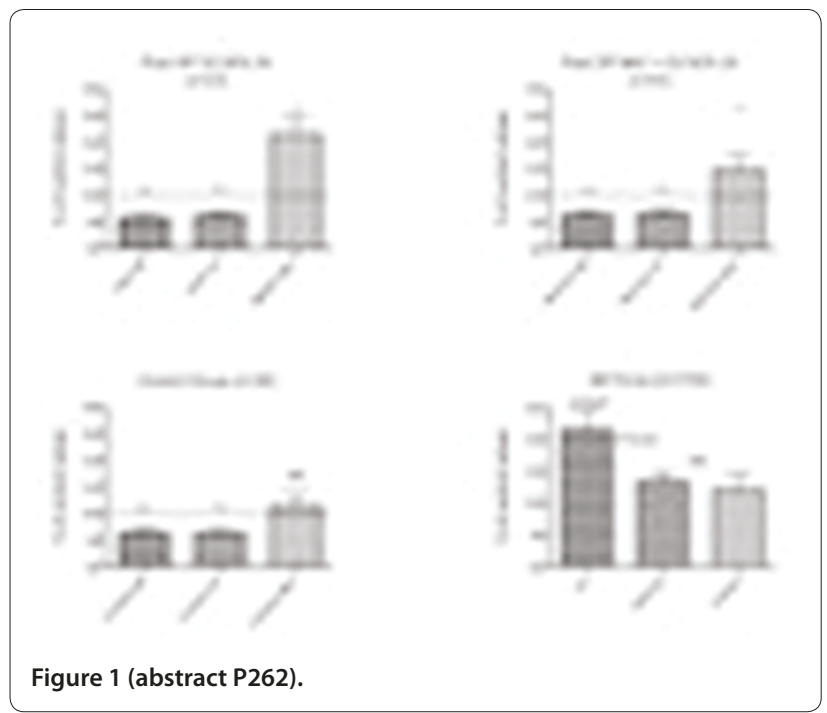

consecutive hours on the first, third, and fifth days postoperatively. The second group (G2; $n=26$ ) in addition to oxygen received NAC $200 \mathrm{mg} /$ day for 5 days. The third group $(\mathrm{G} 3 ; n=26)$ was the control group which did not receive any oxygen variation. On postoperative day 6 , hemoglobin, hematocrit and reticulocytes were measured and compared to the baseline values. A total of five patients (three in G1 and two in G2) were excluded for discontinuing oxygen and/or early discharge from hospital.

Results The reticulocyte count in G1 showed statistically different values compared to $\mathrm{G} 2$ and $\mathrm{G} 3$. These findings correlate with other clinical trials [2]. The fact that no statistical difference of hemoglobin level was recorded could be attributed to the lack of follow-up after patient discharge (postoperative day 6). See Figure 1.

Conclusion Induced relative hypoxia seems to be an effective stimulus for reticulocyte synthesis. However, further investigations are needed to confirm these findings and their impact on hemoglobin.

References

1. Balestra C, et al:. J Appl Physiol 2006, 100:512-518

2. Theunissen et al:: Crit Care 2011, 15(Suppl 1):P422.

P263

Pre-emptive hypothermia during resuscitated porcine hemorrhagic shock

J Matallo', W Stahl', M Gröger', A Seifritz', O Mccook', M Georgieff',

P Asfar' ${ }^{2}$, M Matejovic ${ }^{3}$, E Calzia', P Radermacher', F Simon

'University Medical School, Ulm, Germany; '2University Hospital, Angers,

France; ${ }^{3}$ Charles University, Plzeñ, Czech Republic

Critical Care 2012, 16(Suppl 1):P263 (doi: 10.1186/cc10870)

Introduction The role of hypothermia in hemorrhagic shock is still a matter of debate [1]. Therefore, we studied the effects of deliberate, pre-emptive hypothermia on hemodynamics and organ function during long-term porcine hemorrhage and resuscitation.

Methods Anesthetized and instrumented pigs were randomly assigned to $32^{\circ} \mathrm{C}(n=7), 35^{\circ} \mathrm{C}(n=7)$, and $38^{\circ} \mathrm{C}(n=6)$ of core temperature and subjected to 4 hours of hemorrhage (removal of $40 \%$ of the calculated blood volume, additional removal/retransfusion of blood to maintain mean arterial pressure (MAP) $=30 \mathrm{mmHg}$ ). After 12 hours of reperfusion comprising retransfusion of shed blood, colloid fluid resuscitation and noradrenaline to keep MAP at pre-shock levels, animals were rewarmed to $38^{\circ} \mathrm{C}$. Data (median, quartiles) were obtained before and at the end of the shock phase as well as at 12 and 22 hours of resuscitation, intergroup differences were analyzed using a Kruskal-Wallis ANOVA on ranks. 
Results Fluid balance and noradrenaline requirements did differ between groups. At 12 hours of reperfusion - that is, immediately before rewarming - the $32^{\circ} \mathrm{C}$ group showed the lowest blood levels of creatinine $(P=0.026)$, troponin I $(P=0.053)$, the thrombin-antithrombin complexes $(P=0.012)$, and von Willebrand factor $(P=0.012)$. At the end of the experiment - that is, after rewarming - all these intergroup differences had disappeared, but the $32^{\circ} \mathrm{C}$ group presented with arterial hypotension $(P=0.039)$, the most severe visceral organ acidosis (portal and hepatic venous base excess: $P=0.044, P=0.022$, respectively), and the highest TNFa blood levels $(P=0.030)$.

Conclusion Deliberate, pre-emptive moderate hypothermia slowed but did not protect against hemorrhagic shock and resuscitationinduced organ dysfunction, possibly due to a delayed but not attenuated inflammatory response.

Acknowledgements Supported by the Bundesministerium der Verteidigung (M/SABX/8A004).

Reference

1. Tisherman S: I Intensive Care Med 2010, 25:240-242.

\section{P264}

Carbon monoxide therapy protects against hepatic microvascular injury in a mouse model of murine hemorrhagic shock and resuscitation

H Gomez, I Nassour, P Loughran, J Brumfield, L Otterbein, B Zuckerbraun University of Pittsburgh, PA, USA

Critical Care 2012, 16(Suppl 1):P264 (doi: 10.1186/cc10871)

Introduction The purpose of this study is to evaluate the effects of inhaled carbon monoxide (CO) as an adjunct to resuscitation on hepatic microvascular and endothelial integrity in a murine model of hemorrhagic shock and resuscitation (HSR). Others and ourselves have previously demonstrated that $\mathrm{CO}$ can protect against organ injury in experimental models of HSR [1]. Additionally, CO can prevent tissue hypoxia during hemorrhage. Based upon this we hypothesized that $\mathrm{CO}$ prevents hepatic injury and prevents hepatic hypoxia by maintaining endothelial integrity and the hepatic microvascular circulation.

Methods Male C57BL/6 mice underwent sham operation or hemorrhage to a target MAP of $25 \mathrm{mmHg}$. Mice were maintained at this pressure for 120 minutes and then resuscitated with Ringer's lactate at two times the volume of total shed blood. Mice were sacrificed 4 hours after resuscitation. Mice were randomized to receive room air or inhaled $\mathrm{CO}(250 \mathrm{ppm})$ for 30 minutes starting 90 minutes into the shock period ( $n=6$ to 8 per group). Relative hepatic hypoxia was determined using EF5 immunofluorescence. Sinusoidal integrity was determined by scanning electron microscopy of the hepatic sinusoidal endothelium and Evan's blue tissue levels. Leukocyte stasis, rolling, and adhesion were determined using intravital microscopy of post-sinusoidal hepatic venules. Statistical analysis was determined by ANOVA.

Results EF5 staining demonstrated that hemorrhagic shock induced liver hypoxia, which was prevented by CO treatment. Scanning EM imaging of hepatic sinusoids demonstrated that HSR results in loss of normal endothelium, with loss of fenestrations, rounding of cells, and adherent circulating cells. CO therapy prevented these changes. Relative hepatic levels of Evans blue, suggesting endothelial leak, were increased $1.7 \pm 0.23$-fold in HSR compared to sham-operated mice $(P<0.05)$. CO treatment minimized endothelial leak, resulting in a $1.23 \pm 0.21$-fold increase compared to sham $(P<0.05$ compared to air-treated HSR). In addition, leukocyte rolling and adhesion were significantly diminished by $\mathrm{CO}$ as compared to the air-treated group in HSR.

Conclusion $\mathrm{CO}$ protected the hepatic sinusoidal endothelium from HSR-induced injury. Further investigations into the mechanisms of action are necessary. $\mathrm{CO}$ therapy may prove to be a useful resuscitative adjunct in the treatment of HSR.

Reference

1. Kim HS, Loughran PA, Rao J, Billiar TR, Zuckerbraun BS: Am J Physio/ 2008, 295:G146-G152.
P265

Customized modeling to predict the use of vasopressors in ICUs A Fialho', F Cismondi', S Vieira², S Reti ${ }^{3}$, L Celi ${ }^{3}$, M Howell $^{3}$, J Sousa ${ }^{3}$,

S Finkelstein

'Massachusetts Institute of Technology, Cambridge, MA, USA; ${ }^{2}$ Technical University of Lisbon, Instituto Superior Técnico, Lisbon, Portugal; ${ }^{3}$ Beth Israel

Deaconess Medical Centre, Boston, MA, USA

Critical Care 2012, 16(Suppl 1):P265 (doi: 10.1186/cc10872)

Introduction Vasopressors belong to a powerful class of drugs extremely useful for managing hypotension in patients with systemic shock. Being able to predict a patient's impending use of vasopressors could be beneficial as the central line insertion protocol could be initiated in a safe and timely fashion and, a central line would only be inserted if the patient has a likely future vasopressor need. Our goal in this work was to develop predictive risk models for the impending use of vasopressors in an ICU, and to make model comparisons between the general population and patients with pneumonia and pancreatitis. Methods We performed a retrospective cohort study using data from four different adult ICUs at a tertiary-care hospital. Data contained 1,484 adult ICU patients, including a subgroup of 475 patients with an ICD9 diagnosis of pneumonia and 104 with an ICD9 diagnosis of pancreatitis. Two modeling approaches were used - fuzzy modeling (FM) and logistic regression (LR) - combined with a sequential forward feature selection process. For each group of patients, the selected dataset was divided into two parts: one for feature selection and the other for 10 -fold cross-validation. The models' calibration was assessed using the Hosmer-Lemeshow goodness-of-fit test, and discrimination using the area under the receiver-operating curve (AUC).

Results All models presented good fit $(P>0.05)$ and discrimination. An AUC of 0.83 and 0.86 was obtained for the pneumonia and pancreatitis subgroups, respectively, compared to an AUC of 0.81 obtained for the general population of patients. A set of common predictive variables was found for the general population of patients: arterial base excess, noninvasive blood pressure and lactic acid. Additionally, groupspecific predictive variables were found for each of the two subgroups of patients: white blood cell count for pneumonia patients, and temperature for pancreatitis patients.

Conclusion Generally, accurate and well-calibrated predictive risk models were obtained for the impending use of vasopressors in an ICU. However, significantly more accurate and well-calibrated models were developed for the two subpopulations - pneumonia and pancreatitis than for the general population of ICU patients. This finding challenges one-model-fits-all approaches to overall predictive risk modeling and instead supports tailored modeling that is at least stratified at a disease level.

\section{P266}

Implementation of the fifth link of the Chain of Survival concept for out-of-hospital cardiac arrest

TTagami' ${ }^{1}$ R Tosa ${ }^{2}$, M Omura², H Yokota' ${ }^{1}$, H Hirama'

'Nippon Medical School, Tokyo, Japan; '2Aizu Chuo Hospital, Fukushima, Japan

Critical Care 2012, 16(Suppl 1):P266 (doi: 10.1186/cc10873)

Introduction The 2010 resuscitation guidelines of the American Heart Association-International Liaison Committee on Resuscitation recommend an additional fifth link (post-resuscitation care in a regional center) in the Chain of Survival concept for out-of-hospital cardiac arrest (OHCA) in addition to early access to emergency medical care (first link), early cardiopulmonary resuscitation (second link), early defibrillation (third link), and early advanced cardiac life support (fourth link). However, no direct evidence supports its implementation. Our study aimed to determine the effectiveness of this fifth link.

Methods This multicenter, region-wide, prospective clinical study involved all eligible OHCA patients in the Aizu region $(n=1,482$, suburban/rural, Fukushima, Japan). Primary outcomes before (January 2006 to April 2008) and after (January 2009 to December 2010) the implementation of the fifth link were evaluated. After implementation, 
all post-cardiac arrest syndrome patients were concentrated in a hospital having facilities for post-resuscitation management and provided intensive care, including appropriate hemodynamic and pulmonary management, therapeutic hypothermia, and percutaneous coronary intervention. The primary outcome measure was patient survival at 1 month with a favorable neurological outcome.

Results The primary outcome improved significantly from $0.5 \%$ (before, 4/770) to $3.0 \%$ (after, 21/712) $(P<0.0001)$. The multivariate odds ratio for the primary outcome was $8.3(95 \% \mathrm{Cl}, 2.6$ to 26.6$)$ after the implementation of the fifth link, $7.1(\mathrm{Cl}, 2.0$ to 25.1$)$ for a bystanderwitnessed arrest, and $5.0(\mathrm{Cl}, 2.6$ to 26.6$)$ for early defibrillation. Conclusion The proportion of OHCA patients with a favorable neurological outcome improved significantly after the implementation of the fifth link of the Chain of Survival. This finding may require confirmation in an urban setting and/or with randomized trials.

Trial registration University Hospital Medical Information Network Clinical Trials Registry: UMIN000001607 [http://apps.who.int/ trialsearch/trial.aspx?trialid=JPRN-UMIN000001607]

\section{P267}

Examination of out-of-hospital cardiac arrest patients with the Utstein style in Saga prefecture, Japan

T Iwamura, Y Sakamoto, N Kutsukata, T Hitomi, K Seki, M Koga,

TYamashita, A Nakashima, Y Nishimura, M Yahata, K Yamada

Saga University, Saga, Japan

Critical Care 2012, 16(Suppl 1):P267 (doi: 10.1186/cc10874)

Introduction Saga Prefecture is a small prefecture with an area of $2,439 \mathrm{~km}^{2}$ (place-of-residence $1,339 \mathrm{~km}^{2}$ ), a population of 849,709 , and is located in northwestern Kyushu in the western part of Japan. Saga University has the only medical department in Saga Prefecture, Japan, and it is in charge of both the online and offline medical control of Saga. This report examined the present status of OHCA in Saga, which should be improved, and it aimed at exploring policies that can contribute to the improvement in a ROSC rate.

Methods The study examined 785 OHCA cases using the emergency conveyance record (the Utstein style) submitted for the purpose of MC verification by the fire-fighting organization in Saga from 1 July 2010 to 31 June 2011. The fire-fighting organization was classified into five areas (A to E) for every near medical classification. Comparative examinations were conducted between the background (age, gender, cardiac arrest cause, initial waveform, and hospital waveform, witness, bystander CPR, oral instruction, and pre-hospital medical examination (shock, advanced airway management, and drug use)) and the ROSC rate between the five areas. Statistical analyses included the chi-square test and Fisher's test.

Results Age, gender, cardiac arrest cause, initial waveform, witness, shock and drug use pre-hospital did not differ significantly between the five regions. The ROSC rate was significantly higher in $A$ and $C$ areas than in $D$ and $E$ areas (A: $40.1 \%$ to $D: 24.4 \% P<0.01, A: 40.1 \%$ to E: $26.8 \% P<0.05, C: 39.9 \%$ to $D: 24.4 \% P<0.05$ ), and the ROSC rate of a hospital waveform of asystole was significantly higher in $A$ and $C$ areas than in the other areas (A: $32.0 \%$ to B: $15.3 \%, \mathrm{D}: 13.2 \%, \mathrm{E}: 12.2 \% P<0.01$, C: $27.8 \%$ to B: $15.3 \%, E: 12.2 \% P<0.05)$. There were significantly fewer examples of oral instruction enforcement in the $E$ area in comparison to the other areas (E: $39.7 \%$ to A: $62.5 \%, B: 65.7 \%, C: 65.9 \%$, D: $62.0 \%$ $P<0.01$ ), and there were fewer examples of CPR enforcement in the $D$ and $E$ areas in comparison to the $B$ and $C$ areas (D: $50.8 \%$ to B: $63.9 \%$ $P<0.05$, E: $42.3 \%$ to B: $63.9 \% P<0.01$, E: $42.3 \%$ to C: $59.7 \% P<0.05$ ). CPR was not always delivered without oral instruction because the bystander CPR-less rate of the oral-instruction-less example to citizens was not less than $80 \%$ in all the areas.

Conclusion An improvement of the quality of oral instruction could improve the ROSC rate. BLS education to the area, a re-examination of the oral instruction manual in the applicable areas, and the suitable evaluation of various examples of agonal respiration are together expected to improve the ROSC rate.
P268

Effectiveness and limitations of learning cardiopulmonary resuscitation with an automated external defibrillator in the curriculum of First Aid courses among lay people

U Kovačič ${ }^{1}$, L Kosec $^{2}$

${ }^{1}$ Faculty of Medicine, University of Ljubljana, Slovenia; ${ }^{2}$ General Hospital in

Novo Mesto, Slovenia

Critical Care 2012, 16(Suppl 1):P268 (doi: 10.1186/cc10875)

Introduction The effectiveness and limitations of widespread promotion of cardiopulmonary resuscitation (CPR) with an automated external defibrillator (AED) among the laity was investigated. Early, qualitative and continuous cardiac massage has been stressed in the 2010 ERC guidelines. Since 2009 about 45,000 laypersons attended the mandatory First Aid courses for drivers (organised by Slovenian Red (ross), which include learning CPR with an AED.

Methods One hundred laypersons who attended 4-hour classes in CPR before the driving lessons were compared to 60 motivated laypersons who attended 6-hour classes in CPR before starting to work as lifeguards in pools. Sixty instructors served as the control group. All participants (randomly assigned in pairs) got the same 6-minute case-based scenario on a manikin. Rescuers were changing every 2 minutes. Basic skills in CPR were determined by the two instructors and by a sensored manikin. Massage was assessed as qualitative if at least $90 \%$ of massages were provided with proper hand placement, adequate compression depth and adequate frequency. We measured the response time from the call for help to the start of heart massaging and the percentage of the time of massaging regarding the total time from start of massaging to the end of the scenario.

Results Cardiac massage was not performed adequately in $48 \%$ of laypersons. This was statistically significantly more than among lifeguards (16\%) and instructors (23\%). The median response times of laypersons and lifeguards were 15 seconds and 16 seconds, respectively; this was statistically $(P<0.05)$ longer than instructors $(12$ seconds). The median percentage of the time of massaging in group of laypersons was $51 \%$ ( 56 to $58 \%$, 25 th to 75 th percentiles), which was statistically significantly smaller than in the group of lifeguards $(64 \%, 62$ to $66 \%)$ and in the control group (67\%, 62 to $69 \%)$.

Conclusion The majority of all laypersons approach CPR in about 15 seconds from identification of unconsciousness. However, only about one-half of laypersons after the mandatory CPR course perform qualitative cardiac massage, which is significantly less than among motivated laypersons. The latter perform qualitative massage and achieve the same percentage of the massaging time as instructors. Results suggest that widespread promotion of the CPR protocol with an AED among laypersons has limitations. Therefore, education of laypersons should particularly focus on groups that have intrinsic motivation.

\section{P269}

Survival after out-of-hospital cardiac arrest during nights and weekends

K Maekawa, KTanno, M Hase, K Mori, Y Asai

Sapporo Medical University, Sapporo, Japan

Critical Care 2012, 16(Suppl 1):P269 (doi: 10.1186/cc10876)

Introduction Out-of-hospital cardiac arrest (OOHCA) still has a low survival rate, despite considerable efforts including early applications of basic life support and defibrillation in the pre-hospital setting. Postresuscitation care after hospitalization, influencing the final outcome, may be less available during nights and weekends because of hospital, staffing, and response factors. We sought to determine whether outcomes after OOHCA differ during nights and weekends (off-hours) compared with daytimes of weekdays (on-hours).

Methods We performed a retrospective analysis of 4-year data collected prospectively in a single institute. Adults with witnessed OOHCA of cardiac origin were recruited. The therapeutic strategy after hospitalization, including extracorporeal cardiopulmonary resuscitation (ECPR), therapeutic hypothermia $(\mathrm{TH})$ and primary percutaneous coronary intervention (PCI), was dependent on the critical care physicians in charge. We used a propensity-score matching 
to reduce the differences of pre-hospital variables between patients arriving during off-hours and on-hours. Primary endpoint was 90-day survival after cardiac arrest. We evaluated the survival difference using the log-rank test and identified the significant interventions affecting outcome using the Cox regression model.

Results Of 185 patients, 131 arrived during off-hours (the off-hours group) and 54 arrived during on-hours (the on-hours group). The matching process selected 37 patients each from both groups. The matched off-hours group had a lower survival rate than the matched on-hours group ( $10.8 \%$ vs. $37.8 \%$; log-rank $P=0.025$ ). Multivariate Cox regression analysis showed that TH was associated with 90 -day survival after cardiac arrest (adjusted hazard ratio (HR), $0.43 ; 95 \% \mathrm{Cl}, 0.23$ to 0.79 ), but there were no significant associations of ECPR (adjusted HR, $0.83 ; 95 \% \mathrm{Cl}, 0.50$ to 1.37 ) and primary $\mathrm{PCl}$ (adjusted $\mathrm{HR}, 0.76 ; 95 \% \mathrm{Cl}$, 0.42 to 1.38 )

Conclusion Lower survival rates after OOHCA during nights and weekends were seen at our institute. TH was more likely to be induced in patients arrived during daytimes of weekdays, and independently associated with survival benefit.

Reference

1. Peberdy MA, Ornato JP, Larkin GL, et al: Survival from in-hospital cardiac arrest during nights and weekends. JAMA 2008, 299:785-792.

P270

CPR initiated after telephone-assisted instruction produces a better outcome of bystander-witnessed out-of-hospital cardiac arrests than no bystander CPR but is less effective than CPR on the bystander's own initiative

H Inaba', T Kamikura', KTakase', W Omi², S Sakagami², Y Myojo', J Taniguchi ${ }^{3}$

'Kanazawa University Graduate School of Medicine, Kanazawa, Japan; 2Kanazawa Medical Center, Kanazawa, Japan; ${ }^{2}$ shikawa Prefectural Central Hospital, Kanazawa, Japan

Critical Care 2012, 16(Suppl 1):P270 (doi: 10.1186/cc10877)

Introduction Telephone CPR has been shown to increase the incidence of bystander CPR and is expected to improve the outcomes of out-ofhospital cardiac arrests (OHCAs). The aim of present study was to clarify if the outcomes of bystander-witnessed OHCAs having CC-only and conventional CPR following telephone CPR may be better than those having no bystander CPR and if the type (CC-only and conventional) and origin (following telephone CPR and on bystander's own initiative) may affect the outcomes of bystander-witnessed OHCAs with bystander CPR.

Table 1 (abstract P270). Comparison of survival between OHCAs without bystander CPR and bystander CPR in bystander-witnessed OHCAs

\begin{tabular}{lcc}
\hline Factor & Adjusted odds ratio & $\mathbf{9 5 \%} \mathrm{Cl}$ \\
\hline Type of CPR & & \\
No bystander CPR & Reference & \\
CC-only CPR following telephone-CPR & 1.66 & 1.49 to 1.84 \\
Conventional CPR following telephone-CPR & 1.67 & 1.48 to 1.89 \\
CC-only CPR on bystander's own initiative & 2.22 & 1.99 to 2.49 \\
Conventional CPR on bystander's own initiative & 2.36 & 2.10 to 2.66 \\
Aetiology & & \\
$\quad$ Presumed cardiac & 2.44 & 2.27 to 2.63 \\
$\quad$ Noncardiac & Reference & \\
Time intervals & & \\
$\quad$ Witness-call & 0.98 & 0.97 to 0.98 \\
Witness-first CPR performed either by & 0.97 & 0.96 to 0.98 \\
Citizens or by EMTs & & \\
Call-arrival at patients & 0.93 & 0.92 to 0.94 \\
\hline
\end{tabular}

Comparisons of 1-month survival with favourable neurological outcomes between OHCAs without bystander CPR and with four types of bystander CPR in bystander-witnessed OHCAs (multiple logistic regression analysis).
Table 2 (abstract P270). Effects of type and origin by bystander CPR on survival of bystander-witnessed OHCAs having bystander CPR

\begin{tabular}{|c|c|c|}
\hline Factor & Adjusted odds ratio & $95 \% \mathrm{Cl}$ \\
\hline \multicolumn{3}{|l|}{ Type of CPR } \\
\hline CC-only CPR & 0.96 & 0.88 to 1.04 \\
\hline Conventional CPR & Reference & \\
\hline \multicolumn{3}{|l|}{ Origin of CPR } \\
\hline Following telephone-CPR & 0.73 & 0.67 to 0.80 \\
\hline On bystander's own initiative & Reference & \\
\hline \multicolumn{3}{|l|}{ Aetiology } \\
\hline Presumed cardiac & 2.27 & 2.05 to 2.51 \\
\hline Noncardiac & Reference & \\
\hline \multicolumn{3}{|l|}{ Time intervals } \\
\hline Witness-call & 0.99 & 0.98 to 0.99 \\
\hline $\begin{array}{l}\text { Witness-first CPR performed either by } \\
\text { citizens or by EMTs }\end{array}$ & 0.98 & 0.97 to 0.99 \\
\hline Call-arrival at patients & 0.88 & 0.87 to 0.90 \\
\hline
\end{tabular}

Effects of type and origin by bystander CPR on 1-month survival with favourable neurological outcomes of bystander-witnessed OHCAs having bystander CPR (multiple logistic regression analysis).

Methods From the Japanese nationwide database for 431,968 OHCAs that occurred from January 2005 to December 2008, we extracted and analyzed 112,144 bystander-witnessed OHCAs without any involvement of physicians, using multiple logistic regression analysis. Results The analysis for all bystander-witnessed OHCAs revealed that both CC-only and conventional CPR following telephone CPR produce better outcomes than no bystander CPR (Table 1). The analysis for bystander-witnessed OHCAs with bystander CPR disclosed that CPR on the bystander's own initiative produces a better outcome than CPR following telephone CPR (Table 2).

Conclusion Telephone CPR improves the outcomes of bystanderwitnessed OHCAS. However, efforts to increase the incidence of early CPR on the bystander's own initiative would be necessary to obtain a higher incidence of survival in bystander-witnessed OHCAs.

P271

Critical times in pediatric out-of-hospital cardiac arrest

J Tijssen', C Zhan², C Parshuram', L Morrison², J Hutchison

${ }^{1}$ Hospital for Sick Children, Toronto, Canada; 'University of Toronto, Canado

Critical Care 2012, 16(Suppl 1):P271 (doi: 10.1186/cc10878)

Introduction Pediatric out-of-hospital cardiac arrest (OHCA) has a less than $10 \%$ survival. Studies of the scene time and level of emergency medical services (EMS) training in pediatric OHCA are lacking. The objectives of this study are to describe the scene time, level of training and the order and timing of arrival of first responders to pediatric OHCA in a large, densely populated area, the Toronto region.

Methods The Resuscitation Outcomes Consortium (ROC) EpistryCardiac Arrest database was queried for all patients $<19$ years old from December 2005 to November 2011 in the Toronto region for age, sex, event characteristics, underlying conditions, cause of the cardiac arrest, level of EMS care, time to EMS arrival, scene time, return of spontaneous circulation (ROSC) and survival to hospital discharge. Patients were excluded if they were declared dead at the scene.

Results Four hundred and fifty-two patients with OHCA were included. Thirty-one percent were infants, $29.4 \%$ age 1 to 11 years (child), and $37.4 \%$ age 1 to 18 (adolescent) years with $62.8 \%$ of cases male. Thirty percent had a significant past medical history. The causes of the cardiac arrest were trauma (14.4\%), drowning $(6.2 \%)$, sudden infant death syndrome $(4.0 \%)$, and unknown in $63 \%$. The first EMS responders were fire in $52.2 \%$, advanced care paramedics in $25 \%$, and primary care paramedics in $22.3 \%$. Survival was increased the earlier the EMS arrived $(P=0.015)$. The timing of arrival of advanced paramedics at the scene appeared to be associated with survival although this was not statistically significant $(P=0.22)$. Infants had a shorter scene time ( $P$ $<0.001)$ and an earlier arrival of advanced care paramedics at the scene 
$(P=0.04)$. A shorter scene time was associated with ROSC on arrival at the emergency department $(P<0.001)$ and a nonsignificant trend for improved survival $(P=0.13)$. Adolescents were more likely to have ROSC on arrival at the emergency department $(P<0.001)$ and more likely to survive $(P<0.05)$ compared to children or infants.

Conclusion The timing of arrival of advanced paramedics at the scene may have been associated with survival and a larger study is needed to confirm this trend. A shorter scene time was associated with ROSC and a trend for increased survival. However, infants have shorter scene times but worse outcomes. To provide increased power and scope for this study we will expand it to include all 10 Regional Clinical ROC Centers and future analyses will include the remaining Utstein data fields and compare the effects of advanced versus basic life support interventions during resuscitation.

P272

Don't stop your heart in front of your family: family as a bystander is associated with poor outcome of bystander-witnessed, bystanderCPR-performed out-of-hospital cardiac arrest

H Inaba' ${ }^{1}$ K Takase', T Nishi', T Kamikura', Y Wato², H Hamada ${ }^{3}$

'Kanazawa University Graduate School of Medicine, Kanazawa, Japan;

2Kanazawa Medical University, Uchinada, Japan; '3uzu General Hospital,

Suzu, Japan

Critical Care 2012, 16(Suppl 1):P272 (doi: 10.1186/cc10879)

Introduction Early CPR with a considerable quality is essential for survival from out-of-hospital cardiac arrest (OHCA). This study was conducted to test our hypothesis that the relation of the bystander to the victim may affect the outcomes of OHCAs.

Methods From a Japanese nationwide database for 431,968 OHCAs that occurred from January 2005 to December 2008, we extracted and then analyzed 45,248 bystander-witnessed, bystander-CPR-performed OHCAs without any involvement of physicians. Backgrounds, characteristics and outcomes were compared among the three groups of OHCAs categorized by the bystander's relation to victims. Multiple logistic regression analysis was applied to clarify if the relation may affect the 1-month survival with favourable neurological outcomes. Results When the bystander was family, CPR was more frequently initiated following telephone-assisted instruction and the interval between collapse and bystander CPR was significantly prolonged. Univariate analysis followed by multiple logistic regression analysis revealed that family as a CPR performer significantly decreases the 1 -month survival with favourable neurological outcomes. See Tables 1 and 2.

Conclusion Despite educational efforts, most family members do not appear to be good CPR performers. The first responder system that enables a good CPR performer to reach the scene quickly may be needed for OHCAs witnessed by the family.

Table 1 (abstract P272). Backgrounds, characteristics and outcomes of OHCAs with reference to relation of bystander to victim

\begin{tabular}{|c|c|c|c|c|}
\hline \multirow[b]{2}{*}{ Background, characteristics and outcome } & \multicolumn{3}{|c|}{ Relation of bystander to victim } & \multirow[b]{2}{*}{$P$ value } \\
\hline & $\begin{array}{c}\text { Family } \\
(n=25,119)\end{array}$ & $\begin{array}{c}\text { Friends, colleagues and } \\
\text { passers-by }(n=5,191)\end{array}$ & $\begin{array}{c}\text { Others } \\
(n=14,938)\end{array}$ & \\
\hline Patient's age, median (25 to $75 \%$ ) & $77(66$ to 84$)$ & 61 (50 to 73 ) & 84 (75 to 90$)$ & $<0.001$ \\
\hline Sex - male (\%) & 61.6 & 76.8 & 44.7 & $<0.001$ \\
\hline CPR following telephone CPR (\%) & 75.1 & 42.7 & 36.1 & $<0.001$ \\
\hline Initial rhythm shockable (\%) & 16.3 & 33.4 & 9.8 & $<0.001$ \\
\hline \multicolumn{5}{|l|}{ Tune intervals, minutes, median ( 25 to $75 \%$ ) } \\
\hline Collapse-call & $2(0$ to 5$)$ & $2(0$ to 4$)$ & $2(0$ to 5$)$ & $<0.001$ \\
\hline Collapse-bystander CPR & $3(1$ to 6$)$ & $1(0$ to 4$)$ & $0(0$ to 2$)$ & $<0.001$ \\
\hline Call arrival at patient & $8(6$ to 11$)$ & $8(6$ to 11$)$ & $8(6$ to 10$)$ & $<0.001$ \\
\hline \multicolumn{5}{|l|}{ Outcomes } \\
\hline 1-month survival (\%) & 8.1 & 17.2 & 9.2 & $<0.001$ \\
\hline $\begin{array}{l}\text { 1-month survival with favorable } \\
\text { neurological outcomes (\%) }\end{array}$ & 4.0 & 11.9 & 4.8 & $<0.001$ \\
\hline
\end{tabular}

Table 2 (abstract P272). Relation of bystander to victim as a factor associated with 1-month survival of bystander-witnessed OHCAs having bystander CPR

\begin{tabular}{|c|c|c|c|}
\hline \multirow[b]{2}{*}{ Factor } & \multicolumn{3}{|c|}{ Adjusted odds ratio ( $95 \%$ confidence interval) } \\
\hline & $\begin{array}{l}\text { Bystander-witnessed OHCAs } \\
\text { with bystander CPR }\end{array}$ & $\begin{array}{c}\text { Of presumed } \\
\text { cardiac etiology }\end{array}$ & $\begin{array}{l}\text { Of presumed cardiac etiology } \\
\text { with shockable initial rhythm }\end{array}$ \\
\hline \multicolumn{4}{|l|}{ Etiology of arrest } \\
\hline Presumed cardiac & $1.39(1.24$ to 1.55$)$ & Undefined & Undefined \\
\hline Noncardiac & Reference & & \\
\hline \multicolumn{4}{|l|}{ Initial rhythm } \\
\hline Shockable & 4.38 (3.95 to 4.85$)$ & 4.82 (4.29 to 5.42$)$ & Undefined \\
\hline Nonshockable & Reference & Reference & Reference \\
\hline Patient's age & 0.97 (0.97 to 0.98$)$ & 0.97 (0.97 to 0.97 ) & 0.98 (0.97 to 0.98$)$ \\
\hline \multicolumn{4}{|l|}{ Sex } \\
\hline Male & $1.14(1.02$ to 1.26$)$ & $1.16(1.02$ to 1.32$)$ & $1.07(0.90$ to 1.26$)$ \\
\hline Female & Reference & Reference & Reference \\
\hline \multicolumn{4}{|l|}{ Relation of bystander to victim } \\
\hline Family & Reference & Reference & Reference \\
\hline Friend, colleague and passers-by & $1.70(1.49$ to 1.95$)$ & $1.40(1.19$ to 1.64$)$ & $1.61(1.42$ to 1.81$)$ \\
\hline Others & 1.59 (1.42 to 1.78$)$ & 1.46 (1.27 to 1.68$)$ & $1.32(1.10$ to 1.59$)$ \\
\hline
\end{tabular}


P273

Coronary perfusion pressure in a pig model of prolonged cardiac arrest treated by different modes of venoarterial extracorporeal membrane oxygenation and intraaortic balloon counterpulsation J Bělohlávek', M Mlcek², M Huptych³', S Havranek', P Ostadal'4, A Linhart'1, O Kittnar ${ }^{2}$

'General Teaching Hospital Prague, Czech Republic; ${ }^{2}$ st Medical School,

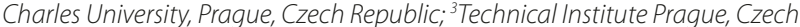
Republic; ${ }^{4}$ Homolka Hospital, Prague, Czech Republic Critical Care 2012, 16(Suppl 1):P273 (doi: 10.1186/cc10880)

Introduction An extracorporeal membrane oxygenation (ECMO)based approach is increasingly used in cardiac arrest (CA). However, little is known about coronary perfusion pressure progress over time in CA managed by ECMO. The aim of this study was to assess femorofemoral (FF) compared to femoro-subclavian (FS) venoarterial ECMO in a pig model of prolonged CA on coronary perfusion pressure (CPP), myocardial metabolic recovery and resuscitability.

Methods A total of 11 female pigs, body weights $50.3 \pm 3.4 \mathrm{~kg}$, were enrolled into a protocol of prolonged cardiac arrest treated by FF or FS ECMO \pm IABP in a randomized fashion. Animals under general anesthesia had undergone 15 minutes of ventricle fibrillation (VF) with basal ECMO flow of 5 to $10 \mathrm{ml} / \mathrm{kg} /$ minute simulating low-flow CA followed by continued VF with ECMO flow of $100 \mathrm{ml} / \mathrm{kg} / \mathrm{minute}$. CPP, myocardial lactate metabolism and myocardial oxygen extraction were determined.

Results CPP decreased from baseline of $85 \mathrm{mmHg}(72,94.5)$ to $15 \mathrm{mmHg}(10,20.5)$ during CA. The first CPP value on ECMO increased to $34 \mathrm{mmHg}(26.5,44)$ and during the further protocol gradually rose to significantly higher CPP of $68 \mathrm{mmHg}(45.5,82)$ before CPR $(P=0.003)$. This phenomenon of gradual rise was even more pronounced in FF ECMO, animals started on FF ECMO completed the protocol with identical CPP values as at baseline $(85 \mathrm{mmHg}(80,99)$ vs. $86 \mathrm{mmHg}$ $(78,86), P=0.55)$. Following $C A$, significantly higher lactate levels were detected in animals started on FS ECMO in all post-arrest periods $(P=0.016$ and $P=0.035$ for difference in arterial and coronary sinus lactate levels, respectively). Oxygen extractions after a steep increase during CA declined immediately after ECMO initiation and remained further with no statistically significant differences between respective ECMO arms ( $P$ for difference $=0.547$ ). Resuscitability was high, we gained 5 minutes return of spontaneous circulation (ROSC) in eight animals (73\%) and 60 minutes ROSC was present still in eight animals (73\%). Conclusion Our experimental study confirmed that, in a pig model of prolonged cardiac arrest, VA ECMO, mainly the FF approach, increases significantly the CPP over time, assures good metabolic recovery and offers sustained reasonable resuscitability.

P274

Capnometry successfully predicts outcome and determination of the cessation of cardiopulmonary resuscitation efforts

EH Hajdinjak', ŠG Grmec², MK Križmarić3 ${ }^{3}$ ETT Torkar², MS Skufca²,

DB Buić-Rerečić ${ }^{2}$ MK Kovač² MZ Zelinka²

'Center for Emergency Medicine Maribor, University of Maribor, University of Ljubljana, Slovenia, Maribor, Slovenia: ${ }^{2}$ Community Health Centre Ljubljana University of Ljubljana, University of Maribor, Ljubljana, Slovenia; ${ }^{3}$ Faculty of Medicine, University of Maribor, Slovenia

Critical Care 2012, 16(Suppl 1):P274 (doi: 10.1186/cc10881)

Introduction Prognosis in patients suffering out-of-hospital cardiac arrest is poor. Higher survival rates have been observed only in patients with ventricular fibrillation who were fortunate enough to have basic and advanced life support initiated soon after cardiac arrest. An ability to predict cardiac arrest outcomes would be useful for resuscitation. Changes in expired end-tidal carbon dioxide levels during cardiopulmonary resuscitation (CPR) may be a useful, noninvasive predictor of successful resuscitation and survival from cardiac arrest, and could help in determining when to cease CPR efforts.

Methods This is a prospective, observational study of 1,080 cases of out-of-hospital cardiac arrest. The patients were intubated and measurements of end-tidal carbon dioxide taken. Data according to the Utstein criteria, demographic information, medical data, and partial pressure of end-tidal carbon dioxide $\left(\mathrm{PetCO}_{2}\right)$ values were collected for each patient in cardiac arrest by the emergency physician. We hypothesized that an end-tidal carbon dioxide level of $1.9 \mathrm{kPa}(14.3 \mathrm{mmHg})$ or more after 20 minutes and $1.8 \mathrm{kPa}$ or more after 15 minutes of standard advanced cardiac life support would predict restoration of spontaneous circulation (ROSC).

Results PetCO after 20 minutes of advanced life support averaged $0.97 \pm 0.33 \mathrm{kPa}$ in patients who did not have ROSC and $4.85 \pm 1.74 \mathrm{kPa}$ in those who did $(P<0.001)$. PetCO 2 after 15 minutes of advanced life support averaged $1.11 \pm 0.39 \mathrm{kPa}$ in patients who did not have ROSC and $3.65 \pm 0.98 \mathrm{kPa}$ in those who did $(P<0.001)$. End-tidal carbon dioxide values of $1.9 \mathrm{kPa}(14.3 \mathrm{mmHg})$ or less discriminated between the 578 patients with ROSC and 502 patients without. When a 20-minute endtidal carbon dioxide value of $1.9 \mathrm{kPa}(14.3 \mathrm{mmHg})$ or less was used as a screening test to predict ROSC, the sensitivity, specificity, positive predictive value, and negative predictive value were all $100 \%$. The 15 -minute petCO value of $1.8 \mathrm{kPa}$ had a sensitivity and NPV of $100 \%$ with high specificity PPV value (98\%).

Conclusion End-tidal carbon dioxide levels of more than $1.9 \mathrm{kPa}(14.3$ $\mathrm{mmHg}$ ) after 20 minutes may be used to predict ROSC with accuracy. End-tidal carbon dioxide levels should be monitored during CPR and considered a useful prognostic value for determining the outcome of resuscitative efforts and when to cease CPR in the field.

P275

Modified clinical decision rule for termination-of-resuscitation in cases of refractory out-of-hospital cardiac arrest

Y Goto', ${ }^{1}$ Maeda', ${ }^{1}$ Goto ${ }^{2}, \mathrm{H}_{\text {Inaba }}{ }^{3}$

'Kanazawa University Hospital, Kanazawa, Japan; ${ }^{2}$ Yawata Medical Center, Komatsu, Japan; 'Kanazawa University Graduate School of Medicine, Kanazawa, Japan

Critical Care 2012, 16(Suppl 1):P275 (doi: 10.1186/cc10882)

Introduction Two international termination-of-resuscitation (TOR) rules for the emergency medical services (EMS) personnel have been proposed to identify nonsurvivors after out-of-hospital cardiac arrest (OHCA). The first is for use by responders providing basic life support (BLS) which includes three criteria: not witnessed by EMS personnel, no shocks are administered and no return of spontaneous circulation (ROSC). The other is for use by responders providing advanced life support (ALS) which adds two criteria: unwitnessed by a bystander and no bystander cardiopulmonary resuscitation. Simpler criteria as a universal TOR rule may be desirable for any level of EMS personnel. We performed this study to validate two TOR rules and a modified BLS TOR rule which includes three criteria: unwitnessed arrest, no shocks administered and no ROSC achieved before arrival at hospital for predicting refractory OHCAs.

Methods We analysed 289,769 OHCA adult patients with presumed cardiac causes, using a prospectively recorded nationwide Utstein-style database in Japan over 5 years (2005 to 2009). The primary endpoint was 1-month survival with unfavourable neurological outcome, or Glasgow-Pittsburgh cerebral performance category (CPC) scale $=3$ to 5 . Results The overall rates of 1-month survival with CPC $=1$ or 2 and collective 1 -month survival were $2.55 \%$ and $5.22 \%$, respectively. The incidences of misclassification in the BLS, ALS and modified BLS TOR rules for 1 -month survival with CPC $=3$ to 5 were $0.20 \%, 0.15 \%$ and $0.13 \%$, respectively. The specificity $(95 \% \mathrm{Cl})$ in the BLS, ALS and modified BLS TOR rules for 1-month survival with CPC $=3$ to 5 were 0.941 (0.935 to 0.946$), 0.981$ (0.978 to 0.984 ) and 0.972 (0.968 to 0.975 ), respectively. The area under the receiver operating characteristic curve in the BLS, ALS and modified BLS TOR rules for 1-month survival with $\mathrm{CPC}=3$ to 5 were $0.865,0.654$ and 0.765 , respectively.

Conclusion We found that each TOR rule had high specificity (ability to predict survivors with favourable neurological outcome) and low misclassification rate as a universal TOR rule. The modified BLS TOR rule is simpler and as reliable as the other two rules. In Japan, as EMS providers are legally prohibited from terminating resuscitation in the field, the amendment of related laws and the establishment of national consensus would be necessary to apply these rules in the Japanese EMS system.

Reference

1. Morrison LJ, et al:: N Engl J Med 2006, 355:478-487. 
P276

Survival benefit for patients receiving antibiotics following out-ofhospital cardiac arrest

KJ Davies, ID Kerslake, J Walters, MJ Thomas

Bristol Royal Infirmary, Bristol, UK

Critical Care 2012, 16(Suppl 1):P276 (doi: 10.1186/cc10883)

Introduction Therapeutic hypothermia $(\mathrm{TH})$ has become standard management following out-of-hospital cardiac arrest (OHCA). Recent evidence suggests $\mathrm{TH}$ increases the risk of pneumonia. We retrospectively assessed infective indicators after OHCA and evaluated the effect of antibiotics on survival.

Methods We identified all patients admitted to the ICU of a regional primary angioplasty hospital following OHCA from May 2007 to December 2010. We recorded ICNARC predicted mortality scores, blood and respiratory (protected catheter aspiration) culture results, white blood cell count (WBC) and C-reactive protein (CRP), hospital outcome and ICU length of stay. All chest radiographs (CXRs) were reviewed by a respiratory consultant (JW). Any antibacterial therapy was recorded. Results A total of 144 patients were admitted to the ICU following OHCA. Mean age was 61.7 years $(95 \% \mathrm{Cl} 59.0$ to 64.4$)$. The mortality rate was $66.67 \%$ (58.62 to 73.84 ) with mean ICNARC predicted mortality of $77.11 \%$ (73.84 to 80.39 ). Of 144 patients, 138 (95.8\%; 91.1 to 98.1 ) had at least one positive marker of infection within 72 hours. Sixty-four had microbiology samples analysed, 34 of which were positive $(53.1 \% ; 41.1$ to $64.8 \%)$. Of 88 patients who had a CXR, $26(29.6 \% ; 21.0$ to 39.8$)$ had consolidation. Ninety-six of 115 patients (83.5\%; 75.6 to 89.1 ) had a CRP $>100 \mathrm{mg} / \mathrm{l}$ (normal value $<10 \mathrm{mg} / \mathrm{l}$ ) within 72 hours and 82 of 115 $(71.3 \% ; 62.5$ to 78.8$)$ had an abnormal WBC ( $<4.0$ or $\left.>11.0 \times 10^{9} / l\right)$. Fiftysix of 144 patients (38.9\%; 31.3 to 47.0 ) received antibiotics during the first 7 days of their ICU stay (mean time to first dose 2.17 days; 1.69 to 2.66). The hospital mortality rate for these patients $53.6 \%$ (40.7 to 66.0 ) was significantly less than those not receiving antibiotics $75.0 \%(65.0$ to 82.9$)\left(X^{2} 6.14, P=0.01\right)$ with absolute risk reduction of 0.214 ( 0.055 to $0.365)$ and NNT of 5 ( 3 to 18$)$. There was no difference in age $(59.9 \pm 4.2$ vs. $62.9 \pm 3.5)$ or ICNARC predicted mortality $(75.1 \pm 5.2$ vs. $78.4 \pm 4.2)$ between the groups.

Conclusion The post-arrest management of OHCA is commonly complicated by infections, the diagnosis of which is delayed by a universal increase in inflammatory markers, body temperature control, delay in the processing of samples and poor quality radiography. We have shown a significant reduction in mortality in patients receiving antibiotics compared with patients who do not, despite there being no difference in age or predicted mortality between the groups. This could be due to treatment of an aspiration pneumonia, an anti-inflammatory effect or that some patients did not survive long enough to receive antibiotics. It suggests that a formal clinical trial is warranted.

\section{P277}

Correlation between IL- 6 and S-100B blood levels and outcome of post-cardiac arrest syndrome and influence of therapeutic hypothermia on these mediator blood levels

K Shinozaki ', S Oda², T Sadahiro², M Nakamura², E Watanabe², R Abe ${ }^{2}$,

T Nakada' ${ }^{2}$, Y Morita ${ }^{2}$, K Nakanishi ${ }^{3}$, N Kitamura ${ }^{4}$, H Hirasawa $^{2}$

'Chiba Aoba Municipal Hospital, Chiba City, Japan; ${ }^{2}$ Chiba University, Chiba

City, Japan; ${ }^{3}$ Narita Red Cross Hospital, Narita City, Japan; ${ }^{4}$ Kimitsu Chuo Hospital, Kisarazu City, Japan

Critical Care 2012, 16(Suppl 1):P277 (doi: 10.1186/cc10884)

Introduction To elucidate the significance of IL-6, S-100B and NSE in pathophysiology of post-cardiac arrest syndrome (PCAS), blood levels of those mediators sampled within the first 24 hours after cardiac arrest (CA) were compared between groups classified according to survival and neurological outcomes. Furthermore, influence of stability of core temperature with therapeutic hypothermia $(\mathrm{TH})$ on these mediator blood levels was also investigated.

Methods Nontraumatic out-of-hospital CA patients were included. Blood was sampled on admission, at 6 hours and 24 hours after CA, respectively. Then, patients that died within 24 hours after CA were excluded. Patients were classified into nonsurvivors (died within 28 days) and survivors (survived for 28 days or longer), and classified into poor neurological outcome (CPC 3 to 5) and favorable neurological outcome (CPC 1 to 2), respectively. Factors significantly correlated with survival and neurological outcomes were investigated by comparing baseline characteristics and mediator blood levels. Patients receiving TH were also included into subgroup analysis. If the core temperature was maintained at $33 \pm 1{ }^{\circ} \mathrm{C}$ for more than 18 hours within the first 24 hours, the patient was classified into maintained, and if not into notmaintained, and mediator blood levels were compared between the subgroups.

Results One-hundred and four patients survived more than 24 hours out of all 1,026 patients analyzed. Mean IL-6, S-100B and NSE levels in nonsurvivors $(n=51)$ were significantly higher than those in survivors $(n=53)$ at all timepoints $(P<0.01)$. Those in poor neurological outcome $(n=74)$ were significantly higher than those in favorable neurological outcome $(n=29)$ at all timepoints $(P<0.01)$. From the results of ROC analysis and multivariate analysis, IL- $6>240 \mathrm{pg} / \mathrm{ml}$ at 6 hours and S-100B $>0.37 \mathrm{ng} / \mathrm{ml}$ on admission were chosen as an independent predictors of nonsurvival, and S-100B $>0.07 \mathrm{ng} / \mathrm{ml}$ at 24 hours was chosen as that of poor neurological outcome. Subgroup analysis of 56 patients showed that mean levels of IL- 6 at 6 hours, S-100B at 6 hours and S-100B at 24 hours in the maintained $(n=29)$ group were significantly lower than those in the not-maintained group $(n=27)$ $(P<0.05)$.

Conclusion IL- 6 and S-100B levels within 24 hours after CA, but not NSE, are related to survival and neurological outcome. IL- 6 and S-100B are considered to be important mediators for the pathophysiology of PCAS and TH may influence blood levels of these mediators.

\section{P278}

Early neurological outcome prediction model after

bystander-witnessed out-of-hospital cardiac arrest: a nationwide population-based study

Y Goto', T Maeda', Y Goto ${ }^{2}$, H Inaba ${ }^{3}$

'Kanazawa University Hospital, Kanazawa, Japan; ${ }^{2}$ Yawata Medical Center

Komatsu, Japan; ${ }^{3 K a n a z a w a}$ University Graduate School of Medicine,

Kanazawa, Japan

Critical Care 2012, 16(Suppl 1):P278 (doi: 10.1186/cc10885)

Introduction Identification of prehospital prognostic factors in out-ofhospital cardiac arrests (OHCAs) with prehospital return of spontaneous circulation (ROSC) and establishment of a prediction model for survival with favorable neurological outcome may minimize the costs and save the medical resources. In this study, we developed a best model for predicting 1-month survival with favorable neurological outcome (defined as Glasgow-Pittsburgh cerebral performance category (CPC) scale $=1$ or 2 ), using a logistic regression analysis.

Methods of 522,801 resuscitation-attempted adult patients after OHCAs, 9,876 bystander-witnessed arrests of presumed cardiac origin with prehospital ROSC were analyzed in a prospectively recorded nationwide Utstein-style database in Japan over 5 years (2005 to 2009). The endpoint was 1-month survival with favorable neurological outcome. We performed multivariate logistic regression analysis to develop a prediction model using the prehospital factors.

Results Overall rates of 1 -month survival and that with favorable neurological outcome were $56.7 \%(n=5,604)$ and $40.6 \%(n=4,013)$, respectively. Multivariate logistic regression analysis revealed that the odds ratio for age, shockable initial rhythm and collapse-ROSC time interval were 0.964 ( $95 \% \mathrm{Cl} 0.961$ to 0.967$), 3.564$ (95\% Cl 3.232 to 3.934 ) and 0.967 (95\% Cl 0.963 to 0.970$)$, respectively, and that these variables were identified as the best variables for developing a prediction model. A statistical outcome prediction model using these three variables was as follows: $\mathrm{Pf}=\exp (\mathrm{B}) /[1+\exp (\mathrm{B})]$, where $\mathrm{Pf}$ is the probability of a favorable outcome and $\exp (B)$ is the exponential function of $B: B=-0.037 \times a g e$ (years) $+0.635 \times$ (shockable rhythm ( 1 or $0))-0.034 \times($ collapse-ROSC time interval (minutes) $)+2.540$. The area under the receiver operating characteristic curve of this model for predicting 1-month favourable neurological outcome was 0.764 .

Conclusion Three prehospital prognostic factors (age, shockable initial rhythm and collapse-ROSC time interval) were identified as the best variables in predicting favorable neurological outcomes in OHCAs with prehospital ROSC. A model using these prehospital prognostic factors 
has shown a good predictive value for estimating 1-month survival with favorable neurological outcome in OHCA patients. Although this novel model needs to be validated using another external dataset, this model may help to minimize the cost and save medical resources.

\section{P279}

Helium ventilation is safe and feasible in ICU patients admitted after cardiac arrest

D Brevoord, C Beurskens, N Juffermans, WVan den Bergh, W Lagrand, B Preckel, J Horn

Academic Medical Centre, University of Amsterdam, the Netherlands

Critical Care 2012, 16(Suppl 1):P279 (doi: 10.1186/cc10886)

Introduction Most patients admitted to the ICU after cardiac arrest die or have an unfavourable neurological outcome due to brain damage. Currently, the only treatment to reduce brain injury after cardiac arrest is mild hypothermia. Helium inhalation has shown promising results as a neuroprotective agent in animal models of cerebral infarction. If helium inhalation ameliorates neurological damage by reducing reperfusion injury in humans as well, this could be of great benefit to patients. As no studies exist that investigate the use of helium ventilation in patients after cardiac arrest we investigated whether this treatment is safe and feasible.

Methods A single-centre open-label intervention study was performed in a mixed 30-bed academic ICU, approved by the local medical ethics committee. Inclusion criteria: admission after a witnessed cardiac arrest, presenting with ventricular fibrillation or tachycardia, return of spontaneous circulation within 30 minutes, treatment with hypothermia. Exclusion criteria: pre-existing neurological disorders or the need for $\mathrm{FiO}_{2}>50 \%$ or $>10 \mathrm{mmHg}$ PEEP on ICU admission. Helium was administered during 3 hours as a 1:1 mixture with oxygen, using a Servo-i ventilator. An independent data safety monitoring board reviewed all problems arising from the helium ventilation itself and all fatalities. Poor outcome was assessed with the Glasgow Outcome Score at 30 days: death and vegetative state were defined as poor outcome. Data are presented as mean \pm SD or numbers and proportions.

Results In total 25 patients were included, $20(80 \%)$ male, age $64.8 \pm 12.1$ years, APACHE II score $20.0 \pm 8.6$, SAPS II $53.6 \pm 18.6$. Helium treatment was started 4:57 $\pm 0: 54$ hours after arrest. In one patient the treatment was stopped due to inadequate ventilation using the preset limits. This was not due to the helium ventilation and no adverse events due to helium ventilation were noted. Overall, nine (36\%) patients had a poor outcome.

Conclusion In this small study, we encountered no problems associated with helium treatment in patients admitted to the ICU after cardiac arrest. This opens the way for studies investigating the hypothesis that helium treatment reduces neurological injury in these patients.

\section{P280}

Therapeutic hypothermia in an out-of-hospital arrest population: are we selecting appropriately?

A Short, M Brett, L Donaldson

Glasgow Royal Infirmary, Glasgow, UK

Critical Care 2012, 16(Suppl 1):P280 (doi: 10.1186/cc10887)

Introduction We question how appropriately we select patients to undergo therapeutic hypothermia following out-of-hospital cardiac arrest.

Methods The population was identified through searching Wardwatcher between August 2006 and February 2011. Inclusion criteria were all patients with an ICU admission of out-of-hospital cardiac arrest. Exclusion criteria were: no CPR within the preceding 24 hours; admission from theatre; insufficient data. Data were gathered from Wardwatcher, Careview and patients' case notes for age, arrest rhythm, downtime (DT) - time from arrest to return of spontaneous circulation, time to initiation of CPR, temperatures at various time points, cause of arrest and outcome. Statistical analysis was performed with Fisher's exact test, significance level of $P<0.05$. Permission for use of patient notes was granted from the consultant group of the ICU audited.
Results Seventy patients had a hospital admission of post-cardiac arrest. Five failed the inclusion criteria and six fulfilled exclusion criteria. A total of $36(51 \%)$ were cooled (Table 1). Twelve (33\%) of the cooled population survived to hospital discharge $(D / C)$, one $(8 \%)$ cooled within 4 hours, three (25\%) cooled for over 12 hours. Ten (28\%) patients were cooled despite not having a cardiac cause. One (4\%) of the 23 noncooled patients survived to hospital discharge, four (17\%) had a cardiac cause. The median age of cooled population was 66 years (quartile range 53.5 to 74 years) and 44 years (quartile range 41 to 52 years) of the noncooled.

Table 1 (abstract P280). Population cooled post cardiac arrest

\begin{tabular}{lccc}
\hline & D/C $(\boldsymbol{n}=\mathbf{1 2})$ & Died $(\boldsymbol{n}=\mathbf{2 6})$ & \\
\hline VF arrest & $10(83 \%)$ & $18(75 \%)$ & $P=0.69$ \\
DT $>30$ minutes & $1(8 \%)$ & $14(58 \%)$ & $P=0.005$ \\
First CPR $<5$ minutes & $11(92 \%)$ & $17(71 \%)$ & $P=0.22$ \\
Cardiac aetiology & $10(83 \%)$ & $16(67 \%)$ & $P=0.44$ \\
\hline
\end{tabular}

Conclusion Survival is improved in patients cooled post-out-ofhospital cardiac arrest $[1,2]$. Downtime is statistically significant in the survival of cooled patients. Achieving optimal timing of cooling was no better in surviving versus dying populations. Cooling post-out-ofhospital cardiac arrest is expensive and time-consuming; selection criteria need to be evaluated to concentrate this resource on patients where there is a higher prospect of a positive outcome [2].

References

1. Holzer M, et al.: Crit Care Med 2005, 33:414-418.

2. Hay A, et al:: Anaesthesia 2008, 63:15-19.

P281

Therapeutic hypothermia for nonventricular fibrillation/ventricular tachycardia cardiac arrest

S Jog', D Patel', M Patel', R Kulkarni', N Chouthai ${ }^{2}$

'Deenanath Mangeshkar Hospital and Research Centre, Pune, India; 'Wayne State University, Detroit, MI, USA

Critical Care 2012, 16(Suppl 1):P281 (doi: 10.1186/cc10888)

Introduction Although efficacy of therapeutic hypothermia (TH) for cardiac arrest following ventricular tachycardia (VT)/ventricular fibrillation (VF) is a recommended therapy, the efficacy of TH for nonVF/VT cardiac arrest is still not well studied. We conducted a study to evaluate efficacy and outcomes of TH in non-VF/VT cardiac arrest patients in terms of survival and neurological outcome.

Methods TH was initiated with intravenous ice-cold saline and maintained with an external servo controlled cooling system (ESCCS); by Blanketrol II Hypo-Hyperthermia system (Cincinnati Sub-Zero Inc.) between 34 and $32^{\circ} \mathrm{C}$ for 24 hours. Gradual rewarming was also done with ESCCS. Non-VF/VT cardiac arrest patients with GCS $\leq 7$ at 60 minutes of return of spontaneous circulation (ROSC) were enrolled. Standard hemodynamic monitoring and management was continued in all patients.

Results A total of 13 patients with average GCS of 3.4 at 1 hour after ROSC were enrolled in the study. Average time for ROSC was 16.5 minutes. Demographic and baseline variables were comparable amongst survivors and nonsurvivors except age (survivors 43 years and nonsurvivors 65 years). Average duration to achieve target temperature was 4.9 hours. Five out of 13 (38.46\%) patients survived without any neurological deficit or cognitive dysfunction (Cerebral Performance Category - 1). Out of eight nonsurvivors, six died due to cardiogenic shock, one died due to refractory hypoxia and in one case relatives opted for withhold of aggressive care. Cardiac arrest was out of hospital in eight patients (three survivors and five nonsurvivors) and intra-hospital in five (two survivors and three nonsurvivors).

Conclusion TH may have beneficial effects in the neurological outcome of patients having non-VT/VF cardiac arrest. Additional controlled studies are warranted to establish efficacy of TH as a treatment for nonVT/VF cardiac arrest. 
P282

Comparison of cold crystalloid and colloid infusions for induction of therapeutic hypothermia

RS Skulec, AT Truhlar, ZT Turek, RP Parizkova, VC Cerny

Charles University in Praque, University Hospital Hradec Kralove, Czech

Republic

Critical Care 2012, 16(Suppl 1):P282 (doi: 10.1186/cc10889)

Introduction While cold crystalloids have been used for induction of therapeutic hypothermia after cardiac arrest [1,2], the effectiveness of cold colloids has not so far been evaluated. Therefore, we investigated the cooling effect of rapid intravenous infusion of cold crystalloid compared to colloid in a porcine model of ventricular fibrillation (VF).

Methods VF was electrically induced in 22 anesthetized domestic pigs $(33 \pm 2 \mathrm{~kg}$ ). Defibrillation was attempted after 15 minutes CPR using the AutoPulse (Zoll Medical, USA) and artificial ventilation. After spontaneous circulation was restored, the animals were randomized to receive either $1,500 \mathrm{ml}$ of $1^{\circ} \mathrm{C}$ cold normal saline (group $A ; n=9$ ) within 20 minutes using a Zoll Power Infuser, or $1,500 \mathrm{ml}$ of $1{ }^{\circ} \mathrm{C}$ cold Voluven (6\% hydroxyethyl starch $130 / 0.4$ in $0.9 \% \mathrm{NaCl}$ ) (group $\mathrm{B} ; n=9$ ), or no infusion (group $C ; n=4$ ). The animals were observed for 90 minutes following infusion. Cerebral, rectal, intramuscular, pulmonary artery, and subcutaneous fat body temperatures (BT) were continuously recorded using GES 130 temperature probes and GMH 3250 digital thermometers (Greisinger Electronic, Germany). Data were analyzed with JMP 3.2 software (SAS Institute, USA) and are expressed as a mean \pm SD. $P<0.05$ was considered statistically significant.

Results In total, $46.6 \pm 3.2 \mathrm{ml} / \mathrm{kg}$ cold normal saline was infused in group A, and $45.7 \pm 2.7 \mathrm{ml} / \mathrm{kg}$ cold colloid in group B. The animals treated with cold fluids achieved a significant decrease of BT in all measurement sites while there was a spontaneous increase in group $C$ $(P<0.05)$. At the time of finishing infusion there was a greater decrease in cerebral and pulmonary artery $\mathrm{BT}$ in group $\mathrm{A}$ compared to group $\mathrm{B}$ $\left(-1.7 \pm 0.4\right.$ vs. $-1.1 \pm 0.3^{\circ} \mathrm{C}, P=0.002$; and $-2.1 \pm 0.3$ vs. $-1.6 \pm 0.2^{\circ} \mathrm{C}$, $P<0.001$ respectively). Area under the curve analysis of the decrease in intracerebral BT revealed a more vigorous cooling effect in group A compared to $B\left(-91 \pm 30\right.$ vs. $-62 \pm 27^{\circ} \mathrm{C} /$ minute, $\left.P=0.047\right)$. There was also a higher calculated enthalpy for crystalloid solution compared to colloid in the time-point of maximal BT decrease $(33.9 \pm 5.7 \mathrm{vs}$. $26.6 \pm 3.4 \mathrm{~kJ} / \mathrm{kg}, P<0.05)$.

Conclusion Cold crystalloid infusions resulted in a more intense cooling effect than colloid infusions of the same temperature and infusion rate in this porcine model of cardiac arrest.

Acknowledgements The study was supported by grant MZO 00179906.

References

1. Kim F, et al: Circulation 2007, 115:3064-3070.

2. Skulec R, et al.: Crit Care 2010, 14:R231.

P283

Cerebral oxygenation during induction of therapeutic hypothermia after cardiac arrest

I Meex, J Dens, F Jans, C De Deyne

Ziekenhuis Oost-Limburg, Genk, Belgium

Critical Care 2012, 16(Suppl 1):P283 (doi: 10.1186/cc10890)

Introduction Induced mild hypothermia $\left(32\right.$ to $34^{\circ} \mathrm{C}$ ) improves survival and neurological outcome after CA. Near-infrared spectroscopy (NIRS) measures cerebral tissue oxygen saturation $\left(\mathrm{SctO}_{2}\right)$. As of today, no data are available on $\mathrm{SctO}_{2}$ monitoring during therapeutic hypothermia (TH). Therefore, $\mathrm{SctO}_{2}$ was measured in this study during the first 36 hours after $C A$.

Methods After IRB approval, data were collected from 23 patients. Cold saline $(30 \mathrm{ml} / \mathrm{kg})$ was administered as soon as possible after hospital admission. TH $\left(33^{\circ} \mathrm{C}\right)$ was induced by endovascular or surface cooling and maintained for 24 hours. All patients were sedated (propofol/ remifentanil) for the duration of TH. NIRS sensors were bilaterally applied to the frontotemporal area before start of TH. Patients were monitored during induction, maintenance and recovery of $\mathrm{TH}$.

Results Of 23 patients, 11 patients did not survive until hospital discharge due to post-ischemic brain damage. Twelve patients survived until hospital discharge, of whom eight without any neurological impairment. Temperature at admission was $34.6^{\circ} \mathrm{C}\left( \pm 0.5^{\circ} \mathrm{C}\right)$. Patients reached the target temperature of $33^{\circ} \mathrm{C}, 4$ hours after induction of TH. Two patients died during maintenance of TH due to refractory hemodynamic shock. In all patients, $\mathrm{SctO}_{2}$ values started above $65 \%$. Two and a half hours after induction of $\mathrm{TH}, \mathrm{SctO}_{2}$ values decreased with $9 \%( \pm 3 \%)$. The decrease in cerebral oxygenation during induction of TH was not associated with a major change in hemodynamic parameters (MAP before induction of TH: $79 \mathrm{mmHg} \pm 19$; at $33^{\circ} \mathrm{C}: 82 \mathrm{mmHg} \pm 9$ ), nor with a major change in systemic oxygenation $\left(\mathrm{SpO}_{2}\right.$ before $\mathrm{TH}: 99 \% \pm 1$; at $33^{\circ} \mathrm{C}: 97 \% \pm 3$ ). In patients who survived until hospital discharge, $\mathrm{SctO}_{2}$ returned to baseline values, 3.5 hours after induction of $\mathrm{TH}$, before the target temperature of $33^{\circ} \mathrm{C}$ was reached. In patients who did not survive the hospital stay, $\mathrm{SctO}_{2}$ remained lower than baseline values until the target temperature was reached. In these nonsurvivors, $\mathrm{SctO}_{2}$ values did only return to baseline values during maintenance of TH (10 hours after induction of TH). During maintenance of TH and rewarming $\left(0.3^{\circ} \mathrm{C}\right)$, no further significant changes in $\mathrm{SctO}_{2}$ values were observed.

Conclusion Noninvasive monitoring revealed a decrease in cerebral oxygenation during induction of mild hypothermia in patients after cardiac arrest. We observed a difference in oxygenation between hospital survivors and nonsurvivors.

\section{P284}

Survey on the management of patients treated with therapeutic hypothermia post cardiac arrest in London hospitals

A Walecka, SC Robert, A Prasad

The Royal Free Hospital, London, UK

Critical Care 2012, 16(Suppl 1):P284 (doi: 10.1186/cc10891)

Introduction The second UK national survey on therapeutic hypothermia $(\mathrm{TH})$ post cardiac arrest demonstrated an impressive increase in its implementation across the UK (from $28 \%$ to $85.6 \%$ ) [1]. Therapeutic hypothermia, however, induces numerous physiological and pathophysiological changes and therefore should be performed in a standardised and controlled manner in order to be safe and effective. We carried out a telephone survey to determine the current $\mathrm{TH}$ practice in London hospitals.

Methods Thirty-two London intensive care units (ITUs) were contacted by telephone. The data were analysed using Excel spreadsheets.

Results of the 32 ITUs contacted, 30 (93.7\%) had been using therapeutic hypothermia following cardiac arrest. Fifteen $(50 \%)$ of them were teaching hospitals and the remaining 15 (50\%) were district general hospitals. Twenty-two (73.3\%) hospitals had a protocol in place. External cooling was the preferred method used by $28(93.3 \%)$ hospitals. The target temperature varied from 32 to $35^{\circ} \mathrm{C}$ with two $(6.7 \%)$ ITUs targeting a temperature of $32^{\circ} \mathrm{C}, 11(36.7 \%)$ of $33^{\circ} \mathrm{C}$, six $(20 \%)$ of $34^{\circ} \mathrm{C}$, one $(10 \%)$ of 32 to $33^{\circ} \mathrm{C}$, seven $(23.4 \%)$ of 32 to $34^{\circ} \mathrm{C}$ and one $(10 \%)$ of 34 to $35^{\circ} \mathrm{C}$. The time of cooling varied between 12 and 48 hours. The cooling period was measured from initiation of cooling by $22(73.3 \%)$ ITUs and from achievement of target temperature by six (20\%) ITUs. Two responders were not sure how it was measured. Twenty-five $(83.3 \%)$ units measured core temperature during the cooling. Passive rewarming was used by $20(66.6 \%)$ responders. Twenty-four $(80 \%)$ units maintain normothermia post therapeutic hypothermia. From additional aspects of the management of the induced hypothermia, 20 (66.6\%) ITUs adjusted drug doses while starting TH, 15 (50\%) monitored the depth of sedation, and $17(56.6 \%)$ regularly checked train of four in paralyzed patients. Shivering was treated with sedation and paralysis by $25(83.3 \%)$ of responders. Pregnancy status of all women younger than 50 years old was checked by $10(33.3 \%)$ units. Fifteen $(50 \%)$ units do not audit the practice regularly.

Conclusion There are significant variations in practice between London hospitals which probably reflect the ongoing debate on the optimal management of patients treated with $\mathrm{TH}$. Of note is that $50 \%$ of surveyed hospitals do not audit the current practice regularly which may have an impact on the quality and effectiveness of therapeutic cooling.

Reference

1. Binks AC, et al:: Therapeutic hypothermia after cardiac arrest -

implementation in UK intensive care units. Anaesthesia 2010, 65:260-265. 
P285

Simplified EEG/aEEG to monitor the injured brain after cardiac arrest

H Friberg', M Rundgren', E Westhall', N Nielsen², T Cronberg'

'Lund University, Lund, Sweden; 'Helsingborg Hospital, Helsingborg, Sweden

Critical Care 2012, 16(Suppl 1):P285 (doi: 10.1186/cc10892)

Introduction Once hemodynamics is stabilized, the main concern in the comatose cardiac arrest patient is the status of the brain and the potential recovery of brain functions. Approximately $30 \%$ of comatose cardiac arrest patients develop electrographic seizures, many of whom have associated clinical seizures that may be concealed by sedation and paralyzers. As part of the Lund coma project, we have continuously monitored and evaluated simplified EEG/aEEG in consecutive hypothermia-treated cardiac arrest patients.

Methods Needle electrodes corresponding to the F3 to P3 and F4 to P4 leads were applied at admission to the ICU. The Nervus NicoletOne ${ }^{\circledast}$ monitor (CareFusion Inc.) was used to display the continuous raw EEG curves as well as the amplitude integrated EEG (aEEG). The EEG data were available to the treating intensivist and were linked to the Department of Neurophysiology, where the accumulated data were interpreted once daily, 5 days a week.

Results Monitoring of aEEG was successfully applied in all patients. Four dominating patterns were defined; flat, continuous, suppressionburst (SB) and electrographic status epilepticus (ESE) [1]. We identified three groups of patients: one group with mild brain injury and a good outcome, characterized by a return of a continuous EEG pattern during the first 24 hours. A second group with severe brain injury and a poor outcome had a flat EEG or a SB pattern during the first 24 hours, which evolved into alfa-coma or a treatment refractory ESE. In this group, early myoclonus was common. The third group with a presumed intermediate brain injury often developed a late ESE during rewarming, from a continuous and sometimes reactive background EEG. In this third group, which presented with low brain damage biomarkers and unremarkable MR brain imaging, there were survivors, some of whom received prolonged care in the ICU [2].

Conclusion Simplified EEG/aEEG is easily applied and well adapted to the ICU environment. In combination with the raw EEG, the aEEG serves as a trend monitor of the injured brain in the comatose patient after cardiac arrest. The simplified EEG/aEEG helps detect ESE and is of importance for guiding anticonvulsive treatment. The evolution of the EEG pattern mirrors the natural recovery of cortical function after cardiac arrest and gives useful positive as well as negative prognostic information. Simplified EEG/aEEG serves the needs of the intensivist and has the potential to become part of a standard monitoring regimen. References

1. Rundgren M, et al:: Crit Care Med 2010, 38:1838

2. Cronberg T, et al:: Neurology 2011, 77:623.

P286

Usefulness of a Bispectral index oriented sedative method without neuromuscular blocker for therapeutic hypothermia after cardiac

arrest

S Shiraishi, Y Ohta, TTagami, Y Ono, T Masuno, H Yokota

Nippon Medical School, Tokyo, Japan

Critical Care 2012, 16(Suppl 1):P286 (doi: 10.1186/cc10893)

Introduction During therapeutic hypothermia (TH) after cardiac arrest (CA), neuromuscular blockers are often used to prevent or treat thermogenic shivering [1]. But the following risks due to neuromuscular paralysis are encountered: prolonged muscle weakness, hypostatic pneumonia and venous thromboembolism. So we evaluated the usefulness of Bispectral index (BIS) oriented sedation without neuromuscular blocker in six cases of post CA patients receiving $\mathrm{TH}$.

Methods Six consecutive patients admitted after CA and treated with $\mathrm{TH}$ by the same attending physicians' group were included. BIS monitoring was applied immediately after the admission to ER. After initial resuscitation and radiological examination, including coronary angiography and angioplasty, patients were admitted to the ICU and cooled down to a target body temperature of $34^{\circ} \mathrm{C}$ using a surface cooling system with an external pad. Target body temperature was maintained for 48 hours and rewarmed to $36^{\circ} \mathrm{C}$ over another 48 hours. As induction of patients' sedation, we injected $5 \mathrm{mg}$ midazolam and $0.2 \mu \mathrm{g}$ fentanyl intravenously just as we recognized patients' movement or immediately before induction of $\mathrm{TH}$. For maintenance of sedation, midazolam at dose $0.1 \mathrm{mg} / \mathrm{kg} / \mathrm{hour}$, dexmedetomidine at dose $0.4 \mu \mathrm{g} /$ $\mathrm{kg} /$ hour and fentanyl at doses $0.8 \mu \mathrm{g} / \mathrm{kg} / \mathrm{hour}$ were administrated continuously. The midazolam and the dexmedetomidine infusion were adjusted to a target BIS value of 40 or less. BIS monitoring was ceased after completion of both rewarming and discontinuation of sedative drugs.

Results In all six patients, TH was completed without severe complication, especially shivering movement and serious hypostatic pneumonia. Three patients presenting unstable BIS values lower than 10 during TH showed poor neurological outcome, while the other three patients presenting stable BIS values about 40 showed favorable neurological outcome. Myoclonic movement or convulsion, regarded as signs of bad outcome, was observed in two poor neurological outcome patients. Cough reflex was observed in two favorable neurological outcome patients throughout their $\mathrm{TH}$.

Conclusion BIS oriented sedation without neuromuscular blocker is feasible in maintaining TH for survivors from CA. By keeping muscular function, both noxious and beneficial movements are preserved and these help us to predict neurological outcome and prevent patients from hypostatic disorders.

Reference

1. Chamorro et al:: Anesth Analg 2010, 110:1328-1335.

\section{P287}

Predictive factors of neurologic outcome in therapeutic

hypothermia after prehospital return of spontaneous circulation

Y Ohta, S Shiraishi, Y Ono, G Matsumoto, T Tagami, T Masuno, H Yokota Nippon Medical School, Tokyo, Japan

Critical Care 2012, 16(Suppl 1):P287 (doi: 10.1186/cc10894)

Introduction Induction of hypothermia is generally accepted to improve neurologic recovery of out-of-hospital cardiopulmonary arrest (CPA). Early prognostication of post-CPA patients is challenging. The aim of the present study was to evaluate the predictive factors for neurologic outcome in out-of-hospital cardiac arrest patients who returned their spontaneous circulation in a prehospital setting (PROSC) and underwent therapeutic hypothermia (TH).

Methods PROSC patients transported to our institution between January 2007 and May 2011 were retrospectively analyzed. TH was performed for all comatose PROSC patients admitted to the hospital for post-resuscitation care, regardless of the etiology of cardiac arrest or patient's age, except for those whose hemodynamic and pulmonary status could not be maintained. Neurological outcome at 1 month was compared as a primary end-point using the Pittsburgh cerebral performance category (CPC) scale and patients were classified into a favorable outcome group (CPC 1 and 2) or poor outcome group (CPC 3 to 5). Clinical parameters were compared between patients whose neurologic outcomes were favorable and poor.

Results There were 33 PROSC patients: 27 (81\%) survived and 14 (42\%) achieved a favorable neurological outcome. The cause of the CPA was cardiac attack in 17, noncardiac attack in 10, and unknown in six patients. Average age in the favorable recovery group was significantly younger than in the poor recovery group $(62.5$ vs. $70.3, P<0.05)$. The favorable group was all the proportion of patients with ventricular fibrillation (VF) at the scene. Of the 14 that achieved a favorable neurological outcome, the cause of the CPA was cardiac attack in 12 and unknown in two patients. On the other hand, electrocardiograms of poor neurological outcome showed VF, pulseless electrical activity, and asystole. The cause of the CPA was cardiac attack in five, noncardiac attack in 10, and unknown in four. Average pH of artery blood gas $(A B G)$ in the favorable recovery group was significantly higher than in the poor recovery group (7.31 vs. 7.17, $P<0.004)$. The receiver-operator characteristic curve for $\mathrm{pH}$ of $\mathrm{ABG}$ on arrival was analyzed. The area under the curve was 0.76 .

Conclusion A suitable $\mathrm{pH}$ at the time of hospital arrival was associated with a favorable neurologic outcome among post-cardiac arrest patients without presumed noncardiac etiology. 
P288

Employment status 1 year after out-of-hospital cardiac arrest in comatose patients treated with therapeutic hypothermia

K Kragholm¹, M Skovmoeller', AL Christensen', K Fonager², HH Tilsted ${ }^{2}$, H Kirkegaard ${ }^{3}$, I De Haas', BS Rasmussen'

'Cardiovascular Research Center, Aalborg, Denmark; ${ }^{2}$ Aarhus University Hospital, Aalborg, Denmark; ${ }^{3}$ Aarhus University Hospital, Skejby, Aarhus, Denmark

Critical Care 2012, 16(Suppl 1):P288 (doi: 10.1186/cc10895)

Introduction Therapeutic-induced mild hypothermia (TIMH) with a core temperature of 32 to $34^{\circ} \mathrm{C}$ for 12 to 24 hours for comatose survivors of out-of-hospital cardiac arrest (OHCA) with ventricular fibrillation or tachycardia has improved survival and neurologic outcome $[1,2]$. The aim of this study was to evaluate the incidence of patients returning to work 1 year after survival of OHCA treated with TIMH.

Methods From 30 June 2004 to 30 June 2009, OHCA patients between 18 and 65 years of age treated with TIMH were identified by the Danish National Patient Registry and intensive unit registrations. Data were collected from ambulance and hospital records. Employment status was registered prior to and 1 year after OHCA from the Danish Ministry of Employment and Welfare database, using five work categories (WC): WC 1 , working full-time and independent of any social welfare; WC 2 , unemployed but able to work; WC 3 , on sick leave and receiving social welfare; WC 4 , substantially reduced ability to work: and WC 5 , on early retirement.

Results One hundred and thirty-three patients were identified. Forty eight patients were excluded from the final analysis, of which 29 patients were not able to work at baseline (WC 3 to 5), 14 patients in WC 1 to 2 at baseline died in hospital, three patients died after hospital discharge and two patients had turned 65 years of age at follow-up and went on regular retirement. A total of 85 patients in WC 1 to 2 at baseline were included in the final analysis, of which $55(64.7 \%)$ of these initially comatose patients with OHCA treated with TIMH had returned to work 1 year after OHCA.

Conclusion Approximately two-thirds of the survivors belonging to WC 1 to 2 at baseline have returned to work at 1 year follow-up after OHCA treated with TIMH. A larger study is needed to confirm these results and to determine predictors of returning to work in comatose patients after OHCA treated with TIMH.

\section{References}

1. Bernard SA, Gray TW, Buist MD, Jones BM, Silvester W, Gutteridge G, Smith K: Treatment of comatose survivors of out of hospital cardiac arrest with induced hypothermia. N Engl J Med 2002, 346:557-563.

2. Hypothemia After Cardiac Arrest Study Group: Mild therapeutic hypothermia to improve the outcome after cardiac arrest. N Engl J Med 2002, 346:549-556.

P289

Changes in cerebrospinal fluid and serum cytokine levels in severe traumatic brain injury patients

TSaito' ${ }^{1}$, H Kushi², J Sato', A Yoshino', KTanjo

'Nihon University, School of Medicine, Tokyo, Japan; ${ }^{2}$ Nihon University, College of Humanities and Sciences, Tokyo, Japan

Critical Care 2012, 16(Suppl 1):P289 (doi: 10.1186/cc10896)

Introduction Inflammatory response following brain injury begins with brain tissue injury triggered neuroinflammation, which induces a systemic inflammatory response syndrome. We investigated the characteristics of the acute inflammatory response following severe traumatic brain injury through changes in cerebrospinal fluid (CSF) and serum cytokine levels.

Methods The subjects were 24 patients with severe traumatic brain injury. We measured levels of the proinflammatory cytokines IL- 6 and $\mathrm{IL}-8$, and the anti-inflammatory cytokine IL-10 in peripheral blood and CSF on four occasions, at the time of admission and after 24 hours, 72 hours and 1 week.

Results CSF and serum IL-6 levels continued to rise until 72 hours after admission. CSF IL- 6 levels were 50 to 400 times serum levels. Serum IL-8 levels remained at 20 to $30 \mathrm{pg} / \mathrm{ml}$. CSF IL-8 levels were 100 to 800 times the serum levels, and remained high after the peak of $23,500 \mathrm{pg} /$ $\mathrm{ml}$ at the time of admission. CSF and serum IL-10 levels were high, but not abnormally high as for IL-6 and IL-8, and decreased with time. The difference in CSF and serum levels, as seen for IL- 6 and IL-8, was not seen for IL-10.

Conclusion We elucidated the following points concerning the acute inflammatory response following severe traumatic brain injury. High levels of IL- 6 and IL-8 are maintained in both CSF and serum. CSF levels of IL- 6 and IL-8 are one or two orders of magnitude greater than serum levels. Upregulation of IL-10 is minimal in comparison with IL- 6 and IL-8, suggesting that in neuroinflammation IL-10 functions poorly as an antiinflammatory cytokine.

\section{P290}

Noninvasive cerebral oxygenation monitoring during rapid ventricular pacing in transcutaneous aortic valve implant

J Dens, I Meex, F Jans, H Gutherman, C De Deyne

Ziekenhuis Oost-Limburg, Genk, Belgium

Critical Care 2012, 16(Suppl 1):P290 (doi: 10.1186/cc10897)

Introduction Most recent attention in interventional cardiology is now directed towards treatment of valvular heart disease. In patients with high-risk cardiac surgery, transcutaneous aortic valve implantation (TAVI) could offer a therapeutic solution. Near-infrared spectroscopy (NIRS) has been introduced as a useful noninvasive cerebral monitoring technique assessing cerebral oxygenation. As of today, no reports have been published on the use of any NIRS technology during TAVI procedures. During valve prosthesis implantation, a cardiac standstill by rapid ventricular pacing (RVP) is induced to minimize cardiac motion. While RVP is advantageous for valve positioning, a combination of rapid heart rate and ventricular hypertrophy can induce a complete loss of cardiac output. In most cases, this hemodynamic deficit is well tolerated, due to the brief duration of RVP. But as of today no data are available on cerebral oxygenation during these critical periods of RVP. Methods We report on 10 consecutive patients ( $>75$ years, major comorbidities) suffering from severe aortic stenosis. Bilateral ForeSight sensors were applied after induction of anesthesia. We were especially interested if any change in cerebral oxygenation ( $\mathrm{SctO}_{2}$ monitoring) occurred during these RVP periods.

Results In all patients, the procedure was technically successfully performed. Mean SctO before RVP was 67\% (59 to 71\%) and immediately decreased during RVP to mean $54 \%$ (37 to $70 \%$ ). In seven patients, $\mathrm{RVP}$ resulted in $\mathrm{SctO}$ decreases below $55 \%$ (mean $44 \%$; range 37 to $52 \%$ ). These decreases lasted for mean 20 minutes (14 seconds to 87 minutes). Systolic blood pressure before RVP was mean $135 \mathrm{mmHg}$ ( 95 to $165 \mathrm{mmHg}$ ) and decreased to mean $74 \mathrm{mmHg}$ (112 to $42 \mathrm{mmHg}$ ) during RVP. In six patients, RVP resulted in a decrease in systolic blood pressure below $90 \mathrm{mmHg}$, which was immediately countered by vasoactive drugs (adrenaline). In two patients, extensive hypotension persisted despite vasoactive support and CPR had to be initiated. In one patient, $\mathrm{SctO}_{2}$ values remained below $55 \%$ for 87 minutes and the patient was declared brain dead 48 hours later.

Conclusion Transcutaneous cardiac interventions, especially those with transient cardiac standstill, can induce longlasting intraprocedural inadequacy of cerebral perfusion, despite immediate restoration of normal blood pressure. Future strategies should therefore be focused on optimalizing cerebral oxygenation before RVP.

\section{P291}

Novel models to predict elevated intracranial pressure during intensive care and long-term neurological outcome after TBI F Guiza', B Depreitere', I Piper², G Van den Berghe', G Meyfroidt 'UZ Leuven, Belgium; 2 2 Southern General Hospital, Glasgow, UK Critical Care 2012, 16(Suppl 1):P291 (doi: 10.1186/cc10898)

Introduction Elevated intracranial pressure (ICP) episodes are associated with poor outcome and should be prevented. We developed models to predict these episodes 30 minutes in advance, and to predict long-term neurological outcome by using dynamic characteristics of continuous ICP and mean arterial pressure (MAP) monitoring. 


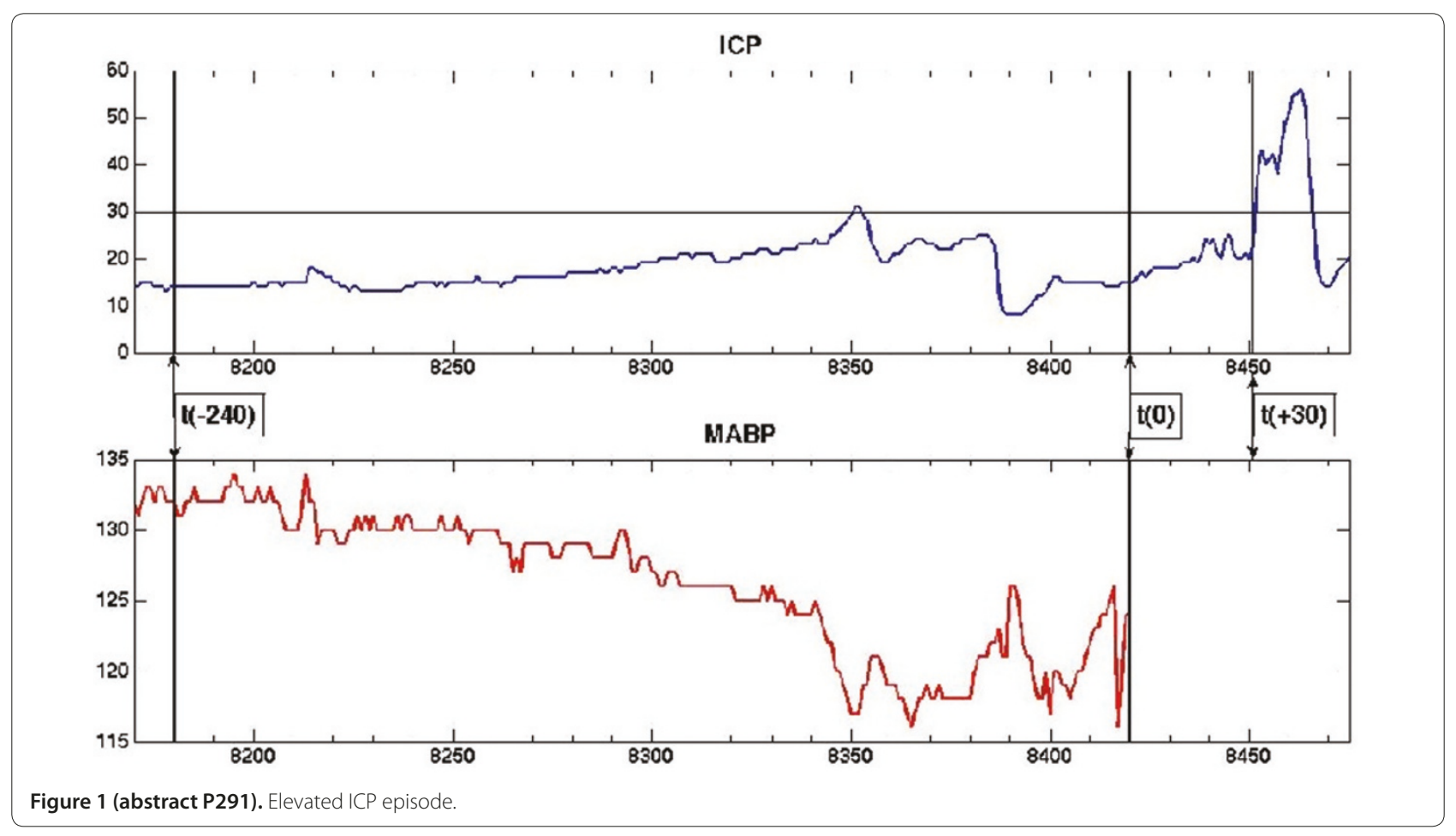

Methods The Brain-IT [1] dataset has records for 264 patients from 22 neuro-ICUs in 11 European countries. Logistic regression and Gaussian processes (machine learning method) were used. CRASH [2] and IMPACT [3] predictors were used together with dynamic data. Results Predictions of elevated ICP episodes (Figure 1) were externally validated with good calibration and discrimination (AUROC 0.87). Prediction of poor neurological outcome at 6 months (GOS 1 to 2) with static data had 0.72 AUROC; adding dynamic information increased performance to 0.9 (Table 1).

Table 1 (abstract P291). Model performance

\begin{tabular}{lccc}
\hline & Elevated ICP & GOS 1 to 2 static & GOS 1 to 2 dynamic \\
\hline AUROC & 0.87 & 0.72 & 0.90 \\
HL P value & 0.12 & 0.51 & 0.95 \\
Brier scaled & $39.4 \%$ & $7.7 \%$ & $46 \%$ \\
\hline
\end{tabular}

Conclusion Dynamic data in continuous MAP and ICP monitoring allows prediction of elevated ICP. Adding information of the first 24 hours of ICP and MAP to known risk factors allows accurate prediction of neurological outcome at 6 months. References

1. Piper I, et al:: Acta Neurochir 2003, 145:615-628.

2. Murray GD, et al:: J Neurotrauma 2007, 24:329-337.

3. CRASH Trial Collaborators: BMJ 2008, 336:425-429.

\section{P292}

Transcranial Doppler pulsatility index is a poor predictor of hydrocephalus in patients with aneurysmal subarachnoid haemorrhage

MH Kiel, AW Oldenbeuving, M Sluzewski, JA Van Oers, D Ramnarain

St Elisabeth Hospital, Tilburg, the Netherlands

Critical Care 2012, 16(Suppl 1):P292 (doi: 10.1186/cc10899)

Introduction Hydrocephalus is a common complication of aneurysmal subarachnoid haemorrhage (aSAH). The increase in intracranial pressure is associated with increased mortality and morbidity. Early recognition and intervention in these patients is essential in order to achieve favourable outcome. In the literature the value of noninvasive measurement of transcranial Doppler (TCD)-derived pulsatility index (PI) in predicting increased intracranial pressure remains questionable. The aim of this study was to examine the value of $\mathrm{PI}$ in predicting hydrocephalus in patients with aSAH.

Methods In a retrospective cohort study from January 2010 to June 2011,61 patients with aSAH were diagnosed with hydrocephalus on CT scan during treatment in our ICU. On 93 occasions of TCD recordings of the middle cerebral artery, PI was calculated on the same day. Results See Table 1 and Figure 1. Ninety-three CT scans could be correlated with PI on the same day of the scan. Using a cut-off value

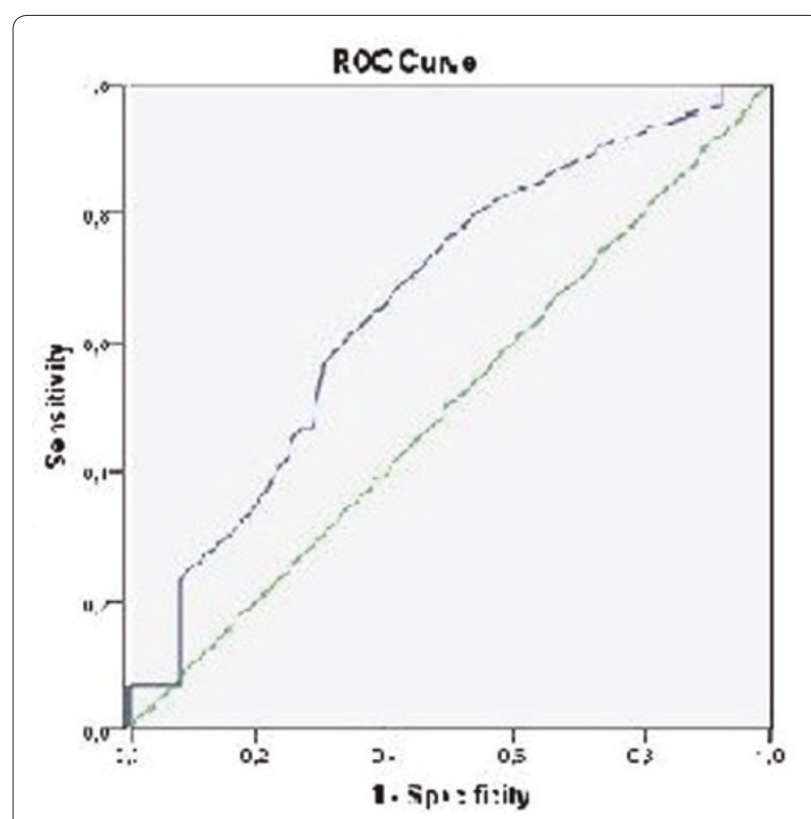

Figure 1 (abstract P292). Receiver operating characteristic curve. 
Table 1 (abstract P292). The $2 \times 2$ table for PI 1.4

\begin{tabular}{lccc}
\hline Index test & Hydrocephalus + & Hydrocephalus - & Total \\
\hline $\mathrm{PI}>1.4$ & 7 & 5 & 12 \\
$\mathrm{PI} \leq 1.4$ & 23 & 58 & 81 \\
Total & 30 & 63 & 93
\end{tabular}

of $\mathrm{PI}>1.4$, sensitivity was low (23.3\%) and specificity was high (92.1\%). Negative and positive predictive values were $71.6 \%$ resp. $58.3 \%$. The receiver operating characteristic curve showed an area under the curve of 0.67. The likelihood ratio for a negative (LR-) resp. positive (LR+) test was 0.83 resp. 2.94 . Pretest probability of $32 \%$ increased to $57 \%$ posttest probability with $\mathrm{PI}>1.4$ and decreased to $28 \%$ with $\mathrm{PI} \leq 1.4$.

Conclusion PI with a cut-off value of 1.4 has a poor sensitivity and a high specificity. PI has limited value in ruling in and out hydrocephalus in aSAH patients due to a low LR+ and LR-

\section{P293}

Transcranial cerebral oximetry in newborn infants on mechanical ventilation as a method for prevention of hyperoxia and oxidative stress

V Estrin, A Simonova

Scientific Research Institute of Obstetrics and Pediatrics, Rostov on Don, Russia

Critical Care 2012, 16(Suppl 1):P293 (doi: 10.1186/cc10900)

Introduction The aim of this research was the effectiveness of respiratory therapy in infants with respiratory distress syndrome (RDS) who are ventilated by correcting the oxygen status and parameter optimization of ventilation by determining the oxygen saturation in the brain by transcranial cerebral oximetry (TCO).

Methods A total of 24 infants born in the physiological department of a maternity hospital in RNIIAP was studied. All of the children were measured for the saturation of brain tissue oxygen (SctL, SctR) with a cerebral oximeter (ForeSight; USA) at 1, 3 and 5 days after birth. Later in the study, two groups of newborn infants on mechanical ventilation were included. Patients of group $1(n=38)$, modes of mechanical ventilation and $\mathrm{FiO}_{2}$ were determined under control TCO to bring rates of cerebral oxygenation to the age norm. Patients of group $2(n=37)$, mode selection and ventilator $\mathrm{FiO}_{2}$ were carried out under the control of pulse oximetry and partial oxygen tension $\left(\mathrm{pO}_{2}\right)$ in acid-base balance, without regard for performance of TCO. In all patients were determined serum peroxides (Oxystat test; BIOMEDICA GRUPPE, Germany), as well as the oxidation products of proteins (AOPP) in the serum of a set of AOPP (Immunodiagnostik, USA) for 1, 5 and 10 days. We measured blood gas parameters with an automatic analyzer (ABL; Denmark).

Results Determined by the age norm, TCO indicators for healthy infants amounted in the left hemisphere of the brain to $79.2 \pm 4.06 \%(P<0.01)$ and in the right hemisphere to $84.89 \pm 5.1 \%(P<0.01)$. We established in the group of infants where the mode selection ventilation and $\mathrm{FiO}_{2}$ were carried out on the basis of TCO indicators, an average $\mathrm{FiO}_{2}$ in the inspired mixture of $21 \%$ with an average $\mathrm{pO}_{2}$ in blood capillaries $61.95 \pm 20.16 \%$, in contrast to $\mathrm{FiO}_{2} 55 \%$ with $\mathrm{pO}_{2}-78.01 \pm 18.93 \%$ in patients of group 2. Patients of group 1 showed significantly (in all cases $P<0.01$ ) decreased length of stay on mechanical ventilation (from 9.4 to 5.6 bed-days), compared with the control group. Investigation of the activity markers of oxidative stress showed three times reduction of the oxidation products of proteins (AOPP) and twofold reduction of peroxides in patients in the study group, compared with the control group, to 10 days of observation $(P<0.05)$.

Conclusion Monitoring of oxygen saturation in the brain tissue by TCO in infants with RDS reduces the mortality rate and the term of mechanical ventilation and hyperoxia.
P294

Cerebral oximetry and brain death in the ICU: data from seven cases N Billet' ${ }^{1}$ I Meex², M Vanderlaenen', R Heylen'1, W Boer ${ }^{1}$, C De Deyne',

FV Jans

'Ziekenhuis Oost-Limburg, Genk, Belgium; '2University of Hasselt, Diepenbeek, Belgium

Critical Care 2012, 16(Suppl 1):P294 (doi: 10.1186/cc10901)

Introduction Cerebral oximetry, using near-infrared spectroscopy to measure cerebral tissue oxygen saturation $\left(\mathrm{SctO}_{2}\right)$, is being increasingly used in the ICU. We hypothesized that if a patient becomes brain dead in the ICU, this must be reflected in $\mathrm{SctO}_{2}$ values. This might help in the timing of invasive procedures such as angiography, sometimes necessary in the confirmation of brain death.

Methods We retrospectively analyzed the cerebral oximetry data of seven patients with severe TBI or diffuse cerebral edema who evolved to brain death while being treated in the ICU. Absolute $\mathrm{SctO}_{2}$ values were continuously measured with ForeSight technology (Casmed) with sensors applied bilaterally to the forehead.

Results Three patients (one TBI and two SAH) with continuous ICP and $\mathrm{SctO}_{2}$ monitoring suffered, despite maximal medical treatment, a sudden (over 1 to 3 hours) increase in mean ICP from $32 \mathrm{mmHg}$ to $91 \mathrm{mmHg}$ (equalization of ICP and MAP). Over the same time period, a parallel decrease in mean $\mathrm{SctO}$ from $71 \%$ to $54 \%$ ICP was observed. One patient (cerebral edema after asphyxia) had continuous EEG and $\mathrm{SctO}_{2}$ monitoring: a sharp decrease in $\mathrm{SctO}_{2}$ from $67 \%$ to $56 \%$ over 30 minutes was accompanied by an increase in suppression ratio from $70 \%$ to $100 \%$. The absence of cerebral blood flow was confirmed by CT angiography. One patient (cerebral edema after prolonged CPR) had only $\mathrm{SctO}_{2}$ measurement for cerebral monitoring: during his stay in the ICU, there was a sudden decrease in SctO from 64\% to 54\% over a 90 -minute period. Shortly after this, the pupils became dilated and fixed. Brain death was confirmed by full EEG. Three brain-dead patients with documented absence of cerebral blood flow were monitored for $\mathrm{SctO}_{2}$ during subsequent organ donation procedure: $\mathrm{SctO}$, remained at a mean value of $59 \%$ during the procedure, and fell sharply only at the onset of circulatory arrest to reach a stable value of $25 \%$.

Conclusion In this small cohort of patients, the onset of brain death was accompanied in all cases by a sharp and large decrease in $\mathrm{SctO}_{2}$ from $67 \%$ to $55 \%$ (mean, $n=5$ ) and remained stable. SctO values only reached minimal values $(25 \%, n=3)$ at complete circulatory arrest. Our data suggest that $\mathrm{SctO}_{2}$ measurement may be helpful in the timing of the diagnosis of brain death, especially in those patients without ICP or continuous EEG monitoring.

\section{P295}

Deoxyhaemoglobin as a biomarker of cerebral autoregulation D Highton', A Ghosh', I Tachtsidis², C Kolyva², J Panovska², C Elwell², M Smith'

'National Hospital for Neurology and Neurosurgery, London, UK; ${ }^{2} U C L$, London, UK Critical Care 2012, 16(Suppl 1):P295 (doi: 10.1186/cc10902)

Introduction Cerebral autoregulation (CA) maintains cerebral blood flow over a range of perfusion pressure. Continuous CA monitoring might define pressure targets minimising secondary brain injury, but application is limited by available monitoring modalities. Near-infrared spectroscopy (NIRS) is a noninvasive optical technique characterising aspects of CA. The NIRS-derived tissue oxygenation index (TOI) is correlated with blood pressure (BP) to produce an index of vascular reactivity (TOx) [1]. The contribution from extracerebral tissues, optical complexity of injured brain and complex physiology represented by NIRS are likely to limit agreement with other techniques. NIRSmeasured deoxyhaemoglobin $(\mathrm{HHb})$ may have advantages as its physiological confounds are less complicated and are predominantly in the cerebral venous circulation. This study compares $\mathrm{HHb}$ with established indices of reactivity - the mean velocity index (Mx) and oxygen reactivity index (ORx).

Methods Thirteen brain-injured patients were studied. Ipsilateral 60 -minute recordings included intracranial pressure, brain tissue oxygen $\left(\mathrm{PbrO}_{2}\right)$, transcranial Doppler and NIRS (NIRO 100; Hamamatsu 
Photonics). ORx and Mx were derived from continuous correlation between BP and neuromonitoring [1]. $\mathrm{HHb}$ was compared identically deriving HHBx. Comparisons used Pearson correlation, subsequent analysis characterised time lags between $\mathrm{BP}$ and monitored variables ( 0.05 to $0.003 \mathrm{~Hz}$ ) with wavelet lag coherence.

Results There was correlation between $\operatorname{HHBx}(r=-0.62, P<0.01)$, ORx $(r=0.52, P<0.05)$ and Mx. TOx showed no significant correlation $(r=0.18)$ as individual recordings demonstrated TOI fluctuations paradoxical to other monitoring. The mean lag between $\mathrm{BP}$ and $\mathrm{HHb}$ (24 seconds) was shorter than $\mathrm{PbrO}_{2}$ (68 seconds).

Conclusion $\mathrm{HHb}$ may provide a surrogate to inform cerebrovascular reactivity assessment. Complexity in the oxyhaemoglobin component of TOI may be introduced by vasopressor-related skin artefact or arterial volume changes [2] explaining poor agreement of TOx. $\mathrm{HHb}$ is theoretically free of this effect but will vary with cerebral metabolism, venous dynamics and oxygenation and demonstrates lag behind BP changes. Future analyses might compensate using model-based analysis [3], potentially describing measures of vascular reactivity from multiple NIRS and neuromonitoring variables, incorporating widely different aspects of cerebral physiology.

References

1. Zweifel C, et al:: Stroke 2010, 41:1963-1968.

2. Ogoh S, et al.: Clin Physiol Funct Imaging 2011, 31:445-451.

3. Banaji M, et al:: PLoS Comput Biol 2008, 11:e1000212.

P296

Study of the acoustic stem evoked potentials in blood circulation disorder in the vertebral basilar basin

I Vlasova, T Vizilo, VTsiuriupa

Scientific Clinical Center of Miners' Health Protection, Leninsk-Kuznetsky,

Russia

Critical Care 2012, 16(Suppl 1):P296 (doi: 10.1186/cc10903)

Introduction Acoustic brainstem evoked potentials (ABEP) offer a possibility to objectivise disorder of the brain stem structure function. Methods There were flicks of $9.5 \mathrm{~Hz}$ with intensity $70 \mathrm{~dB}$ higher than the hearing threshold. The latency time of the I to $V$ peaks, the interpeak intervals (IPI), the peak amplitudes (PA) and the amplitude correlations were measured. The clinical neurophysiological assessment of 30 patients (16 men and 14 women, age from 40 to 70 years) with clinical presentation of ischemic stroke in the vertebral basilar basin (VBB) allowed us to determine the following forms of acute ischemic disorders of the brain circulation: transitory ischemic attacks (TIA) $(n=16)$, lacunar infarction (LI) $(n=10)$, and nonlacunar infarction (NLI) in $\operatorname{VBB}(n=4)$.

Results According to the ABEP the common feature in all groups of patients was the decrease of the correlation of the V PA to I PA that was significant in $56 \%$ cases in NLI, in $47 \%$ cases in $\mathrm{LI}$ and in $15 \%$ cases in TIA; the decrease of all PA (to 0.12 to $0.15 \mathrm{mkV}$ ) was significant in $49 \%$ cases in NLI and in 39\% cases in LI. A distinct tendency to the laterality of the peak latency increase in TIA and LI in 49\% of cases, and a significant laterality of the peak latency increase in 35\% that reflected the dissymmetric disorder of the neuronal acoustic activity of the brainstem were observed. There was a tendency to increase of the I to III and III to V intervals in 46 to $61 \%$ in TIA. The I to III and III to V IPI were significantly increased in LI and NLI, in $35 \%$ and $47 \%$ cases respectively. The patients with NLI demonstrated an increase of the I to V IPI. There was such neurophysiological dynamics. The reconstruction of the amplitude and peak latency in TIA was observed in $100 \%$ of cases in the treatment process. This was not registered in LI and NLI.

Conclusion All strokes in the VBB are characterized by functional changes on the part of the brain stem structures predominantly at the pontomedullary and pontomesencephalic levels. There is a dependence between stroke severity, brainstem structure damage and neurophysiological dynamics. ABEP allow one to objectivise the brain stem structure dysfunction in the VBB's disturbed circulation.

\section{P297}

Examination of the autonomic nervous system in the ICU: a pilot study

L Wieske, E Kiszer, C Verhamme, IN Van Schaik, MJ Schultz, J Horn

Academic Medical Center, Amsterdam, the Netherlands

Critical Care 2012, 16(Suppl 1):P297 (doi: 10.1186/cc10904)

Introduction The most widely used test for autonomic dysfunction in the ICU is the heart rate variability (HRV) test [1]. HRV is thought to be a very sensitive but less specific test [1]. Several other tests are available. For this pilot study we have investigated the ability of two tests, the skin wrinkle test (SWT), a test for postganglionic sympathetic function, and the cold face test (CFT), a reflex slowing heart rate after cold application to the forehead, to detect autonomic dysfunction in critically ill patients alongside the HRV.

Methods ICU patients mechanically ventilated for at least 3 days were included. Exclusion criteria: polynomic or autonomic neuropathy, admission after stroke or cardiac arrest. HRV was investigated using power spectral analysis of continuous 5-minute ECG recordings [1]. The simulated SWT was used and wrinkling was assessed on a five-point scale [2]. Under continuous ECG recording a cold pack was applied to measure the CFT [3]. Changes in SWT and CFT results over time were compared to the changes in the SOFA score. Studies procedures were also performed in 17 healthy controls.

Results Twelve patients were included (mean age: 54 (SD: 15)). HRV analysis showed decreased heart rate variability in all patients (median total power: $32 \mathrm{~ms}^{2}$ (IQR: 11 to 320$)$ ). The SWT could be performed in 10 patients. SWT results were abnormal (score $\leq 2)$ in $60 \%$ of cases $(6 \%$ in healthy controls; $P<0.01$ ). The CFT was done in nine patients. Critically ill patients showed a blunted response on the CFT (2.5\% increase in RR length ( $95 \% \mathrm{Cl}:-0.2 \%$ to $5.2 \%)$ vs. $7.1 \%$ in healthy controls $(95 \% \mathrm{Cl}$ : $3.7 \%$ to $10.5 \% ; P=0.03)$ ). Figure 1 displays the CFT results over time. Conclusion CFT detected autonomic dysfunction in critically ill patients better than the SWT and was easier to perform. Diagnostic accuracy and prognostic value need to be investigated.

References

1. Buchman TG, et al: Curr Opin Crit Care 2002, 8:311-315.

2. Wilder-Smith EP, et al:: Clin Neurophysiol 2009, 120:953-958.

3. Reyners AK, et al:: Eur J Appl Physiol 2000, 82:487-492.
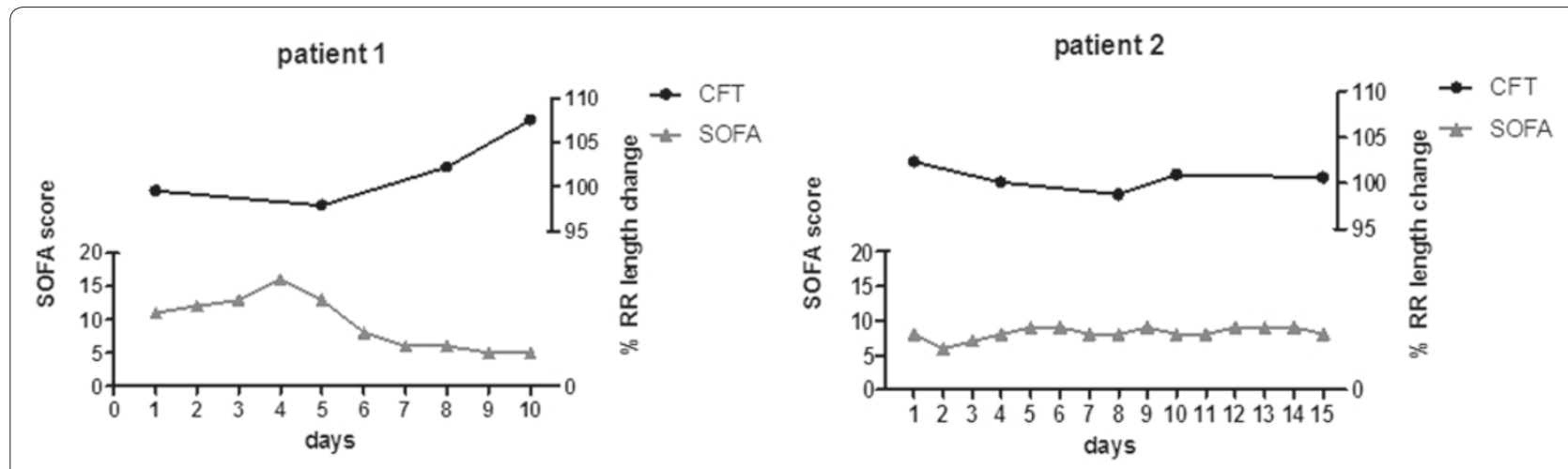

Figure 1 (abstract P297). Changes in cold face test (CFT) results over time. 
P298

Predictive value of glial fibrillary acidic protein for prognosis in patients with moderate and severe traumatic brain injury: a systematic review and meta-analysis

E Laroche', AF Turgeon', A Boutin', E Mercier1, F Lauzier', R Zarychanski²,

L Moore', J Granton³, P Archambault', F Lamontagne ${ }^{4}$, F Rousseau',

F Légaré', E Randell', J Lapointe', J Lacroix ${ }^{6}$, D Fergusson ${ }^{7}$

'Université Laval, Québec, Canada; 'University of Manitoba, Canada; ${ }^{3} U n i v e r s i t y$ of Toronto, Ontario, Canada; ${ }^{4}$ Université de Sherbrooke, Québec, Canada;

${ }^{5}$ Memorial University, NewFoundland, Canada; Université de Montréal, Québec,

Canada; 'Ottawa Hospital Research Institute, Ontario, Canada

Critical Care 2012, 16(Suppl 1):P298 (doi: 10.1186/cc10905)

Introduction Biomarkers have been proposed as potential prognostic indicators following a traumatic brain injury (TBI). Among those, glial fibrillary acidic protein (GFAP) has been one of the most studied. The objective of this study was to assess the prognostic value of GFAP levels in patients with moderate to severe TBI.

Methods We systematically searched Medline, Embase, Cochrane Central, Scopus, BIOSIS, TRIP, conference abstracts, bibliography of selected studies and narrative reviews. Cohort studies including $\geq 4$ patients with moderate or severe TBI and reporting GFAP levels (sampled within the first 24 hours of care) from any biological tissue or fluid, and mortality or Glasgow Outcome Scale (GOS), were eligible. Two independent reviewers screened all citations, selected eligible studies and extracted data using a standardized data extraction form. Pooled results from random effect models are presented using geometric mean ratios (GMRs). $1^{2}$ tests were used to measure statistical heterogeneity.

Results We retrieved 4,709 citations and eight studies were deemed potentially eligible. Among those, one was found to be a duplicate publication. Seven studies were thus included $(n=404)$. Four studies presented data on mortality ( 3 or 6 months) and four studies used the GOS (6 or 12 months) as an outcome measure. We found significant associations between serum GFAP levels and mortality in pooled analysis of three studies (GMR $14.73(95 \% \mathrm{Cl} 5.93$ to 34.12$) ; I^{2}=79 \%$ ), and between GFAP and GOS $\leq 3$ in three studies (GMR 8.80 (95\% Cl 3.94 to 19.66$) ;\left.\right|^{2}=77 \%$ ). Two studies could not be used in pooled analyses: one presented means of GFAP levels from multiple samplings over time (GMR 1.98 (95\% Cl 1.06 to 3.70)) while the other presented the highest peak levels of GFAP during the acute phase of care (GMR $3.20(95 \% \mathrm{Cl}$ 1.82 to 5.65$)$ ).
Conclusion Serum GFAP levels following TBI were significantly higher in patients showing an unfavourable prognosis (death or GOS $\leq 3$ ). The small number of studies included precluded further exploration of statistical heterogeneity. More investigations of the association between serum GFAP levels and prognosis following TBI are needed before recommending for routine use for neuroprognostication.

P299

Prevalence of pituitary disorders associated with traumatic brain injury: a systematic review

F Lauzier1', O Lachance', B Senay², I Côté2, P Archambault², F Lamontagne³, A Boutin', L Moore', F Bernard ${ }^{4}$, C Gagnon'2, D Cook ${ }^{5}$, AF Turgeon'

'CHA-Hôpital de l'Enfant-Jésus, Université Laval, Québec, Canada; '2Université

Laval, Québec, Canada; ${ }^{3}$ Université de Sherbrooke, Canada; ${ }^{4}$ Université de

Montréal, Canada; ${ }^{5}$ McMaster University, Hamilton, Canada

Critical Care 2012, 16(Suppl 1):P299 (doi: 10.1186/cc10906)

Introduction Pituitary disorders are an often-neglected consequence of traumatic brain injury (TBI). We systematically reviewed their prevalence in studies with low risk of bias including moderate/severe TBI patients.

Methods We searched EMBASE, MEDLINE, Scopus, Cochrane Central Register, BIOSIS, Trip Database, references of included studies and narrative reviews. We included cohort studies, cross-sectional studies and RCTs that tested the integrity of $\geq 1$ pituitary axis in adult victims of TBI. Two investigators independently reviewed selected citations, extracted data and assessed the risk of bias. Studies including $<10 \%$ of mild TBI victims were considered as involving mainly moderate/severe TBI patients. Prevalence is reported as weighted mean (lowest and highest prevalence) in three time-frames: acute $(<1$ month post TBI), mid ( 3 to 12 months) and long-term setting ( $>12$ months). Studies were considered at low risk of bias if the authors defined inclusion/exclusion criteria, avoided voluntary sampling, and tested $>90 \%$ of included patients with proper detailed diagnostic criteria. Studies testing all pituitary axes were considered as evaluating hypopituitarism, which was defined as the dysfunction of at least one axis.

Results Among 12,514 citations, we included 55 studies $(4,648$ patients). Patients suffered from mild $(11.9 \%, n=555)$, moderate $(7.9 \%$, $n=367)$ and severe $(30.4 \%, n=1,415) \mathrm{TBI}$, others being of unknown severity. Prevalences of pituitary axis dysfunction are reported in Table 1. Few studies considering mainly moderate/severe TBI patients were at low risk of bias.

Table 1 (abstract P299)

\begin{tabular}{|c|c|c|c|c|c|c|}
\hline & Hypopituitarism & GH & АСTH & TSH & Gonadal & ADH \\
\hline \multicolumn{7}{|l|}{ Acute phase } \\
\hline $\begin{array}{l}\text { All studies, } \\
n \text { (patients) }\end{array}$ & $\begin{array}{c}58.3 \%(32.3 \text { to } 76.1) \\
6(513)\end{array}$ & $\begin{array}{c}28.7 \% \text { (8.8 to } 77.2) \\
9(784)\end{array}$ & $\begin{array}{c}14.3 \% \text { (0.7 to } 45.7) \\
11(958)\end{array}$ & $\begin{array}{c}9.4 \%(0 \text { to } 40.6) \\
12(837)\end{array}$ & $\begin{array}{c}44.3 \% \text { (7.7 to } 91.7) \\
10(827)\end{array}$ & $\begin{array}{l}12.6 \% \text { (0 to } 27.2) \\
13(1821)\end{array}$ \\
\hline $\begin{array}{l}\text { Low risk of bias, } \\
n \text { (patients) }\end{array}$ & $\begin{array}{c}71.3 \% \text { (52.9 to } 76.1) \\
3(216)\end{array}$ & $\begin{array}{c}36.8 \% \text { (8.8 to } 77.2) \\
5(389)\end{array}$ & $\begin{array}{c}14.5 \% \text { (0.7 to 23.6) } \\
4(385)\end{array}$ & $\begin{array}{c}10.1 \%(1.6 \text { to } 32.6) \\
6(523)\end{array}$ & $\begin{array}{c}54.3 \%(23.5 \text { to } 80.0) \\
5(337)\end{array}$ & $\begin{array}{l}18.8 \% \text { (0 to } 27.2) \\
5(739)\end{array}$ \\
\hline $\begin{array}{l}+ \text { moderate/severe, } \\
n \text { (patients) }\end{array}$ & $\begin{array}{c}70.0 \% \text { (52.9 to } 74.3) \\
2(170)\end{array}$ & $\begin{array}{c}36.1 \% \text { (8.8 to } 77.2) \\
4(321)\end{array}$ & $\begin{array}{c}14.5 \% \text { (0.7 to 23.6), } \\
4 \text { (385) }\end{array}$ & $\begin{array}{c}5.2 \%(1.6 \text { to } 14.7) \\
4(406)\end{array}$ & $\begin{array}{c}61.4 \% \text { (23.5 to } 80.0) \\
3(220)\end{array}$ & $\begin{array}{c}23.8 \% \text { (0 to 27.2), } \\
4(303)\end{array}$ \\
\hline \multicolumn{7}{|l|}{ Mid-term } \\
\hline $\begin{array}{l}\text { All studies, } \\
n \text { (patients) }\end{array}$ & $\begin{array}{c}32.1 \%(8.9 \text { to } 56.4) \\
9(608)\end{array}$ & $\begin{array}{l}14.8 \% \text { (6.3 to } 25.0) \\
11(643)\end{array}$ & $\begin{array}{c}9.7 \%(0 \text { to } 50.0) \\
12(669)\end{array}$ & $\begin{array}{l}4.3 \% \text { (0 to 22.2) } \\
\quad 11 \text { (629) }\end{array}$ & $\begin{array}{c}\text { 18.8\% (0 to 66.7) } \\
15(792)\end{array}$ & $\begin{array}{l}3.8 \% \text { (0 to 14.0) } \\
\quad 11 \text { (691) }\end{array}$ \\
\hline $\begin{array}{l}\text { Low risk of bias, } \\
n \text { (patients) }\end{array}$ & - & $\begin{array}{l}12.1 \%(6.3 \text { to } 22.2) \\
5(231)\end{array}$ & $\begin{array}{c}16.7 \%(4.2 \text { to } 50.0) \\
4(215)\end{array}$ & $\begin{array}{l}6.1 \%(0 \text { to } 22.2) \\
5(231)\end{array}$ & $\begin{array}{l}25.2 \%(0 \text { to } 56.3) \\
5(218)\end{array}$ & $\begin{array}{c}11.2 \%(8.3 \text { to } 14.0) \\
2 \text { (98) }\end{array}$ \\
\hline $\begin{array}{l}+ \text { moderate/severe, } \\
n \text { (patients) }\end{array}$ & - & $\begin{array}{c}12.1 \%(6.32 \text { to } 22.2), \\
5(231)\end{array}$ & $\begin{array}{c}16.7 \% \text { (4.2 to } 50.0), \\
4(215)\end{array}$ & $\begin{array}{c}6.1 \%(0 \text { to } 22.2) \\
5(231)\end{array}$ & $\begin{array}{c}25.2 \%(0 \text { to } 56.3) \\
5(218)\end{array}$ & $\begin{array}{c}8.3 \%(-), \\
1(48)\end{array}$ \\
\hline \multicolumn{7}{|l|}{ Long-term } \\
\hline $\begin{array}{l}\text { All studies, } \\
n \text { (patients) }\end{array}$ & $\begin{array}{l}29.1 \%(0.9 \text { to } 73.3) \\
19(1418)\end{array}$ & $\begin{array}{l}15.0 \%(0 \text { to } 51.8) \\
27(1966)\end{array}$ & $\begin{array}{c}10.2 \% \text { (0 to } 64.4) \\
26(1782)\end{array}$ & $\begin{array}{c}6.3 \% \text { (0 to } 31.8) \\
25(1698)\end{array}$ & $\begin{array}{l}12.2 \% \text { (0 to } 50.0) \\
25(1798)\end{array}$ & $\begin{array}{c}2.7 \% \text { (0 to } 18.2) \\
17(1108)\end{array}$ \\
\hline $\begin{array}{l}\text { Low risk of bias, } \\
n \text { (patients) }\end{array}$ & $\begin{array}{l}31.1 \%(-) \\
1(45)\end{array}$ & $\begin{array}{c}16.6 \% \text { (7.2 to } 28.0) \\
8 \text { (499) }\end{array}$ & $\begin{array}{c}6.8 \% \text { (0 to } 18.8) \\
6(369)\end{array}$ & $\begin{array}{c}8.2 \%(1.0 \text { to } 20.0) \\
10(734)\end{array}$ & $\begin{array}{l}12.9 \%(1.5 \text { to } 29.3) \\
9(707)\end{array}$ & $\begin{array}{l}5.0 \%(0 \text { to } 6.9) \\
3(200)\end{array}$ \\
\hline $\begin{array}{l}\text { + moderate/severe, } \\
n \text { (patients) }\end{array}$ & $\begin{array}{c}31.1 \%(-) \\
1(45)\end{array}$ & $\begin{array}{c}15.7 \% \text { (7.2 to } 21.7) \\
5(381)\end{array}$ & $\begin{array}{c}7.3 \% \text { (1.4 to } 18.8) \\
4(301)\end{array}$ & $\begin{array}{c}8.6 \% \text { (1.0 to 20.0), } \\
7(616)\end{array}$ & $\begin{array}{l}12.7 \%(1.5 \text { to } 29.3) \\
6(589)\end{array}$ & $\begin{array}{c}6.7 \% \text { (6.3 to } 6.9) \\
2(150)\end{array}$ \\
\hline
\end{tabular}


Conclusion Pituitary disorders frequently arise after TBI, but prevalence remains uncertain due to low overall quality of available data. Factors other than methodological quality and TBI severity are likely to explain the observed wide prevalence ranges. The clinical significance of TBIassociated pituitary disorders also requires further rigorous evaluation.

\section{P300}

Mannose binding lectin deficiency attenuates neurobehavioral

deficits following experimental traumatic brain injury

L Longhi', F Orsini ${ }^{2}$, N Fedele ${ }^{2}$, N Stocchetti', MG De Simoni ${ }^{2}$

'University of Milano, Milan, Italy; ${ }^{2}$ Mario Negri Institute, Milan, Italy

Critical Care 2012, 16(Suppl 1):P300 (doi: 10.1186/cc10907)

Introduction Mannose binding lectin (MBL) is the activator of the lectin complement pathway. After cerebral ischemia it has been shown that MBL could be a mediator of secondary brain damage, in contrast after traumatic brain injury (TBI) there are data suggesting that it could be linked to neuroprotection. We tested the hypothesis that MBL is involved in the pathophysiology of TBI. We characterized (1) the temporal activation of MBL and (2) the effects of its inhibition in a model of experimental TBI.

Methods (1) Male C57/BI6 mice were subjected to intraperitoneal anesthesia (pentobarbital, $65 \mathrm{mg} / \mathrm{kg}$ ) followed by the controlled cortical impact brain injury model of experimental TBI (injury parameters: velocity of $5 \mathrm{~m} / \mathrm{second}$ and $1 \mathrm{~mm}$ depth of deformation). MBL immunostaining was evaluated at various time points after TBI: 30 minutes, 1, 6, 12, 24, 48, 96 hours and 1 week using anti MBL-A and MBL-C antibodies $(n=3)$. (2) The effects of MBL inhibition were evaluated by comparing functional and histologic outcomes in C57/ Bl6 mice (WT) and in MBL knockout $\left(^{(-)}\right)$mice. Functional outcome was tested using the Composite Neuroscore and Beam Walk test weekly up to 4 weeks postinjury $(n=11)$. Histologic outcome was evaluated by calculating the contusion volume at 4 weeks postinjury $(n=6)$. Shamoperated mice received identical anesthesia without brain injury.

Results We observed a robust MBL-positive immunostaining in the injured cerebral cortex starting at 30 minutes postinjury and up to 1 week, suggestive of an activation of this pathway following TBI. MBL was observed both at endothelial and tissue levels. Consistently, injured WT and MBL $\left(^{(-)}\right)$mice showed neurological motor deficits up to 4 weeks postinjury when compared to their sham controls. Notably, MBL $\left(^{-1-}\right)$ mice showed attenuated behavioral deficits when compared to their WT counterpart at 2 to 4 weeks postinjury $(P<0.01$ for both Neuroscore and Beam Walk test). In contrast we observed similar contusion volumes at 4 weeks postinjury (WT $=15.6 \pm 3.2 \mathrm{~cm}^{3}$ and $\mathrm{MBL}$ $\mathrm{KO}=13.9 \pm 3.2 \mathrm{~cm}^{3}, P=0.3$ ).

Conclusion We observed that: (1) MBL deposition and/or synthesis is increased following TBl; and (2) MBL deficiency is associated with functional neuroprotection, suggesting that MBL modulation might be a potential therapeutic target after TBI.

\section{P301}

Azathioprine and aspirin in treatment of childhood primary arterial stroke: therapeutic benefits and side effects

A Alhaboob, G Ahmed

King Khalid University Hospital and College of Medicine, King Saud University, Riyadh, Saudi Arabia

Critical Care 2012, 16(Suppl 1):P301 (doi: 10.1186/cc10908)

Introduction The objectives were to describe a cohort of children presenting with medium/large vessel childhood primary angiitis of the central nervous system (PACNS); to report their short-term neurological outcome; and to evaluate efficacy and safety of implemented management.

Methods The study included 68 patients, aged less than 16 years. They had their symptoms within 14 days of admission. They received induction therapy with pulses of intravenous steroids and/ or intravenous immunoglobulin followed by maintenance therapy with azathioprine and low-dose aspirin. They were also treated with anticoagulants for 4 weeks along with the induction therapy. They were assessed for; their clinical presentation, disease severity (progressive or nonprogressive), hospital course, adverse effects of the used treatment and outcome. Reports of their neuroimaging studies were also collected.

Results Studied patients were $42(62.76 \%)$ boys and 26 (38.23\%) girls. Their mean age was $8.5 \pm 3.5$ years. The commonest presenting symptoms were motor deficit (70\%), headache (64\%) and fever (20\%), while the commonest presenting neurological signs were hemiparesis $(60 \%$ ), seizure $55 \%$ (focal $35 \%$, generalized $20 \%$ ), and decreased level of consciousness (30\%). Neuroradiological studies of the brain revealed: ischemic strokes in 50 children (73.5\%), hemorrhagic strokes in 10 (14.7\%) and ischemic-hemorrhagic lesions in eight (11.8\%). Conventional angiography (CA) and/or magnetic resonance angiography (MRA) at the time of admission revealed that 51 (75\%) patients had nonprogressive and $17(25 \%)$ had evidence of progressive arteriopathy. Out of the studied patients, 56 (81.5\%) survived and $12(18.5 \%)$ died. Male sex, deep coma and intracerebral bleeding causing severe raised intracranial pressure were poor prognostic signs. Survivors were discharged on oral aspirin and 15 of them commenced also on azathioprine. On follow-up it was found that out of the 56 survivors, 11 were normal (19.65\%), $14(25 \%)$ had minor disabilities, another $11(19.65 \%)$ had moderate disabilities and 20 (35.7\%) had severe disabilities.

Conclusion The spectrum of CPACNS includes progressive and nonprogressive forms. Characteristic features on presentation may predict later progression and outcome; identify a distinct high-risk CPACNS cohort; and guide the selection of patients for immunosuppressive therapy. Further studies are required to substantiate our findings.

\section{P302}

Changes of ribosomal protein S3 immunoreactivity and its new expression in microglia in the mice hippocampus after lipopolysaccharide treatment

JH Cho, CW Park, HY Lee, MH Won

Kangwon National University, Chuncheonsi, South Korea

Critical Care 2012, 16(Suppl 1):P302 (doi: 10.1186/cc10909)

Introduction Lipopolysaccharide (LPS) has been commonly used as a reagent for a model of systemic inflammatory response. Ribosomal protein S3 (rpS3) is a multifunctional protein that is involved in transcription, metastasis, DNA repair and apoptosis. In the present study, we examined the changes of rpS3 immunoreactivity in the mouse hippocampus after systemic administration of $1 \mathrm{mg} / \mathrm{kg}$ LPS.

Methods Six-week-old male ICR mice were purchased from the Jackson Laboratory (Bar Harbor, ME, USA). LPS (Sigma, St Louis, MO, USA) was dissolved in saline, and administered intraperitoneally with $1.0 \mathrm{mg} /$ $\mathrm{kg} / 10 \mathrm{ml}$ dose. The control animals were injected with the same volume of saline. Mice ( $n=7$ at each time point) were sacrificed at designated times $(3,6,12,24,48$ and 96 hours after LPS treatment). The brain tissues were cryoprotected by infiltration with $30 \%$ sucrose overnight. Thereafter, frozen tissues were serially sectioned on a cryostat (Leica, Wetzlar, Germany) into 30- $\mu \mathrm{m}$ coronal sections, and they were then collected into six-well plates containing 0.1 M PBS.

Results From 6 hours after LPS treatment, rpS3 immunoreactivity was decreased in pyramidale cells of the hippocampus proper and granule cells of the dentate gyrus. At this point in time, rpS3 immunoreactivity began to increase in nonpyramidal cells and nongranule cells in the hippocampus. From 1 day after LPS treatment, rpS3 immunoreactivity in pyramidal and granule cells was hardly detected, and nonpyramidal and nongranule cells showed strong rpS3 immunoreactivity. Based on double immunofluorescence staining, microglia, not astrocytes, expressed strong rpS3 immunoreactivity at 1 and 2 days after LPS treatment.

Conclusion These results indicate that changes in rpS3 immunoreactivity in pyramidal and granule cells and rpS3 expression in activated microglia after LPS treatment may be associated with the neuroinflammatory responses in the brain. 
P303

Neuronal damage using Fluoro-Jade B histofluorescence and gliosis in the striatum after various durations of transient cerebral ischemia in gerbils

JH Cho, CW Park, HY Lee, MH Won

Kangwon National University, Chuncheonsi, South Korea

Critical Care 2012, 16(Suppl 1):P303 (doi: 10.1186/cc10910)

Introduction Ischemic damage occurs well in vulnerable regions of the brain, including the hippocampus and striatum. In the present study, we examined neuronal damage/death and glial changes in the striatum 4 days after 5, 10, 15 and 20 minutes of transient cerebral ischemia using the gerbil. Spontaneous motor activity was shown to be increased with the duration time of ischemia-reperfusion (I-R).

Methods To examine neuronal damage, we used Fluoro-Jade B (F-JB, a marker for neuronal degeneration) histofluorescence staining. F-JBpositive cells were detected only in the 20-minute ischemia group, not in the other groups. In addition, we examined gliosis of astrocytes and microglia using antiglial fibrillary acidic protein (GFAP) and anti-ionized calcium-binding adapter molecule 1 (Iba-1), respectively.

Results In the 5-minute ischemia group, GFAP-immunoreactive astrocytes were distinctively increased in number, and the immunoreactivity was stronger than that in the sham group. In the 10-minute, 15-minute and 20-minute ischemia groups, GFAP immunoreactivity was more increased with the duration of I-R. On the other hand, the immunoreactivity and number of Iba-1-immunoreactive microglia were distinctively increased in the 5-minute and 10-minute ischemia groups. In the 15-minute ischemia group, microglia were largest in size, and the immunoreactivity was highest; however, in the 20-minute ischemia group, the immunoreactivity was low compared to the 15-minute ischemia group. The results of western blotting for GFAP and Iba-1 were similar to the immunohistochemical data.

Conclusion These findings indicate that neuronal death was detected only in the 20-minute ischemia group 4 days after I-R; in addition, the change pattern of astrocytes and microglia were apparently different according to the duration time of I-R.

\section{P304}

Molecular, histological and microcirculatory modeling of cerebral

ischemia in pigs

O Suchadolskiene', A Pranckunas', B Kumpaitiene', P Dobozinskas'1,

Z Dambrauskas', V Veikutis², K Stasaitis', G Baliutyte' ${ }^{3}$, D Vaitkaitis',

$\checkmark$ Borutaite $^{3}$

'Lithuanian University of Health Sciences, Kaunas, Lithuania; ${ }^{2}$ Institute of Cardiology, Lithuanian University of Health Sciences, Kaunas, Lithuania; ${ }^{3}$ Institute of Neurosciences, Lithuanian University of Health Sciences, Kaunas, Lithuania

Critical Care 2012, 16(Suppl 1):P304 (doi: 10.1186/cc10911)

Introduction Ischemic brain injury due to stroke and/or cardiac arrest is a major health issue in modern society requiring urgent development of new effective therapies. The use of appropriate animal models is essential to study the mechanisms of ischemia-induced injury and neuroprotection. The goal of our study was to establish a reliable and reproducible model of brain ischemia in pigs (with the ischemiainduced microcirculatory, mitochondrial and structural alterations) for further research.

Methods Eighteen pigs ( 18 to $22 \mathrm{~kg}$ ) were anesthetized and randomly assigned to the one of the following groups: 1 - control, 2 - unilateral carotid occlusion, 3 - bilateral carotid occlusion, 4 - bilateral carotid occlusion + hypotension (MAP 40 to $50 \mathrm{mmHg}$ ). In order to investigate the effects and mechanisms of cerebral ischemia, we assessed the mitochondrial respiration (high-resolution respirometry), microcirculation (in vivo SDF videomicroscopy) and histological structure (light microscopy) of brain tissue in healthy control animals and after 3 hours of brain ischemia (three different models).

Results LEAK respiration (measured in the presence of pyruvate + malate but without ADP) was not affected by ischemia in any model. The OXPHOS capacity with pyruvate + malate as substrates decreased by $20 \%$ and $79 \%$ compared to the control level after bilateral carotid artery occlusion and bilateral carotid occlusion + hypotension, respectively, resulting in the decrease of $\mathrm{RCI}$ (ADP/PM) by $14 \%$ and $73 \%$. The OXPHOS capacity with succinate as substrate remained constant after unilateral carotid artery occlusion but decreased by $53 \%$ after bilateral carotid artery occlusion and hypotension compared to the control level $(P<0.05, n=3$ to 6$)$. Mitochondrial respiration rates after addition of atractyloside and cytochrome $\mathrm{c}$ were the same in all experimental groups, suggesting that intactness of mitochondrial outer membrane was not affected by cerebral ischemia. Microcirculatory and histological alterations also demonstrated increasing derangement and reversible structural changes after bilateral carotid occlusion and vascular occlusion combined with systemic hypotension.

Conclusion The experimental model of bilateral carotid artery occlusion and systemic hypotension-induced cerebral ischemia in pigs is a useful tool to investigate the mechanism of cerebral ischemia and/ or neuroprotection (medications, hypothermia, and so forth).

\section{P305}

Delayed post-ischaemic administration of xenon reduces brain damage in a rat model of global ischaemia

V Metaxa', R Lagoudaki², S Meditskou², O Thomareis², A Sakadamis ${ }^{2}$ 'St Bartholomew's Hospital, London, UK; ${ }^{2}$ Aristotle University, Thessaloniki, Greece

Critical Care 2012, 16(Suppl 1):P305 (doi: 10.1186/cc10912)

Introduction Cerebral ischaemia is among the leading causes of death, disability and economic expense in the world. Xenon has been shown to be neuroprotective both in vivo and in vitro, predominantly when administered as a preconditioning agent. We have used a rat model of global ischaemia to investigate whether xenon-induced neuroprotection is observed following an ischaemic insult.

Methods Adult male Wistar rats underwent bilateral common carotid artery occlusion and were ventilated for 1 hour with $21 \% \mathrm{O}_{2} / 78 \% \mathrm{~N}$. The animals were randomized to receive $21 \% \mathrm{O}_{2} / 78 \% \mathrm{~N}_{2^{\prime}} 50 \% \mathrm{O}_{2} / 50 \%$ $\mathrm{N} \mathrm{O}$ or $50 \% \mathrm{O} / 50 \%$ xenon $(n=10)$. After a further 45 minutes, they were killed and their brains were removed for histological, immunochemical and molecular analysis. The numbers of ischaemic neurons in the cortex and the hippocampus as well as the expression of c-fos were evaluated on adjacent brain sections.

Results Both $\mathrm{N}_{2} \mathrm{O}$ and xenon administration reduced the number of ischaemic neurons in the cortex. In xenon-treated rats, fewer ischaemic neurons were also observed in the $C A 1$ region of the hippocampus. The xenon group demonstrated a significant reduction of c-fos expression compared to control and $\mathrm{N}_{2} \mathrm{O}$ groups. See Figure 1.

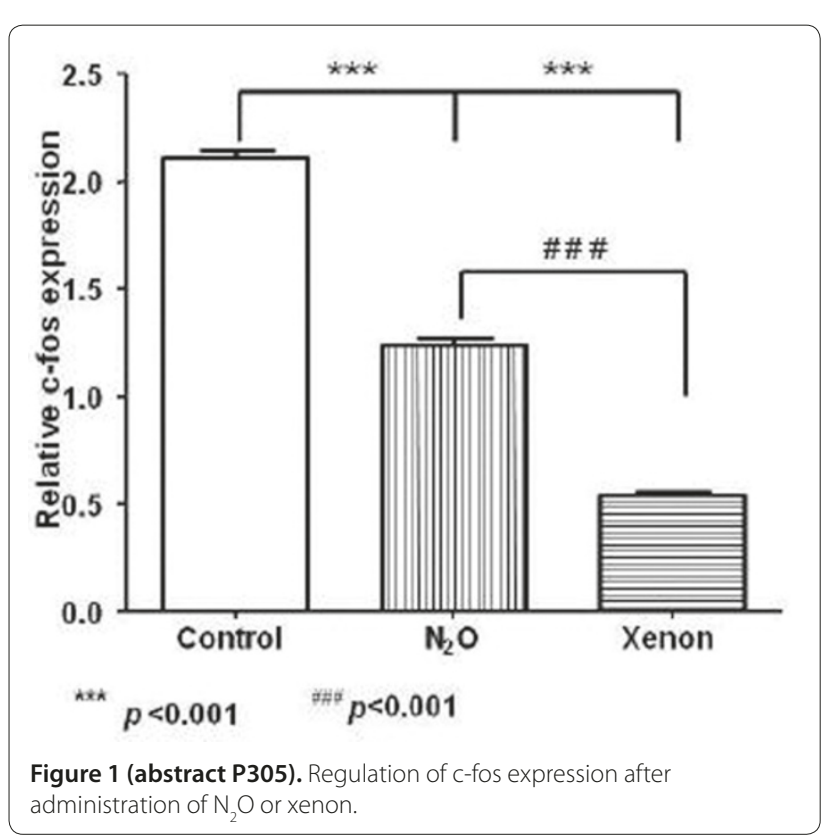


Conclusion In our model of global cerebral ischaemia, the administration of xenon reduced the number of ischaemic neurons compared to control, both in the cerebral cortex and in the hippocampus.

Reference

1. Wilhelm S, et al: Effects of xenon on in vitro and in vivo models of neuronal injury. Anesthesiology 2002, 96:1485-1489.

P306

Seizures in the respiratory ICU: single-center study of patients with new-onset seizures

D Talwar, V Nair, J Chudiwal

Metro Center for Respiratory Diseases, Noida, India

Critical Care 2012, 16(Suppl 1):P306 (doi: 10.1186/cc10913)

Introduction New-onset seizures in the ICU are a diagnostic and management challenge as patients have multiple comorbidities and receive various antibiotics. In the respiratory ICU with different patient profiles, etiopathogenesis of seizures is unreported.

Methods We retrospectively analyzed the profile of 3,342 patients admitted to the RICU from 2006 to 2011. A computerized search revealed 79 patients $(2.4 \%)$ with new-onset seizures. Complete clinical, laboratory, radiological and treatment profiles were recorded and statistically analyzed using the chi-square test, odds ratio and relative risk of individual variable.

Results Of 79 patients, 44 patients (55.7\%) were males and the mean age was $61.28 \pm 19.57$ years. Severe sepsis was diagnosed in $32(40.5 \%)$ and multiorgan failure in 19 (24.1\%). Head CT done in $65(82.3 \%)$ patients was reported abnormal in $34(52.3 \% ; P=0.072)$ patients. Lumbar puncture was done in $40(50.6 \%)$ with five $(12.5 \%)$ patients having meningitis. Thirteen of 37 (35.1\%) patients showed focal activity on EEG $(P=0.27 ; \mathrm{OR}=1.73)$. Electrolyte abnormalities were: hypermagnesemia in 20 patients $(25.3 \%)$, hypocalcemia in 17 patients $(21.5 \%)$, and hypernatremia in 13 patients $(16.5 \%)$, hyponatremia in three patients (3.8\%) and hypomagnesia in four $(5.17 \%)$ cases. The antibiotics received revealed $27(34.2 \%$; $R R=1.27)$ patients on levofloxacin alone or in combination. Twenty-eight of 79 (35.4\%) patients were on carbapenems with meropenem in 23/79 (29.1\%; $R R=1.21)$ and imipenem in 5/79 $(6.32 \% ; R R=0.41)$. See Table 1 .

Table 1 (abstract P306). Attributable causes of seizures in RICU cases $(n=79)$

\begin{tabular}{lcc}
\hline Anoxia & 8 & $10.1 \%$ \\
Metabolic & 15 & $19.0 \%$ \\
Drugs only & 16 & $20.3 \%$ \\
CNS infection & 5 & $6.3 \%$ \\
Trauma & 2 & $2.5 \%$ \\
Alcohol & 5 & $6.3 \%$ \\
Multiple & 22 & $27.8 \%$ \\
Miscellaneous & 6 & $7.6 \%$
\end{tabular}

Conclusion New-onset seizure in RICU cases is multifactorial in origin. Use of levofloxacin in combination had the highest relative risk of developing seizure although when given alone the risk is rare (2.1\%). Severe sepsis with multiorgan failure being seen in nearly one-half of $\mathrm{RICU}$ cases may decrease seizure threshold in these patients.

P307

Early treatment with intravenous immunoglobulins in patients with critical illness polyneuropathy: a randomized controlled, doubleblinded study

R Brunner, W Rinner, R Kitzberger, T Sycha, J Warszawska, U Holzinger,

C Madl

Medical University of Vienna, Austria

Critical Care 2012, 16(Suppl 1):P307 (doi: 10.1186/cc10914)

Introduction Critical illness polyneuropathy (CIP) is a severe complication of critical illness. The clinical features of CIP are muscle weakness and atrophy causing delayed weaning and prolongation of the mobilization phase. Although the exact etiopathogenesis has not yet been fully elucidated, sepsis, systemic inflammatory response syndrome, and multiple organ failure seem to play an important role. CIP is diagnosed by signs of denervation in electromyography. Although there is no causal treatment for CIP, retrospective data suggest that early IgM-enriched intravenous immunoglobulin (IVIG) application may prevent or mitigate CIP. Therefore we aimed to investigate the use of IVIG in the early treatment of CIP in critically ill patients in a prospective, randomized, double-blind and placebocontrolled setting.

Methods In this prospective, randomized, double-blind and placebocontrolled trial critically ill patients with clinical evidence for incipient CIP, a diagnosis of SIRS/sepsis and failure of at least two organ systems were randomized to be treated either with IgM-enriched IVIG or with human albumin $1 \%$ as placebo over a period of 3 days. The primary objective was to demonstrate that administration of IVIG prevents and/or mitigates CIP in critically ill patients, measured by electrophysiological stimulation of the median, ulnar and tibial nerves on days 0, 4, 7 and 14. Electrophysiological measures were graded according to compound muscle action amplitude size (CIP score) of the respective nerve. Secondary objectives were mortality from any cause within a 28-day period and lengths of ICU stay.

Results Thirty-eight critically ill patients were included and randomized to either receiving IgM-enriched IVIG $(n=19)$ or placebo $(n=19)$. Baseline characteristics including CIP score on day 0 were similar between the two groups. CIP could not be improved significantly by IVIG treatment for three consecutive days, represented by similar CIP scores of all three measured nerves on days 4, 7 and 14 in the IVIG and the placebo group. Mean CIP score levels of all three nerves significantly increased from baseline to day 4 in both groups.

Conclusion Results suggest that early treatment with IVIG neither significantly improves CIP nor influences the length of stay or mortality in critically ill patients. Consistent with the literature, CIP deteriorated during the course of disease in critically ill patients with a diagnosis of SIRS/sepsis and failure of two organ systems.

\section{P308}

Intracranial pressure monitoring in acute liver failure: a retrospective cohort study

C Karvellas', O Fix², H Battenhouse ${ }^{3}$, V Durkalskiं, C Sanders ${ }^{4}$, W Lee ${ }^{4}$ 'University of Alberta, Edmonton, Canada: ${ }^{2}$ UCSF, San Francisco, CA, USA; ${ }^{3}$ Medical University of South Carolina, Charleston, SC, USA; ${ }^{4}$ University of Texas-Southwestern, Dallas, TX, USA

Critical Care 2012, 16(Suppl 1):P308 (doi: 10.1186/cc10915)

Introduction Intracranial hypertension (ICH) complicates roughly $25 \%$ of acute liver failure (ALF) patients with grade III/IV encephalopathy. Intracranial pressure (ICP) monitoring is controversial due to complications in 5 to $20 \%$ and absence of documented mortality benefit.

Methods Using prospectively collected data from the US Acute Liver Study Group registry, we reviewed 630 ALF patients with severe encephalopathy (grade III/IV) and INR $>1.5$ enrolled between 1 March 2004 through 31 August 2011. ICP monitoring was used in 143 patients (23\%); 487 control patients with grade III/IV hepatic coma $(n=487)$ were not monitored.

Results The most common etiology of ALF was acetaminophen (51\%, $P=0.13$ between groups). Of ICP monitored (ICPM) patients, $85 \%$ $(n=121)$ received devices within 24 hours of admission to study. ICPM patients were significantly younger ( $36 \pm 6$ years vs. $43 \pm 15$ years, $P<0.001)$ than controls, more likely to be on renal replacement therapy ( $48 \%$ vs. $31 \%, P<0.001$ ) but less likely to be on vasopressors ( $20 \%$ vs. $32 \%, P=0.008)$. ICPM patients were given more ICH directed therapies (mannitol $43 \%$ vs. $13 \%$, hypertonic saline $21 \%$ vs. $6 \%$, hypothermia $29 \%$ vs. $11 \%, P<0.001$ for each comparison). For ICPM patients, the median INR on the day of monitor insertion was 2.2 (1.6 to 2.9) and platelet count 116 (84 to 171 ); $74 \%$ were given FFP (vs. $46 \%$ controls, $P<0.001$ ) and $19 \%$ (vs. $14 \%$ controls, $P=0.14$ ) received platelets. ICP monitoring was also strongly associated with listing $(78 \%$ vs. $27 \%, P<0.001)$ and receipt of liver transplant $(42 \%$ vs. $18 \%, P<0.001)$. Twenty-one-day mortality was similar between ICPM patients (33\%) and controls (37\%, 
$P=0.33$ ) when all or only nontransplanted patients ( $46 \%$ vs. $45 \%$, 0.8 ) were considered. Of 66 ICPM patients with detailed information, $18(29 \%)$ had evidence of ICH (ICP $>25 \mathrm{mmHg}$ ) at the time of ICPM insertion (maximum ICP on day $1 \sim 18$ (12 to 26 ) $\mathrm{mmHg}$ ). Of 49 patients with a known ICPM device, 14 patients received epidural catheters, six subdural, 11 intraparenchymal, seven intraventricular and 11 lumbar monitors. In only one of 49 ICPM patients was intracranial hemorrhage reported, and this patient survived.

Conclusion In ALF patients, ICP monitor placement is strongly associated with liver transplantation but not with overall or transplant free mortality. In the absence of ICP monitoring, ALF patients may be less aggressively treated for intracranial hypertension. The value of ICP monitoring in ALF remains to be determined but ICPM placement clearly affects the frequency of interventions for elevated ICP.

P309

Retrospective observation of 6-month survival following decompressive craniectomy in a London major trauma and stroke centre

J Dawson, P Hopkins, J Ling, D Walsh, C Tolias

King's Health Partners, London, UK

Critical Care 2012, 16(Suppl 1):P309 (doi: 10.1186/cc10916)

Introduction This study describes 5.5 years of retrospective data examining hospital and 6-month outcome of patients following decompressive craniectomy (DC). The effectiveness of DC remains uncertain with conflicting results in patients with TBI and stroke $[1,2]$. Methods Data were drawn (1 January 2006 to 30 June 2011) from three hospital databases following approval by the institutional board. Results There were 2,148 neurosurgical admissions with 71 undergoing DC. Forty-eight of 71 (67.6\%) survived to hospital discharge and 21/33 in both TBI and stroke groups survived to 6 months. See Table 1.

Table 1 (abstract P309)

\begin{tabular}{lccccccc}
\hline Year & $\begin{array}{c}\text { Neurosurgical/ } \\
\text { total }\end{array}$ & $\begin{array}{c}\text { Total Hospital } \\
\text { DC }\end{array}$ & $\begin{array}{c}\text { 6-month } \\
\text { survival }\end{array}$ & $\begin{array}{c}\text { MCA } \\
\text { survival }\end{array}$ & TBI & stroke & Other \\
\hline 2006 & $292 / 1,839$ & 2 & 2 & 2 & 1 & 0 & 1 \\
2007 & $298 / 1,652$ & 2 & 2 & 2 & 0 & 2 & 0 \\
2008 & $286 / 1,563$ & 11 & 10 & $8(1 \mathrm{~N} / \mathrm{A})$ & 7 & 4 & 0 \\
2009 & $493 / 1,840$ & 18 & 11 & 10 & 7 & 8 & 3 \\
2010 & $505 / 1,835$ & 21 & 16 & $15(1 \mathrm{~N} / \mathrm{A})$ & 11 & 9 & 1 \\
2011 & $274 / 918$ & 17 & 7 & $5(2 \mathrm{~N} / \mathrm{A})$ & 7 & 10 & 0 \\
5.5 -year & $2,148 / 9,647$ & 71 & 48 & $42(4 \mathrm{~N} / \mathrm{A})$ & 33 & 33 & 5 \\
data & & & & & & &
\end{tabular}

Conclusion Survival following DC in this institution compares favourably with published data. Reduced survival in 2011 may be a case-mix effect related to increased tertiary referrals. We will now prospectively collect these data including quality-of-life measures. References

1. Cooper DJ, et al:: N Engl J Med 2011, 364:1493-1502.

2. Hofmeijer J, et al.: Lancet Neurol 2009, 8:326-333.

P310

Feasibility of a multicenter prospective cohort study on the evaluation of prognosis in severe traumatic brain injury

AF Turgeon', F Lauzier', M Thibodeau', A Rigamonti², M Meade ${ }^{3}$,

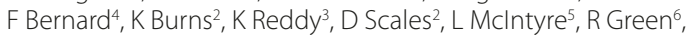

D Griesdale', L Moore', M Savard', D Jichici³, J Paquet', D Zygun',

D Fergusson ${ }^{5}$, for the Canadian Critical Care Trials Group ${ }^{5}$

'Université Laval, Québec, Canada; ${ }^{2}$ University of Toronto, Ontario, Canada; ${ }^{3}$ McMaster University, Ontario, Canada; ${ }^{4}$ Université de Montréal, Québec

Canada; ${ }^{5}$ University of Ottawa, Ontario, Canada; ${ }^{6}$ University Dalhousie, Nova Scotia, Canada; ${ }^{7}$ University of British Columbia, Canada; ${ }^{8}$ University of Calgary, Alberta, Canada

Critical Care 2012, 16(Suppl 1):P310 (doi: 10.1186/cc10917)

Introduction Conducting prospective research in severe traumatic brain injury (TBI) patients is challenging. To prepare for a large-scale multicenter study to evaluate long-term prognosis in severe $\mathrm{TBI}$, we conducted a prospective pilot study evaluating the patterns of enrollment, the compliance to the schedule of prognostic tests and the completeness of follow-up for 6-month functional outcome measures. Methods We conducted a pilot study in nine level I trauma centers in Canada. Adult patients with severe TBI expected to require mechanical ventilation for $\geq 48$ hours were enrolled on their first day in the ICU. Prognostic tests were performed on arrival (CT scan), day 1 (serum biomarker), day 3 (serum biomarker, CT scan) and day 7 (serum biomarker, CT scan, MRI, SSEP, EEG) with time windows of 24 or 48 hours depending on the test. Prognostic measures were collected during the first week in the ICU to examine the association with the extended Glasgow Outcome Scale score. We considered as appropriate a compliance to the schedule of prognostic tests $\geq 90 \%$ and a proportion of lost to follow-up $<10 \%$. We obtained REB approval from participating centers and written informed consent from SDMs.

Results Among 116 consecutive eligible patients, 50 were enrolled over a total of 204 weeks of screening between May 2010 and May 2011. Two centers used a deferred consent approach. Patients were primarily male with a median age of 45 years and a GCS of 5 (25th to 75th: 3 to 7). The two main reasons for nonenrollment were the time window for inclusion being after regular working hours $(35 \%, n=23)$ and oversight $(24 \%, n=16)$. The compliance to the different tests ranged from 93 (three missing tests) to $100 \%$. All blood samples but one (day 7) were performed. The main reason for missing a test was the patient's instability (hemodynamic or increased ICP) $(n=5)$. In six patients, the MRI had to be delayed due to the presence of material not compatible with the procedure. No patient was lost to follow-up at 6 months.

Conclusion These results demonstrate the feasibility of enrollment and complying to a structured protocol of prognostic tests in a prospective multicenter study in severe TBI patients.

P311

Predictive value of neuron-specific enolase following moderate and severe traumatic brain injury: a systematic review and meta-analysis

E Mercier', AF Turgeon', A Boutin', F Lauzier', R Zarychanski²,

P Archambault', J Granton³, F Lamontagne', L Moore', F Rousseau',

F Légaré1, E Randell ${ }^{5}$, J Lacroix ${ }^{6}$, J Lapointe', D Fergusson ${ }^{7}$

'Université Laval, Québec, Canada; ${ }^{2}$ University of Manitoba, Canada; ${ }^{3}$ Université of Toronto, Ontario, Canada; ${ }^{4}$ Université de Sherbrooke, Québec, Canada;

${ }^{5}$ Memorial University, NewFoundland, Canada; 'Université de Montréal, Québec, Canada: ${ }^{7}$ Ottawa Hospital Research Institute, Ontario, Canada

Critical Care 2012, 16(Suppl 1):P311 (doi: 10.1186/cc10918)

Introduction Biomarkers such as the neuron-specific enolase (NSE) have been proposed as potential prognostic markers following traumatic brain injury (TBI) $[1,2]$. However, the use of NSE is not currently recommended for prognostic evaluation. Our objective was to systematically review the prognostic value of NSE levels following moderate or severe TBI.

Methods We systematically searched MEDLINE, Embase, Cochrane, Biosis, Scopus, Trip, references of eligible studies, reviews and conference proceedings. Eligible studies were cohort studies including $\geq 4$ patients with moderate or severe TBI having measured the association between NSE levels (first 24 hours) and mortality or the Glasgow Outcome Scale (GOS). Independently, two reviewers selected studies and extracted data using a standardized form. Pooled results using random-effect models were used using weighted mean differences (WMD); heterogeneity was assessed using $I^{2}$ tests. Sensitivity analyses were planned to explain statistical heterogeneity (for example, extracerebral injuries).

Results We retrieved 4,711 citations and included 22 studies $(n=757)$. Seventeen studies used the GOS as an outcome measure while 10 studies reported mortality. Most studies evaluated outcomes at 6 months or beyond (range: 1 to 12 months). Ten studies could not be included in the pooled analyses: three reported mean levels of serial samplings, two presented peak levels, two reported medians, one did not report any measure of dispersion and data could not be extracted from two studies. We observed a significant association between serum NSE levels and mortality (five studies: WMD 25.90 (95\% 
Cl 15.97 to 35.83$\left.), I^{2}=60 \%\right)$ and GOS $\leq 3$ (10 studies: WMD $17.69(95 \%$ $\mathrm{Cl} 12.14$ to 23.24$), I^{2}=64 \%$ ). Similar results were found with or without extracerebral injuries. The number of studies included in pooled analyses precluded performing relevant sensitivity analyses.

Conclusion We observed a significant association between serum NSE levels and unfavorable outcomes (mortality or GOS $\leq 3$ ) not influenced by extracerebral injuries. Further studies need to evaluate the usefulness of serum NSE levels for prognosis assessment in TBI and its potential impact on clinical decision-making.

\section{References}

1. Papa $L$, et al:: Use of biomarkers for diagnosis and management of traumatic brain injury patients. Exp Opin Med Diagn 2008, 2:937-945.

2. Zitnay GA, et al:: Traumatic brain injury research priorities: the Conemaugh International Brain Injury Symposium. J Neurotrauma 2008, 25:1135-1152.

P312

Blood-brain barrier permeability following traumatic brain injury M Jungner, P Bentzer

Lund University, Lund, Sweden

Critical Care 2012, 16(Suppl 1):P312 (doi: 10.1186/cc10919)

Introduction Brain edema and intracranial hypertension is deleterious after traumatic brain injury (TBI), but the underlying pathophysiology is complex and poorly understood. One major subject of controversy is the time course and extent of blood-brain barrier dysfunction following trauma, and previous studies in humans have only provided semi-quantitative data. The objective of the present study was therefore to quantify changes in blood-brain barrier permeability in the early course of TBI.

Methods Seventeen nonconsecutive brain trauma patients and two controls were included in this prospective observational study. Following i.v. injection of iohexol and CT perfusion scans, patients were scanned eight times from 4 to 25 minutes. The blood-to-brain transfer constant (K) for iohexol, reflecting permeability and area available for diffusion, was calculated by Patlak plot analysis of the enhancement curves of intracerebral large venous vessels and pericontusional brain parenchyma.

Results Fourteen patients were included within 1 day and three were included within 5 days of the injury. In nonischemic tissue surrounding contusions and hematomas, $\mathrm{K}$ was focally increased in 11 of all included trauma patients and in six of seven patients with raised intracranial pressure. In noninjured areas and in controls, $\mathrm{K}_{\mathrm{i}}$ was about $0.06 \mathrm{ml} /$ minute/100 $\mathrm{g}$ and increased by 100 to $2,000 \%$ in pericontusional tissue. See Figure 1

Conclusion TBI is associated with early focal increases in blood-brain barrier permeability. The results suggest that in the injured brain, capillary hydrostatic and oncotic pressures are likely to influence edema formation.

\section{References}

1. Patlak et al:: Graphical evaluation of blood-to-brain transfer constants from multiple-time uptake data. J Cereb Blood Flow Metab 1983, 3:1-3.

2. Maeda et al:: Ultra-early study of edema formation in cerebral contusion using diffusion MRI and ADC mapping. Acta Neurochir Supp/ 2003, 86:329-331.

P313

Can urinary 8-OHdG be a good indicator of vasospasm occurrence following subarachnoid hemorrhage?

K Ikeda, T Ikeda, H Taniuchi, S Suda, Y Ikeda, H Jimbo

Tokyo Medical University, Hachioji Medical Center, Tokyo, Japan

Critical Care 2012, 16(Suppl 1):P313 (doi: 10.1186/cc10920)

Introduction There is substantial evidence to suggest that oxidative stress is associated with cerebral vasospasm following subarachnoid hemorrhage $(\mathrm{SAH})$. Urinary 8-OHdG is the most common biomarker of DNA damage by oxidative stress. The aim of this study was to determine whether $8-\mathrm{OHdG}$ is a good indicator of vasospasm occurrence following $\mathrm{SAH}$.

Methods The subjects were 23 patients who received surgical clipping or endovascular coiling within 24 hours after the onset of SAH. We classified the patients according to the occurrence of angiographic vasospasm. We examined the urinary $8-\mathrm{OHdG}$ levels with highperformance liquid chromatography for 10 days following $\mathrm{SAH}$. The urinary $8-\mathrm{OHdG}$ levels were adjusted according to serum creatinine levels.

Results The urinary 8-OHdG levels were elevated on day 2 compared with those on day 1 only in the vasospasm (+) group. The urinary 8-OHdG levels in the vasospasm (+) group were significantly higher than those in the nonvasospasm (-) group on days 1,2,8 and 9. Furthermore, we examined the correlations between the urinary $8-\mathrm{OHdG}$ levels on admission to the ICU and the grades of the World Federation of Neurologic Surgeons and Fisher, but none were observed. Discussion An elevated urinary 8-OHdG level on day 2 was observed only in the vasospasm group. Therefore, we speculated that free radicals may have a role in inducing vasospasm in the early phase following SAH. The urinary 8-OHdG levels were higher in the vasospasm group than in the nonvasospasm group, but we did not find any correlation with severity of SAH. We suspect that the higher urinary 8-OHdG levels on days 8 and 9 in the vasospasm group indicated ischemic brain injury after vasospasm.

Conclusion We believe that oxidative stress has a role in the development of cerebral vasospasm and that urinary 8-OHdG may be a good indicator of vasospasm occurrence following $\mathrm{SAH}$.

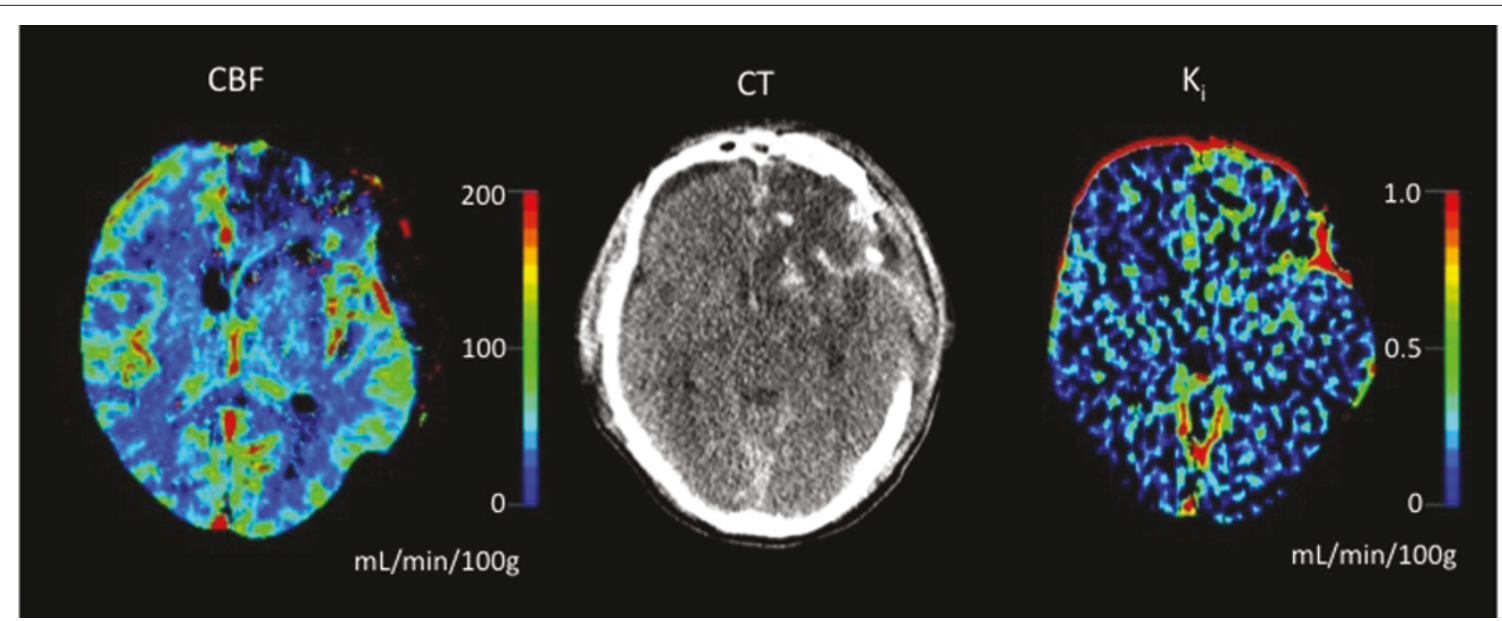

Figure 1 (abstract P312). CBF (left) and permeability (right) maps, and contrast-enhanced CT scan (middle). 
P314

Cortical capillary recruitment by rosuvastatin in experimental brain trauma is associated with increased NO production

P Bentzer, M Jungner

Lund University, Lund, Sweden

Critical Care 2012, 16(Suppl 1):P314 (doi: 10.1186/cc10921)

Introduction Microvascular dysfunction, characterized by edema formation secondary to increased blood-brain barrier (BBB) permeability and decreased blood flow, contributes to poor outcome following brain trauma. Recent studies have indicated that statins may counteract edema formation following brain trauma but little is known about other circulatory effects of statins in this setting. The objective of the present study was to investigate whether statin treatment improves brain microcirculation early after traumatic brain injury, and whether microvascular effects are associated with altered production of nitric oxide and prostacyclin.

Methods After fluid percussion injury, rats were randomized to intravenous treatment with $10 \mathrm{mg} / \mathrm{kg}$ rosuvastatin or vehicle. Brain edema (wet/dry weight), BBB integrity $\left({ }^{51} \mathrm{Cr}\right.$-EDTA blood to brain transfer), cerebral blood flow $\left({ }^{14} \mathrm{C}\right.$-iodoantipyrine autoradiography), and the number of perfused cortical capillaries (FITC-albumin fluorescence microscopy) were measured at 4 and 24 hours. Production of NO and prostacyclin was estimated by measuring the stable degradation products nitrite and nitrate $\left(\mathrm{NO}_{\mathrm{x}}\right)$, and 6-keto-PGF-1a in plasma. Sham injured animals were treated with vehicle and analyzed at 4 hours.

Results Trauma resulted in brain edema, BBB dysfunction, and reduced cortical blood flow, and no effect of treatment on these parameters could be detected. Trauma also induced a reduction in the number of perfused capillaries, which was improved by statin treatment. Statin treatment led to increased plasma $\mathrm{NO}_{\mathrm{x}}$ levels and reduced mean arterial blood pressure. The 6-keto-PGF-1a levels tended to increase after trauma, and were significantly reduced by rosuvastatin.

Conclusion Rosuvastatin treatment improves microcirculation after traumatic brain injury by increasing the number of perfused capillaries. This effect is associated with increased NO and reduced prostacyclin production.

References

1. Béziaud et al.: Crit Care Med 2011, 39:2300-2307.

2. Cherian, Robertson: J Neurotrauma 2003, 20:77-85.

3. Prinz, Endres: Anesth Analg 2009, 109:572-584.

\section{P315}

Effects of sinvastatin in prevention of vasospasm in nontraumatic subarachnoid hemorrhage: preliminary data

S Macedo, V Aguiar, PF Rosa, IT Ladeia, YK Castro, LA Ferreira, DR De Melo, LG Rezende

São Jose do Avai Hospital, Itaperuna, Brazil

Critical Care 2012, 16(Suppl 1):P315 (doi: 10.1186/cc10922)

Introduction Some trials have shown that statins in the acute phase of aSAH reduce the incidence, morbidity and mortality of cerebral vasospasm. Independent of their cholesterol-lowering effect, statins have multiple biological properties, including downregulating inflammation and upregulating endothelial NO synthase. The purpose of this study is to evaluate the potential of sinvastatin (SVT) as prevention against vasospasm.

Methods We realized a prospective study, randomized, nonblind, with the use of $80 \mathrm{mg}$ SVT (night) in the first 72 hours of the beginning of bleeding, and a control group that did not use SVT, for 21 days, between January and December 2008. Informed consent was obtained for all patients. CT scans were performed as control and another CT scan in patients with altered neurological signals. In the presence of changes suggestive of vasospasm or correlation in clinical and CT scans, the patients were taken for cerebral arteriography examination followed by an angioplasty procedure if necessary. Liver and renal function and LDL cholesterol were evaluated every 3 days. Exclusion criteria: liver and renal disease, pregnancy, elevation of serum transaminases (three times the value of normality), creatinine $\geq 2.5$, rhabdomyolysis or CK total $\geq 1,000 \mathrm{U} / \mathrm{l}$.
Results We excluded two patients with bleeding for more than 72 hours. There was no significant change in the levels of CK total, renal or liver function. We included 21 patients, 11 in the SVT group and nine in the control group. The mortality was eight patients (38\%), six patients in the control group and two of the SVT group. Vasospasm was confirmed by cerebral arteriography examination in four patients in the control group and one patient in the SVT group. All patients that had a bad outcome (death) had Fisher IV scale.

Conclusion SVT at a dose of $80 \mathrm{mg}$ was effective in reducing the mortality (18.1\% against 66\%) compared to the group that did not use SVT, and also decreased the incidence of cerebral vasospasm despite the APACHE II score being higher in the group that used SVT (14.3 vs. 10.7). There was less morbidity in the SVT group with an average Glasgow Outcome Scale of 3.25 vs. 2.1.

References

1. Lynch JR, Wang H, et al: Simvastatin reduces vasospasm after aneurysmal subarachnoid hemorrhage: results of a pilot randomized clinical trial. Stroke 2005, 36:2024-2026.

2. McGirt MJ, Lynch JR: Simvastatin increases endothelial nitric oxide synthase and ameliorates cerebral vasospasm resulting from subarachnoid hemorrhage. Stroke 2002, 33:2950-2956.

P316

Evaluation of arterial and venous ophthalmic hemodynamics in preeclamptic pregnant women

EM Shifman, NV Khramchenko, SV Sokologorskiy

Federal Centre for Obstetrics, Gynecology \& Neonatology, Moscow, Russia Critical Care 2012, 16(Suppl 1):P316 (doi: 10.1186/cc10923)

Introduction The aim of the study was to evaluate arterial and venous ophthalmic blood flow parameters in mild and severe preeclampsia pregnancies and in normotensive pregnancies.

Methods A total of 117 women 25 to 30 years old with singleton pregnancies 30 to 40 weeks of gestation were recruited. Among them 40 pregnant women developed severe preeclampsia, 42 mild preeclampsia, and 35 were normotensive. Using color flow mapping (CFM) and pulse-wave Doppler imaging (PWD), maximum blood flow velocity $(\mathrm{mFV})$ in the right/left arterial and venous ophthalmics along with Gosling's Doppler pulsatility index (PI) [1] in both arterial ophthalmics were evaluated. Mean blood pressure in all patients was also registered.

Results The highest mFV values $(59.2 \pm 4.61$ and $23.6 \pm 4.03 \mathrm{~cm} / \mathrm{second})$ were in the severe preeclampsia group while in the mild preeclampsia group $\mathrm{mFV}$ increased slightly or remained normal $(35.6 \pm 2.97$ and $13.6 \pm 0.81 \mathrm{~cm} / \mathrm{second}$ ). There was no $\mathrm{mFV}$ increase in the normotensive pregnancy group $(31.5 \pm 2.21 \mathrm{~cm} /$ second). No significant correlation was found between gestation age and mentioned hemodynamic parameters in the normotensive pregnancy group. PI values in the arterial ophthalmic in normotensive pregnant women were $2.92 \pm 0.59$ and the highest in all groups. In group with mild preeclampsia this parameter was $1.47 \pm 0.30$ and the lowest one was in patients with severe preeclampsia $-1.17 \pm 0.08$.

Conclusion In women with preeclampsia significant changes in ophthalmic hemodynamics take place - $\mathrm{mFV}$ in arterial and venous ophthalmics increases while PI values go down. This might be evidence of orbital hyperperfusion in preeclamptic pregnant women. Low PI values may be used as the markers of severe preeclampsia.

Reference

1. Gosling RG, King DH: Arterial assessment by Doppler shift ultrasound. Proc RSoc Med 1974, 67:447-449.

\section{P317}

Data classification of magnetic resonance tomography and computer tomography images of brain in parturients with neurological complications of eclampsia

GTikhova, E Shifman

Kulakov Scientific Center of Obstetrics, Gynecology and Perinatology, Moscow, Russia

Critical Care 2012, 16(Suppl 1):P317 (doi: 10.1186/cc10924)

Introduction The goal of the study was to classify protocol data recorded during magnetic resonance tomography (MRT) and 
computer tomography $(\mathrm{CT})$ examinations of the brain in patients with neurological complications of eclampsia; to define the MRT/ CT examination data structure; and to perform frequency analysis of main MRT/CT characteristics and estimate their frequency distributions defined by studied pathology. The data included in the study were reported in medical journals and met definite criteria for inclusion.

Methods We collected cases of neurological complications of eclampsia reported in English-language medical journals from 1980 to 2008. The study methods include structural and frequency analysis of brain MRT/ CT image protocols.

Results The analyzed sample included 77 cases of neurological complications of eclampsia. We extracted the following positions from the plain texts of MRT/CT descriptions: brain injury areas (occipital, temporal, parietal and frontal lobes); injury depth (cortical and/or subcortical matter); brain structures undergoing injury (classification was too complicated); injury nature (vasogenic/ischemic edema, hemorrhage). Abnormalities in occipital (84.6\%) and parietal (70.7\%) lobes were the most frequent, injuries in temporal lobes were quite rare $(26.9 \%)$, but damage in frontal lobes was the most uncommon (24.4\%). Combined injury in occipital and parietal lobes was recorded in more than two-thirds of cases (72.4\%). Combined injury in occipitalfrontal lobes (29.3\%) and occipital-temporal (27.6\%) lobes were observed in almost one-third of patients. Synchronous injury in the temporal and frontal lobes was the least common (6.9\%). Simultaneous damage of three and more lobes was observed quite rarely (14.6\%). Most abnormalities were bilateral with frequency not less than $78.0 \%$. Unsymmetrical injury observed in some patients was located in the right lobe in most cases. All analyzed cases include only $7.1 \%$ of single left injury and all of them were located in the occipital lobe. Vasogenic edema occurred in $83.5 \%$ of cases, while ischemic damage was observed in $10.4 \%$. The incidence of hemorrhage was $6.1 \%$.

Conclusion The analysis reveals a general picture of the most distinctive features of brain damage following neurological complications of eclampsia.

P318

Eleven years of critical obstetric pathology: epidemiologic study

L Calejman, V Nunes Velloso, E Canedo, M Deheza

Hospital Rivadavia, Capital Federal, Argentina

Critical Care 2012, 16(Suppl 1):P318 (doi: 10.1186/cc10925)

Introduction The objective was to describe the characteristics of pregnant and puerperal women admitted to the ICU from February 2000 to February 2011.

Methods Patients admitted between the mentioned periods were grouped by age, sex, nationality, APACHE II score, days in the ICU, cause of admission: hypertensive syndromes in pregnancy (HSP), preeclampsia $(\mathrm{P})$, eclampsia (E), HELLP syndrome $(\mathrm{H})$, gestational diabetes, sepsis and placentary disorders; need for mechanical ventilation (MV) and dialysis, maternal mortality, and if they had proper prenatal care.

Results A total of 3,568 patients were admitted, from which 471 patients (13.2\%) were of obstetric cause; average age was 24 years, APACHE II score of 6 . There were 39 cases of arterial hypertension and 26 of diabetes mellitus before pregnancy. Sixty-eight percent were first pregnancy. The most frequent causes of admission were hypertension secondary to pregnancy (HSP) in 353 patients (75\%): $\mathrm{P} 44 \%(n=156), \mathrm{E}$ $8 \%(n=28), \mathrm{H} 33 \%(n=116), \mathrm{H} / \mathrm{E}$ combined $15 \%(n=53)$. Other causes of admission: sepsis $16 \%(n=75)$, placental disorders $7 \%(n=33)$, and neurological deterioration (CVA/S SHEEHAN) $2 \%(n=10)$. They required an average of two drugs to control blood pressure for the patients who needed it in $68 \%(n=320)$. The average stay in the ICU was 6.5 days. From a total of 471 patients, 73 patients required mechanical ventilation $(15 \%)$ and $118(25 \%)$ patients presented high levels of urea and creatinine, 11 patients (2\%) required dialysis. With respect to nationality 301 patients (64\%) were Argentinean, the others reported were from Bolivia, Paraguay and Peru. Prenatal checks occurred in only $35 \%(n=165)$ of the patients. The mortality rate was $6 \%(n=28)$.

Conclusion Critical obstetrical pathology is common in the ICU, HSP as the main cause. A high number of first pregnancy patients with little prenatal care was observed. This type of patient requires low levels of life support.
P319

Clinical outcomes in neonates following maternal magnesium sulfate therapy in preeclampsia/eclampsia

GTikhova, E Shifman

Kulakov Scientific Center of Obstetrics, Gynecology and Perinatology, Moscow, Russia

Critical Care 2012, 16(Suppl 1):P319 (doi: 10.1186/cc10926)

Introduction Magnesium sulfate therapy (MST) is the method of choice in prophylaxis and treatment of eclamptic seizures in many countries. A lot of high-quality clinical trials and meta-analyses proved its efficacy and safety for mothers. But the effect of maternal MST on the fetus and neonate is still controversial. The goal of the study was to analyze available trials concerning this problem in order to prove statistically that maternal MST given as prophylaxis or treatment of eclamptic seizures has no adverse effects on the mature fetus and term neonate. Methods Trials were searched for in the PubMed database among English-language articles published in 1990 to 2010. Analysis includes randomized controlled prospective clinical trials comparing MST with no treatment, placebo or other anticonvulsant. The following neonatal outcomes were chosen as the main endpoints of the study: neonatal death, neonatal hypotonia, Apgar score $<7$ at 1 and 5 minutes, intubation at place of delivery, admission to the NICU, treatment in NICU $>7$ days. The total effect of MST was measured as the relative risk of adverse outcome in the MST group compared with control and its $95 \% \mathrm{Cl}$. Meta-analysis of neonatal outcomes was performed under a random-effect model for seven endpoints and a fixed-effect model for three endpoints.

Results Neonatal mortality in the MST group was compared with different control groups. Each of these studies showed no significant difference between two groups: MST/mixed $(0.89,95 \% \mathrm{Cl} 0.80$ to 0.99$)$, MTS/no treatment-placebo $(0.99,95 \% \mathrm{Cl} 0.93$ to 1.05$), \mathrm{MTS} /$ diazepam $(1.09,95 \% \mathrm{Cl} 0.91$ to 1.29$), \mathrm{MTS} /$ fenitoin $(0.75,95 \% \mathrm{Cl} 0.56$ to 1.02$)$. The neonatal hypotonia rate is significantly higher in the MST group (3.57, $95 \% \mathrm{Cl} 2.89$ to 4.42 ), although significant heterogeneity of the control group may be a valuable confounding factor. There was no evidence for changing incidence of Apgar $<7$ at 1 and 5 minutes in the MTS group compared with control $(0.79,95 \% \mathrm{Cl} 0.70$ to 0.89 and $0.80,95 \%$ $\mathrm{Cl} 0.64$ to 0.99 correspondingly). The same results were observed for intubation at place of delivery $(1.04,95 \% \mathrm{Cl} 0.90$ to 1.29$)$ and admission to $\mathrm{NICU}(0.96,95 \% \mathrm{Cl} 0.85$ to 1.08$)$. The incidence of treatment in the $\mathrm{NICU}>7$ day was significantly lower in MST group than in control $(0.54$, $95 \% \mathrm{Cl} 0.52$ to 0.78$)$.

Conclusion Maternal MST given as prophylaxis or treatment of eclamptic seizures does not affect neonatal mortality and incidence of neonatal hypotonia, Apgar $<7$ at 1 and 5 minutes, intubation at place of delivery and admission to the NICU in a population of term newborns. Maternal MST significantly reduces the risk of neonate treatment in $\mathrm{NICU}>7$ days in this population.

\section{P320}

Sleep monitoring by actigraphy in short-stay ICU patients

AW Van der Kooi' ${ }^{1}, J H$ Tulen², AW De Weerd ${ }^{3}$, MM Van Eijk', MJ Van Uitert ${ }^{4}$, SE De Rooij ${ }^{4}$, BC Van Munster ${ }^{4}$, AJ Slooter $^{1}$

'University Medical Center Utrecht, the Netherlands; ${ }^{2}$ Erasmus MC, University Medical Center Rotterdam, the Netherlands; ${ }^{3}$ Sein, Zwolle, the Netherlands: ${ }^{4}$ Academic Medical Center, Amsterdam, the Netherlands

Critical Care 2012, 16(Suppl 1):P320 (doi: 10.1186/cc10927)

Introduction Sleep deprivation is common in ICU patients, but difficult to investigate [1]. The gold standard for sleep monitoring, polysomnography (PSG), is impractical for use in ICU patients [2]. Actigraphy proved to be a good alternative in non-ICU patients [3]. However, in prolonged mechanically ventilated patients, actigraphy was inaccurate, probably due to ICU-acquired weakness and resulting inactivity [2]. Short-stay ICU patients do not suffer from ICU-acquired weakness, and the accuracy of actigraphy in these patients has not yet been studied [4]. The aim of this study was to investigate actigraphy for sleep assessment in short-stay ICU patients.

Methods PSG and actigraphy measurements were conducted in seven postcardiothoracic surgery patients. Total sleep time, sleep 
efficiency, number of awakenings and wake time after sleep onset were determined with actigraphy and compared to PSG. The accuracy, sensitivity (percentage correctly scored as sleep) and specificity (percentage correctly scored as awake) were calculated for actigraphy using high, medium, low and automatic threshold sensitivity settings of the actigraphy software.

Results The only parameter that showed a significant correlation between PSG and actigraphy was the number of awakenings ( $r=0.76$, $P=0.049$, high threshold setting). Actigraphy underestimated wake time after sleep onset and overestimated total sleep time and sleep efficiency. The median specificity for actigraphy was below 19\% and the median sensitivity above $94 \%$ for all threshold settings.

Conclusion Actigraphy is not reliable for one-night sleep-wake detection in short-stay postoperative ICU patients.

References

1. Figueroa-Ramos M, et al:: Intensive Care Med 2009, 35:781-795.

2. Beecroft J, et al:: Intensive Care Med 2008, 34:2076-2083.

3. de Souza L, et al.: Sleep 2003, 26:81-85.

4. Schweickert WD, et al:: Chest 2007, 131:1541-1549.

\section{P321}

Quality and quantity of sleep in multipatient versus single-room ICUs

M Van Eijk, A Slooter

University Medical Center Utrecht, the Netherlands

Critical Care 2012, 16(Suppl 1):P321 (doi: 10.1186/cc10928)

Introduction Sleep fragmentation and deprivation is common in ICU patients [1]. It is assumed that the ICU environment (overexposure to sound and light during night-time) leads to disturbed sleep [2]. In our hospital, a new ICU was built with quiet, single-patient rooms with much daylight. This created an opportunity to study the effects of nursing environment on sleep quality and quantity in ICU patients. Methods We included 21 postcardiothoracic surgery patients: 11 subjects were admitted to the old, ward-like ICU, and 10 patients to the new, single-room ICU (see Figure 1). Hypnograms were derived from a polysomnography from 07:00 p.m. to 07:00 a.m.

Results Both groups did not differ with respect to age, duration of surgery or use of psychoactive medication. Polysomnography recordings showed no differences in total sleep time and awakenings (63 \pm 26 in the old ICU and $56 \pm 30$ in the new ICU). The mean percentage of sleep stages in the old versus new situation did not essentially different either: N1: $12.9 \%$ versus $8.0 \%, P=0.21$, ANOVA; N2: $80.3 \%$ versus $87.2 \%, P=0.07$, ANOVA; N3: $5.2 \%$ versus $2.5 \%, P=0.18$, ANOVA. Only REM sleep latency was longer in the old ICU: 314.7 versus 633.5 minutes, $P=0.02$, ANOVA.

Conclusion Except for REM onset latency, sleep improvement was not achieved by changing a ward-like into a single-patient-room ICU environment. When striving for more natural sleep, attitudes towards

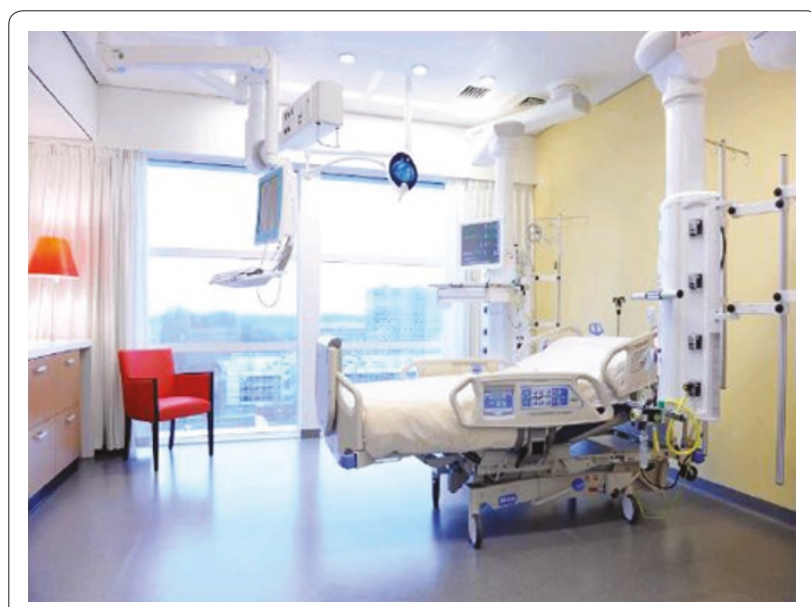

Figure 1 (abstract P321). New, single-room ICU. nursing and medication may play a more important role than ICU design.

References

1. Cooper AB, et al:: Chest 2000, 117:809-818.

2. Gabor JY, et al:: Am J Respir Crit Care Med 2003, 167:708-715.

\section{P322}

Oral melatonin in high-risk critically ill patients: quality of sedative effect

G Sabbatini, G Mistraletti, B Cerri, S Miori, I Galluccio, M Tozzi, C Villa, M Umbrello, F Fraschini, G lapichino

Università degli Studi di Milano, Milan, Italy

Critical Care 2012, 16(Suppl 1):P322 (doi: 10.1186/cc10929)

Introduction Analgesic/sedative therapy is necessary in ICU patients; however, it presents important side effects. Critically ill patients have altered circadian rhythm, delirium and agitation often requiring additional sedation. The dramatically reduced endogenous blood melatonin level (basal and night peaks) could play a role in this context. We evaluated the effects of oral melatonin administration on the adaptation to critical illness and invasive procedures in high-risk critically ill patients [1] consciously sedated [2].

Methods Double-blind RCT between placebo and melatonin ( $3 \mathrm{mg}$ bid, 8:00 and 12:00 p.m., from third ICU day until discharge). Inclusion: age $>18$, SAPS II $>32$, expected mechanical ventilation (MV) $>4$ days, practicability of the gastroenteric tract. Patients were treated according to local guidelines [2], titrating sedatives to a conscious target (Richmond Agitation Sedation Scale (RASS) $=0$ ) as early as possible. Each day, the physician in charge stated the RASS target; nurses assessed the actual RASS.

Results Eighty-two patients enrolled: age 72 (60 to 77), SAPS II 41 (34 to 54), MV length 11 (6 to 22) days. Fifteen pancreatitis, 33 acute lung diseases, 13 acute heart diseases, 21 other. The analgesic/sedative therapy during the first 3 days was not different between groups. Melatonin administration determined early weaning from sedatives and analgesics. The prevalence of conscious sedation (RASS $=0$ ) was higher in the melatonin group ( 67.9 vs. $60.1 \%, P<0.01)$, while deeper levels of sedation (RASS $=-3 /-4$ ) were lower in the melatonin group (RASS -3: 2.4 vs. $7.7 \%, P<0.01$; RASS $-4: 1.9$ vs. $4.3 \%, P<0.01$ ). Melatonin administration caused no oversedation ( 26.3 vs. $24.2 \%, P=0.94)$, while decreased undersedation ( $18.6 \%$ vs. $26.2 \%, P=0.05)$. RASS targets were joined more frequently in the melatonin group, even if not significantly (55.1 vs. $49.6 \%, P=0.12$ ).

Conclusion Oral melatonin increased the prevalence of conscious sedation in high-risk critically ill patients; it allowed a better achievement of RASS target, particularly decreasing undersedation episodes.

Clinicaltrial.gov NCT00470821

References

1. lapichino et al:: Crit Care Med 2006, 34:1039.

2. Cigada et al:. J Crit Care 2008, 23:349.

\section{P323}

Sedation depth and mortality in mechanically ventilated critically ill adults

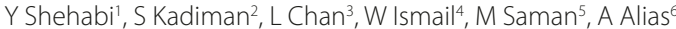

'University New South Wales, Randwick, Australia; ${ }^{2}$ National Heart Institute, Kuala Lumpur, Malaysia; ${ }^{3}$ University Malaya, Kuala Lumpur, Malaysia; ${ }^{4}$ Raja Perempuan Zainab II Hospital, Kota Bharu, Malaysia; ${ }^{5}$ Sarawak General Hospital, Kuching, Malaysia; ${ }^{M}$ Malacca General Hospital, Malacca, Malaysia Critical Care 2012, 16(Suppl 1):P323 (doi: 10.1186/cc10930)

Introduction Deep sedation is common in ventilated patients, particularly in the first 48 hours in the ICU, which may adversely affect outcomes such as mortality. This period is usually unobserved in clinical trials due to late randomisation. We investigated the relationship between early sedation depth, sum of Richmond Agitation Sedation Scale (RASS) -3 to -5 and clinical outcomes, including mortality. Methods A waiver of consent was granted. In collaboration with the Australian New Zealand Intensive Care Research Centre, we conducted 
a multicentre prospective longitudinal cohort study in 11 centers in Malaysia. Critically ill patients ventilated and sedated $\geq 24$ hours were followed from ICU admission to hospital discharge. The administration of all sedatives was measured daily. Four-hourly RASS assessments were conducted and delirium assessed daily (CAM-ICU during light sedation RASS -2 to +1 ). Multivariable Cox regression proportional hazard was used to quantify relationships between early deep sedation and time to extubation and delirium occurring after 48 hours and hospital mortality adjusting for diagnosis, age, gender, APACHE II score, operative, elective, early use of vasopressors and dialysis.

Results We studied 259 patients with mean (SD) age 53.1 (15.9) years and APACHE II score 21.3 (8.2), ventilated for median (IQR) 5 (3 to 8.8) days. Hospital mortality was 82 (31.7\%). Midazolam and morphine were the commonest agents used, given to 241 (93.1\%) and 201 (77.6\%) patients respectively. Over 2,657 study days, 13,836 assessments were conducted. Deep sedation was recorded in $187(72 \%)$ patients within 4 hours of commencing ventilation and in 159 (61\%) patients at 48 hours. Daily interruption was used on $20 \%$ of study days. Delirium occurred in $114(43 \%)$ of assessed patients with a mean (SD) duration of 1.3 (2.2) days. Early deep sedation independently predicted time to hospital death ( $\mathrm{HR} 1.11,95 \% \mathrm{Cl} 1.05$ to $1.18, P<0.001)$ and time to extubation (HR $0.93,95 \% \mathrm{Cl} 0.89$ to $0.96, P=0.001$ ) but not time to delirium occurring after 48 hours (HR $0.98,95 \% \mathrm{Cl} 0.93$ to $1.03, P=0.46$ ). Midazolam cumulative dose in the first 48 hours was significantly associated with the number of RASS assessments $\leq-3(P<0.001)$.

Conclusion Early ICU sedation depth is a modifiable risk factor for delayed extubation and increased risk of death and should be considered in future sedation trials.

Reference

1. Devlin JW: The pharmacology of over sedation in mechanically ventilated adults. Curr Opin Crit Care 2008, 14:403-407.

P324

Sedation in the ICU: nurses' perceptions of practices and influencing factors

B Sneyers' ${ }^{1}$, PF Laterre ${ }^{2}$, MM Perreault ${ }^{3}$, A Spinewine ${ }^{1}$

'Université catholique de Louvain, Louvain Drug Research Institute, Brussels,

Belgium; 'Université catholique de Louvain, Cliniques Universitaires St-LuC,

Brussels, Belgium; ${ }^{3}$ Université de Montreal, Canada

Critical Care 2012, 16(Suppl 1):P324 (doi: 10.1186/cc10931)

Introduction Our goals are to describe adherence to sedation recommendations [1] in Belgian ICUs and to identify major factors influencing practices.

Methods A national survey including all nurses working in Belgian ICUs was conducted with seven nurses sampled per hospital. A validated self-administered paper survey was designed based on a literature review and data from a previous qualitative study. Topics addressed were current practices and reasons for (non)compliance to sedation recommendations such as use of sedation scales and daily sedation interruption (DSI). Four postal reminders were sent.

Results The response rate was $70 \%(n=587 / 840$ nurses from $99 / 120$ hospitals). Sedation scales are available to $89 \%$ of nurses and frequency of use is variable ( $\leq 1 \times /$ day: $13 \%, 3$ to $4 \times /$ day: $31 \%, \geq 6 \times /$ day: $56 \%)$. When sedation scales are available, perceived indications are monitoring of sedation and analgesia ( $96 \%$ and $31 \%$ of nurses respectively) and dosing adjustment for sedatives and analgesics (14\% and $28 \%$ of nurses respectively). DSI is infrequently used (never used: $38 \%$ of respondents, used for $<25 \%$ of patients: $47 \%$ of respondents, used for 25 to $75 \%$ of patients: $12 \%$ of respondents, used for $>75 \%$ of patients: $3 \%$ of respondents). Numerous barriers for wide implementation are identified, mainly lack of outcome expectancy, as DSI is perceived to impair patient outcomes. It is perceived that DSI increases the risk of complications such as unplanned extubation and pulling of lines and tubes ( $79 \%$ of nurses agree), impairs patients' comfort ( $59 \%$ of nurses agree), and creates traumatic memories in the intubated patients ( $36 \%$ of nurses agree). Moreover, $63 \%$ of nurses agree that they would prefer no DSI if they were an intubated patient. Other barriers are related to knowledge, as $26 \%$ of nurses do not know the practice, and to behaviour, as $53 \%$ of respondents feel DSI is difficult to implement because of organizational constraints.
Conclusion Sedation scales are widely used in Belgium, while use of DSI is low. Barriers impairing adherence to recommendations were identified. Perception that sedation scales are not used for sedative dosing adjustments is present, as well as inadequate use for analgesia. Fear of worsening patient outcomes using DSI is present, contrasting with current literature. A similar survey addressing physicians' perceptions is ongoing.

Reference

1. Jacobi et al:: Crit Care Med 2002, 30:119-141.

\section{P325}

Implementation of a national guideline for analgesia and sedation: how often can a RASS of 0 to -2 be achieved?

R Riessen, P Tränkle, R Pech, G Blumenstock, M Haap

University Hospital Tübingen, Germany

Critical Care 2012, 16(Suppl 1):P325 (doi: 10.1186/cc10932)

Introduction Based on a new national guideline we implemented in our medical ICU an interdisciplinary algorithm for the management of analgosedation, in which nurses had to adjust the dose of the analgesics and sedatives based on sedation goals given by the physicians. Within this project we investigated in what portion of mechanically ventilated patients a sedation level of Richmond Agitation and Sedation Scale (RASS) of 0 to -2 , which is generally recommended by the guideline, can be achieved. We also asked the nurses for an explanation when this goal was not reached.

Methods After an educational program the level of sedation was measured 364 times in 37 mechanically ventilated patients at different time points by an independent observer. In all cases in which the RASS was outside the desired level of 0 to -2 , the nurse in charge was asked to fill out a structured as well as open questionnaire, in which the reasons for this deviation could be stated.

Results The independent observer documented only in 13\% (47/364) of all measurements a RASS of 0 to -2 . We analyzed 295 questionnaires, in which 368 reasons for a deviation from a RASS of 0 to -2 were stated (multiple answers were possible). In 113 questionnaires (38\%) the nurses mentioned that a short-term increase in sedation depth was required for nursing procedures or medical interventions. In 89 questionnaires (30\%) a RASS of 0 to -2 was considered reasonable but could not be achieved at the time of measurement with the current medication ( $n=32$ ) or the consciousness was impaired by CNS diseases $(n=52)$. In 100 questionnaires $(34 \%)$ a RASS of 0 to -2 was not considered reasonable. The following reasons were stated: disease with coma $(n=25)$, controlled ventilation $(n=32)$, distressed patient $(n=12)$, increased intracranial pressure $(n=7)$, status epilepticus ( $n=7)$, hypothermia $(n=4)$, dying patient $(n=4)$, delirium/(auto) aggression $(n=4)$. Other reasons were mentioned in 66 questionnaires $(22 \%)$, most commonly a physician order for a deeper sedation $(n=19)$ or a missing sedation goal $(n=14)$.

Conclusion In mechanically ventilated patients of a medical ICU including also patients with neurologic diseases, a sedation goal of RASS 0 to -2 , as recommended by a current guideline, could only be achieved in a minority of patients despite intensive instructions and a motivated team. In most cases the nurses were able to provide reasonable medical explanations for a deeper sedation or an otherwise impaired consciousness.

\section{P326}

Comparison of the RAMSAY score and the Richmond Agitation

Sedation Score for the measurement of sedation depth

R Riessen, R Pech, PTränkle, G Blumenstock, M Haap

University Hospital Tübingen, Germany

Critical Care 2012, 16(Suppl 1):P326 (doi: 10.1186/cc10933)

Introduction We implemented an interdisciplinary algorithm for the management of analgosedation in mechanically ventilated patients based on a new national guideline. As part of this project we investigated whether the newly introduced Richmond Agitation Sedation Score (RASS) allowed a better monitoring of sedation depth than the formerly used RAMSAY score. 
Methods During the baseline phase of the study we investigated the RAMSAY score, which had been routinely used for several years in our unit. Following an educational program the RAMSAY was replaced by the RASS. During both study phases the actual sedation score was determined within a short period of time by the nurse in charge and an independent observer. In addition, the nurses were asked to evaluate on a six-point Likert scale whether the score appeared to be suitable to describe the actual state of sedation or to discriminate between different levels of sedation $(1=$ very good $)$. The measurements took place at three defined time points (7,9 and 12 o'clock) during the morning shift on weekdays.

Results In the baseline phase (36 patients/422 measurements) using the RAMSAY score, sedation depth documented by the nurses and the observer matched in only $39 \%$ of the measurements. The nurses documented in 246 (58\%) measurements a lighter sedation and in 12 measurements (3\%) a deeper sedation than the observer. In the postimplementation phase (37 patients/346 measurements) using the RASS, we found a significantly higher matching rate of $76 \%$ between nurses and observer compared to RAMSAY $(P<0.001)$. Nurses documented in 47 measurements a lighter (14\%) and in 37 measurements (11\%) a deeper sedation than the observer. The nurses evaluated the RASS in terms of the ability to describe the actual depths of sedation with a mean of 1.7 on the six-point Likert scale significantly better than the RAMSAY score with $3.2(P<0.001)$. Similar results were found regarding the discrimination between different levels of sedation (RASS 1.7, RAMSAY $3.1, P<0.001$ ).

Conclusion In routine use the RAMSAY score showed a poor performance regarding the measurement of sedation depth. After implementation of the RASS, measurement of sedation depth appeared significantly improved.

\section{P327}

Dexmedetomidine is associated with better outcomes in patients undergoing cardiac surgery

PG Brandão', S Lobo', M Nassau Machado', J Duarte', F Lobo', Y Sakr²

${ }^{\prime}$ Hospital de Base de São José do Rio Preto, Brazil;'²Friedrich Schiller University Hospital, Jena, Germany

Critical Care 2012, 16(Suppl 1):P327 (doi: 10.1186/cc10934)

Introduction Cardiac anesthesia has changed over the years from highdose opioids to fast-track surgery. The use of high doses of opioids was justified based on the hemodynamic stability [1] at a cost of prolonged mechanical ventilation support. Our study aims to analyze the use of dexmedetomidine as an anesthesia adjuvant during the induction and maintenance of anesthesia for patients undergoing coronary artery bypass graft (CABG) and valvular heart surgeries.

Methods This study is a retrospective analysis from a prospective database collected from January 2003 to April 2011. The patients were divided into two groups, based on the use of dexmedetomidine (DEX group) intraoperatively or conventional opioid-based technique (Control group). Isoflurane was used for anesthesia maintenance in both groups.

Results We included 1,302 consecutive patients undergoing cardiac surgery during the study period ( $63 \%$ male; median age $=57$ years), 796 patients in the DEX group and 506 patients in the control group. CABG was the most commonly performed surgery (63\%) followed by valve surgeries (37\%). The overall 30 -day hospital mortality rate was $5.8 \%$. Length of stay was significantly lower for patients in the Dex group (3.7 \pm 4.4 days) than for patients in the control group ( $4.5 \pm 6.3$ days) $(P=0.02)$. Thirty-day mortality rates were $3.4 \%$ in the Dex group and $9.7 \%$ in the control group $(P<0.001)$. In the multivariable Cox regression analysis with in-hospital death as the dependent variable, dexmedetomidine (OR $=0.39,95 \% \mathrm{Cl}: 0.23$ to $0.64, P \leq 0.001)$, a high L-EuroSCORE (OR=1.05, 95\% Cl: 1.01 to $1.10, P=0.004)$ and older age ( $\mathrm{OR}=1.03,95 \% \mathrm{Cl}: 1.01$ to $1.05, P=0.003)$ were independently related to in-hospital death. Need for reoperation $(2.0 \%$ vs. $2.8 \%, P=0.001)$, neurologic lesion type $1(2.0 \%$ vs. $4.7 \%, P=0.005)$ and prolonged hospitalization ( $3.1 \%$ vs. $7.3 \%, P=0.001)$ were significantly less frequent in the DEX group than in the control group.

Conclusion Use of dexmedetomidine as anesthesia adjuvant was associated with better outcomes in patients undergoing cardiac surgery.
References

1. Lowenstein $E$, et al:: Cardiovascular response to large doses of intravenous morphine in man. N Engl J Med 1969, 281:1389-1393.

2. Jalonen J, et al.: Dexmedetomidine as an anesthetic adjuvant in coronary artery bypass grafting. Anesthesiology 1997, 86:331-345.

\section{P328}

Cerebral ischemia-reperfusion model in rabbits: relationship between dexmedetomidine and biochemical parameters in lowering intraparenchymal pressure

A Tavlan', ME Ustun', A Yosunkaya', A Ak', A Kiyici', HK BardakCl², F Gok 'Selcuk University, Meram Medical Faculty, Konya, Turkey; ${ }^{2}$ Farabi Hospital, Konya, Turkey

Critical Care 2012, 16(Suppl 1):P328 (doi: 10.1186/cc10935)

Introduction The effect of dexmedetomidine in two different doses on the levels of endothelin-1 (ET-1) and prostoglandin $\mathrm{I}_{2}\left(\mathrm{PGI}_{2}\right)$ in blood and cerebrospinal fluid (CSF) of rabbits via the transient global cerebral ischemia model was studied to determine its intraparenchymal pressure (IPP) reduction mechanism.

Methods Twenty-four New Zealand type rabbits were employed and randomly distributed into four groups. Group I (sham group, $n=6$ ): craniotomy was performed only. Group II (control group, $n=6$ ): bilateral carotid arteries were clamped for 60 minutes after craniotomy, then reperfusion was performed for 60 minutes. In Group III $(n=6)$ and Group IV $(n=6), 80 \mu \mathrm{kg}^{-1}$ and $320 \mu \mathrm{kg}^{-1}$ dexmedetomidine was administered within the first 10 minutes of the reperfusion procedure respectively. Blood and CSF samples were collected 120 minutes after craniotomy. Mean arterial pressures (MAP), heart rates (HR), IPP and temperature values were recorded.

Results There was no significant difference in MAP values between groups $(P \geq 0.05)$. A decrease of HR in Group IV was significantly lower after reperfusion $(P<0.05)$. IPP values after the reperfusion in Groups II and IV were significantly higher than Group I $(P<0.05)$, but no significant increase in Group III $(P \geq 0.05)$. ET-1 levels of both blood and CSF were increased in the group with performed ischemia and reperfusion and no treatment (Group II) and the group administered high-dose dexmedetomidine (Group IV) $(P<0.05)$, while the group administered low-dose dexmedetomidine (Group III) was similar to the sham group $(P \geq 0.05)$. However, $\mathrm{PGI}$ levels of CSF were significantly decreased in the group administered low-dose dexmedetomidine $(P<0.05)$.

Conclusion Dexmedetomidine could decrease intraparenchymal pressure in the transient global cerebral ischemia model when administered at low doses $[1,2]$. It probably contributed to this reduction by preventing an increase of endothelin levels in blood and CSF as well as decreasing $\mathrm{PGI}_{2}$ levels in CSF.

References

1. Zornow MH, Scheller MS, Sheehan PB: Intracranial pressure effects of dexmedetomidine in rabbits. Anesth Analg 1992, 75:232-237.

2. Jolkkonen J, Puurunen K, Koistinaho J, et al: Neuroprotection by the alpha 2-adrenoceptor agonist, dexmedetomidine in rat focal cerebral ischemia. EurJ Pharmacol 1999, 7:31-36.

\section{P329}

Evaluation of sedation using pupilometry in ICUs: a pilot study O Rouche, A Wolak-Thierry, Q Destoop, L Milloncourt, T Floch, P Raclot, J Cousson Centre Hospitalier Universitaire, Reims, France Critical Care 2012, 16(Suppl 1):P329 (doi: 10.1186/cc10936)

Introduction The depth of hypnosis is correlated with the decrease in photomotor reflex (PMR) [1]. It would be beneficial to develop an automated, noninvasive, simple and reproducible technique allowing one to efficiently evaluate the depth of sedation in ICUs. The objective of this observational study is to evaluate the effectiveness of pupilometric video in comparison to the Bispectral index (BIS).

Methods Sedation level was based on the Richmond Score (RASS between -4 and -5 ). Exclusion criteria were neurological pathologies interfering with the PMR. Following a 320 lux flash of light, the PMR was measured by the Neurolight (IDmed). Three measurements a day 
were taken during 48 hours along with the collection of the BIS value (Bis Vista Anandic Medical Systems). The data collected included the variation of pupillary diameter (PD), latency time (LT) and maximal speed of pupillary constriction $\left(\mathrm{V}_{\max }\right)$. These parameters were analyzed after having classified BIS values into three groups.

Results A total of 186 analyses of PMR and BIS were conducted on 31 patients. The averages and standard deviations for each class of BIS were as shown in Table 1. We conducted an analysis of variance in order to compare these three groups of BIS. For the values $\mathrm{V}_{\max }$ and the PD, the ANOVA was significant. Therefore, we proceeded to compare the groups two by two using Bonferroni tests. They revealed significant difference between the BIS $<40$ and $40 \leq$ BIS $\leq 60$ group $(P<0.0001$ for both variables) and between BIS $<40$ and BIS $>60\left(\mathrm{~V}_{\max } P<0.0001\right.$ and PD $P<0.05)$. There was no correlation between any of the BIS groups and the LT variable.

Table 1 (abstract P329). Values of $\mathrm{V}_{\text {max }}, P D$ and TL

\begin{tabular}{lccc}
\hline & BIS $<\mathbf{4 0}(\boldsymbol{n}=\mathbf{6 8})$ & $\mathbf{4 0} \leq \mathrm{BIS} \leq \mathbf{6 0}(\boldsymbol{n}=\mathbf{6 2})$ & BIS $>\mathbf{6 0}(\boldsymbol{n}=\mathbf{3 7})$ \\
\hline$V_{\max }(\mathrm{mm} /$ second $)$ & $0.98 \pm 0.44$ & $1.45 \pm 0.73$ & $1.66 \pm 0.95$ \\
$\mathrm{TL}(\mathrm{ms})$ & $253.8 \pm 68.6$ & $241.6 \pm 41.8$ & $240.6 \pm 52.2$ \\
$\mathrm{PD} \%$ & $12.95 \pm 5.58$ & $18.3 \pm 6.12$ & $17.7 \pm 6.72$ \\
\hline
\end{tabular}

Conclusion The $\mathrm{V}_{\text {max }}$ and the PD seem to be relevant criteria when compared to the BIS. This noninvasive technique of monitoring the depth of sedation could be beneficial especially with patients under myorelaxant drugs. A larger study is necessary in order to confirm these results and enable one to set cut-off values for the $\mathrm{V}_{\max }$ and PD.

Reference

1. Leslie K, et al.: Anesthesiology 1996, 84:52-63.

P330

Effect of critical illness on the pharmacokinetics and dose-response relationship of midazolam

D Ovakim, KJ Bosma, GB Young, M Sen, LE Norton, F Priestap, RG Tirona, R Kim, GK Dresser

University of Western Ontario, London, Canada

Critical Care 2012, 16(Suppl 1):P330 (doi: 10.1186/cc10937)

Introduction Critically ill patients require sedation to tolerate the interventions necessary to facilitate their care. There is growing evidence, however, that use of sedatives, such as the benzodiazepine midazolam, is associated with delirium and other complications that can lead to prolonged ICU stay and increased mortality. The pharmacokinetics of midazolam in healthy populations has been well characterized, and pharmacodynamic studies demonstrate a predictable dose-response relationship. However, in critical illness, where midazolam is often administered as a continuous infusion, the pharmacokinetic properties are often altered. We sought to investigate whether analysis of midazolam plasma concentrations in combination with electroencephalography (EEG) will better define the effect of critical illness on the pharmacokinetics and clinical response to midazolam, while providing a method to assess the adequacy of sedation thereby minimizing the risks associated with prolonged or over-sedation.

Methods For this observational study, patients admitted to the ICU with a diagnosis of sepsis and receiving a continuous infusion of midazolam were screened for inclusion. Upon enrollment, a continuous subhairline EEG was applied and blood samples were collected daily for plasma midazolam quantification. Clinical data and laboratory parameters were followed. Plasma midazolam levels were quantified using liquid chromatography with tandem mass spectroscopy.

Results Data were available for nine patients. Midazolam clearance demonstrated wide intersubject variability (range $31 \mathrm{ml} /$ minute to $1,157 \mathrm{ml} /$ minute) although average clearance among all patients (418 $\mathrm{ml} /$ minute) was comparable to that of healthy controls. Mean midazolam concentrations for patients with coma were significantly higher than for patients without coma $(218 \pm 185 \mathrm{ng} / \mathrm{ml}$ vs. $106 \pm 107 \mathrm{ng} / \mathrm{ml}$ ). The plasma midazolam concentration inversely correlated with EEG frequency, with maximal slowing in the delta $(\leq 4 \mathrm{~Hz})$ range.

Conclusion Midazolam concentrations while on continuous infusion were associated with EEG tracings suggestive of deep sedation. Although clearance was relatively preserved, it varied over a wider range than found in healthy populations. The apparent lower threshold for onset of coma may be a reflection of illness severity, concomitant medication use, and variable clearance during the course of illness. These preliminary results suggest that the combination of continuous bedside EEG and therapeutic drug monitoring may be useful for titrating midazolam infusions and to guide tapering to avoid prolonged coma in patients with variable clearance of midazolam.

\section{P331}

Effect of propofol and midazolan on microcirculation of septic shock patients

G Penna', F Fialho², A Japiassu³, D Salgado', P Kurtz', G Nobre', M Kalichsztein', N Villela ${ }^{5}$, E Bouskela ${ }^{5}$

'Casa de Saúde São José, Rio de Janeiro, Brazil; 'IFF-FIOCRUZ, Rio de Janeiro, Brazil; 3IPEC-FIOCRUZ, Rio de Janeiro, Brazil; ${ }^{4}$ UFRJ, Rio de Janeiro, Brazil; ${ }^{5} U E R J$, Rio de Janeiro, Brazil

Critical Care 2012, 16(Suppl 1):P331 (doi: 10.1186/cc10938)

Introduction Septic shock patients are submitted to many therapeutic strategies, including sedation. It is unknown if different sedative drugs influence microcirculation.

Methods We performed a prospective observational study, using sidestream dark-field imaging (SDF), to evaluate sublingual mucosa of septic shock patients admitted to our ICU. SDF was applied in two settings: continuous sedation with propofol and with midazolan. We repeated each examination after an interval of 30 minutes. Eight fields (videos) were analyzed during propofol and midazolan infusion. Two videos were obtained from each side of the tongue. The Bispectral index was monitored along with the Richmond Agitation Sedation Scale: the dose of both sedatives was titered to maintain light sedation. All demographic and severity of illness data were collected. Vasopressor agents were maintained to a mean arterial pressure of $70 \mathrm{mmHg}$ and the cardiac index was kept stable through the protocol study.

Results We included 15 patients; APACHE II score was (median) 17.5 points and SOFA score 9 points. The Bispectral index was lower in the midazolan group (43 vs. 48.5 points, $P=0.005$ ), although RASS was the same for both groups. Large-vessel perfusion was similar for both groups. The small perfusion vessel proportion was significantly reduced with propofol (92 vs. $96.3 \%, P=0.003$ ). The microvascular flow index was also lower during propofol infusion (MFI - 2.4 vs. 2.7, $P=0.002$ ). We observed a higher heterogeneity index when patients were sedated with propofol (0.4 vs. $0.19, P=0.01)$.

Conclusion Propofol reduces small-vessel perfusion and increases the heterogeneity of circulation in the sublingual mucosa, when compared with the use of midazolan in septic shock patients.

\section{P332}

Current use of pain scores in Dutch ICUs: a postal survey in the Netherlands

M Van der Woude', L Bormans'1, J Hofhuis², P Spronk²

${ }^{1}$ Atrium Medical Center, Heerlen, the Netherlands; ${ }^{2}$ Gelre Hospitals, Apeldoorn, the Netherlands

Critical Care 2012, 16(Suppl 1):P332 (doi: 10.1186/cc10939)

Introduction Pain is a common problem for patients admitted to the ICU, causing patient discomfort, agitation and accidental selfextubation. For this reason the recognition of pain and its severity is extremely important. Several pain scores and protocols are in use. We aimed to elucidate current practice of pain measurements and treatment in Dutch ICUs.

Methods In March 2011, a questionnaire was sent to all Dutch adult ICUs irrespective of the number of ICU beds with active follow-up by telephone calls to optimize the participation rate.

Results A total of 84 ICUs (84/107) returned the survey, representing a response rate of $87 \%$. Most ICUs are community teaching hospitals 
and nonteaching hospitals (85\%) in comparison to academic hospitals (15\%). Most ICUs (94\%) use a standardized pain score in the group of patients who are capable of verbal communication: the Visual Analogue Scale (57\%), Numerical Rating Scale (48\%) and Faces Pain Scale (5\%) being the most frequently used scores. In the group of patients who are unable to communicate, ICUs less frequently use pain scores (19\%), with the Critical-Care Pain Observation Tool (6\%) and Behaviour Pain Scale $(5 \%)$ being used most frequently. Measurement of pain was considered most important for patients with burn wounds $(67 \%)$, trauma patients (64\%), postoperative patients (57\%) and those who receive end-of-life care (64\%). Barriers to use pain measurements included the patient's inability to communicate ( $82 \%)$, interference with pain assessment due to sedation (79\%), hemodynamic instability (64\%), insufficient dosages of analgesics $(60 \%)$ and the unavailability of a standard pain scoring system (51\%). In addition, guidelines for management of sedation and analgesics from the Netherlands Association for Intensive Care (NVIC) had been read by only $20 \%$ of the respondents. Factors that were mentioned to be useful in contributing to an improvement in pain assessment and effective pain control included adequate analgesic dosage (87\%), utilization of protocols and directives $(86 \%)$, enthusiastic and motivated personnel (81\%) and the utilization of standardized pain measurement tools.

Conclusion Most Dutch ICUs measure pain frequently (94\%) in patients who are able to communicate. However, in the group of patients who cannot communicate only $19 \%$ of the Dutch ICUs use a standardized pain score. This finding applied to both academic and nonacademic ICUs, which suggests that efforts should be put into implementing pain measures in Dutch ICUs.

\section{P333}

Efficiency estimation of intrapleural and thoracic paravertebral block in combination with general anesthesia at thoracoscopic interventions

J Sabirov, A Khadjibaev, V Sharipova

Republican Reseach Centre of Emegency Medicine, Tashkent, Uzbekistan Critical Care 2012, 16(Suppl 1):P333 (doi: 10.1186/cc10940)

Introduction Chest injuries and traumas have become one of the most common reasons for admitting patients to emergency surgical hospitals in recent years.

Methods Ninety patients admitted to the RRCEM urgently with chest traumatic injuries have been examined. They were divided into two groups against the applied method of anesthesia. First (control) group (47 patients, $38.5 \pm 2.4$ years): IPA was done before the induction of anesthesia into the second intercostal space from the damaged side with bupivakain at a dose of 75 to $100 \mathrm{mg}$. Analgesic component maintained by the abovementioned IPA and phentanyl bolus dosing. The second group (43 patients, $36.8 \pm 5.4$ years): one-sided TPVB maintained before the induction at ThIV, ThVII levels $0.5 \%-5 \mathrm{ml}(25 \mathrm{mg})$ bupivakain dosing (at the average total 75 to $100 \mathrm{mg}$ ) with posterior paravertebral area catheterization. Analgesic component maintained by paravertebral analgesia and phentanyl bolus dosing.

Results The differences in hemodynamics indexes appeared at the traumatic moment of operation. In the group using IPA, medium hypertension with $A B P$ rise in $25.5 \%$, higher rate of HR in $26.1 \%$ and GPVR in $22 \%$ were observed and were followed by the decrease of SV on $24.6 \%$ and $\mathrm{EF}$ on $13 \%$ compared with the second group. Conducting anesthesia in the first group, hyperdynamic reactions of the systemic hemodynamics at the separate traumatic levels of operation were followed by unbalance of hemodynamic rhythms indicating insufficient prevention from surgical aggression. In the second group, as the result of development of segmental sympathetic block the indexes of ABP, HR and GPVR were not higher than normal.

Conclusion Both methods of regional anesthesia cut short pain syndrome sufficiently and safely in patients with chest injuries before an operative intervention. Introduction of the TPVB component into the anesthesia scheme of thoracoscopic operative interventions allows one to provide additional antinociceptive protection in the intraoperative period with minimal stress of central and peripheral parameters and promotes the reduction of narcotic analgesic use due to significant analgesic efficiency and neurovegetative protection.
P334

Preoperative diclofenc reduces postcraniotomy headache: a randomized, placebo-controlled trial

C Molnár, É Simon, J Gál, P Siró, Á Kazup, B Fülesdi

University of Debrecen, Hungary

Critical Care 2012, 16(Suppl 1):P334 (doi: 10.1186/cc10941)

Introduction We tested the hypothesis that $100 \mathrm{mg}$ oral preoperative diclofenac reduces postcraniotomy headache.

Methods A total of 145 patients having elective craniotonomies were randomly assigned to diclofenac or placebo. Severity of pain was assessed by an independent observer using a visual analogue scale on the day of surgery, on the first postoperative day, and on the fifth postoperative day. The total amount of analgesics administered during the first five postoperative days was converted to intramuscular morphine equivalents. Results were compared using unpaired, twotailed $t$ tests; $P<0.05$ was considered statistically significant.

Results In total, 104 patients had supratentorial and 41 had infratentorial interventions. Sixty-two patients were assigned to placebo and 83 were assigned to diclofenac. The results of VAS scores are shown in Table 1.

Table 1 (abstract P334). Results of VAS scores

\begin{tabular}{lccc}
\hline & Placebo & DICLO & P value \\
\hline Day of surgery & $4.9 \pm 3.5$ & $2.2 \pm 3.5$ & $<0.001$ \\
First postoperative day & $5.5 \pm 3.4$ & $3.7 \pm 3.5$ & $<0.01$ \\
Fifth postoperative day & $4.3 \pm 3.8$ & $2.6 \pm 2.9$ & $<0.01$ \\
\hline
\end{tabular}

The relative efficacy of diclofenac was similar in patients having supratentorial and infratentorial surgery. Diclofenac also appeared to be comparably effective in both men and women. Systemic analgesic requirements were reduced during the initial five postoperative days in patients assigned to diclofenac (intramuscular morphine equivalents: placebo $=5.3 \pm 4.3 \mathrm{mg}$ vs. diclofenac $=3.6 \pm 3.3 \mathrm{mg}$ ).

Conclusion Preoperatively diclofenac reduces postcraniotomy headache compared to placebo, and reduces postoperative analgesic requirements.

\section{P335}

Long-term adverse neuropsychological functioning in children who survived meningococcal septic shock: is there a relationship with sedation and analgesia during paediatric ICU admission? HL Van Zellem', E Utens', SN De Wildt', WC Hop ${ }^{2}$, NJ Vet', KF Joosten'1, C Buysse

'Erasmus MC - Sophia Children's Hospital, Rotterdam, the Netherlands; 'Erasmus MC, Rotterdam, the Netherlands

Critical Care 2012, 16(Suppl 1):P335 (doi: 10.1186/cc10942)

Introduction Our objective was to evaluate the association between the use of sedative and analgesic agents during paediatric intensive care unit (PICU) treatment and long-term neuropsychological outcome in children who survived meningococcal septic shock (MSS).

Methods This study is part of a medical and psychological followup study of all consecutive MSS survivors requiring PICU treatment between 1988 and 2001 at the Erasmus MC - Sophia Children's Hospital, a tertiary-care university hospital. This follow-up study revealed that MSS survivors showed long-term (at least 4 years after PICU admission) impairments on several domains of neuropsychological functioning. Severity of illness was no significant predictor of adverse neuropsychological outcome. The use (type, number and dose) of sedatives and analgesics was retrospectively evaluated.

Results The study population consisted of 77 patients (52\% male $(n=40)$, median age 25 months at time of PICU admission). In 45 patients (58\%) one or more analgesic and/or sedative drugs were administered during PICU admission. Benzodiazepines were the most commonly used drugs $(n=39 ; 51 \%)$, followed by opioids $(n=23 ; 30 \%)$. In total 15 different kinds of analgesic or sedative drugs were given. There was a statistically significant correlation between the use of opioids (both as continuous (cumulative dose) and dichotomous variable) 
and adverse outcome on multiple domains of neuropsychological functioning (full-scale IQ $(P=0.02 ; Z=-2.28)$, verbal IQ $(P=0.02$; $Z=-2.32)$, verbal reasoning $(P=0.02 ; Z=-2.34)$, social comprehension $(P=0.01 ; Z=-2.56)$, visual-motor integration $(P=0.03 ; Z=-2.17))$. After univariate analysis, correcting for socioeconomic status, age at followup and severity of illness, these correlations remained significant. Conclusion The use of opioids during PICU admission was significantly associated with long-term adverse neuropsychological outcome in MSS survivors.

P336

Delirium could be an indicator of sepsis in patients under 65 years old with urinary tract infections

U Yamada, K Yokota, D Ohta, K Furukawa

St Luke's International Hospital, Tokyo, Japan

Critical Care 2012, 16(Suppl 1):P336 (doi: 10.1186/cc10943)

Introduction Delirium, known as sepsis-associated encephalopathy, is a frequent complication of sepsis and may be an independent predictor of mortality of septic patients [1]. A recent study reported delirium could be a predictor or an early marker of sepsis in CABG patients [2]. Urinary tract infection (UTI) often complicates sepsis and delirium; however, relations between delirium and sepsis in UTI patients have not been well investigated. We assessed the relationship between delirium and sepsis in patients with UTI.

Methods This study was conducted at St Luke's International Hospital in Tokyo, Japan between January 2009 and October 2011. UTI and sepsis were diagnosed based on positive bacterial cultures and clinical symptoms. Delirium was screened with the Delirium Screening Tool (the 11 -item questionnaire, sensitivity $98 \%$ and specificity $76 \%$ ) by trained physicians and nurses. Medical records of patients were reviewed to collect information including age, sex and complications. The association between possible risk factors and delirium was analyzed by chi-squared tests and $t$ tests. Statistical analysis was performed using SPSS software version 15.0J.

Results Of all 1,727 UTI patients, 905 were men and the mean age was $73.65 \pm 14.1$. In total, 425 patients (24.6\%) became delirious, and 247 patients $(14.3 \%)$ had sepsis. There was no significant association between sepsis and delirium $(P=0.051)$. However, in the younger population (age $<65$ ) delirium occurred significantly more frequently in septic patients than in nonseptic patients $(22.9 \%$ vs. $10 \%, P<0.001)$.

Conclusion Among UTI patients, sepsis may increase the complication of delirium. Especially in patients under 65 years old with UTI, delirium symptoms can be a marker for complication of sepsis. In contrast, delirium of patients aged 65 or over could be associated with not only sepsis but also other factors such as dementia, aging and UTI itself.

References

1. Ebersoldt M, Sharshar T, Annane D: Sepsis-associated delirium. Intensive Care Med 2007, 33:941-950.

2. Martin BJ, Buth KJ, Arora RC, Baskett RJ: Delirium as a predictor of sepsis in post-coronary artery bypass grafting patients: a retrospective cohort study. Crit Care 2010, 14:R171.

P337

Delirium screening in critically ill patients: a systematic review and meta-analysis

A Serpa Neto', AP Nassar Júnior², SO Cardoso', JA Manetta', VG Pereira', DC Esposito', MC Damasceno', AJ Slooter ${ }^{3}$

'ABC Medican School, Santo André, Brazil; '2São Camilo Hospital, São Paulo,

Brazil; ${ }^{3}$ University Medical Center Utrecht, the Netherlands

Critical Care 2012, 16(Suppl 1):P337 (doi: 10.1186/cc10944)

Introduction Despite its frequency and impact, delirium in critically ill patients is poorly recognized. Our aim was to systematically review the accuracy of delirium screening instruments in critically ill patients.

Methods Systematic review and meta-analysis of publications between 1966 and 2011. The Medline and Embase databases were searched for studies on delirium in critically ill patients in ICUs, surgical wards or emergency rooms. The delirium screening tool had to be feasible in a clinical setting for use by a nonexpert. As the gold standard, delirium

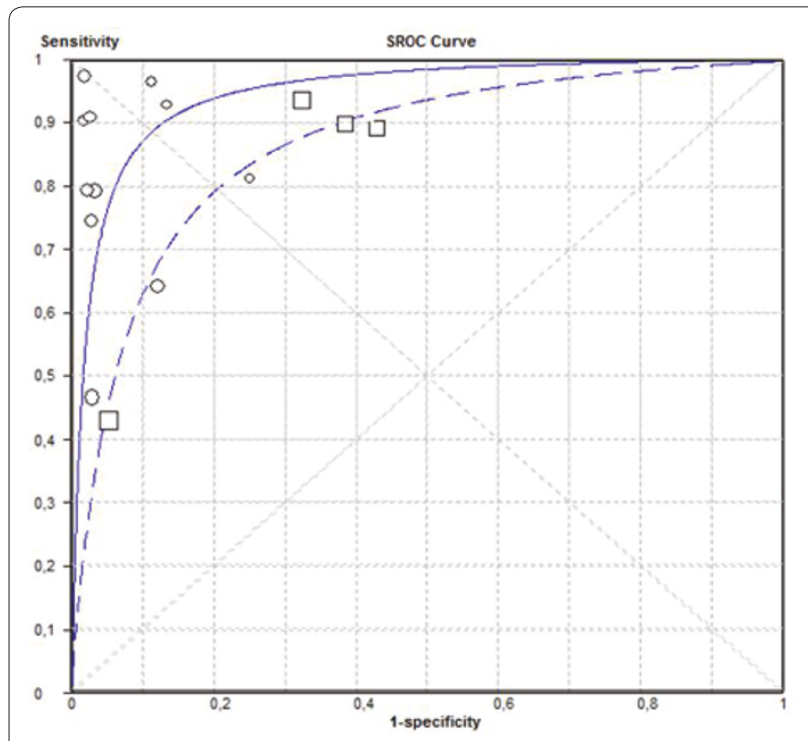

Figure 1 (abstract P337). ROC curve: CAM-ICU (solid line and circles) and ICDSC (dashed line and squares).

had to be diagnosed based on appropriate criteria by a delirium expert. The outcomes assessed were: sensitivity, specificity, likelihood ratios and summary receiver-operating characteristic (ROC) curves.

Results Fifteen studies covering 1,404 participants and five screening tools were included in the systematic review. The pooled sensitivities and specificities for CAM-ICU for detection of delirium in critically ill patients were $76.0 \%$ and $95.7 \%$ and for ICDSC were $74.4 \%$ and $75.2 \%$, respectively. All but one study was performed in a research setting, and that one study suggested that, with routine use of CAM-ICU, one-half of the patients with delirium were not detected. See Figure 1.

Conclusion The CAM-ICU was the most specific bedside tool for assessment of delirium in critically ill patients. However, there was significant heterogeneity of the results. These findings were largely obtained in research settings, and the low sensitivity of the CAM-ICU in routine, daily practice may limit its use as a screening test.

\section{P338}

Electroencephalography-based monitoring of delirium in the ICU: what are the opportunities?

AW Van der Kooi, FS Leijten, RJ Van der Wekken, AJ Slooter

University Medical Center Utrecht, the Netherlands

Critical Care 2012, 16(Suppl 1):P338 (doi: 10.1186/cc10945)

Introduction Up to $80 \%$ of patients experience delirium during their ICU stay $[1,2]$. The most sensitive screenings tool for delirium in a research setting is the Confusion Assessment Method for the ICU (CAMICU), but the low sensitivity of the CAM-ICU in daily practice (47\%) hampers early detection of delirium and thereby delays treatment $[3,4]$. Therefore, there is a need for an objective tool for continuous delirium monitoring. Diagnosis of delirium can also be conducted using electroencephalography (EEG) [5]. EEG with a limited number of electrodes and automatic processing may be a more sensitive approach for delirium monitoring. The aim of this systematic review is to explore opportunities for automatic detection of delirium by summarizing EEG characteristics of delirium.

Methods A systematic literature search was conducted in Embase and Medline. Articles concerning quantitative EEG and delirium were included. Per article, the differences between delirious and nondelirious subjects in EEG characteristics were noted.

Results Fourteen studies were included, which were predominantly conducted in older patients. The relative power of the theta frequency band was most often and without exception significantly different (7/14 studies) in delirious subjects. Other frequently measured parameters 
were the relative power of the delta and alpha frequency band and the peak frequency. None of these studies addressed the optimal electrode deviation or the question of how to distinguish sleep from delirium.

Conclusion Given the feasibility for continuous EEG monitoring in ICU, EEG delirium monitoring in ICU patients seems to be promising.

References

1. Ely EW, et al:. JAMA 2004, 291:1753-1762.

2. Girard TD, et al:: Lancet 2008, 371:126-134.

3. van Eijk MM, et al:: Am J Respir Crit Care Med 2011, 184:340-344.

4. van Eijk MMJ, et al: Crit Care Med 2009, 37:1881-1885.

5. Romano J, et al: Arch Neurol Psychiatry 1944, 51:356-377.

\section{P339}

Performance of SAPS 3 in predicting delirium among critically ill patients

T Cosentino, J Biatto, I Souza, M Dutra, L Ilnicki, P Martins, G Schettino,

F Machado

Hospital Sírio-Libanês, São Paulo, Brazil

Critical Care 2012, 16(Suppl 1):P339 (doi: 10.1186/cc10946)

Introduction Delirium is a common complication in critically ill patients, occurring in up to $80 \%$ of patients on mechanical ventilation [1] Recent studies showed that sicker patients at ICU admission, assessed by severity scores, are more susceptible to developing delirium [2]. To further evaluate this hypothesis, we undertook this study to assess the performance of SAPS 3 in predicting delirium, among adult patients admitted to a general ICU.

Methods This was a prospective observational cohort study performed between June 2010 and June 2011, in a 26-bed ICU at Hospital SírioLibanês, São Paulo, Brazil. All consecutive adult patients admitted to the ICU were included. Patients with a previous diagnosis of advanced dementia and those with acute neurological disease (Glasgow $<13$ ) were excluded. The evaluation of delirium was performed using the CAM-ICU during routine bedside rounds in the morning. Discrimination and calibration of SAPS 3 in predicting delirium were assessed by the area under the receiver operating curve (AUR ROC) and the goodness of fit (GoF) test, respectively. Secondary outcomes were hospital mortality and lengths of stay among patients with delirium.

Results A total of 225 patients were included. The incidence of delirium was $24 \%$. Patients who develop delirium during the ICU stay were older (OR 1.04, 1.02 to 1.07) and more likely to have a previous diagnosis of hypertension (OR 2.36, 1.24 to 4.52 ). The SAPS 3 (OR 1.09, 1.06 to 1.13) score, SOFA (OR 1.23, 1.09 to 1.39) score, and mechanical ventilation requirement (OR $3.6 ; 1.35$ to 9.60 ) were higher among patients with delirium. These patients had longer ICU and hospital length of stay, and a higher crude mortality rate (24.07 vs. $7.02 \%)$. In a multivariate analysis, age (OR 1.03, 1.00 to 1.05), use of mechanical ventilation (OR 3.91, 1.22 to 12.96) and SAPS 3 score (OR 1.08, 1.04 to 1.12 ) were independently associated with delirium. SAPS 3 performed well in predicting delirium with an AUR ROC of 0.785 ( 0.714 to 0.856 , best cut-off value $\geq 54$ points) and a GoF of 0.175 .

Conclusion We found that SAPS 3 was a good parameter for predicting delirium during the ICU stay. Future studies are needed to confirm our results in a larger and different patient sampling.

References

1. Ely EW, et al.: JAMA 2001, 286:2703-2710.

2. Van Rompaey B, et al:: Crit Care 2009, 13:R77.

P340

Incidence of delirium and inadequacy of the clinical diagnosis in

patients in intensive care

A Okada, R Azevedo, F Freitas, A Bafi, M Jackiu, M Assunção, B Mazza,

F Machado

Universidade Federal de São Paulo, Brazil

Critical Care 2012, 16(Suppl 1):P340 (doi: 10.1186/cc10947)

Introduction This study aims to assess the incidence, risk factors and impact of delirium on outcome and to analyze the concordance between the Confusion Assessment Method for the Intensive Care (CAM-ICU) and clinical diagnosis.
Methods A prospective observational study in a university hospital including patients over 18 years old, in the first 48 hours of ICU admission, with an expected ICU stay of at least 72 hours and signed informed consent. Pregnancy, cognitive impairment prior to admission, hepatic encephalopathy, Glasgow Coma Scale $\leq 9$, active psychiatric illness, need for sedation or neuromuscular blockade, aphasia, foreign language, deafness and brain death were exclusion criteria. CAM-ICU was applied and doctors and nurses asked about the presence of delirium. Demographic data, SOFA score, mechanical ventilation and drugs used were determined. Patients were followed for 14 days or until discharge from the ICU. The agreement between CAM-ICU and clinical diagnosis was assessed using Cohen's kappa statistic (к). Risk factors were assessed by a multivariate regression model.

Results In the 119 patients included, the incidence of delirium was $24.4 \%$ (29 patients) and time to development of delirium was $68.3 \pm 63.6$ hours. The agreement between clinical diagnoses and CAMICU was better for medical residents (Table 1). Patients with delirium had a longer ICU $(10.83 \pm 15.08$ and $4.98 \pm 9.57, P=0.015)$ and hospital $(36.93 \pm 31.33$ and $19.10 \pm 19.48, P=0.0004)$ length of stay, higher ICU mortality $(13.79 \%$ and $2.22 \%, \mathrm{OR}=7.04$ (1.22 to 40.7$))$ and hospital mortality $(27.6 \%$ and $6.66 \%, \mathrm{OR}=5.33(1.67$ to 17.04$))$ than patients without delirium. Risk factors were: mechanical ventilation $(P=0.018$, $\mathrm{OR}=3.09(1.21$ to 7.86$))$ and APACHE II score greater than $8.5(P=0.011$, $\mathrm{OR}=5.35$ (1.48 to 19.43$)$ ).

Table 1 (abstract P340). $\mathrm{K}$ values

\begin{tabular}{lcc}
\hline Health provider & Delirium & Hypoactive \\
\hline Attending physicians & 0.530 & 0.019 \\
Medical residents & 0.615 & 0.018 \\
Nurses & 0.588 & 0.025 \\
\hline
\end{tabular}

Conclusion Delirium had a higher incidence in intensive care patients and was related to longer hospital stay and higher mortality. Specific tests should be used for diagnosis, since the clinical suspicion has low sensitivity, especially in cases of hypoactive delirium and among attending physicians.

Reference

1. Ely EW, et al:: Crit Care Med 2001, 29:1370-1379.

\section{P341}

Investigation into detection and treatment rates of hyperactive and hypoactive delirium in the ICU setting

S Kudsk-Iversen, J Wong, H Kingston, L Poole

The Royal Liverpool University Hospital, Liverpool, UK

Critical Care 2012, 16(Suppl 1):P341 (doi: 10.1186/cc10948)

Introduction We aimed to investigate the link between the type of delirium (that is, hyperactive or hypoactive), its detection by the day staff and the subsequent treatment. The morbidity related to delirium is well known to critical care medical staff; however, some findings suggest insufficient and inconsistent recognition and management of delirium [1]. Hypoactive delirium, despite being more common in the ICU setting, often goes undetected and undertreated due to its withdrawn and drowsy presentation [2].

Methods A prospective cohort study over 8 weeks in a 25-bed ICU setting. Daily CAM-ICU assessments were done by three trained doctors. It was noted whether the ICU team had assessed the individual patient for delirium. If the patient was delirious, the team was informed and their management was noted. Eligible patients had to have a RASS score above -4 and be able to comply with the assessment. The Fisher's exact test was used to calculate statistical significance of detection and treatment.

Results A total of 139 patients were included, providing a total of 507 patient-days. On 32 occasions (6\%) the patient assessed was found to be delirious. Twelve patients in ITU (19\%) and nine in HDU (9\%) were delirious at least once. Of the 32 cases of delirium, 53\% were hyperactive. Seventy-six percent of the hyperactive and $27 \%$ of the hypoactive cases had been detected by the day team $(P=0.0118)$. Once 
Table 1 (abstract P341). Hyperactive and hypoactive cases and their detection rate

\begin{tabular}{lcc}
\hline & $\begin{array}{c}\text { Number of } \\
\text { hyperactive (\%) }\end{array}$ & $\begin{array}{c}\text { Number of } \\
\text { hypoactive (\%) }\end{array}$ \\
\hline Detected & $13(76)$ & $4(27)$ \\
Not detected & $4(24)$ & $11(73)$ \\
\hline
\end{tabular}

Table 2 (abstract P341). Hyperactive and hypoactive cases and their treatment rate

\begin{tabular}{lcc}
\hline & $\begin{array}{c}\text { Number of } \\
\text { hyperactive (\%) }\end{array}$ & $\begin{array}{c}\text { Number of } \\
\text { hypoactive (\%) }\end{array}$ \\
\hline Treated & $13(76)$ & $3(20)$ \\
Not treated & $4(24)$ & $12(80)$ \\
\hline
\end{tabular}

the delirium was recognised, $76 \%$ of the hyperactive and $20 \%$ of the hypoactive cases were started on targeted treatment $(P=0.0038)$. See Tables 1 and 2 .

Conclusion Although the study had a higher rate of hyperactive delirium compared to otherwise available research, the findings confirmed that a significant proportion of hypoactive delirium goes undetected and remains largely undertreated.

References

1. Gong Z, et al:: Chin J Traumatol 2009, 12:328-333.

2. Spronk P, et al.: Intensive Care Med 2009, 35:1276-1280.

P342

Memories and post-traumatic stress-related symptoms in older, post-cardiac surgery patients: substudy of an RCT

N Hammond, F Bass, Y Shehabi

Prince of Wales Hospital, Randwick, Australia

Critical Care 2012, 16(Suppl 1):P342 (doi: 10.1186/cc10949)

Introduction The majority of ICU survivors display little evidence of severe psychological sequelae. However, there is evidence of posttraumatic stress disorder (PTSD)-related symptoms such as anxiety, depression, panic attacks, distressing memories and flashbacks within the first 3 months post ICU discharge [1,2]. This substudy of the DEXCOM trial 3 (randomised controlled trial of neurobehavioural effects of dexmedetomidine or morphine for sedation and analgesia in patients 60 years or older, undergoing coronary artery bypass grafting and/or valve replacement) aims to explore any negative memories of the ICU and development of PTSD-related symptoms between treatment groups of patients at high risk of developing delirium.

Methods At 8 weeks post ICU discharge, patients completed three assessment tools, by mail or telephone. Tools used were Depression Anxiety Stress Scale, ICU memory assessment tool and impact of events scale.

Results A total of 153 patients completed the substudy; 72 patients in the $[M]$ orphine group and 81 in the [D]exmedetomidine group. The mean age (years) in the M group was 72 (SD 5) and in the D group 69 (SD 6), with $71 \%(n=51)$ males in the M group and $84 \%(n=68)$ in the D group. The mean ICU hours for M and D were 58 (SD 40) and 48 (SD 32) respectively. No significant differences of memories or PTSDrelated symptoms between the two treatment groups, for each of the three assessment tools, were found. From the ICU memory tool, 21\% $(n=15 / 70)$ of M group patients and $15 \%(n=12 / 81)$ of the D patients remember being in the ICU. Just over one-half of the patients in both groups did not remember all of their ICU stay with clarity (M group: $54 \%, n=39 / 72$; D group: $51 \%, n=40 / 78)$. Furthermore, $23 \%(n=15 / 64)$ of $M$ patients and $14 \%(n=10 / 73)$ of $D$ patients had intrusive memories whilst in the ICU.

Conclusion Patients undergoing cardiac surgery with ICU stay do not have clear memories of this episode. A small number had intrusive memories, which are more common in $\mathrm{M}$ patients. The study used a convenience sample so was not powered to detect a significant difference. No differences in factual or delusional memories or PTSDrelated symptoms between the treatment groups were found. These data could be the basis of a sample size calculation for a larger study. References

1. Schelling G, et al:: Crit Care Med 1998, 26:651-659.

2. Stoll C, et al.: J Thorac Cardiovasc Surg 2000, 120:505-512.

3. Shehabi Y, et al.: Anesthesiology 2009, 111:1074-1083.

P343

Using tramadol to monitor hepatic drug metabolism in the critically ill

KLane1, JJ Dixon', D McKeown², A Johnston², I MacPhee1, BJ Philips'

'Acute Kidney Injury Research Group, St George's, University of London, UK; ${ }^{2}$ Analytical Services International Ltd, St George's, University of London, UK Critical Care 2012, 16(Suppl 1):P343 (doi: 10.1186/cc10950)

Introduction Previously, we have demonstrated significant inhibition of hepatic drug metabolism by the enzymes cytochrome P450 (CYP) $3 \mathrm{~A} 4$ and $3 \mathrm{~A} 5$ in acute kidney injury (AKI) using midazolam as a probe drug $[1,2]$. We are now developing the use of tramadol as a probe drug to test the hypothesis that CYP2D6 function is also inhibited by AKI in critical illness. In this study we sought to determine whether a single timepoint tramadol concentration could be identified as a reliable surrogate for measurement of a full area under the concentration time curve after intravenous administration in adults.

Methods We conducted a study of 10 critically ill patients in our hospital's general critical care unit. Tramadol $10 \mathrm{mg}$ was given intravenously, and serum was taken at $0.5,1,2,3,4$ and 8 hours for determination of concentrations of tramadol ([tramadol]) and its two main metabolites. Inclusion criteria: age $>18$ years, predicted ICU stay $>48$ hours. Exclusion criteria: recent receipt of tramadol or major CYP2D6 inhibitors, hepatic failure, pregnancy/breastfeeding.

Results There was a strong correlation between the area under the curve (AUC) of the [tramadol]-time graph and $t=4$ hours [tramadol], $P<0.0001, r=0.983$. See Figure 1. The [tramadol] at other timepoints correlated less strongly with the AUC. The mean [tramadol] at 4 hours was $29.7 \mathrm{ng} / \mathrm{ml}$ (24.3 to 35.1) and the mean AUC was $257 \mathrm{ng} / \mathrm{hour} / \mathrm{ml}$ (211 to 303). Analysis of tramadol metabolites confirmed that CYP2D6 was predominantly responsible for tramadol metabolism.

Conclusion A single blood sample, taken 4 hours post-intravenous tramadol injection, reliably predicts integral tramadol exposure in critically ill adults and may be useful for assessing CYP2D6 function.

A larger study of the influences of AKI and CYP genotype on hepatic drug metabolism in the critically ill is underway.

References

1. Kirwan CJ, et al:: Intensive Care Med 2009, 35:1271-1275.

2. Kirwan CJ, et al: Intensive Care Med 2012, 38:76-84.

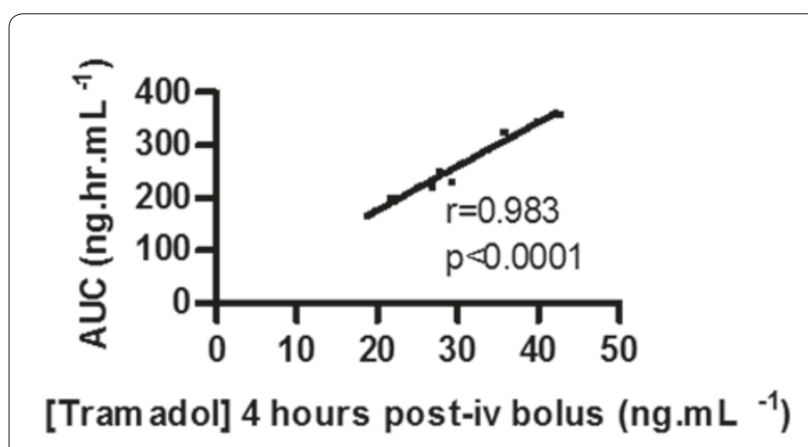

Figure 1 (abstract P343). Correlation of [tramadol] at $t=4$ hours and AUC [tramadol]-time graph. iv, intravenous. 
P344

Data mining techniques for predicting acute kidney injury after elective cardiac surgery

J Van Eyck, J Ramon, F Guiza, G Meyfroidt, M Bruynooghe,

G Van den Berghe

K.U. Leuven, Heverlee, Belgium

Critical Care 2012, 16(Suppl 1):P344 (doi: 10.1186/cc10951)

Introduction Development of acute kidney injury (AKI) during the postoperative period is associated with increases in both morbidity and mortality. The aim of this study is to develop a statistical model capable of predicting the occurrence of AKI in patients after elective cardiac surgery.

Methods A total of 810 adult ( $>18$ years) elective cardiac surgery patients, admitted to the surgical ICU of the University Hospital of Leuven between 18 January 2007 and 8 January 2009, were retrospectively selected for this study. Patients with an ICU stay of less than 24 hours, as well as patients suffering from chronic kidney disease, were excluded. Relevant patient records were extracted from an electronic database system and analyzed using data mining techniques [1]. The main advantage of these techniques is that they are capable of automatically selecting the variables that are relevant to a particular problem. Using such a data mining algorithm, predictive models were built on a development cohort of 385 patients and validated on a separate cohort of 425 patients.

Results In this study, two separate models were developed for predicting the occurrence of AKI (defined as RIFLE stage three or need for renal replacement therapy) within a week after the patient's admission. An initial model was built using only readily available admission data (including demographic information, previous treatments and preadmission values for physiological variables). This resulted in an AUC of $0.6056(95 \% \mathrm{Cl}, 0.4874$ to 0.7239$)$ on the validation cohort. The initial model was then extended by adding information on administered medication, measurements of physiological parameters and laboratory results available during the first four hours of the patient's ICU stay. This new model resulted in an AUC of $0.8339(95 \% \mathrm{Cl}, 0.7364$ to 0.9315$)$ on the validation cohort.

Conclusion In this study, we have shown that data mining techniques are a viable option for developing predictive models in a clinical setting. Furthermore, we have shown that by adding information gathered during the patient's stay, a model's performance can drastically improve compared to a model using only admission data. Thus, it might be possible to further improve existing scoring systems such as the Thakar score [2] and the simplified renal index [3].

References

1. Meyfroidt G, et al:: Best Pract Res Clin Anaesthesiol 2009, 23:127-143.

2. Thakar C, et al.: J Am Soc Nephrol 2005, 16:162-168.

3. Duminda N, et al:. JAMA 2007, 297:1801-1809.

P345

Acute kidney injury in critically ill patients with A/H1N1 pneumonitis in 2010/11

M Atkinson, A Krige, S Chukkambotla

East Lancashire Hospitals NHS Trust, Blackburn, UK

Critical Care 2012, 16(Suppl 1):P345 (doi: 10.1186/cc10952)

Introduction $\mathrm{A} / \mathrm{H} 1 \mathrm{~N} 1$ infection is a major seasonal cause of illness requiring critical care admission. A high proportion of these patients develop acute kidney injury (AKI) [1].

Methods We studied all A/H1N1-positive admissions to a district general hospital (DGH) ICU during the months of December 2010 and January 2011. The study aimed to describe the incidence of AKI using the creatinine score from the RIFLE criteria and its associations with mortality, incidence and duration of intermittent positive pressure ventilation (IPPV), length of stay in the ICU and provision of renal replacement therapy (RRT).

Results Twenty-seven patients were admitted to the ICU who tested positive for $\mathrm{A} / \mathrm{H} 1 \mathrm{~N} 1$. Fourteen $(52 \%)$ met the RIFLE criteria for AKI. Of these, three (11\%) met the RIFLE criterion for Risk $(>150 \%$ change in creatinine), three $(11 \%)$ met the criterion for Injury (>200\% change in creatinine), and eight (30\%) met the criterion for Failure $(>300 \%$ change in creatinine). Nine patients (33\% of all patients, $64 \%$ of AKI patients) received RRT. ICU mortality was three out of 14 (21\%) patients with AKI and one out of $13(8 \%)$ patients without AKI. This difference was not statistically significant. Thirteen out of $20(65 \%)$ ventilated patients developed AKI, compared with one out of seven (14\%) nonventilated patients. This difference was statistically significant $(P=0.0329)$. Excluding fatalities, the duration of IPPV was longer in patients with AKI (median 11 days, range 0 to 54 days) than in patients without AKI (median 1 day, range 0 to 20 days). This difference was statistically significant $(P<0.05)$. Excluding fatalities, the length of stay was longer in patients with AKI (median 19 days, range 10 to 68 days) than in patients without AKI (median 5 days, range 2 to 29 days). This difference was statistically significant $(P<0.02)$.

Conclusion We noted a higher incidence of AKI in critical illness associated with $\mathrm{A} / \mathrm{H} 1 \mathrm{~N} 1(52 \%)$ compared to that of a larger study [1]. AKI was associated with the incidence as well as duration of mechanical ventilation and length of stay in the ICU. The use of RRT in the current study (60\%) was much higher than in the modeling study (16\%). We found a trend towards greater mortality with AKI, although (unlike Petillä and colleagues [1]) this failed to reach significance.

Reference

1. Pettilä V, et al:: Acute kidney injury in patients with influenza A (H1N1)

2009. Intensive Care Med 2011, 37:763-767.

P346

A RIFLE score-based trigger for renal replacement therapy and survival after cardiac surgery

A Schneider, G Eastwood, S Seevanayagam, G Matalanis, R Bellomo

Austin Health, Heidelberg, Australia

Critical Care 2012, 16(Suppl 1):P346 (doi: 10.1186/cc10953)

Introduction It is controversial whether all critically ill patients with RIFLE-F class acute kidney injury (AKI) should receive renal replacement therapy (RRT). We reviewed the outcome of open-heart surgery patients with severe AKI who did not receive RRT.

Methods We identified all patients who developed AKI after cardiac surgery during a 4-year period, and obtained baseline characteristics, intraoperative details and in-hospital outcomes. We analyzed physiological and biochemical features at the time of RRT initiation or at peak creatinine if no RRT was provided.

Results We reviewed 1,504 patients. Of these, 137 (9.1\%) developed postoperative AKI with 71 meeting RIFLE-F criteria and 23 (32.4\% of RIFLE-F cases) not receiving RRT. Compared with RRT-treated RIFLE-F patients, no-RRT patients had lower APACHE III scores, less intraaortic balloon pump requirements, shorter intensive care stay and a trend toward lower mortality. At peak creatinine, their urinary output, arterial $\mathrm{pH}$ and $\mathrm{PaO} / / \mathrm{FIO}_{2}$ ratio were all significantly higher. Their serum creatinine was also higher ( 304 vs. $262 \mu \mathrm{mol} / \mathrm{l}, P=0.02$ ). Only three died in-hospital. Detailed review of cause and mode of death was consistent with non-RRT-preventable deaths. In contrast, 27 patients with RIFLE-R or RIFLE-I class received RRT. Compared with RRT-treated RIFLE-F patients, they had a trend towards a more severe presentation and a higher mortality (51.8\% vs. $29.2 \%, P=0.02)$. See Figure 1 .

Conclusion After cardiac surgery, RRT is typically applied to patients with the most severe clinical presentation irrespective of creatinine levels. A RIFLE score-based trigger for RRT is unlikely to improve patient survival.

\section{P347}

Effect of off-pump versus on-pump coronary artery bypass grafting in patients with chronic kidney disease

ME Schroeder', L Chawla', Y Zhao' ${ }^{1}$, F Lough'1, F Najam', M Seneff', JM Brennan ${ }^{3}$

'George Washington University Hospital, Washington, DC, USA; ${ }^{2}$ Duke Clinical Research Institute, Raleigh, NC, USA; ${ }^{3}$ Duke University Medical Center, Raleigh, NC, USA Critical Care 2012, 16(Suppl 1):P347 (doi: 10.1186/cc10954)

Introduction Patients with chronic kidney disease (CKD) have been largely excluded from clinical trials of off-pump coronary artery bypass 


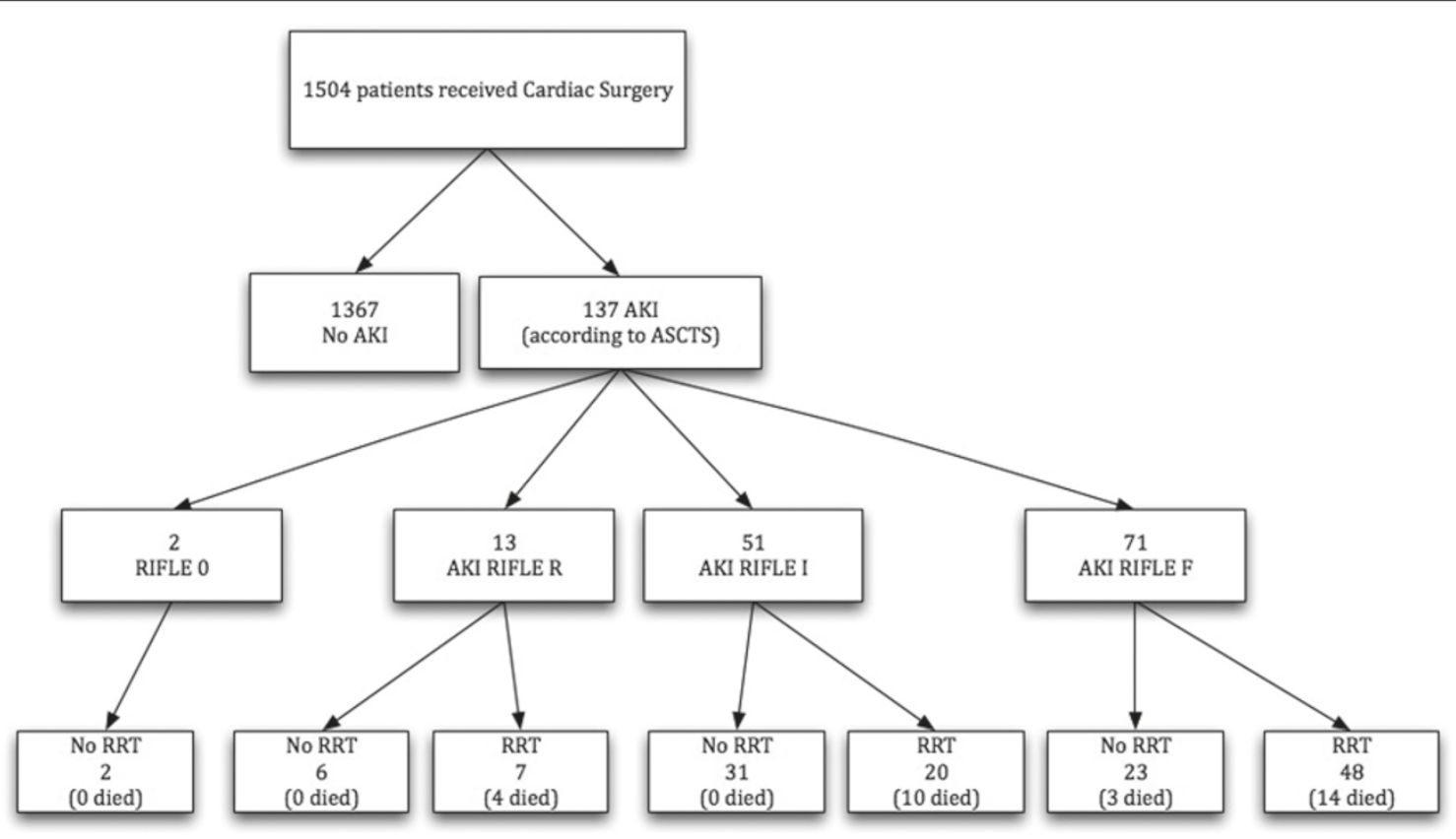

Figure 1 (abstract P346). Flow chart. ASCTS, Australian Society of Cardio Thoracic Sugery.

grafting (OPCAB). We sought to determine if the pump status affected outcomes in patients with CKD.

Methods Using a nonrandomized cohort of 742,909 nonemergent, isolated CABG cases (including 158,561 OPCAB cases) in the Society of Thoracic Surgery Database from 2004 through 2009, we evaluated the association between pump status (off-pump vs. on-pump) and in-hospital death or incidence of renal replacement therapy (RRT) across strata of preoperative renal function. We used both propensity methods and an instrumental variable (IV) approach to account for imbalances in baseline patient risk.

Results Compared with on-pump cases, off-pump cases were of similar age (65.6 vs. 64.9 years) with a similar distribution of preoperative estimated glomerular filtration rate (eGFR). In a propensity weighted analysis, OPCAB was associated with a reduction in composite inhospital death or RRT, with a progressively increased benefit among those with lower preoperative renal function (eGFR $\geq 90 \mathrm{ml} /$ minute: risk difference $=0.05$ per 100 patients (on-pump minus off-pump), $95 \%$ confidence interval $=-0.06$ to $0.16 ; 60$ to $89 \mathrm{ml} /$ minute: $0.14,0.05$ to 0.23 ; 30 to $59 \mathrm{ml} /$ minute: $0.66,0.45$ to 0.87 ; and 15 to $29 \mathrm{ml} /$ minute: $3.66,2.14$ to 5.18). A similar trend was observed for both component endpoints. However, while the IV analysis confirmed the protective effect of OPCAB on composite in-hospital death or RRT among patients with a reduced eGFR, this result was driven by an effect on RRT and not mortality.

Conclusion Patients with CKD experience less death or incidence of RRT when treated with off-pump versus on-pump CABG; however, this composite effect is driven by a reduction in incidence of RRT (not death) among low eGFR patients. Prospective trials comparing these procedures in patients with impaired preoperative renal function are warranted.

\section{P348}

Effects of renal-dose dopamine on renal tubular functions following coronary artery bypass grafting surgery

P Zeyneloglu, H Ozdemir, O Komurcu, N Bayraktar, A Sezgin, A Pirat, G Arslan

Baskent University, Ankara, Turkey

Critical Care 2012, 16(Suppl 1):P348 (doi: 10.1186/cc10955)

Introduction Cardiopulmonary bypass (CPB) is regarded as an important contributor to acute kidney injury and use of renal-dose dopamine to protect the kidneys against hypoperfusion injury following cardiac surgery remains controversial. Cystatin $\mathrm{C}$ has been described as a sensitive biomarker of early renal tubular injury. We aimed to evaluate the effect of renal-dose dopamine on renal tubular functions in patients undergoing coronary artery bypass grafting (CABG) surgery. Methods Thirty-six patients undergoing CABG surgery were prospectively randomized to receive either $2 \mu \mathrm{g} / \mathrm{kg} /$ minute dopamine infusion (Group $\mathrm{D}, n=19$ ) or saline as placebo (Group $\mathrm{P}, n=17$ ) starting from induction of anesthesia for 48 hours. Serial blood and urine samples after induction of anesthesia and 2, 12, 24, 48 hours post CPB were collected to measure serum cystatin $C$, creatinine levels and urinary $\beta_{2}$-microglobulin. Intraoperative and daily measurements of hemodynamic parameters and urine output were recorded.

Results The groups were similar in terms of physical characteristics, perioperative hemodynamic measurements, urine outputs and surgical times. Serum cystatin $C$ levels demonstrated similar increases during 12,24 and 48 hours post CPB in the dopamine and placebo groups $(P>0.05$ for all). See Table 1. No differences were detected with respect to serum creatinine and urine $\beta_{2}$-microglobulin levels between the groups ( $P>0.05$ for both). GFR was preserved equally in both groups on postoperative day $2(104.1 \pm 23.1$ vs. $101.4 \pm 35.8 \mathrm{ml} /$ minute, $P>0.05)$.

Table 1 (abstract P348). Serum cystatin C levels $(\mathrm{ng} / \mathrm{ml}$ ) of the patients

\begin{tabular}{lccc}
\hline & Group D $(n=19)$ & Group P $(n=17)$ & $P$ value \\
\hline Induction & $803 \pm 173$ & $789 \pm 285$ & 0.987 \\
2 hours CPB & $857 \pm 236$ & $861 \pm 347$ & 0.664 \\
12 hours CPB & $807 \pm 239$ & $1,132 \pm 396$ & 0.052 \\
24 hours CPB & $906 \pm 211$ & $1,158 \pm 432$ & 0.149 \\
48 hours CPB & $1,296 \pm 341$ & $1,129 \pm 350$ & 0.296 \\
\hline
\end{tabular}

Conclusion The results suggest that renal-dose dopamine does not exacerbate the severity of renal tubular injury when compared with the untreated controls during the early postoperative period of patients undergoing $C A B G$ surgery.

Reference

1. Sumeray M, et al:. J Nephrol 2001, 14:397-402. 
P349

Nurses' knowledge regarding the early identification of acute kidney injury

S Agege Lobo, RM Matheus

Faculdade de Medicina de São José do Rio Preto - FAMERP, São José do Rio

Preto, Brazil

Critical Care 2012, 16(Suppl 1):P349 (doi: 10.1186/cc10956)

Introduction The objective was to evaluate nurses' knowledge on the early identification of acute kidney injury (AKI) in an ICU, inpatient care unit, and emergency unit.

Methods This was a multicenter, prospective, longitudinal study. The study population included 216 nurses who work in the ICU, inpatient care unit, and emergency unit at six public and private hospitals. Data collection was performed from October 2010 to February 2011 using a 10-question questionnaire related to prevention, diagnosis, and treatment of AKI.

Results Data showed that $81.7 \%$ of the nurses gave correct answers regarding the association of urine volume rate in the identification of AKI; $57.2 \%$ did not know how to identify the clinical manifestations of AKI; $67.1 \%$ made a mistake by answering that the subtle increase of creatinine has no great impact on a mortality rate; $66.8 \%$ answered the question incorrectly on measures to prevent AKI; $60.4 \%$ were correct when they answered that the use of loop diuretics in the prevention of AKI is not recommended; and $92.5 \%$ said they had no knowledge of the Acute Kidney Injury Network classification.

Conclusion The results showed that most nurses do not have enough knowledge for the early identification of AKI. This highlights the importance of training programs for nurses who work at hospital units, with the purpose of developing professional competences and aptitudes regarding both prevention and detection of AKI.

\section{P350}

Neutrophil gelatinase-associated lipocalin predicts postoperative fluid overload, a potentially modifiable risk factor for mortality after cardiac surgery

M Haase', P Mertens ${ }^{1}$, M Plaß², R Bellomo ${ }^{3}$, A Haase-Fielitz'

'Otto-von-Guericke University Magdeburg, Germany; ${ }^{2}$ German Heart Center, Berlin, Germany: ${ }^{3}$ Austin Health, Melbourne, Australia

Critical Care 2012, 16(Suppl 1):P350 (doi: 10.1186/cc10957)

Introduction In most previous studies, neutrophil gelatinaseassociated lipocalin (NGAL), measured immediately following cardiac surgery, has been demonstrated to predict postoperative increases in serum creatinine and decline in urine output. In patients undergoing cardiac surgery, postoperative fluid overload is a typical complication. In this study, we investigated the early postoperative value of NGAL to predict subsequent fluid overload, a potentially modifiable risk factor in these patients.

Methods We studied 100 adult cardiac surgery patients assigned to the control arm of a randomized controlled trial. Urine and serum were sampled immediately after admission to the ICU. Urine NGAL was measured on the ARCHITECT laboratory platform (Abbott Diagnostics) and serum creatinine using an enzymatic assay. Postoperative fluid overload was defined as positive fluid balance with $>10 \%$ excess of preoperative body weight within 48 hours. An area under the curve of the receiver-operating characteristics (AUC-ROC) of 0.5 indicates the predictive ability equaling the toss of a coin and $>0.7$ of a useful biomarker.

Results Postoperative fluid overload was present in $15 \%$ of patients with a mean positive fluid balance of $12 \pm 9 \mathrm{~kg}$. Patients who survived the hospital stay had a lower positive fluid balance of $2.8 \mathrm{I}$ (25th to 75th percentiles: 1.5 to 5.5 ) compared to patients who died (23.0 I (25th to 75th percentiles: 14.5 to 24.0 )); $P=0.010$. A positive fluid balance predicted in-hospital mortality with AUC $0.94(95 \% \mathrm{Cl} 0.83$ to 0.99 ), sensitivity $100 \%$ and specificity $80 \%$ at a cut-off $>6 \mathrm{I}$. Urine NGAL predicted fluid overload (AUC-ROC 0.80 (95\% Cl 0.64 to 0.93$)$ ) and mortality (AUC-ROC 0.88 ( $95 \% \mathrm{Cl} 0.78$ to 0.97$)$ ). Serum creatinine did not predict fluid overload (AUC-ROC 0.51 (95\% CI 0.24 to 0.78$)$ ) or mortality (AUC-ROC 0.61 (95\% Cl 0.16 to 0.99$)$ ).

Conclusion Fluid overload frequently occurs during the first 48 hours after cardiac surgery and is strongly correlated with postoperative mortality. Early postoperatively measured urine NGAL is a good predictor of fluid overload and mortality whereas measurement of serum creatinine at the same time equals the toss of a coin. Early NGALguided adjustments of fluid management might reduce organ edema and potentially improve patient outcomes after cardiac surgery. Our findings should be validated in larger patient cohorts.

P351

Plasma and urine neutrophil gelatinase-associated lipocalin as markers of acute kidney injury in critically ill adults

R Matsa', E Ashley², J Osypiw², V Sharma', A Walden², L Keating²

'Oxford University Hospitals NHS Trust, Oxford, UK; ${ }^{2}$ Royal Berkshire NHS

Foundation Trust, Reading, UK

Critical Care 2012, 16(Suppl 1):P351 (doi: 10.1186/cc10958)

Introduction Acute kidney injury (AKI) has significant impact both on the morbidity and mortality in patients on the ICU. The current definition and classification of AKI [1] uses changes in both the serum creatinine and urine output. This occurs late in the evolution of AKI and so the diagnosis can be delayed. Early detection of AKI could allow earlier recognition and treatment of the condition. Neutrophil gelatinase-associated lipocalin (NGAL) is a $25-\mathrm{kDa}$ protein produced in response to inflammation, infection and kidney injury [2] and is found in blood and urine samples obtained from patients soon after the onset of AKI [3]. Earlier studies have shown that NGAL can be detected as early as 2 hours following AKI [2]. The predictive value of NGAL in the ICU may help the earlier recognition of AKI. The aim of the study is to determine whether plasma and/or urine NGAL levels can predict the earlier incidence of AKI (as defined by RIFLE criteria) in critically ill patients.

Methods This single-centre prospective observational study is currently recruiting 200 consecutive adult patients with no AKI on presentation to the ICU. Serial samples of plasma and urine are collected on all patients included in the study at 0 hours and then every 24 hours in the ICU stay up to 72 hours and assayed for NGAL using a turbidimetric assay on the standardised automated analyser.

Results Results on the first 27 patients are currently available. The predictive performance of pNGAL at admission for AKI (24 hours prior to creatinine-based (RIFLE) diagnosis) was good (AUC-ROC 0.8 (95\% $\mathrm{Cl} 0.88$ to 1.03$)$ ). The predictive performance of UNGAL at admission for the occurrence of AKI (24 hours prior to creatinine-based (RIFLE) diagnosis) (AUC-ROC 0.77 ( $95 \% \mathrm{Cl} 0.47$ to 1.07)) was fair. See Table 1.

Table 1 (abstract P351). Sensitivity and specificity of pNGAL (time '0) to diagnose AKI occurrence at 24 hours

\begin{tabular}{lcc}
\hline Cut-off $(\mathbf{n g} / \mathbf{m l})$ & Sensitivity $(\mathbf{9 5 \%} \mathrm{Cl})$ & Specificity $(\mathbf{9 5 \%} \mathrm{Cl})$ \\
\hline$<270.0$ & $82.6(61.2$ to 95$)$ & $100(37.6$ to 100$)$ \\
$<316.5$ & $86.9(66.4$ to 97.2$)$ & $100(37.6$ to 100$)$ \\
$<381.0$ & $91.3(71.9$ to 98.9$)$ & $100(37.6$ to 100$)$ \\
\hline
\end{tabular}

Conclusion Early results on pNGAL suggest that it could be an independent predictor of AKI in an unselected population of critically ill adults. Further results are awaited.

References

1. Bellomo R, et al:: Crit Care 2004, 8:R204-R212.

2. Mishra J, et al:: Lancet 2005, 365:1231-1238.

3. Mishra J, et al.: Pediatr Nephrol 2006, 21:856-863.

\section{P352}

Plasma and urine neutrophil gelatinase-associated lipocalin in septic and nonseptic ICU patients

CS Vrettou', S Kokkoris², K Apostolou², M Parisi², E Haritatos²

S Dimopoulos ${ }^{2}$, S Nanas'

'National and Kapodistrian University of Athens, Greece; ${ }^{2}$ Evaggelismos

Hospital, Athens, Greece

Critical Care 2012, 16(Suppl 1):P352 (doi: 10.1186/cc10959)

Introduction In this prospective cohort study we investigate how admission plasma and urine neutrophil gelatinase-associated lipocalin 
(pNGAL and uNGAL) levels are affected by the presence of sepsis in a general ICU population. These novel biomarkers are currently being evaluated for acute kidney injury (AKI) prediction. However, they are also increased in sepsis, which can be a confounding factor regarding their specificity for AKI $[1,2]$.

Methods Ninety-six patients consecutively admitted to the ICU were included in the study. Exclusion criteria were chronic renal failure, AKI prior to ICU admission, brain death, pregnancy, age $<18$ years and predicted ICU stay less than 48 hours. Patients' demographic characteristics, APACHE II and SOFA score, existing comorbidities, primary reason for admission to intensive care, pNGAL, UNGAL, white cell count and C-reactive protein levels were recorded on admission while the RIFLE score and sepsis status were recorded until day 7 post admission. The Mann-Whitney $U$ test was used to compare pNGAL and uNGAL levels in septic and nonseptic patients.

Results Out of 96 patients included, 56 were male, 12 had AKI and 30 had sepsis on admission. The mean age was $55.5 \pm 19.6$ years, the mean APACHE II score was $14.8 \pm 5.6$ and the mean admission SOFA score was $6.6 \pm 2.9$. There were 43 medical admissions, 17 elective surgical, and 36 emergency surgical including trauma.

Both pNGAL and UNGAL were higher in patients with AKI on admission $(P<0.001)$. Their levels were also found to be higher in septic compared with nonseptic patients (septic pNGAL $=153.13 \pm 144.86$ vs. nonseptic pNGAL $=102.45 \pm 95.65, P=0.076$; septic uNGAL $=306.66 \pm 532.88$ vs nonseptic UNGAL $=123.41 \pm 354.07, P=0.002$ ). When patients with AKI as well as patients who developed AKI within the first 7 days post admission were excluded from the analysis, higher UNGAL and pNGAL values in the group of septic patients were not significant at the level of $5 \%$. The estimated sample size for significance $5 \%$ and power $80 \%$ is 74 for uNGAL $(2,200$ for pNGAL). Moreover pNGAL and UNGAL had a similar area under the ROC curve (0.773 and 0.779 respectively) for predicting AKI in septic patients.

Conclusion Both biomarkers are increased in the case of sepsis in our population. Septic AKI affecting UNGAL more than pNGAL could explain the smaller $P$ value for UNGAL in the group of patients with sepsis.

References

1. Int Care Med 2010, 36:1333-1340,

2. Am J Respir Crit Care Med 2011, 183:907-914.

P353

Urinary neutrophil gelatinase-associated lipocalin as an early marker of acute kidney injury complicating circulatory shock

H Sherif, M Foda, M Shehata, A Ibrahim

Faculty of Medicine, Cairo University, Cairo, Egypt

Critical Care 2012, 16(Suppl 1):P353 (doi: 10.1186/cc10960)

Introduction We evaluated the novel urinary neutrophil gelatinaseassociated lipocalin (NGAL) as an early biomarker that rapidly releases in acute kidney injury (AKI) complicating circulatory shock.

Methods We measured the urinary NGAL level from collected urine in 45 patients with circulatory shock, during the first 6 hours and after 24 hours. Eleven patients responded to fluid infusion \pm vasopressors and were considered as a separate control group.

Results The estimated urinary NGAL at day 1 and day 2 post circulatory shock could predict AKI presented at days 2 and 3 and days 3 and 4 $(P<0.05, P<0.001$ and $P<0.001, P<0.001)$ respectively. Apart from all conventional kidney parameters and biomarkers, significant inverse correlations could be detected only between urinary NGAL at days 1 and 2 with the corresponding urine output in the patient group ( $r=-0.51$ and $-0.64, P<0.05$ and $P<0.001$, respectively). The best cutoff value of urinary NGAL at day 1 was $26 \mathrm{ng} / \mathrm{ml}$, for which sensitivity was $62 \%$ and $69 \%$ and specificity was $75 \%$ and $80 \%$ for prediction of AKI presented at days 2 and 3, respectively. While the best cut-off at day 2 was $29 \mathrm{ng} / \mathrm{ml}$, for which sensitivity was $70 \%$ and $74 \%$ and specificity was $90 \%$ and $80 \%$ for prediction of AKI presented at days 3 and 4 , respectively. Urinary NGAL at day 2 could significantly predict mortality complicating AKI rather than at day $1(P<0.05)$.

Conclusion Urinary NGAL seems to be a potential early and sensitive biomarker for AKI and a persistently increased level at day 2 can predict mortality following circulatory shock.
P354

Additive value to clinical judgement of blood neutrophil gelatinaseassociated lipocalin in diagnosis of acute kidney injury and prediction of mortality in patients hospitalized from the emergency department

L Magrini', B De Berardinis', R Marino', G Gagliano', E Ferri', P Moscatelli²,

P Ballarino' ${ }^{2}$ B Gliozzo ${ }^{3}$, G Carpinteri', S Di Somma'

'S. Andrea Hospital 'Sapienza' University, Rome, Italy;' 'S. Martino Hospital,

Genoa, Italy; 'S. Elia Hospital, Catania, Italy

Critical Care 2012, 16(Suppl 1):P354 (doi: 10.1186/cc10961)

Introduction Acute kidney injury (AKI) is a common and difficult to diagnose complication among hospitalized patients with increasing incidence.

Methods A total of 665 (357 M:308 F; mean age $74 \pm 14$ years) emergency department (ED) patients designated for hospitalization were included in a multicenter prospective study to evaluate the utility of blood neutrophil gelatinase-associated lipocalin (NGAL) assessments as an aid in the early risk evaluation for AKI. NGAL and serum creatinine $(\mathrm{s} C \mathrm{Cr}$ ) were determined at ED presentation (T0), 6, 12, 24 and 72 hours after hospitalization. The clinical certainty of AKI was determined by ED physician (Ph) while blinded to NGAL results.

Results Preliminary diagnosis of AKI by the ED Ph occurred in 218/665 patients (33\%). Final adjudicated AKI clinical diagnosis was confirmed in $49 / 665$ patients (7\%). The AUC for NGAL alone in the final diagnosis of AKI was $0.80( \pm 0.07)$. When NGAL was added to the ED Ph's clinical judgement in a logistic model, the AUC was increased to 0.89 ( \pm 0.06$)$. The AUCs for the additional endpoints are shown in Table 1. When the same model combining NGAL with the ED Ph's clinical judgement was compared to a clinical model combining TO sCr results with the ED Ph's clinical judgement, the net reclassification index was $32.4 \%$, meaning that the correction classification of AKI improved 32.4 percentage points.

Table 1 (abstract P354)

\begin{tabular}{lccc}
\hline & Event & No event & AUC (95\% CI) \\
\hline Diagnosis of AKI & 49 & 616 & $0.80(0.07)$ \\
RIFLE & 25 & 640 & $0.72(0.12)$ \\
sCr bump & 10 & 655 & $0.85(0.10)$ \\
Oliguria & 14 & 651 & $0.81(0.14)$ \\
Mortality & 27 & 638 & $0.76(0.11)$ \\
\hline
\end{tabular}

Conclusion Our study demonstrated that blood NGAL measurements in patients hospitalized from the ED for critical conditions may improve the clinical diagnosis of AKI development. The routine use of NGAL in the ED may provide utility in deciding the appropriate treatment and management strategies for patients at risk for AKI development.

\section{P355}

Is cystatin C reliable in the anesthetized pig? An experimental study with special reference to septic shock

M Eriksson', E Söderberg', M Lipcsey', J Sjölin², M Castegren³, M Sjöquist', A Larsson

'Surgical Sciences, Anesthesia \& Intensive Care, Uppsala, Sweden; ${ }^{2}$ Medical Sciences, Infectious Disease, Uppsala, Sweden; ${ }^{3}$ Centre for Clinical Research, Eskilstuna, Sweden; ${ }^{4}$ SLU, Uppsala, Sweden; ${ }^{5}$ Medical Sciences, Clinical

Chemistry, Uppsala, Sweden

Critical Care 2012, 16(Suppl 1):P355 (doi: 10.1186/cc10962)

Introduction Our aim was to investigate renal function during 24 hours of endotoxemic shock with special focus on the reliability of analysis options in kidney damage.

Methods Twenty anesthetized pigs received randomly a continuous 24-hour endotoxin infusion at $0.063 \mu \mathrm{g} / \mathrm{kg} / \mathrm{hour}(n=8)$ or $0.25 \mu \mathrm{g} / \mathrm{kg} /$ hour $(n=9)$ or $\mathrm{NaCl}$ (controls $n=3$ ). Boluses $(10 \mathrm{ml} / \mathrm{kg}$ ) of succinylated gelatin were given when the arterial blood pressure was $50 \mathrm{mmHg}$ or below. Samples for analysis of cystatin $\mathrm{C}$ as well as clearances of inulin, $\mathrm{PAH}$ and creatinine were noted and urine was collected. 
Results Cystatin C was, already at baseline, not normally distributed. This was in contrast to the other renal variables. Five pigs had baseline values of cystatin $C$ in plasma $>0.6 \mathrm{mg} / \mathrm{l}$ (one control; four endotoxemic pigs), whereas 15 pigs had plasma levels $<0.3$. When individual values were noted over time, it became obvious that, with the exception of the four endotoxemic animals, which shifted considerably over time, there appears to be two subgroups of pigs regarding their cystatin $C$ values. There were only minor differences in cystatin $C$ over time for each individual pig compared with the baseline value, except for the four pigs that shifted considerably over time. There were no major differences in urinary output between untreated controls and any of the two endotoxemic groups of pigs during the 24-hour experimental period. There was no obvious relation between the administration of bolus doses of gelatin and subsequent diuretic response. Cystatin C did not correlate to creatinine clearance $\left(r^{2}=0.06\right)$, PAH clearance $\left(r^{2}=0.05\right)$, inulinxurine $\left(r^{2}=0.04\right)$ or diuresis $\left(r^{2}=0.004\right)$. No similar subgroupings were noted for any of the other renal variables, although it should be noted that correlations between all variables were weak.

Conclusion In this experiment, we noted that there appears to be two populations of pigs regarding their cystatin $C$ values. This result is in contrast to a previous study from our group [1]. Our findings may be explained by the alterations that occur in renal vascular resistance [2], although these findings may also indicate a genetic variation influencing either the levels of cystatin $\mathrm{C}$ or the antigen determinants of cystatin C. Until our data have been confirmed or disproved, we strongly suggest that porcine Cystatin $C$ values should be interpreted with great care as a marker for glomerular filtration rate in pigs.

References

1. Lipcsey, et al:: Crit Care Med 2009, 37:2782-2790.

2. Benes J, et al.: Crit Care 2011, 15:R256.

P356

Are serum cystatin-C-based estimates better than those derived

from serum creatinine in critically ill patients?

JP Baptista, SC Teixeira, J Pimentel

Coimbra Universitary Hospital, Coimbra, Portugal

Critical Care 2012, 16(Suppl 1):P356 (doi: 10.1186/cc10963)

Introduction The aim of our study was to evaluate the utility of two cystatin-C-based equations, as a surrogate of the renal function (glomerular filtration rate (GFR)) in a group of critically ill patients. Methods This was a monocentric, prospective and observational study including 146 samples respecting 22 ICU patients. Daily evaluation of seric creatinine, seric cystatin C (CC) and 24-hour creatinine clearance $(24 \mathrm{CrCL})$ was performed during the ICU stay. Comparisons were done between two CC-based equations (Hoek $(\mathrm{H})$ and Larsson (L) formulas) and: 24CrCL; Cockroft-Gault (CG); modified Cockroft-Gault (mCG); and six-variable Modification of Renal Disease (MDRD6) formulas. Patients with chronic renal failure were excluded. Correlation, precision, bias and discrimination power were assessed using Spearman coefficient, Bland-Altman plots and receiver operating characteristic curves.

Results The average age of the patients was 63.4 years and male gender was predominant (68\%). The APACHE II score was $16.8 \pm 5.7$. The medians of $\mathrm{H}$ and $\mathrm{L}$ estimates were 50.5 (28 to 77.6 ) and 47.7 (24.5 to 79.2$)$ respectively, as compared to 69.8 (29.8 to 115.7$), 60.7(42.6$ to 101.4 ), 58.9 (42.6 to 65.1 ) and 59.2 (40.6 to 106.8$) \mathrm{ml} / \mathrm{minute} / 1.73$ $\mathrm{m}^{2}$, respectively to $24 \mathrm{CrCL}$ (reference method), $\mathrm{CG}, \mathrm{mCG}$ and MDRD6. Correlation ( $r$ ) between $\mathrm{H}, \mathrm{F}, \mathrm{CG}, \mathrm{mCG}, \mathrm{MDRD} 6$ and $24 \mathrm{CrCL}$ was $0.83 / 0.83 / 0.73 / 0.70 / 0.74$, respectively. $H$ and $L$ formulas showed the smallest bias and limits of agreement, when compared with formulas based on serum creatinine, respectively $-17.5 / \pm 52 \mathrm{ml} /$ minute $/ 1.73 \mathrm{~m}^{2}$ and $-21 / \pm 52.8 \mathrm{ml} / \mathrm{minute} / 1.73 \mathrm{~m}^{2}$. The sensibility for the identification of acute renal dysfunction $\left(24 \mathrm{CrCL}\right.$ under $60 \mathrm{ml} /$ minute $/ 1.73 \mathrm{~m}^{2}$ ) was high for $\mathrm{H}$ and $\mathrm{L}$ formulas (area under the curve of 0.94 for both). In the subgroup of 29 samples with $24 \mathrm{CrCL}$ above $130 \mathrm{ml} /$ minute $/ 1.73 \mathrm{~m}^{2}$ (patients with hyperfiltration) these two formulas had low sensibility (between 8 and 22\%) for identification of this condition.

Conclusion In this population of critically ill patients, cystatin-C-derived Hoek and Larsson equations underestimated $24 \mathrm{CrCL}$; however, they have a better performance than the classic estimates (CG and MDRD6). Nevertheless, they are inaccurate when applied to ICU patients with hyperfiltration (GFR >130 ml/m/1.73 $\mathrm{m}^{2}$ ).
P357

Assessment of glomerular filtration rate in trauma patients in early resuscitation phase

M Bhattacharyya ${ }^{1}$, R Kumar $^{2}$, STodi

${ }^{1}$ AMRI Hospitals, Kolkata, India; ${ }^{2}$ Jadavpur University, Kolkata, India

Critical Care 2012, 16(Suppl 1):P357 (doi: 10.1186/cc10964)

Introduction An estimate of the glomerular filtration rate (GFR) is important to individualize drug dosages. Trauma leads to systemic inflammatory response syndrome, which has an effect on GFR. The main objective of this study was to assess the GFR in trauma patients during the first 24 hours of admission.

Methods A prospective observational study of 50 trauma patients aged between 18 and 90 years admitted to the ICU. Exclusion: patients with chronic kidney disease and structural kidney damage. The study population was assessed for GFR by the measurement of creatinine clearance from 24-hour urine creatinine and from serum creatinine. Demographic parameters were documented.

Results Total patients admitted to the ITU during July 2010 to April 2011 with trauma were 67 , of which 50 patients were included in the study. The mean age of the study group was 39 years, male $86 \%$, mean APACHE IV score 32 and mean Injury Severity Score 10. Out of 50 trauma patients, 13 (26\%) patients developed glomerular hyperfiltration (GHF) within 24 hours of admission. Mean creatinine clearance in the GHF group was $177.92 \pm 29$ and minimum/maximum values were 151.4 and $254.3 \mathrm{ml} /$ minute/1.73 $\mathrm{m}^{2}$ respectively. Compared to the GHF group, mean creatinine clearance levels were considerably lower in nonGHF patients (86.03 \pm 29$)$ with a range of values from 41 to $138.5 \mathrm{ml} /$ minute $/ 1.73 \mathrm{~m}^{2}$.

Conclusion Incidence of glomerular hyperfiltration is relatively common in critically ill multitrauma patients in the first 24 hours. This should be taken into account while deciding drug dosing in this group of patients.

P358

Validation of a continuous low-dose iohexol infusion to measure

the glomerular filtration rate

J Dixon', K Lane', N Dalton², I MacPhee', B Philips

'St George's Hospital, London, UK; ${ }^{2}$ King's College, London, UK

Critical Care 2012, 16(Suppl 1):P358 (doi: 10.1186/cc10965)

Introduction We have designed a method of continuous measurement of the glomerular filtration rate (GFR) with the intention of applying the method in patients with acute kidney injury (AKI). The aim of the study was to prove the method in healthy volunteers (HV) and patients with chronic kidney disease (CKD).

Methods HV and patients with CKD were randomly allocated to measurement of GFR using iohexol, either by the established method of single injection and measurement of its rate of elimination (gold standard), or by the continuous infusion of a very low dose of iohexol ( $0.5 \mathrm{ml} /$ hour for 12 hours). The GFR was measured again, using the other method, after a washout period of 4 to 28 days. Plasma iohexol concentration was measured at 10 time points and plotted on a twophase exponential decay graph. The GFR was calculated by dividing the infusion concentration by the plateau concentration. The $t$ test compared results with 4-hour creatinine clearance $(4-\mathrm{CrCl})$, and the CKD-EPI equation.

Results Six HV and seven CKD patients volunteered, with five in each group completing the study. There was no difference between the two groups $(P=0.79)$. In $\mathrm{HV}$, the mean GFR measured by single injection was $105 \pm 7.3$ and $109.4 \pm 9.9 \mathrm{ml} /$ minute $/ 1.73 \mathrm{~m}^{2}$ by infusion (Pearson $r=0.95, P=0.0002$ ). In CKD patients, the mean GFR measured by single injection was $40 \pm 5.4$ and $44.8 \pm 6.2 \mathrm{ml} /$ minute $/ 1.73 \mathrm{~m}^{2}$ by infusion (Pearson $r=0.99, P<0.0001$ ). The infusion method depends on reaching a steady plasma concentration, which took $165 \pm 84$ minutes in HV and $483 \pm 127$ minutes in CKD patients to be within $10 \%$ of the steady state. The GFR is overestimated by $4-\mathrm{CrCl}$ (by $13.9 \pm 12.9 \mathrm{ml} /$ minute $/ 1.73 \mathrm{~m}^{2}$, $P<0.0001$ ) and by CKD-EPI (by $8.4 \pm 9.6 \mathrm{ml} /$ minute $/ 1.73 \mathrm{~m}^{2}, P<0.0001$ ). Conclusion In future work, we aim to validate this method in critically ill patients with AKI. We predict the steady state achieved will be increased. Anticipated problems include increased time or failure 
to reach steady state. However, given the simplicity of the method we hypothesise that changes in iohexol concentration may provide valuable real-time information about the GFR in AKI. Changes are likely to occur before serum creatinine rises. In conclusion, the continuous iohexol infusion method of measuring GFR appears to be accurate and precise. In stable subjects, a steady plasma concentration is achieved before it is observed with creatinine changes.

\section{P359}

Investigation into the effects of commencing haemodialysis in the critically ill

R Docking', L Moss', M Sim', D Sleeman², J Kinsella'

'University of Glasgow, UK; 'niversity of Aberdeen, UK

Critical Care 2012, 16(Suppl 1):P359 (doi: 10.1186/cc10966)

Introduction We aimed to describe haemodynamic changes when haemodialysis is instituted in the critically ill. Three hypotheses are tested: (1) the initial session is associated with cardiovascular instability; (2) the initial session is associated with more cardiovascular instability compared to subsequent sessions; and (3) looking at unstable sessions alone, there will be a greater proportion of potentially harmful changes in the initial sessions compared to subsequent ones.

Methods Data were collected for 209 patients, identifying 1,605 dialysis sessions. Analysis was performed on hourly records, classifying sessions as stable/unstable by a cut-off $> \pm 20 \%$ change in baseline physiology (HR/MAP). Data from 3 hours prior to and 4 hours after dialysis were included, and average and minimum values derived. Three time comparisons were made (pre-HD:during, during HD:post, preHD:post). Initial sessions were analysed separately from subsequent sessions to derive two groups. If a session was identified as being unstable, then the nature of instability was examined by recording whether changes crossed defined physiological ranges. The changes seen in unstable sessions could be described as to their effects: being harmful/potentially harmful, or beneficial/potentially beneficial.

Results Discarding incomplete data, 181 initial and 1,382 subsequent sessions were analysed. A session was deemed to be stable if there was no significant change $(> \pm 20 \%)$ in the time-averaged or minimum MAP/ HR across time comparisons. By this definition 85/181 initial sessions were unstable (47\%, 95\% CI SEM 39.8 to 54.2$)$. Therefore Hypothesis 1 is accepted. This compares to $44 \%$ of subsequent sessions $(95 \% \mathrm{Cl} 41.1$ to 46.3). Comparing these proportions and their respective $\mathrm{Cl}$ gives a $95 \% \mathrm{Cl}$ for the standard error of the difference of $-4 \%$ to $10 \%$. Therefore Hypothesis 2 is rejected. In initial sessions there were 92/1,020 harmful changes. This gives a proportion of $9.0 \%$ (95\% CI SEM 7.4 to 10.9). In the subsequent sessions there were 712/7,248 harmful changes. This gives a proportion of $9.8 \%$ ( $95 \%$ CI SEM 9.1 to 10.5). Comparing the two unpaired proportions gives a difference of $-0.08 \%$ with a $95 \% \mathrm{Cl}$ of the $\mathrm{SE}$ of the difference of -2.5 to +1.2 . Hypothesis 3 is rejected. Fisher's exact test gives a result of $P=0.68$, reinforcing the lack of significant variance. Conclusion Our results reject the claims that using haemodialysis is an inherently unstable choice of therapy. Although proportionally more of the initial sessions are classed as unstable, the majority of MAP and HR changes are beneficial in nature.

\section{P360}

Is the AKIN score useful as an indicator of the optimum time for intervention with renal replacement therapy in critically ill patients? S Mousdale, J Bannard-Smith

Royal Blackburn Hospital, Blackburn, UK

Critical Care 2012, 16(Suppl 1):P360 (doi: 10.1186/cc10967)

Introduction Acute kidney injury represents a significant workload and economic burden for critical care units. In the critical care setting AKI is usually associated with a variety of aetiologies such as septic shock, major surgery and heart failure [1]. Controversy exists as to the optimal time for the institution of renal replacement therapy (RRT) [2]. Scoring systems such as AKIN have used the rise in serum creatinine combined with reduced urine output over a period of 48 hours as indicative of the degree of injury [3]. We used this scoring system to see if ITU mortality correlated with increasing AKIN score.
Methods The Critical Care Minimum Dataset records of patients admitted to our mixed general ICU were investigated. Those patients who received renal organ support were investigated further. The change in serum creatinine levels in the 48 hours prior to institution of RRT was used to determine the AKIN score. Patients in whom there was not a significant rise in creatinine, but who received RRT, were staged zero. Unfortunately, urine output data were not available to improve accuracy.

Results There were a total of 276 patients whose records were adequate for this audit. Several records were incomplete and not used. Demography and APACHE II scores were similar across all groups. Length of stay and days of RRT were similar across the groups. ICU survival was as follows: AKIN stage: (0) $42.2 \%$, (1) $50.6 \%$, (2) $51.7 \%$, (3) $70.4 \%$. Pearson chi-square $P<0.001$.

Conclusion We were not able to demonstrate improved survival when RRT was initiated at an earlier AKIN stage. A small nonsignificant trend was observed with increasing stage and the differences between groups were significant. Very early initiation of RRT was associated with increased mortality. Stage (3) included patients with chronic kidney disease, which probably skewed the results in this group. We cannot recommend the use of the AKIN score as a pointer to when to initiate RRT, based on these data.

References

1. Uchino S, et al.: Intensive Care Med 2007, 33:1563-1570.

2. Bagshaw SM, et al.: J Crit Care 2009, 24:129-140.

3. Mehta RL, et al: Crit Care 2007, 11:R31.

\section{P361}

Timing for initiation of continuous renal replacement therapy in patients with septic shock and acute kidney injury

HP Shum, KC Chan, MC Kwan, WT Yeung, WS Cheung, WW Yan

Pamela Youde Nethersole Eastern Hospital, Hong Kong

Critical Care 2012, 16(Suppl 1):P361 (doi: 10.1186/cc10968)

Introduction The optimal timing for initiation of renal replacement therapy (RRT) in septic acute kidney injury (AKI) remains controversial. The aim of this study is to investigate the impact of early versus late initiation of continuous RRT (CRRT), as defined using the simplified RIFLE classification, on organ dysfunction among patients with septic shock and AKI.

Methods Patients were divided into early (sRIFLE Risk) or late (sRIFLE Injury or Failure) initiation of RRT. Patients with chronic kidney disease stage 5 or on dialysis were excluded.

Results One hundred and twenty patients admitted within a 3.5-year period fulfilled inclusion criteria. Thirty-one (26\%) underwent early, 89 (74\%) had late CRRT. No significant difference was noted between the two groups with respect to change in total SOFA score/non-renal SOFA score in the first 24/48 hours after initiation of CRRT, vasopressor use, dialysis requirement and mortality (at 28 days, 3 months and 6 months). The change of nonrenal SOFA score 48 hours after CRRT correlated with the SOFA score at the start of CRRT $(P=0.034)$ and the APACHE IV risk of death $(P=0.000)$, but not the glomerular filtration rate (GFR) at the start of CRRT $(P=0.348)$. See Tables 1 and 2.

Table 1 (abstract P361). Baseline characteristics and parameters on initiation of CRRT

\begin{tabular}{lccc}
\hline & Early & Late & $P$ value \\
\hline Age & $70.7 \pm 15.1$ & $69.3 \pm 13.1$ & 0.614 \\
APACHE IV & $119 \pm 31$ & $131 \pm 37$ & 0.110 \\
Starting GFR & $36.2 \pm 20.9$ & $18.1 \pm 8.2$ & $<0.001$ \\
Start SOFA & $11.6 \pm 3.3$ & $13.3 \pm 2.7$ & 0.006 \\
\hline
\end{tabular}

Table 2 (abstract P361). Outcome parameters

\begin{tabular}{lccc}
\hline & Early & Late & $P$ value \\
\hline NR SOFA 0 to 48 & $-0.52 \pm 3.91$ & $-0.71 \pm 3.57$ & 0.827 \\
Hospital death & $17(54.8 \%)$ & $48(53.9 \%)$ & 0.931 \\
28-day survival & $16(51.6 \%)$ & $46(51.7 \%)$ & 0.994 \\
\hline
\end{tabular}


Conclusion For septic shock with AKI, no significant difference in organ function and outcome was noted when the timing of initiation of CRRT was classified using SRIFLE criteria. Subsequent improvement of organ function correlated with initial SOFA and APACHE scores instead of the GFR (which determine sRIFLE class) on starting of CRRT. The use of more global assessment tools, such as the SOFA score, for stratification purposes on appropriate timing of CRRT warrants further investigation.

P362

Early application of CVVH In the complex treatment of patients with early severe acute pancreatitis

I Aleksandrova, M Ilynsky, S Rei, G Berdnikov, L Marchenkova, V Kiselev

Hospital Research Institute for Emergency Medicine N.V. Sklifosovsky, Moscow,

Russia

Critical Care 2012, 16(Suppl 1):P362 (doi: 10.1186/cc10969)

Introduction A large population-based study of 1,024 deaths from acute pancreatitis (AP) has revealed that the median time lapse between the onset of AP and death was 6 days [1]. A number of authors considered the patients with persistent or progressive early multiple organ failure (MOF) as patients with early severe acute pancreatitis (ESAP) [2].

Methods The aim of current study was to evaluate the efficiency of early CVVH in a complex treatment of ESAP. The retrospective analysis involved 106 patients. The patients were divided into three groups: the first group $(n=45)$ received CVVH dose $<30 \mathrm{ml} / \mathrm{kg} /$ hour, the second group $(n=20)$ received the dose $>30 \mathrm{ml} / \mathrm{kg} / \mathrm{hour}$, and in the third group ( $n=41) \mathrm{CVVH}$ was not used during the early phase of disease (Table 1). In the first and second groups the median time interval between admission and start of CVVH was $2(2 ; 3)$ days.

Table 1 (abstract P362)

\begin{tabular}{lccc}
\hline Variable & First group & Second group & Third group \\
\hline Age & $42 \pm 15$ & $39 \pm 13$ & $47 \pm 16$ \\
BMI & $31 \pm 5$ & $29 \pm 4$ & $29 \pm 5$ \\
APACHE II score & $17(5)$ & $17(9)$ & $15(7)$ \\
SOFA score & $5(4)$ & $5(3)$ & $5(3)$ \\
Ranson score & $8(6)$ & $8(7)$ & $10(9)$ \\
Early mortality (\%) & 27 & $10^{*}$ & 42 \\
Infection (\%) & 47 & 35 & 29 \\
Overall mortality (\%) & 49 & $25^{*}$ & 51 \\
\hline
\end{tabular}

Data presented as median (IQR). ${ }^{P} P<0.05$, second group versus third group.

Results As compared to reference group 3 , significant $(P=0.022)$ reduction of early mortality (14 days) was observed in the second group, and decreasing tendency $(P=0.093)$ of mortality rate was detected in the first group. The median time interval between admission and death was 14 days (in the first and second groups) and 5 days in the third group.

Conclusion The early application of the CVVH increases time interval for care delivery and allows reducing early mortality. The best results were obtained in the group of patients who were treated with the higher dose of $\mathrm{CVVH}$ (earlier restoration of homeostasis and decreased severity of early MOF).

References

1. Mole DJ, et al:: HPB 2009, 11:166-170.

2. Isenmann R, et al:: Pancreas 2001, 22:274-278.

\section{P363}

Timing of the initiation of continuous renal replacement therapy and clinical outcome in patients with severe sepsis and septic shock $\mathrm{SCho}$

Seoul Asan Hospital, Seoul, South Korea

Critical Care 2012, 16(Suppl 1):P363 (doi: 10.1186/cc10970)

Introduction Timing of renal replacement therapy (RRT) in critically ill severe sepsis and septic shock patients with acute kidney injury is highly subjective and may influence outcome. The aim of this study is to evaluate the relationship between timing of RRT and 28-day mortality in patients with severe sepsis and septic shock.

Methods All patients diagnosed with severe sepsis and septic shock and treated at the medical ICU in a university-affiliated, tertiary-referral center, from January 2005 to December 2006 were reviewed. Timing of RRT was stratified into early and late by RIFLE (Risk, Injury, Failure, Loss, and End-stage) criteria and blood urea nitrogen (BUN) at the time RRT was started. The primary outcome was 28 -day death from any cause.

Results Of the 326 patients diagnosed with severe sepsis and septic shock and admitted to the medical ICU during the study period, 78 patients received RRT. The mean age of the patients was $61.5 \pm 14.7$ years and 54 patients were male (69.2\%). The timing of RRT was categorized into early (Risk, and Injury) and late (Failure) by RIFLE criteria and also categorized into early (BUN $<75 \mathrm{mg} / \mathrm{dl}$ ) and late (BUN $\geq 75 \mathrm{mg} / \mathrm{dl}$ ). Comparing the relationship between RIFLE criteria (Risk and Injury vs. Failure) and 28-day mortality showed no significant difference $(70.8 \%$ vs. $73.3 \%, P=0.81)$. The timing of RRT by serum BUN also showed no significant difference in 28-day mortality before start of RRT by BUN $\geq 75 \mathrm{mg} / \mathrm{dl}$ versus BUN $<75 \mathrm{mg} / \mathrm{dl}(77.3 \%$ vs. $69.6 \%, P=0.50)$.

Conclusion Timing of RRT, stratified into early and late by RIFLE and BUN, showed no significant difference in 28-day mortality in patients with severe sepsis and septic shock.

P364

Amino acid concentrations in serum, urine and dialysate/ultrafiltrate solutions of continuous venovenous hemodiafiltration patients

JM Lee, YJ Lee, J Hong

Ajou University School of Medicine, Suwon, Kyeonggi-do, South Korea

Critical Care 2012, 16(Suppl 1):P364 (doi: 10.1186/cc10971)

Introduction A prospective study was performed for evaluating the amino acid losses during continuous venovenous hemodiafiltration (CVVHDF).

Methods Serum, 24-hour urine and dialysate/ultrafiltrate solutions of CVVHDF were obtained on days 1,3 , and 5 from 11 critically ill patients (five males, six females, mean age $63.0 \pm 18.1$ (24 to 90 )) in the surgical ICU. We analyzed 40 kinds of amino acid concentrations in serum (34 samples), urine (15 samples) and dialysate/ultrafiltrate solutions (30 samples) by high-performance liquid chromatography analysis. The mean dialysate amount was $918.2 \mathrm{ml}(600$ to $1,500 \mathrm{ml})$, mean replacement fluid amount $1,136.4 \mathrm{ml}(1,000$ to $2,000 \mathrm{ml})$ and mean blood flow rate $175 \mathrm{ml}$ (100 to $200 \mathrm{ml}$ ), respectively. Nutritional support for CVVHDF patients was guided as protein intake at 1.2 to $1.5 \mathrm{~g} / \mathrm{kg} /$ day, caloric intake at $30 \mathrm{kcal} / \mathrm{kg} /$ day.

Results Among the analyzed 40 amino acids, the five highest mean concentration levels of 24-hour dialysate/ultrafiltrate solutions were glutamine $(65,178.3 \mu \mathrm{mol} / \mathrm{l}$ (hereafter, all units for amino acids are $\mu \mathrm{mol} / \mathrm{I})$ ), alanine $(48,633.3)$, glycine $(33,959.5)$, proline $(27,701.5)$, lysine $(26,519.4)$; of serum were glutamine (694.4), alanine (438.1), glycine (349.7), lysine (275.7), proline (262.4); and of 24-hour urine were glycine $(1,523.0)$, histidine (957.5), alanine (920.7), glutamine (904.6), lysine (699.1), respectively. Amino acid concentrations of 24-hour dialysate/ ultrafiltrate solutions showed significant correlation with amino acid concentrations of serum $(P=0.000)$. The mean amount of total amino acid loss on day 5 of CVVHDF was 2.8 times that of day 1 and 1.7 times that of day 3. The increase of amino acid loss according to CVVHDF progression was most prominent in glutamic acid (8.9 times from day 1 to day 5).

Conclusion The highest concentration level of 24-hour dialysate/ ultrafiltrate solution was glutamine. The amount of amino acid loss after CVVHDF was correlated with the serum amino acid amount and increased according to CVVHDF progression.

P365

Evaluation of the potential adverse effects associated with calcium carbonate precipitate during continuous venovenous hemofiltration J McKee, B Brooks, J Daller, J Gass, D Pantaleone, P Zieske

Baxter, Round Lake, IL, USA

Critical Care 2012, 16(Suppl 1):P365 (doi: 10.1186/cc10972)

Introduction This study evaluated the potential adverse effects associated with exposure to calcium carbonate precipitate during 
continuous venovenous hemofiltration $(\mathrm{CVVH})$. The clinical use of Accusol 35 Solution (Accusol 35) has been associated with occasional formation of calcium carbonate precipitate in the tubing set during therapy.

Methods Fourteen mongrel dogs were anesthetized, instrumented, and received $\mathrm{CVVH}$ with the test $(n=6)$ or negative control article $(n=8)$ for 6 hours. The test article was Accusol 35 with induced precipitate formation prior to CVVH. The test article contained visible particles and subvisible particles $36 \times$ higher than the maximum concentration specified in the European Pharmacopoeia (EP). The negative control article was Accusol 35 containing no visible particles and subvisible particles within EP specification. One-half of the dogs in the negative control article group received a central venous injection of Sephadex G-50 beads $(10 \mathrm{mg} / \mathrm{kg})$ following $\mathrm{CVVH}$ as a positive control. Select cardiovascular (CV) parameters were monitored continuously or were calculated at predetermined times. Arterial samples were obtained at predetermined times for analysis of blood gases and electrolytes. Samples of the test and negative control articles were obtained hourly during $\mathrm{CVVH}$ for determination of $\mathrm{pH}$ and subvisible particles. Dogs were euthanized and lung tissue samples were examined histologically. Results All CV parameters remained stable and no differences were observed between the test and negative control articles. Sephadex beads caused an increase $(P<0.01)$ in mean pulmonary arterial pressure due solely to a similar increase $(P<0.01)$ in pulmonary vascular resistance. No differences in blood gases or electrolytes were observed between the test and negative control articles. Sephadex beads caused a decrease $(P>0.05)$ in arterial blood $\mathrm{PO}_{2}$ and an increase $(P>0.05)$ in arterial blood $\mathrm{PCO}_{2}$. No differences in lung histology were observed between the test and negative control articles. The lungs from all dogs given Sephadex beads contained multiple intravascular particles in large-caliber blood vessels.

Conclusion CVVH performed on anesthetized dogs for 6 hours using Accusol 35 containing visible and subvisible particles $36 \times$ higher than the maximum concentration specified in the EP resulted in no adverse effects on CV parameters, blood gases and electrolytes, and lung histology as compared with Accusol 35 containing no visible particles and subvisible particles that were within EP specification.

\section{P366}

Regional citrate anticoagulation in CVVH: a new protocol combining citrate solution with a phosphate-containing replacement fluid

S Morabito', V Pistolesi', L Tritapepe', E Vitaliano², E Strampelli',

F Polistena', L Zeppilli', A Pierucci'

'Policlinico Umberto I, Rome, Italy; 2 Pertini H, Rome, Italy

Critical Care 2012, 16(Suppl 1):P366 (doi: 10.1186/cc10973)

Introduction Regional citrate anticoagulation (RCA) is a highly effective anticoagulation (AC) method in CRRT and different combinations of citrate (Citr) and CRRT solutions can affect the acid-base (A-B) balance. Regardless of the $A C$ protocol, hypophosphatemia occurs frequently in CRRT (80\%). The aim was to evaluate safety and effects on A-B balance of a new RCA-CVVH protocol using $18 \mathrm{mmol} / \mathrm{l}$ Citr solution combined with a phosphate-containing hemofiltration (HF) solution.

Methods In our center, RCA-CVVH is routinely performed with a $12 \mathrm{mmol} / \mathrm{l}$ predilution Citr solution (Prismocitrate 10/2) and a postdilution $\mathrm{HF}$ solution $\left(\mathrm{HCO}_{3}{ }^{-} 32, \mathrm{Ca}^{2+} 1.75, \mathrm{Mg}^{2+} 0.5, \mathrm{~K}^{+} 2 \mathrm{mmol} / \mathrm{l}\right)$ (protocol A). In the case of persistent acidosis, not related to Citr accumulation, $\mathrm{NaHCO}_{3}$ infusion is started. In order to optimize the buffer balance, a new protocol has been designed throughout a mathematical model developed to estimate Citr and $\mathrm{HCO}_{3}^{-}$mass transfer. Recently introduced solutions have been adopted: $18 \mathrm{mmol} / \mathrm{l}$ predilution Citr solution (Prismocitrate 18), postdilution HF solution (Phoxilium, $\mathrm{HCO}_{3}{ }^{-} 30$, phosphate $1.2, \mathrm{Ca}^{2+} 1.25, \mathrm{Mg}^{2+} 0.6, \mathrm{~K}^{+} 4 \mathrm{mmol} / \mathrm{l}$ ) (protocol $\mathrm{B}$ ). In relation to $\mathrm{Qb}$, the Citr solution rate was set to meet the target circuit Citr concentration $(3 \mathrm{mmol} / \mathrm{l})$. To maintain systemic $\mathrm{Ca}^{2+}(1.1$ to $1.25 \mathrm{mmol} / \mathrm{l}), \mathrm{CaCl}_{2} 10 \%$ was started according to estimated $\mathrm{Ca}^{2+}$ loss.

Results In a cardiac surgery patient with AKI, A-B status and electrolytes have been evaluated comparing protocol A (five circuits, 301 hours) versus protocol $B$ (two circuits, 97 hours): $\mathrm{pH} 7.39 \pm 0.03$ versus $7.44 \pm 0.03(P<0.0001)$, blood $\mathrm{HCO}_{3}{ }^{-} 22.3 \pm 1.8$ versus $22.6 \pm 1.4 \mathrm{mmol} / \mathrm{l}$
$(P=\mathrm{NS}), \mathrm{BE}-2.8 \pm 2.1$ versus $-1.6 \pm 1.2(P<0.01)$, serum phosphate $0.85 \pm 0.2$ versus $1.3 \pm 0.5 \mathrm{mmol} / \mathrm{l}(P=0.027)$, serum $\mathrm{K}^{+} 4 \pm 0.2$ versus $4.2 \pm 0.3 \mathrm{mmol} / \mathrm{l}(P=\mathrm{NS})$ with $\mathrm{KCl}$ infusion $4 \pm 0.2$ versus $1.4 \pm 1.5 \mathrm{mmol} /$ hour $(P<0.0001)$. Protocol $\mathrm{A}$ required $\mathrm{NaHCO}_{3}$ and $\mathrm{Na}$-phosphate infusion $(8.9 \pm 2.8 \mathrm{mmol} / \mathrm{hour}$ and $5 \mathrm{~g} /$ day, respectively) while protocol B allowed one to stop both supplementations. Systemic and circuit $\mathrm{Ca}^{2+}$ were easily maintained in the target range with both protocols.

Conclusion Although needing confirmation in an adequate number of patients, protocol $B$ was able to provide a buffer balance more positive than protocol $A$ and allowed one to adequately control the A-B status without additional $\mathrm{NaHCO}_{3}$ infusion and in the absence of alkalosis, despite the use of a standard $\mathrm{HCO}_{3}{ }^{-}$concentration $\mathrm{HF}$ solution. Furthermore, the combination of a phosphate-containing replacement fluid appeared effective to prevent hypophosphatemia. Finally, the use of a mathematical model allowed predicting the effects of different replacement solutions and/or RCA-CVVH settings on the mass balance of the main solutes.

\section{P367}

Regional citrate anticoagulation with a low-concentration solution in predilution-postdilution CVVH

$\checkmark$ Pistolesi, S Morabito, L Tritapepe, L Cibelli, M Ambrosino, F Polistena, L Zeppilli, E Strampelli, MI Sacco, A Pierucci

Policlinico Umberto I, Rome, Italy

Critical Care 2012, 16(Suppl 1):P367 (doi: 10.1186/cc10974)

Introduction Systemic anticoagulation (AC) can increase the bleeding risk in CRRT. However, regional citrate anticoagulation (RCA) is a valid alternative to heparin (Hep) in patients at high risk of bleeding. The aim was to evaluate efficacy and safety of RCA-CVVH using a lowconcentration citrate (Citr) solution.

Methods In cardiac surgery patients with AKI we adopted RCA-CVVH as an alternative to Hep or no-AC CRRT. Criteria for switching to RCA: early circuit clotting (24 hours) or Hep-related complications. RCA$\mathrm{CVVH}$ was performed with a predilution Citr solution $(12 \mathrm{mmol} / \mathrm{l})$ and a postdilution hemofiltration solution $\left(\mathrm{HCO}_{3}{ }^{-} 32 \mathrm{mEq} / \mathrm{l}\right)$. In relation to blood flow rate $(\mathrm{Qb})$, the Citr solution rate was set to meet a circuit Citr concentration of $3 \mathrm{mmol} / \mathrm{l}$ and modified to obtain circuit $\mathrm{Ca}^{2+}$ $<0.4 \mathrm{mmol} / \mathrm{l} . \mathrm{CaCl}_{2}(10 \%)$ was infused to maintain systemic $\mathrm{Ca}^{2+}\left(\mathrm{s}^{-} \mathrm{Ca}^{2+}\right)$ of 1.1 to $1.25 \mathrm{mmol} / \mathrm{l}$. To facilitate CVVH settings, we developed a mathematical model to estimate the metabolic Citr load, buffer balance and $\mathrm{Ca}^{2+}$ loss.

Results In 30 patients at high bleeding risk (age $70.5 \pm 9.3$, SOFA score $13.7 \pm 2.5$ ) the AC modality was switched to RCA-CVVH from no AC or Hep. CVVH initial settings: dialysis dose $33.6 \pm 3.4 \mathrm{ml} / \mathrm{kg} / \mathrm{hour}$; Qb $135 \pm 14 \mathrm{ml} /$ minute; Q Citr 1,703 $\pm 250 \mathrm{ml} /$ hour; Q postdilution $761 \pm 181 \mathrm{ml} /$ hour; Citr load $11.6 \pm 2.1 \mathrm{mmol} / \mathrm{hour}$; $\mathrm{CaCl}_{2} 3.7 \pm 1.5 \mathrm{ml} /$ hour. Target circuit $\mathrm{Ca}^{2+}$ and $\mathrm{s}-\mathrm{Ca}^{2+}$ were maintained $(0.37 \pm 0.09$ and $1.18 \pm 0.13 \mathrm{mmol} / \mathrm{l})$ with few modifications of $\mathrm{Citr}$ and $\mathrm{CaCl}_{2}$ infusion rates. We used 146 RCA-CVVH circuits with filter life $50.5 \pm 35.8$ hours (median 41 ; total 7,372). RCA-stopping causes: $34 \%$ CVC malfunction, $24 \%$ alarm handling/technical issues, $20 \%$ scheduled, $14 \%$ medical procedures, $8 \%$ others. Before starting RCA, we used 69 Hep circuits ( 2,015 hours) and 74 no-AC circuits $(1,827$ hours) with a filter life of $29.2 \pm 20.7$ hours (median 22) and $24.7 \pm 20.6$ hours (median 20), shorter than RCA $(P<0.0001)$. Circuits running at 24,48 and 72 hours $(\%)$ : RCA 73, 42 and 28; Hep 43, 23 and 10; and no-AC 38, 12 and 5 (log-rank test $P<0.0001)$. During RCA-CVVH no patients had bleeding complications and the transfusion rate was lower if compared to other $A C$ modalities ( 0.29 vs. 0.69 blood units/day, $P=0.001)$. PLT count $(P=0.018)$ and AT-III activity $(P=0.009)$ increased throughout days of RCA, reducing supplementation needs. RCA has been stopped for Citr accumulation in one patient (calcemia/s- $\mathrm{Ca}^{2+}>2.5$ ).

Conclusion In this experience, RCA allowed one to safely prolong the filter life, decreasing the transfusion rate and supplementation needs for AT-III and PLT. The use of a mathematical model allowed one to simplify the CVVH settings. Therefore, RCA should be worthy of more consideration as the first-choice CRRT AC modality in patients at high risk of bleeding. 


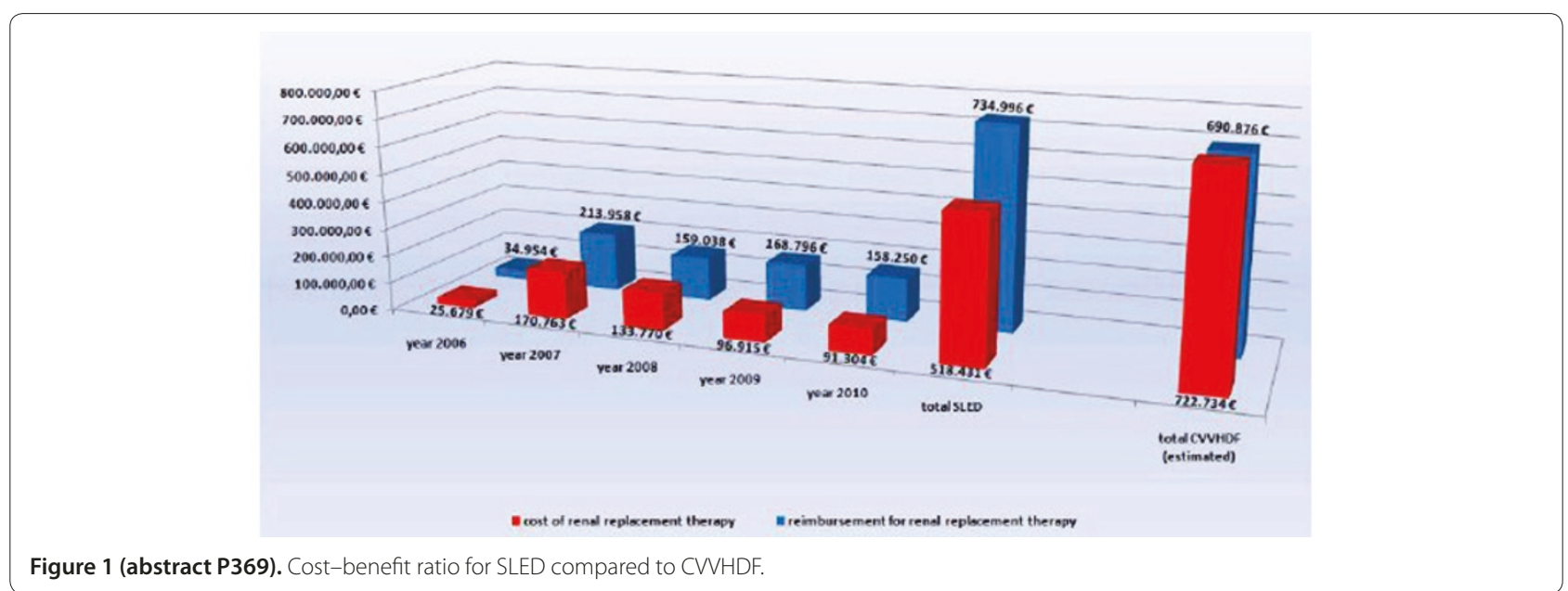

P368

Exposure to intermittent hemodialysis and renal recovery after acute kidney injury: a systematic review

A Schneider1', M Bagshaw², NJ Glassford', R Bellomo

'Austin Health, Heidelberg, Australia; 'University of Alberta, Edmonton, Canada

Critical Care 2012, 16(Suppl 1):P368 (doi: 10.1186/cc10975)

Introduction Renal replacement therapy (RRT) in critically ill patients can be applied in a continuous (CRRT) or intermittent (IRRT) fashion. To date, there is no systematic comparison on the impact of these two modalities on renal recovery after an episode of acute kidney injury (AKI). We sought to compare the rates of renal recovery with RRT independence between CRRT and IRRT as an initial modality for RRT in AKI.

Methods We searched MEDLINE and EMBASE. We retrieved all studies published between 2000 and 2010 that report original data on renal recovery to RRT dependence after AKI in adults. Authors of studies with incomplete data were contacted. Search date: January 2011. Two authors independently assessed the trial quality and extracted data. Pooled analyses were performed and a chi-square test performed. Sensitivity analyses were performed after stratification by premorbid chronic kidney disease, number of centers, type of study and illness severity index. In a subsequent analysis we pooled the studies according to the percentage of patients exposed to IHD into low-exposure $(<50 \%$ of patients exposed) or high-exposure ( $>50 \%$ patients exposed). Results We identified 50 studies ( 14,796 patients). Overall, as compared with those that received IRRT as an initial modality (IRRT group), those that received CRRT (CRRT group) had higher average illness severity scores (mean APACHE III equivalent 88 vs. $72, P<0.01$ ) and higher inhospital mortality $(57.7 \%$ vs. $37.9 \%, P<0.0001)$. When reported at 28 days after initiation of RRT (outcome reported in 25 studies), $19.4 \%$ of survivors were RRT dependent in the CRRT group versus $26.9 \%$ in the IRRT group $(P=0.004)$. At hospital discharge (reported in 26 studies), RRT dependence was present in $10.9 \%$ of the CRRT group versus $20.8 \%$ in the IRRT group $(P<0.0001)$. At day 90 (reported in 22 studies), RRT dependence was $7.8 \%$ in the CRRT group versus $36.1 \%$ in the IRRT group $(P<0.0001)$. The sensitivity analyses confirmed these findings in all subgroups. The rates of RRT dependency in the low-exposure group and the high-exposure group at days 28,90 and hospital discharge were $19.6 \%, 8.8 \%$ and $12.4 \%$ versus $43.2 \%, 26.8 \%$ and $14.0 \%$ respectively (all $P<0.0001$, except for hospital discharge $P=$ NS).

Conclusion The preponderance of the available evidence suggests that CRRT is associated with a higher rate of renal recovery in AKI survivors compared with IRRT.
P369

Sustained low-efficiency dialysis for renal replacement therapy in the ICU: a cost-benefit analysis of the years 2006 to 2010

T Neuenfeldt, HB Hopf

Asklepios Klinik, Langen, Germany

Critical Care 2012, 16(Suppl 1):P369 (doi: 10.1186/cc10976)

Introduction Sustained low-efficiency dialysis (SLED) as primary renal replacement therapy in acute renal failure is still not widely used compared to continuous venovenous hemodiafiltration (CVVHDF), despite possible economical advantages. Based on one key paper [1] we use SLED as primary renal replacement therapy. However, since medical and economical data with SLED are scarce, we evaluated costs and outcome in a 5-year retrospective study on our ICU.

Methods During 2006 to 2010 we performed a search on our KIS selecting all patients with the diagnoses $\mathrm{N} 17$ and $\mathrm{N} 18$ who were treated with SLED or CVVHDF on our ICU. We excluded all patients with a stay $<2$ days or with an extrarenal indication for dialysis or with preexisting chronic dialysis. The following variables were extracted from the chart: number of SLED, stay in ICU and hospital, mortality in ICU and hospital, SAPS II, TISS 28, blood urea and creatinine, C-reactive protein, mechanical ventilation, and diagnoses. We evaluated the long-term outcome by sending all discharged patients a questionnaire.

Results During the period from 2006 to 2010, 3,247 SLED treatments in 421 patients (mean SAPS II was 52 patients) were performed. ICU mortality was $36 \%$ and hospital mortality was $46 \%$. A persistent need for dialysis (end-stage kidney disease) was registered in $9 \%$. Total costs for SLED were $€ 518.431$ and total reimbursements amount to $€ 734.996$ (Figure 1). Assuming for cost comparisons also 3,247 CVVHDF-days, we estimated costs of $€ 722.734$ with reimbursements of $€ 690.876$ for CVVHDF.

Conclusion Thus, since short-term and long-term outcome of our patients was comparable to published outcome data with CVVHDF, SLED is at least comparable to CVVHDF even in a busy ICU environment. Moreover, in view of costs, SLED is the preferable dialysis form for renal replacement therapy also in the ICU.

Reference

1. Vinsonneau C, et al.: Lancet 2006, 368:379-385.

\section{P370}

The new dialysis method Mini-SLED is useful for dialyzing acute brain stroke patients

F Taki, Y Komatsu

St Luke's International Hospital, Tokyo, Japan

Critical Care 2012, 16(Suppl 1):P370 (doi: 10.1186/cc10977)

Introduction Hemodialysis (HD) patients are known to be a high-risk population for brain stroke. On acute phase of stroke, standard HD treatment may increase cerebral damage by changing serum and 
tissue osmolarity. For low clearance dialysis, CRRT, PD or low Qb HD were used but there are some complications. To dialyze these patients more safely and simply, we modified a new dialysis method, Mini-SLED (sustained low-efficiency dialysis).

Methods We conducted a retrospective observational study from June 2006 to October 2011. Maintenance HD patients who onset acute brain stroke, including hemorrhage and ischemic infarction, were observed. We divided patients into four groups by dialysis modality and compared the clinical parameters. Determination of Mini-SLED was Qb $200 \mathrm{ml} /$ minute, QD 100 to $200 \mathrm{ml} /$ minute, duration for 2 to 3 hours. Results Sixty-one patients were observed in this study. Mean age 72.5 years, 39 patients were male, 45 patients had diabetes. Major clinical parameters and outcomes are presented in Table 1. Patients treated with Mini-SLED have lower risk of rebleeding compared to low $\mathrm{Qb} H D$ or CRRT, and were more cost-effective than PD. Delivered Kt/V of MiniSLED was $0.72 \pm 0.23$. Modality difference did not affect mortality.

Table 1 (abstract P370). Dialysis methods and clinical parameter

\begin{tabular}{lcccc}
\hline & $\begin{array}{c}\text { CRRT } \\
(\boldsymbol{n}=\mathbf{2 5})\end{array}$ & $\begin{array}{c}\text { PD } \\
(\boldsymbol{n}=\mathbf{3})\end{array}$ & $\begin{array}{c}\text { Low Qb HD } \\
(\boldsymbol{n}=\mathbf{2 1})\end{array}$ & $\begin{array}{c}\text { Mini-SLED } \\
(\boldsymbol{n}=\mathbf{1 2})\end{array}$ \\
\hline NIHSS (score) & $30.8 \pm 17.2$ & $34.6 \pm 16.4$ & $31.7 \pm 20.8$ & $32 \pm 19.8$ \\
Rebleed $(n, \%)$ & $6,24 \%$ & $1,33 \%$ & $4,19.0 \%$ & $0,0 \%$ \\
Mortality $(n, \%)$ & $5,20 \%$ & $1,33 \%$ & $5,23.8 \%$ & $2,16 \%$ \\
Kt/N (daily) & $0.68 \pm 0.32$ & $0.25 \pm 0.16$ & $0.86 \pm 0.23$ & $0.72 \pm 0.23$ \\
Cost (\$/1 treat) & $498 \pm 30.2$ & $92.4 \pm 22.6$ & $82.5 \pm 12.5$ & $86.7 \pm 14.3$ \\
\hline
\end{tabular}

Conclusion Our Mini-SLED methods are effective and safe for dialyzing acute brain stroke patients.

\section{P371}

Investigation into haemodynamic stability during intermittent haemodialysis in the critically ill

R Docking', L Moss', M Sim', D Sleeman², J Kinsella'

'University of Glasgow, UK; 'niversity of Aberdeen, UK

Critical Care 2012, 16(Suppl 1):P371 (doi: 10.1186/cc10978)

Introduction Studies that have reported cardiovascular (CVS) instability with haemodialysis (HD) are outdated and small. By analysing sessions in detail it will be possible to identify the frequency and nature of CVS instability. Hypothesis 1: haemodialysis is associated with CVS instability in the majority of sessions. Hypothesis 2: the majority of CVS changes in unstable sessions will be harmful/potentially harmful.

Methods Data were collected for 209 patients, identifying 1,605 dialysis sessions. Analysis was performed on hourly records, classifying sessions as stable/unstable by a cut-off $> \pm 20 \%$ change in baseline physiology (HR/MAP). Data from 3 hours prior to and 4 hours after dialysis were included, and average and minimum values derived. Three time comparisons were made: pre-HD:during, during HD:post, pre-HD:post-HD. If a session was identified as being unstable, then the nature of instability was examined by recording whether changes crossed defined physiological ranges. The changes seen in unstable sessions could be described as to their effects: being harmful/ potentially harmful, or beneficial/potentially beneficial.

Results Discarding incomplete data, 1,563 sessions were analysed. A session was deemed to be stable if there was no change $> \pm 20 \%$ in time-averaged or minimum MAP/HR across three time comparisons. In 1,563 sessions there was stability in 874 sessions $(55.8 \%, 95 \%$ $\mathrm{Cl}$ for SEM 53.2 to 58.4). Hypothesis 1 is rejected. Each session had 12 potential comparisons of MAP, HR and time, therefore in the 689 unstable sessions there were 8,268 potential changes $\pm 20 \%$ $(689 \times 12)$. There were $804 / 8,268$ harmful/potentially harmful changes, $922 / 8,268$ beneficial/potentially beneficial changes and 6,542/8,268 opportunities for change where none occurred. Therefore, looking at harmful/potentially harmful changes there were 804/8,268 (9.7\%, 95\% $\mathrm{Cl}$ for SEM 9.1 to 10.4). Looking at potentially beneficial changes this occurred in $922 / 8,268(11.2 \%, 95 \% \mathrm{Cl}$ for SEM 10.5 to 11.9$)$, and if these were combined with the nonsignificant changes this gave a proportion of 7,464/8,268 (90.3\%, 95\% CI SEM 89.6 to 90.9). Therefore Hypothesis 2 is rejected.

Conclusion The results above are encouraging, especially given the stringent definitions of instability used. By making multiple time-period comparisons the validity of the claims of haemodynamic stability are enforced, compared to previous papers. The number of sessions and measurement points combine to add weight to our findings, supported by robust confidence interval data.

\section{P372}

Evaluation of microcirculation before and during continuous renal replacement therapy and the impact of dose prescription C Pipili', CS Vrettou ${ }^{2}$, S Poulaki ${ }^{3}$, A Papastylianou ${ }^{3}$, M Parisi $^{3}$, ES Tripodaki $^{3}$, S loannidou ${ }^{3}$, S Kokkoris ${ }^{3}$, E Douka ${ }^{3}$, S Nanas ${ }^{2}$

'Aretaieion University Hospital, Athens, Greece; 'National and Kapodistrian University of Athens, Greece; ${ }^{3}$ Evaggelismos Hospital, Athens, Greece Critical Care 2012, 16(Suppl 1):P372 (doi: 10.1186/cc10979)

Introduction Microcirculation (MC) might provide evidence for the solute exchange taking place during the dialysis process. Near-infrared spectroscopy (NIRS) with combination of vascular occlusion technique (VOT) allows evaluation of peripheral tissue oxygen utilization and restoration mainly depending on integrity and functionality of vascular endothelium. Our purpose was to evaluate the acute effect of continuous renal replacement therapy (CRRT) on the MC as assessed by NIRS and VOT and to explore the impact of delivered CRRT dose on $M C$ alterations.

Methods A total of 43 critically patients who underwent CRRT were eligible to participate in the study. The mean age of our population was $66 \pm 17$ years and $40 \%$ were females. The APACHE II score was $20 \pm 6$, the mean serum creatinine before the CRRT initiation was $2.6 \pm 1.6$ $\mathrm{mg} / \mathrm{dl}$ and the mean CRRT delivered dose was $23 \pm 6 \mathrm{ml} / \mathrm{kg} / \mathrm{hour}$. The median value of dose was used to form groups of high (>22.5 ml/kg/ hour) and low ( $\leq 22.5 \mathrm{ml} / \mathrm{kg} / \mathrm{hour})$ delivered dose. NIRS parameters were evaluated before CRRT initiation ( $\mathrm{HO})$, at 6 hours $(\mathrm{H} 6)$ and at 24 hours $(\mathrm{H} 24)$ during the CRRT process. Tissue oxygen saturation $\left(\mathrm{StO}_{2}, \%\right)$, defined as the percentage of hemoglobin saturation in the microvasculature compartments, was measured with a probe placed on the thenar muscle. A 3-minute brachial VOT was applied to evaluate the oxygen consumption rate (OCR, \%/minute), the recovery slope (RS, $\% /$ minute), and the hyperemia recovery area as the area (units/minute) under the $\mathrm{StO}_{2} \%$ curve above baseline values.

Results Two-way repeated-measures ANOVA were performed for $\mathrm{StO}_{2}, \mathrm{OCR}, \mathrm{RS}$ and hyperemia recovery area at $\mathrm{HO}, \mathrm{H} 6$ and $\mathrm{H}_{2} 4$. StO $\mathrm{S}_{2}$ correlated with RIFLE on admission and at the time of CRRT initiation ( $r=0.283, P=0.03$ and $r=0.45, P<0.0001$ respectively). There was a significant decrease in OCR with time (hours on CRRT process) (withinsubjects ANOVA $F=4.83, P=0.014$ ) and especially between $\mathrm{H} 0$ and $\mathrm{H} 24$ $(-10.5 \pm 9.4$ vs. $-12 \pm 8.3, P=0.008)$. Furthermore, a significant increase in RS was found in patients who received a high CRRT dose (betweensubjects ANOVA $F=4.5, P<0.05)$, especially at $\mathrm{H} 6$ post CRRT initiation (76 \pm 117 vs. $86 \pm 128, P=0.05$ ).

Conclusion Critically ill patients, receiving a dialysis dose higher than $22.5 \mathrm{ml} / \mathrm{kg} /$ hour, showed improved MC. Further studies are needed to investigate the role of NIRS technology as a tool to assess the need for CRRT initiation in acute renal failure.

\section{P373}

Ultrafiltration during continuous hemofiltration in stabilized ICU patients is not associated with microcirculatory perfusion derangements

B Scheenstra, G Veenstra, M Koopmans, WP Kingma, H Buter, HM Hemmelder, EC Boerma

Medical Centre Leeuwrden, the Netherlands

Critical Care 2012, 16(Suppl 1):P373 (doi: 10.1186/cc10980)

Introduction Ultrafiltration during intermittent haemodialysis has been associated with reduction in microcirculatory perfusion, as observed with sidestream dark-field (SDF) imaging [1]. This technique has also been useful in the evaluation of volume status in critically 
ill patients [2]. To date no data are available on the influence of ultrafiltration during continuous venovenous hemofiltration (CVVH) on microcirculatory perfusion.

Methods In this single-centre, prospective, observational study patients with acute renal failure on CVVH were included after hemodynamic stabilization and written informed consent $A$ fixed dose of net ultrafiltration was calculated for each patient, aiming at a negative total fluid balance of $50 \mathrm{ml} /$ hour. Microcirculatory perfusion was observed with sublingual SDF imaging after 1 hour of zero balance $\mathrm{CVVH}$ (T1) and additionally after 1 hour of negative fluid balance ultrafiltration (T2). The primary outcome was a change in microvascular flow index (MFI) between T1 and T2. Data are presented as median (IQR). Differences are calculated with a nonparametric test for paired data.

Results Eleven patients were eligible for the study; one denied informed consent. One patient could not be evaluated due to the unavailability of the research team and in two patients we were unable to obtain images of proper quality. The median APACHE II score was 26 (21 to 29); at baseline LOS ICU was 5 (3 to 6) days and fluid balance +7.9 (5.1 to 14.2 ) I. Hemodynamic and microcirculatory variables are depicted in Table 1.

Table 1 (abstract P373). (Micro)circulatory variables during ultrafiltration

\begin{tabular}{lccc}
\hline & T1 & T2 & $P$ value \\
\hline RR mean & 71 (65 to 94$)$ & 66 (63 to 95$)$ & 0.87 \\
Heart rate & $97(78$ to 126$)$ & $94(75$ to 123$)$ & 0.03 \\
MFI & $2.9(2.7$ to 3$)$ & 3 (3 to 3] & 0.34 \\
TVD & $20(18$ to 22$)$ & 21 (17 to 23) & 0.5 \\
\hline
\end{tabular}

Conclusion A negative net fluid balance of $50 \mathrm{ml} /$ hour during ultrafiltration in CVVH is not associated with microcirculatory perfusion alterations.

References

1. Bemelmans et al.: Nephrol Dial Transplant 2009, 24:3487-3492.

2. Pottecher et al:: Intensive Care Med 2010, 36:1867-1874.

P374

Plasmapheresis without apparatus in complex care of victims with crush syndrome during the first hours after extrication in a field hospital of EMERCOM of Russia in emergency areas

A Popov', I Yakirevich', A Skorobulatov', V Shabanov ${ }^{2}$

'EMERCOM of Russia, Zhukovsky, Moscow Region, Russia; ${ }^{2}$ All-Russian Centre of Disaster Medicine, Moscow, Russia

Critical Care 2012, 16(Suppl 1):P374 (doi: 10.1186/cc10981)

Introduction This is the generalization of the experience of membranous plasmapheresis without apparatus (MPPA) application in the complex care of victims with crush syndrome (CS) in the field hospital (FH) of EMERCOM of Russia during elimination of medical consequences of earthquakes (Pakistan, 2005; China, 2008; Haiti, 2010). Methods Thirty-eight victims with CS (19 males, 19 females, age $34.5 \pm 4$ ) were in the resuscitation department of the $\mathrm{FH}$. Compound fractures of tubular bones and crushed tissues necrosis were observed. Joint movement was severely restricted and artery pulsation was uncertain. Condition severity: according to the Glasgow Coma Scale $12 \pm 1$, according to APACHE II score $29 \pm 4$. The tendency to hypotension and tachycardia, increase of body temperature and dyspnea intensification were observed, diurnal diuresis decreased. Plasmapheresis treatment was carried out by the MPPA method. A total of $2 \pm 1$ procedures were conducted to each patient with the removal of $70 \pm 10 \%$ of the plasma circulation volume per session. Removed plasma volume was calculated for each victim individually on the basis of average volume before plasma exchange. The procedure frequency was once per day. Substitution means: crystalloids, hydroxyethylized starch, proteins. The MPPA procedure time was from 60 to 120 minutes. MPPA was carried out in all victims during complex care for CS: elimination of painful impact and stressful situation; restoration of acid-alkaline conditions and water-electrolytic balance of blood, maintenance of hemodilution with 25 to $30 \%$ hematocrit; correction of the blood coagulation system; detoxication with the application of active methods of homeostasis correction; prevention and elimination of purulent and septic complications; primary surgical debridement and excision of necrotic mass areas carried out with general anesthesia, no excision conducted; and transport immobilization before evacuation.

Results Among all victims, hemodynamics stabilization was noted in $28 \pm 6$ hours, and dieresis increased up to $1,200 \pm 100 \mathrm{ml} /$ day in $18 \pm 8$ hours. Acute renal failure cases were not noted. All victims in stable condition were evacuated to specialized hospitals by helicopter. No mortality rate during medical aid rendering was noted.

Conclusion MPPA application allows one to reduce the rate of complications and mortality. MPPA application is the method of extracorporeal homeostasis correction option for victims with $\mathrm{CS}$ in a $\mathrm{FH}$ in emergencies.

\section{P375}

Degree of impaired kidney function at hospital discharge has a major impact on long-term survival of critically ill patients recovered from renal failure

S Stads, G Fortrie, J Van Bommel, R Zietse, M Betjes

Erasmus MC, Rotterdam, the Netherlands

Critical Care 2012, 16(Suppl 1):P375 (doi: 10.1186/cc10982)

Introduction Renal replacement therapy (RRT) in critically ill patients with acute kidney injury (AKI) is associated with high mortality. However, little is known about the prognosis of renal function after ICU discharge and the effect of persisting impaired kidney function on longterm survival. The objective of this study was to evaluate the overall long-term mortality in a cohort of ICU patients with AKI necessitating RRT. We hypothesized that both patient characteristics and the degree of renal insufficiency at hospital discharge will influence long-term mortality.

Methods A retrospective cohort study was performed including all patients older than 18 years admitted to the ICU of a tertiary-care center between 1994 and 2010, who underwent continuous RRT during their ICU stay $(n=1,220)$.

Results In-hospital mortality was $54.9 \%$. After hospital discharge, the overall mortality was $75.3 \%$ after a median follow-up of 8.5 years (range 1 to 17 years). Univariate analysis showed that age, surgical or nonsurgical reason for ICU admission and kidney function at discharge were associated with overall survival. Multivariate Cox regression analysis of the association of kidney function at hospital discharge with patient survival was performed, adjusting for age, sex and surgical or nonsurgical admission type. The eGFR at hospital discharge remained independently associated with long-term survival $(P<0.001)$. Only 87 (15.8\%) patients were discharged with an eGFR $>90 \mathrm{ml} /$ minute (using the MDRD formula). In this group 5-year and 10-year survival were respectively $77.6 \%$ and $66.7 \%$. The mortality risk increased for every increase in stage of chronic kidney disease (hazard ratio $1.25, P<0.001$ ). Patients discharged with an eGFR $<30 \mathrm{ml} /$ minute (CKD 4 to $5,37.3 \%$ of patients at hospital discharge) had a 5-year and 10-year survival of only $42.5 \%$ and $28.5 \%$.

Conclusion ICU patients with AKI who received CRRT have a high mortality risk. This is more outspoken for patients who experience incomplete recovery of renal function at hospital discharge. Impaired kidney function at discharge has a major negative impact on their longterm survival. These results stress the importance of preserving kidney function in ICU patients and the need for long-term nephrological follow-up. Future research will have to identify possible determinants in the period following hospital discharge that can be used to prolong survival in these patients.

P376

Long-term survival for ICU patients with acute kidney injury

D Scott', F Cismondi'2, J Lee', T Mandelbaum³', LA Celi', RG Mark', D Talmor ${ }^{2}$ 'MIT, Cambridge, MA, USA; 'Beth Israel Deaconess Medical Center, Boston, MA, USA; '3Sheba Medical Center, Tel Hashomer, Israel Critical Care 2012, 16(Suppl 1):P376 (doi: 10.1186/cc10983)

Introduction A recently published study [1] validated the criteria used in the Acute Kidney Injury Network (AKIN) definitions [2] of the 
three stages of acute kidney injury (AKI) using in-hospital mortality. In the present study, we validate the clinical applicability of the AKIN classifications through long-term survival analysis of AKI patients.

Methods From over 17,000 adult ICU patients in the MIMIC II database $[3,4](V 2.5)$, we excluded patients having end-stage renal disease and those with insufficient data and determined AKI stages for each patient. Multivariate Cox regression was used to determine hazard ratios (HRs) for 2-year survival, controlling for: age, sex, nonrenal Sequential Organ Failure Assessment (SOFA) score and selected co-morbidities.

Results Among the final cohort of 14,525 patients, $43 \%$ had no AKI and $39 \%, 14 \%$ and $4 \%$ developed AKI 1, 2 and 3 respectively. The results of the regression analysis show that AKI 1 (HR 1.12, P<0.05), AKI 2 (HR 1.10, $P=0.05)$ and AKI $3(H R 1.64, P<0.001)$ were significantly associated with increased 2-year mortality. In addition, age (HR 1.04, $P<0.001)$, gender (M) (HR 0.93, $P<0.05)$, nonrenal SOFA score (HR 1.05, $P<0.001)$ and all co-morbidities were significant predictors. Adjusted and unadjusted Kaplan-Meier curves for patients with AKI 3 are remarkably different from each other, suggesting that in these most severely ill patients AKI is only one aspect of their illness.

Conclusion AKI stages 1, 2 and 3 are significant indicators of 2-year mortality. The difference between AKI 1 and 2 is smaller than that between AKI 2 and 3 and it may be prudent to re-examine the criteria used to define AKI to provide better separation among the three classes.

\section{References}

Mandelbaum T, et al.:. Crit Care Med 2011, 39:2659-2664.

2. Mehta RL, et al: Crit Care 2007, 11:R31.

3. Saeed M, et al:: Crit Care Med 2011, 39:952-960.

4. MIMIC II databases [http://physionet.org/mimic2]

\section{P377}

Super high-flux continuous hemodialysis: an efficient compromise for blood purification in sepsis

T Rimmelé, M Page, C Ber, F Christin, J Baillon, J Crozon, C Chapuis-Cellier, R Ecochard, B Allaouchiche

Edouard Herriot Hospital, Hospices Civils de Lyon, France

Critical Care 2012, 16(Suppl 1):P377 (doi: 10.1186/cc10984)

Introduction High cut-off membranes are proposed for blood purification therapy in septic shock. However, albumin loss related to these membranes is a major drawback limiting their clinical acceptance. Super High-Flux membranes with an optimized cut-off may combine enhanced middle molecule clearances (inflammatory mediators) with limited albumin loss. The aim of our study was to compare small, middle molecule clearances and albumin loss between continuous hemodialysis using a Super High-Flux membrane (SHF-HD) and conventional continuous hemofiltration (CVVH).

Methods After approval by the ethics committee, patients were enrolled in a single-blind RCT. Patients with septic shock and acute kidney injury received either SHF-HD (EMiC2 ${ }^{\circledR}$ filter; Fresenius Medical Care) (cutoff $=40 \mathrm{kDa}$, dialysate flow rate of $40 \mathrm{ml} / \mathrm{kg} / \mathrm{hour}$ ) or conventional $\mathrm{CVVH}$ (cut-off $=30 \mathrm{kDa}$, UF flow rate of $40 \mathrm{ml} / \mathrm{kg} / \mathrm{hour}$ ). Each patient received a maximum of three sessions of 48 hours each. Creatinine (113 Da), $\beta_{2}$-microglobulin $\left(\beta_{2}-\mathrm{M}\right)(11.8 \mathrm{kDa})$, kappa free light chain of
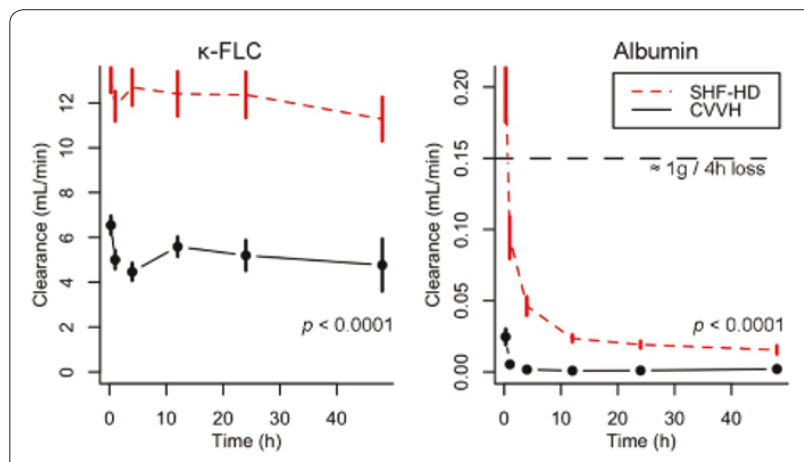

Figure 1 (abstract P377). immunoglobulins ( $\mathrm{k}-\mathrm{FLC})(23 \mathrm{kDa})$ and albumin (68 kDa) clearances were measured at 15 minutes, 1 hour, 4 hours, 12 hours, 24 hours and 48 hours. $\beta_{2}-\mathrm{M}$ and $\mathrm{k}-\mathrm{FLC}$ were chosen as a middle molecular weight marker. A linear mixed-effects model compared clearances between groups.

Results Twenty-four patients were included, 12 in the SHF-HD group (32 sessions) and 12 in CVVH (30 sessions). $\mathrm{K}$-FLC and albumin clearances were higher in the SHF-HD group over time. No difference was observed for creatinine $(P=0.18)$ and $\beta_{2}-\mathrm{M}(P=0.98)$ clearances. Plasma albumin levels and the amount of albumin infused did not differ between groups. See Figure 1.

Conclusion The removal of middle molecular weight molecules is higher with SHF-HD. Albumin loss was limited in both groups, even with SHF-HD. Therefore, SHF membranes seem to represent an alternative to high cut-off membranes for blood purification therapies.

P378

Efficacy of continuous haemodiafiltration using a

polymethylmethacrylate membrane haemofilter in the treatment of sepsis and acute respiratory distress syndrome

M Sakai

Shintakeo Hospital, Takeo, Japan

Critical Care 2012, 16(Suppl 1):P378 (doi: 10.1186/cc10985)

Introduction CHDF using a polymethylmethacrylate membrane is currently widely applied for nonrenal indications in Japan; this technique is used in the treatment not only of patients with sepsis but also of those with cytokine-induced critical illness such as acute respiratory distress syndrome (ARDS) and pancreatitis. This study aimed to investigate the clinical efficacy of continuous haemodiafiltration using a polymethylmethacrylate membrane haemofilter (PMMA-CHDF) in the treatment of patients with sepsis and ARDS.

Methods Thirty-five patients diagnosed with sepsis (ARDS $(n=10)$, pyelonephritis $(n=5)$, cholangitis $(n=5)$, tsutsugami in Scrub typhus disease $(n=1)$, mamushi snake bite $(n=1)$, haemophagocytic syndrome $(n=1)$, antineutrophil cytoplasmic antibody lung disease $(n=1)$, beriberi heart disease $(n=1)$ and unknown causes $(n=8))$ were enrolled in this study between August 2010 and November 2011.The common cause for ARDS in older patients was aspiration pneumonia. Our study group comprised 15 men and 20 women, aged 35 to 85 years (median age 68 years).

Results Before initiating treatment with the PMMA-CHDF, the average APACHE score of these patients was $17.5 \pm 3.6$, whereas the average Sepsis-related Organ Failure Assessment score was $6.5 \pm 1.3$. The duration of PMMA-CHDF treatment was $5.2 \pm 2.3$ days. Following initiation of PMMA-CHDF treatment, early improvement of haemodynamics was observed, along with an increase in the urine output. The average survival rates of patients were $75.6 \%$. The lowest survival rate among diseases (35\%) belonged to the unknown group. The highest survival rate for patients with ARDS was $95 \%$. Moreover, the urine output significantly increased in the survival group.

Conclusion The present study suggests that cytokine-oriented critical care using PMMA-CHDF might be effective in the treatment of sepsis and ARDS, particularly in the treatment of ARDS associated with aspiration pneumonia in older patients.

P379

Possible adsorption mechanism of high mobility group box 1 protein on a polyacrylonitrile (AN69ST) membrane filter O Nishida', M Yumoto', K Moriyama', Y Shimomura', T Miyasho², SYamada

'Fujita Health University School of Medicine, Toyoake, Japan; 'Rakuno Gakuen University, Ebetsu, Japan; ${ }^{3}$ Shino-Test Corporation, Sagamihara, Japan Critical Care 2012, 16(Suppl 1):P379 (doi: 10.1186/cc10986)

Introduction At ISICEM 2011, we reported that AN69ST showed the highest capacity to adsorb high mobility group box 1 protein (HMGB1) when compared with polymethylmethacrylate, polysulfone and high cut-off membrane [1]. Here we focus on whether filtration or surface heparin on AN69ST by a priming circuit with a heparinized saline 


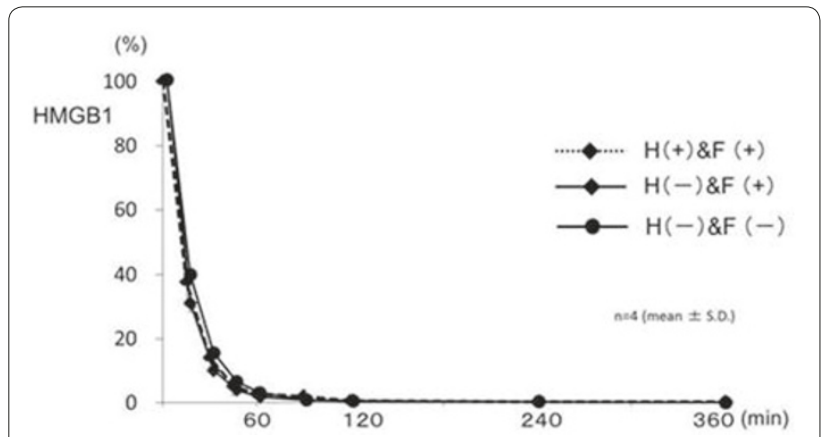

Figure 1 (abstract P379). Time course of HMGB1 levels in the test solution.

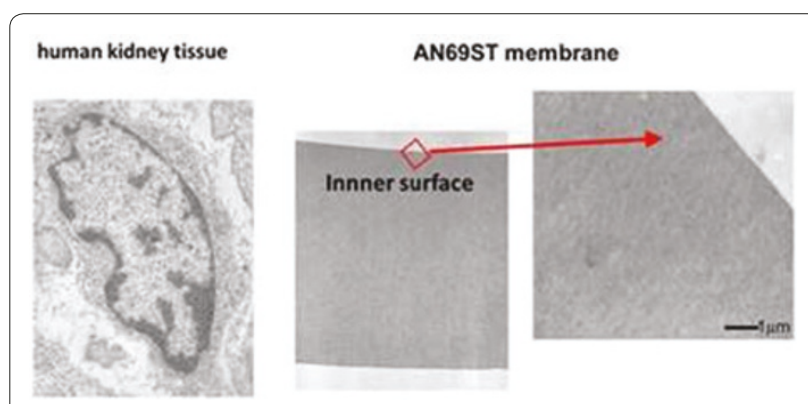

Figure 2 (abstract P379). Immunoelectron microscopy using antiHMGB1 polyclonal antibodies.

contributes to HMGB1, with a heparin-binding protein, adsorption on AN69ST.

Methods The test solution contained $100 \mathrm{~g} \mathrm{HMGB} 1$ and $35 \mathrm{~g}$ albumin in $1,000 \mathrm{ml}$ substitution fluid. We executed three different experimental hemofiltrations with solution flow of $100 \mathrm{ml} /$ minute and: ultrafiltrate flow 1,000 $\mathrm{ml} /$ hour using AN69ST primed with a heparinized saline, $\mathrm{F}(+)$ and $\mathrm{H}(+)$; ultrafiltrate flow $1,000 \mathrm{ml} /$ hour using AN69ST with no heparinized saline, $\mathrm{F}(-)$ and $\mathrm{H}(+)$; and ultrafiltrate flow of $0 \mathrm{ml} /$ hour using AN69ST with no heparinized saline, $\mathrm{F}(-)$ and $\mathrm{H}(-)$. In addition, AN69ST membrane was immunostained using an antibody that confirmed dying on human kidney tissue.

Results The concentration decreases of HMGB1 at 0,60 and 360 minutes indicated no significant differences among the three different hemofiltration experiments (Figure 1). At 60 minutes, reduction rates of HMGB1 were: $\mathrm{F}(+)$ and $\mathrm{H}(+), 97.3 \% ; \mathrm{F}(+)$ and $\mathrm{H}(-), 94.8 \%$; and $\mathrm{F}(-)$ and $\mathrm{H}(-), 96.4 \%$ respectively. $\mathrm{HMGB} 1$ was not detected in bulk layers by immunostaining (Figure 2).

Conclusion Surface heparin or filtration might not contribute to HMGB1 adsorption on the AN69ST membrane. Remarkable adsorption on AN69ST is likely to be influenced by material characteristics, hydrogel structure with moisture content, or negative electric charge and may occur not in bulk layers but on large surfaces of membranes. Reference

1. Yumoto M, et al: Ther Apher Dial 2011, 15:385-393.

P380

High mobility group box 1 levels in septic disseminated intravascular coagulation patients undergoing Polymixin-B immobilized fiber-direct hemoperfusion

Y Ishibe, Y Suzuki, H Sato, G Takahashi, M Kojika, N Matsumoto, Y Inoue, SEndo

Iwate Medical University, Morioka, Japan

Critical Care 2012, 16(Suppl 1):P380 (doi: 10.1186/cc10987)

Introduction The serum levels of high mobility group box 1 (HMGB1) were examined in patients with septic disseminated intravascular coagulation (DIC) undergoing Polymixin-B immobilized fiber-direct hemoperfusion (PMX-DHP).

Methods The subjects were 16 patients with serum endotoxin levels of $1.1 \mathrm{pg} / \mathrm{ml}$ or over. The average APACHE II score was 32.2, the average SOFA score 12.4, and the average DIC score 5.5.

Results Following PMX-DHP, the serum endotoxin level decreased to below the limit of detection in all patients. The serum HMGB1 level decreased significantly to $31.2,16.6$ and $7.9 \mathrm{ng} / \mathrm{ml}$ on days 0,1 , and 2 , respectively. The average of the DIC score improved from 5.6 to 3.9 to 2.9 . Overall, the 30 -day, 60 -day, 90 -day and 180 -day mortality rates were $0,6.3 \%, 12.5 \%$ and $12.5 \%$, respectively.

Conclusion Following initiation of PMX-DHP, the serum HMGB1 level decreased and the DIC score also decreased accordingly.

P381

Polymyxin B-immobilized fiber column hemoperfusion has the ability of endotoxin removal during 24 hours

C Mitaka, Y Ueda, Y Miyawaki, M Yamauchi, T Toyofuku, G Haraguchi, T Kudo

Tokyo Medical and Dental University, Tokyo, Japan

Critical Care 2012, 16(Suppl 1):P381 (doi: 10.1186/cc10988)

Introduction Endotoxin plays an important role in the pathogenesis of septic shock. Endotoxin adsorption therapy by Polymyxin B-immobilized fiber column (PMX) hemoperfusion has been used for the treatment of septic shock patients in Japan. According to the company's recommendation, the standard duration of PMX treatment for patients with septic shock is 2 hours. However, we have shown that greater than 2 hours duration of PMX treatment significantly improved hemodynamics and significantly decreased administration of norepinephrine than 2 hours duration of PMX treatment. Our hypothesis was that PMX treatment had the ability of endotoxin removal during 24 hours. Therefore, the purpose of this study was to evaluate the endotoxin adsorption ability of 24 hours duration of PMX treatment.

Methods In this study, we measured plasma endotoxin concentrations of blood drawn from the radial artery and the outlet circuit of the PMX column after 24 hours duration of PMX treatment in septic shock patients. The assay for endotoxin was performed with separated plasma from heparinized whole blood samples centrifuged at 3,000 rpm for 40 seconds. The high-sensitivity assay was performed by kinetic turbidimetric Limulus assay using a MT-358 Toxinometer (Wako Pure Chemical Industries, Ltd, Japan). This Limulus assay test is specific to endotoxin and has no cross-reaction to $\beta$-glucan. The endotoxin removal rate was defined by the equation: ((radial artery endotoxin concentration - outlet circuit of PMX column endotoxin concentration) / radial artery endotoxin concentration) $\times 100 \%$. The endotoxin removal rate represents endotoxin adsorption ability. Five patients with septic shock were studied.

Results The APACHE II scores of these patients were $26.2 \pm 5.9$ (mean \pm SD, range 18 to 34 ) at admission to the ICU. Three patients survived and two patients died. Before the start of PMX treatment, heart rates were $119 \pm 19 \mathrm{bpm}$, mean arterial pressures were $60 \pm 19$ $\mathrm{mmHg}$, and plasma endotoxin concentrations of radial arterial blood were $91.4 \pm 7.4 \mathrm{pg} / \mathrm{ml}$ (mean $\pm \mathrm{SD}$ ). After 24 hours duration of PMX treatment, plasma endotoxin concentrations decreased from $55.0 \pm 58.9 \mathrm{pg} / \mathrm{ml}$ (radial arterial blood) to $19.4 \pm 29.5 \mathrm{pg} / \mathrm{ml}$ (outlet circuit of PMX column). The endotoxin removal rate was $62.8 \pm 22.1 \%$, suggesting that endotoxin adsorption ability is still retained during 24 hours PMX treatment.

Conclusion These findings suggest that 24 hours duration of PMX treatment is effective to remove endotoxin. Further studies are needed to confirm this ability.

References

1. Mitaka C, et al:: Shock 2009, 32:478-483.

2. Kambayashi J, et al.: J Biochem Biophys Methods 1991, 22:93-100. 
P382

Polymyxin B-direct hemoperfusion therapy could contribute to hemodynamics and outcomes in emergency surgical patients

M Yokota, T Goto, T Harada, M Takeda, R Moroi, M Namiki, A Yaguchi Tokyo Women's Medical University, Tokyo, Japan

Critical Care 2012, 16(Suppl 1):P382 (doi: 10.1186/cc10989)

Introduction Polymyxin B-direct hemoperfusion (PMX-DHP) (Toraymyxin ${ }^{\oplus}$; Toray Medical Co., Tokyo, Japan) has been approved to treat patients with endotoxemia and/or severe sepsis due to Gramnegative infection since 1994 in Japan. However, its efficacy and indication are still controversial. Recently, randomized controlled studies were performed in other countries. Our hypothesis is that PMX-DHP may be useful for emergency-operated patients to eliminate endotoxins from the systemic circulation after removal of the source of infection.

Methods From July 1994 to May 2011, all adult patients treated with PMX-DHP in our ICU were included in this retrospective observational study. Patients' clinical and microbiological data were collected from medical archives. The emergency postoperation patients and the medical patients were compared for severity, mortality, and hemodynamic status. Values are expressed as mean \pm SD. Data were analyzed by Mann-Whitney $U$ test, chi-square test and Fisher's exact probability test. $P<0.05$ was considered statistically significant.

Results One hundred and sixty-six patients (98 men, 68 women; age range 24 to 92 years (mean $64.7 \pm 13.3$ ) ) were studied. The mortality rate was $34.9 \%$ at 28 days after PMX-DHP.There were 129 (77.7\%) emergency surgical patients and $37(22.3 \%)$ medical patients. The APACHE II score on the day of PMX-DHP was not significantly different between surgical and medical patients $(20.3 \pm 7.0$ vs. $19.2 \pm 8.1, P=0.417)$. Mean arterial pressure (MAP) significantly improved in emergency surgical patients before and after PMX-DHP therapy $(73.7 \pm 24.8$ vs. $79.7 \pm 26.0 \mathrm{mmHg}$, $P=0.017)$, while MAP was not statistically different in medical patients $(69.7 \pm 24.2$ vs. $76.7 \pm 27.1 \mathrm{mmHg}, P=0.178)$. The inotropic score had no statistical difference between before and after PMX-DHP in both surgical and medical patients $(13.2 \pm 19.8$ vs. $12.6 \pm 19.2, P=0.61$; $16.8 \pm 27.3$ vs. $13.8 \pm 23.6, P=0.65$, respectively). The mortality rates at 28 days, 90 days, 0.5 year and 1 year after PMX-DHP were significantly different between surgical and medical patients ( 28.7 vs. $56.8,43.8$ vs. $83.3,52.2$ vs. $85.7,54.5$ vs. $91.2 \%, P<0.0001$, respectively).

Conclusion MAP increased in surgical patients but did not change in medical patients after PMX-DHP, and the inotropic score was not significantly different in both sets of patients. The mortality was significantly lower in surgical patients than in medical patients.
P383

Clinical impact of enhanced cytokine clearance with sustained highefficiency daily diafiltration using a mediator-adsorbing membrane (SHEDD-fA) in patients with severe sepsis

O Nishida', T Nakamura', N Kuriyama', K Moriyama', T Miyasho², SYamada

${ }^{1}$ Fujita Health University School of Medicine, Toyoake, Japan; 2Rakuno Gakuen University, Ebetsu, Japan; 'Shino-Test Corporation, Sagamihara, Japan Critical Care 2012, 16(Suppl 1):P383 (doi: 10.1186/cc10990)

Introduction SHEDD-fA is an effective modality that makes the best use of three principles in the treatment of severe sepsis: diffusion, convection and adsorption. We reported the efficacy of SHEDD-fA for the treatment of severe sepsis at the 31st ISICEM 2011 [1]. Here we present the blood clearance $(\mathrm{CL})$ of seven important cytokines with SHEDD-fA.

Methods Ten critically ill patients were studied who were on SHEDD$\mathrm{fA}$, at $\mathrm{QB}=150 \mathrm{ml} /$ minute, $\mathrm{QF}=1,500 \mathrm{ml} /$ hour (post dilution) and $\mathrm{QD}=300$ to $500 \mathrm{ml} /$ minute as a nonrenal indication. In order to maximize cytokine adsorption efficiency, we used a large-size $\left(2.1 \mathrm{~m}^{2}\right)$ PMMA dialyzer. Blood samples were taken to measure the $C L$ of plasma cytokines (HMGB-1, IL-6, IL-8, IL-10, G-CSF, MCP-1 and MIP-1) at 1 hour and 3 hours after initiation (in one cytokine by 62 to 107 samples).

Results The median values of $\mathrm{CL}$ with interquartile ranges of each cytokine (molecular weight: kDa) were: HMGB1 (30 kDa), $53.1 \mathrm{ml} /$ minute (2.1 to 12.5$)$; IL-6 (21 kDa), $39.9 \mathrm{ml} /$ minute (12.4 to 70.6$)$; IL-8 ( $8 \mathrm{kDa}), 64.1 \mathrm{ml} /$ minute $(-0.5$ to 82.0$) ; \mathrm{IL}-10 \mathrm{ml} /$ minute (35 to $40 \mathrm{kDa}$ ), $45.6 \mathrm{ml} /$ minute $(0.5$ to 88.3$)$; G-CSF ( $19 \mathrm{kDa}), 33.2 \mathrm{ml} /$ minute $(9.3$ to 60.8); MCP-1 (8.7 kDa), $68.5 \mathrm{ml} /$ minute (-14.4 to 125.4$)$; and MIP-1 (7.8 $\mathrm{kDa}), 66.5 \mathrm{ml} /$ minute (18.6 to 100.0 ). In particular, CL of HMGB1 was positively correlated with pre-SHEDD-fA blood levels, indicating the mechanism of HMGB1 removal was through adsorption. As a result of enhancing the intensity of the dosage, $\mathrm{CL}$ (53 $\mathrm{ml} /$ minute) of HMGB1 was higher than that $(25 \mathrm{ml} /$ minute) of an in vitro experiment that we reported at the 31st ISICEM 2011. See Figure 1.

Conclusion Taking into account the fact that the creatinine $\mathrm{CL}$ of native kidney function is $100 \mathrm{ml} /$ minute, our findings suggest that SHEDD-fA is a feasible adjusted modality for the treatment of patients with severe sepsis, with or without acute kidney injury. Considering our other laboratory findings, deep filtration may enhance blood clearance.

Reference

1. Nishida O, et al:: Contrib Nephrol 2011, 173:172-181.
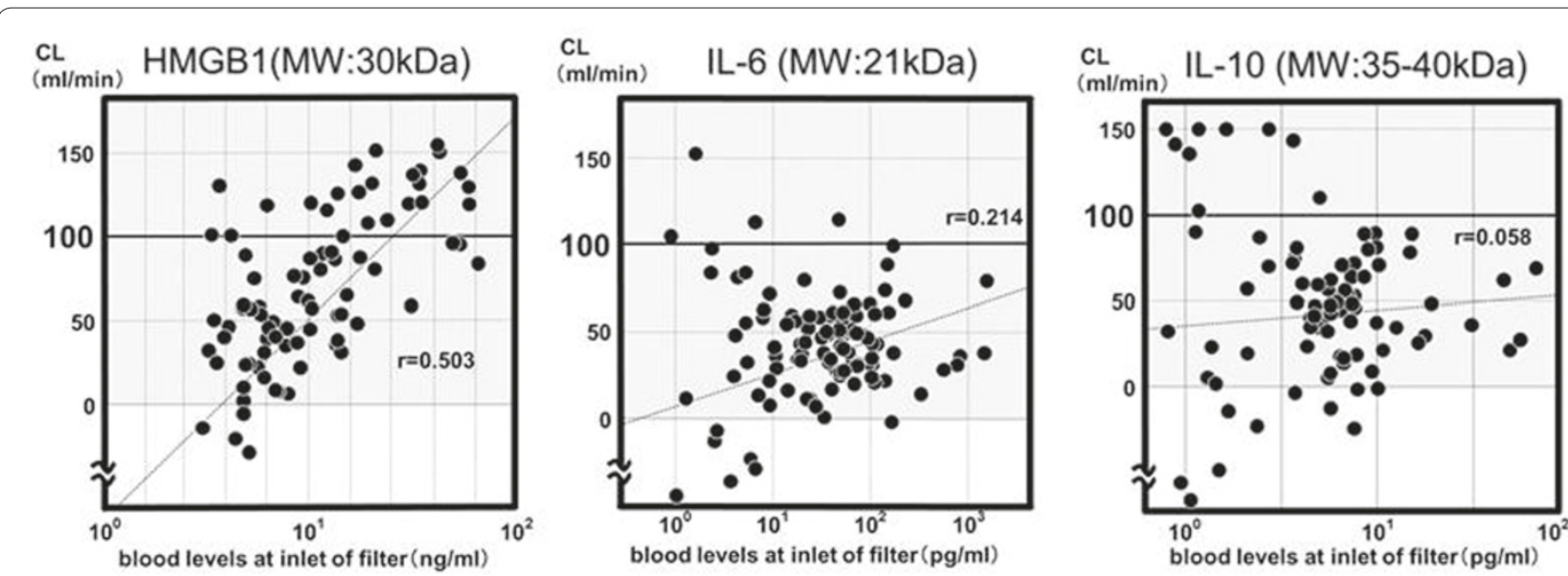

Figure 1 (abstract P383). Correlation between clearance and blood level of cytokines. 
P384

Mortality and priority level for ICU admission in the setting of limited critical care beds in El Salvador

V Segura, NR Reyes, ME Tejada, EM Zolano

Hospital San Rafael, Santa Tecla, El Salvador

Critical Care 2012, 16(Suppl 1):P384 (doi: 10.1186/cc10991)

Introduction In El Salvador there are a limited number of ICU beds. The ICU bed per inhabitant ratio is only 0.7 per 100,000 in a country with a population of $6,071,774$ [1]. The aim of this study was to show the impact that the ICU bed deficit has on the mortality of the patients admitted to the internal medicine floor.

Methods We conducted a descriptive, cross-sectional study. A nonprobabilistic sample was estimated using EPIDAT 4.0 (mortality rate $16 \%, 95 \% \mathrm{Cl}, P<0.05$ ). We enrolled 513 patients admitted to the Internal Medicine ward, from June to November 2011. All patients were evaluated using the ICU admission priority criteria of the Society of Critical Care Medicine (SCCM). We divide the patients into high priority (SCCM priority levels 1 and 2) and low priority (SCCM priority levels 3 and 4) for ICU admission. The probability of death using APACHE II score and mortality rate was calculated for each group, in order to obtain the Standardized Mortality Ratio (SMR). A t test and a Mantel-Haenszel test were used for statistical analysis between groups.

Results A total of 513 patients were included in the study; 101 patients in the high priority group and 412 patients in the low priority group. There was a significantly higher mortality $(P=0.048)$ in the high priority level group especially with an APACHE score less than 9.0 (Figure 1).

Conclusion The study shows that there is an increased mortality rate in patients with high priority level for admission to the ICU with an APACHE II score less than 9 points. This represents 90 patients/year whose survival and prognosis could be improved by increasing the number of ICU beds available.

Reference

1. Boletin de indicadores del Sistema Nacional de_Salud 2009 [http://www. salud.gob.sv/archivos/pdf/Boletin_de_indicadores_del_Sistema_Nacional_ de_Salud_2009.pdf]

\section{P385}

Mainz Emergency Evaluation Scoring in combination with capnometry predicts outcome in trauma patients

EH Hajdinjak', ŠG Grmec ${ }^{2}$, MK Križmarić3 ${ }^{3}$ ET Torkar², DB Buić-Rerečić ${ }^{2}$ MZ Zelinka², MŠ Škufca

'Center for Emergency Medicine, University of Ljubljana, University of Maribor, Maribor, Slovenia; ${ }^{2}$ Community Health Centre Ljubljana, University of Ljubljana, University of Maribor, Ljubljana, Slovenia; ${ }^{3}$ Faculty of Medicine, University of Maribor, Slovenia

Critical Care 2012, 16(Suppl 1):P385 (doi: 10.1186/cc10992)

Introduction This prospective study assessed the efficacy of the predicting power for mortality of two different prehospital scoring systems in trauma patients. We present an improved Mainz Emergency Evaluation Scoring (MEES) in combination with capnometry (MEESC). MEESc is a new scoring system. We compared the prognostic role of outcome of these two prehospital descriptive scoring systems with the prognostic scoring system APACHE II.

Methods In a prehospital setting, the values of MEES and capnometry (initial and final) were collected from each patient. We added final values of petCO $\mathrm{C}_{2}$ to the MEES scoring system and ranked from 0 to 2 so that the final maximum sum of the scoring system would be 30 without any change in the minimal score being 10 . This study was performed over 10 years (from January 2000 to July 2010) and included 231 consecutive patients hospitalized for major trauma, requiring intubation at the roadside and in whom prehospital pet $\mathrm{CO}_{2}$ has been recorded. Patients younger than 16 years and those with severe hypothermia were excluded from the study. There were 156 males and 75 females, age range 16 to 84 , mean $43.6 \pm 17.8$ years. In hospital we calculated the APACHE II scoring system for each patient. For every scoring system, the sensitivity, specificity, correct outcome prediction and area under the ROC curve were determined.

Results For prediction of mortality, the best cut-off points were 19 for MEES and 22 for MEESC. The area under the ROC curve was 0.63 for MEES, 0.81 for MEESc ( $P=0.02$ vs. MEES) and 0.84 for APACHE II $(P<0.01$ vs. MEES).

Conclusion There were significant differences between MEES and MEESc. MEESc improved the results of MEES in predicting outcome for severe trauma patients. The prehospital use of the improved MEESC could be an efficient communication protocol between the prehospital and hospital settings (MEESc is comparable with APACHE II).

\section{P386}

Predicting outcome in the ICU: comparison of Ranson criteria and Ranson + CRP levels in acute pancreatitis

$\checkmark$ Inal, L Yamanel, B Comert

GATA, Ankara, Turkey

Critical Care 2012, 16(Suppl 1):P386 (doi: 10.1186/cc10993)

Introduction The aim of this study was to investigate and compare Ranson criteria (RC) and RC + serum CRP levels as a feasible, practical and precise method in acute pancreatitis (AP) cases admitted to the ICU in respect of length of stay (LOS) predicting severity of disease.

Methods This study was based on determination of RC scores in AP cases in a retrospective manner. On the other hand, this study included only the patients' zero-time RC scores, not the 48-hour scores, for the sake of more practical precision. Serum CRP levels were found to have prognostic importance in AP, significantly more than $150 \mathrm{mg} / \mathrm{l}$ in necrotizing $A P$, at $50 \mathrm{mg} / \mathrm{l}$ in this study. Therefore, patients' were evaluated for RC and RC + CRP scores for comparison. However, RC had been etiologically modified for presence of gall bladder stones (GBS); only the cases without GBS were included in order to prevent bias of

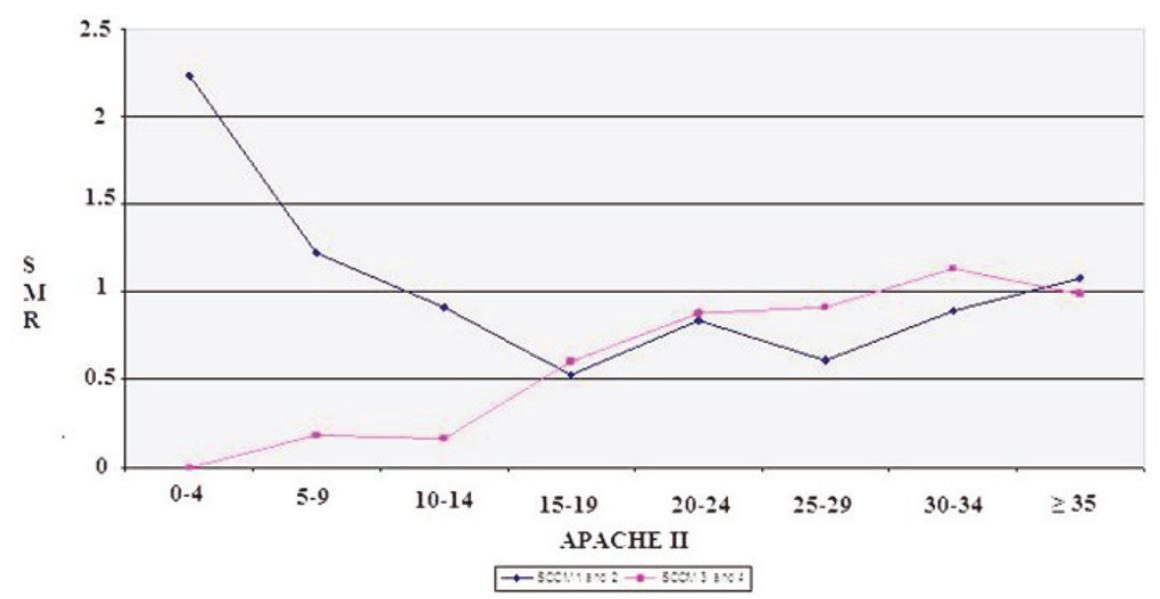

Figure 1 (abstract P384). SMR according to APACHE II and SCCM criteria. 
results. In addition, necrotizing cases were assumed to increase CRP levels more than predicted and were also excluded. After the exclusion of cases, 89 patients' data were collected and compared for LOS in the ICU between 2005 and 2009.

Results Statistical analysis of patients' data for significance and receiver operating curve (ROC) analysis to predict LOS, therefore pointing to disease severity, was executed. All of the statistical comparisons were found significant for predicting LOS; RC $(P<0.05), \mathrm{RC}+\mathrm{CRP}$ together $(P<0.01)$ and CRP alone $(P<0.04)$. Severity of the disease and therefore LOS were increased for RC score $>3$ and CRP levels $>50 \mathrm{mg} / \mathrm{l}$. ROC analysis resulted in RC (AUC 0.895), RC + CRP (AUC 0.901) and CRP (AUC 0.823) for LOS.

Conclusion AP cases usually require ICU care and treatment. There are some consented scoring systems such as RC, APACHE II and Glasgow in predicting disease severity and guiding the physician's approach. Although the most sensitive and specific method seemed to be APACHE II scoring, it is time consuming and complex. On the other hand, RC and Glasgow scorings need to be evaluated in 48 hours. In the end, in the hardworking hours on the ICU, we need a more practical method of provision. In this study, we have found no priority of RC, RC + CRP and CRP alone in predicting AP outcome, excluding GBS disease and necrotizing cases. We conclude that, practically, ICU physicians could substantially depend on CRP levels alone in the evaluation and approach in these specific cases of AP.

\section{P387}

Number of failed organs and response to therapy determine outcome in patients with acute pancreatitis requiring level 1 organ support

G Morris-Stiff, A Baker, A Breen, A Smith

Leeds Teaching Hospitals, Leeds, UK

Critical Care 2012, 16(Suppl 1):P387 (doi: 10.1186/cc10994)

Introduction The aim of the study was to establish if the number of organs failing at admission to the ICU and the response to support had a bearing on outcome in patients with severe acute pancreatitis (SAP). Methods Only SAP patients requiring organ support were included in the analysis. Gallstones (55\%) and alcohol were the commonest aetiologies. The proportion of patients with one, two or three system failures at baseline, 24,48 , and 72 hours were calculated and related to outcome.

Results A total of 123 patients ( 85 male and 38 female) with a mean age of 58 years met the study criteria. The numbers of patients presenting with one, two and three organ failures were 29,70 and 24 respectively, of which the mortality was six (21\%), $29(41 \%)$ and $14(48 \%)$. Subsequent figures were 24,57 and 39 with mortalities of four (17\%), 19 (33\%), and $24(62 \%)$ at 24 hours; 21,53 and 43 with mortalities of two (10\%), 18 (34\%), and $26(60 \%)$ at 48 hours; and 17,49 and 45 with mortalities of zero $(0 \%), 16(33 \%)$, and $28(62 \%)$ at 72 hours.

Conclusion These data allow prognostication of patients with SAP requiring organ support. At 72 hours, the prognosis of patients with single organ failure is excellent and that of patients with three-organ failure remains poor.

\section{P388}

Mortality predictors in acute pancreatitis admitted to the ICU

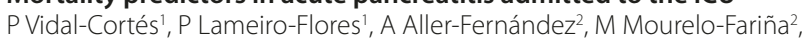
R Gómez-López', P Fernández-Ugidos', M Alves-Pérez',

E Rodríguez-García

${ }^{1}$ CHU Ourense, Spain: ${ }^{2}$ CHU A Coruña, Spain

Critical Care 2012, 16(Suppl 1):P388 (doi: 10.1186/cc10995)

Introduction Patients diagnosed with acute pancreatitis (AP) are usually admitted to our units. Despite using a lot of scores, none has proved an acceptable yield to identify patients with higher mortality risk. Our purpose is to identify mortality predictors of patients admitted to our ICU diagnosed with AP.

Methods We performed a retrospective study in which we analyzed patients diagnosed with AP admitted to a 24-bed ICU between January 2000 and December 2009. Postcardiopulmonary bypass pancreatitis and readmissions were excluded. Demographic characteristics, comorbidities and parameters included in severity scores (APACHE II, SAPS II, SOFA) were studied. A Cox proportional hazard regression model was used to assess the effect of each variable on patient survival. Results A total of 122 patients diagnosed with AP were admitted to our ICU between January 2000 and December 2009 (68.9\% men, mean age: $60.5 \pm 14$ years); $43.4 \%$ were smokers and $41.8 \%$ alcohol consumers. The most frequent comorbidity was hypertension $(41.8 \%)$, followed by dyslipemia $(24.6 \%)$, cardiac disease $(17.2 \%), \mathrm{DM}$ and pulmonary pathology $(13.1 \%)$. Solid or hematologic malignancy $(10.6 \%)$, chronic renal failure (9\%) and hepatic pathology (5.7\%) were other comorbidities. Biliary etiology was the most frequent (48.5\%), followed by alcoholic AP (20.5\%) and unknown etiology (17.2\%); $3.3 \%$ were postbiliary manipulation (surgery or ERCP) AP. The mean APACHE II score at admission was $16.42 \pm 7.64$. In total, $56.6 \%$ patients needed mechanical ventilation, $50.8 \%$ vasopressors and $40.2 \%$ renal support during their ICU stay. The ICU length of stay (LOS) was $16.55 \pm 21.6$, hospital LOS $45.39 \pm 45.42$ days. A total of $28.7 \%$ patients died in the ICU, and $38.5 \%$ during their hospital stay. We did not find any relation between comorbidities or AP etiology and outcome. Mortality predictors in AP patients were: PaFi relation $(-0.007, P=0.006)$, mean and systolic arterial pressure $(-0.39, P=0.019$ and $-0.038, P=0.001$ respectively), $\mathrm{pH}(-5.641, P=0.001), \mathrm{HCO}_{3}(-0.081, P=0.050)$, creatinine $(0.347$, $P<0.001)$, urea $(0.008, P=0.002)$, 24 -hour diuresis $(-0.001, P=0.002)$ and Glasgow Coma Scale $(-0.312, P=0.050)$.

Conclusion Comorbidities and AP etiology are not predictors of ICU mortality. Of the variables included in severity scores, only those related to organ dysfunction (hemodynamic - SAP, MAP, $\mathrm{pH}, \mathrm{HCO}_{3}^{-}$; respiratory-PaFi relation; and renal - $\mathrm{Cr}$, urea and 24-hour diuresis) are ICU mortality predictors in AP patients.

P389

System biology prediction model based on clinical data: highly accurate outcome prediction in patients with acute-on-chronic liver failure

MJ McPhail, DL Shawcross, RD Abeles, T Chang, GL Lee, MA Abdulla,

C Willars, E Sizer, G Auzinger, W Bernal, JA Wendon

King's College Hospital, London, UK

Critical Care 2012, 16(Suppl 1):P389 (doi: 10.1186/cc10996)

Introduction Present outcome prediction tools for patients with acuteon-chronic liver failure during critical illness are only of moderate accuracy. Regression methods on latent variables (usually applied to top-down system biology applications with spectroscopic data) may offer significant advantages over logistic regression techniques as multiple cross-correlations are acceptable in this form of modelling.

Methods Between 1 January 2000 and 31 December 2010 all patients admitted to the Liver Intensive Therapy Unit (LITU) at King's College Hospital had daily prospective collection of demographic, biochemistry and bedside physiology. Logistic regression modelling (LRM) and partial least-squares discriminant analysis (PLSDA), Model for Endstage Liver Disease (MELD) and APACHE II scores were compared using receiver operating characteristic (ROC) curve analysis.

Results A total of 986 patients (median age 52 (range 16 to 90 ) years; $603(62 \%)$ male) with cirrhosis and emergency LITU admission were identified. The median APACHE II score was 21 ( 5 to 50 ) and the median MELD score 23 (3 to 50 ). Overall LITU survival was $63 \%$ and survival to hospital discharge $51 \%$. Predictive accuracy at day 3 was improved in all models over admission values. The AUROC for LITU survival for MELD and APACHE scores on day 3 was 0.78 (95\% Cl 0.75 to 0.82 , sensitivity $72 \%$, specificity $75 \%$ ) and 0.83 (0.78 to 0.83 , sensitivity $83 \%$, specificity $63 \%$ ) respectively. A LRM utilising nine variables had an AUROC of 0.85 ( $95 \% \mathrm{Cl} 0.82$ to 0.87 , sensitivity $72 \%$, specificity $83 \%$ ). Two-component PLSDA identified 30 variables with independent prognostic significance. Performance in outcome prediction was improved over logistic regression at day 3 - sensitivity $86 \%$, specificity $81 \%$, AUROC 0.91 ( 0.89 to $0.93, P<0.001$ for all comparisons) in a model incorporating 30 variables. Cross-validation and permutation analysis confirmed the internal validity of this method.

Conclusion This application of latent variable regression modelling techniques to intensive care datasets demonstrates high accuracy of 
prediction. Liver-specific outcome schema based on logistic regression may not fully describe the complex cross-correlating interactions that PLS techniques are designed to incorporate. Further validation in other centres and disease groups is warranted.

\section{P390}

Prognostic relevance of arterial ammonia levels in different acute and acute-on-chronic liver diseases

V Fuhrmann, A Drolz, B Jaeger, M Wewalka, R Saxa, T Horvatits,

TPerkmann, C Zauner, P Ferenci

Medical University Vienna, Austria

Critical Care 2012, 16(Suppl 1):P390 (doi: 10.1186/cc10997)

Introduction Increased levels of arterial ammonia in patients with acute liver failure (ALF) are associated with increased mortality. There is a lack of data for prognostic impact of arterial ammonia in patients with acute-on-chronic liver failure (AoCLF) and hypoxic hepatitis $(\mathrm{HH})$. We evaluated arterial ammonia levels and their prognostic relevance in patients with $\mathrm{HH}, \mathrm{ALF}, \mathrm{AoCLF}$ and without evidence for any liver disease.

Methods One-hundred and ninety-seven critically ill patients were studied at the Medical University Vienna: 72 patients with $\mathrm{HH}, 22$ with ALF, 58 with AoCLF and 45 critically patients without evidence for liver disease. Arterial ammonia concentrations were assessed on a daily basis in all patients and compared among the four study groups as well as between 28-day survivors and nonsurvivors.

Results The 28-day mortality rates in HH, ALF, AoCLF and in the control group were $54 \%(n=39), 27 \%(n=6), 53 \%(n=31)$ and $27 \%(n=12)$, respectively. Peak arterial ammonia levels in patients with $\mathrm{HH}$ were significantly higher in 28-day nonsurvivors compared to survivors $(P<0.01)$. Cox regression identified peak arterial ammonia concentrations as an independent predictor for 28 -day mortality $(P<0.01)$. Peak arterial ammonia levels in 28-day transplant-free ALF survivors were significantly lower compared to ALF patients who died or underwent liver transplantation $(P<0.05)$. There was no association between outcome and arterial ammonia in AoCLF patients and in the control group.

Conclusion Elevated arterial ammonia levels are frequently observed in critically ill patients with liver injury but not in patients of comparable severity of illness without hepatic impairment. They indicate poor prognosis in $\mathrm{HH}$ and ALF, but not AoCLF.

\section{P391}

Liver failure secondary to alcoholic liver disease carries a worse prognosis than other aetiologies of liver failure: retrospective analysis of routine biochemical markers in critically ill patients with liver failure

E McCarron, I Welters

Royal Liverpool University Hospital, Liverpool, UK

Critical Care 2012, 16(Suppl 1):P391 (doi: 10.1186/cc10998)

Introduction Patients with liver failure in the critical care unit frequently provide physicians with problems about management and prognosis. Alcoholic liver disease (ALD) in particular is showing an increase in admission and mortality in the UK [1]. Current biochemical tests make it difficult to differentiate between types and severity of liver damage and fail to give a true idea about prognosis and outcome, often only showing low-grade derangements before hyperacute decompensation of liver function. The aim of this study was to look at various liver function tests (LFTs) routinely recorded in patients admitted to critical care with liver failure, to see whether they differed between ALD and nonalcoholic aetiologies (NALD); that is, drug overdose and nonalcoholic steatohepatitis, and so forth. We also aimed to assess their prognostication value and relation to severity of disease scores. Methods A total of 119 patients admitted to the ITU with liver failure ( 66 ALD and 53 NALD) between 2008 and 20011 were included. Each patient had admitting electrolytes, haematology, LFTs and clotting studies along with APACHE II score, length of stay and ventilation and vital organ support requirement.

Results ALD patients were found to have lower sodium (mean 135.56; $P=0.004)$ and be hypocalcaemic $(P=0.015)$, as well as having more deranged LFTs $(P<0.001)$ and clotting studies $(P<0.001)$. ALD patients also had longer ITU stays $(P<0.001)$ and higher mortality rates $(45.45 \%$ ALD vs. $13.2 \%$ NALD). Receiver-operated curve analysis revealed that current biochemical markers (ALT, PT, GGT, albumin) are not sensitive and specific enough in detecting ALD. The prothrombin time yielded the best area under the curve with $80.4 \%$ in ALD versus $71.7 \%$ in NALD. None of the markers was discriminatory for determining the type of liver damage.

Conclusion Our results suggest that currently used markers of liver disease are neither sensitive nor specific enough in patients with failure secondary to ALD. Research is needed to develop novel biomarkers to better prognosticate outcome. Aetiology of acute-on-chronic liver failure plays a major role in determining outcome, and subgroups of liver patients should be analysed individually. Studies [2,3] have shown that various markers are released depending on the type of damage and differ in acute liver damage of different origin. Better understanding of their role could prove useful in these patients.

References

1. Thomson SJ, et al: Alcohol Alcohol 2008, 43:416-422.

2. Antoine DJ, et al:: Keratin-18 and HMGB1 as predictive biomarkers for mode of cell death and clinical prognosis during acetaminophen hepatotoxicity in man. $J$ Hepatol 2012. [Epub ahead of print]

3. Zhou RR, et al:: BMC Gastroenterol 2011, 11:21.

P392

Incidence, morbidity and mortality of admissions related to alcohol consumption on critical care: a single-centre experience

A Retter, F Tait, M Stockwell

St Helier Hospital, London, UK

Critical Care 2012, 16(Suppl 1):P392 (doi: 10.1186/cc10999)

Introduction Excessive alcohol consumption is a major challenge to public health. In 2000 it accounted for $4 \%$ of the global disease burden. However, the relationship between alcohol and health is complex and the burden it places on admissions to critical care is uncertain.

Methods We conducted a retrospective analysis of prospectively collected data on the influence of excess alcohol consumption on the outcome of patients admitted from July 2009 to July 2011. The admitting physician determined the relationship between alcohol use and admission. No patients were excluded. All continuous data are expressed as medians and were compared using the Wilcoxon MannWhitney $\mathrm{U}$ test. Categorical data were compared using the chi-squared test.

Results A total of 1,150 patients were admitted, 129 cases (11.2\%) were identified as having excess alcohol consumption. Of these cases $34 \%$ were women, whilst $48 \%$ of the controls were female. The median age of the cases was 54 years versus 68 years for the controls $(P<0.001)$. The cases had a lower APACHE II score, 14.3 vs. $15.8(P=0.002)$. Twentyfour $(18.6 \%)$ of the cases with excess alcohol consumption died on the ICU compared to 141 controls (13.8\%) $(P>0.1)$. The hospital mortality was similar between the two groups, 28 (21.7\%) against $215(21.1 \%)$ controls $(P>0.5)$. The cases spent longer on the ICU, median 3.95 days versus 2.9 in the controls $(P<0.001)$. On admission the cases required a median of 2.0 organ supportive therapies compared to 1.8 in the control group $(P<0.001)$. The cases were ventilated for a mean of 4.1 days compared to 2.4 days in the controls $(P<0.001)$. There was no difference in the rate of sepsis between either group, $10 \%$ in the cases and $9.8 \%$ in the controls. Twenty-six patients were admitted with known alcoholic cirrhosis $(0.23 \%), 10$ with oesophageal varices and three with acute pancreatitis related to alcohol.

Conclusion To our knowledge this is the largest single-centre assessment of the burden of excess alcohol consumption on patients admitted to critical care. Eleven per cent of all admissions to the ICU were complicated by excess alcohol consumption. The ITU mortality of these patients was increased when compared to the controls, despite the patients having an equivalent APACHE score on admission and tending to be younger. The cases spent less time in hospital than the controls. This was due to a bimodal distribution of their survival curve. Our study is limited by its retrospective design and the risk of selection bias. 
P393

Changing outcomes in patients with chronic liver disease in intensive care: a decade of experience

MJ McPhail, DL Shawcross, RD Abeles, G Huei-Lee, M Abdulla, T Chang,

CWillars, E Sizer, G Auzinger, W Bernal, J Wendon

King's College Hospital, London, UK

Critical Care 2012, 16(Suppl 1):P393 (doi: 10.1186/cc11000)

Introduction Patients with chronic liver disease requiring intensive care are thought to carry a poor prognosis in comparison with noncirrhotic patients with similar severity of illness. During the last decade improvements in multiple areas of management in patients in the ICU have occurred but improvement in outcomes in patients with cirrhosis has not been shown.

Methods Between 1 January 2000 and 31 December 2010 all patients admitted to the Liver Intensive Therapy Unit (LITU) at King's College Hospital had daily prospective collection of demographic, biochemistry and bedside physiology. These data were used to quantify the severity of illness (APACHE II and Model for End-stage Liver Disease (MELD)) and outcomes in these patients.

Results A total of 958 patients (median age 52 (range 16 to 90 ) years; $603(62 \%)$ male) with cirrhosis and emergency LITU admission were identified. Aetiology of cirrhosis was alcohol in $43 \%$, viral in $10 \%$, autoimmune disease in $10 \%$ and nonalcoholic fatty liver disease/ metabolic in the remainder. The pattern of aetiology of cirrhosis did not change over time and a viral aetiology was associated with improved outcome (OR $0.53,95 \% \mathrm{Cl} 0.34$ to $0.81, P=0.003$ ); alcohol was not associated with poorer outcome $(P=0.09)$. The primary reasons for admission were bleeding (33\%), sepsis (27\%), hepatic encephalopathy (17\%), metabolic (7\%) and other (16\%). The median APACHE II score was 21 (5 to 50) and the median MELD score 23 (3 to 50). Overall LITU survival was $63 \%$ and survival to hospital discharge $51 \%$. LITU survival increased from $47 \%$ to $73 \%$ over the study period (2000 to 2010) with hospital outcome improving from $40 \%$ to $63 \%$. The median admission APACHE II score fell from 23.4 to 21.9 over the study period $(P<0.001)$ with the MELD score falling from 23.4 to $18.3(P<0.001)$. Length of LITU stay did not change significantly over the study period $(P=0.092)$. The reduction in illness severity was predominantly due to a smaller percentage of patients with renal failure and those with three or more organs in failure (32\% up to 2005 and $24 \%$ post $2005, P=0.004)$. The reduction in MELD score related to decreased renal dysfunction; creatinine over the study period $(1.9 \mathrm{mg} / \mathrm{dl}$ to $1.6 \mathrm{mg} / \mathrm{dl}, P<0.001)$ with no change in bilirubin, and by contrast a small rise in international normalised ratio (INR 1.8 to $2.2, P=0.07$ ).

Conclusion Survival of patients with cirrhosis admitted to the specialist LITU is improving over time. The factors relating to this may be resultant upon earlier admission to critical care and a lower incidence of renal dysfunction. Alcohol aetiology is not relevant to outcome.

P394

Multivariate regression analysis of outcomes following orthotopic liver transplantation in decompensated cirrhotics transplanted

from the ICU

THughes, M McPhail, M Al-Freah, D Abeles, W Bernal, G Auzinger, J Wendon, CWillars

Institute of Liver Studies, King's College Hospital, London, UK

Critical Care 2012, 16(Suppl 1):P394 (doi: 10.1186/cc11001)

Introduction Patients listed for orthotopic liver transplantation (OLT) frequently develop complications resulting in transfer to the ICUs of tertiary centres. The ICU mortality for cirrhotics has been variously reported from $38 \%$ to in excess of $90 \%$ [1]. The APACHE II score, MELD score and bacteraemia are independent predictors of mortality [2]. The aim of this study was to identify the risk factors relating to early mortality after OLT in cirrhotics transplanted from the ICU.

Methods A retrospective analysis of 1,284 patients transplanted between the dates of 1 January 2000 and 31 December 2008 in a major UK liver transplant centre was performed. Patient characteristics were recorded at transplant assessment and on the day of transplant: age, MELD score, UKELD score, serum sodium, creatinine, bilirubin, albumin INR. Organ support (including ventilation, inotropic support and haemofiltration), lactate and APACHE II score on ICU admission and at the time of transplantation were also analysed. The primary outcome measure was patient survival at 3 months. Statistical analysis was by Mann-Whitney test, logistic regression and area under the receiveroperator curve analysis.

Results Eighty-one patients were transplanted from the ICU with cirrhosis complications. Statistical significance was demonstrated for admission lactate $(P=0.032)$, transplant lactate $(P<0.000)$, transplant APACHE II score $(P=0.001)$, admission inotropic support $(P=0.019)$, transplant inotropic support $(P<0.000)$ and transplant renal support $(P<0.000)$ when comparing 3-month survival with death on univariate analysis. On multivariate logistic regression analysis, high lactate (OR $1.28,95 \% \mathrm{Cl} 1.08$ to $1.51, P=0.003)$ and use of renal replacement therapy (OR 3.52, 95\% Cl 1.42 to $8.74, P=0.006$ ) at the time of transplantation were independently associated with poor outcome. A combination of these two measures had an AUROC of 0.883 ( 0.791 to $0.945, P<0.001$, sensitivity $86 \%$, specificity $86 \%$ ) for prediction of death within 3 months.

Conclusion Patients with chronic liver disease transplanted from the ICU have a worse outcome if they require renal support or demonstrate hyperlactataemia on the day of transplantation.

References

1. Austin MJ, et al:: Curr Opin Crit Care 2008, 14:202-207.

2. Karvellas CJ, et al:: Crit Care Med 2010, 38:121-126.

\section{P395}

Liver transplantation in the critically ill: a Canadian collaboration C Karvellas', T Lescot ${ }^{2}$, H Vahidy', P Goldberg ${ }^{3}$, P Chaudhury ${ }^{3}$, P Metrakos ${ }^{3}$, N Kneteman', G Meeberg', M Sharpe ${ }^{4}$, J Ronco ${ }^{5}$, E Renner ${ }^{6}$, E Cook S Bagshaw

'University of Alberta, Edmonton, Canada; ${ }^{2}$ Hôpital Saint-Antoine, Paris, France; ${ }^{3}$ Mc Gill University, Montreal, Canada; ${ }^{4}$ University of Western Ontario, London, Canada; ${ }^{5}$ University of British Columbia, Vancouver, Canada; ${ }^{6}$ University of Toronto, Canada: ${ }^{7}$ Harvard School of Public Health, Boston, MA, USA

Critical Care 2012, 16(Suppl 1):P395 (doi: 10.1186/cc11002)

Introduction Critically ill cirrhotic patients awaiting liver transplantation (LT) often receive prioritization for organ allocation. Outcomes in these patients are multifactorial, and identification of patients most likely to benefit is essential. Despite the need for evidence-based allocation criteria based on patient factors and physiology scores, few data currently exist on outcomes. Scoring systems such as MELD and SOFA (Sequential Organ Failure Assessment) are in use, but have not been evaluated in predicting outcome with LT.

Methods In a five-center Canadian study (Edmonton, Montreal, Toronto, London and Vancouver), all cirrhotics admitted to the ICU requiring organ support (mechanical ventilation, vasopressors or renal replacement therapy (RRT)) prior to undergoing LT between January 2000 and December 2009 were examined. MELD and SOFA scores were evaluated at ICU admission and the day of LT along with other donor factors.

Results A total of 198 cirrhotics (mean age 53 years, $66 \%$ male) were reviewed. The most common etiologies were hepatitis C (31\%) and alcohol (15\%). LT occurred a median time of 29 (5 to 101) days from listing and 5 ( 3 to 10 ) days from ICU admission. In total, $88 \%$ of patients required vasopressors, $56 \%$ received RRT prior and $87 \%$ were ventilated prior to LT. The median MELD score was 34 (26 to 39) on ICU admission and 34 ( 27 to 40 ) on the day of LT respectively. SOFA scores were 12 (10 to 15) and 13 (10 to 17) on ICU admission and on the day of LT respectively. Comparing patients who were alive $(n=166,84 \%)$ versus dead $(n=32,16 \%)$ at 90 days, there were no statistically significant differences in MELD score on admission or day of LT ( $P>0.6$ for both). There were also no statistically significant differences between SOFA score on admission or day of LT $(P>0.17$ for both). Patients alive at 90 days were significantly younger ( 52 vs. 56 years, $P=0.007$ ). Patients over 60 had significantly higher 90 -day mortality ( $27 \%$ vs. $13 \%, P=0.04)$ and a trend towards increased 1 -year mortality ( $37 \%$ vs. $23 \%, P=0.09)$. There were no significant differences in donor characteristics (donor age $>60$, cold ischemia time $>8$ hours, split graft, donor cerebrovascular event) comparing patients alive at 90 days to nonsurvivors. 
Conclusion Older critically ill cirrhotics (over 60 years) undergoing liver transplantation have significantly worse post-LT outcomes. MELD and SOFA scores do not appear to predict outcome post LT in this cohort.

\section{P396}

Acute respiratory distress syndrome: analysis of incidence and mortality in a university hospital critical care unit

JF Figueira, MO Oliveros, JL López, BC Civantos, LF Fernández Hospital Universitario La Paz, Madrid, Spain

Critical Care 2012, 16(Suppl 1):P396 (doi: 10.1186/cc11003)

Introduction The aim was to determine the incidence of acute respiratory distress syndrome (ARDS) in patients admitted to a university hospital ICU, analyse the ICU and the in-hospital mortality, and evaluate the associated factors.

Methods A prospective study in an ICU from October 2008 to January 2011. The ICU comprises 20 beds in a medical-surgical area, 10 in a critical burns area. All patients who underwent mechanical ventilation (MV) during 48 hours or more and who fulfilled ARDS criteria as defined by the 1994 American-European Consensus Conference on ARDS were included. All patients were ventilated following the protective MV strategy recommended.

Results During this period 1,900 patients were admitted, 697 needed MV for at least 48 hours and 108 fulfilled the ARDS criteria $(5.6 \%$ of those admitted, $17 \%$ of the group on MV); $63 \%$ were male. The patients' age was $52 \pm 12$. The APACHE II score on admission was $23 \pm 7$, in survivors (S) $20 \pm 7$ and $24 \pm 6$ in nonsurvivors (NS) $(P=0.002)$. ARDS was primary in $70 \%$ and secondary in $30 \%$. The most common aetiology was pneumonia (53\%) followed by sepsis of intra-abdominal origin (15\%). Duration of MV was $32.7 \pm 30.2$ days in S, $20.79 \pm 20.73$ in NS $(P=0.019)$. Survivors' mean length of stay was $35 \pm 24$ days, $23 \pm 20$ for NS $(P=0.007)$. ICU mortality was $49 \%$ and in-hospital mortality was $55 \%$. Primary ARDS had an ICU mortality of $47 \%$, an inhospital mortality of $52 \%$. Secondary ARDS had a $55 \%$ ICU mortality, an in-hospital mortality of $64 \%$. Duration of primary ARDS was longer, $15.3 \pm 12.2$ versus $8.7 \pm 79$. Globally the main cause of death was multiple organ dysfunction, predominantly respiratory failure (55\%). In primary ARDS the main cause of death was chiefly pulmonary (69\%), while in secondary ARDS it was mainly multiple organ dysfunction associated with septic shock (71\%). Factors associated with increased mortality were APACHE II score $>23$ and the presence of multiple organ dysfunction.

Conclusion Certain controversy remains regarding a decrease in ARDSrelated mortality. Despite the fact that its incidence is not very high, it is still a clinical entity with a high mortality, and with a prognosis influenced not only by the degree of pulmonary involvement but by the association with multiple organ dysfunction.

References

1. Roca O, et al: Estudio de cohortes sobre incidencia de SDRA en pacientes ingresados en UCly factores pronósticos de mortalidad. Med Intensiva 2006, 30:6-12

2. Zambon M, Vincent JL: Mortality for patients with ALI/ARDS have decreased over time. Chest 2008, 133:151-161.

3. Frutos-Vivar et al.: Epidemiology of ALI and ARDS. Curr Opin Crit Care 2004, 10:1-6.

P397

Epidemiology and outcome of sepsis syndromes in Italian ICUs: a regional multicenter observational cohort L Laudari', Y Sakr², C Elia', L Mascia', B Barberis ${ }^{3}$, S Cardellino ${ }^{4}$, S Livigni ${ }^{5}$, G Fiore ${ }^{6}$, C Filippini', VM Ranieri

'San Giovanni Battista-Molinette Hospital, University of Torino, Turin, Italy; ${ }^{2}$ Friedrich Schiller University Hospital, Jena, Germany; ${ }^{3}$ Ospedale degli Infermi, Revoli, Italy; ${ }^{4}$ Ospedale Cardinal Massaia, Asti, Italy; ${ }^{5}$ Ospedale Giovanni Bosco, Turin, Italy: ${ }^{6}$ Ospedale Santa Croce, Moncalieri, Italy

Critical Care 2012, 16(Suppl 1):P397 (doi: 10.1186/cc1 1004)

Introduction We assessed the epidemiology of sepsis syndromes in patients admitted to ICUs of the Piedmont region in northern Italy and investigated the impact of sepsis on ICU mortality in these patients.
Methods In this prospective, multicenter, observational study, all 3,902 patients (mean age \pm SD: $64.3 \pm 15.7$ years, $63.5 \%$ male) admitted to one of 24 medical or surgical ICUs between 3 April and 29 September 2006 were included.

Results Four hundred and forty-six of the patients had sepsis, including 160 patients with severe sepsis $(4.1 \%)$ and 145 patients (3.7\%) with septic shock. ICU mortality was $20 \%(n=780)$ and median ICU length of stay was 3 (1 to 9 ) days. ICU mortality was higher (41.3 vs. $17.2 \%$, $P<0.001)$ and the median ICU LOS longer (15 (7 to 26$)$ vs. 2 ( 1 to 7 ), $P<0.001)$ in patients with sepsis than in those without sepsis. The mortality rate increased with the severity of sepsis (sepsis without organ failure, severe sepsis, and septic shock: 19.9, 44.4, and 58.6\%, respectively). ICU-acquired sepsis was associated with higher ICU mortality rates than sepsis occurring within 48 hours of ICU admission (49.8 vs. $33.0 \%, P<0.001)$. In multivariate logistic regression analysis, the occurrence of severe sepsis (OR, $1.70(1.06$ to 2.72$) ; P=0.026)$ and septic shock (OR, 2.25 (1.49 to 3.49); $P<0.001)$ were independently associated with an increased risk of ICU death.

Conclusion In this large multicenter cohort, severe sepsis and septic shock were independently associated with an increased risk of death. Our data underscore the regional variability in the epidemiology and outcome of sepsis syndromes and may be useful for resource allocation.

\section{P398}

Outcome of faecal peritonitis in the ICU

J Sayer, G Simpson, L Mccrossan, I Welters

Royal Liverpool University Hospital, Liverpool, UK

Critical Care 2012, 16(Suppl 1):P398 (doi: 10.1186/cc11005)

Introduction Faecal peritonitis often leads to intensive care admission. Anecdotally, patients with co-existing malignancy had an improved outcome. A retrospective analysis of all patients admitted to intensive care over 7 years was conducted to investigate this observation and identify factors that are associated with outcome from faecal peritonitis in intensive care.

Methods A retrospective analysis of all cases of faecal peritonitis admitted to the Royal Liverpool University Hospital ICU over 7 years. Clinical records, laboratory results, histology reports and radiological data were accessed. Statistical analysis was performed using chisquared and Student's $t$ tests.

Results A total of 133 patients were admitted to intensive care in 7 years. Thirty-six patients had underlying malignancy. Predicted mortality, indicated by APACHE II score, was similar in both groups (malignancy: 17.1, nonmalignancy: 16.2). Inpatient mortality was lower in patients with malignancy than those without (malignancy: $21.6 \%$, nonmalignancy: $38.1 \%, P<0.1$ ) and shorter ITU stay (malignancy: 6.8 days, nonmalignancy: 12.7 days, $P \leq 0.0005)$. Cancer patients required a shorter period of TPN or NG feeding (malignancy: 4.29 days, nonmalignancy: 7.7 days, $P<0.05)$, and a shorter duration of inotropic support (malignancy: 2.54 days, nonmalignancy: 4.44 days, $P<0.05$ ). Peak inflammatory markers are lower in patients with malignancy, notably neutrophil count (malignancy: 21.15, nonmalignancy: 24.9, $P<0.05)$.

The mean APACHE II score was significantly lower in cases who survived, compared to those who did not (nondeaths: 15.3, deaths: 19.3, $P<0.005$ ). Mean albumin at admission was similar for patients who survived compared to those who did not (deaths: 18.2, nondeaths: 18.6); however, minimum albumin during admission is significantly lower in patients who died than those who survived (deaths: 10.33, nondeaths: $13.24, P<0.005$ ). Duration of feeding support (TPN or NG feeding) and time to commencement of feeding showed no difference between patients who survived and those who did not.

Conclusion Underlying malignancy is associated with an increased survival, shorter ITU stay, less requirement for inotropic support and decreased inflammatory markers potentially due to a less aggressive inflammatory response as a consequence of the presence of malignancy. In this series, delay to introduction of nutrition and length of nutritional support are not associated with outcome; however, low albumin is associated with a poor outcome, although it is not clear if this is secondary to nutrition or inflammation. 
P399

HIV patients in the ICU: our experience

$\checkmark$ Nunes Velloso, L Calejman, E Canedo, M Deheza

Hospital Rivadavia, Capital Federal, Argentina

Critical Care 2012, 16(Suppl 1):P399 (doi: 10.1186/cc11006)

Introduction The objective was to describe characteristics of HIVpositive patients admitted to the ICU.

Methods HIV-positive patients admitted between February 2000 and February 2011, and demographic data, APACHE II score, cause of admission, days of internment, need for mechanical ventilation (MV), previous antiretroviral therapy of high efficacy before admission (HAART), viral load and CD4 count.

Results A total of 3,568 patients were admitted; 715 patients (20.03\%) were HIV-positive, 413 patients $(57.76 \%)$ were masculine and 302 patients (42.23\%) feminine, and average age was 33 for men and 35 for women. The APACHE II average score was 13 versus 15.28 for the general population. The most frequent cause of admission was respiratory failure in 329 patients $(46 \%), 57 \%$ due to Pneumocystis jivoreci and bacterial pneumonias in $35 \%$, the most frequent bacteria isolated were Streptococcus, Staphylococcus aureus and Haemophilus influenzae. There were two cases of respiratory Kaposi sarcoma and 26 cases of Mycobacterium tuberculosis. Other causes were decrease in mental state in 157 patients (22\%), with the most frequent causes reported being toxoplasmosis, cryptococcus neoformans and brain lymphoma, immediately post surgery in 79 patients (11\%), COPD reagudization and asthma (9\%), digestive bleeding in 36 patients (5\%) and renal insufficiency in 50 patients (7\%). From the 715 HIV-positive patients admitted, 479 required MV (67\%). Regarding nationality, 276 (38.6\%) patients were Argentinean, and the other nationalities were Bolivian, Paraguayan, Peruvian and Korean. The average length of stay was 10.5 days and the mortality was $43 \%$. The viral load average was inferior to $10^{4} \mathrm{RNA} / \mathrm{ml}$ in just 44 known patients and the CD4 count was determined in 75 patients, from which the average was $400 / \mathrm{mm}^{3}$. The proportion of patients receiving HAART was just $26 \%$.

Conclusion HIV-positive patients have a high frequency of admission to the ICU, and they have a lower risk score in comparison with non-HIV patients. The two main causes of admission where respiratory disease and infectious CNS disease. Significant results were the prevalence of patients from limited countries, high mortality and prolonged stay in the ICU, and poor adherence to antiretroviral therapy.

\section{P400}

Impact of congestive heart failure on severe sepsis and septic shock survivors: outcomes and performance status after 1-year hospital discharge

M Alkhalaf', N Abd-Aziz'ㄹ Y Arabi³, B Tangiisuran'

'School of Pharmaceutical Sciences, Penang, Malaysia; ${ }^{2}$ University Technology MARA, Puncak Alam, Malaysia; ${ }^{N}$ National Guard Hospithal,

Riyadh, Saudi Arabia

Critical Care 2012, 16(Suppl 1):P400 (doi: 10.1186/cc1 1007)

Introduction The objective of this study was to evaluate the impact of $\mathrm{CHF}$ on severe sepsis and septic shock survivor outcomes after 1 year of hospital discharge.

Methods A retrospective cohort and cross-sectional study was conducted at a tertiary-care hospital in Saudi Arabia. All patients ( $\geq 18$ years) with severe sepsis/septic shock admitted for more than 1 day to the medical-surgical and trauma ICU between April 2007 and March 2010 and alive at hospital discharge were included in the study. Patients who died during admission, could not be contacted and with multiple ICU admission within the same hospitalization were excluded. Data were collected using the electronic ICU database, hospital information system and systematic review of medical records to determine hospital outcomes and performance status pre sepsis. Assessment of the vital status and performance at 1-year hospital discharge were performed via structured telephone interviews using the Karnofsky Performance Status Scale.

Results A total of 195 hospital survivors from 364 patients were included in the final analysis. More than $70 \%$ of severe sepsis/septic shock with congestive heart failure cases died, $70 \%$ of them dead within 3 months. Patients with CHF as compared to patients without CHF had a higher percentage of comorbidity disease $(P<0.01)$ and poor performance status $(P<0.05)$. The majority of these patients $(85.7 \%)$ who were older $(P<0.001)$, and required a higher dose of dobutamine $(P<0.0001)$, had higher urine output $(P<0.001)$ and prolonged INR $(P<0.01)$ were unable to care for self at 1 year of hospital discharge. Survivors with CHF who died (OR 4.7, Cl 1.52 to 14.33) had higher dose of dopamine $(P<0.045)$ and poor performance status pre sepsis $(P<0.028)$.

Conclusion About three-quarters of survivors of severe sepsis/septic shock with congestive heart failure died after 1 year of hospital discharge. Many of them (70\%) died within 3 months of hospital discharge. The majority had poor performance status and only $14 \%$ were able to carry on normal activity at 1 year after hospital discharge. These data highlight the need for different strategies to care for sepsis survivors with congestive heart failure.

\section{P401}

Predictive value of $\mathrm{N}$-terminal pro-brain natriuretic peptide among critically ill patients

M Cubrilo-Turek, N Maric, I Mikacic, N Tolj Karaula, N Budinski, M Mackovic Clinical Hospital Sveti Duh, Zagreb, Croatia

Critical Care 2012, 16(Suppl 1):P401 (doi: 10.1186/cc11008)

Introduction N-terminal pro-brain natriuretic peptide (NT-proBNP) represents a useful cardiac marker in evaluating heart failure. However, its role in the assessment of critically ill patients is not clear. The aim of this study was to evaluate survival of infected and noninfected patients according to the measurements of NT-proBNP.

Methods Serum NT-proBNP measurements were done in 89 (46 males/43 females, $68.20 \pm 13.80$ years) consecutive critically ill patients within 6 hours after admission to the ICU. NT-proBNP was determined with a sandwich immunoassay on an Elecsys 2010 (Roche Diagnostics, Mannheim, Germany). Logarithmic transformation of data was required because of the skewed distribution of NT-proBNP.

Results The median NT-proBNP (pg/ml) was $2,485.1 \mathrm{pg} / \mathrm{ml}$ (range 31.5 to $12,041 \mathrm{pg} / \mathrm{ml}$ ) (log NT-proBNP mean $3.34 \pm 0.71 \mathrm{pg} / \mathrm{ml})$. Mean log NTproBNP levels were higher at admission to the hospital in nonsurvivors $(3.73 \pm 0.67 \mathrm{pg} / \mathrm{ml})$ compared with survivors $(3.12 \pm 0.65 \mathrm{pg} / \mathrm{ml})$, which was statistically significant $(P<0.0001)$. Higher concentrations were found in proven infection $(X \pm S D)(3.43 \pm 0.68)$ than in bacteriological negative patients $(3.30 \pm 0.72)$, but it was statistically insignificant $(P<0.42)$. From 57 survivors seven were mechanically ventilated (12.28\%) while $14(43.75 \%)$ from 32 nonsurvivors were ventilated, which was statistically significant $(P<0.001)$. More nonsurvivors were taking vasoactive medications ( $n=12$ or $37.5 \%)$ than survivors $(n=3$ or $5.26 \%$ ), which was statistically significant $(P<0.001)$. NT-proBNP showed no correlation for any analyzed parameters (age, erythrocytes, leucocytes, body temperature, systolic and diastolic blood pressure, C-reactive protein, fibrinogen, lactates or procalcitonin). The use of ROC curve analysis reveals for serum NT-proBNP high sensitivity (75\%), low specificity (57.9\%) and low accuracy (64\%) for discriminating survivors from nonsurvivors.

Conclusion Our results showed that cardiac NT-proBNP levels can be elevated in critically ill patients and may also serve as markers of severity and prognosis for survival. Mean baseline levels of log NT-proBNP were different in critically ill patients with proved bacteriological infection than in patients without proven infection.

\section{P402}

Low preoperative hepcidin concentration is a risk factor for mortality but not for acute kidney injury after cardiac surgery A Haase-Fieiltz', P Mertens' ${ }^{1}$ M Plaß², M Westerman³', R Bellomo ${ }^{4}$, M Haase 'Otto-von-Guericke University, Magdeburg, Germany; ${ }^{2}$ German Heart Center, Berlin, Germany; ${ }^{3}$ ntrinsicLifeSciences, La Jolla, CA, USA; ${ }^{4}$ Austin Health,

Melbourne, Australia

Critical Care 2012, 16(Suppl 1):P402 (doi: 10.1186/cc11009)

Introduction Hepcidin - expressed in renal proximal tubular cells - is a key regulator of iron homeostasis and was recently described as a renal 
biomarker that early postoperatively predicts protection from acute kidney injury (AKI).

Methods We studied 100 adult patients at increased risk of AKI (RIFLE) after cardiac surgery. Plasma and urine were sampled before induction of anesthesia and hepcidin 25-isoforms were quantified by competitive enzyme-linked immunoassay. Our objective was to assess the predictive indices of preoperatively measured urine and plasma hepcidin for the development of postoperative AKI and other patientrelated outcomes, including the need for renal replacement therapy (RRT) and in-hospital mortality.

Results Preoperatively, patients not developing AKI presented with nonsignificantly higher urine and plasma hepcidin concentrations compared to patients that developed AKI which did not translate into a good predictive value for postoperative AKI (AUC-ROC $<0.70$ for both biomarkers). Also, the preoperative urine and plasma hepcidin concentrations as well as serum creatinine concentration did not distinguish patients requiring postoperative RRT from those who did not require RRT (urine: AUC-ROC 0.62 ( $95 \% \mathrm{Cl} 0.38$ to 0.86 ), plasma: AUCROC $0.63(95 \% \mathrm{Cl} 0.34$ to 0.91$)$, serum creatinine: AUC-ROC $0.61(95 \% \mathrm{Cl}$ 0.22 to 0.99$)$ ). However, a low preoperative hepcidin concentration in urine (median $5 \mathrm{ng} / \mathrm{ml}, 25$ th to 75 th percentiles 4 to $15 \mathrm{ng} / \mathrm{ml}$ ) and in plasma (median $50 \mathrm{ng} / \mathrm{ml}$, 25th to 75 th percentiles 30 to $55 \mathrm{ng} / \mathrm{ml}$ ) was a good predictor for postoperative mortality with an AUC-ROC for urine hepcidin of 0.89 ( $95 \% \mathrm{Cl} 0.73$ to 0.99 ) (cut-off: $130 \mathrm{ng} / \mathrm{ml}$, sensitivity $73 \%$ and specificity $100 \%$ ) and an AUC-ROC for plasma hepcidin of 0.90 ( $95 \% \mathrm{Cl} 0.80$ to 0.99 ) (cut-off: $55 \mathrm{ng} / \mathrm{ml}$, sensitivity $83 \%$ and specificity $100 \%$ ). Preoperative serum creatinine did not predict mortality (AUCROC 0.50 ( $95 \% \mathrm{Cl} 0.10$ to 0.94 ). Patients who survived the hospital stay had a median preoperative hepcidin concentration in urine of $330 \mathrm{ng} /$ $\mathrm{ml}$ (25th to 75 th percentiles 140 to $760 \mathrm{ng} / \mathrm{ml}$ ), and plasma of $115 \mathrm{ng} /$ $\mathrm{ml}$ ( 25 th to 75 th percentiles 80 to $200 \mathrm{ng} / \mathrm{ml}$ ).

Conclusion Our findings suggest that low preoperative hepcidin concentration indicates mortality but not renal endpoints in patients undergoing cardiac surgery. Thereby, hepcidin may contribute to early risk stratification. Findings should be validated in independent patient cohorts with a larger number of events.

\section{P403}

Outcomes and resource use for over $\mathbf{8 0}$ year olds admitted to a UK critical care unit after an emergency laparotomy over a 3-year period

$\checkmark$ Banks, C Scott

Northern General Hospital, Sheffield, UK

Critical Care 2012, 16(Suppl 1):P403 (doi: 10.1186/cc11010)

Introduction There are few data on older people emergency surgical critical care (CC) admissions and the potential implications for future resource demands and service planning.

Methods Retrospective data were collected from a cohort of patients $>80$ years old admitted after emergency surgery between 2009 and 2011. CC and hospital information databases were used. Data included mortality, length of stay (LOS) and duration of renal replacement therapy (RRT) and advanced respiratory support (ARS).

Results A total of 118 patients were admitted; $52 \%$ female: mean age 85 years, male mean age: 84 years. In total, $69 \%$ were general surgical, $22 \%$ vascular, and $9 \%$ hepatobiliary. Eleven per cent required RRT for a mean of 3.6 days, and $41 \%$ needed ARS for a mean of 3.8 days. See Table 1.

Conclusion CC and hospital mortality was $17 \%$ and $33 \%$ respectively. This study concurs with another which demonstrated that age is not a good predictor of outcome post surgery [1]. These patients did not have a significant impact on RRT or ARS resources or CC LOS.

\section{Reference}

1. Ford $\mathrm{P}$, et al:: Determinants of outcome in critically ill octogenarians after surgery. Br J Anaesth 2007, 99:824-829.

P404

Correlation between APACHE II score and quality of life among patients discharged from the ICU

L Zubek', L Szabó', L Horváth', A Mesterházi², J Gál'1, G Élő

'Semmelweis University, Budapest, Hungary; 'Markusovszky Hospital,

Szombathely, Hungary

Critical Care 2012, 16(Suppl 1):P404 (doi: 10.1186/cc11011)

Introduction The goal of intensive therapy is not only saving the patient's life, but also to restore their quality of life. Based on expected quality of life improvement, a fair allocation of limited available resources can be provided. The assessment scores for the physical state of ICU patients, which correlate with survival, are widely known. However, it would be useful to know if these score systems also correlate with the long-term quality of life. The aim of our study was to investigate the correlation between the APACHE II score and the longterm quality of life after ICU treatment.

Methods We have collected data retrospectively from patients treated in our department during the first quarter of 2008. The APACHE II score was calculated for all patients, after which we examined the correlation between this value and the survival of the patients. One year after ICU therapy, the Hungarian version of the EQ-5D questionnaire (measurement consist of five dimensions: mobility, self-care, usual activities, pain/discomfort, anxiety/depression and a visual analog scale about health state) developed by EuroQol Group was sent out by post. The correlation between the APACHE score and quality of life was calculated, the Spearmann rank-order correlation was used.

Results During this period, 190 patients were treated in our department. The average of the APACHE II score was $13.23( \pm 6.99)$. In total, $25.3 \%$ of patients died during treatment; $22.1 \%$ died during the first posttreatment year; $27.9 \%$ surely survived and $24.7 \%$ of patients were unattainable. In our cohort, every patient below 11 points survived and none above 24. The average APACHE score of patients completing the questionnaire was $9.30( \pm 3.85)$. They assessed their health as $66 \%$ at VAS, although correlation between this value and the APACHE score could not be shown. However, we found statistically significant correlation between the APACHE score and the current mobility of the patients $(P=0.021)$. Based on our data, $34 \%$ of the patients had problems with mobility, $36 \%$ with usual activity, $62 \%$ of patients complained about pain or discomfort, $50 \%$ felt anxiety or depression and $18 \%$ had problems with self-care.

Conclusion ICU admission is associated with a high mortality, a poor physical quality of life and low quality-adjusted life-years for 1 year after discharge. We found that the APACHE II score did not show significant correlation with patient's long-term quality of life, but we detected significant correlation between the APACHE II score and the current mobility of the patients.

Table 1 (abstract P403)

\begin{tabular}{|c|c|c|c|c|}
\hline Patient group & CC mortality, $n$ (\%) & Hospital mortality, $n(\%)$ & LOS CC (days) & LOS hospital (days) \\
\hline All patients, $n=118$ & $20(17 \%)$ & $39(33 \%)$ & 1 to $34,4.5$ mean & 1 to 247,29 mean \\
\hline Age 80 to $84, n=70$ & $15(21 \%)$ & $29(41 \%)$ & 1 to $34,4.9$ mean & 1 to 247,28 mean \\
\hline Age $>85, n=48$ & $5(10 \%)$ & $10(21 \%)$ & 1 to $32,4.1$ mean & 1 to 171,31 mean \\
\hline ARS, $n=49$ & $15(30 \%)$ & $22(45 \%)$ & 1 to $34,7.5$ mean & 1 to 79,20 mean \\
\hline No ARS, $n=69$ & $5(7 \%)$ & $17(25 \%)$ & 1 to $24,3.8$ mean & 1 to 247,35 mean \\
\hline RRT, $n=13$ & $7(54 \%)$ & $9(69 \%)$ & 1 to 34,8 mean & 1 to 247,35 mean \\
\hline No RRT, $n=105$ & $13(12 \%)$ & $30(29 \%)$ & 1 to 32,4 mean & 1 to 247,30 mean \\
\hline
\end{tabular}


P405

Parameters that affect outcome in surgical ICU patients

A Vakalos, M Petkopoulou, D Jannussis

Xanthi General Hospital, Xanthi, Greece

Critical Care 2012, 16(Suppl 1):P405 (doi: 10.1186/cc11012)

Introduction Surgical ICU patients have a lower severity illness score on ICU admission day. The aim of our study was to compare the length of stay (LOS), ventilation days (VD) and parameters that affect the APACHE II-III scoring system between surgical patients who died in the ICU and surgical patients who survived and discharged from the ICU.

Methods During November 2005 and May 2011, 310 patients were admitted to our medical and surgical ICU. From these, 122 were surgical patients (39.35\%) and were included retrospectively in our study. Mean age was 64 years, mean APACHE II score 14.5, actual mortality rate $12.29 \%$. The patients were separated into two groups. Group A involved 107 surgical patients who survived the ICU and group B 15 surgical patients who died in the ICU. We looked for statistical significant difference (two-tailed $P$ value) between the mean APACHE values at admission of group $A$ and group B, using the unpaired MannWhitney test (nonparametric) or the unpaired $t$ test Welch corrected (parametric), according to the normality test.

Results The mortality rate of surgical patients was $12.29 \%$. We detected no statistical difference between the two groups according to age $(P=0.27)$, heart rate $(P=0.13)$, temperature $(P=0.57), \mathrm{Na}(P=0.44), \mathrm{K}$ $(P=0.18), \mathrm{WBC}(P=0.56), \mathrm{Ht}(P=0.7), \mathrm{PaO}_{2}(P=0.28), \mathrm{PaCO}_{2}(P=0.7)$, albumin $(P=0.21)$, glucose $(P=0.68)$ and GCS $(P=0.26)$. We detected statistically significant higher group $B$ values according to BUN $(P=0.015)$, creatinine $(P=0.005)$, bilirubin $(P=0.0032)$, APACHE ॥ score $(P=0.0018)$, LOS $(P<0.0001)$ and VD $(P<0.0001)$. We detected statistically significant higher group $A$ values according to mean arterial pressure $(P=0.0052)$ and $\mathrm{PH}(P=0.0027)$.

Conclusion According to our data, surgical patients who died (group B) had higher severity score on admission. Nevertheless, the main difference between surgical patients who died and who survived the ICU was hemodynamic instability, which was severe enough to cause hypoperfusion, metabolic acidosis, early acute kidney injury and early multiple organ dysfunction. As a result, the length of stay and the ventilation days were higher in group B patients, assuming that early and effective surgical management is important in order to avoid early multiple organ dysfunction on ICU admission.

P406

Relationship between illness severity scores in the ICU

A Schneider ${ }^{1}$ M Lipcsey ${ }^{1}$, M Bailey ${ }^{2}$, D Pilcher ${ }^{3}$, R Bellomo

'Austin Health, Heidelberg, Australia; ${ }^{2}$ Monash University, Melbourne,

Australia; ${ }^{3}$ ANZICS, Melbourne, Australia

Critical Care 2012, 16(Suppl 1):P406 (doi: 10.1186/cc11013)

Introduction Many different illness severity scores are used to report the estimated risk of death (ROD) of patients in clinical research. Such variability makes mortality comparison between studies difficult. Accordingly, it would be desirable to establish a methodology to translate the value obtained from one scoring system into an estimated equivalent value for another scoring system.

Methods We used the adult patient database of the Australian and New Zealand (ANZ) Intensive Care Society to obtain simultaneous APACHE II (APII), APACHE III (APIII) and SAPS II scores. We used linear regression analyses to create models enabling translation of one score into another. These analyses were performed for the whole cohort, after exclusion of cardiac surgery patients and after matching for similar risk of death.

Results We obtained complete data for three illness severity scores (SAPS II, APII, and APIII) in 636,431 admissions. There was a good correlation between the APIII and APII scores $\left(r^{2}=0.76\right)$. The overall model was APIII $=3.09 \times \mathrm{APII}+5.8$. The APIII/APII coefficient $(\mathrm{SE})$ was $3.09(0.002)$ for the whole cohort, $3.1(0.002)$ after exclusion of cardiac surgery patients and $2.98(0.01)$ after exclusion of patients with an absolute difference in ROD $>1 \%$ between the two scores. There was a similar correlation between the APIII and the SAPS II scores $\left(r^{2}=0.76\right)$. The overall model was APIII $=1.47 \times$ SAPS $\|+8.6$. The APIII/SAPS $\|$ coefficient (SE) was 1.47 (0.001) for the whole cohort, 1.49 (0.001) after exclusion of cardiac surgery patients and $1.55(0.006)$ after exclusion of patients with an absolute difference in ROD $>1 \%$ between the two scores. Finally, the correlation between the APII and SAPS II scores was moderate $\left(r^{2}=0.63\right)$. The overall model was APII $=0.36 \times$ SAPS $\|+4.4$. The APII/SAPS II coefficient (SE) was $0.36(0.0003)$ for the whole cohort, $0.37(0.0004)$ after exclusion of cardiac surgery patients and $0.39(0.002)$ after exclusion of patients with an absolute difference in ROD $>1 \%$.

Conclusion Simple and robust translational formulas can be developed to allow clinicians to compare illness severity in intensive care studies of similar patients when such illness severity is expressed with different scoring systems.

P407

Predicting hospital mortality: comparing accuracy of SAPS II and clinical staff prognosis

I Patrício', M Marques'1, A Costa-Pereira², O Ribeiro², I Aragão', T Cardoso' 'Hospital Geral de Santo António, University of Porto, Portugal;' ${ }^{2}$ Faculty of Medicine, University of Porto, Portugal

Critical Care 2012, 16(Suppl 1):P407 (doi: 10.1186/cc11014)

Introduction The purpose of this study is to compare the accuracy of Simplified Acute Physiology Score (SAPS) II with the subjective opinion of clinical staff in predicting hospital mortality, in critically ill adult patients.

Methods A prospective study in a mixed ICU, at a university hospital, using SAPS II to assess the risk of death. Patient outcome was also predicted subjectively by the clinical staff (consultants, residents and nurses), including the possibility of return to prior physical activity. The subjective predictions were compared with SAPS II predictions using logistic regression analysis and receiver operating characteristic curve (ROC) measurement, as well as sensitivity and specificity analysis for each group of participants.

Results Over the study period 72 patients were included, with a mean age of $56.5 \pm 16.8$ years; $55 \%$ were male. The mean SAPS II was $47.3 \pm 15.4$. Eighteen patients died in hospital (25\%). Discriminations analysis showed the following areas under ROC: SAPS II 0.84 (95\% Cl: 0.741 to 0.945 ); consultants 0.77 ( $95 \% \mathrm{Cl}: 0.632$ to 0.908 ); residents 0.67 ( $95 \% \mathrm{Cl}: 0.513$ to 0.828 ); nurses 0.62 ( $95 \% \mathrm{Cl}: 0.453$ to 0.777 ). See Figure 1. Conclusion In our study, contrary to previous descriptions of similar studies, SAPS II was more accurate in predicting hospital mortality than clinical staff opinion. Differences were also found between different groups of clinical staff, partially related to previous ICU clinical experience.

References

1. Scholz N, et al:. Eur J Anaesthesiol 2004, 21:606-611.

2. Sinuff T, et al:: Crit Care Med 2006, 34:878-885.

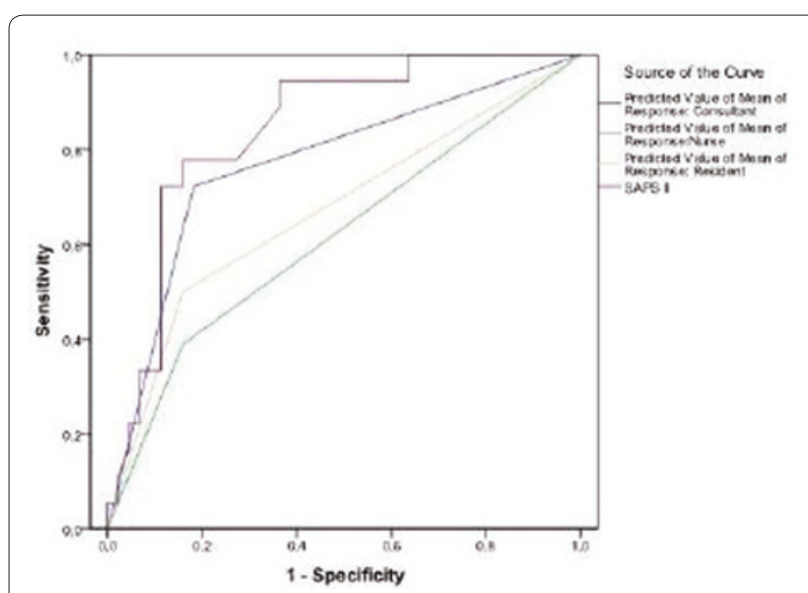

Figure 1 (abstract P407). ROC curve for SAPS II, consultants, nurses and residents, for hospital mortality. 
P408

Predictors of mortality in patients from a hematological ICU in Brazil

OB Silva, L Correa, P Loureiro, E Araujo, D Teles, LA Vasconcelos,

T Salvattori, P Schwambach, GT Henriques-Filho

HEMOPE, Recife, Brazil

Critical Care 2012, 16(Suppl 1):P408 (doi: 10.1186/cc11015)

Introduction The study was designed to analyze the factors responsible for increased mortality in an ICU specialized in hematological patients. There are few ICUs specialized in hematological diseases, with reports of high mortality rates (45 to $85 \%$ ) [1], mostly related to severity of patients with blood cancer [2], mechanical ventilation (MV) and multiple organ failure [2-4]. The most prevalent disease differs among studies [1-4] and acute leukemia seems to have the worst prognosis [2]. Methods A retrospective cohort was conducted at HEMOPE's ICU. Data were collected from the medical records of patients admitted from January 2006 to December 2009.

Results Of the 576 admissions, 396 (68.75\%) could be analyzed. The average age was $48.3 \pm 19.4$ years ( 11 to 88 years), $54 \%$ were female and there was no association between mortality and age or gender. Acute leukemia occurred in $43 \%$ (65.3\% acute myeloid leukemia). Sepsis was the major cause of admission (55.3\%). The overall mortality rate was $57.5 \%$ and the specific one was $42.7 \%$. The mean APACHE II score for this population was $13.4 \pm 1.0$ (7 to 43 ) and was statistically higher in the group that died $(14.6 \pm 0.7$ vs. $11.8 \pm 0.8 ; P=0.013)$. Mean SOFA at day 1 (D1) and day 3 (D3) was $2.8 \pm 0.2$ and $2.1 \pm 0.2$ respectively, also significantly higher in those that died (D1 $3.9 \pm 0.3$ and D3 $2.9 \pm 0.3$; $P<0.0001$ ). Almost $60 \%$ used vasoactive drugs (VAD) on admission and had a higher mortality rate $(P<0.0001)$. MV was used in $86 \%$ and $69 \%$ died $(P<0.001)$. Of those with renal substitutive therapy (RST), $81.9 \%$ died $(\mathrm{OR}=3.12 ; 99 \% \mathrm{Cl}=1.5$ to 6.91$)$. Mortality was also associated with the completion of chemotherapy before ICU admission $(P=0.003)$ and severe neutropenia $(P<0.0001)$. In multivariate analysis, $\mathrm{MV}(\mathrm{RR}=13.1$; $99 \% \mathrm{Cl}=5.14$ to 33.45$)$ and a one-unit increase in SOFA D1 (RR $=1.26$; $99 \% \mathrm{Cl}=1.15$ to 1.37 ) were associated with an increase in mortality. Conclusion For this population, in univariate analysis mortality was related to SOFA, RST, MV, use of VAD on admission, chemotherapy before ICU admission, and severe neutropenia. Although there was a relation between APACHE II score and mortality, this score underestimates it. In multivariate analysis, needing MV and a high SOFA D1 were independent predictors of death.

\section{References}

1. Shelton BK: Crit Care Clin 2010, 26:1-20.

2. Kress JP, et al.: Am J Respir Crit Care Med 1999, 160:1957-1961.

3. Taccone FS, et al:: Crit Care 2009, 13:R15.

4. Thiéry G, et al.: J Clin Oncol 2005, 23:4406-4413.

P409

Retrospective study of the outcomes of patients admitted to the ICU with a hematological malignancy

H Lewis, J Patel, N Lonsdale

Birmingham Heartlands Hospital, Birmingham, UK

Critical Care 2012, 16(Suppl 1):P409 (doi: 10.1186/cc1 1016)

Introduction The UK prevalence of haematological malignancy is increasing. Seven percent of these patients become critically ill, necessitating ITU care [1]. The past decade has seen significant advances in the treatment and outcomes of patients with hematological malignancies. This has challenged the preconception that these patients are poor candidates for ICU admission. This study evaluated the trends in admission and outcomes of patients admitted to a general ICU with a diagnosis of hematological malignancy.

Methods A retrospective study of the last 50 consecutive admissions of patients with a hematological malignancy admitted to the ICU. Patients were identified from the ICNARC database. Demographic data, APACHE II, SOFA scores on admission, baseline neutrophil count and organ support data were collected. The primary outcome was ICU and hospital mortality. Data were compared against the cohort of patients admitted between April 2010 and April 2011.
Results The last 50 patients were admitted between January 2004 and August 2011. Overall the number of admissions increased throughout this period, with only one admission in 2004, peaking at 10 in 2009. In 2011, patients with a hematological malignancy represented $0.5 \%$ of all the ICU admissions. The commonest malignancies were acute myeloid leukemia (43\%) and lymphoma (31\%). The primary reason for admission was sepsis (61\%), with pneumonia the commonest source (27\%) and $42 \%$ admitted with neutropenic sepsis. Compared to the 2010/11 cohort the patients admitted with a hematological malignancy had significantly higher mean APACHE II scores (24 (SD 8) vs. 15 (SD 6) $P<0.0001$ ), a longer mean ICU stay (10 days (SD 17) vs. 6 days (SD 10) $P<0.0001)$ and greater ICU $(50 \%$ vs. $27 \% P<0.0001)$ and hospital mortality $(61 \%$ vs. $29 \% P<0.0001)$. However, the overall trend was a considerable fall in mortality from $91 \%$ (2004 to 2007) to $36 \%$ (2008 to 2011). The mean SOFA score on admission for the hematological patients was 9 (SD 3). Twenty patients required two levels of organ support with only three patients receiving renal replacement therapy. No independent risk factors for outcome were identified.

Conclusion The outcomes of patients with hematological malignancies admitted to the ICU are improving with rates approaching that of our general ICU population. Patients with hematological malignancy requiring ICU admission continue to increase and admission should be based on their physiological derangement and overall prognosis. Further prospective studies are required to investigate potential predictors of outcomes in these patients.

\section{Reference}

1. Hampshire P, et al:: Admission factors associated with hospital mortality in patients with haematological malignancy admitted to UK adult, general critical care units: a secondary analysis of the ICNARC Case Mix

Programme Database. Crit Care 2009, 13:R137.

P410

Six-month survival of patients with lung cancer admitted to a medical ICU: a retrospective study

O Keller, GL Laplatte, H Lessire

CHPasteur, Colmar, France

Critical Care 2012, 16(Suppl 1):P410 (doi: 10.1186/cc11017)

Introduction ICU admission of patients with lung cancer remains debated because of the poor short-term prognosis. We evaluated the duration of survival of patients admitted to our ICU and looked for factors associated with better survival.

Methods All patients with nonresectable lung cancer admitted to our ICU between 1 January 2008 and 31 December 2010 were included in a retrospective study. Postoperative patients were not included.

Results Twenty-two patients were included. Seventeen had nonsmallcell lung cancer (NSCLC). One had small cell lung cancer. Fifteen patients $(65 \%)$ had metastatic disease. Twelve patients were in palliative therapy. The reason for ICU admission was acute respiratory failure in 12 patients (55\%), hemorrhage in five patients (23\%). Nine patients $(41 \%)$ had an infection. Fourteen patients (64\%) needed invasive mechanical ventilation. One-month survival was $45 \%$ (10/22). Six-month survival was $13 \%(3 / 22)$. One-year survival was $0 \%$. Onemonth survivors showed a nonsignificant trend to lower performance status and severity of disease. All 6-month survivors had metastatic disease. Six-month survivors had nonsignificantly lower performance status (1.7 \pm 0.6 vs. $2.7 \pm 1.2 ; P=\mathrm{NS})$. IGS II, SOFA score and duration of mechanical ventilation were significantly shorter in survivors (see Table 1).

Conclusion Prognosis of patients with nonresectable lung cancer admitted to the ICU was poor. Metastatic disease did not influence

Table 1 (abstract P410)

\begin{tabular}{lccc}
\hline & IGS & SOFA & $\begin{array}{c}\text { Number of } \\
\text { ventilation days }\end{array}$ \\
\hline Nonsurvivors & $53.2 \pm 6.5$ & $5.6 \pm 3.8$ & $6.1 \pm 6.4$ \\
6-month survivors & $36.3 \pm 9.8$ & $1.3 \pm 2.3$ & $0.7 \pm 1.2$ \\
& $P<0.05$ & $P<0.05$ & $P<0.01$ \\
\hline
\end{tabular}


survival in our survey. Patients admitted for a critical illness requiring more than a few days of mechanical ventilation were very unlikely to survive over 6 months.

References

1. Toffart $A C$, et al:: Use of intensive care in patients with nonresectable lung cancer. Chest 2011, 139:101-108.

2. Roques $\mathrm{S}$, et al:. Six month prognosis of patients with lung cancer admitted to the intensive care unit. Intensive Care Med 2009, 35:2044-2050.

P411

Health-related quality of life and survival of cancer patients admitted to ICUs: results of the QALY study

AB Cavalcanti', UV Silva², KN Normílio-Silva', AN Silva', R Zancani', MJ Giorgi', AD Dias', AT Simone', PL Safra', AC Fiqueiredo',

G Tunes-da-Silva', AC Lima33, LA Hajjar', JO Auler', J Eluf-Neto', FR Galas' 'São Paulo State Cancer Institute, São Paulo, Brazili, ${ }^{2}$ Barretos Cancer Hospital, Barretos, Brazil; ${ }^{3}$ Instituto de Matemática e Estatística - Universidade de São Paulo, Brazil; ${ }^{4}$ Faculdade de Medicina da Universidade de São Paulo, Brazil Critical Care 2012, 16(Suppl 1):P411 (doi: 10.1186/cc11018)

Introduction Very limited data are available regarding postdischarge health-related quality of life (HRQL) of cancer patients needing intensive care. Our objective is to describe $\mathrm{HRQL}$ and survival in an unselected population of cancer patients who were admitted to ICUs. Methods In this prospective cohort study conducted at two cancer hospitals in Brazil, we enrolled a random sample of adult patients with cancer admitted to the ICUs. We collected data at ICU admission, including $\mathrm{HRQL}$ before the acute process that led to ICU admission, and followed patients up on 15,90 and 180 days after ICU admission to assess $\mathrm{HRQL}$ and vital status. We determined $\mathrm{HRQL}$ with the EQ-5D questionnaire, and the results were presented as summary measures with values between -1 and 1, with 0 meaning HRQL similar to death and 1 perfect HRQL. Summary measures were calculated using timetrade-off value sets obtained from the UK population. Survival was calculated with the Kaplan-Meier estimator.

Results We enrolled 805 patients. Mean age was $61.4 \pm 14.3$ and $42.5 \%$ were female. Elective surgeries represented $52.2 \%$ of admissions, urgent surgeries represented $5.0 \%$ and $42.8 \%$ were admitted due to clinical reasons. Survival at 180 days was $51.2 \%(95 \% \mathrm{Cl} 47.4$ to 54.9$)$. The HRQL summary measure (median (interquartile range)) before ICU admission was 0.64 ( 0.12 to 0.81 ), on the 15 -day follow-up 0.73 (0.19 to $0.92)$, on the 90 -day follow-up 0.73 ( 0.20 to 0.85 ) and on the 180 -day follow-up 0.70 ( 0.35 to 0.89 ).

Conclusion HRQL is, on average, moderately impaired before ICU admission and through the 180-day follow-up in cancer patients needing intensive care. Only about one-half of the patients were alive after 180 days. However, there is large variability on both HRQL and length of survival; thus, methods to estimate quality-adjusted life-years on an individual basis are necessary.

\section{P412}

Characteristics, resource consumption and outcome of cancer patients admitted to ICUs

R Garcia, L Terceros, I Saez, J Flordelis, L Colino, C Mudarra, STemprano,

J Montejo

12 de Octubre Hospital, Madrid, Spain

Critical Care 2012, 16(Suppl 1):P412 (doi: 10.1186/cc11019)

Introduction The development of cancer treatment has improved the prognosis for cancer patients and they need more support measures in the ICU. Our objective is to evaluate the characteristics and evolution of cancer patients admitted to a general ICU of a university hospital. Methods A retrospective study of cancer patients admitted to an ICU from January 2008 to December 2010. We collected demographic and cancer characteristics, reason for admission, complications, resource consumption and mortality. We compared quantitative variables with the Student $t$ test and the qualitative variables with the chi-square test, statistical significance accepted as $P<0.05$.

Results A total of 108 patients were admitted with cancer, 23 with cured cancer were excluded, so we selected 85 patients $(4.38 \%$ of total admissions). Sixty-eight percent were male, with a mean age of $60.21 \pm 14.31$ years and with an APACHE score of $22.21 \pm 9.13$. Solid cancer was more frequent, $76.6 \%$ (urogenital $20 \%$, lung $15.4 \%$ and low intestinal $15.4 \%$ were the most common). In the hematologic cancers (23.5\%), the most frequent were non-Hodgkin lymphoma and acute leukemia (both 7\%). Active cancer (new diagnosis, recurrence or progression) was presented in $75.3 \%$. The main reason for admission was respiratory failure $(52.9 \%)$, shock (18.8\%) or neurological impairment $(16.5 \%)$. The most common diagnoses were pulmonary sepsis (23.5\%), other sepsis $(21.2 \%)$ and heart failure (8.2\%). The ICU stay was $7.20 \pm 12.32$ days; with a mortality of $41.2 \%$ (hospital mortality $50.6 \%)$. The mortality was higher in the active disease ( $91 \%$ vs. $64 \%)$, $P<0.01$. Patients who died developed more respiratory ( $88.6 \%$ vs. $48 \%$ ), hemodynamic $(91.4 \%$ vs. $44 \%)$, renal $(68.6 \%$ vs. $16 \%)$ or hematologic failure $(45.7 \%$ vs. $16 \%), P<0.03$. Septic patients were those with higher ICU mortality (55.3\% vs. $29.8 \%$ ) and hospital mortality $(63.2 \%$ vs. $40.4 \%), P<0.05$. By contrast, the patients with the longest survival were the neurological $(90 \%$ vs. $54.7 \%)$ and cardiology patients $(88.9 \%$ vs. $55.3 \%), P<0.05$. Patients who died needed more MV $(88.6 \%$ vs. $52 \%)$, vasopressors $(91.4 \%$ vs. $46 \%$ ) or dialysis ( $34.3 \%$ vs. $4 \%), P<0.01$. The hematologic cancer had more cardiovascular $(85 \%$ vs. $56.9 \%)$ or hematologic failure ( $65 \%$ vs. $16.9 \%$ ) and neutropenia ( $45 \%$ vs. $9.2 \%)$ with $P<0.03$, but this is not reflected in more consumption of resources or mortality.

Conclusion The mortality was associated with organ failure and greater need for resources. Hematologic cancer develops more organ failure without affecting resource consumption or their outcome in our series. Septic patients have higher ICU and hospital mortality, and neurological patients lower.

\section{P413}

Managing critically ill oncological patients in hospital: a survey across all ICUs in the UK

C Gore, TWigmore

Royal Marsden Hospital, London, UK

Critical Care 2012, 16(Suppl 1):P413 (doi: 10.1186/cc11020)

Introduction The survival rates for oncology patients admitted to the ICU have improved significantly. The prognostic influence of the preadmission oncological and treatment history is being questioned, the most significant impact being related to acute physiological status. In

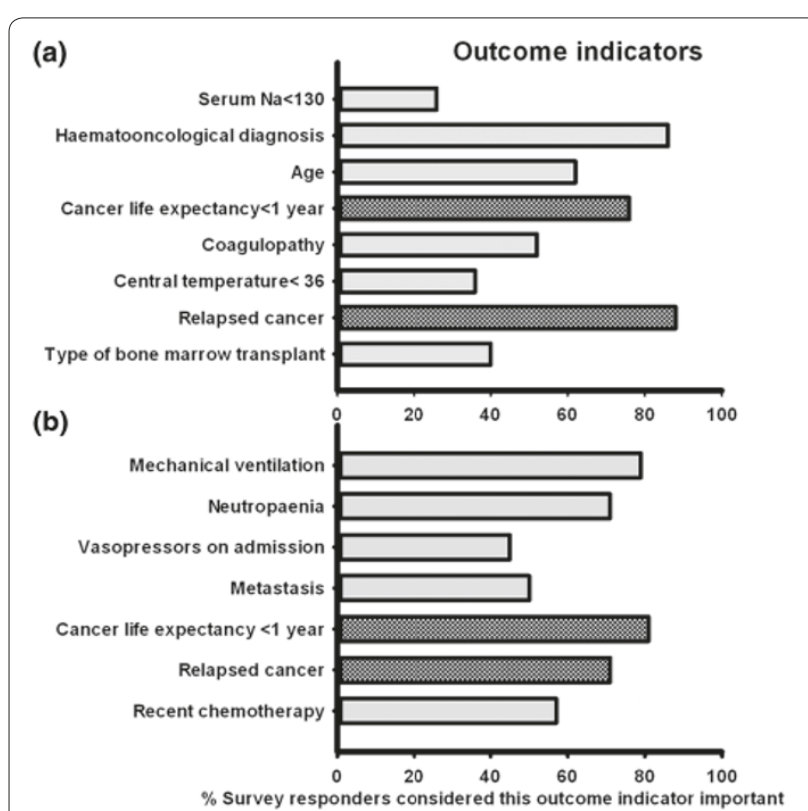

Figure 1 (abstract P413). (a) Hematological cancer. (b) Solid tumor. Checked bars, not proven in the literature. 
this survey, we sought to evaluate the awareness of overall mortality rates in critically ill cancer patients among intensivists in the UK. Methods We surveyed intensive care lead clinicians in December 2011 in order to establish: a profile of the hospital and ICU they work in; their estimate of overall ICU mortality for critically ill cancer patients; the value of six outcome indicators in predicting mortality in two subgroups of oncological candidates for ICU admission; and the local management of acutely deteriorating cancer patients potentially requiring ICU care.

Results The ICU mortality rates estimated by survey respondents differed from those reported in the literature: for solid tumor 21\% (SEM 3 ) versus 10 to $23 \%$, for metastatic solid tumor $38 \%$ (SEM 4) versus $23 \%$, hematological malignancy $45 \%$ (SEM 3 ) versus 33 to $43 \%$ with allograft transplant $54.8 \%$ (SEM 5) versus 39 to $50 \%$ and autograft transplant $56 \%$ (SEM 5) versus $44 \%$. Regarding the management of cancer patients, there were conflicts reported between teams (rarely $44 \%$, occasionally $56 \%$, commonly $0.2 \%$ ). Few units had established triage policies for the acutely ill cancer patient (5\%) and it was also not common that plans were made prior to the patient's deterioration (never $11 \%$, rarely $38 \%$, occasionally $41 \%$, commonly $9 \%$ ). Figure 1 shows those outcome indicators thought to be important by responders in forecasting ICU prognosis.

Conclusion The awareness of improved outcome in critically ill cancer patients differs among physicians, and in general estimated mortalities were far higher than those reported in the literature.

Reference

1. Azoulay E, et al:: Ann Intensive Care 2011, 1:5.

Role of illness severity scores in predicting mortality in the coronary care unit

G Argyriou, J Terrovitis, G Sainis, V Papas, C Marvaki, S Nanas, C Routsi

Medical School, University of Athens, Greece

Critical Care 2012, 16(Suppl 1):P414 (doi: 10.1186/cc11021)

Introduction Several illness severity scores have been developed in order to predict outcome in multidisciplinary ICUs. However, the role of these scores has not been thoroughly investigated in coronary care units (CCUs) and the results are conflicting [1,2]. The aim of this study was to evaluate the utility of two of the most widely used scores - that is, APACHE II and Sequential Organ Failure Assessment (SOFA) - for the prediction of mortality in patients admitted to CCUs.

Methods All patients consecutively admitted to an eight-bed CCU from April 2010 to May 2011 were prospectively studied. Demographic, clinical and laboratory data were recorded. Illness severity on admission was measured using the APACHE II and SOFA scores. For the calculation of the scores, the worst values for each variable on admission day were used.

Results A total of 200 patients (age $70 \pm 17$ years, 65\% males) were admitted to the CCU during the study period; diagnoses included acute coronary syndrome $(65 \%)$, pulmonary edema $(11.5 \%)$, congestive heart failure $(5.5 \%)$ and other (18\%). The median length of CCU stay was $5 \pm 3$ days and the median length of hospital stay $9 \pm 7$ days. The CCU mortality rate was $20 \%$ and in-hospital mortality $24.2 \%$. Both APACHE II and SOFA scores were independently associated with mortality $(\mathrm{OR}=1.30 ; \mathrm{Cl}: 1.21$ to $1.40, P<0.001$ and $\mathrm{OR}=1.82 ; \mathrm{Cl}: 1.53$ to $2.16, P<0.001$ respectively). The receiver operating characteristic curves confirmed both scores as equally effective predictors of clinical outcome with areas under the curve of $0.92, P<0.001$ and $0.91, P<0.001$ for APACHE II and SOFA score respectively.

Conclusion The APACHE II and SOFA scores on admission are independent predictors of mortality in patients hospitalized in a CCU. Both scores demonstrate excellent performance in discriminating high-risk patients and thus are useful tools to predict clinical outcome in CCUs.

References

1. Clin Cardiol 1999, 22:366.

2. Minerva Anestesio/ 2011, 77:305
P415

New severity score of acute respiratory failure

S Allal, A Khedher, I Ben Saida, A Azouzi, A Farjallah, I Chouchen,

SBouchoucha, M Boussarsar

CHU Farhat Hached Hospital, Sousse, Tunisia

Critical Care 2012, 16(Suppl 1):P415 (doi: 10.1186/cc11022)

Introduction Acute respiratory failure (ARF), a common syndrome, is still poorly clinically appreciated. Literature review reports only a few attempts in neonatology (Silverman score) and in adults (Patrick score [1]) constructed by authors in 1996 for scientific research purposes. Both scores have never been validated. Instead, clinicians developed specific scores. We constructed a new respiratory failure score, organized in a trimodal manner (Table 1). Items were selected on the basis of pathophysiological and clinical expertise. Particular attention was paid to formulation and scaling to make the score both simple, noninvasive, inexpensive, didactic, and with interesting clinimetric properties. The objective of this study is to validate this score already in use for several years in our ICU.

Table 1 (abstract P415). Score of respiratory failure

\begin{tabular}{|c|c|c|c|}
\hline Grade & Respiratory rate & Accessory muscle use & Hypoxemia \\
\hline I & $<30$ & Intercostal & Normal \\
\hline$\|$ & 30 to 40 & $\begin{array}{l}\text { Supraclavicular and/or } \\
\text { suprasternal }\end{array}$ & Cyanosis \\
\hline III & $>40$ & $\begin{array}{l}\text { Thoraco-abdominal } \\
\text { swing/nasal flaring }\end{array}$ & $\begin{array}{l}\text { Circulatory and/or } \\
\text { consciousness disorders }\end{array}$ \\
\hline IV & Gasp & $\begin{array}{c}\text { Exhaustion/ventilatory } \\
\text { arrest }\end{array}$ & $\begin{array}{c}\text { Cardio-circulatory } \\
\text { arrest }\end{array}$ \\
\hline
\end{tabular}

Methods A prospective study among 70 patients with ARF on previously healthy lungs. ARF was rated in a randomized blinded manner respectively by residents and seniors. An inter-rater reliability analysis using the kappa statistic was performed to determine consistency among raters. Clinimetric properties were assessed by examining the prognostic prediction by the ROC curve using a composite gold standard $\left(\mathrm{PaO}_{2} /\right.$ $\mathrm{FiO}_{2}<250$ and/or ventilatory support).

Results The inter-rater reliability for the raters was found to be $k=0.82$ $(P<0.001)$, indicating an almost perfect agreement [2]. The area under the ROC curve was revealed very interesting $(A \cup C=0.88)$ indicating an excellent prognostic predictive power.

Conclusion This new and validated score could drive some advantages in daily practice, allowing accurate assessment of ARF severity, more objective monitoring of patients and easier communication between care providers. It may accurately guide oxygen supplementation and ventilatory support and afford accurate monitoring of pathophysiological and etiological treatment of ARF. It could be a valuable tool in randomized clinical trials or physiological studies evaluating treatments in ARF. Finally it could be used as an educational tool.

References

1. Patrick W, Webster K, Ludwig L, Roberts D, Wiebe P, Younes M: Non-invasive positive pressure ventilation in acute respiratory distress without prior chronic respiratory failure. Am J Respir Crit Care Med 1996, 153:1005-1011.

2. Landis JR, Koch GG: The measurement of observer agreement for categorical data. Biometrics 1977, 33:159-174.

P416

Validity of six prognostic scoring systems for septic shock patients admitted to a medical ICU

B Khwannimit, R Bhurayanontachai

Prince of Songkla University, Hat Yai, Thailand

Critical Care 2012, 16(Suppl 1):P416 (doi: 10.1186/cc11023)

Introduction This study aimed to assess the validity of the APACHE II, SAPS II, and SAPS 3, along with each of their customized scores, in predicting hospital mortality in patients with septic shock admitted to our ICU.

Methods A prospective cohort study was conducted over a 6-year period in a medical ICU of a tertiary referral university teaching hospital in Thailand. 


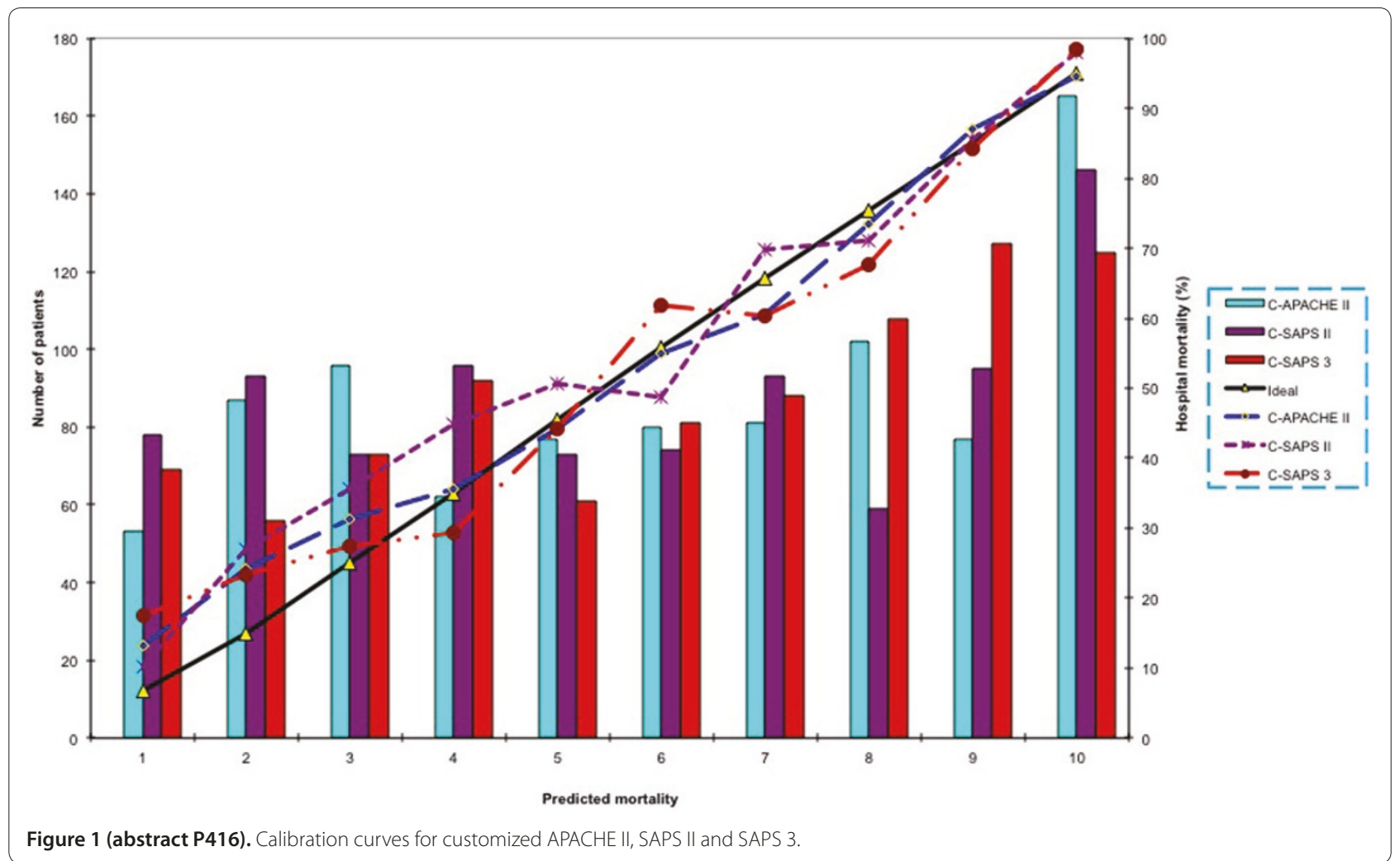

Results A total of 880 patients were enrolled and a hospital mortality rate of $57.4 \%$ was found. Community-acquired infections accounted for $57.2 \%$ and $32.8 \%$ of patients had positive blood culture. The respiratory tract was the most common site of infection (48.7\%). The predicted mortality of all the scores was close to the observed mortality, with a standardized mortality ratio (95\% confidence interval) of 0.94 ( 0.86 to 1.02) for APACHE II, 1.01 (0.92 to 1.1) for customized APACHE II, 0.93 (0.85 to 1.01) for SAPS II, 1.07 (0.98 to 1.17) for customized SAPS II, 0.97 (0.89 to 1.06) for SAPS 3 and 1.02 (0.93 to 1.11) for customized SAPS 3. All six scores were well discriminated, with areas under the receiver operating characteristic curves of $0.82,0.813,0.819,0.815,0.817$ and 0.813 , respectively. The Hosmer-Lemeshow goodness-of-fit showed good calibration in only the customized APACHE II (H-statistic 12.4, $P=0.26)$. See Figure 1 .

Conclusion In this study, the customized APACHE II was found to be accurate in predicting hospital mortality in septic shock patients requiring ICU admission.

\section{P417}

Risk factors of venous thrombosis in knee joint endoprosthesis SV Vlasov, IV Vlasova

Scientific Clinical Center of Miner's Health Protection, Leninsk-Kuznetsky, Russia Critical Care 2012, 16(Suppl 1):P417 (doi: 10.1186/cc11024)

Introduction The high risk of thromboembolic complications after knee joint prosthetics is conditioned by the series of surgical intervention particularities. The influence of intraoperative tourniquet usage on the leg deep venous thrombosis frequency was studied.

Methods The study included 125 patients with gonarthrosis of degree III who received total knee joint endoprosthesis. There were 26 men and 99 women at the age of 36 to $77(60.7 \pm 8.03)$. For all patients, spinal anesthesia in combination with long-term epidural blockade for postsurgical pain relief was performed. The antithrombotic measures included Klexan $40 \mathrm{mg}, 12$ to 15 hours before surgery and 8 hours after it. Color mapping of the lower leg vessels with an Acuson 128XP/10c scanner was performed before surgery, on 4 to 5 days after prosthetics and before discharge from the in-patient department. In addition, all patients underwent study of endothelial vasodilating function using the method proposed by Celermajer and colleagues [1].

Results On 4 to 5 days after surgery, leg deep venous thrombosis was found in 11 patients (8.8\% of all patients after prosthetics). For decrease of intraoperative blood loss the tourniquet was applied onto the middle third of the leg in 77 patients (60.6\%). In this group DVT was found in $10.4 \%$ of cases. In the nontourniquet group (48 patients) DVT was found in $6.25 \%$. The differences in the complication frequency were not statistically valid. The data from duplex scanning showed that 43 patients (34.4\%) before surgery had changes in the lower leg veins in view of varicose subcutaneous veins and post-thrombophlebitic syndrome combined with disorders of endothelial vasodilating function and low venous tone. Tourniquet use in patients with venous pathology resulted in DVT in 30\% (five of 15 patients). When a tourniquet was not used in patients with venous disease, DVT was found only in one of 28 patients (3.5\%). The test showed a significant difference in the frequency of thromboembolic complications in these groups $(P<0.001)$.

Conclusion Therefore, using a tourniquet in patients with evident base venous pathology in terms of varicose subcutaneous veins or postthrombophlebitic syndrome in total knee joint endoprosthetics is a risk factor for venous thrombosis development.

Reference

1. Celermajer DS, et al:: Non-invasive detection of endothelial dysfunction in children and adults at risk of atherosclerosis. Lancet 1992, 340:1111-1115.

\section{P418}

Proximal and distal deep venous thrombosis in critically ill patients: incidence and prevalence

S Yus Teruel, J Camacho Oviedo, L Cachafeiro Fuciños,

M Hernandez Bernal, A Agrifoglio Rotaeche, M Irazábal Jaimes,

L Fernandez Rodriguez, B Civantos Martín, J Díez

Hospital Universitario La Paz, Madrid, Spain

Critical Care 2012, 16(Suppl 1):P418 (doi: 10.1186/cc11025)

Introduction The aim of this study was to detect deep venous thrombosis (DVT) in patients admitted to a critical care unit (ICU) by 
compression ultrasonography, and to determine the incidence and prevalence of proximal and distal DVT in this setting.

Methods This was a prospective observational study conducted in our medical-surgical and trauma ICU from October 2009 to September 2010. The inclusion criterion was $\geq 72$ hours of ICU stay. Exclusion criteria were admitting diagnosis of pulmonary embolism or DVT, readmission, and patients with support withdrawal orders. The study was approved by the Research Ethics Board of La Paz Hospital. Bilateral lower extremity compression ultrasound was performed within 48 hours of admission to evaluate the prevalence, and twice weekly until discharge to assess the incidence. We collected demographic data, body mass index (BMI), APACHE II score, SOFA score, diagnostic categories, classic risk factors for DVT, femoral catheter and the use of mechanical ventilation and muscle relaxants. For the statistical analysis chi-square and Fisher tests were used, as well as Mann-Whitney and Student tests for data comparison. For the probability of DVT and its relation with the associated factors, the odds ratio and confidence interval were used. Statistical significance was $P<0.05$.

Results We enrolled 182 patients, with male predominance (57.3\%), 135 were mechanically ventilated (74.2\%) and the mean APACHE II score was 19.3 ( \pm 7.8 ). The mortality in the ICU was $15.4 \%(28)$, and 20.9 (38) in hospital. The prevalence of proximal DVT was $29.1 \%$ (53/182), and the incidence $24.0 \%(31 / 129)$. Seventy-nine percent of patients received DVT prophylaxis. The localization of incidentally diagnosed DVT was proximal in $29 \%$ and distal in 35\%; 19 (64\%) of these were identified on day 5 of admission. In four patients DVT was clinically suspected and only in one of them was DVT confirmed. The most frequently involved were soleal veins (67\%). Independent risk factors for incidental DVT were: older age (62 \pm 15.4 years vs. $54.5 \pm 17.1$; $P=0.032)$; $B M I\left(27.7 \pm 5.5 \mathrm{~kg} / \mathrm{m}^{2}\right.$ vs. $\left.24.9 \pm 5.2 \mathrm{~kg} / \mathrm{m}^{2} ; P=0.014\right) ;$ and mechanical ventilation: (OR: $3.3,95 \% \mathrm{Cl}=1.0$ to 10.26 ). Patients with incidental DVT had a higher hospital mortality $(P=0.03)$.

Conclusion In our study DVT was an early, asymptomatic and frequent event ( $46 \%$ of the ICU patients). In the presence of risk factors, a diagnostic ultrasound test might have a role.

P419

Saddle embolism is associated with the major adverse events in patients with nonhigh-risk pulmonary embolism

H Kim, W Kim

Asan Medical Center, Seoul, South Korea

Critical Care 2012, 16(Suppl 1):P419 (doi: 10.1186/cc11026)

Introduction In some patients with acute pulmonary embolism (PE), thrombi may lodge at the levels of bifurcation of the pulmonary trunk and extend into both main pulmonary arteries, forming so-called saddle embolism (SE). The aim of this study was to assess the incidence of SE and whether it is associated with an increased risk of complicated clinical course in patients with nonhigh-risk PE.

Methods Between January 2006 and June 2010, 297 consecutive patients with nonhigh-risk PE that was confirmed with contrastenhanced spiral computed tomography (CT) in the emergency department were studied. One experienced radiologist evaluated the presence of SE. The clinical information, echocardiographic and CT parameters were reviewed. Patients were divided into SE and nonSE. Multivariate logistic regression was applied to determine factors associated with occurrence of major adverse events (MAE).

Results Twenty-seven out of 297 patients (9.1\%) were found to have a SE. Overall mortality at 1 month was $12.5 \%$ with no difference between the groups $(11.9 \%$ vs. $18.5 \%, P=0.32)$, although SE patients were more likely to receive thrombolytic therapy $(8.1 \%$ vs. $29.6 \%$, $P<0.01)$. SE patients had $\mathrm{s}$ significantly higher rate of MAE $(59.3 \% \mathrm{vs}$. $25.6 \%, P<0.01)$. Presence of SE and the ratio of right ventricular to left ventricular diameter were associated with an odds ratio of MAE within 1 month of 2.48 (95\% Cl: 1.10 to $6.04, P=0.03)$ and $3.34(95 \% \mathrm{Cl}: 1.46$ to $7.46, P<0.01$ )

Conclusion SE by CT angiography was associated with PE-related shock, intubation, mortality, thrombolysis, and thrombectomy within 1 month in patients with nonhigh-risk PE and may be a useful method for simple risk stratification.
P420

Efficacy and safety of enoxaparin as deep vein thrombosis prophylaxis in critically ill patients

R Al-Hubail, $N$ Hassan

King Fahad Specialist Hospital, Dammam, Saudi Arabia

Critical Care 2012, 16(Suppl 1):P420 (doi: 10.1186/cc11027)

Introduction Critically ill patients are at high risk of developing deep vein thrombosis (DVT). DVT cannot be detected in most cases, leading to fatal embolic manifestations [1]. The goal of this study was to review the occurrence of DVT in patients receiving enoxaparin during their length of stay in the ICU (ICU LOS). In addition we review the occurrence of major bleeding and thrombocytopenia secondary to enoxaparin.

Methods This was a retrospective database analysis including medical and surgical patients admitted to a tertiary hospital (King Fahad Specialist Hospital Dammam) critical care department from 1 January to 31 December 2010, aged 17 to 70 years, excluding patients with: platelets <50,000/l; evidence of active bleeding; new ischemic or haemorrhagic stroke; spinal or epidural catheter who were already on anticoagulant when admitted to the ICU and who were previously diagnosed with DVT or with pulmonary embolism (PE); DNR (do not resuscitate). The APACHE II score, predicted mortality and ICU LOS were calculated for included patients in the study. The hospital electronic system and critical care database were reviewed with the physician order sheet according to the ICU protocol for DVT prophylaxis (enoxaparin $40 \mathrm{mg}$ subcutaneously once daily).

Results Five hundred and ninety-seven patients were investigated, from which 22 (3.5\%) fulfilled exclusion criteria, 220 (36\%) were on a sequential decompression device (SD), and 26 (4\%) were not on DVT prophylaxis (protocol violation). This gave a study population of 329 $(55 \%)$ cases that were on enoxaparin thromboprophylaxis. In this population there were no recorded cases of DVT and two cases (0.6\%) of PE. Major bleeding was recorded in seven cases $(2.1 \%)$, platelets $<50,000 / \mathrm{l}$ in eight cases (2.4\%), and $\mathrm{Hb}$ level $<1.5 \mathrm{~g} / \mathrm{dl}$ from baseline without bleeding in 47 cases (14.2\%). See Table 1.

Table 1 (abstract P420)

\begin{tabular}{lccc}
\hline Type of case & ICU LOS (days) & APACHE II & $\begin{array}{c}\text { Predicted } \\
\text { mortality (\%) }\end{array}$ \\
\hline Total cases on enoxaparine & $5.29 \pm 7.3$ & $16.7 \pm 10.5$ & $28.4 \pm 23.7$ \\
Low platelets cases & $11.75 \pm 9.7$ & $23 \pm 2.3$ & $48.75 \pm 11.18$ \\
Major bleeding cases & $13.5 \pm 13.1$ & $22.4 \pm 17.4$ & $30 \pm 17.5$ \\
\hline
\end{tabular}

Conclusion Using the hospital and critical care databases, we observed that the critically ill patients receiving enoxaparin as thromboprophylaxis did not experience DVT, and two $(0.6 \%)$ had PE during their ICU stay. However, thrombocytopenia and major bleeding were recorded at very low frequencies $(2.5 \%)$.

Reference

1. Greets et al:: Chest $2003,124: 357 s-363 s$.

P421

Reducing the level of postoperative thrombotic complications by the combination of low molecular weight heparin and epidural anesthesia at the patients after total hysterectomy

O Tarabrin, V Dubinina, A Turenko, S Tarasenko, S Shcherbakov,

D Gavrychenko, G Mazurenko

Odessa National Medical University, Odessa, Ukraine

Critical Care 2012, 16(Suppl 1):P421 (doi: 10.1186/cc11028)

Introduction Each year in the world, cancer of the reproductive system is diagnosed in more than 600,000 women. $\ln 8$ to $35 \%$ of patients with cancer of the reproductive system, pulmonary embolism was the cause of death - and in $43 \%$ the background for other fatal complications. Methods The results of surgical treatment of 79 patients after hysterectomy under prolonged epidural anesthesia during the period from 2008 to 2010 entered the study. The condition of hemostasis was monitored by 12 standard biochemical tests, as well as the new 
instrumental method - hemoviscoelastography preoperatively, intraoperatively and every day during 10 days after surgery. Prevention of thrombotic complications in group 1 (37 patients), conducted by bemiparin 3,500 units: the first injection 12 hours before surgery, then at 6 hours after the operation in the future once a day for 10 days; group 2 (42 patients) received heparin 5,000 units: the first injection 6 hours before surgery, then 6 hours after the operation, then four times per day for 10 days.

Results All patients included in the study before surgery had detected hypercoagulation and inhibition of fibrinolysis: increasing of MA (maximum density of the clot, fibrin-platelet constant of the blood) to $20.7 \%(P<0.001)$ and ICD (intensity of coagulation drive (the intensity of clot formation)) to $15.6 \%$; reduction of IRCL (intensity of the retraction and clot lysis) to $13.6 \%(P<0.05)$ in both groups compared to normal rates. At the first day after surgery in patients treated with bemiparin (group 1) MA and ICD decline to $12.7(P<0.05)$ and $9.6 \%(P<0.001)$ respectively, and IRCL increased by $4.6 \%(P<0.05)$ compared with preoperatively. In group 2 there was a similar picture: the reduction of MA and ICD to 10.3 $(P<0.001)$ and $6.6 \%(P<0.05)$ respectively, and IRCL increased by $4.4 \%$ $(P<0.001)$. At the fifth day the condition of hemostasis in both groups came almost to the same value - a moderate hypocoagulation, normal activity of fibrinolysis. At 7 days of the postoperative period, thrombotic complications developed in one patient of the first group (2.70\%). In the second group, complications developed in four $(9.52 \%)$ patients: in three cases deep venous thrombosis, and in one case coagulopathic bleeding. Conclusion Using a combination of bemiparin and epidural anesthesia reduces the level of postoperative thrombotic complications, such as deep venous thrombosis, and massive bleedings in the patients after total hysterectomy. Using hemoviscoelastography enables quick identification of disorders of hemostasis in patients after hysterectomy before, during and after the surgery.

\section{P422}

Cost-effectiveness analysis of two thromboprophylactic strategies

following major surgery

C Ebm, M Cecconi, A Rhodes, M Grounds

St George's Healthcare Trust, London, UK

Critical Care 2012, 16(Suppl 1):P422 (doi: 10.1186/cc11029)

Introduction Patients recovering from major surgery are at high risk of developing life-threatening deep venous thrombosis, which is a key source of postoperative morbidity and mortality. Our objective was to assess the cost-effectiveness of two different thromboprophylactic agents for patients admitted to the ICU after high-risk surgery: intermittent pneumatic compression (IPC) and anti-embolism stockings (AES).

Methods A decision model (TreeAge Software 2010) was constructed simulating the impact of AES and IPS on patient outcomes and costs following high-risk surgery in the UK. Probabilities were assessed from published data [1]. ICU and item costs were derived from NHS reference costs tablets. Assessed outcomes were cost per deep vein thrombosis (DVT) and pulmonary embolism (PE) prevented, net monetary benefit and incremental costs per quality-adjusted life expectancy (QALY) gained.

Results Total costs for in-patients receiving AES were $£ 923$ and $£ 1,010$ for patients treated with IPC. Equipment costs and cost of initial care were higher in patients who received IPC, but this was partly offset by a reduction in costs related to treatment of early (DVT and PE) and late complications (post-thrombotic syndrome and pulmonary hypertension). IPC treatment increased QALY by approximately 0.01 years. The incremental cost-effectiveness of the IPC device was $£ 12,650$ per QALY gained. One-way sensitivity analysis revealed that the most sensitive variables were probability of developing a DVT resulting from the insignificant difference in treatment efficacy.

Conclusion Based on UK cost-effectiveness guidelines, our results indicate that IPC stockings should be used for patients at high risk of developing DVT. IPCs decrease the incidence of developing DVT and therefore result in cost savings related to preventive and therapeutic actions. For patients at low risk of developing DVT, AES are favoured due to higher utility and lower maintenance costs associated with AES. Due to the lack of reliable data on the incidence of PE as well as the absence of reliable head-to-head studies between IPC and AES, no generalisable conclusion to favour either strategy can be made.

\section{Reference}

1. Roderick P, Ferris G, Wilson K, Halls H: Towards evidence-based guidelines for the prevention of venous thromboembolism: systematic reviews of mechanical methods, oral anticoagulants, dextran and regional anaesthesia for thromboprophylaxis. Health Technol Assess 2005, 9(49):iii-iv, 1-78.

P423

Consequences of suspected heparin-induced thrombocytopenia in

the ICU

M Ostermann', L Mclntyre ${ }^{2}$, F Lauzier ${ }^{3}$, J Alhashemi ${ }^{4}$, I Qushmaq ${ }^{5}$,

S Langevin ${ }^{3}$, P Dodek ${ }^{6}$, M Albert 7 , K Khwaja ${ }^{8}$, J Kutsiogiannis? $L_{\text {B Burry }}^{10}$, J Granton ${ }^{10}$, J Friedrich ${ }^{10}$, N Ferguson ${ }^{10}$, J Marshall10, S Finfer ${ }^{11}$, D Heels-Ansdell'12, N Zytaruk'2, D Cook'2, J Sheppard'2, T Warkentin ${ }^{12}$, M Crowther ${ }^{12}$

${ }^{1}$ King's College London, UK; ${ }^{2}$ University of Ottawa, Canada; ${ }^{3} \mathrm{CHA}$-Hôpital de I'Enfant-Jésus, Université Laval, Québec, Canada; ${ }^{*}$ King AbdulAziz University, Jeddah, Saudi Arabia; ${ }^{5}$ King Faisal Hospital, Jeddah, Saudi Arabia; ${ }^{6}$ University of British Columbia, Vancouver, Canada; 'Université de Montréal, Canada; ${ }^{8}$ Université McGill, Montréal, Canada; ${ }^{9}$ University of Alberta, Edmonton, Canada; ${ }^{10}$ University of Toronto, Canada; "The George Institute, Sidney, Australia; ${ }^{12}$ McMaster University, Hamilton, Canada

Critical Care 2012, 16(Suppl 1):P423 (doi: 10.1186/cc11030)

Introduction Clinical suspicion of heparin-induced thrombocytopenia (HIT) may prompt changes in drug management and alert clinicians to an increased risk of thrombosis. However, thrombocytopenia in the ICU occurs in about $50 \%$ of patients, is multifactorial and is due to HIT in $<1 \%$. We aimed to describe the consequences of suspected HIT among medical-surgical critically ill patients in terms of drug and device management, and thrombotic outcomes.

Methods We enrolled 3,746 patients in the PROTECT trial comparing prophylactic dalteparin to unfractionated heparin. We defined HIT as occurring in patients with a clinical or laboratory-driven suspicion of HIT and a positive serotonin release assay (SRA). We defined suspected HIT as patients whose clinicians were sufficiently concerned about HIT to withhold heparin. We defined consequences of HIT as occurring from 1 day before it was suspected to 30 days thereafter.

Results One hundred and thirty patients (3.5\%) had heparin held due to clinical suspicion of HIT. Of these, $10(7.7 \%)$ had a positive SRA test. The drugs and devices used for thromboprophylaxis, as well as thrombotic events, are outlined in Table 1. At least one new thrombotic event developed in $23.8 \%$ of patients with suspected HIT and $40.0 \%$ of patients with HIT.

Table 1 (abstract P423)

\begin{tabular}{lc}
\hline & $\begin{array}{c}\text { 1 day before to } 30 \text { days after } \\
\text { heparin held for suspect HIT }\end{array}$ \\
\hline Intervention & $34(26.2)$ \\
Danaparoid & $8(6.2)$ \\
Lepirudin & $11(8.5)$ \\
Fondaparinux & $19(14.6)$ \\
Argatroban & $67(51.5)$ \\
Any of the above drugs & $25(19.2)$ \\
Anti-embolic stockings & $37(28.5)$ \\
Pneumatic compression device & $49(37.7)$ \\
Anti-embolic stockings or pneumatic & \\
compression device & $96(73.8)$ \\
Any of the above interventions & \\
Incident thromboses & $30(23.1)$ \\
Venous thrombosis (including PE) & $1(0.8)$ \\
Arterial thrombosis & $2(1.5)$ \\
Progression of a previous thrombus & $31(23.8)$ \\
Any of the above &
\end{tabular}

Conclusion Over $90 \%$ of patients with suspected HIT did not have HIT. One-half of patients with suspected HIT were prescribed another 
anticoagulant and one-third received mechanical prophylaxis. Thrombotic rates are higher in patients with HIT and suspected HIT than other patients. The frequent suspicion of HIT in critically ill patients and initiation of other interventions may create a greater clinical and economic burden than HIT itself.

P424

Evaluation of iron, transferrin and ferritin serum levels in patients with severe sepsis and septic shock

M Missano Florido, M Assunção, B Mazza, M Jackiu, F Freitas, A Bafi,

F Machado

UNIFESP, São Paulo, Brazil

Critical Care 2012, 16(Suppl 1):P424 (doi: 10.1186/cc1 1031)

Introduction Iron metabolism is altered in critically ill patients leading to hypoferremia. Several studies related it to inflammatory response $[1,2]$. The present study aims to evaluate iron, transferrin and ferritin serum levels in patients with severe sepsis and septic shock and its association with severity of organ dysfunction.

Methods A prospective observational cohort study, unicentric, in a tertiary teaching hospital. From November 2010 to October 2011 patients over 18 years old with severe sepsis or septic shock with up to 72 hours of organ dysfunction were included. Exclusion criteria were blood transfusion or iron supplementation in the last 90 days, previous inclusion and pregnancy. After obtaining informed consent, blood samples were taken at baseline and on day 7. Demographic and APACHE II and SOFA data were also collected. Patients who were transfused with red blood cells between the two periods were excluded from the day 7 sample. Patients were followed until hospital discharge or death.

Results Thirty patients were included, with a mean age of $59.6 \pm 19.3$, APACHE II score $19.1 \pm 7.2$, SOFA at baseline $8.5 \pm 4.0$, and most patients had septic shock (63.3\%). Baseline iron and transferrin levels were low in $83.3 \%$ ( 14.0 (5.0 to 25.5$)$ ) and in $96.7 \%(94.1 \pm 31.6)$ of the patients, while ferritin was high in $63.3 \%$ (954.0 (508.4 to 5,394.0)). In the 19 patients where a day 7 sample was available, variation between baseline and day 7 was statistically significant for transferrin $(97.9 \pm 37.5$ to $132.7 \pm 48.3$, $P=0.013$ ) and ferritin (478.0 (224.5 to $1,741.0)$ to 376.0 (187.0 to 886.7) $P=0.018)$, while iron levels showed a trend towards increasing levels at day 7 (17.0 (6.5 to 44.3) to 29.0 (21.0 to 54.0), $P=0.061)$. Baseline SOFA score trends to be lower in hypoferrinemic patients (7.7 \pm 3.8 vs. $12.4 \pm 1.9, P=0.098)$. The Spearman test showed a weak correlation only between SOFA and iron levels $\left(P=0.008 ; r^{2}=0.48\right)$.

Conclusion Septic patients have low iron and transferrin levels, associated with high ferritin levels, and those levels improved during the course of disease. Low iron levels might be associated with low SOFA scores.

References

1. Lagan AL, et al: Am J Physiol Lung Cell Mol Physiol 2008, 294:L161-L174.

2. Quinlan GJ, et al:: Am J Respir Crit Care Med 1997, 155:479-484.

P425

Using angiogenic factors and their soluble receptors to predict organ dysfunction in patients with disseminated intravascular coagulation associated with severe trauma

TWada', S Jesmin², S Gando 3, S Zaedi², H Yokota'

'Nippon Medical School, Tokyo, Japan; ${ }^{2}$ National Center for Global Health and Medicine, Tokyo, Japan; ${ }^{3}$ Hokkaido University Graduate School of Medicine, Sapporo, Japan

Critical Care 2012, 16(Suppl 1):P425 (doi: 10.1186/cc11032)

Introduction Disseminated intravascular coagulation (DIC) is observed after not only sepsis but also trauma. DIC is associated with concomitant activation of coagulofibrinolytic disorder and systemic inflammation with endothelial dysfunction and microvascular permeability. The angiogenic factors, including vascular endothelial growth factor (VEGF), angiopoietin (Ang), and their receptors, play crucial roles in angiogenesis and microvascular permeability. The aim of the study was to assess: the relationship between angiogenic factors, their soluble receptors and organ dysfunction associated with DIC precipitated by severe trauma; and the effects of DIC-induced platelet consumption, thrombin generation and tissue hypoxia on the expression of these factors and receptors.
Methods Fifty-seven patients with severe trauma were divided into two subgroups: 30 DIC patients and 27 non-DIC patients. The serum levels of angiogenic factors were measured on admission (day 1), day 3 , and day 5 . We compared serum levels of these angiogenic factors between with and without DIC groups and evaluated their predictive value for organ dysfunction and outcome.

Results DIC patients showed higher Sequential Organ Failure Assessment (SOFA) scores, soluble fibrin and lactate levels. The serum levels of VEGF, Ang1, and the sTie2 levels were lower in the DIC patients than the non-DIC patients. The serum levels of SVEGFR1, Ang2 and the Ang2/Ang1 ratio in the DIC patients were higher than in those without DIC. The sVEGFR2 levels showed no statistically significant difference between the patients with and without DIC. The levels of sVEGFR1, Ang 2 and the Ang2/Ang1 ratio correlated with the SOFA score. In particular, sVEGFR1 and Ang2 were independent predictors of an increase in the SOFA score. The lactate levels independently predicted increases in the levels of sVEGFR1 and Ang2 and platelet consumption also independently predicted the increase in Ang2 levels in severe trauma patients with DIC.

Conclusion Angiogenic factors and their soluble receptors, particularly sVEGFR1, play pivotal roles in the development of organ dysfunction in $\mathrm{DIC}$ associated with severe trauma. The DIC-induced tissue hypoxia and platelet consumption plays crucial roles in inducing sVEGFR1 and Ang2, and in determining the prognosis of the severity of organ dysfunction.

\section{P426}

A simple blood-saving bundle reduces diagnostic blood loss in mechanically ventilated patients

R Riessen, M Behmenburg, G Blumenstock, D Guenon, S Enkel, M Haap

University Hospital Tübingen, Germany

Critical Care 2012, 16(Suppl 1):P426 (doi: 10.1186/cc11033)

Introduction By introducing a blood-saving-bundle (BSB) consisting of a closed-loop arterial blood sampling system, smaller tubes and an attempt to reduce the number of blood samples, we aimed to reduce blood loss caused by diagnostic blood sampling and to minimize the development of anemia in a high-risk group of mechanically ventilated intensive care patients.

Methods Included were all patients from our medical ICU who were ventilated for more than 72 hours. Exclusion criteria were acute or chronic anemia on admission, a bleeding episode during the ICU stay or end-of-life therapy. The BSB was introduced in 2009 with training and educational support. Patients treated in the year 2008 before the introduction of the BSB served as a control group and were compared to patients treated in 2010 after introduction of the BSB (BSB group). Daily blood loss was calculated on the basis of the documentation of blood samples and laboratory values in the patient data management system and by using data from two representative study periods in which the sample volumes of all diagnostic blood tests were measured. Results The control group comprised of 41 patients (614 observation days), the BSB group of 50 patients (559 observation days). Mean blood loss per ICU day decreased from $43.3 \mathrm{ml}(95 \% \mathrm{Cl} 41.2$ to $45.3 \mathrm{ml})$ in the controls to $15.0 \mathrm{ml}(14.3$ to $15.7 \mathrm{ml})$ in the BSB group $(P<0.001)$. The introduction of a closed-loop arterial blood sampling system contributed most to this effect. Mean hemoglobin values showed a similar decrease in both groups during the ICU stay. However, hemoglobin values $<9 \mathrm{~g} / \mathrm{dl}$ were measured in $21.2 \%$ of observation days in the controls versus $15.4 \%$ in the BSB group $(P=0.01)$. In the control group $31.7 \%$ (18.1 to $48.1 \%$ ) of the patients received red blood cell transfusions in contrast to only $8.0 \%$ (2.2 to $19.2 \%$ ) in the BSB group $(P=0.006)$, while the hemoglobin concentration triggering transfusion was not significantly different $(8.2 \mathrm{vs.} 7.8 \mathrm{~g} / \mathrm{dl})$. The mean number of intubation days was 7.1 days (6.1 to 8.3 days) in the controls and 7.5 days $(6.6$ to 8.5$)$ in the BSB group $(P=N S)$. However, patients in the BSB group stayed with a mean of 9.8 days (8.6 to 11.3 days) significantly shorter in the ICU than controls with 13.2 days (10.9 to 15.4 days) $(P=0.014)$.

Conclusion Our BSB could easily be implemented and was able to reduce diagnostic blood loss by $65 \%$. After introduction of the BSB we observed less transfusions and a shorter ICU stay in mechanically ventilated patients; this, however, has to be interpreted with caution due to the longitudinal study design. 
P427

Comparative assessment of invasive and noninvasive methods for detection of total hemoglobin in gynecological patients' blood

AV Pyregov, SV Petrov

Research Center for Obstetrics, Gynecology and Perinatology, Moscow, Russia Critical Care 2012, 16(Suppl 1):P427 (doi: 10.1186/cc11034)

Introduction Safety of patients is possible to increase applying early detection of intraoperative and postoperative hemorrhage using the widening array of monitoring opportunities; not only the hemodynamic parameters, but the detection of total hemoglobin. Continuous noninvasive monitoring of total hemoglobin content is possible due to the Masimo Rainbow SET technology, using multiwave spectrophotometry.

Methods Seventy-eight patients aged 15 to $59(35.9 \pm 1.62)$ with laparoscopic gynecological operations were included in the research after permission of the ethics committee and signing the informing agreement. Total hemoglobin was detected by laboratory method invasively, discretely and delayed. Total hemoglobin was detected by another method oximetrically $(\mathrm{SpHb})$ during the monitoring process on the platform Rainbow SET technology noninvasive, continuous, and promptly. SpHb was compared with total hemoglobin on the following stages of the research: before the operation, during the operation and in the early postoperative period. Statistical analysis was fulfilled by comparing real and tabular (critical) criteria of reliability - Student test. Results During the detection of total hemoglobin by the laboratory method, the mean value was $121.5 \pm 17.28 \mathrm{~g} / \mathrm{l}$, while oximetrically it occurred $118.6 \pm 17.41 \mathrm{~g} / \mathrm{l}$. The real criterion of reliability (tr) was 0.85 , the critical criterion of reliability (tcr) was 2.63 .

Conclusion We did not discover statistically significant differences of total hemoglobin determined by two different methods. Thereby, noninvasive monitoring of total hemoglobin contention using multiwave spectrophotometry by Masimo Rainbow SET technology can serve as an appropriate replacement for the laboratory screening of hemoglobin.

\section{P428}

Use of coagulation screening in the critical care unit

A Rice, R Paterson, C Cairns

Forth Valley Royal Hospital, Larbert, UK

Critical Care 2012, 16(Suppl 1):P428 (doi: 10.1186/cc11035)

Introduction The aim of this audit is to compare the effectiveness of indiscriminate coagulation testing versus selective testing based on clinical indications within the HDU setting. Coagulation tests (PT and APTT) are often taken as a matter of routine alongside patient's daily blood tests in the critical care setting. Abnormal coagulation results rarely alter patient management while repeated testing has significant detrimental financial implications.

Methods Over a 14-day period, the blood results of HDU patients were prospectively analysed in order to assess whether or not a coagulation screen was conducted and whether or not this was appropriate based on clinical indications. Following targeted education towards medical and nursing staff, including publicising a list of clinical indications within the unit, the audit cycle was repeated.

Results Prior to education, only $37 \%$ of coagulation screens were clinically indicated. Following implementation of the indications this rose to $50 \%$. Using the guidelines in the second round there was $100 \%$ identification of abnormal results compared to only $81 \%$ prior to education. On review of all these data we were able to extrapolate that prior to targeted education there was a 2:1 ratio of appropriate to inappropriate coagulation testing, post intervention this rose to 5:1. Conclusion With local targeted education of staff we significantly reduced the number of inappropriate coagulation tests undertaken within our unit from $65 \%$ to $27 \%$. Along with this we had a $100 \%$ detection rate for abnormal results using our list of clinical indications for testing. In our high turnover critical care unit this would indicate potential savings of around $£ 10,000$ per annum; a significant amount in an organisation with longstanding financial constraints.
P429

Templating effect of clot structure can predict clot development and outcome in diluted blood: a comparison with thromboelastography

M Lawrence', J Kaczynski², S Stanford ${ }^{1}$, R Morris ${ }^{3}$, P Evans ${ }^{2}$

${ }^{1}$ Swansea University, Swansea, UK; ${ }^{2} A B M U$ LHB, Swansea, UK; ${ }^{3}$ UWIC, Cardiff, UK

Critical Care 2012, 16(Suppl 1):P429 (doi: 10.1186/cc11036)

Introduction Treatment of major hemorrhage with colloids is known to have an effect on clot outcome. However, determining both the rate and extent of these changes is difficult. Development of a new biomarker has shown that it can detect structural development earlier and quantifies these changes to clot outcome accurately when compared to other methods. This study compares the fractal dimension, Df [1], found when the clot first forms to measures of mature clot firmness obtained from thromboelastography.

Methods Forty healthy blood samples were obtained; each sample was allocated a random dilution ratio $(10 \%, 20 \%, 40 \%, 60 \%)$ and diluted with gelofusine. These were matched with 40 healthy samples that were undiluted. An oscillatory shear technique was applied to the blood using an AR-G2 measuring Df (clot structure). Additionally the clot development in terms of firmness was measured using a ROTEM analyser measuring at 5, 10, 15 minutes and its maximum (A5, A10, A15, MCF).

Results Df significantly decreases with increasing dilution. The decrease in structural complexity indicates that gelofusine even at $40 \%$ dilution is producing poor quality clots. See Table 1.

Table 1 (abstract P429). Change in Df with dilution

\begin{tabular}{ll}
\hline Dilution \% & Df \\
\hline 0 & $1.74(0.05)$ \\
10 & $1.72(0.04)$ \\
20 & $1.70(0.06)$ \\
$40^{*}$ & $1.63(0.05)$ \\
$60^{*}$ & $1.59(0.06)$ \\
\hline
\end{tabular}

*Significant decrease from $0 \%$.

Conclusion Df that is measured at the incipient clot is found much sooner than the mature clot parameters, between 5 and 30 minutes earlier. Df is significantly correlated $(P<0.05)$ with the mature clot parameters of clot firmness (A5, A10, A15 and MCF) and elasticity $\left(G^{\prime}\right)$. This suggests that in dilution Df can determine the eventual clot outcome very early. Measurement of Df could guide fluid replacement and component therapy more accurately and earlier than conventional markers.

Reference

1. Evans P, et al:: Blood 2010, 116:3341-3346.

P430

Fractal analysis: a new biomarker for determining clot

characteristics in critically ill patients

GR Davies, SN Stanford, MJ Lawrence, D Gill, PR Williams, K Morris,

DThomas, PA Evans

NISCHR Haemostasis Biomarker Research Unit, Swansea, UK

Critical Care 2012, 16(Suppl 1):P430 (doi: 10.1186/cc11037)

Introduction Recent research [1] has highlighted a novel new biomarker of haemostasis: the fractal dimension (Df). This new biomarker relates the kinetics of clot formation to clot outcome in whole blood and allows us to quantify the complexity of the fibrin network microstructure which is believed to be the template for development of the mature clot. It is well established that abnormalities in haemostasis contribute to the pathogenicity of critical illness [2]. This prospective study aims to assess the effect of critical illness on clot structure and monitor the sensitivity of Df to therapeutic intervention.

Methods Patients with critical illness inducing SIRS were recruited on admission to the intensive therapy unit in a large teaching hospital in Wales. Blood was taken for routine coagulation testing, ROTEM thromboelastometry and rheological analysis (Df and Tgel) on 
Table 1 (abstract P430)

\begin{tabular}{lcc}
\hline & Mean Df & Mean MCF (mm) \\
\hline Pre enoxaparin & $1.79 \pm 0.08$ & $68.0 \pm 8.0$ \\
Post enoxaparin & $1.64 \pm 0.10$ & $64.3 \pm 4.2$ \\
\hline
\end{tabular}

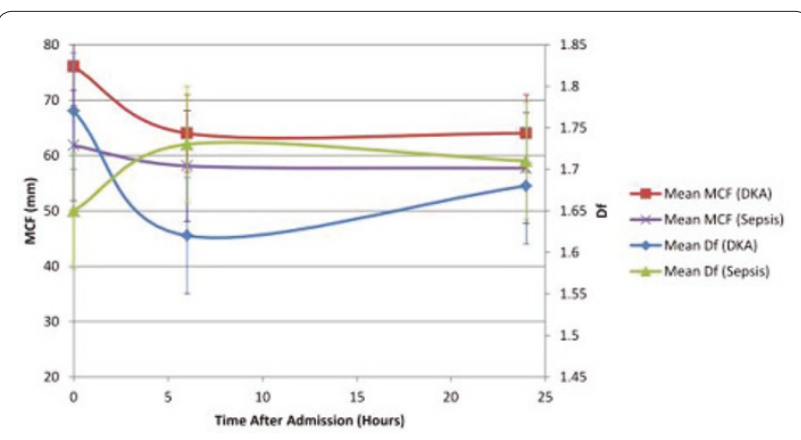

Figure 1 (abstract P430).

admission, at 6 hours and 24 hours to assess pathophysiological state and progression. Twelve patients were recruited: nine severe sepsis and three severe DKA with metabolic disorder. Twelve healthy volunteers were recruited as a matched control.

Results Mean Df in the control group was $1.73 \pm 0.03$ whereas mean Df in DKA and sepsis was found to be $1.77 \pm 0.07$ and $1.65 \pm 0.05$ respectively. Marked differences were observed in Df and maximum clot firmness (MCF) in response to treatment intervention (Figure 1). Furthermore, patients saw a dramatic decrease in Df post enoxaparin, but no significant change in MCF was observed (Table 1).

Conclusion Df shows specificity between severe DKA and sepsis. Df shows sensitivity to treatment intervention and illness progression in the critically ill.

\section{References}

1. Evans PA, et al.: Blood 2010, 116:3341-3346

2. Levi M, Opal SM: Crit Care 2006, 10:222.

P431

Fractal dimension: a biomarker for detecting acute thromboembolic disease

K Hawkins ${ }^{1}$, N Badiei', J Weisel², I Chernysh², PR Williams', MJ Lawrence', PA Evans ${ }^{1}$

'Swansea University, Swansea, UK; ${ }^{2}$ University of Pennsylvania, Philadelphia, PA, USA

Critical Care 2012, 16(Suppl 1):P431 (doi: 10.1186/cc11038)

Introduction This study investigates the potential use of rheometry to provide a structural biomarker for acute critical illness. Previous studies have reported an association of altered fibrin clot network
Table 1 (abstract P431). Results of the fractal dimension obtained by rheometry of fibrin clots

\begin{tabular}{lc}
\hline Thrombin $(\mathrm{NIH})$ & Fractal dimension \\
\hline 0.02 & 1.85 \\
0.1 & 1.95 \\
0.3 & 2.13 \\
\hline
\end{tabular}

architecture with several diseases including sepsis, bleeding or acute thromboembolic disease [1]. We investigate our biomarker by examining the relationship between thrombin generation and clot architecture in an in vitro model.

Methods Rheometry and confocal laser scanning microscopy (CLSM) were used to monitor and image the formation of fibrin clots. Clotting was initiated by the addition of different levels of thrombin to solutions of a fixed concentration of fibrinogen. Each sample was divided into two aliquots; one added to the measuring geometry of an AR-G2 rheometer and one to the microscope slide for the spinning disk CLSM (Olympus IX71).

Results The micrographs of formed clots (Figure 1) show marked qualitative differences in clot architecture. Upon increasing the available thrombin, the clot network (visually) appears more dense. Table 1 shows the value of the structural biomarker, the fractal dimension, that corresponds to the clots formed in Figure 1.

Conclusion We demonstrate, for the first time, that the fractal dimension obtained by rheometry is a sensitive measure of visually observed structural differences within the fibrin network. Rheometrical detection of incipient clots formed in whole blood provides the clinician with a powerful tool for the diagnosis of thromboembolic disease.

\section{Reference}

1. Scott et al:: Arterioscler Thromb Vasc Biol 2004, 2:1558-1566.

P432

Thromboelastography (platelet contribution to clot strength) for

the assessment of platelet residual function

D Haxhiademi', S Parri', A Cerillo', P Del Sarto', R Paniccia², D Prisco ${ }^{2}$

${ }^{1}$ Fondazione Toscana G. Monasterio, Massa, Italy; ${ }^{2}$ Thrombosis Centre,

University of Florence, Italy

Critical Care 2012, 16(Suppl 1):P432 (doi: 10.1186/cc11039)

Introduction In the early postoperative period after cardiac surgery, platelet dysfunction is one of the main causes of excessive bleeding; there is still controversy regarding the timing of antiplatelet therapy discontinuation [1]. The Clinical Practice Guidelines of the Society of Thoracic Surgeons recommend that point-of-care $(P O C)$ testing may help identify patients who can safely undergo urgent operations [2]. This study was designed to test the relationship between platelet function as revealed by POC tests and postoperative bleeding in patients that undergo cardiac surgery without suspending thienopyridines at least 5 days prior to surgery.
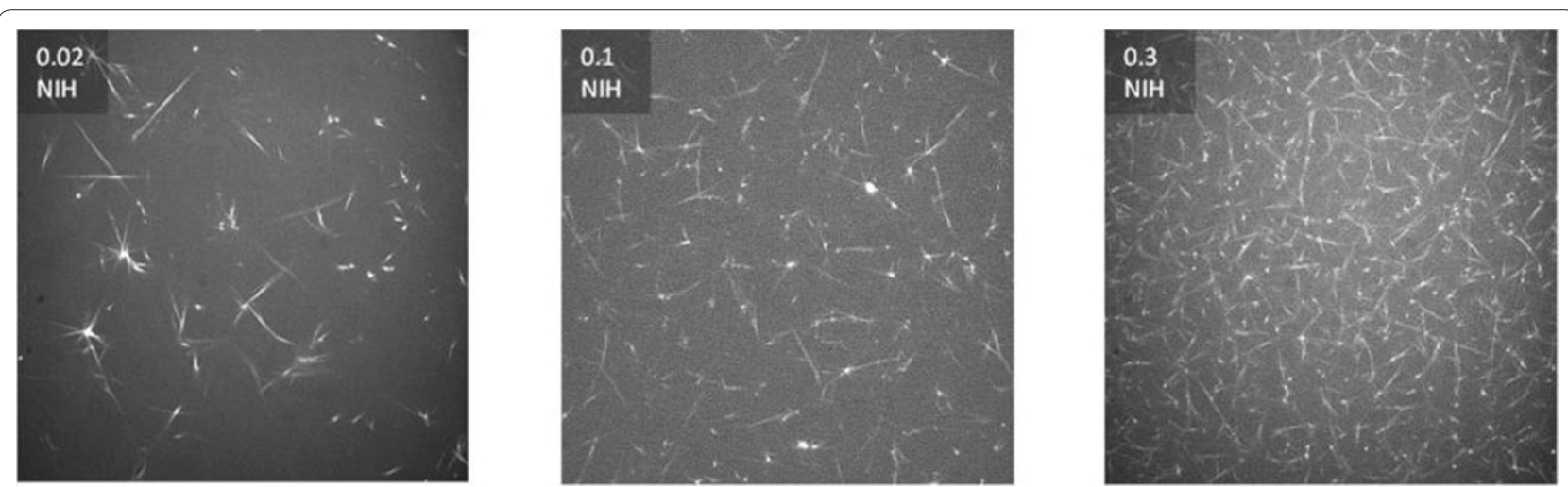

Figure 1 (abstract P431). CLSM micrographs of formed fibrin clots at thrombin levels of $0.02,0.1$ and $0.3 \mathrm{NIH}$. 
Methods Adult patients scheduled for cardiac operations in which thienopyridines were not discontinued at least 5 days before surgery were included. From November 2010 to February 2011, 20 patients were enrolled in this pilot study. Samples were taken before induction of anesthesia (T0) and 2 hours after arrival in the ICU (T1). Standard laboratory tests and the following POCs were performed: multiple electrode aggregometry (MEA), PFA 100 and thromboelastography (TEG). Functional fibrinogen level (FFL) is a recent modification of TEG used to investigate the function of fibrinogen [3]. We used the combination of TEG and FFL to detect platelet contribution to the clot strength.

Results There was no significant association between bleeding at 4, 6 and 12 hours and any of the preoperative tests. There was no significant association between bleeding at 4, 6 and 12 hours and any of the standard laboratory tests. Platelet contribution to clot strength (\%pltMA) detected by TEG showed a significant association with postoperative blood loss (at 4, 6 and 12 hours, respectively $P=0.02$, $P=0.02, P=0.01$.

Conclusion Our data confirm the utility of perioperative evaluation of platelet contribution to clot strength evaluated by TEG. It helps to understand the mechanism behind the surgical bleeding and reduce empirical transfusions.

\section{References}

1. Hartmann M, et al:: Transfus Med Rev 2006, 20:230-241.

2. Ferraris V, et al: Ann Thorac Surg 2011, 91:944-982

3. Mousa SA, et al.: Thromb Res 2001, 104:49-56.

P433

Measurement of hemoglobin in the operating room: what methods can we trust?

B Giraud, D Frasca, O Mimoz

CHU La Milétrie Poitiers, France

Critical Care 2012, 16(Suppl 1):P433 (doi: 10.1186/cc1 1040)

Introduction A noninvasive and continuous monitoring of total hemoglobin $(\mathrm{Hb})$ by spectrophotometry was recently marketed $(\mathrm{SpHb}$; Masimo, Irvine, CA, USA). The main objective of this study was to determine the absolute and trend accuracy of $\mathrm{SpHb}$ compared to $\mathrm{Hb}$ assessment at the laboratory ( $\mathrm{HbLab}$ ) used as the reference method.

Methods After obtaining ethics committee approval and informed consent, 51 adult patients ( 29 men, 22 women, age 18 to 90 years) undergoing major surgery with expected large blood loss were enrolled in the study. Patients wore Rainbow adult resposable sensors (R2-25, Revision E) connected to a Radical-7 Pulse CO-Oximeter, software version 7.6.0.1. HbLab values were obtained by analyzing arterial blood samples at the laboratory using a Sysmex XT-2100i automated hematology analyzer (Roche Diagnostics, Paris, France). The same samples were also analyzed with a satellite laboratory COOximeter (Siemens RapidPoint 405 CO-Oximeter; Siemens, Munich, Germany), HbSat, and a point-of-care hemoglobinometer (HemoCue, Hb201; Ångelholm, Sweden), HcueArt. At the same time, a fourth drop of blood after skin puncture on the ear or finger was taken for capillary blood sampling tested also with the HemoCue: HcueCap. Invasive $\mathrm{Hb}$ values were compared to Sphb obtained at the time of the blood draw. An initial set was collected before surgery. Then blood samples were taken on approximately an hourly basis or more often if clinically indicated. Bland-Altman method plots were used to compare absolute accuracy of test devices to laboratory values. The ability of the test devices to follow the trend of the changes in $\mathrm{Hb}$ values reported by the reference device was assessed by plotting the difference between subsequent measurements reported by each device to the difference in subsequent measurements reported by the reference device, and a coefficient of determination $\left(R^{2}\right)$ was calculated.

Results The study included 210 measurements. HbLab ranged between 6.8 and $16.3 \mathrm{~g} / \mathrm{dl}$. Compared to the reference method, the average bias was $0.96 \pm 2.78 \mathrm{~g} / \mathrm{dl}$ for SpHb, $0.16 \pm 0.45 \mathrm{~g} / \mathrm{dl}$ for HcueArt, $0.5 \pm 1.71$ $\mathrm{g} / \mathrm{dl}$ for HcueCap and $0.81 \pm 1.04 \mathrm{~g} / \mathrm{dl}$ for HbSat. $R^{2}$ values were 0.39 for $\mathrm{SpHb}, 0.93$ for HcueArt, 0.53 for HcueCap and 0.47 for HbSat.

Conclusion This study shows that HcueArt seem the most reliable method of $\mathrm{Hb}$ assessment. The $\mathrm{SpHb}$ has a lower accuracy, but its ability to monitor $\mathrm{Hb}$ continuously and noninvasively remains attractive and development of this method should be encouraged.
P434

Retrospective comparison study of warfarinised trauma patients and an age-matched control group of nonwarfarinised patients

M Omar, P Stevens, T Jenkins, K Morris, H Hussain, A Evans

Morriston Hospital, Swansea, UK

Critical Care 2012, 16(Suppl 1):P434 (doi: 10.1186/cc11041)

Introduction There are several studies stating the association of mortality between trauma and anticoagulation; however, it is difficult to ascertain a credible conclusion due to the small number of data and inconclusive results. Some studies have showed a significant increased risk of morbidity and mortality. We analysed retrospective data of 45,798 trauma patients, out of which 254 were on warfarin. The incidence of death continues to rise and there are no specific strategies to reduce haemodilution and coagulopathy which maybe the underlying cause of mortality.

Methods A retrospective analysis of a national database collected in 2009 and 2010, from the Trauma Audit and Research Network (TARN) UK. The data also contain vital information including age, Glasgow Coma Scale (GSC), Injury Severity Score (ISS), INR, blood products given, number of days in hospital and clinical outcome. We evaluated trauma patients who were on warfarin therapy and compared their clinical outcome and mortality to age-matched patients with similar injuries not on warfarin.

Results A total of 45,780 adult patients were analysed. These were subdivided into 32,225 young patients under 65 years with median age 60.5 , of which 59 were on warfarin; and 13,555 older patients aged over 65 with median age 80.4, of which 195 were on warfarin. The mortality rate in warfarinised patients was significantly higher than in the nonwarfarinised age-matched group aged $<65(5 / 59,8.5 \%$ vs. $1,223 / 32,163,3.8 \% ; P<0.001 ; 95 \% \mathrm{Cl})$. The group age $>65$ included 13,555 , of which 195 were warfarinised $(4.7 / 195,24.1 \%$ vs. 1,501/13,360, $11.3 \% ; P<0.001 ; 95 \% \mathrm{Cl})$.

Conclusion This data analysis proves that mortality is significantly higher in warfarinised patients compared to the nonwarfarinised agematched group. Future research needs to focus on both developing a practical procedure reducing risks of morbidity and mortality by exploring coagulopathy and early correction of coagulations. Anticoagulated patients are more likely to receive aggressive i.v. fluid resuscitations as the result of haemorrhage which leads to haemodilution and further exacerbates coagulopathy. This cascade of events is the underlying mechanism causative to mortality.

References

1. Kauvar DS: Impact of haemorrhage on trauma outcome: an overview of epidemiology clinical presentation and therapeutic considerations. J Trauma 2006, 60:S3-S11.

2. WHO: Injuries and Violence: Facts. Geneva, Switzerland: WHO; 2010.

3. Dawn M, Mark D, Pasquale M, Thomas W: Impact of pre-injury warfarin use in elderly trauma patients. J Trauma 2000, 48:3.

\section{P435}

In medical-surgical ICU patients, major bleeding is common but independent of heparin prophylaxis

F Lauzier', D Arnold ${ }^{2}$, C Rabbat ${ }^{2}$, D Heels-Ansdell2, P Dodek', B Ashley ${ }^{3}$, M Albert ${ }^{4}$, K Khwaja $^{5}$, M Ostermann ${ }^{6}$, Y Skrobik ${ }^{4}$, R Fowler ${ }^{7}$, L Mclntyre $^{8}$, J Nates ${ }^{9}$, T Karachi ${ }^{2}$, R Lopes ${ }^{10}$, N Zytaruk ${ }^{2}$, M Crowther ${ }^{2}$, D Cook ${ }^{2}$ 'CHA-Hôpital de l'Enfant-Jésus, Université Laval, Québec, Canada; 'McMaster University, Hamilton, Canada; 'University of British Columbia, Vancouver, Canada: ${ }^{4}$ Université de Montréal, Canada: ${ }^{5}$ Université McGill, Montréal, Canada; 'King's College London, UK; 'University of Toronto, Canada; ${ }^{8}$ Université d'Ottawa, Canada; ${ }^{M}$ MD Anderson Hospital, Houston, TX, USA. ${ }^{10}$ Duke Clinical Research Institute, Durham, NC, USA

Critical Care 2012, 16(Suppl 1):P435 (doi: 10.1186/cc11042)

Introduction Bleeding frequently complicates critical illness. Our objectives were to describe the incidence, locations and predictors of major bleeding in patients with low risk of bleeding receiving thromboprophylaxis.

Methods In the PROTECT trial comparing dalteparin to unfractionated heparin for thromboprophylaxis in medical-surgical ICU patients, research coordinators used a validated ICU-specific tool to describe the 
site and severity of each bleeding event, which was reevaluated by two independent blinded adjudicators. Patients with trauma, orthopedic surgery or neurosurgery were excluded. Major bleeding was defined as life threatening, occurring in critical sites, requiring $\geq 2$ units of red blood cells or an invasive intervention, or associated with an unexplained decrease in systolic blood pressure $(\geq 20 \mathrm{mmHg})$ or increase in heart rate ( $\geq 20$ beats/minute). We used Cox proportional hazard models adjusting for age, APACHE II, reason for ICU admission, end-stage renal disease, drugs affecting coagulation, coagulation parameters and lifesupport interventions to identify predictors of bleeding.

Results Among 3,746 patients, 208 had major bleeding $(5.6 \%, 95 \% \mathrm{Cl}$ 4.9 to $6.3 \%)$. The commonest bleeding sites were: gastrointestinal tract $(51.9 \%)$, surgical site $(30.3 \%)$, respiratory tract $(15.9 \%)$, retroperitoneal (8.2\%) and intracranial (3.4\%). Independent predictors of major bleeding (expressed as hazard ratio with $95 \% \mathrm{Cl}$ ) were: prolonged activated partial thromboplastin time (aPTT) $(1.10,1.05$ to 1.14 per 10 -second increase), thrombocytopenia $\left(1.16,1.09\right.$ to 1.24 per $50 \times 10^{9} / \mathrm{l}$ decrease in platelet count), therapeutic heparin $(3.26,1.72$ to 6.17$)$, antiplatelet agents (that is, acetylsalicylic acid and/or clopidogrel) (1.38, 1.02 to 1.88$)$, renal replacement therapy $(1.75,1.20$ to 2.56$)$ and surgery in the preceding 3 days (1.64, 1.01 to 2.65). Prophylactic dalteparin in the preceding 3 days was not associated with bleeding.

Conclusion Major bleeding occurred in 5.6\% of medical-surgical ICU patients. Prolonged aPTT, thrombocytopenia, therapeutic (but not prophylactic) heparin, anti-platelet agents and recent surgery are potentially modifiable and independent predictors of bleeding.

P436

Reducing ICU blood draws with artificial intelligence

FC Cismondi', AS Fialho', SM Vieira², LA Celi', SR Reti ${ }^{3}$, JM Sousa², SN Finkelstein'

'Massachusetts Institute of Technology, Cambridge, MA, USA; ${ }^{2} I S T$, Lisbon,

Portugal: ${ }^{3}$ BIDMC, Brookline, MA, USA

Critical Care 2012, 16(Suppl 1):P436 (doi: 10.1186/cc11043)

Introduction Recent studies have demonstrated that frequent laboratory testing does not necessarily relate to better outcomes. Our aim is to reduce unnecessary blood draws for ICU laboratory tests by predicting which tests are likely to return as normal or abnormal and therefore influence clinical management around gastrointestinal (Gl) bleeding.

Methods An artificial intelligence tool, namely fuzzy systems, was applied to 1,092 Gl bleed patients extracted from a large ICU database with over 32,000 patients. A classification approach for laboratory test outcome was utilized for a total of seven outcome variables shown in Table 1. The outcome for each test was binarized as normal or abnormal. Input variables included 10 physiological variables such as heart rate, temperature and urine output, as well as further data on transfusions for platelets, red blood cells and plasma.

Results Classification accuracy of greater than $80 \%$ was achieved for all of the seven outcome variables (Table 1). Sensitivity and specificity were satisfactory for all the outcomes. Input variables frequently selected as most predictive of normal or abnormal results include urine output and red blood cell transfusion.

Table 1 (abstract P436). Classification results

\begin{tabular}{lccc}
\hline Outcome & ACC (\%) & Sensitivity & Specificity \\
\hline Calcium & $85 \pm 2.3$ & $0.88 \pm 0.3$ & $0.81 \pm 0.1$ \\
PTT & $86 \pm 1.2$ & $0.89 \pm 0.1$ & $0.82 \pm 0.2$ \\
Hematocrit & $82 \pm 1.6$ & $0.84 \pm 0.2$ & $0.78 \pm 0.1$ \\
Fibrinogen & $84 \pm 2.8$ & $0.87 \pm 0.3$ & $0.80 \pm 0.4$ \\
Lactate & $80 \pm 2.2$ & $0.82 \pm 0.2$ & $0.77 \pm 0.4$ \\
Platelets & $88 \pm 1.3$ & $0.90 \pm 0.1$ & $0.85 \pm 0.2$ \\
Hemoglobin & $84 \pm 3.1$ & $0.85 \pm 0.3$ & $0.81 \pm 0.2$ \\
\hline
\end{tabular}

ACC, accuracy of classification.
Conclusion Reducing frequent laboratory testing, and potential phlebotomy complications, is a major concern in critical care medicine. If one could predict in advance whether a laboratory test would be normal or abnormal then that particular laboratory test may not be ordered, and thereby reducing potential complications and costs. In this work we present an artificial intelligence method for the classifying the likelihood of a blood test being normal or abnormal. Our results show acceptable classification accuracy both in terms of sensitivity and specificity.

\section{P437}

Hemostasis system condition in infectious complication

development in severe burned patients

M Presnyakova, $\vee$ Kuznetsova

Nizhny Novgorod Research Institute of Traumatology and Orthopedics,

Nyzhny Novgorod, Russia

Critical Care 2012, 16(Suppl 1):P437 (doi: 10.1186/cc11044)

Introduction Over the period of the history of combustiology one of the main problems for treatment of patients with burns is infection, both local - bacterial pneumonia - and generalized - sepsis characterized by extremely severe course, complex diagnostics and high lethality rate. However, the role of hemostasis disorders in infectious complication development in severe burned patients is taken into consideration insufficiently. The aim of the study is to reveal the most relevant hemostasis system changes in sepsis and pneumonia in patients with serious heat injury in an acute period of burn disease. Methods Hemostasis and biochemical blood parameters were studied in 169 patients with over $20 \%$ of the body burned, from the first to 12th days after burn. Sepsis developed in 33 patients, 69 patients had pneumonia, and in 67 patients there were no complications of sepsis and pneumonia. Infectious septic complications were diagnosed in the clinic on the basis of clinical and laboratory findings, as well as confirmed by morphological studies in casualties ( 44 from 102 patients). Diagnosis of disseminated intravascular coagulation (DIC) syndrome was made based on standard criteria.

Results The analysis of findings showed both sepsis and pneumonia development in an acute period of burn disease to be accompanied by disorders of anticoagulant, fibrinolytic and procoagulant parts of the hemostasis system typical for DIC syndrome. The changes of hemostasis system indices were not only the characteristic of infection in burned patients but they preceded the diagnosis of sepsis and pneumonia in the clinic on average by 2 to 4 days. In patients with pneumonia, relevant and statistically significant were the activity changes of XIladependent fibrinolysis, from the second to sixth days. And on the third to seventh days there was reliable pneumonia development with decreased activity of antithrombin III. In patients with sepsis were revealed changes of XIla-dependent fibrinolysis activity - from the third to seventh days - and antithrombin III activity - from the third to the sixth days.

Conclusion The development of both local and generalized infection in an acute period of burn disease occurs against the background of DIC syndrome induced by a serious heat injury. The indices of hemostasis system can be included into a complex of clinic and laboratory studies aimed at detecting infection and early intensive etiopathological therapy.

\section{P438}

Randomized comparison of fibrinogen concentrate versus cryoprecipitate for bleeding control in pediatric cardiac surgery (FICCS study)

F Galas' , L Hajjar', B Sorensen², J Almeida', M Sundin'1, V Guimaraes', S Zefferino', L Camara', F Maua', M Moreira', C Puttini', M Carmona', J Auler Jr' ${ }^{1}$, R Nakamura

'Heart Institute, São Paulo, Brazil; ' $G u y$ 's and St Thomas' NHS Foundation Trust \& King's College London School of Medicine, London, UK Critical Care 2012, 16(Suppl 1):P438 (doi: 10.1186/cc11045)

Introduction We compared hemostatic outcomes after treatment with fibrinogen concentrate or cryoprecipitate in pediatric cardiac surgery patients with intraoperative bleeding. 
Methods This was a single-center, randomized, open-label study. Key inclusion criteria were age $<15$ years, cardiac surgery involving cardiopulmonary bypass, intraoperative bleeding after neutralization of heparin, and hypofibrinogenemia. Patients received fibrinogen concentrate $\left(60 \mathrm{mg} / \mathrm{kg}\right.$ body weight; Haemocomplettan $\left.{ }^{\circledR} \mathrm{P}\right)$ or cryoprecipitate (10 ml/kg body weight). After study medication, allogeneic blood products were administered as required. Blood samples taken immediately before randomization and 1, 24 and 48 hours after study medication were subjected to laboratory and thromboelastometry (ROTEM) coagulation tests.

Results Sixty-three patients (fibrinogen concentrate: 30 ; cryoprecipitate: 33) completed the study. The median age was 3 years 5 months and the median weight was $6.7 \mathrm{~kg}$. Median fibrinogen doses were 504 $\mathrm{mg}$ (fibrinogen concentrate) and $402 \mathrm{mg}$ (cryoprecipitate). Plasma fibrinogen concentrations increased after study medication and were similar in the two groups. No significant between-group differences were observed in PT, aPTT or platelet count. In both groups, all ROTEM parameters showed significant improvement after study medication, with no clinically relevant between-group differences in any of the EXTEM, INTEM or FIBTEM clotting parameters. Total avoidance of allogeneic blood product transfusion was achieved in $70 \%$ of patients in the fibrinogen concentrate group versus $18.2 \%$ in the cryoprecipitate group $(P<0.001)$. The mean bleeding mass was significantly lower in the fibrinogen concentrate group than in the cryoprecipitate group after 30 minutes. The thorax was opened after study medication in zero patients $(0 \%)$ in the fibrinogen concentrate group and in six patients (18.2\%) in the cryoprecipitate group $(P=0.025)$.

Conclusion Fibrinogen concentrate raised fibrinogen levels and improved coagulation measures to a similar degree as cryoprecipitate. Bleeding and transfusion of allogeneic blood products were lower in the fibrinogen concentrate group. Fibrinogen concentrate may be a valuable option for controlling bleeding and avoiding transfusion in cardiac surgery.

P439

Efficacy of tranexamic acid in decreasing blood loss during cesarean section

O Tarabrin, V Kaminskiy, S Galich, R Tkachenko, A Gulyaev, S Shcherbakov,

D Gavrychenko

Odessa National Medical University, Odessa, Ukraine

Critical Care 2012, 16(Suppl 1):P439 (doi: 10.1186/cc11046)

Introduction Despite significant progress in obstetric care, the problem of bleeding during labor remains unfinished. Annually in the world 125,000 women die from obstetric hemorrhage.

Methods We performed a randomized, double-blind study in 37 patients who underwent cesarean section. Patients were divided into two groups: the first group $(n=19)$ received preoperative (30 minutes before operation) tranexamic acid $10 \mathrm{mg} / \mathrm{kg}$; the second group $(n=18)$ received preoperative placebo. The condition of hemostasis was monitored by haemoviscoelastography.

Results All patients included in the study before the surgery had moderate hypercoagulation and normal fibrinolysis: increasing of the intensity of clot formation (ICF) to $11.4 \%$ compared to normal rates; the intensity of the retraction and clot lysis (IRCL) was $16.45 \pm 1.40$ in both groups. At the start of the operation in patients (group 1) - ICF decreased $9.7 \%(P<0.05)$, and IRCL decreased $27.6 \%(P<0.05)$ compared with preoperatively. In group 2, ICF decreased $8.8 \%(P<0.05)$, and IRCL increased $11.4 \%(P<0.05)$ compared with preoperatively. At the end of the operation, the condition of hemostasis in both groups came almost to the same value - moderate hypocoagulation, depressed fibrinolysis. In both groups there were no thrombotic complications. Intraoperative blood loss in the first group was $300 \pm 40.5$ and in the second was $500 \pm 60.6$

Conclusion Using tranexamic acid before surgery significantly reduces intraoperative blood loss by $40 \%$, without thrombotic complications.
P440

Reduced EPO receptor expression may contribute to limited pleiotropic effects of EPO during critical illness

O McCook, S Matějková, J Matallo, A Scheuerle, P Moeller, M Georgieff,

E Calzia, P Radermacher, H Schelzig

University Medical School UIm, Germany

Critical Care 2012, 16(Suppl 1):P440 (doi: 10.1186/cc11047)

Introduction We showed neuroprotective and renoprotective effects of recombinant human erythropoietin (rhEPO) after kidney and spinal cord ischemia/reperfusion (I/R) injury $[1,2]$, but clinical studies using rhEPO to prevent acute kidney injury yielded equivocal results $[3,4]$. Increased cytokine release and/or oxidative stress can cause EPO resistance due to receptor modification and/or downregulation [5]. Since we recently failed to confirm rhEPO-related kidney protection in atherosclerotic swine [6], we compared kidney EPO receptor expression in swine strains with and without pre-existing vascular disease and kidney dysfunction.

Methods EPO receptor expression was quantified with immunohistochemistry (densitometric image analysis) of formalin-fixed paraffin sections from pre-injury kidney biopsies taken in young and healthy pigs (German Landrace, up to now $n=4$ ) as well as swine (FBM strain, up to now $n=6)$ with familial hypercholesteremia $(11.1(7.4 ; 12.3)$ vs. $1.4(1.3 ; 1.5) \mathrm{mmol} / \mathrm{l}, P<0.001, n=20$ and 15 , respectively, $P<0.001)$ and consecutive, diet-induced atherosclerosis [7].

Results Atherosclerotic swine presented with reduced glomerular filtration rate (creatinine clearance $76(60 ; 83)$ vs. $103(79 ; 120) \mathrm{ml} /$ minute, $n=19$ each, $P=0.004$ ) and chronic histological kidney injury (dilatation of Bowman's space, swelling of Bowman's capsule, tubular dilatation and necrosis). EPO receptor expression was reduced by nearly two orders of magnitude in this strain $\left(94.6(8.3 ; 112.5) \times 10^{7} \mathrm{vs}\right.$. $1.7(0.0 ; 4.7) \times 10^{7}$ densitometric units, $\left.P=0.010\right)$.

Conclusion Even pretreatment with rhEPO did not influence I/Rinduced acute kidney in swine with pre-existing impairment of kidney function and histological damage. The lacking beneficial effect of rhEPO was most likely due to the reduced expression of the EPO receptor, which may also explain contradictory results in clinical trials due to the frequent underlying kidney disease in the patients recruited. References

1. Simon F, et al:: Crit Care Med 2008, 36:2143-2150.

2. Simon F, et al.: Intensive Care Med 2011, 37:1525-1533.

3. Song YS, et al:: Am J Nephrol 2009, 30:253-260.

4. Endre ZH, et al:: Kidney Int 2010, 77:1020-1030.

5. van der Putten K, et al:: Nat Clin Pract Nephrol 2008, 4:47-57.

6. Simon F, et al:: Shock 2011, 36(Suppl 1):S24.

7. Thim T: Dan Med Bull 2010, 57:B4161.

P441

Recognition and management of haemophagocytic

lymphohistiocytosis on the ICU: a case series

R Baumber, B Agarwal, M Carrington

Royal Free Hospital, London, UK

Critical Care 2012, 16(Suppl 1):P441 (doi: 10.1186/cc11048)

Introduction Haemophagocytic lymphohistiocytosis $(\mathrm{HLH})$ is a rare haematological condition, with a reported incidence of the familial variety of the disease being 1.2 cases per 10,000 children per year. The acquired form of HLH predominantly affects adults and is almost always precipitated by infection, with Epstein-Barr virus (EBV) being the commonest trigger. This results in abnormal activation and proliferation of histiocytes and macrophages. Widespread phagocytosis of blood cell components leads cytopenias, a strong proinflammatory response and cytokine release leading to tissue necrosis and multiple organ failure. A large majority of these patients will present to the ICU for organ support and the aim of this report is to provide an update on $\mathrm{HLH}$ and raise awareness of this rare condition amongst the critical care community.

Methods A single-centre retrospective review of case records of all patients admitted to our ICU, in a tertiary haematology referral centre, in the last 5 years with a confirmed or suspected diagnosis of $\mathrm{HLH}$, based on HLH-2004 guidelines. Data were collected on demographic 
variables, $\mathrm{HLH}$ disease characteristics, acute physiological derangement (APACHE II and SAPS II), and outcome.

Results Twenty-four patients were identified with a diagnosis of $\mathrm{HLH}$, 18 males and six females, with mean age 42.6 years. A history of prior haematological malignancy, HIV infection and immunosuppressive therapy was present in six, five and four patients respectively; no underlying medical condition was found in $5 / 24$ patients. Infective causes were identified in 15/24 patients, EBV in eight out of 15 . Other infective causes were Cytomegalovirus, Toxoplasma gonadii, Mycobacterium tuberculosis and Schistosomiasis. All patients were pancytopenic at ICU admission and had significantly elevated serum ferritin $(15,771 \pm 17,718)$ and triglyceride $(3.8 \pm 2.05)$ levels. Eleven out of 24 patients displayed features of acute liver involvement. Mean APACHE II score was $20.5 \pm 5.1$ and mean SAPS II was $51.3 \pm 12.1$. Ten out of 24 survived to ICU discharge, and 6/24 (25\%) were alive at the time of hospital discharge. The survivors had lower APACHE and SAPS scores, and were associated with a non-EBV infection and a lower incidence of liver involvement.

Conclusion HLH is a rare but fatal haematological syndrome that in its acquired form may present to ICU clinicians for organ support. Diagnosis of HLH in the intensive care setting may be difficult because sepsis may cause similar clinical and laboratory abnormalities. Presence of more severe acute physiological derangement, EBV aetiology and features of liver failure portend a poor prognosis in $\mathrm{HLH}$.

Reference

1. Henter Jl, et al.: HLH 2004: diagnostic and therapeutic guidelines for haemophagocytic lymphohistiocytosis. Pediatr Blood Cancer 2007, 48:124-131.

\section{P442}

Blood transfusion is an independent predicting factor for poor outcome after cardiac surgery

J Almeida, S Zeferino, F Galas, J Fukushima, L Camara, M Lima, T Santos,

M Ferreira, J Auler Jr, R Kalil Filho, L Hajjar

Heart Institute, São Paulo, Brazil

Critical Care 2012, 16(Suppl 1):P442 (doi: 10.1186/cc11049)

Introduction Blood transfusion is associated with worse outcome in critically ill patients. A restrictive strategy of blood transfusion has been advocated in patients undergoing cardiac surgery in order to avoid clinical complications related to exposure to blood components. Nevertheless, the blood transfusion rate remains elevated in clinical practice.

Methods We performed a retrospective study with 750 patients undergoing elective coronary arterial graft bypass (CABG) surgery, valvar surgery or combined procedure under cardiopulmonary bypass (CPB) between October 2010 and October 2011 at a university hospital cardiac surgery referral center in Brazil. We collected baseline characteristics and preoperative laboratory data, EuroSCORE, type of surgical procedure, intraoperative characteristics, blood transfusion exposure, postoperative severe complication as bleeding, low output cardiac syndrome, vasoplegia syndrome, myocardial ischemia, stroke, ventricular or supraventricular tachyarrhythmia, respiratory failure, acute renal failure, infection, ICU length of stay, hospital length of stay and mortality in 30 days.

Results A total of 512 patients (68.4\%) was exposed to blood transfusion components. Transfused patients presented a higher number of severe clinical complications in the postoperative period compared to nontransfused patients $(74(34.1 \%)$ vs. $312(61.9 \%), P<0.0001)$. Also, the mortality rate was higher in transfused patients than nontransfused patients $(1(0.5 \%)$ vs. $18(3.6 \%), P<0.016)$. In a multivariate analysis, age, obesity, perioperative myocardial ischemia, valve disease, heart failure, blood transfusion and CPB duration are independently associated with mortality.

Conclusion Blood component exposure is associated with poor outcome and mortality in patients undergoing cardiac surgery. Despite the evidence that blood transfusion is associated with worse outcome, the blood transfusion rates remain unacceptably high in clinical practice. Reference

1. Hajjar LA, Vincent JL, Galas FR, Nakamura RE, Silva CM, Santos MH, et al: Transfusion requirements after cardiac surgery: the TRACS randomized controlled trial. JAMA 2010, 304:1559-1567.
P443

Red blood transfusion is a predictor of poor outcome in pediatric cardiac surgery

C Colognesi, R Maia, L Hajjar, F Galas

Heart Institute, São Paulo, Brazil

Critical Care 2012, 16(Suppl 1):P443 (doi: 10.1186/cc11050)

Introduction Red blood cell transfusion is associated with morbidity and mortality among adults undergoing cardiac surgery. We aimed to evaluate the association of transfusion with morbidity in pediatric cardiac surgical patients. The purpose of this study was to assess whether red blood cell transfusions result in worse outcomes after cardiac surgery in pediatric patients.

Methods We studied an observational and prospective cohort of 200 patients undergoing cardiac surgery for congenital heart disease. We recorded baseline characteristics, RACHS-1 score, intraoperative data, cardiopulmonary bypass length, type of surgery, transfusion requirement and postoperative complications as need for reoperation, time of mechanical ventilation, cardiovascular complications, acute renal failure, infection, readmission at ICU and death during 28 days. Results One hundred and twenty-four patients were exposed to blood components. Seventy-seven percent of patients presented at least one major complication. There was no difference between transfused and nontransfused patients regarding baseline or intraoperative characteristics. Transfused patients presented a higher incidence of major complications than nontransfused patients $(93.5 \%$ vs. $54.5 \%$, $P=0.002$ ). In a multivariate analysis, red blood cell transfusion was an independent risk factor for clinical complications including death in 28 days $(\mathrm{OR}=2.2(95 \% \mathrm{Cl} 1.4$ to 23.4$)$ ).

Conclusion Blood transfusion after pediatric cardiac surgery is a risk factor for worse outcome. Avoiding blood transfusion may reduce mortality in this population.

P444

No impact of a massive transfusion protocol on coagulopathy and mortality at a level 1 trauma center: why?

C Bourassa-Fulop, J Chauny, J Paquet, R Daoust, E Notebaert

Hôpital Sacré-Coeur, Montreal, Canada

Critical Care 2012, 16(Suppl 1):P444 (doi: 10.1186/cc11051)

Introduction In 2010 we studied the mortality and coagulopathy of all massively transfused patients at our hospital since 2004. We compared those who were transfused before the implementation of our massive transfusion protocol (MTP) (from 2004 to 2006) to those transfused with MTP. We found that our MTP did not lower mortality (35.7\%) and our incidence of coagulopathy was high $(72.6 \%)$. The aim of the present study is to explain those results, while concentrating uniquely on trauma patients.

Methods We conducted a retrospective nested case-control study from our trauma registry. We included trauma patients who received 10 packed red blood cells (pRBC) or more in 24 hours and excluded those who died within the very first hours of massive trauma. We extracted supplementary demographic and clinical data from the laboratory database and the hospital files. Chi-square tests and multivariate logistic regression were used to compare the effect of the two approaches (MTP vs. non-MTP) on mortality and coagulopathy, defined as an INR $\geq 1.8$, a PTT $\geq 54$, a fibrinogen $<1 \mathrm{~g} / \mathrm{l}$ or a platelet count $<50,000$, while controlling for acidosis (defined as a $\mathrm{pH} \leq 7.1$ ), hypothermia (defined as $\leq 35^{\circ} \mathrm{C}$ ) and Injury Severity Score (ISS) (critically injured if ISS $\geq 30$ ).

Results Of the 84 trauma patients, 23 were transfused with the MTP and 61 without. The average ISS score was very high (29.2), most were male (73.8\%) and the average age was 41 years. The MTP versus non-MTP groups were similar in regards to age, sex, $\mathrm{pH}$, temperature, ISS and Revised Trauma Score, but the MTP group received more transfusions ( $40 \%$ vs. $22 \%$ when dichotomized in two groups: above 20 pRBC and between 10 and $20 \mathrm{pRBC}$ ). The mortality and coagulopathy were similar in both the MTP and non-MTP group (39\% vs. $34 \%$ and $65 \%$ vs. $75 \%$ respectively). PTM did not affect mortality or coagulopathy, even when controlling for all other variables. Individually, both hypothermia $(\mathrm{OR}=2.6,95 \% \mathrm{Cl}: 1.1$ to 6.8$)$ and acidosis $(\mathrm{OR}=4.3,95 \% \mathrm{Cl}: 1.6$ to 13.0$)$ significantly affected mortality, while the number of pRBC $(\mathrm{OR}=3.8$, $95 \% \mathrm{Cl}: 1.1$ to 14.1 ) was the main determinate for coagulopathy. 
Conclusion In our population of severely injured patients, the MTP was not found to be beneficial in regards to mortality nor coagulopathy. Hypothermia and acidosis seem to be the main determinants for mortality and should be among the priorities in caring for trauma patients.

Reference

1. Cotton BA, et al.: J Trauma 2009, 66:41-49.

P445

Massive transfusion practice

M Campbell, G Yakandawala, S Liddle, K Mehta, J Chooi

BHR NHS Trust, London, UK

Critical Care 2012, 16(Suppl 1):P445 (doi: 10.1186/cc11052)

Introduction Management of massive blood loss requires a multidisciplinary team approach. Current guidelines are varied and generic with a lack of adherence when it comes to management of massive haemorrhage. The aim of our survey was to assess the transfusion practice in the management of massive haemorrhage in a busy district general hospital with a tertiary neurosurgical centre and the busiest obstetric unit in London.

Methods $\mathrm{A}$ retrospective analysis of cases requiring transfusion of more than 6 units of red blood cells (RBC), between January 2009 and January 2010. Sixty-eight cases of massive transfusion were identified, and data collected included causes of the haemorrhage, patient's demographics and past medical background, investigations (FBC, clotting), use of blood products and patient outcome.

Results There were 21 gastrointestinal, 17 vascular, 12 general surgical, seven trauma, six obstetric, and five haematology-oncology patients. Thirty-one per cent of patients were 61 to 80 years old. Overall mortality was $35 \%$, highest mortality among vascular patients. Average blood products per patient: RBC 9 units, fresh frozen plasma (FFP) 4 units, platelets (PLT) 1.2 units, cryoprecipitate 0.67 units. Tranexamic acid was used in eight cases and factor VII in one case. At the time of haemorrhage, FBC, clotting screen and fibrinogen levels were requested in $56 \%$ of patients. In this group, FFP, PLTs and cryoprecipitate were used more frequently with mean use of blood products: RBC 9 units, FFP 5 units, PLT 1.5 units, and cryoprecipitate 1 unit.

Conclusion Blood product use varied widely irrespective of speciality, the dependent factor being individual doctors involved in patient management. Due to difficulty of accessing and their complexity in emergency situations, it was noted that hospital guidelines were disregarded. FFP was the commonly used blood product while cryoprecipitate and tranexamic acid were underused. Only $56 \%$ of patients had FBC and clotting screen to guide transfusion management. In these patients the ratio of cryoprecipitate and PLTs to RBCs was higher. This survey showed the need for revised, easily accessible and user-friendly guidelines for the management of massive haemorrhages. The results of this survey helped to establish pointof-care testing (thromboelastography) to provide a target controlled therapy and make the use of blood and blood products cost-effective. References

CRASH-2 Trial Collaborators et al:: Lancet 2010, 376:23-32.

Johansson PI, Stensballe J: Transfusion 2010, 50:701-710.

3. Zink KA, Sambasivan CN, Holcomb JB, Chisholm G, Schreiber MA: Am J Surg 2009, 197:565-570.

4. Enriquez LJ, Shore-Lesserson L: Br J Anaesth 2009, 103(Suppl 1):i14-i22.

\section{P446}

Red blood cell transfusion improves microdialysis-assessed interstitial lactate/pyruvate ratio in critically ill septic patients

P Kopterides, N Nikitas, M Theodorakopoulou, A Diamantakis,

D Vassiliadi, A Kaziani, S Assoti, F Drakopanagiotakis, A Antonopoulou,

P Papadopoulos, E Mavrou, C Georgiadou, A Tsantes, A Armaganidis, I Dimopoulou

'Attiko' University Hospital, Haidari - Athens, Greece

Critical Care 2012, 16(Suppl 1):P446 (doi: 10.1186/cc11053)

Introduction Even though red blood cell (RBC) transfusion is a common intervention in the critical care setting, there is a paucity of data regarding its impact on tissue metabolism. The aim of this study was to explore the effect of RBC transfusion on microdialysis-assessed interstitial fluid metabolic parameters in septic patients.

Methods We conducted an observational, clinical study in a 25 bed, medical-surgical ICU of a university hospital. We analyzed the effect of transfusion of either 1 or 2 RBC units on interstitial fluid metabolic activity by means of a microdialysis (MD) catheter inserted in the subcutaneous adipose tissue of the upper thigh. Samples were collected before (T0) and after (T1a and T1b; spaced out by 4 hours) transfusion. Lactate, pyruvate, glycerol and glucose concentrations were measured with a bedside analyzer and the lactate/pyruvate (LP) ratio was calculated automatically.

Results We enrolled 37 patients with severe sepsis/septic shock requiring RBC transfusion. After transfusion, the mean arterial pressure increased from $79 \pm 9$ to $82 \pm 10$ (T1a vs. T0: $P<0.05$ ) and $83 \pm 10$ $\mathrm{mmHg}$ (T1b vs. T0: $P<0.001$ ). Besides a nonstatistically significant drop in arterial partial oxygen pressure, we observed no change in arterial blood gases and vital signs. Overall, RBC transfusion did not alter any of the MD-assessed parameters (that is, lactate, pyruvate, glycerol and glucose) or blood lactate, but it decreased the tissue LP ratio from (T0) 18.80 (interquartile range (IQR), 14.85 to 27.45 ) to (T1a) 17.80 (IQR, 14.35 to 25.20$)(P<0.05)$ and (T1b) $17.90(\mathrm{IQR}, 14.45$ to 22.75$)(P<0.001)$. The post-transfusion changes in $\mathrm{LP}$ ratio at $\mathrm{T} 1 \mathrm{a}(r=-0.42 ; 95 \% \mathrm{Cl},-0.66$ to $-0.098 ; P=0.01)$ and $\mathrm{T} 1 \mathrm{~b}(r=-0.68 ; 95 \% \mathrm{Cl},-0.82$ to $-0.44 ; P<0.001)$ were significantly correlated with the pre-transfusion LP ratio but not with baseline demographic characteristics, vital signs, severity scores, hemoglobin level and blood lactate. Finally, 39.0\% of the transfused RBC units were leukoreduced and their median storage time was 16 days (IQR, 11 to 24). RBC storage time and leukocyte reduction had no influence on the tissue metabolic response to transfusion.

Conclusion Tissue oxygenation is improved by red blood cell transfusion in critically ill septic patients. Monitoring of the tissue LP ratio by microdialysis may represent a useful method for individual clinical management.

\section{P447}

Blood transfusion after cardiac surgery increases the hospital length of stay in adult patients

L Hajjar', JL Vincent'ㄹ, J Almeida', F Jatene'1, A Rodrigues', J Fukushima', R Nakamura', C Silva', E Osawa', R Kalil', F Galas', J Auler Jr'

'Heart Institute, São Paulo, Brazil;'Erasme Hospital, Université libre de Bruxelles, Belgium

Critical Care 2012, 16(Suppl 1):P447 (doi: 10.1186/cc11054)

Introduction Transfusion of allogeneic red blood cells (RBC) is a recognized risk factor for adverse outcomes following cardiac surgery. A potential endpoint to assess clinical complications and incremental use of resources is the measurement of hospital length of stay (LOS). The primary objective of this study was to evaluate the relationship between blood transfusion and increased hospital LOS after cardiac surgery.

Methods A prospective observational substudy that analyzed data from the overall 502 patients enrolled in the Transfusion Requirements After Cardiac Surgery (TRACS) study [1]. Patients who received blood transfusion during surgery or ICU stay were further categorized according to the number of prescribed RBC units: nontransfusion group, low transfusion requirement group (3 units or less), and high transfusion requirement group (more than 4 units).

Results Patients who received any RBC unit had longer median LOS than patients in the nontransfusion group: 15 days $(95 \% \mathrm{Cl}, 12.66$ to $17.34)$ in high transfusion requirement group versus 10 days $(95 \% \mathrm{Cl}$, 9.1 to 10.9$)$ in low transfusion group versus 8 days (95\% $\mathrm{Cl}, 7.4$ to 8.6$)$ in nontransfusion group $(P<0.001)$. In a multivariate Cox proportional hazards model the following factors were considered predictive: age older than 65 years (hazard ratio (HR), $1.38(95 \% \mathrm{Cl}, 1.11$ to 1.73$)$; $P=0.004)$, EuroSCORE 3 to 5 (HR, 1.44 (95\% Cl, 1.12 to 1.86$) ; P=0.005)$, EuroSCORE higher than $5(\mathrm{HR}, 1.7(95 \% \mathrm{Cl}, 1.26$ to 2.28$) ; P<0.001)$, valvular surgery (HR, $1.57(95 \% \mathrm{Cl}, 1.26$ to 1.95$) ; P<0.001)$, combined procedure ( $\mathrm{HR}, 1.6(95 \% \mathrm{Cl}, 1.03$ to 2.46$) ; P=0.034)$, bypass duration higher than 100 minutes (HR, $1.23(95 \% \mathrm{Cl}, 1.01$ to 1.51$) ; P=0.046)$, LVEF lower than $40 \%(\mathrm{HR}, 1.69(95 \% \mathrm{Cl}, 1.24$ to 2.32$) ; P=0.001)$, LVEF 40 to $59 \%(\mathrm{HR}, 1.36(95 \% \mathrm{Cl}, 1.1$ to 1.69$) ; P=0.004)$, RBC transfusion 
of 1 to 3 units ( $\mathrm{HR}, 1.24(95 \% \mathrm{Cl}, 1.01$ to 1.53$) ; P<0.001)$, and $\mathrm{RBC}$ transfusion $>3$ units ( $\mathrm{HR}, 1.96(95 \% \mathrm{Cl}, 1.45$ to 2.66$) ; P<0.001)$. In an adjusted model for age, EuroSCORE, type of surgical procedure, LVEF and cardiopulmonary bypass time, the exposure to RBC transfusion was associated with an elevated LOS.

Conclusion Blood transfusion is an independent risk factor for prolonged hospital LOS after cardiac surgery. This finding can support the development of blood conservation strategies in order to avoid deleterious outcomes of blood exposure.

Reference

1. Hajjar $L A$, Vincent $J L$, Galas FR, et al: Transfusion requirements after cardiac surgery: the TRACS randomized controlled trial. JAMA 2010, 304:1559-1567.

P448

Transfusion of blood stored for longer periods of time does not alter the reactive hyperemia index in healthy volunteers

A Coppadoro', L Berra², B Yu², C Lei², E Spagnolli², AU Steinbicker², KD Bloch², T Lin'2, HS Warren², FY Sammy², BO Fernandez ${ }^{3}$, M Feelisch ${ }^{3}$,

WH Dzik², CP Stowell2, WM Zapol²

'University of Milan-Bicocca, Monza, Italy; 'Massachusetts General Hospital,

Boston, MA, USA; ${ }^{3}$ University of Warwick, Coventry, UK

Critical Care 2012, 16(Suppl 1):P448 (doi: 10.1186/cc11055)

Introduction The purpose of this study is to investigate the effects of transfusing human packed red blood cells (PRBC) after prolonged storage, as compared to short storage. Retrospective data suggest that transfusion of PRBC stored for over 2 weeks is associated with increased mortality and morbidity. During storage, PRBC progressively release hemoglobin, which avidly binds nitric oxide (NO). We hypothesized that the NO-mediated hyperemic response following ischemia would be reduced after transfusion of PRBC stored for 40 days.

Methods We conducted a cross-over randomized interventional study, enrolling 10 healthy adults. Nine volunteers completed the study; one volunteer could not complete the protocol because of anemia. Each volunteer received 1 unit of 40-day and 1 unit of 3-day stored autologous leukoreduced PRBC, on different study days according to a randomization scheme. Blood withdrawal and reactive hyperemia index $(\mathrm{RHI})$ measurements were performed before and 10 minutes, 1 hour, 2 hours, and 4 hours after transfusion.

Results The change of RHI after transfusion of 40-day stored PRBC did not differ as compared to 3 -day stored PRBC $(P=0.67)$. Plasma hemoglobin and bilirubin levels were higher after transfusion of 40-day than after 3-day stored PRBC ( $P=0.02$ and 0.001 , respectively). Plasma levels of potassium, LDH, haptoglobin, cytokines, as well as blood pressure, did not differ between the two transfusions and remained within the normal range. Plasma nitrite concentrations increased after transfusion of 40-day stored PRBC, but not after transfusion of 3-day stored PRBC $(P=0.01)$.

Conclusion Transfusion of 1 unit of autologous PRBC stored for longer periods of time is associated with increased hemolysis, an unchanged $\mathrm{RHI}$ and increased levels of plasma nitrite in healthy volunteers.

\section{P449}

Liberal use of platelet transfusions in the acute phase of trauma resuscitation: a systematic review

J Hallet', F Lauzier', O Mailloux², VTrottier', P Archambault²,

R Zarychanski ${ }^{3}$, AF Turgeon'

'CHA-Hôpital de l'Enfant-Jésus, Université Laval, Québec, Canada; 'Université

Laval, Québec, Canada; 'University of Manitoba, Winnipeg, Canada

Critical Care 2012, 16(Suppl 1):P449 (doi: 10.1186/cc11056)

Introduction With the recognition of early trauma coagulopathy, trauma resuscitation has recently shifted towards early and aggressive transfusion of platelets (PLTs). However, the clinical benefits of this strategy remain controversial. This systematic review examined the impact of an aggressive approach (higher PLT:RBC ratios) compared to restrictive PLT transfusions (lower PLT:RBC ratios) in the acute phase of trauma resuscitation.

Methods We systematically searched Medline, Embase, Web of Science, Biosis, Cochrane Central and Scopus to identify relevant randomized controlled trials (RCTs) and observational studies comparing the effect of two or more different PLT:RBC ratios in trauma resuscitation. We excluded studies using whole blood or systematically addressing the use of hemostatic products. Two independent reviewers selected the studies, extracted data using a standardized form, and assessed the risk of bias using the Newcastle-Ottawa scale and a checklist of key methodological elements (for example, use of massive transfusion protocol, survival bias). Disagreements were solved by consensus or a third party. The primary outcome was mortality. Secondary outcomes were multiple organ failure (MOF), lung injury and sepsis. A metaanalysis using random effects models was planned.

Results From 6,123 citations, seven observational studies were included ( $n=4,230$ patients). No RCT was identified. All studies were considered to be at low risk of bias and addressed confoundings through multivariate regression or propensity scores. Four studies $(n=1,978)$ reported a decrease in mortality with higher PLT:RBC ratios in patients requiring massive transfusion and one study observed no mortality difference $(n=1,181)$ in nonmassively transfused patients. Two studies reported on the implementation of a massive transfusion protocol with higher PLT:RBC ratios; only one revealed a survival benefit $(n=211)$. Of the three studies accounting for survival bias, two demonstrated a survival benefit $(n=1,300)$. Among two studies reporting on the secondary outcomes $(n=854)$, one observed an increase in MOF with higher PLT:RBC ratios. Clinical heterogeneity between studies and methodological limitations precluded the use of a meta-analysis.

Conclusion There is insufficient evidence to strongly support the use of a specific PLT:RBC ratio for acute trauma resuscitation, especially considering survival bias and nonmassively transfused patients. RCTs examining both safety and efficacy of liberal PLT transfusions are warranted.

\section{P450}

Impact on early trauma mortality of the adoption of the Updated European Guidelines on the management of bleeding

E Cingolani', G Nardi', G Ranaldi', C Siddi', S Rogante', A Ciarlone ${ }^{2}$ 'Azienda Ospedaliera San Camillo Forlanini, Roma, Italy; ${ }^{2}$ S. Camillo Hospital, Roma, Italy Critical Care 2012, 16(Suppl 1):P450 (doi: 10.1186/cc11057)

Introduction Post-traumatic bleeding is the leading cause of potentially preventable death among trauma patients. The Updated European Guidelines (UEG), published at the beginning of 2010, were aimed to provide an evidence-based multidisciplinary approach to improve the management of the critically injured bleeding trauma patients. The aim of this study is to evaluate the impact of the implementation of UEG recommendations on early hospital mortality for severe trauma in a high-flow trauma center.

Methods S. Camillo Hospital is a level 1 trauma center based in downtown Rome, with a catchment population of 2.5 million people. UEG recommendations were formally adopted and implemented since 1 April 2010. The pre-existing hospital guidelines were modified as follows: immediate pelvic ring closure for all unstable patients with a suspected pelvic fracture; early administration of plasma with a higher rate of plasma/blood units; early use of thromboelastometry to monitor bleeding patients; and early use of antifibrinolitics for all bleeding patients. Data on trauma admissions and early hospital (6 hours) mortality before (2009) and after the adoption of the UEG were collected using the hospital registry, and were subsequently analysed. Results A total of 1,617 patients met the criteria for full trauma team activation (551 in 2009, 528 in 2010 and 538 during the first 11 months of 2011). There were no differences for gender, age, mechanism of injury and average ISS. In 200921 patients died within the first 6 hours versus 17 in 2010 and 12 in 2011; $P=0.3, P$ for trend $=0.1$ Hemorrhage was the most important cause of death within this time-span. All early trauma deaths occurred in the operating room or in the emergency room during the initial stabilization.

Conclusion This is a retrospective cohort study based on the data of the S. Camillo Hospital registry and the emergency department electronic shift. With the limitations of all retrospective studies, our data suggest that the implementation of the European Guidelines recommendations might contribute to a relevant reduction in early trauma mortality.

Reference

1. Rossaint et al.: Crit Care 2010, 14:R52. 
P451

Hemodynamics in the severely injured patient with significant hemorrhage

G Nardi, D Piredda, A Cossu, E Cingolani, M Cristofani, I Ghezzi

S. Camillo Hospital, Roma, Italy

Critical Care 2012, 16(Suppl 1):P451 (doi: 10.1186/cc1 1058)

Introduction Very little is known about the hemodynamic impairments induced by trauma and severe hemorrhage. The aim of this study is to contribute to a better understanding of this topic. A recent paper has shown that about $50 \%$ of the hemorrhagic patients receive vasopressors [1] together with fluids, blood and plasma. Fluids and vasopressors are aimed to restore patients' hemodynamics; however, they might be detrimental.

Methods The setting was a 10-bed trauma ICU in a level 1 trauma center with a catchment population of over 2.5 million people. This is a retrospective cohort study based on the data of the ICU electronic shift. During a 24-month period (2009 and 2010), 780 patients with major trauma (ISS $>15$ ) were admitted to the hospital; 410 of them were subsequently admitted to the shock and trauma ICU. All patients with ISS $>15$, who had received $\geq 5$ blood units before ICU admission, and who were submitted to semi-invasive hemodynamic monitoring (PICCO), were entered into the study.

Results Thirty patients (mean age $42.7 \pm 17$, mean $37.5 \pm 12$ ) met the study criteria. At the time of insertion of the PICCO catheter (TO) the 30 patients had already received an average of $8,760 \mathrm{ml}$ fluids $(3,239 \mathrm{ml}$ blood, plasma and platelets, $4,870 \mathrm{ml}$ crystalloids and $685 \mathrm{ml}$ colloids). Systemic blood pressure, central venous pressure and heart rate at T0 were, as an average, in the normal range. Nevertheless, six patients (20\%) had a Cardiac Index lower than $2.5 \mathrm{l} /$ minute, and $76 \%$ had a $\mathrm{DO}_{2}$ significantly lower than the normal range. In the subsequent 24 hours following the information of the PICCO, these patients received, as an average, an additional $6,070 \mathrm{ml}$ fluids, blood and plasma. All vasopressors were discontinued, but $40 \%$ of the patients received dobutamine. Within 24 hours (T24), oxygen transport ( $\mathrm{DO}_{2}$ ) and lactate were back to the normal values in all patients but one. ICU mortality and hospital mortality were respectively $13.3 \%$ and $16 \%$.

Conclusion A high percentage of the severely injured patients who received $\geq 5$ units of PRC have a low oxygen transport at the time of ICU admission. A high percentage of them is treated with vasopressors. However, as $20 \%$ of the patients in our study had a low cardiac index in spite of a normal blood pressure and a highly positive fluid balance, vasopressors might be harmful. In our experience, hemodynamic monitoring with PICCO allowed the early recognition of inappropriate oxygen transport and a goal-directed treatment. Our data do not support the use of vasopressors to increase blood pressure in trauma patients.

Reference

1. J Trauma 2011, 71:17-19.

P452

Critical older trauma patients

M Irazábal, S Yus, L Fernández

Hospital La Paz, Madrid, Spain

Critical Care 2012, 16(Suppl 1):P452 (doi: 10.1186/cc1 1059)

Introduction The aim of this study was characterize the older injured patient in our setting and identify risk factors that might predict mortality. Trauma is the fifth leading cause of death over the age of 65. In Spain, it has become a major public health problem as a result of the increase of this population. It represents $30 \%$ of the trauma admissions to our ICU. Geriatric patients may have comorbidities, limited physiologic reserve, may be taking chronic medication and the injury pattern is different [1].

Methods We retrospectively analyzed trauma patients aged 65 years and older admitted to our ICU from January 2000 through December 2010. Three groups were formed on the basis of age: 65 to 70,71 to 78 and older than 78 years. The Injury Severity Score (ISS) was categorized into three ranges: $>12,12$ to 18 and $>18$. Variables studied include: age, gender, mechanism of injury, anticoagulant therapy (ACT), ISS, Glasgow Coma Scale (GCS) or presence of pupillary abnormalities and need for emergent neurosurgery (ENS) at admission. Primary outcome measures were in-hospital mortality and time to death. The secondary endpoint was to identify the effect of chronic medication on mortality. Categorical variables were compared by chi-squared test and continuous variables by Student's t/Mann-Whitney tests. Multiple logistic regression analysis was used to predict mortality. $P<0.05$ was considered statistically significant.

Results The inclusion criteria were met by 261 patients. Age average was 75.57 years (SD 5.7). Male gender was more prevalent (58.5\%) for all age groups. The median ISS was 17. The most frequent trauma mechanism was low-energy type (58.2\%). Patients with chronic ACT numbered 41 (15.7\%). The mean ICU stay was 12.8 days (SD 2.8). Global mortality was $34.1 \%$. Age $>78$ years and ISS $>18$ were predictive of mortality $(P<0.05)$ with a HR of $6.0(\mathrm{Cl} 2.5$ to 14.6$)$ and $1.01(\mathrm{Cl} 1.01$ to 1.05$)$ respectively. Furthermore, the time to death was found to be earlier in both of the latter groups $(P<0.05)$. GCS $<4$ or bilateral mydriasis was associated with $100 \%$ mortality. About $15 \%$ of patients with low-energy trauma (LET) underwent ENS compared to $7.8 \%$ with high-energy trauma. For the same ISS category, ACT increases the risk with HR 2.7 (Cl 1.2 to 6.3) of ENS compared with nonanticoagulated patients.

Conclusion LET accounted for most of the older trauma patients admitted to our ICU and had increased risk of death, especially with ACT. Although this is not necessarily secondary to alarming mechanisms. Reference

1. Spaniolas et al:: Ground level falls are associated with significant mortality in elderly patient. J Trauma 2010, 69:821-825.

P453

Outcomes in older blunt chest wall trauma patients: a retrospective study

C Battle, H Hutchings, PA Evans

Swansea University, Swansea, UK

Critical Care 2012, 16(Suppl 1):P453 (doi: 10.1186/cc11060)

Introduction Blunt chest wall trauma accounts for over 15\% of all trauma admissions to emergency departments in the UK and has high morbidity and mortality rates [1]. Reported risk factors for morbidity and mortality in blunt chest trauma patients include patient age, pre-existing disease and three or more rib fractures [2]. No guidelines exist for management of this patient group unless the patient has severe immediate life-threatening injuries. The aim of this study was to investigate whether blunt chest wall trauma patients aged 65 years or more have higher rates of mortality, morbidity (respiratory complications), ICU admissions and hospital length of stay (HLOS) than patients aged less than 65 years.

Methods A retrospective study was completed in which the notes of 1,056 blunt chest wall trauma patients who presented in 2010 to the emergency department of a large regional trauma centre in Wales were examined. A total of 94 out of the 1,056 (9\%) patients were admitted to hospital in 2010 with blunt chest wall trauma. Data were recorded for each of the admitted patients including patient age, severity of injury, morbidity, mortality, ICU admission and HLOS. Patients were grouped according to age; group one included all blunt chest wall trauma patients aged 65 years or more and group two included all patients aged less than 65 years. Pearson's chi-square analyses were performed to determine whether any differences existed between the two groups and significance set at $P<0.05$.

Results There was no significant difference in severity of injury between the groups. The mortality rate and HLOS in the patients aged 65 years or more were significantly higher $(P<0.05)$ than in the younger patient group. There were no significant differences between the morbidity rates and number of ICU admissions.

Conclusion Blunt chest wall trauma patients have a significantly higher rate of mortality and hospital length of stay if aged 65 years or more when compared to those patients aged less than 65 years. Older blunt chest wall trauma patients should be considered for a higher level of care on admission to hospital from the emergency department.

References

1. Trauma Audit and Research Network: Blunt Chest Trauma Admissions in the UK in 2010. TARN; 2011. (Kindly provided by Tom Jenks.)

2. Blecher GE, Mitra B, Cameron PA, et al.: Failed emergency department disposition to the ward of patients with thoracic injury. Injury 2008, 39:586-591. 
P454

Mortality trend alteration of thoracic injury after rapid response trauma team establishment

K Chittawatanarat, C Ditsatham, K Chandacham, T Jirapongchareonlap, N Chotirosniramit

Chiang Mai University, Chiang Mai, Thailand

Critical Care 2012, 16(Suppl 1):P454 (doi: 10.1186/cc11061)

Introduction The Department of Surgery, Faculty of Medicine, Chiang Mai University established a rapid response trauma team (RRTT) in July 2006. The aims of this study were to verify mortality rate alteration after setting up the RRTT.

Methods We retrospectively collected data between January 2004 and September 2009. The month before July 2006 was defined as before RRTT and after July 2006 as after RRTT. The monthly mortality rate, severity injury score (ISS) and demographic data were collected.

Results A total 951 patients were included (427 (30 months) before RRTT and 524 (39 months) after RRTT). Of these, 83 patients (8.8\%) were dead after admission and analyzed for characters of mortality. The average age of mortality patients was $38.7 \pm 16.3$ years. Male was the predominant gender. The most common mechanism of injury was a motorcycle accident. Although there were no differences of character and mechanism of injuries between the two periods, patients associated with maxillofacial injury had significant lower mortality after RRTT (28.5\% vs. $10.5 \% ; P=0.04)$. However, the after RRTT group had significantly higher occurrence of urinary complication and acute renal failure. The average adjusted monthly mortality rate was lower after RRTT $(9.0 \pm 6.1$ vs. $6.9 \pm 4.0 \%)$. Time series analysis between two periods demonstrated a decrease trend in monthly mortality after RRTT (coefficient $(95 \% \mathrm{Cl})=-0.61(-1.13$ to -0.23$) ; P<0.01)$ ).

Conclusion Rapid response trauma team establishment could decrease the mortality trend. A protective effect was predominant in patients associated with maxillofacial injury.

P455

Trauma patients and cervical spine protection in critical care: the impact of a spinal checklist on clinical care and documentation A Chick, C Scott, H Ellis, A Tipton

Sheffield Teaching Hospitals NHS Foundation Trust, Sheffield, UK

Critical Care 2012, 16(Suppl 1):P455 (doi: 10.1186/cc11062)

Introduction In October 2010 a specific online proforma for cervical spine (C-spine) assessment in the context of trauma was introduced in critical care in a large UK teaching hospital. The aim of this study is to assess the impact of the Metavision Spinal Checklist (MSC) on clinical care and documentation. Prior to October 2010, the documentation of $\mathrm{C}$-spine status on admission to critical care was incomplete or unclear in over $40 \%$ of these patients.

Methods Patients were identified from a comprehensive critical care database. Inclusion criteria: age $>16$; polytrauma or traumatic brain injury; other trauma where mechanism of injury suspicious for C-spine injury; admission date after 1 October 2010, before 30 November 2011. Exclusion criteria: pre-existing spinal injury; mechanism of trauma not consistent with C-spine injury. Clinical and MSC details were recorded, including sequential forms for individual patients where the C-spine status changed (for example, C-spine cleared and hard collar removed). Results A total of 62 patients met the inclusion criteria; $47 \%$ of these had been transferred from a district hospital. In patients with an MSC completed, there was $100 \%$ documentation of time, date and name of the completing critical care consultant. Seventy-five per cent of initial MSCs indicated the name of the responsible consultant spinal surgeon. Seventy-nine per cent of patients with a completed MSC required their $\mathrm{C}$-spines to be cleared after critical care admission. When completed, the initial MSC allowed clearance of C-spine and immediate removal of hard collar in $67 \%$ of those patients. There were clearly documented instructions for C-spine care from a spinal consultant in $92 \%$ of patients with a completed MSC. Overall, an MSC was completed for only $39 \%$ of patients, despite $53 \%$ of patients having sustained a spinal fracture at some level (for example, lumbar, thoracic or cervical). The median time from critical care admission to MSC completion was 36 hours (range 3 hours to 12 days, mean 48 hours).
Conclusion The uptake of this checklist has not been optimal, but the MSC provides an excellent tool for clear documentation of C-spine status. During this initial trial phase, October 2010 to December 2011, the MSC has been consultant-only. Further action will involve rollingout the checklist to critical care trainee doctors to improve the rate of documentation of $\mathrm{C}$-spine status and improve patient safety in this area of significant clinical risk [1].

Reference

1. Morris CG, et al:: BMJ 2004, 329:495-499.

\section{P456}

Effect of instrumented spinal fixation on outcome in polytrauma

patients in the ICU

G Simpson, C Menakaya, A Bidwai, R Pillay, M Dematas

Royal Liverpool University Hospital, Liverpool, UK

Critical Care 2012, 16(Suppl 1):P456 (doi: 10.1186/cc11063)

Introduction Spinal injuries in polytrauma patients carry high morbidity and mortality often necessitating intensive care admission. A review of polytrauma patients admitted to the ICU at The Royal Liverpool University Hospital was undertaken to investigate the effect of spinal instrumentation on outcome in the ITU.

Methods A retrospective review of all polytraumatized patients admitted to the RLUH ICU over 3 years with a thoraco-lumbar spinal fracture. Clinical records, laboratory results and radiological records were accessed. Patients were grouped according to the use of instrumented spinal fixation versus conservative management and outcomes compared.

Results Fourteen polytrauma patients with spinal fractures were admitted to the ICU over 3 years, five managed conservatively with a TLSO brace and nine managed operatively with instrumented spinal fixation. The degree of injury as graded by the Injury Severity Scale (ISS) was lower in the nonoperative group (mean: 27, range: 14 to 59) compared to the operative group (mean: 36.1, range: 14 to 57). Mortality was significantly higher in patients conservatively managed (nonoperative: $60 \%$, operative: $0 \%)(P<0.01)$. The intubation time was lower in patients who underwent spinal instrumentation (mean: 12.3 days, range: 1 to 27 days), when compared to conservative management (mean: 16 days, range: 11 to 27 days), and similarly the ITU length of stay was reduced in the operative group (operative: mean 20.6 days, nonoperative: 32.25 days). Development of respiratory failure was decreased in patients treated with instrumented fixation (operative $33.3 \%$, nonoperative: $71 \%$ ).

Conclusion Surgical stabilization of spinal fractures avoids restrictive spinal braces and permits mobilization. Surgical fixation of spinal fractures appears to decrease mortality and ITU stay and has a beneficial effect on respiratory function, with regards to degree of ventilatory support and development of respiratory failure.

\section{P457}

Whole body computed tomography scanning for severe blunt polytrauma: analysis of Trauma Audit and Research Network database 2005 to 2010

PA Hunt ${ }^{1}$, F Lecky², O Bouamra²

${ }^{1}$ James Cook University Hospital, Middlesbrough, UK; ${ }^{2}$ Hope Hospital, Salford, UK

Critical Care 2012, 16(Suppl 1):P457 (doi: 10.1186/cc11064)

Introduction There is growing evidence to recommend the use of whole body computed tomography (WBCT) scanning in the early management of severe blunt polytrauma patients. One recent study reported a survival advantage when using WBCT compared to a conventional imaging approach [1]. A number of UK NHS institutions already utilise WBCT protocols based upon either injury mechanismrelated or physiological factors, or a combination of these. However, the UK Royal College of Radiologists is yet to provide recommendations on the use of WBCT in polytrauma. We present the results of our analysis of a large retrospective case series from 2005 to 2010 taken from the Trauma Audit and Research Network (TARN) database. We believe this is the first analysis of its kind involving UK trauma cases and provides 
important evidence to support the use of WBCT and guide best clinical practice.

Methods We utilised retrospective, multicentre data of severe blunt polytrauma (ISS $>15$ ) direct ED admissions aged $>15$ years recorded in the UK TARN database to compare survival at 30 days between two groups of patients: those who underwent WBCT scans, and those who received a focused CT scan approach as part of their initial management in the emergency department. A total of 12,792 cases were included in the final dataset.

Results A total 2,822 (22\%) of 12,792 cases underwent WBCT from the $\mathrm{ED}$. The median ISS for the WBCT group was 22 (IQR 14 to 33 ) compared to 16 (IQR 9 to 25) for the focused CT group. The calculated crude mortality rate for the WBCT group was $10.1 \%$ compared to $8.7 \%$ in the focused CT group $(P=0.0124)$. Multivariate analysis with adjustments for potential confounding factors demonstrated an OR of $1.313(95 \%$ $\mathrm{Cl}=1.083$ to $1.592, P=0.006$ ) in favour of survival in the WBCT group. Conclusion Despite the crude mortality rates appearing to demonstrate a poorer outcome in the WBCT group, correcting for confounding factors revealed an around $30 \%$ improvement in survival for the WBCT group. However, when also correcting for the potential effect of clustering, the benefit of WBCT is less clear, with an around $20 \%$ improvement in survival and a lower level of significance $(P=0.084)$. This effect may, in part, be due to differing trauma systems and logistical organisation between institutions. Overall, the results of our investigation appear to suggest a potential survival benefit from the use of WBCT in severe blunt polytrauma.

Reference

1. Huber-Wagner S, et al: Lancet 2009, 373:1455-1461.

P458

Post-traumatic rhabdomyolysis: an observational study in seven patients

M Alezrah, A Berger, P Bentzinger, C Sassot, L Profumo, B Saumande, O Collange, A Meyer, B Calon

réanimation chirurgicale, Strasbourg, France

Critical Care 2012, 16(Suppl 1):P458 (doi: 10.1186/cc11065)

Introduction In the ICU, post-traumatic rhabdomyolysis is a relatively rare $(1 / 5)$ cause of crush syndrome [1]. Early aggressive treatment is quintessential to avoid complications such as renal failure and death [2]. This observational study intends to assess the incidence of complications after traumatic crush injury in a tertiary trauma center ICU.

Methods During 24 months, seven patients admitted to our surgical intensive care after polytrauma (ISS > 15) suffered severe rhabdomyolysis (CPK $>5,000 \mathrm{U} / \mathrm{I})$ treated by intensive fluid resuscitation, bicarbonate and furosemide.

Results The following data are reported in Table 1: renal function (initial creatinine, renal replacement therapy (RRT), rhabdomyolysis (maximal CPK and myoglobin), acidosis (lowest $\mathrm{pH}$, highest lactate $(\mathrm{HL})$, time lactate $>5 \mathrm{mmol} / \mathrm{l}$ ) and complications (mortality, neurological sequelae).

Conclusion Survival was $100 \%$ but neurological impairment in the limbs is a major complication. The two RRT patients had a wide range

\section{Table 1 (abstract P458). Results}

\begin{tabular}{lc}
\hline Initial creatinine $(\mu \mathrm{mol} / \mathrm{l})$ & 69 to 198 \\
RRT & $2 / 7$ \\
Maximal CPK $\left(10^{3} \mathrm{U} / \mathrm{l}\right)$ & 11 to 144 \\
Maximal myoglobin $\left(10^{3} \mathrm{U} / \mathrm{l}\right)$ & 4 to 159 \\
$\mathrm{pH}$ & 7 to 7.3 \\
Highest lactate $(\mathrm{mmol} / \mathrm{l})$ & 2 to 28 \\
Time lactate $>5 \mathrm{mmol} / \mathrm{l}$ (hours $)$ & 0 to 84 \\
Mortality & 0 \\
Neurological sequelae & $6 / 7$ \\
\hline
\end{tabular}

of maximal CPK levels (15,780 to 52,600 U/l), but more severe acidosis (lowest pH 7.0 to 7.2 , maximum lactate: 7.5 to $28 \mathrm{mmol} / \mathrm{l}$, acidosis duration: 72 to 84 hours). This acidosis turned out to be due to intraabdominal complications: post-traumatic pancreatitis and mesenteric ischemia. The vital prognosis of post-traumatic crush injury was good but the sequelae of the compartment syndrome were major. The need for RRT was not linked to CPK levels but rather to acidosis due to intraabdominal complications.

References

1. Bagley WH, Yang H, Shah KH: Rhabdomyolysis. Intern Emerg Med 2007, 2:210-218

2. Bosch X, Poch E, Grau JM: Rhabdomyolysis and acute kidney injury. N EnglJ Med 2009, 361:62-72

P459

Exertional rhabdomyolysis in female amateur triathletes

$\checkmark$ Meighan

Galway University Hospital, Galway, Ireland

Critical Care 2012, 16(Suppl 1):P459 (doi: 10.1186/cc11066)

Introduction Multisport endurance events are becoming increasingly popular in Ireland. Overexertion, especially in the heat, of overweight or poorly conditioned athletes increases the risk of rhabdomyolysis. This study presents a case series of three female amateur triathletes presenting with acute abdominal pain caused by rhabdomyolysis.

Methods The medical case notes of three female athletes presenting to the emergency department were reviewed.

Results All three patients presented with abdominal pain after triathlon training. On admission, creatinine kinase levels were over 30,000 in all three cases and all required acute hospital admission for pain relief and intravenous fluids to prevent renal failure.

Conclusion Exertional rhabdomyolysis is not rare, but rarely do such patients present to the emergency department with acute abdominal pain. Whilst triathlon training is popular among amateur sports people, awareness must be raised to train appropriately under proper conditions.

P460

Controlled mechanical ventilation tactics in patients with polytrauma during interhospital transportation to the specialized center

A Shatalin', S Kravtsov², V Agadzhanyan² D Skopintsev²

'Federal State Budgetary Medical Prohylactic Institution, Leninsk-Kuznetsky, Russia: ${ }^{2}$ Federal State Budgetary Medical Prohylactic Institution 'Scientific Clinical Center of the Miners Health Protection', Leninsk-Kuznetsky, Russia Critical Care 2012, 16(Suppl 1):P460 (doi: 10.1186/cc11067)

Introduction This study is an analysis of the influence of controlled mechanical ventilation (CMV) with PEEP in conditions of pneumocompression of the Chestnut antishock suit on the hemodynamics and blood oxygenation in patients with polytrauma during interhospital transportation.

Methods Seventy-two patients with polytrauma complicated by II and III stage ARDS were included in the study. The mean age was $33 \pm 2$ years. All patients were divided into two equal groups. The control group (CG) CMV was carried out with no PEEP. The experimental group (EG) CMV was carried out with PEEP 8 to $10 \mathrm{mbar}$. Both groups received the CMV regimen with $V 7 \mathrm{ml} / \mathrm{kg}, \mathrm{P} 30 \mathrm{mbar}$. The injury severity according to the ISS was $37.6 \pm 1$ points in the EG and $39.1 \pm 1$ in the CG. The transportation time was $135 \pm 7$ minutes, the distance was $136 \pm 10 \mathrm{~km}$. Immobilization in the lower extremity fractures and pelvis fractures was carried out using the Chestnut suit with pneumocompression over the damaged parts of the body of $40 \mathrm{mmHg}$ and over the remaining parts of the body of $20 \mathrm{mmHg}$. Statistical analysis was performed using Statistica 6.1. We used Student's criterion.

Results In the EG there were the high values of $\mathrm{SpO}_{2}$ during all observation periods and $\mathrm{PaO}_{2} / \mathrm{FiO}_{2}$ after completion of the transportation in 1 and 12 hours $(P<0.05)$. $\mathrm{PaCO}_{2}$ in the EG was lower after completion of the transportation in 1 and 12 hours compared to the CG $(P<0.05)$. In the $\mathrm{EG}$ the value of $\mathrm{FiO}_{2}$ decreased from $0.5 \pm 0.01$ in 
the early examination to $0.4 \pm 0.01$ in 12 hours after transportation. In the $\mathrm{CG}, \mathrm{FiO}_{2}$ did not change. Hemodynamics differences between the groups were not documented, except for HR $(P>0.05)$. Tachycardia was less expressed in the EG. The difference from the CG according to this index occurred 12 hours after completion of the transportation, $83 \pm 1$ and $87 \pm 0.7$ beats/minute respectively $(P<0.05)$. The lactate rate was lower in the EG during all periods of observation $(P<0.05)$. After completion of the transportation, the lactate rate in the EG was $2.2 \pm 0.1 \mathrm{~mol} / \mathrm{l}$ and in the $C G$ was $2.7 \pm 0.1 \mathrm{~mol} / \mathrm{l}$.

Conclusion Use of CMV with PEEP in patients with polytraumacomplicated ARDS provided more expressed improvement of the blood oxygenation. Improvement of the blood gas exchange was accompanied by lactate decrease in both groups: by $24 \%$ in the EG, and by $13 \%$ in the CG. Application of the Chestnut allowed one to level the hemodynamic disorders using CMV with PEEP by means of preload maintenance and of the systolic output as a consequence.

\section{P461}

Impact of fluid resuscitation volume on the severity of organ failures in severely burned patients

N Depaye, G Minguet, A Magnette, D Jacquemin, D Ledoux, P Damas University Hospital of Liege, Belgium

Critical Care 2012, 16(Suppl 1):P461 (doi: 10.1186/cc1 1068)

Introduction Adequacy of fluid resuscitation remains a cornerstone of early burn management. The Parkland formula - that is, administration of $4 \mathrm{ml} / \mathrm{kg} / \%$ total of the body surface area (TBSA) burned with Ringer's lactate for the first 24 hours post injury - has been used for decades. The purpose of this study was to evaluate the effect of adherence with the Parkland protocol and its impact on the severity of organ failure during the first week post injury using the Sequential Organ Failure Assessment (SOFA) score.

Methods We conducted a retrospective review of burns' resuscitation data, from 2000 to 2007, on 101 adult patients (aged $\geq 16$ years) admitted within the first 24 hours following injury, with a \%TBSA burned of 20 or more. A classification of patients into four groups, according to fluids administered, was done for comparison between these groups. The SOFA score was calculated daily for the first week after admission. The neurological component of SOFA was left out because of the difficulty to assess the actual Glasgow Coma Scale in sedated patients. Organ failures were defined by partial SOFA $\geq 3$. Data are expressed as median (Q1 to Q3) and are analyzed using the chisquare test $(P<0.05$ was considered statistically significant).

Results A total of 62 patients with complete data on fluid administration were included in the analysis. Median age was 41 (28 to 54) years, median TBSA burned was 35.5 ( 25 to 50 ); median ICU stay was 38 (12 to 62$)$ days and $13(21 \%)$ patients died. Ten patients suffering from inhalation injury were excluded from further analysis. Median fluids administered was $4.9(4.1$ to 6.2$) \mathrm{ml} / \mathrm{kg} / \%$ TBSA at 24 hours. Five patients received $<3.5 \mathrm{ml} / \mathrm{kg} / \%$ TBSA, 15 between 3.5 and $4.5 \mathrm{ml} / \mathrm{kg} / \%$ TBSA, 17 between 4.5 and $6 \mathrm{ml} / \mathrm{kg} / \%$ TBSA and 15 patients $>6 \mathrm{ml} / \mathrm{kg} / \%$ TBSA. No differences existed between groups concerning the cause and surface of injury, age, sex, and comorbidities. Compared to others, patients who received $>6 \mathrm{ml} / \mathrm{kg} / \%$ TBSA had a significant increase in respiratory failure $(P=0.03)$. The amount of fluids administered had no impact on the incidence of cardiovascular $(P=0.89)$, renal $(P=0.11)$, liver $(P=0.52)$ and coagulation $(P=0.86)$ failure.

Conclusion This single-centre retrospective study indicates that fluid resuscitation volumes frequently overcome those previously established by the Parkland protocol. This fluid over-resuscitation may have deleterious effects on patient outcome by increasing the incidence of respiratory failure.

Reference

1. Jeffrey RS: The phenomenon of 'fluid creep' in acute burn resuscitation. J Burn Care Res 2007, 28:382-395.
P462

Organ dysfunction in the resuscitation phase of critical burn patients

A Agrifoglio, M Sánchez, M Hernández, J Camacho, L Cachafeiro,

M Asensio, E Herrero, A García de Lorenzo, M Jiménez

Hospital Universitario La Paz, Madrid, Spain

Critical Care 2012, 16(Suppl 1):P462 (doi: 10.1186/cc11069)

Introduction Sequential Organ Failure Assessment (SOFA) is useful to assess organ dysfunction in burn patients [1]. The aim of this study was to determine the change in organ dysfunction from admission to day 3 . Methods We performed a prospective observational cohort study with critical burn patients (total body surface area (TBSA) $>20 \%$ and/ or inhalation injury) admitted to our burn ICU from September 2008 to December 2010. Epidemiological data and SOFA score at admission (day 0) and days 1,2 and 3 were collected.

Results Sixty-four patients were enrolled (70\% men) with mean age of $48.2 \pm 19.0$ years; Abbreviated Burn Severity Index (ABSI): $8.78 \pm 2.59$; APACHE II score: $13.5 \pm 6.5$. Twenty-three patients $(35.9 \%)$ had inhalation injury and 19 patients (29.7\%) died. The SOFA score was increased from day 0 to day 3 . At admission the most frequent dysfunctions were cardiovascular and respiratory. The respiratory was similar in the next days and the cardiovascular dysfunction worsened (Table 1). Haematological dysfunction appeared at day $3(1.05 \pm 1.0)$ and neurological, renal and hepatic dysfunction were uncommon in the resuscitation phase.

Table 1 (abstract P462). SOFA during the resuscitation phase

\begin{tabular}{lcccc}
\hline & Day 0 & Day 1 & Day 2 & Day 3 \\
\hline SOFA & $3.40 \pm 2.48$ & $4.26 \pm 2.99$ & $4.95 \pm 3.04$ & $5.25 \pm 3.25$ \\
Respiratory & $1.38 \pm 1.12$ & $1.32 \pm 1.09$ & $1.81 \pm 1.09$ & $1.76 \pm 1.07$ \\
Cardiovascular & $1.19 \pm 1.76$ & $2.06 \pm 1.94$ & $2.10 \pm 1.85$ & $2.22 \pm 1.89$ \\
\hline
\end{tabular}

Conclusion In the resuscitation phase of our critical burn patients the initial dysfunction was respiratory and cardiovascular, progressing later to cardiovascular dysfunction and haematological dysfunction appearing at the third day of admission. Knowing the possible evolution of organ dysfunction may help early detection and treatment.

Reference

1. Latenser B: Crit Care Med 2009, 37:2819-2826.

P463

Epidemiological study of critical burn patients in an ICU

L Cachafeiro, M Sanchez, E Herrero, J Camacho, M Hernandez,

A Agrifoglio, A García de Lorenzo, M Jimenez

Hospital La Paz, Madrid, Spain

Critical Care 2012, 16(Suppl 1):P463 (doi: 10.1186/cc11070)

Introduction Burn injuries remain a significant problem with high associated morbidity and mortality, long average stays and high costs. The aim of our study is to analyze the epidemiology and mortality of critical burn patients admitted to the ICU at a university hospital in Madrid, Spain.

Methods We performed a prospective, observational and descriptive study in patients admitted with burns over $20 \%$ of the total body surface area (TBSA), from October 2008 to December 2009. Demographic data were collected, TBSA, location and mechanism of burns, severity scores (ABSI, APACHE II, SOFA at admission, and next 3 days) length of stay, complications and mortality. Data are presented as number and percentage or as median and interquartile range, and they were analyzed with the Fisher exact test and Mann-Whitney test.

Results During this period, 64 patients were admitted to our unit, 45 (70.3\%) were men and $19(29.7 \%)$ were women. The mean age was $48 \pm 19$. SOFA score at admission was $3 \pm 2$, APACHE II score $15 \pm 6$ (range 4 to 38 ) and ABSI 8 (range 5 to 16). The TBSA average was $40 \pm 20 \%$ and the mechanism of burn was by flame in 60 patients $(93.8 \%)$, scald in four (6.3\%), electrical in two (3.1\%) and chemical in one (1.6\%). The most frequent location was in the upper limbs in 60 patients $(93.8 \%)$, followed by thorax in 50 (78.19\%), head and neck in 43 (67.2\%), lower 


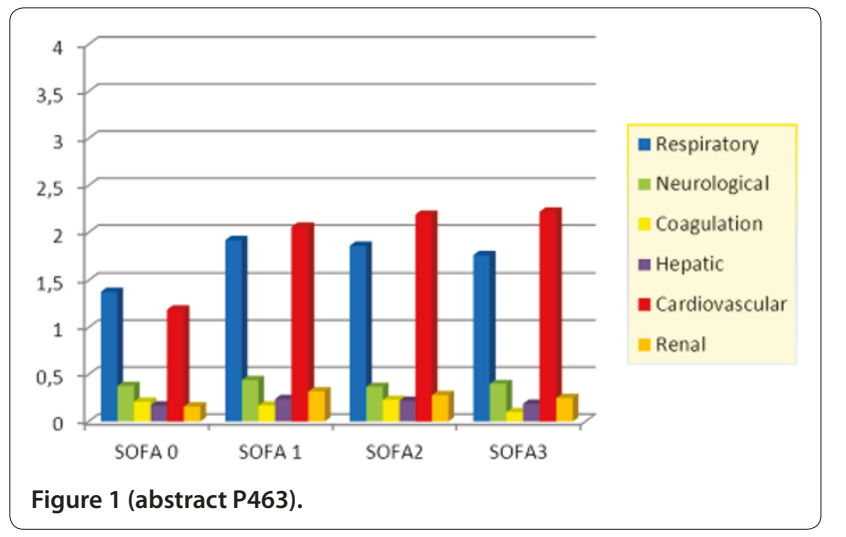

limbs in 43 (67.2\%), and back in 29 (45.3\%). Six patients had trauma associated and 23 had inhalation injury. Thirty-two patients (50.0\%) required escharotomy at admission and $16(25.0 \%)$ had compartment syndrome. Forty-four patients (68.8\%) needed mechanical ventilation, and $20(31.3 \%)$ tracheostomy. Fifty-six patients had complications. The most frequent were: shock (70.3\%), ARDS (31.3\%), sepsis (35.9\%) and renal failure (26.6\%). All complications increased significantly the mortality $(P<0.001)$. The length of stay was 30 days and global mortality was $29.7 \%$ (19 patients). See Figure 1.

Conclusion In our study the most common burns were caused by flame in the upper limbs, chest, neck and face. Eighty-nine percent of our patients had complications, and they increased significantly the length of stay and mortality. Based on the SOFA score, patients had higher scores for respiratory and cardiovascular systems. However, mortality was lower than expected in severity scores.

\section{P464}

Fluid creep in burn resuscitation: the tide has not yet turned

E James, M Hayes, P McCabe, G Williams, M Takata, MP Vizcaychipi Chelsea and Westminster Hospital and Imperial College, London, UK Critical Care 2012, 16(Suppl 1):P464 (doi: 10.1186/cc11071)

Introduction The purpose of this study was to examine the fluid resuscitation of severely burned patients admitted to our regional centre and to review whether our practice had changed over the last 5 years in light of concerns of fluid creep. Fluid creep is the term coined by Pruitt used to describe fluid resuscitation in excess of that predicted by the Parkland formula and which is associated with abdominal compartment syndrome (ACS) [1].

Methods We completed a retrospective review in accordance with clinical governance guidance of patient notes evaluating all admissions in two groups (Group A: 1 May 2005 to 30 April 2006 and Group B: 1 May 2010 to 30 April 2011). The review examined the first 72 hours of fluid resuscitation in patients with $\geq 15 \%$ TBSA burns who were admitted less than 24 hours post burn injury.

Results There were 12 patients in each group. Both groups were comparable in both admission (Table 1) and resuscitation data. The total fluid (mean \pm SD) given in the first 24 hours post burn-centre admission was $5.36 \pm 2.22 \mathrm{ml} / \mathrm{kg} / \%$ TBSA in Group A and $5.72 \pm 3.00 \mathrm{ml} / \mathrm{kg} / \%$ TBSA in Group $B(P=0.817)$ with three patients in each group receiving in excess of $250 \mathrm{ml} / \mathrm{kg}$. Almost one-third of the fluid administered was colloid in each group. The hourly urine output (mean $\pm \mathrm{SD}$ ) was $1.34 \pm 0.72 \mathrm{ml} / \mathrm{kg} /$ hour in Group A and $1.53 \pm 1.47 \mathrm{ml} / \mathrm{kg} /$ hour in Group B $(P=0.817)$. Inhalational injury was present in six patients in Group $A$ and three in Group B. The inhalational injury group (mean \pm SD) received $6.64 \pm 2.51 \mathrm{ml} / \mathrm{kg} / \%$ TBSA whilst the noninhalational injury group received $4.88 \pm 2.50 \mathrm{ml} / \mathrm{kg} / \%$ TBSA $(P=0.101)$. There was no reported incidence of ACS.

Conclusion Despite our awareness of fluid creep, our practice has not changed significantly over the last 5 years. Fluid was administered in excess of that predicted by the Parkland formula despite almost one-third being given as colloid and no cases of ACS being reported. A multicentre randomised control trial is required to examine stricter
Table 1 (abstract P464)

\begin{tabular}{lccc}
\hline Patient data & Group A & Group B & P value \\
\hline Number $(n)$ & 12 & 12 & \\
Age (years) & $49(18$ to 69$)$ & $38.5(21$ to 77$)$ & 0.260 \\
Weight $(\mathrm{kg})$ & $72(55$ to 109$)$ & $75(60$ to 99$)$ & 0.794 \\
\% TBSA & $37.5(16$ to 70$)$ & $31(18$ to 60$)$ & 0.602 \\
Inhalation injury $(n)$ & $6 / 12$ & $3 / 12$ & 0.206 \\
Trauma $(n)$ & $1 / 12$ & $0 / 12$ & 0.307 \\
Admission base deficit & $-5.95(-15$ to +1$)$ & $-6.55(-11.7$ to +2.5$)$ & 0.931 \\
Admission lactate $(\mathrm{mmol} / \mathrm{l})$ & $3.03(0.98$ to 5.4$)$ & $2.05(0.5$ to 4.1$)$ & 0.081 \\
Survival $(n)$ & $6 / 12$ & $9 / 12$ & 0.206 \\
\hline Data pres
\end{tabular}

Data presented as median (range).

titration of fluid administration to urine output and the specific role of colloids in early resuscitation.

Reference

1. Pruitt BA Jr: J Trauma 2000, 49:567-568.

P465

Early administration of parenteral estrogen suppresses the deleterious local and systemic inflammatory response in severe burns

JG Wigginton', PE Pepe', JW Simpkins², JW Gatson', KG Wigginton', KR Kareem'1 , JP Minei', D Maass'

'University of Texas Southwestern Medical Center, Dallas, TX, USA; ${ }^{2}$ University of North Texas, Fort Worth, TX, USA

Critical Care 2012, 16(Suppl 1):P465 (doi: 10.1186/cc11072)

Introduction Soon after severe burns, deleterious cytokines are produced and found in the burned skin, including dead tissue in thirddegree injuries. This is followed by a systemic surge in these markers and correlated with subsequent multiorgan failure (MOF). In animal models, this response can be somewhat blunted by early debridement, but such early intervention is not usually feasible in most clinical settings. As estrogen is a powerful anti-inflammatory/anti-apoptotic agent, we tested parenteral $17 \beta$-estradiol $\left(E_{2}\right)$ as a feasible early alternative intervention to dampen the proinflammatory response.

Methods Male rats $(n=168)$ were assigned randomly to one of three groups: (1) sham (no) burn $(n=8)$; (2) burn given placebo $(n=80)$; and (3) burn given $\mathrm{E}_{2}$ (estrogen). Groups 2 and 3 had $40 \%$ TBSA third-degree dorsal burns, early fluid resuscitation and $0.5 \mathrm{mg} / \mathrm{kg}$ i.p. estrogen (or placebo) 15 minutes post burn. From each group of 80 , eight animals were sequentially sacrificed (and burn tissue and blood sampled for IL- 6, TNFa, IL-1 $\beta$ ) at one of 10 time points as follows: $0.5,1,2,4,6,8,18$ and 24 hours and 7 days ( 7 days only for the eight shams).

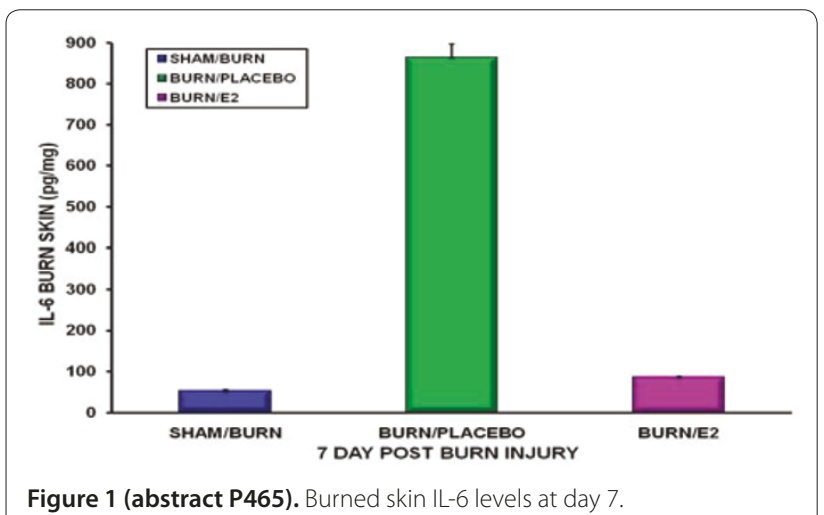


Results In placebos, very high levels of cytokines appeared almost immediately in the echars and circulation, persisting 7 days post burn. In the estrogen group, cytokines, including tissue and circulating IL-6, the greatest predictor of MOF, remained suppressed at all time points, even day 7 (Figure 1).

Conclusion Early single-dose parenteral estrogen can dramatically suppress both the local and systemic massive proinflammatory responses in severe burns. Based on these data, estrogen may not only be an inexpensive, simple, adjunctive therapy in burn management, it may obviate the need for many subsequent interventions altogether. References

1. Crit Care Med 2010, 38:5620-S629

2. J Neuroinflamm 2009, 6:30-36

P466

Reducing the indication of ventilatory support in the severely burnt patient and improving outcomes: results of a new protocol approach within a regional burns centre

J Gille', H Taha², R Blankenburg', T Raff', A Sablotzki

'St Georg Hospital, Leipzig, Germany; 'Royal Devon \& Exeter Hospital

Foundation Trust, Exeter, UK

Critical Care 2012, 16(Suppl 1):P466 (doi: 10.1186/cc11073)

Introduction Initial management of the severely burnt patient often includes sedation and mechanical ventilatory support as routine. Conversely it is documented in the literature that nonjudiciously applied mechanical ventilatory support can itself lead to poorer patient outcomes [1]. Exploring means to reduce this iatrogenic risk, a standardised in-house five-point protocol offering clinical guidance on the use and duration of ventilation was introduced, analysed and the impact on outcome assessed.

Methods A clinical observation study, approved by the local ethical committee, was designed and executed. Criteria for early spontaneous breathing were defined. These were formulated into a protocol for the management of severely burnt patients and trialled over 2 years in clinical practice on all admitted patients (group A). The ventilation period, complications and final outcomes were recorded and compared with a retrospective control group of patients (group B) collated prior to implementation of the protocol. Initial study analysis revealed high inclusion rates of superficial burns in the intervention group. To achieve comparability these were excluded and further analysis was conducted only for patients with an abbreviated burn severity index $(A B S I) \geq 7$.

Results In total 118 patients were included. The demographics and injury characteristics of both groups were similar. Patients of group A $(n=61)$ had fewer ventilator days in the time course of treatment $(3.9 \pm 11.7$ vs. $17.1 \pm 19.6$ days, $P<0.01)$. Affiliation to group $A$ correlated with a shorter time of ventilation after admission $(P<0.01) ; 61.1 \%$ of these patients were extubated within 6 hours after admission (vs. 14.3\% in group B). Group A showed lower mortality rates (1 (1.4\%) vs. 8 (14\%), $P=0.01)$, shorter total hospital stay $(34.2 \pm 23.9$ vs. $50 \pm 38.4, P=0.014)$ and lower incidence of sepsis (24 (39.3\%) vs. $39(68.4 \%), P<0.01)$.
No patients fulfilling the inclusion criteria required re-intubation or emergency intubation.

Conclusion Extended periods of mechanical ventilatory support are known to be associated with poorer outcomes in the severely burnt patient. Guidance on minimising ventilator dependency through introduction of a protocol has led to improved outcomes of such patients within a regional burns centre. This study suggests that many burns patients are overtreated through routine ventilation.

\section{Reference}

1. Mackie D, Spoelder E, Paauw R, et al.: Mechanical ventilation and fluid retention in burn patients. J Trauma 2009, 67:1233-1238.

\section{P467}

Cardiopulmonary exercise testing and elective open abdominal aortic aneurysm surgery over a 6-year period in a UK teaching hospital

AH Raithatha, S Smith, K Chakrabarti, A Tridente, K Kerr Sheffield Teaching Hospitals NHS Trust: Northern General Hospital, Sheffield, UK

Critical Care 2012, 16(Suppl 1):P467 (doi: 10.1186/cc11074)

Introduction A reduced oxygen uptake at anaerobic threshold (AT) and an elevated ventilatory equivalent for carbon dioxide (VE/VCO $)$ have been shown to be predictors of outcome after major surgery [1]. We report the demographic and outcome data of patients undergoing elective open abdominal aortic aneurysm (AAA) surgery who underwent cardiopulmonary exercise testing (CPET) testing within our unit and examine the relationship between age, AT and VE/VCO on survival outcomes.

Methods A retrospective observational analysis of our unit's CPET Excel database was conducted to identify patients who underwent CPET testing for elective open AAA repair over a 6-year period. Demographic data and survival at 30 days, 90 days and 1 year were extracted. Logistic regression analysis was undertaken using STATA statistical software to determine if age, AT or VE/VCO $\mathrm{CO}_{2}$ were predictors of survival at 30 days, 90 days or 1 year.

Results CPET was performed in 259 patients who subsequently underwent an elective open AAA repair. Outcome data were available for 185 patients from a potential 222 in whom 1-year follow-up was available (83\%). Baseline demographics included AT $\leq 10.9 \mathrm{ml} / \mathrm{kg} /$ minute in $39 \%$ and $>10.9 \mathrm{ml} / \mathrm{kg} /$ minute in $61 \%$ of patients with respective median ages in these groups being 73 and 72 . Regression analysis demonstrated that AT was the only predictor of survival at 30 days, 90 days and 1 year. Age and AT remained independent predictors of survival at 90 days and 1 year following multivariate analysis. Of note, 87 patients underwent elective endovascular aneurysm repair and CPET, median age 76 , during the period analysed. In particular, $26.4 \%$ were older than 80 years old, versus $14.7 \%$ in the AAA group. See Figure 1.

Conclusion Our data support existing evidence that AT can be used as a predictor of survival in open elective AAA surgery. In addition, age at
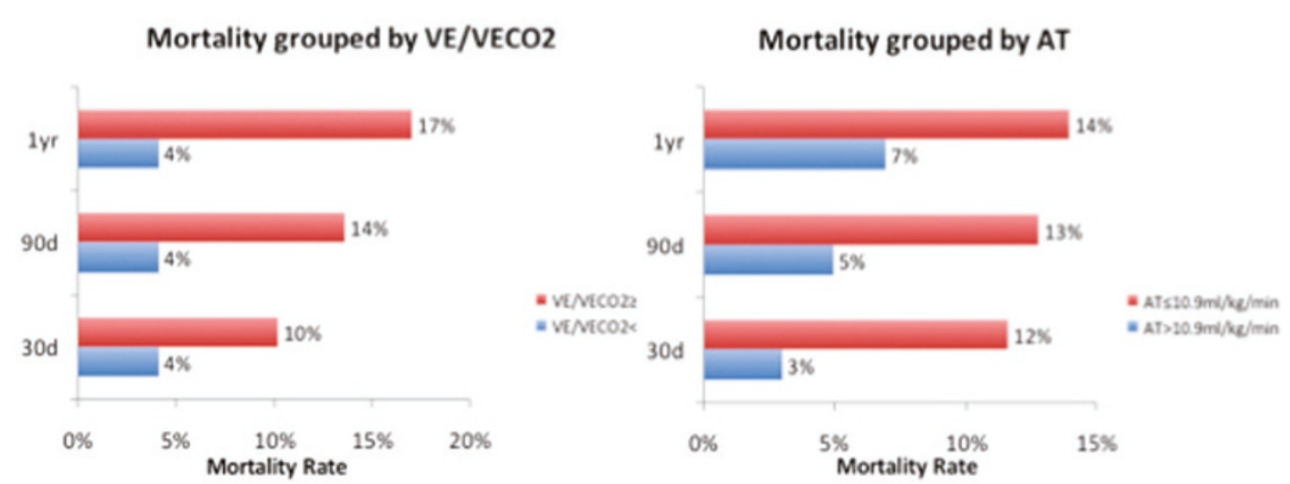

Figure 1 (abstract P467). Elective AAA mortality rates by group 
CPET also predicted 90-day and 1-year survival; however, VE/VCO was not a predictor of survival in this cohort.

Reference

1. Carlisle J, et al:: Mid-term survival after abdominal aortic aneurysm surgery predicted by cardiopulmonary exercise testing. Br J Surg 2007, 94:966-969.

P468

Perioperative evaluation of elective surgical patients: is it possible to plan ICU admission?

LM Mozzoni, FR Ruggeri, MN Nastasi

Ospedale Ceccarini Riccione, Italy

Critical Care 2012, 16(Suppl 1):P468 (doi: 10.1186/cc1 1075)

Introduction The aim of the study is to evaluate the possibility to predict ICU admission in elective surgical patients, studying the perioperative period variables.

Methods This is a prospective, nonintervention study concerning 207 patients, who have been operated on under elective conditions from January to October 2011. The group we studied was affected by thoracic $(n=78)$ or abdominal $(n=129)$ cancer. Mean age was 67.8 (SD 11.3; limits 24 to 91 ). ASA score III concerned 107 patients (51.7\%) and score II 98 patients (47.3\%). A senior anesthetist screened all patients before operation, assigning them to one of these three possible groups: G0 (patient who does not need ICU admission), G1 (patients who could need ICU admission), G2 (patients who definitely need ICU admission). Scheduling of patients into groups was made considering medical history, laboratory data, physical evaluation and type of surgery. Patients were studied from surgical intervention to discharge. All data were analyzed using IBM SPSS statistics v19 (SPSS Inc.), using adequate test and accepting $P<0.05$.

Results Sixty-six patients (31.9\% of all patients) were in G0, 70 (33.8\%) in G1 and 71 (34.3\%) in G2. The ASA score can distinguish patients in $\mathrm{G} 0$ and $\mathrm{G} 2$, but not in G1 $(P<0.05)$. The decision to schedule patients in a group arises mainly from the coexistence of both cardiovascular and respiratory diseases [1]. Ninety patients (43.5\%) entered the ICU; $30(42.8 \%)$ of these were in G1 and $34(47.9 \%)$ in G2; 26 (39.4\%) were in G0. Distribution in the three groups of ICU-admitted patients was similar $(P=\mathrm{NS})$ and there was no significant relationship between the ASA score (and its distribution in the three groups) and ICU admission $(P=\mathrm{NS})$. Patients admitted had undergone surgery of longer duration or had problems in the theater (low output syndrome, difficult weaning at the end of procedure, bleeding) or organizational problems $(P<0.05)$. ICU-admitted patients show a lower number of postoperative complications as arrythmias and wound infections $(P<0.05)$. Four patients died, all had been hospitalized in the ICU. The mortality rate was $1.9 \%$ ( $75 \%$ were in G2). Patients with complications requiring further surgery were $15(7.2 \%)$, seven of which had been hospitalized in the ICU.

Conclusion Preoperative evaluation does not appear to be a significant predictor for ICU admission, which is determined by intraoperative or organizational factors. The ICU admission reduces the incidence of postoperative complications; mortality is mainly due to the immediate perioperative period.

Reference

1. Rhodes A, et al: Intensive Care Med 2011, 37:1466-1472.

P469

Cardiac-specific biomarkers and life-threatening complications of off-pump versus on-pump coronary bypass surgery in Egyptian patients

H Elabd', A Alsherif2, T El Gohary², M Hagras², S Salah Eldin²

'Student Hospital, Cairo University, Giza, Egypt; '2Kasr Alaini Hospitals, Cairo,

Egypt

Critical Care 2012, 16(Suppl 1):P469 (doi: 10.1186/cc11076)

Introduction Coronary artery bypass grafting (CABG) has traditionally been performed with the use of cardiopulmonary bypass (ONCAB). This study aims to compare between on-pump and off-pump surgery concerning postoperative morbidity and mortality, and also to evaluate 6-month graft patency in Egyptian patients.
Methods This nonrandomized single-centre control trial was prospectively conducted on 65 patients who were subjected to coronary artery bypass surgery followed by stay in the Open Heart Intensive Care Center of the Police Authority Hospital, in the period from July 2009 to January 2010. Patients were divided into two groups; group A, 25 patients underwent surgery using cardiopulmonary bypass pump (on coronary artery bypass pump (ONCAB)); and group $B, 40$ patients underwent surgery without using cardiopulmonary bypass pump (off-pump coronary artery bypass (OPCAB)). All of the demographic, operative and postoperative data were prospectively collected and analyzed statistically. Six months later, the patients underwent coronary angiography.

Results There was no significant difference between both groups intraoperatively concerning arrhythmias, blood transfusion, and hemodynamic support. Off-pump patients had a significantly higher mean number of constructed grafts than in the ONCAB group (mean, $3.30 \pm 0.88$ vs. $2.84 \pm 0.80, P=0.02$ ). There were no significant differences between off-pump and on-pump regarding postoperative blood loss, blood transfusion, length of the ICU and the hospital stay, the ventilation time, the use of intraaortic balloon pump, renal complications, respiratory complications, and reopening. However, graft occlusion, Ml, raised cardiac enzymes, ventricular tachycardia, cardiogenic shock, and disturbed conscious level were significantly higher in the OPCAB group. The postoperative mortality rate was significantly higher in the OPCAB group than in the ONCAB group $(15 \%$ vs. $0 \%, P=0.046)$. Follow-up angiograms in 40 patients out of $65(61.5 \%)$ who underwent 124 grafts revealed that no significant difference between off-pump and on-pump groups regarding the overall rate of graft patency $(83.5 \%$ vs. $84.4 \%, P=0.84)$. No mortality was reported in both groups at 6-month follow-up.

Conclusion There was a higher incidence in postoperative complications and mortality in the off-pump procedure than the onpump. At 6-month follow-up, no significant differences between both techniques were found in graft patency and mortality. Hence, longerterm mortality from randomized trials of off-pump versus on-pump CABG is needed.

\section{Reference}

1. Shroyer AL, Grover FL, Hattler B, et al:: On-pump versus off-pump coronaryartery bypass surgery. N Eng/J Med 2009, 361:1827-1837.

P470

Aortic aneurysm disease versus aortic occlusive disease: differences in postoperative ICU requirements after open elective abdominal aortic surgery

J Bisgaard', HK Jørgensen'1, T Gilsaa', E Ronholm', P Toft ${ }^{2}$

'Littlebaelt Hospital Kolding, Denmark; ${ }^{2}$ Odense University Hospital, Odense, Denmark

Critical Care 2012, 16(Suppl 1):P470 (doi: 10.1186/cc11077)

Introduction Open elective abdominal aortic surgery is a highrisk procedure involving clamping of the aorta. Indications include abdominal aortic aneurysm (AAA) or aortic occlusive disease (AOD) causing lower limb ischaemia. These patients are often regarded as one entity in postoperative study settings. However, previous studies indicate that risk profiles, inflammatory activity, and haemodynamic capacity may differ between these groups $[1,2]$. The aim of this study was to evaluate postoperative ICU requirements after open elective abdominal aortic surgery, hypothesising that AAA patients had longer ICU stays and needed more mechanical ventilation or acute dialysis than did patients with AOD.

Methods This cohort study was based on prospectively registered data from the Danish National Vascular Registry and the Danish ICU Database between 1 January 2007 and 1 May 2010. The study population comprised all patients $(n=1293)$ undergoing open elective, primary aorto-iliac bypass, or aorto-femoral bypass procedures $(n=363)$ or abdominal aortic aneurysm repair $(n=930)$ in the eight hospitals performing these procedures in Denmark. The primary endpoints were: ICU stay $>24$ hours, mechanical ventilation, and acute dialysis. Results Patients in the AAA group were older $(70 \pm 7$ vs. $62 \pm 9$ years, $P<0.001)$, predominantly males ( 80 vs. $49 \%, P<0.001)$, with a higher prevalence of preoperative cardiac co-morbidity (34 vs. $24 \%, P=0.001$ ), 
and malignant disease ( 2.7 vs. $0.6 \%, P=0.02$ ). In contrast, AOD patients had a higher prevalence of smoking ( 95 vs. $86 \%, P<0.001$ ), and diabetes (16 vs. $9 \%, P<0.001)$. AAA patients had larger intraoperative blood losses $(1,610(1,000$ to 2,500$)$ vs. $1,200(750$ to 1,800$) \mathrm{ml}, P<0.001)$, but duration of surgery was shorter (161 (130 to 205) vs. 194 (160 to 240) minutes, $P<0.001)$. Postoperatively, more AAA patients had ICU stays $>24$ hours ( 62 vs. $45 \%, P<0.001)$, tended to need mechanical ventilation more often ( 16 vs. $12 \%, P=0.08$ ), and more needed acute dialysis ( 3.8 vs. $0.9 \%, P<0.03$ ).

Conclusion Compared to the AOD group, more AAA patients had ICU stays $>24$ hours and more often needed acute dialysis. Distinguishing between these two diseases may be useful in planning and distribution of ICU resources. Furthermore, considering these two patient groups as different pathological entities may be advised in future studies.

References

1. Shteinberg D, et al.: Eur J Vasc Endovasc Surg 2000, 20:462-465.

2. Johnston WE, et al:: Anesthesiology 1987, 66:386-389.

P471

High postoperative blood levels of macrophage migration inhibitory factor are associated with less organ dysfunction in patients after cardiac surgery

C Stoppe', G Grieb', D Simons', R Rossaint', J Bernhagen', S Rex² 'University Hospital of the RWTH, Aachen, Germany; ${ }^{2}$ University Hospital

Gasthuisberg, KU Leuven, Belgium

Critical Care 2012, 16(Suppl 1):P471 (doi: 10.1186/cc11078)

Introduction Macrophage migration inhibitory factor (MIF) is a structurally unique inflammatory cytokine [1] that exerts protective effects during ischemia and reperfusion [2]. We hypothesized that elevated MIF levels in the early postoperative time course might be inversely associated with postoperative organ dysfunction as assessed by SAPS II and SOFA score in patients after cardiac surgery.

Methods Fifty-two cardiac surgical patients (mean age $( \pm$ SD) $67 \pm 10$ years; EuroSCORE: 7 (2 to 11)) were enrolled in this monocenter, prospective, observational study. Serum levels of MIF and clinical data were obtained after induction of anesthesia, at admission to the ICU, 4 hours thereafter and at the first and second postoperative day (POD). Patient outcome was assessed using the SAPS II at POD1 and SOFA score for the first 3 days of the eventual ICU stay.

Results MIF_AUC, the computed area under the curve of MIF serum levels from admission until POD1, was inversely correlated with SAPS II and SOFA score on POD1 (Table 1). MIF at admission $(r=0.296$; $P=0.041)$ and MIF at 4 hours $(r=0.367 ; P=0.012)$ correlated inversely with the $\mathrm{paO}_{2} / \mathrm{FiO}_{2}$ ratios at POD1. Moreover, postoperative MIF values were inversely correlated with SAPS II $(r=0.528 ; P=0.044)$ and SOFA scores during the early postoperative stay (Table 1). In addition, MIF values on POD1 were related to the calculated Cardiac Power Index $(r=0.420 ; P=0.009)$.

Table 1 (abstract P471)

\begin{tabular}{lccc}
\hline MIF level & SOFA 1.POD & SOFA 2. POD & SOFA 3. POD \\
\hline ICU admission & $r=-0.2 ; P=0.18$ & $r=-0.4 ; P=0.11$ & $r=-0.6 ; P=0.05$ \\
4 hours later & $r=-0.4 ; P=0.40$ & $r=-0.5 ; P=0.05$ & $r=-0.8 ; P=0.01$ \\
MIF_AUC & $r=-0.4 ; P=0.01$ & $r=-0.2 ; P=0.55$ & $r=-0.4 ; P=0.19$ \\
1.POD & $r=-0.3 ; P=0.08$ & $r=-0.6 ; P=0.03$ & $r=-0.7 ; P=0.02$
\end{tabular}

Conclusion Elevated postoperative MIF levels are inversely correlated with organ dysfunction in patients after cardiac surgery.

References

1. Calandra T, et al:: Macrophage migration inhibitory factor: a regulator of innate immunity. Nat Rev Immunol 2003, 3:791-800

2. Koga $\mathrm{K}$, et al: Macrophage migration inhibitory factor provides cardioprotection during ischemia/reperfusion by reducing oxidative stress. Antioxid Redox Signal 2011, 14:1191-1202.
P472

Predictors of prolonged mechanical ventilation after heart transplantation

M Turker, P Zeyneloglu, A Pirat, A Sezgin, G Arslan

Baskent University, Ankara, Turkey

Critical Care 2012, 16(Suppl 1):P472 (doi: 10.1186/cc11079)

Introduction Several studies have reported that prolonged mechanical ventilation is associated with high mortality and morbidity rates, length of hospital stay, and costs after coronary artery and valvular surgeries. However, no study has focused on the incidence and risk factors of prolonged mechanical ventilation after heart transplantation. The aim of this study was to determine the incidence and predictors of prolonged mechanical ventilation after heart transplantation.

Methods We retrospectively analyzed the records of 38 out of 45 patients who underwent heart transplantation from February 2003 to November 2010 at our center. Patients under 12 years of age and those who died before extubation were excluded. We defined prolonged mechanical ventilation as mechanical ventilation longer than 36 hours. Preoperative, intraoperative, and postoperative variables were collected.

Results The mean age of the patients ( $71 \%$ male) was $31.5 \pm 16.8$ years and the incidence of prolonged mechanical ventilation was $40 \%$. Compared with patients who did not require prolonged mechanical ventilation, those who did had significantly lower preoperative hemoglobin levels $(12.0 \pm 1.5$ vs. $13.7 \pm 2.4 \mathrm{mg} / \mathrm{dl}, P=0.03)$, higher intraoperative lactate levels (7.14 \pm 4.13 vs. $3.5 \pm 1.82 \mathrm{mmol} / \mathrm{l}$, $P=0.006)$, higher postoperative day 1 serum creatinine levels $(2.2 \pm 0.9$ vs. $1.2 \pm 0.7 \mathrm{mg} / \mathrm{dl}, P=0.002)$, and longer cardiopulmonary bypass times $(143.0 \pm 24.2$ vs. $122.8 \pm 29.1$ minutes, $P=0.005)$. Binary logistic regression revealed that the postoperative day 1 serum creatinine level was an independent risk factor for prolonged mechanical ventilation after heart transplantation (OR: $5.109 ; 95 \% \mathrm{Cl}: 1.362$ to $19.159, P=0.016$ ). Length of hospital stay was significantly longer in patients with PMV than those who did not require prolonged mechanical ventilation ( $36.4 \pm 30.4$ vs. $21.8 \pm 12.7, P=0.049$ ). The respective mortality rates for patients with prolonged mechanical ventilation and those without prolonged mechanical ventilation were $60 \%$ versus $40 \%, P=0.15$.

Conclusion Prolonged mechanical ventilation occurred in $40 \%$ of our patients after heart transplantation. A higher creatinine level during the first 24 hours after the surgery was associated with prolonged mechanical ventilation in this study.

Reference

1. Cislaghi F, et al:: Minerva Anestesiol 2007, 73:615-621.

\section{P473}

Atrial fibrillation following major noncardiac thoracic surgery: significance and impact on morbidity

H Michalopoulou, PS Stamatis, M Michaloliakou, N Baltagiannis, D Stamatis

'Metaxa' Hospital, Athens, Greece

Critical Care 2012, 16(Suppl 1):P473 (doi: 10.1186/cc11080)

Introduction Atrial fibrillation (AF) is a common complication after noncardiac thoracic surgery. Its impact on overall mortality has not yet been fully assessed and few data are available on the effects of the noncardiac post-thoracotomy AF on clinical outcomes.

Methods From July 2006 to July 2011, 226 consecutive patients undergoing lung resection for lung cancer were studied retrospectively. Preoperative data and serial electrocardiograms were evaluated. Hypertension, dyslipidaemia, diabetes mellitus, smoking and advanced age ( $>75$ years) were considered as risk factors. Patients $(n=97)$ who had structural heart disease or $\geq 2$ risk factors were considered a highrisk group whereas those with $<2$ risk factors constituted the low-risk group.

Results Thirty-two patients (14.16\%) experienced new-onset postoperative AF. The high-risk group had a 58\% incidence of AF compared with $23 \%$ in the low-risk group $(P<0.001)$. Moreover, following $\beta$-blocker administration, more of the high-risk group required antiarrhythmic treatment with amiodarone than did the low-risk group ( $67 \%$ vs. $35 \%$ respectively, $P=0.02$ ). Patients who developed AF had 
a significantly longer hospital stay $(P<0.01)$. The 30 -day mortality rate was significantly higher in the high-risk group $(11 \%$ vs. $2 \% ; P=0.03)$ but AF was not an independent risk factor for death. In the multivariate analysis, major resection (pneumonectomy) and advanced age were identified as independent risk factors for the development of postoperative $\operatorname{AF}(P=0.004$ and $P=0.008$ respectively).

Conclusion Atrial fibrillation occurrence after lung resection does not independently affect the short-term mortality but is associated with a prolonged length of hospital stay.

P474

Oxygen delivery index during goal-directed therapy predicts complications and hospital length of stay in patients undergoing high-risk surgery

M Cecconi, N Arulkumaran, R Suleman, D Shearn, M Geisen, J Mellinghoff, D Dawson, J Ball, M Hamilton, M Grounds, A Rhodes

St George's Hospital, London, UK

Critical Care 2012, 16(Suppl 1):P474 (doi: 10.1186/cc11081)

Introduction The aim of this study was to evaluate the efficacy of a goal-directed therapy (GDT) protocol designed to augment the oxygen delivery index $\left(\mathrm{DO}_{2} \mathrm{I}\right)$ and to assess the relationship between $\mathrm{DO}_{2} \mathrm{I}$ measurements and postoperative complications and length of stay.

Methods A single-centre retrospective cohort study assessing the data obtained during an 8-hour post-operative GDT protocol in consecutive major surgical patients admitted to the ICU.

Results Thirty-seven patients were included. The median DO I increased over the 8-hour protocol from a baseline level of $407 \mathrm{ml} / \mathrm{minute} / \mathrm{m}^{2}$ to a maximum of $537 \mathrm{ml} /$ minute $/ \mathrm{m}^{2}(P<0.0001)$ (Figure 1). Twenty-one (57\%) patients developed a postoperative complication. Patients who developed zero or one complication had a higher maximum oxygen delivery index $\mathrm{DO}_{2}$ I than patients who had more than one complication (602 vs. $477 \mathrm{ml} / \mathrm{minute}^{2} / \mathrm{m}^{2}, P=0.018$ ) (Table 1). The proportion of patients with a length of stay greater than 2 weeks was less in patients who achieved a $\mathrm{DO}_{2}$ l of at least $600 \mathrm{ml} / \mathrm{minute} / \mathrm{m}^{2}(P=0.035)$.

Conclusion Postoperative GDT was able to increase $\mathrm{DO}_{2} \mathrm{I}$ in the postoperative period. Patients who achieved a $\mathrm{DO}_{2} \mathrm{l}$ of $600 \mathrm{ml} /$ minute/ $\mathrm{m}^{2}$ were less likely to suffer postoperative complications and have a significantly reduced length of hospital stay.

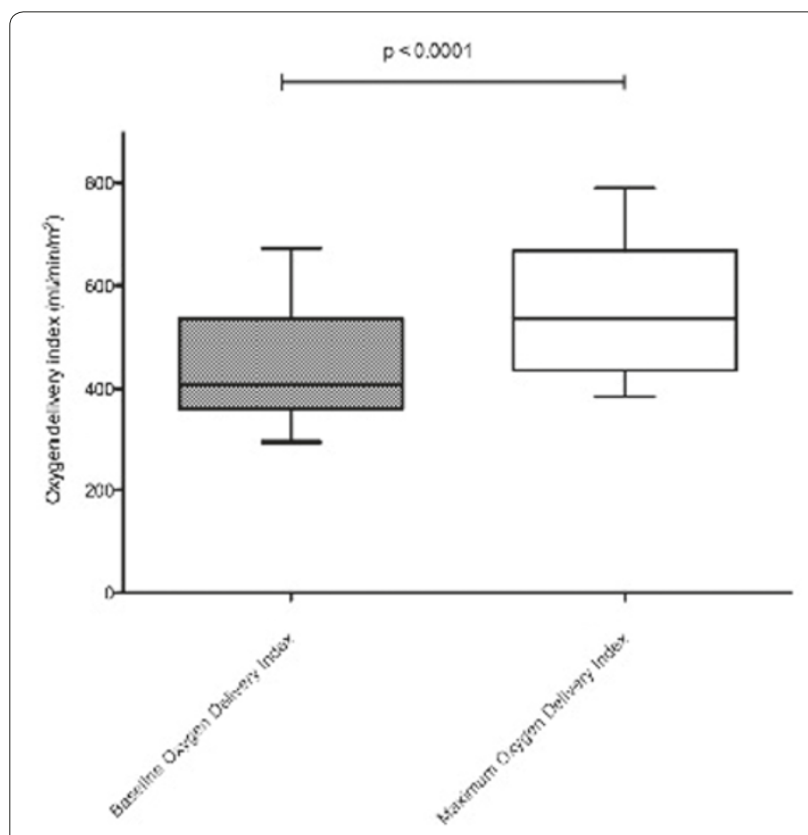

Figure 1 (abstract P474). Increase in $\mathrm{DO}_{2}$ I from baseline to maximum over the 8-hour protocol.
Table 1 (abstract P474). Postoperative complications by achievement of an oxygen delivery index of 600

\begin{tabular}{lccc}
\hline & DO $_{\mathbf{2}} \mathbf{I}>600$ & DO $_{2} \mathbf{I}<600$ & $P$ value \\
\hline Number of patients & $16(43 \%)$ & $21(57 \%)$ & - \\
Complications & $13(29 \%)$ & $32(71 \%)$ & $P=0.003$ \\
Mortality & $0(0 \%)$ & $4(100 \%)$ & $P=0.12$ \\
\hline
\end{tabular}

P475

Transfer delays in patients referred for neurosurgical intervention with traumatic brain injury

L Smith', B Jordan², J Paddle

${ }^{1}$ Royal Cornwall Hospital NHS Trust, Truro, UK; ${ }^{2}$ Derriford Hospital, Plymouth, UK

Critical Care 2012, 16(Suppl 1):P475 (doi: 10.1186/cc11082)

Introduction National guidance for patients presenting to the emergency department (ED) with a traumatic head injury advises that head computed tomography (CT) should be performed and reported within 1 hour [1]. The operative intervention or injury to knife time should be within 4 hours [2]. With more than $50 \%$ of patients requiring neurosurgical intervention in the UK taken to hospitals without onsite neurosurgical services [3], secondary transfer is necessary prior to definitive intervention. Are we achieving timely transfers in rural England?

Methods The Royal Cornwall Hospital is a district general hospital serving a population of 300,000 . The regional neurosurgical unit is $100 \mathrm{~km}$ away. All patients undergoing transfer to the neurosurgical unit during 2009 were identified. A notes review was undertaken of all these patients transferred to the care of neurosurgeons. The operative logs were also reviewed. Time lines were created of their care from ambulance call to neurosurgical intervention.

Results Ten patients in total were transferred for neurosurgical intervention. Two of these patients required two transfers as they were initially seen in satellite minor injury units. No patient had CT within 1 hour of arriving in the ED. The median time was 2 hours 56 minutes. The CT report was available at a median of 3 hours 17 minutes. None of these patients arrived in the tertiary referral centre within 4 hours of their injury. The fastest time to intervention was 8 hours 29 minutes, median 22 hours 59 minutes after injury.

Conclusion We are not meeting targets for $\mathrm{CT}$ head acquisition and transfer for neurosurgical intervention. Prompt transfer of a trauma patient from a rural district general hospital in the UK to a tertiary referral centre for neurosurgical intervention is a multifactorial problem. The introduction of trauma centres and of protocols for direct admission to tertiary centres by paramedics may reduce the delays that our audit has highlighted.

References

1. Head Injury Triage: Assessment, Investigation and Early Management of Head Injury in Infants, Children and Adults, Methods Evidence and Guidance. Commissioned by the National Institute for Health and Clinical Excellence. [http://guidance.nice.org.uk/CG56/NICEGuidance/pdf/English]

2. Better Care for the Severely Injured. Joint Report. Royal College of Surgeons of England and British Orthopaedic Association. [http://www.rcseng.ac.uk/ publications/docs/severely_injured.html]

3. Trauma: Who Cares? Report. National Confidential Enquiry into Patient Outcome and Death. [http://www.ncepod.org.uk/2007report2/Downloads/ SIP_report.pdf]

P476

Performances of ventilator at simulated altitude

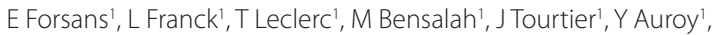

C Bourrilhon²

${ }^{1}$ HIA Val-de-Grâce, Paris, France; ${ }^{2}$ Institut de Recherche Biomédicale des Armées, Brétigny sur Orges, France

Critical Care 2012, 16(Suppl 1):P476 (doi: 10.1186/cc11083)

Introduction We have assessed the ability of three ventilators to deliver to a normal lung model a set tidal volume $\left(V_{t}\right)$ at different simulated cabin altitudes. We studied the performance of the LTV-1200 (Viasys Healthcare, USA), the Elisée 350 (Resmed, Australia) and the Medumat transport (Weinmann, Germany). 


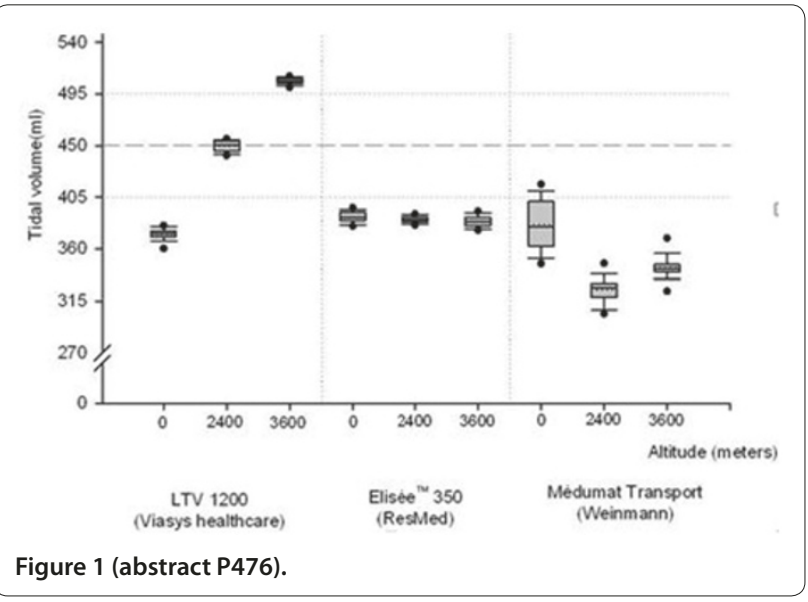

Methods We used a decompression chamber to mimic the hypobaric environment at a range of simulated cabin altitudes of 2,438 and $3,657 \mathrm{~m}(8,000$ and 12,000 feet). Ventilators were tested with a set fraction of inspired oxygen of $50 \%$ and $V_{t}$ set at 450 . Respiratory rate was 12 breaths/minute. Comparisons of preset to actual measured values were accomplished using a $t$ test for each altitude. The protocol included 36 measurements for each $V$ set at each simulated altitude. $A$ significant difference was defined by $P<0.05$.

Results Figure 1 summarizes the data. Comparisons of actual delivered $V_{t}$ in altitude and set $V_{t}$ demonstrated a significant difference for the three ventilators.

Conclusion The LTV-1200 showed a very significant increase in V delivered with increasing altitude (suggesting a lack of efficacy of altimetric correction in hypobaric conditions), whereas the Elisée 350 and Medumat transport delivered respectively a stable and a rather stable $V_{\text {. }}$.

\section{P477}

Impact of H1N1 influenza on critical care and dependent services in Wales during winter 2010/2011

A Jones, D Hope, E Farley-Hill, J Parry-Jones

Welsh Critical Care Networks, Newport, UK

Critical Care 2012, 16(Suppl 1):P477 (doi: 10.1186/cc1 1084)

Introduction Influenza H1N1 admissions to critical care from December 2010 to January 2011 had a significant impact on intensive care bed occupancy across Wales. Wales is relatively underprovided in critical care capacity and as a consequence the surge in admissions had a significant impact on both critical care and critical care dependent hospital services.

Methods Data were collected prospectively through the Critical Care Minimum Data Set: the number of critical care admissions with confirmed or highly suspected influenza, co-morbidities, mortality rate, level 3 bed day occupancy, number and mode of advanced respiratory support days, numbers of nonclinical and clinical transfers, and numbers of cancelled operations requiring critical care.

Results In a 10-week period 128 patients in Wales required critical care with influenza. A total of 1,692 level 3 bed days were required. There are 95 potential level 3 beds across Wales per day. Therefore $>25 \%$ of level 3 beds over 10 weeks were occupied by influenza patients. Fifty percent of patients had significant comorbidities; pregnancy, COPD, morbid obesity, immunocompromise (Figure 1). The overall mortality rate for all affected critical care patients was $23.4 \%$. Mortality was $25 \%$ in those with comorbidities and $22 \%$ in those without. The overall mortality rate for all affected patients treated in Wales during the 2009/10 influenza pandemic was $9.6 \%$. The UK has fewer critical care beds per head of population than comparable nations, and Wales fewer still so critical care in Wales is more vulnerable to surges in admissions. This was apparent in the peak in nonclinical critical care transfers seen during this period, performed due to units exceeding their capacity, and in an increase in cancellations of elective surgery requiring critical care.

Conclusion The shortage of critical care capacity in Wales is made more apparent during times of increased critical care requirement such as the influenza in the winter $2010 / 2011$. Hospital services are increasingly dependent on critical care, and government and health boards need to provide targeted increases in critical care bed provision to match those levels in other similar nations to mitigate the effect on critical care and dependent services due to surges in demand.

\section{P478}

Effects of levels of clinical supervision during simulated ICU scenarios on resident learning and patient care: a qualitative study D Piquette', M Mylopoulos ${ }^{2}$, VR LeBlanc ${ }^{3}$

'Sunnybrook Health Sciences Centre, Toronto, Canada; ${ }^{2}$ SickKids Hospital,

Toronto, Canada; ${ }^{3}$ Wilson Centre, Toronto, Canada

Critical Care 2012, 16(Suppl 1):P478 (doi: 10.1186/cc11085)

Introduction Closer clinical supervision of residents is often perceived as a double-edged sword, improving patient safety but limiting resident participation in patient care. There has been little empirical research on the educational effects of closer supervision. We examined the impact of levels of clinical supervision on clinical learning and patient care during acute simulated resuscitation.

Methods Fifty-four ICU residents (PGY1 to 4) were randomly assigned to complete a simulated ICU scenario in one of three levels of

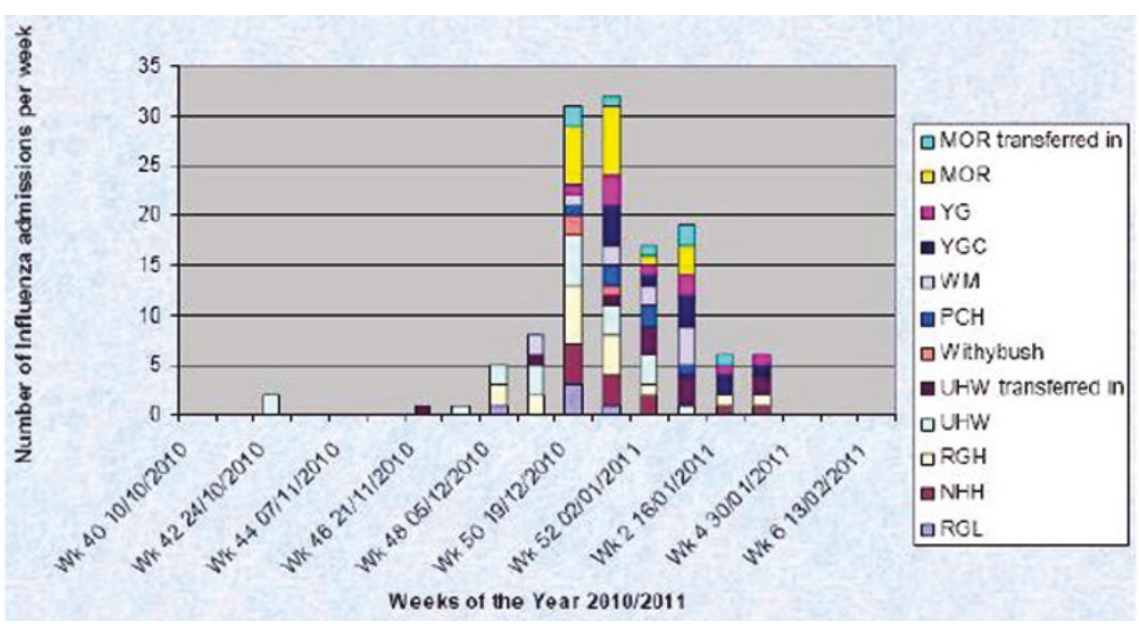

Figure 1 (abstract P477). Influenza admissions into critical care in winter 2010/2011. 
supervision (physical proximity of supervising ICU fellow: distant, immediately available, direct). In-person and telephone interactions between participants were recorded and transcribed. We conducted an inductive thematic analysis of anonymized transcripts using constant comparison within and between scenarios. Distributed cognition theory was used as a framework to guide analysis.

Results Both distant and direct levels of supervision resulted in variable involvement of residents in patient care. A shift of control over patient care from residents to fellows often occurred regardless of the physical distance of the fellow. Direct supervision did not always result in decreased resident contributions. Fellows were found to facilitate more elaborated cognitive contributions from the residents during direct supervision. In addition, practicing in the presence of a supervisor was more likely to lead to timely feedback. However, a presence at the bedside allowed fellows to influence the nature of resident involvement by delegating specific tasks such as technical procedures. During distant supervision, fellows had to use residents as proxies to obtain information about patients and to deliver care, with potentially serious consequences: when residents' interpretations of the clinical information were problematic, the quality of fellows' clinical decisions was negatively affected. Higher cognitive work required of fellows during distant supervision appeared to limit their ability to invest cognitive resources in teaching.

Conclusion Level of clinical supervision was not the main determinant of resident engagement in patient care. Both distantly and directly supervised scenarios presented learning opportunities for residents. Given the observed negative effects of distant supervision on patient care, strategies to optimize unique learning opportunities offered by direct supervision should be investigated.

\section{P479}

Virtual reality and live scenario simulation: options for training medical students in mass casualty incident triage

PL Ingrassia, L Ragazzoni, L Carenzo, FL Barra, D Colombo, G Gugliotta,

F Della Corte

CRIMEDIM Research Center in Disaster and Emergency Medicine, Novara, Italy Critical Care 2012, 16(Suppl 1):P479 (doi: 10.1186/cc11086)

Introduction Multicasualty triage is the process of establishing the priority of care among casualties in disaster management. Recent mass casualty incidents $(\mathrm{MCl})$ revealed that health personnel are unfamiliar with the triage protocols. The objective of this study is to compare the relative impact of two simulation-based methods for training medical students in mass casualty triage using the Simple Triage and Rapid Treatment (START) algorithm.

Methods A prospective randomized controlled longitudinal study. Medical students enrolled in the emergency medicine course were randomized into two groups (A and B). On day 1, group A students were exposed to a virtual reality (VR) scenario and group $B$ students were exposed to a live scenario (LS), both exercises aiming at triaging 10 victims in a limited period of time (30 seconds/victim). On day 2 all students attended a 2-hour lecture about medical disaster management and START. On day 3 group A and B students were exposed to a LS and to a VR scenario respectively. The vital signs and clinical condition of the 10 victims were identical in the two scenarios. Ability of the groups to manage a simulated triage scenario was then compared (times and triage accuracy).

Results Groups A and B were composed of 25 and 28 students respectively. During day 1 group A LS triage accuracy was $58 \%$, while the average time to assess all patients was 4 minutes 28 seconds. The group B VR scenario triage accuracy was $52 \%$, while the average time to complete the assessment was 5 minutes 18 seconds. During day 3 the triage accuracy for group A VR simulation was $92 \%$, while the average time was 3 minutes 53 seconds. Group B triage accuracy during the LS was $84 \%$, with an average time of 3 minutes 25 seconds. Triage scores improved significantly during day $3(P<0.001)$ in the two groups. The time to complete each scenario decreased significantly from day 1 to day 3.

Conclusion The study demonstrates that the training course generates significant improvement in triage accuracy and speed. It also reveals that VR simulation compared to live exercises has equivalent results in prompting critical decisions in mass casualty drills. In the beginning the average time to complete the VR scenario was higher than the LS. This could be due to the fact that on day 1 very detailed VR victims created a higher challenge for untaught students. However, the higher triage accuracy recorded at the end of day 3 in VR could be explained by a lower stress level compared to the LS, which could be creating a more stressful environment in taught students.

\section{P480}

Utilization of iPad in the system of emergency demand and acceptance

K Yamada', Y Sakamoto', Y Enjiyouji²

'Saga University, Saga, Japan;' 'Saga Prefectural Government, Saga, Japan Critical Care 2012, 16(Suppl 1):P480 (doi: 10.1186/cc11087)

Introduction This study reports that the transportation time by ambulance was shorter following the introduction of iPad (Apple, Inc.) to the current system of emergency demand and acceptance in Saga Prefecture, Japan. There were about over 5,000,000 ambulance dispatches in Japan, and the time for transportation is increasing (the national average: 36.1 minutes) [1]. The administration has made various efforts nationwide that did not achieve any positive results. Although the information system of medical institutions and the emergency medical service ( 99 Saga Net) was established in 2003 in Saga, it has been underutilized. The Saga prefectural government renewed the previous system as the real-time system of emergency demand and acceptance for the first time in Japan in April 2011.

Methods Cloud computing has provided new system to facilitate Internet access from ambulances. In addition, iPads were put into all ambulances (about 55) and emergency medical technicians can get the picture of acceptable hospitals in real time. Emergency personnel who arrive on the scene select the patient's symptoms with an iPad, and this new system displays an up-to-date list of acceptable hospitals. The data that the emergency personnel entered into the system from the iPad are uploaded to 99 Saga Net immediately. Therefore, both the emergency personnel and medical staff in the hospital share the information of where the emergency occurred, the transportation and the medical institutes to which patients were transferred in real time. Results The transportation time by ambulance was shorter for the first time since statistics were first kept in 1999, the mean time was 33.7 minutes in 2009 and 33.2 minutes in April 2011. Furthermore, the new system is expected to reduce the operational costs by $40,000,000$ yen a year. The data on the transportation time by ambulance are continually stored in the system and analyses are continuing.

Conclusion The introduction of iPad to the new 99 Saga Net has three beneficial points. First, the utilization of information and communication technology is useful for a realistic emergency medical setting. Second, the situation of a realistic emergency medical setting is visualized in real time. Finally, both the emergency personnel and the medical staff in the hospital share the information in an emergency medical setting by eliminating vertically divided administrative functions. Medical personnel will work with local governments in the future to analyze the data from this new system.

Reference

1. Fire and Disaster Management Agency: White Paper on Fire and Disaster (2010) [in Japanese]. [http://www.fdma.go.jp/html/hakusho/h22/h22/html/2-4-5_4.html]

\section{P481}

Mass evacuation of victims from emergency areas by medical modules aboard the aircraft of EMERCOM of Russia

I Yakirevich, A Popov

EMERCOM of Russia, Zhukovsky, Moscow Region, Russia Critical Care 2012, 16(Suppl 1):P481 (doi: 10.1186/cc11088)

Introduction During elimination of medical consequences of various emergencies the issues concerning victims' mass evacuation to a specialized hospital base are constantly brought up. The physicians of the Central Airmobile Rescue Service of EMERCOM of Russia and the specialists of Kazan Helicopter Plant 'Zarechye' developed two types of modules. The Medical Airplane Module (MMS) is used for medical 
evacuation of four victims aboard Ilyushin 76 aircraft. The Medical Helicopter Module (MMV) is used for medical evacuation of two victims aboard an MI 8 helicopter. MMS and MMV advantages are: mobility - the possibility of installation in various aircraft cabins types; and versatility - the possibility of any required equipment installation for the treatment of victims with various trauma severity, safe fixation of medical equipment straight on the module, equipment operation offline as well as using the aircraft power supply network.

Methods From December 2008 until now 28 medical evacuations were carried out using MMS aboard Iluyshin 76 aircraft: traffic accident victims, terrorism act victims and manmade catastrophes. In total, 198 patients were evacuated (including 12 children), 55 victims with artificial lung ventilation (ALV). Medical evacuation of severely injured children and adults from regional hospitals to Moscow specialized hospitals in order to provide efficient and modern medical aid was carried out using MMV. In total, 27 patients were evacuated (including five children), five patients with ALV. The majority of victims were in severe and extremely severe conditions with associated multisystem trauma. Closed craniocerebral injury was observed in $75 \%$ of victims with mass affection of locomotor apparatus, mine and explosion trauma, gunshot wounds, burn shock and burn disease. Constant monitoring, oxygen therapy, ALV, analgesia and sedation, intensive and anti-shock care as well as wound dressing were carried out in flight. The victims' general condition was evaluated according to the Glasgow Coma Scale, APACHE II and SOFA scales.

Results MMS and MMV application in case of mass evacuation in flight ensures spare victims' transportation, total monitoring and treatment continuity. It enables one to carry out anesthetic and resuscitation treatment, intensive care, monitoring and treatment of all the victims. Conclusion The quality of mass medical evacuation of extremely injured victims has considerably improved and the time of transportation from emergency area to specialized hospitals to render them efficient medical aid has reduced.

\section{P482}

Reliability and validity of an Italian four-level emergency triage system N Parenti', G Rastelli ${ }^{2}$, C Ferri' ${ }^{2}$, V Serventi ${ }^{1}$, R Lazzari ${ }^{3}$, L Sarli' ${ }^{1}$ 'University of Parma, Bologna, Italy; ${ }^{2}$ Ospedale Fidenza, Italy; 3 University of Modena, Italy

Critical Care 2012, 16(Suppl 1):P482 (doi: 10.1186/cc11089)

Introduction The goal of this study is to assess the reliability and validity of a four-level emergency triage system (Urgency Category (UC) $1=$ immediate response; UC 2, 3 and 4 assessment within 20,60 and 120 minutes respectively) used in an Italian large urban hospital with 60,000 emergency department (ED) visits annually.

Methods Three triage nurses, using our triage system, independently assigned, at the same time, triage scores to each patient admitted to the ED from June to August 2011. We collected demographic and clinical characteristics, nurse triage category, resources used for each triage code (for example, laboratory tests, EKG, radiographs, procedures), admission status and site, nurse triage forms that included presenting complaint, vital signs, and pain score. For each scenario, the most frequent UC (the mode) has been considered as true triage. Weighted kappa (K) was used to calculate inter-rater reliability. Validity was evaluated by studying the relationships between the triage category assigned by the nurses and resource consumption.

Results A total of 315 patients admitted to the ED were included in the study randomly ( 35 were excluded for incomplete data). Mean age was 47 years. Five patients were admitted to the ICU, 48 to nonintensive units. Trauma was the most frequent symptom at triage (44\%). The mean time of rating was 2 minutes. The UCs assigned were: $14 \%$ with UC $4,60 \%$ UC $3,25.7 \%$ UC $2,0.3 \%$ UC 1 . We found $2 / 315(0.6 \%)$ cases with a marked discordance (2 or more points), 69/315 (21.9\%) cases with partial agreement $(2 / 3)$ and $244 / 315(77.5 \%)$ cases with a complete agreement $(5 / 5)$ among nurses who used the triage method. Interrater reliability among the three nurses was $\mathrm{K}=0.71$ (Cl: 0.58 to $0.84)$. Hospital admission by our triage system was as follows: $1(100 \%)$, $2(30 \%), 3(12 \%), 4(2 \%)$. The mean of resources used for each triage code was: 4.5 (SD 2.2) for UC 2; 3.2 (SD = 1.67) for UC 3; 1.89 (SD 0.84) for UC 4.
Conclusion Our triage system shows a good interrater reliability and validity in predicting resource consumption. To our knowledge, this is the first prospective Italian study that tests the relationships between the triage category assigned by the nurses (using a four-level triage method) and resource consumption.

\section{P483}

Coordination of emergency resources after Lorca's earthquakes

L Escobar', A Ferrández ${ }^{2}$, J Jimenez', A Peláez', A Corbatón ${ }^{3}$, R Alvaro ${ }^{4}$

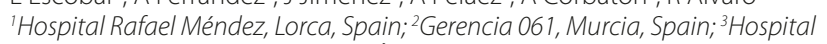
Clínico San Carlos, Madrid, Spain; ${ }^{4}$ Área ll Servicio Cantabro de Salud,

Santander, Spain

Critical Care 2012, 16(Suppl 1):P483 (doi: 10.1186/cc11090)

Introduction This work's purpose is to describe the coordination of different medical resources after Lorca's 2011 earthquakes. They caused 11 deaths, including two pregnant women and their babies, many injured, moderate or severe damage to $80 \%$ of the buildings, and more than 30,000 people without shelter.

Methods A descriptive study of the files of Murcia's Emergency Coordination Center (ECC) on the activation of resources after the earthquakes.

Results Time 17:06 hours: first call. Local resources and city emergency plan are activated. Four medical teams (UME) are pre-activated. 18:49 hours: incoming calls alert of buildings crumbling, dead among the rubble, and hundreds of injured. 18:55 hours: seven UME from five cities are sent to Lorca. 19:00 hours: telephone communications collapse. The ECC uses its internal network. An Advanced Command Point (ACP) is established with a field hospital. 19:10 hours: Rafael Mendez Hospital (225 patients) has to be evacuated. Medical personnel of the hospital, private ambulances and UMEs begin the evacuation. The emergency service of the hospital continues to be operative in the building until evacuation is completed and in a field hospital later. 19:20 hours: the Military Emergencies Unit is required for activation. 19:30 hours: the military and emergency services field hospitals are sent to Lorca. 19:50 hours: Virgen del Alcázar Hospital has to be evacuated (145 patients). 20:25 hours: at the ACP field hospital of the Red Cross, Civil Protection and Emergency Services are being set. 20:30 hours: 11 hospitals in six provinces are contacted to relocate evacuated patients. 20:40 hours: all buildings in Lorca are have been evacuated. Thirty thousand people need shelter. Ten camps with tents are set throughout Lorca by the Red Cross, Emergency Services and Civil Protection to give shelter to 16,000 people. See Figure 1.

Conclusion Coordination of the different medical and emergency services by the ECC made possible correct use of resources and fast attention to the population that minimized the effects of the catastrophe.

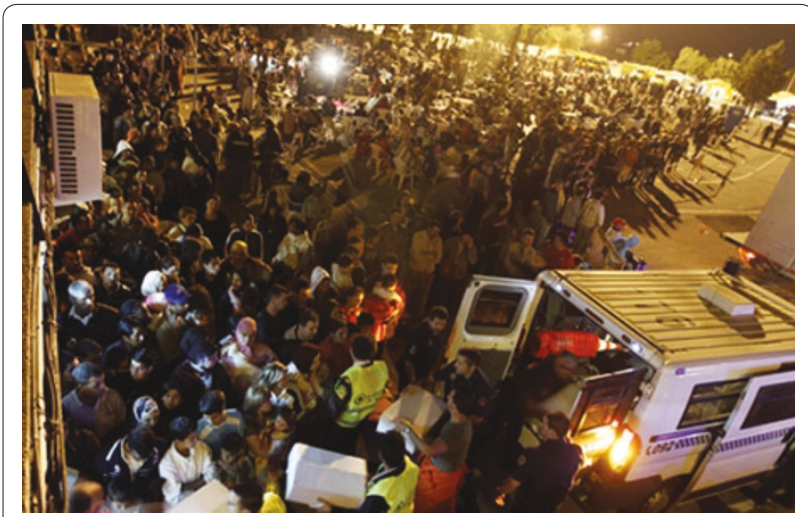

Figure 1 (abstract P483). Thirty thousand people need shelter after the earthquakes. 
P484

Lightning injuries in a lightning city: a district hospital experience in Singapore

Y Mok, P Tan, R Jagadesan

Changi General Hospital, Singapore

Critical Care 2012, 16(Suppl 1):P484 (doi: 10.1186/cc1 1091)

Introduction Tropical Singapore's meterological profile makes it one of the world's lightning capitals. This study aims to assess the profile of the at-risk patient, and possibly identify factors predicting the length of hospital stay in patients with lightning injuries over a period of 11 years. Methods This is an 11-year retrospective study of patients who were admitted to Changi General Hospital, the only hospital located in eastern Singapore, from 2000 to 2011 with the diagnosis of lightning injuries.

Results There were a total of 27 subjects, with 25 (95.6\%) males and two $(7.4 \%)$ females in the sample. Their age ranged from 17 to 62 years; $63 \%$ of the subjects were between 20 and 40 years old. All except three subjects had no comorbidities, with the latter having only hypertension or hyperlipidemia. Most of the events occurred during two periods, March to April and October to December, which is consistent with previously observed seasonal peaks. The length of hospital stay ranged from 1 to 10 days for all patients, except one who stayed for 78 days and one who was transferred to another hospital. Six patients $(22.2 \%)$ required admission to the ICU or high dependency. There were three mortalities, all found in asystole at the incident site and also suffered hypoxic ischemic encephalopathy (HIE). Seventeen (63\%) events were occupation related with all occurring either at the airbase or open construction sites. Although there were reportedly six mechanisms of lightning injuries (direct strike, contact injury, side flash, ground current, upward streamer and blast injury) this study only established two types of mechanisms - direct strike and contact injury - amongst our patients. Clinical and biochemical parameters that were studied included cardiovascular morbidity, rhabdomyolysis, otologic injuries, burns, acute kidney injury and neurological complications. The small numbers limited a statistical analysis for any correlations between clinical factors and prognosis as well as hospital length of stay. Nevertheless, it is notable that all three deaths had asystole arrest at presentation, developed $\mathrm{HIE}$, and a trend towards a higher serum creatinine on admission.

Conclusion The results of this study add to the small but increasing literature on lightning injuries and may serve to increase physician awareness in this medical niche.

References

1. Chao TC, et al:: A study of lightning deaths in Singapore. Singapore Med J 1981, 22:150-157.

2. Feng Z, et al:: Lightning city. The Straits Times, 22 November 2011, B1.

P485

Satisfaction survey among medical staff involved in relief operations following the Great East Japan Earthquake and Tsunami Y Kondo', T Abe ${ }^{2}$, S Deguchi ${ }^{3}$, Y Kuba ${ }^{4}$, H Mitsunaga ${ }^{5}$, H Sekiguchi',

K Kohshi', I Kukita'

'University of the Ryukyus, Nishihara, Japan; '2Mito Kyodo General Hospital, University of Tsukuba, Mito, Japan; ${ }^{3}$ Graduate School of Health Sciences, Meio University, Nago, Japan; ${ }^{4}$ Medical Corporation Kariyushi Heart-Life Hospital, Nishihara, Japan; ${ }^{5}$ Tokyo Institute of Technology, Tokyo, Japan

Critical Care 2012, 16(Suppl 1):P485 (doi: 10.1186/cc11092)

Introduction We conducted an attitude survey regarding satisfaction among medical staff involved in relief operations following the Great East Japan Earthquake (magnitude 9.0) and Tsunami, which struck Japan on 11 March 2011. The damage was enormous and a number of medical relief teams visited the affected area to rescue victims. Our Okinawa medical relief team visited Otuchi, Iwate, on 15 March and provided medical support to the victims for 2.5 months.

Methods We conducted an anonymous paper survey using selfdeveloped questionnaires. The 79 participants included medical doctors, nurses, and logisticians from medical relief teams involved in rescuing victims of the 2011 Great East Japan Earthquake and Tsunami. Data were analyzed using descriptive statistics. We also performed factor analysis to analyze responses with regard to factors such as face wash, toilets, sleep, clothes, and food.

Results The overall response rate was 59.5\% ( $n=47 / 79)$; the response rate was $38.3 \%$ ( $n=18 / 47)$ for medical doctors, $36.2 \%(n=17 / 47)$ for nurses, and $25.5 \%(n=12 / 47)$ for logisticians. The mean length of career was 16.5 years (standard deviation, 9.75). Descriptive statistics revealed that the participants reported high satisfaction with regard to the command system and consistent satisfaction with regard to membership. However, some were unsatisfied with the deployment length. Almost all participants wanted to be part of a relief team if given an opportunity again. Factor analysis derived one factor (eigenvalue shows 3.48 (one factor), 0.33 (two factors), 0.17 (three factors), and 0.13 (four factors)) as comfort. Face wash $(-0.95)$ contributed the most satisfaction compared to other factors such as toilets $(-0.86)$, sleep $(-0.81)$, clothes $(-0.74)$, and food $(-0.69)$.

Conclusion Almost all participants were satisfied with their level of comfort, and the influence of factors responsible for this comfort in descending order were face wash, toilets, sleep, clothes, and food. References

1. Singh G, et al: Psychiatry Clin Neurosci 2003, 57:333-336.

2. Kondo Y, et al:. Jpn J Reanimatol 2011, 30:77-81.

P486

Nuclear disaster and the medical problems during the earthquake in Japan, 2011

Y Haraguchi, Y Tomyasu, H Nishi, M Sakai, E Hoshino, M Hoshino, TTakeda-Nozawa

Japanese Compendium Team for Disaster Medicine, Kawasaki, Japan Critical Care 2012, 16(Suppl 1):P486 (doi: 10.1186/cc11093)

Introduction The roles of medicine including intensivists against natural mega-disaster followed by artificial disaster are discussed.

Methods The Higashinihon earthquake caused more than 2,000 deaths or missing, which was followed by the Fukushima Daiichi nuclear plant explosion. This study was mainly studied based upon on the actual experience in and around the nuclear station.

Results Many medical teams, rescue teams and public officials worked hard. However, many serious problems are revealed, even if they are limited to the medical fields, which are as follows: inappropriate basic preparedness against the largest degree of mega-disaster; lack of official education for medical teams against special disaster, such as nuclear disaster (that is, most members of the Japan DMAT or disaster medical assistance team seemed to be laypersons); incorrect standard/ rules of Japan DMAT, which were thought to be excessively focused upon the cure of the injured patients and a planned short period or nearly 48 hours; and insufficient consideration for the weak people or CWAP: children, (pregnant) women, aged people, and the poor people/ sick patients. Many CWAP seemed not to have survived.

Conclusion In order to cope with the mega-disaster, it became evident that it is insufficient to take makeshift measures or use cheap tricks. Working out the systematization of disaster medicine, based upon the academic viewpoints and philosophy/reliability, is essential to protect the people and the nation too.

\section{P487}

Stressors in the ICU: different perceptions of patients, relatives and staff members

M Umbrello', G Mistraletti', B Cerri', E Carloni', V Mariani², A Di Carlo',

F Martinetti', L D'Amato', S Miori', G lapichino

'Università degli Studi di Milano, Milan, Italy; ${ }^{2} \mathrm{AO}$ San Paolo - Polo

Universitario, Milan, Italy

Critical Care 2012, 16(Suppl 1):P487 (doi: 10.1186/cc11094)

Introduction The high-risk critically ill are exposed to significant stressors, along with difficulties in communicating them to relatives and members of the staff. The aim of this study was to compare the perception of stressors as reported by patients $(P)$, relatives $(R)$ and ICU staff members (S).

Methods A validated questionnaire [1] was used to quantitatively assess discomforts related to the ICU stay. Items were clustered into 
categories; higher scores refer to a higher stressfulness. The median (IQR) was calculated for each category. Twenty-eight high-risk critically ill at discharge, 55 relatives 48 hours after admission of their next of kin, and a total of 125 staff members ( 55 attending physicians, 40 nurses and 30 medical students/specialist trainees) were interviewed. Fifty-six of the staff members were used to keep patients consciously sedated as for local guidelines; the remaining used deeper levels of sedation. Nonparametric tests were used as needed.

Results All stressor categories were differently reported by the three groups analysed: environmental ( $S=17$ (15 to 19$), R=15$ (13 to 18 ), $\mathrm{P}=10$ (8 to 11), $P<0.01$ ), relationships ( $\mathrm{S}=23$ ( 21 to 25 ), $\mathrm{R}=20.5$ (17 to $24.5), P=14$ (11 to 17$), P<0.01$ ), emotional ( $S=25.5$ ( 23 to 28$), R=24$ (20 to 26$), P=18$ (15 to 22$), P<0.01$ ), and physical ( $S=35$ (31 to 38 ), $R=33$ ( 26.5 to 37 ), $P=27$ ( 21 to 30$), P<0.01$ ). Among the staff members, nurses overestimated more than attending physicians, while trainees are closer to relatives' perception $(P=0.03)$. Staff members used to conscious sedation overestimate less the impact of environmental stressors $(P=0.03)$. Years of experience $(r=0.24, P=0.03)$ and age $(r=0.27, P=0.01)$ are related to stressor overestimation among staff members.

Conclusion Members of the staff should reconsider their beliefs on patients' perception of stressors. We argue that such an overestimation may bring inappropriate administration of analgesic and sedative drugs, particularly for nurses and older members of staff. Relatives might be useful intermediaries to have a better insight of patients' perception.

Reference

1. Novaes: Intensive Care Med 1997, 23:1282.

P488

Role of ICU nurses in the confrontation of post-traumatic stress disorder in relatives of ICU patients in a general hospital of Athens, Greece

M Kourti', T Katostaras², G Kallergis², E Christofilou', I Floros', G Fildisis² 'Laiko General Hospital, Athens, Greece; '2University of Athens, Faculty of Nursing, Athens, Greece

Critical Care 2012, 16(Suppl 1):P488 (doi: 10.1186/cc11095)

Introduction This study was planned to assess post-traumatic stress disorder (PTSD) in relatives of ICU patients and to evaluate the role of ICU nurses in the confrontation of these symptoms.

Methods The Impact of Event Scale - Revised (IES-R) was translated and distributed to the family members of patients that were hospitalized in the ICU from August 2008 to September 2010. Two measurements took place: the first one 7 to 10 days from the admission of the patient to the ICU and the second one (to the same relative) after 15 to 20 days from the admission. The maximum IES-R score is 88 ( 0 to 4 for each one of the 22 questions that constitute the scale). Scores over 33 were interpreted as severe cases of PTSD. Patients' health condition was evaluated with the APACHE II score before each measurement.

Results From the first measurement it occurred that $66.7 \%$ of the relatives faced severe symptoms of PTSD (scores $>33$ ) and from the second measurement it occurred that $70 \%$ of family members were identified as cases of severe stress symptoms too. No correlation was found between these symptoms and APACHE II score $(P>0.05)$, indicating that such symptoms exist in family members during the whole patient's stay in the ICU, regardless of the seriousness of the patient's condition.

Conclusion Relatives of ICU patients seem to suffer from symptoms of PTSD. Nurses who work in the ICU, and have direct and longer contact with patients and relatives too, need to recognize, evaluate and minimize these symptoms in order that further disorders and damage to the relatives' mental health be prevented.

\section{References}

1. Azoulay E, et al:: Am J Respir Crit Care Med 2005, 171:987-994.

2. Azoulay E, et al: Am J Respir Crit Care Med 2001, 163:135-139.

3. Horowitz MJ, et al.: Psych Med 1979, 41:209-218.
P489

Family meetings and end-of-life decision-making in Thai critically ill patients

P Chatrkaw ${ }^{1}$, R Champunot $^{2}$, W Riyakul ${ }^{3}$

'Faculty of Medicine, Chulalongkorn University, Bangkok, Thailand;

${ }^{2}$ Buddhachinaraj Hospital, Phitsanulok, Thailand; ${ }^{3}$ Chulalongkorn Hospital,

Bangkok, Thailand

Critical Care 2012, 16(Suppl 1):P489 (doi: 10.1186/cc1 1096)

Introduction Limitation of life-sustaining therapy after critical illnesses in Thailand is uncommon. The barriers may be uncertain prognosis, wrong sense of doctor duty, guilty feeling, conflicts on the goals of care and fear of liability. Therefore we set a formal healthcare team meeting followed by a family meeting to find the balance between curative and palliative intention. The objective was to determine the nature and effects of family meetings in the Thai social context.

Methods A descriptive, retrospective analysis of charts and preference forms after family meetings in the surgical ICU during 2003 to 2005. Close family members were invited and encouraged to express their ideas and feelings.

Results Thirty-one family meetings were analysed. The mean age of the patients was $65.5( \pm 12.9)$ with mean SAPS II of $55.6( \pm 14.9)$. Three patients were post CPR. Metastatic cancer was the most common underlying condition (45.2\%). Most families requested to have full support, except CPR. Around 20\% were not ready to make a decision, but finally agreed not to escalate therapy. See Figure 1.

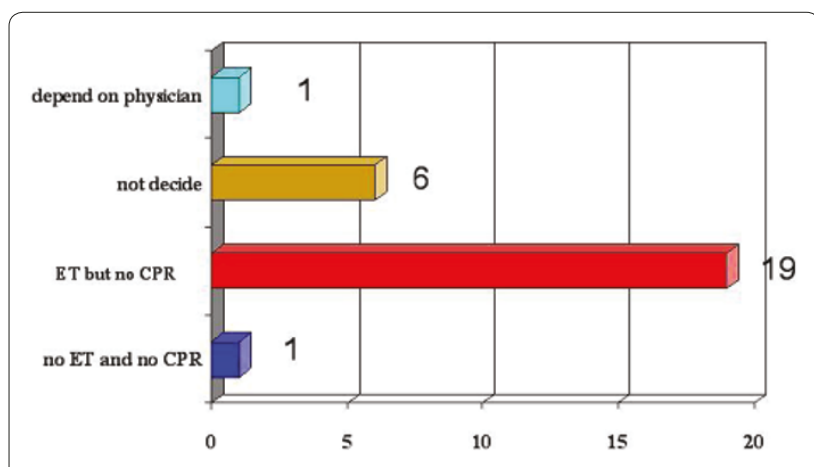

Figure 1 (abstract P489). Preferences for care at the end of life after family meetings.

Conclusion Withholding intubation and withdrawal therapy are uncommon in Thai people. However, most Thai families prefer not to escalate therapy including CPR. All of them died peacefully and the families were satisfied with the care at the end of life.

\section{Reference}

1. Truog RD, et al.: Crit Care Med 2001, 29:2332-2348.

\section{P490}

Influence of burnout on attitudes of ICU doctors and nurses towards liberalization of visiting polices

A Giannini', G Miccinesi², E Prandi', M Audisio 3 , A Bencivinni', E Biagioni ${ }^{5}$, E Castenetto ${ }^{6}$, I Laganà', R Oggioni ${ }^{4}, V_{\text {Porta }}{ }^{8}$, R Salcuni ${ }^{9}$, A Sarti10,

MG Visconti ${ }^{11}$, C Borreani ${ }^{12}$

'Fondazione IRCCS Ca' Granda, Ospedale Maggiore Policlinico, Milan, Italy; ${ }^{2}$ Istituto per lo Studio e la Prevenzione Oncologica, Florence, Italy: ${ }^{3}$ Ospedale di Ciriè, Italy; ${ }^{4}$ Ospedale Nuovo del Mugello, Borgo San Lorenzo, Italy; ${ }^{5}$ Azienda Ospedaliera Universitaria di Modena, Italy; ${ }^{6}$ Ospedale Civico, Chivasso, Italy;

${ }^{7}$ Azienda Ospedaliera 'G. Salesi', Ancona, Italy; ${ }^{8}$ Ospedale Civile, Legnano, Italy; ${ }^{9}$ Ospedale di Ivrea, Italy; ${ }^{10}$ Ospedale S. Maria Nuova, Florence, Italy; ${ }^{11}$ Ospedale 'A. Uboldo', Cernusco sul Naviglio, Italy; ${ }^{12}$ Istituto Nazionale per lo Studio e la Cura dei Tumori, Milan, Italy

Critical Care 2012, 16(Suppl 1):P490 (doi: 10.1186/cc11097)

Introduction The staff working in the ICU have a complex and stressful job and are at risk of burnout [1]. We conjectured that the presence of a burnout profile may also influence the views of ICU doctors and nurses regarding the liberalization of visiting policies. We investigated 
this issue in the course of a survey about the impact on ICU staff of liberalization of visiting policies.

Methods We administered an anonymous closed-question questionnaire to nurses and doctors at eight ICUs that were about to increase the daily visiting time to at least 8 hours, soliciting their views on policy changes in their unit. The ICU staff were asked to fill in the same questionnaire a year after implementation. On both occasions we also administered the Maslach Burnout Inventory (a 22-item self-completed questionnaire) to survey the incidence of burnout.

Results The first response rate was $91 \%$ (234/258), the second $76 \%$ (197/258). Most doctors and nurses gave a favourable opinion regarding changes to visiting policy in both the first (72\%) and the second survey (71\%). In both phases of the study, the percentage of respondents presenting a profile compatible with burnout was $36 \%$ and $41 \%$ respectively. In subjects without burnout there was a marked predominance of a favourable opinion ( $80 \%$ vs. $61 \%)$, and this favourable attitude was also maintained a year after the implementation of policy change (79\% vs. $59 \%$ ).

Conclusion The presence of burnout has a strong influence on the opinion of doctors and nurses regarding liberalization of visiting policies in the ICU. A favourable opinion predominates among ICU staff members without burnout symptoms. In preparing for and assisting the opening of ICUs it is important also to be aware of this aspect and to offer nurses and physicians appropriate support.

Acknowledgments The study was supported by Associazione per il Bambino Nefropatico (Milan, Italy).

Reference

1. Embriaco N, et al.: Curr Opin Crit Care 2007, 13:482-488.

P491

Prevalence, risk factors and impact of severe burnout syndrome in 12 Uruguayan ICUs

G Burghi', J LAmbert², M Chaize 2 , C Quiroga ${ }^{3}$, G Pittini ${ }^{4}$, M Cancela ${ }^{5}$,

H Bagnulo', S Chevret' ${ }^{2}$, E Azoulay ${ }^{2}$

'Hospital Maciel, Montevideo, Uruguay: ${ }^{2}$ Saint Louis Hospital, Paris, France:

${ }^{3}$ Hospital Español, Montevideo, Uruguay; ${ }^{4}$ CAAMEPA, Pando, Uruguay;

${ }^{5}$ Hospital de Clínicas, Montevideo, Uruquay

Critical Care 2012, 16(Suppl 1):P491 (doi: 10.1186/cc11098)

Introduction Burnout syndrome (BOS) is defined as a state of emotional fatigue that leads to a loss of motivation, usually progressing towards feelings of inadequacy and failure. Severe BOS is relevant as it leads to loss of psychological well-being, increased absenteeism and turnover, and deterioration in the quality of care provided to patients. The objective was to determine the prevalence of BOS among Uruguayan ICU clinicians. To evaluate personal or organization characteristics associated with the development of severe BOS.

Methods A survey was conducted in 12 Uruguayan adult ICUs. The level of BOS was evaluated on the basis of the Maslach Burnout Inventory (MBI score). ICU, patient, and clinician characteristics were assessed for their association with the prevalence of severe BOS (that is, highest MBI scores). All variables with $P<0.2$ in univariate analysis were included in a model of ordinal regression. $P<0.05$ was considered statistically significant.

Results A total of 364 questionnaires were evaluated, including 282 nurses and 82 ICU physicians. The prevalence of severe BOS was $51 \%$ among ICU physicians and $42 \%$ in nursing staff. For ICU nurses, factors independently associated with lower $\mathrm{MBI}$ scores were the following: work on fixed days (OR $0.6 ; 95 \% \mathrm{Cl} 0.3$ to $0.9 ; P=0.01$ ), integrated in-ICU working groups (OR $0.6 ; 95 \% \mathrm{Cl} 0.3$ to $0.9 ; P=0.02)$, good relationships with physicians (OR $0.8 ; 95 \% \mathrm{Cl} 0.7$ to $0.9 ; P=0.008$ ) and good relationships with supervisors (OR $0.8 ; 95 \% \mathrm{Cl} 0.7$ to $1 ; P=0.05$ ). In contrast, at least one death over the last week was associated with higher $\mathrm{MBI}$ score (OR 2; $95 \% \mathrm{Cl} 1.2$ to 3.2; $P=0.008)$. For ICU physicians, not being partnered was independently associated with higher $\mathrm{MBI}$ scores. Conversely, good relationships with colleagues was associated with lower $\mathrm{MBI}$ scores (OR 0,$5 ; 95 \% \mathrm{Cl} 0.3$ to $0.8 ; P=0.004)$. Interestingly, this study confirms that clinicians with severe BOS had increased burden such as sleep disorders, libido troubles, lack of memory, inadequate money management as well as the wish to leave the ICU. Conclusion The prevalence of severe BOS is very high among ICU workers in Uruguay. We have identified different risk factors associated with the development of severe BOS. These results confirm previous findings and highlight that strategies to decrease BOS in ICU clinicians are urgently warranted.

\section{P492}

Opening the ICU: views of ICU doctors and nurses before and after liberalization of visiting policies

A Giannini ${ }^{1}$, G Miccinesi ${ }^{2}$, E Prandi ${ }^{1}$, M Audisio $^{3}$, A Bencivinni ${ }^{4}$, E Biagioni ${ }^{5}$, E Castenetto ${ }^{6}$, I Laganà7, R Oggioni ${ }^{4}, V^{7}$ Porta $^{8}$, R Salcuni $^{9}$, A Sarti $^{10}$,

MG Visconti ${ }^{11}$, C Borreani ${ }^{12}$

'Fondazione IRCCS Ca' Granda, Ospedale Maggiore Policlinico, Milan, Italy; ${ }^{2}$ Istituto per lo Studio e la Prevenzione Oncologica, Florence, Italy; ${ }^{3}$ Ospedale di Ciriè, Italy; ${ }^{4}$ Ospedale Nuovo del Mugello, Borgo San Lorenzo, Italy; ${ }^{5}$ Azienda Ospedaliera Universitaria di Modena, Italy; ${ }^{6}$ Ospedale Civico, Chivasso, Italy; ${ }^{7}$ Azienda Ospedaliera 'G. Salesi,' Ancona, Italy; ${ }^{8}$ Ospedale Civile, Legnano, Italy; ${ }^{9}$ Ospedale di Ivrea, Italy; ${ }^{10}$ Ospedale S. Maria Nuova, Florence, Italy; ${ }^{11}$ Ospedale 'A. Uboldo', Cernusco sul Naviglio, Italy; ${ }^{12}$ Istituto Nazionale per lo Studio e la Cura dei Tumori, Milan, Italy

Critical Care 2012, 16(Suppl 1):P492 (doi: 10.1186/cc11099)

Introduction Italian ICUs still impose restrictive visiting policies (with a median visiting time of about 2 hours/day); however, a revision of current policies is underway [1-3]. No data are available on the views of Italian ICU teams following an at least partial liberalization of visiting policies. We investigated this issue in the course of a survey about the impact on ICU teams of the liberalization of visiting policies.

Methods We administered an anonymous closed-question questionnaire to nurses and doctors at eight ICUs that were about to increase daily visiting time to at least 8 hours, soliciting their views on policy changes in their unit. The ICU staff were asked to fill in the same questionnaire a year after implementation.

Results The first response rate was 91\% (234/258), the second $76 \%$ $(197 / 258)$. In the first instance, $83 \%$ of doctors and $67 \%$ of nurses expressed a favourable opinion regarding the change in visiting policy. After 1 year a positive opinion was expressed by $84 \%$ of doctors and $63 \%$ of nurses. Both phases of the study show a significant predominance of positive opinions among doctors $(P=0.032$ and 0.005$)$.

Conclusion Most ICU staff members view the opening of the unit positively, and on the whole maintain this opinion 1 year after the policy change. Overall, the attitude of doctors is more favourable than that of nurses. It is essential to build up a picture of the difficulties that liberalizing visiting could create for ICU staff (and particularly for nurses), and to explore the causes and extent of such difficulties, in order to identify possible solutions and offer nurses and doctors appropriate support.

Acknowledgments The study was supported by Associazione per il Bambino Nefropatico (Milan, Italy).

References

1. Giannini et al:: Intensive Care Med 2008, 34:1256-1262.

2. Giannini et al.: Intensive Care Med 2011, 37:1890

3. Giannini et al.: Pediatr Crit Care Med 2011, 12:e46-e50.

\section{P493}

A family-based satisfaction survey on the ICU

D Moult, R Breeze, A Molokhia

University Hospital Lewisham, London, UK

Critical Care 2012, 16(Suppl 1):P493 (doi: 10.1186/cc11100)

Introduction We conducted a prospective survey to determine satisfaction amongst relatives of patients on our ICU. Patient-reported outcome measures have become widely used and accepted in the pursuit of improved quality of care [1]. However, assessing patient satisfaction is difficult on the ICU, an environment where we more commonly communicate with the family of patients regarding the care of their relative. Therefore, a more family-centred approach is indicated, for which family satisfaction questionnaires have already been validated [2].

Methods We utilised a 35-point questionnaire-based survey of relatives of patients in our ICU over 10 weeks. Questionnaires were distributed to family members when the decision to discharge from the ICU was made. We limited this to two family members per patient who were in the ICU for more than 48 hours. 
Results We received 29 completed questionnaires. Relatives of 24 of the respondents had survived to ICU discharge. Responses were linearly transformed to give percentage scores. Higher values represented a greater degree of satisfaction. Overall care in the ICU, $88.8 \%$. Courtesy, respect and compassion to the patient $(93.8 \%)$, to relatives $(92.2 \%)$; assessment and treatment of pain (94.4\%), breathlessness $(92.9 \%)$, agitation (88.9\%); emotional support (89.4\%); care from nurses (92.0\%), doctors (95.5\%); frequency of communication nurses (92.9\%), doctors (89.7\%). Overall decision-making, $91.3 \%$. Willingness of staff to answer questions ( $90.5 \%)$; honesty $(90.5 \%)$, completeness $(91.4 \%)$, consistency of information (90.5\%); inclusion in decision-making, 78.7\%; support during decision-making, 78.7\%; time to think about information given, $96.2 \%$.

Conclusion Family satisfaction with our ICU is high, with satisfaction high in both care and decision-making domains. Appropriate inclusion with and support during the decision-making process were areas with lower satisfaction scores. The structuring of options for answering these questions may have been a confounding factor in this finding. However, this may represent genuine lower levels of satisfaction and steps should be taken to improve this. In response to these findings we have invited families to join their relatives' part of the consultant ward round to improve inclusion and support in decision-making. We are currently repeating the survey with these changes in place and will present our findings in the future.

\section{References}

1. Dodek et al: Crit Care Med 2004, 32:1922-1927.

2. Wall et al:: Crit Care Med 2007, 35:271-279.

\section{P494}

Immediate needs and level of anxiety of families with traumatic

brain injury patients admitted to ICUs

S Gholamzadeh, R Abdoli, F Shariff, R Gholamzadeh,

A Maraghian Mohammadi

Shiraz University of Medical Sciences, Shiraz, Iran

Critical Care 2012, 16(Suppl 1):P494 (doi: 10.1186/cc11101)

Introduction Meeting the needs of family members of patients in the ICU is an important criterion in assessment of quality of care in the ICU. Therefore this study was conducted to determine the immediate needs and level of anxiety of families with traumatic brain injury patients admitted to ICUs in Shiraz, Iran in 2008.

Methods In this descriptive cross-sectional study, a convenience sample of 60 family members was recruited over a period of 4 months. On the second day of ICU admission, one family member for each patient who met the study criteria were asked to complete three questionnaires, consisting of the Critical Care Family Need Inventory (CCFNI), the Statetrait Anxiety Inventory (STAI) and a demographic data sheet.

Results The mean ages of the subjects were 32.2 years. A total of 10 needs statements in the CCFNI were rated to be important or very important needs by 50 of the 60 families (83.3\%); seven were needs for assurance, two were needs for information, and one of them was needs for proximity. The mean of CCFNI satisfaction scores were low $(16.5 \pm 1.5)$ for needs to comfort, and high for needs to support $(38.1 \pm 4.7)$. Also the mean score of state anxiety $(56.75 \pm 5.7)$ and trait anxiety score ( $52 \pm 6.2)$ was higher than previous studies.

Conclusion A needs-based education program can decrease the level of family anxiety and increase the level of satisfaction.

P495

Families: the newest members of the ICU multidisciplinary team

R Santhirapala, J Lipton, T Hall, R Breeze, A Molokhia

University Hospital Lewisham, London, UK

Critical Care 2012, 16(Suppl 1):P495 (doi: 10.1186/cc11102)

Introduction We have started inviting the relatives of our patients to remain present during our multidisciplinary team ICU ward round. The aim is to improve their understanding of the complex activity on an ICU and reduce inconsistencies in communication. In the UK it is becoming expected practice that patient satisfaction is an endpoint we should be measuring and improving [1]. Assessing this on the ICU is often very difficult due to the confounding factors inherent to critical illness. We often seek assent from families for procedures and to provide some history as a surrogate to patient interview. We think the care we provide should encompass both the patient and their family. This is already accepted practice in the paediatric ICU setting [2]. Communication between family and clinical staff, ideally on a daily basis, is clearly imperative and a systematic approach to improve this is good practice. Increasing insight into relatives' perceptions and expectations will aid the delivery of high-quality care. We believe that involving relatives in the ward round will be of benefit for us in our professional relationships with them and improve their understanding during an extremely difficult time.

Methods This was a prospective study over 2 months formally inviting up to four families per day to be present for that part of the ward round involving their relative. Subsequently they were asked to complete a questionnaire anonymously on the experience.

Results The results that reflected 31 ward round attendances were unanimous: every family agreed that their attendance had a positive impact, alleviating misconceptions about the intensive care environment and clarifying the processes involved in the care of their relative. The survey also revealed that attendance at the ward round provided an excellent opportunity to have their questions answered by consultants. All those invited wished to attend and all respondents said the experience was valuable and they would like to attend again. Comments included: 'Explanations very helpful to deal with the stress of the situation' and 'Reassuring to have information delivered professionally and compassionately'.

Conclusion In this single-centre survey we have demonstrated that inviting families to ICU ward rounds is feasible and we believe that this intervention could improve family satisfaction on the ICU. We are investigating the impact of this intervention with a detailed comparative survey, which we will present in the future.

References

1. Patient Satisfaction [www.patientsatisfaction.co.uk]

2. Aronson et al:: Paediatrics 2009, 124:1120-1125.

P496

Family satisfaction in an interdisciplinary ICU: a quality audit

UM Schmid, R Alpiger, T Rizzo, CK Hofer

Triemli City Hospital, Zurich, Switzerland

Critical Care 2012, 16(Suppl 1):P496 (doi: 10.1186/cc11103)

Introduction The quality of intensive care medicine depends on multiple indicators $[1,2]$. Meeting relatives' needs in the challenging situation of ICU visits is crucial. The aim of this audit was to assess next of kin's satisfaction and influencing factors.

Methods With institutional approval, questionnaires were distributed to family members of ICU patients. The survey included two visual analogue scale ratings (VAS 1: patient care, VAS 2: decision-making) and 24 questions with four dimensions D1 to D4 (general impression, treatment and patient care quality, professional quality) on a five-point Likert scale, transformed into values 1 to 100 . Patient-specific and relatives' sociodemographic data were recorded. Data are presented as the mean $\pm S D$, median (Q.5), interquartile range (IQR) and range (minimum/maximum). Subgroup analysis (relative's and patient's age, sex, education, marital status, length of stay, visit frequency and mortality) was performed using the Mann-Whitney U test.

Results Questionnaires of 159 patients were analyzed (patients: age $=$ $66.1 \pm 13.0$ years, $64 \%$ female, SAPS $=38.8 \pm 17.5$, LOS $=13.5 \pm 11.8$

Table 1 (abstract P496)

\begin{tabular}{lccc}
\hline & Mean \pm SD & Q.5/IQR & Minimum/maximum \\
\hline VAS1 & $9.1 \pm 0.9$ & $9.3 / 0.9$ & $5.5 / 10$ \\
VAS2 & $8.6 \pm 1.5$ & $9.0 / 1.5$ & $3.1 / 10$ \\
ESatisf & $87 \pm 15.1$ & $80 / 20$ & $20 / 100$ \\
D1 & $91.1 \pm 15$ & $100 / 20$ & $20 / 100$ \\
D2 & $89.2 \pm 13$ & $100 / 20$ & $40 / 100$ \\
D3 & $86 \pm 15.4$ & $80 / 20$ & $20 / 100$ \\
D4 & $85.5 \pm 15$ & $80 / 40$ & $20 / 100$ \\
\hline
\end{tabular}


days, mortality $=16.5 \%$; relatives: age $=44.5 \pm 26.9$ years, $63.7 \%$ female, $13 \%$ medical $/ 25.5 \%$ higher education). High satisfaction (VAS 1/2, D1 to D4) was observed (Table 1). Significant differences within subgroups were found: relatives with healthcare education showed higher D1 to D4 satisfaction than the ones with a graduate degree only. Higher VAS scorings were observed from next of kin with high visit frequency $(\geq 5 \times /$ week).

Conclusion Relatives of ICU patients were in general highly satisfied. The educational status and ICU visit frequency of the next of kin were revealed to be influencing factors.

References

1. Intensive Care Med 2007, 33:1913-1920.

2 Intensive Care Med 2009, 35:2051-2059

P497

Incidence of post-traumatic stress, anxiety and depression symptoms in patients and relatives during the ICU stay and after discharge

R Fumis, P Martins, G Schettino

Hospital Sirio-Libanes, São Paulo, Brazil

Critical Care 2012, 16(Suppl 1):P497 (doi: 10.1186/cc11104)

Introduction To study the incidence and predictors of post-traumatic stress, anxiety and depression symptoms in medical and surgical patients and relatives during the ICU stay and at 30 and 90 days post ICU discharge. Methods A prospective study of 72 patients and 99 family members that completed the Hospital Anxiety and Depression Scale during the ICU stay and at 30 and 90 days after discharge. The Impact of Event Scale at 30 and 90 days after ICU discharge was used to evaluate posttraumatic stress disorder (PTSD).

Results The prevalence of symptoms of anxiety, depression or both in patients during the ICU stay was $10 \%, 2.8 \%$ and $6.9 \%$ respectively. Among family members prevalence was $17.3 \%, 6.5 \%$ and $14.4 \%$ respectively, and was significantly higher compared to patients $(P=0.034)$. PTSD symptoms were present in $39.8 \%$ and $32.7 \%$ of family members respectively at 30 and 90 days after discharge. Among patients symptoms were significantly lower $(P<0.001)$. Factors associated with symptoms of anxiety and depression during the ICU stay in a multivariate model included patient-related factors as SAPS 3 (OR $1.1,95 \% \mathrm{Cl} 1.01$ to 1.24 ) and length of family member stay in the ICU (OR $1.39,95 \% \mathrm{Cl} 0.89$ to 2.16 ) and family-related factors as female gender (OR $5.43,95 \% \mathrm{Cl} 0.67$ to 43.8 ) and oncologic diagnosis (OR 0.25 , $95 \% \mathrm{Cl} 0.05$ to 1.31 ). The multivariate model also identified patient age (OR $0.97,95 \% \mathrm{Cl} 0.93$ to 1 ) and oncologic diagnosis (OR $0.27,95 \% \mathrm{Cl}$ 0.09 to 0.79 ) associated with symptoms of post-traumatic stress after discharge among family members.

Conclusion At least one-third of family members visiting patients in the ICU suffer from symptoms of anxiety, depression or both. The level of post-traumatic stress symptoms in family members was high after ICU discharge. Depression, anxiety and post-traumatic stress symptoms were higher among family members compared to patients. Female gender and oncologic diagnosis were strongly associated with depression and post-traumatic stress. Further actions might be adopted to diminish the incidence of these disorders.

References

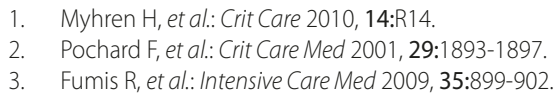

P498

Application of a new German law as a basis for end-of life decisions in a medical ICU

R Riessen, C Bantlin, M Haap

University Hospital Tübingen, Germany

Critical Care 2012, 16(Suppl 1):P498 (doi: 10.1186/cc11105)

Introduction In 2009 a new German law came into effect that clarified issues regarding end-of-life decisions, especially the role of patient autonomy and the importance of a medical indication in the course of treating patients with terminal illness. In this study we analyzed the end-of-life (EOL) policies in our medical ICU with a focus on the practicability of this law.
Methods A retrospective analysis of all patients that were treated in the medical ICU of a large German university hospital in 2009 and 2010 and died during their hospital stay.

Results During the observation period 3,401 patients were treated in our ICU. The ICU mortality was 15\% $(n=501)$, hospital mortality was $19 \%$ $(n=658)$. The mean predictive mortality derived from the SAPS 2 score was $29 \%$ for all patients (standardized mortality ratio 0.67$)$, deceased patients had a predictive mortality of $56 \%$. Of all deceased, $232(35 \%)$ had received CPR, 170 of those (73\%) outside the ICU. Of all patients who died in the hospital, $126(19 \%)$ had received unlimited therapy. Life support was withdrawn in 245 patients (37\%) and life support was withheld in 241 patients (36\%). In 46 patients (7\%) palliative care was instituted right from the beginning of the ICU stay. In 104 cases (16\%) the patients themselves made the EOL decision, in 78 cases $(12 \%)$ an advance directive was present. A legally designated healthcare proxy was involved in $8 \%$. In 541 cases (82\%) the relatives were integrated in EOL decisions with the objective of finding a broad consensus; however, in these cases the assessment of the medical indication and the prognosis by the medical team was of particular importance. Cases in which relatives were not involved in EOL decisions were in $76 \%$ cases with short unsuccessful maximal therapy, for example CPR (median ICU stay 5 hours). The rate of life support withdrawal was highest (60\%) in patients with CNS diseases. We did not experience any serious or unsolvable conflicts with relatives. Involvement of a law court was necessary in none of the cases.

Conclusion EOL policies were applied in $81 \%$ of our intensive care patients who died during their hospital stay. The new German law regulations served as a practical and realizable basis for EOL policies in our medical ICU.

\section{P499}

Effect of a full moon on mortality of patients admitted to the ICU

R Nadeem', A Nadeem ', E Madbouly', J Molnar'2, J Morrison² 'Captain James A. Lovell Federal Healthcare Center, North Chicago, IL, USA: ${ }^{2}$ Rosalind Franklin University of Medicine and Science, North Chicago, IL, USA Critical Care 2012, 16(Suppl 1):P499 (doi: 10.1186/cc11106)

Introduction The effect of the full moon (the lunar effect) on human behaviour has occupied researchers for centuries. We aim to determine such a lunar effect on mortality among patients admitted to the ICU. Methods We analyze the electronic medical records of patients admitted to the ICU. The subjects were divided into two groups: patients who died on full moon days (14th,15th, and 16th days of the lunar month) and the patients who died on other days of the lunar month. The mortality rates were calculated for patients in both groups. Parameters including age, gender, acute physiology and chronic health evaluation (APACHE) III scores, predicted mortality, type of ICU, and actual mortality were compared between the two groups. Student's $t$ test was performed to determine whether there were any differences between the groups.

Results Data from 4,387 patients who were followed for 23 months were analyzed. Overall, 297 patients died during this period, including 31 patients on full moon days and 266 patients on the other days of the month. Both groups were similar in terms of age ( 73 vs. 71 years, $P=0.39)$, APACHE III scores ( 82.06 vs. $76.52, P=0.28)$, and predicted mortality $(0.405$ vs. $0.370, P=0.48)$. There was no difference in the frequency of death between the full moon days and the other days (10.33 vs. $9.85, P=0.81$ ). See Table 1 .

Conclusion The full moon does not seem to affect the mortality of patients admitted to the ICU.

Table 1 (abstract P499). Characteristics of patients who died on full moon days versus other days

\begin{tabular}{lccc}
\hline & Full moon & Other days & $P$ value \\
\hline Age & $73.6 \pm 14.59$ & $71.07 \pm 16.1$ & 0.39 \\
Male/female & $15 / 16$ & $133 / 133$ & 0.86 \\
APACHE III & $82.06 \pm 24.1$ & $76.52 \pm 27.4$ & 0.28 \\
Mortality & $0.405 \pm 0.249$ & $0.370 \pm 0.268$ & 0.48 \\
\hline
\end{tabular}




\section{References}

1. Wolbank S, Praus G, Smolle-Juettner F, et al.: The influence of lunar phenomena on the incidence of emergency cases. Resuscitation 2003, 58:97-102.

2. Alves DW, Allegra JR, Cochrane DG, Cable G: Effect of lunar cycle on temporal association in cardiopulmonary arrest in seven emergency departments during eleven years. Eur J Emerg Med 2003, 10:225-228.

\section{P500}

Potential association of gender with mortality and withdrawal of life-sustaining therapies in patients with severe TBI: a Canadian multicentre cohort study

AF Turgeon', F Lauzier', A Boutin'1, N Côte', R Zarychanski², R Fowler³,

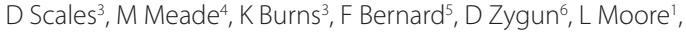

D Fergusson 7

'Laval University, Quebec, Canada; ${ }^{2}$ University of Manitoba, Winnipea,

Canada; ${ }^{3}$ University of Toronto, Canada; ${ }^{4}$ McMaster University, Hamilton,

Canada; ${ }^{5}$ Université de Montréal, Canada; ${ }^{6}$ University of Calgary, Canada;

'Ottawa Hospital Research Institute, Ottawa, Canada

Critical Care 2012, 16(Suppl 1):P500 (doi: 10.1186/cc11107)

Introduction Differences in admission patterns, delivery of care and outcomes between women and men admitted to the ICU have been previously identified [1]. However, these observations have not been well described in patients with traumatic brain injury (TBI). Our objective was to identify differences in outcomes between women and men with severe TBI.

Methods We used data from a large retrospectively cohort study in which adults with severe TBI $(\mathrm{GCS} \leq 8)$ admitted to six Canadian level I trauma centres (2005 to 2006) were identified through health records using ICD-10 codes [2]. Demographic, severity of illness, and outcome data were collected by trained abstractors. The primary outcome was the difference in mortality and withdrawal of life-sustaining therapies (WLST) between women and men; secondary outcome was the impact of age ( $<55$ vs. $\geq 55$ years old) among genders. Analyses included chisquare tests and Cox regression analyses adjusted for GCS motor and pupillary reactivity, with stratification for age.

Results Among 720 patients, 165 were women (22.9\%), 506 (70.3\%) aged $<55$ years old and $214(29.7 \%) \geq 55$ years old. Overall mortality was $31.7 \%$ and $70.2 \%$ of deaths occurring following the WLST [2]. Unadjusted mortality was $41.2 \%$ in women versus $28.8 \%$ in men $(P=0.003)$. We observed similar findings in patients $<55$ years old $(30.5$ vs. $21.4 \%, P=0.06$ ), but not among men and women aged $\geq 55$ years old (55.7 vs. $55.0 \%, P=0.43$ ). Adjusted hazard ratios (HRs) showed a nonsignificantly increased risk of death in women aged $<55$ years old as compared to men (1.51 ( 0.92 to 2.47$)$ ), and in women aged $\geq 55$ years old (1.53 (0.94 to 2.50)). We observed no difference both in the overall unadjusted incidence of WLST between women and men (73.5 vs. $68.8 \%, P=0.47)$ and in women and men aged $<55$ years old, while there was a nonsignificantly increased rate of WLST in women $\geq 55$ years old (HR 1.53 (0.94 to 2.50)).

Conclusion There may be gender-based differences in outcome among patients with severe TBI. Overall, mortality for women tended to be higher, as were decisions for WLST. These differences may be due to unmeasured confounders, biologic responses to $\mathrm{TBI}$, or differences in level of care decision-making.

References

1. Fowler RA, Sabur N, Li P, et al.: CMAJ 2007, 177:1513-1519.

2. Turgeon AF, Lauzier F, Simard JF, et al.; Canadian Critical Care Trials Group: CMAJ 2011, 183:1581-1588

P501

Making drug delivery in the ICU safer: the implementation of advanced computerised intravenous infusion pumps

A Dimech, A Le Page, P Gruber, T Wigmore

The Royal Marsden, London, UK

Critical Care 2012, 16(Suppl 1):P501 (doi: 10.1186/cc11108)

Introduction Drug administration errors account for approximately $78 \%$ of all medical errors occurring in ICUs $[1,2]$. The aim of this project was to introduce advanced computerised infusion pumps with in-built drug safety software, so-called smart pumps, in the ICU to facilitate safer drug administration.

Methods The working group consisted of an ICU pharmacist, clinical nurse specialist and consultant intensivist. A drug library was constructed by the ICU pharmacist and consultant intensivist and loaded onto the infusion pumps. The selection of drugs and dose limits were carefully considered to ensure that they were within the boundaries of normal usage so as not to impede patient management whilst maximising patient safety. Super users then provided individualised training to 85 ICU nurses. In the UK there have been 50 system implementations to date. The national average for compliance with the use of the software is $50 \%$.

Results Feedback following training with the new system was very positive. In our ICU, utilisation of the drug safety software during drug administration was $94 \%$ within the first 6 months. Of 18,000 drug infusions, only 1,000 were used outside the drug safety software. There were seven over-rides from the high limit. Of these, two were for furosemide where there was a genuine clinical need for a higher dose. On two other occasions the software prevented insulin being administered at 30 units/hour and potassium at $100 \mathrm{mmol} /$ hour. The number of drug errors reduced from three to zero during the study period. This demonstrated that the design of our package was sensitive enough to ensure safe drug administration and sufficiently practical to enable consistent use of the system.

Conclusion We have demonstrated that the introduction of an advanced computerised infusion pump system in the ICU can provide a safer drug administration environment if the appropriate health professionals are selected to implement the system, the drug library is constructed carefully and a comprehensive training package is applied. References

1. Upton D: 2011 Why are so few infusions smart? Hosp Pharm Eur 57:39-42.

2 Camire E, et al:: Medication errors in critical care: risk factors, prevention and disclosure. CMAJ 2009, 180:936-943

\section{P502}

Growing a positive culture in an ICU antimicrobial stewardship program

KWalker, J Litynsky, J Powis

Toronto East General Hospital, Toronto, Canada

Critical Care 2012, 16(Suppl 1):P502 (doi: 10.1186/cc11109)

Introduction A 3-month pilot antimicrobial stewardship program (ASP) was initiated in a 490-bed urban community hospital medical/surgical ICU. The ASP continued post pilot. ASP goals are to optimize/reduce antimicrobial (AM) usage, improve clinical outcomes and reduce nosocomial Clostridium difficile rates $[1,2]$.

Methods The pilot had one pharmacist (Ph) providing ICU clinical service and one AMPh, both working as ICUPhs. The AMPh collected standardized data on patients and were reviewed with the ID physician; then the AMPh and ID physician discussed with the ICU care team for optimal AM use. Post pilot, the ICUPh assumed the AM stewardship role. The ASP reduced from 5 to 3 days/week. Data collection included the ASP time required and interventions. The same metrics were collected pre/post pilot.

Results The ASP total patient recommendations/100 patient-days were 5-day mean 9.3, 3-day mean $13.5(P=0.030)$ with an increased ICU physician acceptance ( 5 days $=95.9 \%, 3$ days $=99.7 \%$ ). Statistically significant was an increase in recommendations to broaden therapy (Table 1) and nonstatistically significant was a reduction in recommendations to de-escalate therapy (5-day mean 1.4 recommendations/100 patient-days, 3-day mean 1.2 recommendations/100 patient-days; $P=0.601$ ). Also, there was an increase in recommendations for duration optimization (5-day mean 4.0 recommendations/100 patient-days, 3 -day mean 6.0 recommendations $/ 100$ patient-days; $P=0.055)$ and discontinue AMs (5-day mean 2.7 recommendations/100 patient-days; 3-day mean 3.7 recommendations $/ 100$ patient-days; $P=0.181$ ). The ASP mean time required (minutes/month) was reduced (5 days 864, 3 days $771 ; P=0.267)$.

Conclusion ASP reduction from 5 to 3 days/week was successful. Necessary skills were developed by the ICUPh. ASP reduction increased 
Table 1 (abstract P502)

\begin{tabular}{lccc}
\hline & \multicolumn{2}{c}{ ASP days } & \\
\cline { 2 - 3 } $\begin{array}{l}\text { Recommendations/ } \\
\text { 100 patient-days }\end{array}$ & 5-day mean & 3-day mean & P value \\
\hline Broaden & 0.4 & 1.6 & 0.003 \\
\hline
\end{tabular}

recommendations for discontinuation and prospective duration optimization goals of AMs. A reduction in recommendations to deescalate therapy and an increase in broadening therapy may reflect an increased acceptance goals. The 3-day ASP also demonstrated an increase in total recommendations/100 patient-days and a reduction in the total time required which enhanced use of resources, both financial and human.

References

1. Dellit TH, et al:: Clin Infect Dis 2007, 44:159-177.

2. Polk RE, et al:: Clin Infect Dis 2007, 44:664-670.

P503

Injectional anthrax: the inflammatory response

M Booth, A Hart, L Donaldson

Royal Infirmary, Glasgow, UK

Critical Care 2012, 16(Suppl 1):P503 (doi: 10.1186/cc11110)

Introduction From December 2009 to July 2010 there were 47 cases of anthrax amongst injecting drug users in Scotland with 13 fatalities. The majority presented as severe soft tissue infection following i.v. injection or muscle popping as described by Ringertz and colleagues [1]. At first they were diagnosed as necrotising fasciitis (NF) until the diagnosis of anthrax was made. With experience they appeared to have a milder inflammatory response to their infection compared to other soft tissue infections such as NF. To investigate this the anthrax group was compared to a cohort of confirmed NF cases.

Methods Patients admitted to the ICU with NF or injectional anthrax from 1 January 2008 to 30 June 2011 were identified. The white blood count (WBC) and C-reactive protein (CRP) at presentation were recorded. Demographic data (sex, age, ICU and hospital LOS, APACHE II score, predicted and actual hospital mortality and drug-injecting history) were retrieved. All data were collected prospectively for routine ICU management.

Results There were six patients with injectional anthrax and 16 with NF. The results are presented in Table 1. There was a marked difference in the inflammatory response between the two groups with the CRP being highly statistically significant. The anthrax group was also younger (35.5 vs. 43.2) with a lower severity of illness, lower predicted mortality ( $18.6 \%$ vs. $31.7 \%$ ) but much higher actual mortality.

Conclusion Anthrax releases three factors: lethal factor (LF), edema factor (EF) and protective antigen (PA). PA and LF form lethal toxin which kills macrophages and inhibits B-cell and T-cell function so minimising the immune response to anthrax. This is reflected in the inappropriately low CRP levels at presentation. Severe soft tissue infection in an injecting drug user associated with subjectively poor inflammatory response should raise the possibility of anthrax infection. Reference

1. Ringertz SH, et al:. Injectional anthrax in a heroin skin popper. Lancet 2000, 356:1574-1575.

Table 1 (abstract P503)

\begin{tabular}{lccc}
\hline & Anthrax & NF & \\
\hline Number & 6 & 16 & \\
APACHE II score & 12.2 & 19.4 & $P<0.05$ \\
Died (\%) & 66.6 & 18.8 & $P<0.05$ \\
WBC & 11.6 & 16.0 & NS \\
CRP & 71.2 & 287.3 & $P<0.001$ \\
\hline
\end{tabular}

P504

Multicenter consensus development of a checklist for lung injury prevention

JM Litell', O Gajic', J Sevransky², M Gong ${ }^{3}$, DJ Murphy²

'Mayo Clinic, Rochester, MN, USA; '2Emory University School of Medicine,

Atlanta, GA, USA; ${ }^{3}$ Montefiore Medical Center, Bronx, NY, USA

Critical Care 2012, 16(Suppl 1):P504 (doi: 10.1186/cc11111)

Introduction Acute lung injury (ALI) is linked to almost 75,000 US deaths annually. The syndrome is defined clinically by criteria that identify only patients with established $A L I$, at which point treatment options are limited and largely supportive. After 40 years and more than $25 \mathrm{NIH}-$ funded trials of ALI interventions, only supportive therapy with lung protective ventilation has been associated with a mortality benefit. The US Critical IIIness and Injuries Trials Group lung injury prevention subgroup seeks to standardize best practices for patients at risk of ALI. The recently validated lung injury prediction score (LIPS) identifies patients at risk of ALI, and can prompt the early use of preventative interventions. This may attenuate the progression to ALI. This study seeks expert consensus about best practices in patients at risk of ALI, as determined by their LIPS. These practices will be incorporated into a checklist for lung injury prevention. Standardization of care may protect patients against ALI development and provide a uniform background for enrollment in other ALI trials.

Methods This study employed a Delphi selection process involving 38 intensivist participants using a web-based survey tool. In Round 1 , participants were presented with 15 interventions proposed by investigators. Using a five-item Likert scale, they responded to the question: 'In your opinion as an expert, how sufficient is the evidence that this intervention reduces the risk of ALI in eligible patients?' Participants were also prompted to comment and submit additional items for consideration. In Round 2, participants followed the same approach to rate and comment on items submitted by the group. Finally, in Round 3, participants reviewed aggregated ratings and comments for all items, and voted for or against inclusion in the draft checklist. Inclusion was limited a priori to items with at least $70 \%$ agreement among participants.

Results Following Round 1, items submitted by participants were aggregated with minimal change into six additional items for Round 2. In Round 3, of the 21 total items, nine were endorsed by $70 \%$ of participants for inclusion in a draft checklist. These items were grouped conceptually into two domains: respiratory support and resuscitation. Conclusion The Delphi process of expert consensus can be employed to develop a checklist of time-sensitive interventions, in a manner that combines available evidence with the perspective of expert clinicians.

P505

Impact of the Paediatric Intensive Care Outreach Network service on mortality within intensive care

K Sadasivam, S Skellett

Great Ormond Street Hospital for Children, London, UK

Critical Care 2012, 16(Suppl 1):P505 (doi: 10.1186/cc11112)

Introduction We audited the mortality rate by admission source in our paediatric ICU, a paediatric tertiary referral centre, from 2004 to 2008 and found that the group of emergency unplanned internal admissions had a higher Standardised Mortality Ratio (SMR) of 1.55 compared to a SMR of 1.00 overall for patients admitted to the paediatric ICU. This was in keeping with data from other large paediatric centres [1]. The reasons for the increased mortality for this internal group were not clear and possibly multifactorial. To help address this, a Paediatric Intensive Care Outreach Network (ICON) team was developed and introduced in September 2009.

Methods A before-and-after study design was used to determine differences in percentage of admissions, mortality rate and SMR. Data were collected using the PICANet database for emergency unplanned internal admissions before (August 2004 to August 2008) and after implementation of the ICON team (August 2009 to February 2011). PICANet is a national database that audits all paediatric intensive care admissions in the UK [2]. 
Results A total of 3,629 admissions during a 4-year period pre ICON (August 2004 to August 2008) and 1,446 admissions during 18 months post ICON (August 2009 to February 2011) were audited. Following the introduction of ICON the percentage of unplanned admissions fell from $36.68 \%$ to $22.9 \%$. These patients also had a lower mortality rate $(14.57 \%$ vs. 9.36\%) and the SMR decreased from 1.55 to 1.35 .

Conclusion Our data show that the mortality rate has decreased since the introduction of ICON although a confounding factor could be a concurrent decreased crude mortality rate $5.5 \%$ in 2003 to 2004 vs. $4.2 \% 2008$ to 2010 ) in all paediatric intensive care patients in the UK [2]. Despite this we believe that ICON is a significant contributing factor in identifying and rescuing patients on the wards before further significant deterioration requiring intensive care. Further ongoing audit is required.

References

1. FO Odetola, et al.: Do outcomes vary according to the source of admission to the PICU? Pediatr Crit Care Med 2008, 9:20-25.

2. Paediatric Intensive Care Audit Network [http://www.picanet.org.uk]

\section{P506}

In-hospital rapid response system: effects on outcome and workload

D Liberti, C Di Maria, P De Luca, M Alberico, C Popa, O Sagliocco, MR Scalzulli, E De Blasio

Hospital G. Rummo, Benevento, Italy

Critical Care 2012, 16(Suppl 1):P506 (doi: 10.1186/cc11113)

Introduction The implementation of an in-hospital rapid response system (RRS) could improve the outcome of a deteriorating patient but could increase the medical emergency team (MET) and ICU staff workload $[1,2]$.

Methods $\mathrm{A}$ retrospective analysis of the years pre, during and post implementation of a RRS in a 480-bed hospital with a mean of 17,500 admissions/year.

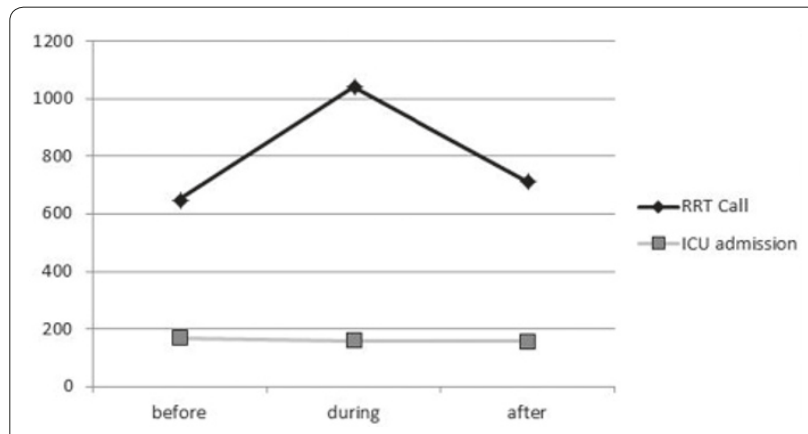

Figure 1 (abstract P506). MET calls and ICU admission before, during and after the RRS implementation.

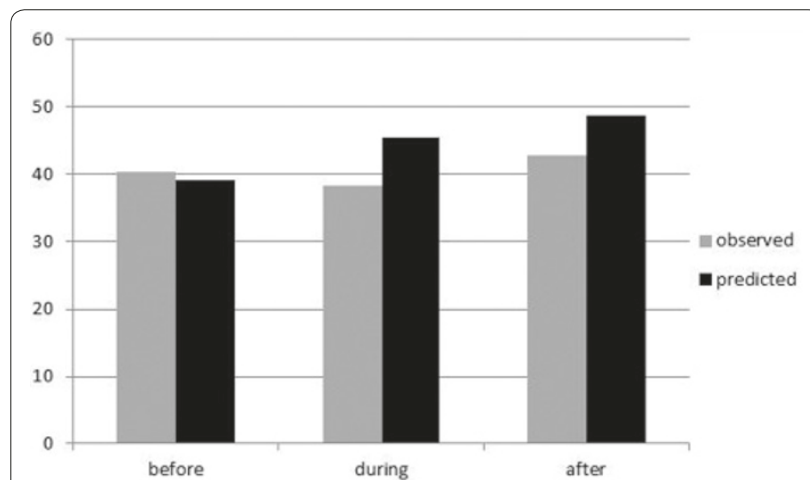

Figure 2 (abstract P506). Hospital mortality predicted and observed before, during and after the RRS implementation.
Results The number of MET calls initially increased from 34 to 56 and then decreased to 39 calls/1,000 admissions/year. Most of the calls were from the emergency department and less from medical and surgical wards. The number of ICU admissions did not increase (Figure 1). During the period of study there was a reduction of observed mortality compared to that predicted from SAPS II score, especially in surgical patients (Figure 2). Finally, there was an increase of ICU length of stay (LOS) from 11.5 to 13.7 days and a reduction of hospital LOS from 24 to 23.1 days.

Conclusion The implementation of RRS could result in a temporary increase of MET calls but not of ICU admissions; moreover, it could lead to a reduction of mortality and hospital LOS, but not of ICU LOS.

References

1. Acutely III Patients in Hospital: Full Guideline [http://guidance.nice.org.uk/ CG50/Guidance]

2. DeVita MA, et al.: Crit Care Med 2006, 34:2463-2478.

P507

Medical emergency team admittance to intensive care versus conventional admittance: characteristics and outcome

G Jäderling, M Bell, CR Martling, A Ekbom, M Bottai, D Konrad Karolinska Institutet, Stockholm, Sweden

Critical Care 2012, 16(Suppl 1):P507 (doi: 10.1186/cc11114)

Introduction The purpose of the medical emergency team (MET) is to find and treat deteriorating ward patients. Suboptimal care and delays on general wards before admission to intensive care have an effect on mortality [1] and patients admitted from general wards have a worse outcome than from the operating room (OR) or emergency department (ED) [2]. MET patients have a high rate of ICU admissions but whether their outcome differs from other patients admitted from the wards has not been studied before. We evaluated characteristics and outcome of ICU patients based on mode of admittance, via the MET versus the conventional way.

Methods An observational prospective study of patients admitted from general wards to the central ICU at Karolinska University hospital, Stockholm, Sweden in 2007 to 2009. Two groups were identified: admissions directly following a MET call or the conventional way, usually on request from the ward physician. Patients were analyzed for age, gender, co-morbidities, length of stay, severity scoring system (APACHE II) and mortality.

Results Of 2,571 ICU admissions, 694 admissions in 643 patients came from the wards. In total, 355 were admitted by the MET and 339 were conventional admissions. Median age was 65 years in the MET group versus 58 years in the conventional group, hospital LOS prior to ICU admission was median 3 days versus 1 day and APACHE II score was a mean of 26 versus 21. They did not differ as to proportion of invasive ventilator treatment or dialysis but MET patients more often received noninvasive ventilation, $57.2 \%$ versus $29.2 \%(P<0.01)$. ICU mortality was $14.5 \%$ versus $8.9 \%(P=0.04)$ and 30 -day mortality $27.0 \%$ versus $19.1 \%(P=0.02)$. MET patients also had a higher proportion of comorbidities, with a prevalence of heart failure in $17.3 \%$ versus $11.7 \%$ $(P=0.0 .4)$ and malignancy in $45.3 \%$ versus $35.1 \%(P<0.01)$ as well as a higher proportion of limitation of medical treatment (LOMT), $23.0 \%$ versus $15.7 \%(P=0.02)$. When LOMT patients were excluded, mortality rates were no longer significantly different, ICU mortality then being $5.7 \%$ versus $3.3 \%(P=0.2)$.

Conclusion Two distinct groups of patients with intensive care needs are found in general wards. Those admitted by the MET are older, have more severe co-morbidities and have been in hospital longer. We find the MET to be an important tool to identify patients with multiple problems and at high risk of an adverse outcome.

References

1. McQuillan et al:: BMJ 1998, 316:1853-1858.

2. Goldhill et al:: Crit Care Med 1998, 26:1337-1345.

P508

Factors affecting critical care admission to a UK university hospital A Tridente', A Chick', S Keep', S Furmanova², S Webber', DC Bryden' 'Sheffield Teaching Hospitals, Sheffield, UK; '2University Hospital Wales, Cardiff, UK Critical Care 2012, 16(Suppl 1):P508 (doi: 10.1186/cc11115)

Introduction Access to critical care is limited, with disparity existing between availability and demand. Guidance to inform triage decisions 
has been published but may no longer reflect current pressures [1,2]. We aimed to identify a set of criteria able to reliably predict likelihood of admission to a critical care unit in a large UK tertiary care centre. Methods Consecutive patient referrals were prospectively enrolled in a review cohort. Data were collected using a predefined case report form (CRF). The CRF included information on the referral, acute physiological parameters, hospital length of stay (LOS), demographic and functional status, dependency and comorbidities. Logistic regression was performed to identify factors predicting admission, employing STATA [3]. Results Between 17 July and 27 November 2011, 201 patients were referred to critical care, of whom 85 (42.7\%) were declined. Median age (interquartile range) was 67 (54 to 79$)$ years, 121 (60.8\%) were male, median LOS (interquartile range) was 1 (1 to 3 ) day. Age, gender, ethnic origin, LOS, referral reason, and markers of acute physiological derangement did not impact on likelihood of admission to critical care. Odds ratios $(95 \% \mathrm{Cls})$ for admission were 3.1 (1.72 to 5.56) for exercise tolerance $>100$ yards $(P<0.001), 3.03$ (1.56 to 5.89 ) for self-caring status $(P=0.001), 0.38$ ( 0.2 to 0.71$)$ for house-bound status $(P=0.003), 0.28$ (0.1 to 0.76$)$ for wheelchair-bound status $(P=0.013), 0.41$ ( 0.23 to 0.74 ) for cardiovascular $(P=0.003), 0.36$ (0.18 to 0.72$)$ for renal system $(P=0.004), 0.34(0.14$ to 0.85$)$ for malignant $(P=0.021)$, and 0.49 ( 0.25 to $0.94)$ for neurological $(P=0.033)$ comorbidities, respectively.

Conclusion Our data suggest that critical care admission decisions are made based mainly on the assessment of patients' pre-morbid state and functional capacity, rather than on the extent of acute physiological derangement. This behaviour is more consistent with the application of a prioritization model, defining those patients who will benefit most from critical care admission (Priority 1 ) to those who will not benefit at all (Priority 4) and consistent with pressured resources, rather than an objective parameters model or a diagnostic model [1].

References

1. Guidelines for intensive care unit admission, discharge, and triage. ACCCM, SCCM. Crit Care Med 1999, 27:633-638.

2. Fair allocation of intensive care unit resources. ATS. Am J Respir Crit Care Med 1997, 156:1282-1301.

3. STATA 10.1. College Station, TX: StataCorp.

\section{P509}

Intensive care services in Hungary 2000 to 2010: an analysis of bed numbers, occupancy rates, case mix and economics

A Csomos 1 , B Fulesdi², M Gresz

'Semmelweis University, Budapest, Hungary; 'University of Debrecen, Hungary: ${ }^{3}$ National Institute for Quality and Organisational Development in Healthcare, Budapest, Hungary

Critical Care 2012, 16(Suppl 1):P509 (doi: 10.1186/cc11116)

Introduction The purpose of this study is to describe the changes in pattern of intensive care (ICU) use over a 10-year period in Hungary. We attempt to analyze national data in order to improve resource use. Methods A retrospective analysis of national data provided by the hospitals for reimbursement of care to the National Healthcare Fund of Hungary between 2000 and 2010.

Results The total number of active hospital beds decreased by $33.4 \%$ (from 65,532 to 44,300); however, the number of ICU beds increased by $9.8 \%$ (from 1,189 to 1,306 ) between 2000 and 2010. As a result, the percentage of ICU beds to hospital beds increased from $1.89 \%$ in 2000 to $2.95 \%$ in 2010 . The ICU bed occupancy rate ranged between $58.43 \%$ and $63.78 \%$; it showed no correlation with the case mix index $\left(r^{2}=0.2799\right)$. The number of ventilator days increased from $28.9 \%$ to $66.1 \%$; it showed good correlation with the case mix index $\left(r^{2}=0.9125\right)$. Analysing 2010 data, we found significantly lower mortality in level III units $(30 \pm 18 \%)$ compared to level II $(51 \pm 20 \%)$ and level I $(56 \pm 19 \%)$ care $(P=0.001$ and 0.003$)$, without significant differences in case mix index (Table 1). The mean ICU bed occupancy rate was $59.5 \%$ (SD $\pm 12 \%$ ), and length of hospital stay was $12.3(\mathrm{SD} \pm 3.0)$ in 2010. Geographic distribution of ICU beds per 100,000 population ranged between 7.3 and 27.4 (nationwide 12.9/100,000); it showed no correlation with regional gross domestic product values $\left(r^{2}=0.4593\right)$.

Conclusion Our data suggest that intensive care beds are not utilized; a progressive level of care does not function and also there are unnecessary regional differences in intensive care provision in Hungary.
Table 1 (abstract P509). Distribution of intensive care services in 2010

\begin{tabular}{lcccc}
\hline National data, & $\begin{array}{c}\text { Total } \\
\text { number } \\
\text { of units }\end{array}$ & $\begin{array}{c}\text { Total } \\
\text { number } \\
\text { of beds }\end{array}$ & $\begin{array}{c}\text { Case mix } \\
\text { index } \\
\text { (mean } \pm \text { SD) }\end{array}$ & P value \\
\hline University hospitals (level III) & 10 & 412 & $7.67( \pm 4.06)$ & 0.204 \\
County hospitals (level II) & 30 & 584 & $8.08( \pm 2.89)$ & 0.376 \\
City hospitals (level I) & 39 & 280 & $6.05( \pm 1.97)$ & 0.093 \\
\hline
\end{tabular}

P510

Data acquisition for the UK Critical Care Minimum Data Set:

validation of a computer model for automatic calculation from an electronic patient record

A Clarke, M Thomas, T Gould, C Bourdeaux

Bristol Royal Infirmary, Bristol, UK

Critical Care 2012, 16(Suppl 1):P510 (doi: 10.1186/cc11117)

Introduction This study reports the accuracy of a computer and a manual system at collecting data for the UK Critical Care Minimum Data Set (CCMDS). This is required by the Department of Health to compare performance, to facilitate funding and to plan future resource provision. There are 14 data fields in the mandatory dataset, and the full compliment extends to 34 fields. At present this is collected manually, which is laborious and subjective. We use an electronic patient record (Innovian, Draeger, Germany) to store all the measured patient observations and laboratory results. We have written a program to interrogate Innovian for the CCMDS data, thereby reducing the administrative time.

Methods A stratified sample of 50 patients' data (elective and emergency surgical and medical patients) was analysed. Both manual and computer systems collected the mandatory 14 items of the CCMDS. This consists of six demographic variables (for example, admission date, discharge date, date of birth) and eight organ support variables (for example, duration of either advanced or basic cardiovascular, respiratory, renal or neurological support or duration of level 2 or 3 support). Where the computer and manual systems returned different values, a blinded physician analysed the patient records and created a gold standard value. The frequency of these differences was analysed. Results Both computer and manual systems returned all the required data, giving a total of 700 data variables. Different values were returned for $183(26 \%)$ variables. The systems had good concordance in the demographic variables, with only 4/300 (1.3\%) discrepancies between the computer and manual systems. In the organ support variables, there were $179 / 400(45 \%)$ discrepancies. Days of renal support had most concordance, with discrepancies in $3 / 50$ patients $(6 \%)$. Days of level 2 support had least concordance, with discrepancies in 37/50 patients (76\%). Overall, the computer method returned the correct variable for 544 (78\%) variables, where the manual system returned the correct variable on 591 (84\%) variables.

Conclusion This study shows that both computer and manual data collection methods could be improved, but at present both have similar accuracy. This may be because the criteria for some organ support can be subjective (for example, risk of deterioration), which can be interpreted in different ways between manual data collectors but not by a computer. We plan to rewrite the computer program, aiming for $>95 \%$ concordance with the gold standard.

\section{P511}

To admit or not to admit? The suitability of critical care admission criteria

D Marriott, ZTurner, N Robin, S Singh

Countess of Chester Hospital, Chester, UK

Critical Care 2012, 16(Suppl 1):P511 (doi: 10.1186/cc11118)

Introduction During the 2010/2011 winter the H1N1 influenza pandemic placed increased demand on critical care services, prompting our department to devise a modified triage tool for the ICU to be implemented at a time of exceptional bed crisis [1]. Scoring systems such as APACHE or Sequential Organ Failure Assessment 
(SOFA) have been used to predict mortality and optimize critical care service utilization [2]. This audit aimed to validate our triage tool for patients admitted to the ICU.

Methods We retrospectively examined patient notes for all admissions to our adult ICU during December 2010 and January 2011. Patient admission criteria $\left(\mathrm{SpO}_{2}<90 \%\right.$ on $\mathrm{FiO}_{2}>85 \%$, respiratory acidosis $\mathrm{pH} \leq 7.2$, respiratory failure or airway compromise, systolic pressure $<90 \mathrm{mmHg}$, SOFA score $\geq 7$ ) or refusal criteria (SOFA score $\geq 12$, severe trauma, unwitnessed or non-VF arrest, severe life-limiting condition) were recorded with outcome data.

Results We analysed 27 sets of notes. Twenty-two patients (81\%) fulfilled at least one admission and no refusal criteria. Two patients (7\%) had documented refusal criteria. The first of these had a severe life-limiting condition, staying 29 days in the ICU and a further 65 days in hospital. The second was admitted post non-VF arrest, dying after 2 days in the ICU. Three patients (11\%) met no admission criteria. These patients stayed between 4 and 6 days in critical care with total hospital stays of 18 to 98 days, one requiring 30 days of rehabilitation.

Conclusion The proposed admission criteria concurred with clinical decision-making in $81 \%$ of admissions. The patients that met refusal criteria required either prolonged hospital stay or had short survival times and may not represent optimal utilization of critical care facilities during a time of increased demand. Those patients not meeting the admission criteria had short critical care stays illustrating that rigid admission requirements may exclude patients who could benefit from critical care. A standardized set of admission criteria may supplement decision-making during times of increased critical care demand and strengthen documentation of those decisions. However, no set of criteria can replace clinical judgement in critical care admission. References

1. Christian MD, et al.: Development of a triage protocol for critical care during an influenza pandemic. CMAJ 2006, 175:1377-1381.

2. Ling CY, et al.: Outcome scoring systems for acute respiratory distress syndrome. Shock 2010, 34:352-357.

\section{P512}

Out-of-hours discharge from the ICU: defining the out-of-hours period and its effect on mortality

YL Bramma, R Allan, R Sundaram

Royal Alexandra Hospital, Paisley, UK

Critical Care 2012, 16(Suppl 1):P512 (doi: 10.1186/cc11119)

Introduction Out-of-hours discharge from the ICU is associated with increased mortality. In Scotland, approximately $15 \%$ of discharges occur out of hours [1]. The aim of this study was to determine the reasons behind out-of-hours discharges in our hospital and the effect this has on mortality.

Methods We carried out a retrospective analysis of all patients admitted to our ICU over a 3-year period. Patients who died during their ICU stay, patients $<16$ years, patients transferred to another ICU, and those with missing data were excluded. Data collected: patient demographics, APACHE II score, time of discharge from the ICU, reason for out-of-hours discharge, and hospital mortality. The out-of-hours period was defined as per the Scottish Intensive Care Society (SICS) as 20:00 to 07:59 hours, then later re-defined as 17:00 to 07:59 hours.

Results A total of 766 patients were included: 607 discharged between 08:00 and 19:59 hours, 159 discharged between 20:00 and 07:59 hours. Data are expressed as mean values (SD) or percentages, 'in hours' versus 'out of hours'. Both groups were similar: age 51.9 (18.1) versus 54.0 (17.7) years, males $48.9 \%$ versus $50.9 \%$, APACHE II score 15.8 (8.7) versus 17.4 (8.0). Hospital mortality following ICU discharge was 9.9\% (55/607 deaths) versus $10.0 \%$ (16/159 deaths), RR 1.11 (95\% Cl 0.66 to 1.88 ). Discharge was delayed due to a shortage of ward beds in $28.5 \%$ versus $43.4 \%$ of cases. No early discharges were recorded. With the out-ofhours period re-defined: 393 patients were discharged between 08:00 and 16:59 hours, 373 between 17:00 and 07:59 hours. Both groups were similar: age 51.0 (18.4) versus 53.8 (17.5) years, males $49.9 \%$ versus $48.8 \%$, APACHE II 14.9 (8.7) versus 17.4 (8.2). Hospital mortality was $7.7 \%$ (28/393 deaths) versus $11.5 \%$ (43/373 deaths), RR 1.62 (95\% Cl 1.03 to 2.55 ). Discharge was delayed due to a shortage of ward beds in $22.7 \%$ versus $41.0 \%$ of cases. ICU step-down is most safely performed when medical staffing levels on the wards are highest. The SICS define the out-of-hours period based on the time of handover to nightshift. For discharges at this time, there was no increase in mortality. In our hospital, evening ward cover is the same as overnight. For an outof-hours period of 17:00 to 07:59, there was a significant increase in mortality following out-of-hours discharge.

Conclusion Our data show increased mortality following ICU stepdown in the evening as well as at night. Discharge was most often delayed due to a lack of ward beds. To reduce mortality, efforts must therefore be made to improve bed management and ensure discharge from the ICU before 17:00.

\section{Reference}

1. Scottish Intensive Care Society Audit Group: Audit of Critical Care in Scotland 2011, Reporting on 2010; 2011. Edinburgh: ISD Scotland.

P513

Delayed discharges revisited: impact of a liaison post on patients' transition from ICU to ward care

J Mellinghoff, P O'Shea, D Dawson, J Ball, A Rhodes, M Grounds

St George's Healthcare NHS Trust, London, UK

Critical Care 2012, 16(Suppl 1):P513 (doi: 10.1186/cc11120)

Introduction This audit reviewed the discharge process of patients from an adult general ICU to the general wards before and after the introduction of a liaison nurse post over a 3-year, 3-month time period. Methods The audit utilised routinely collected retrospective data from a 17-bed ICU. We examined the impact of a liaison post on the length of delays on discharge of patients from the ICU to the general wards. Results The study period was from April 2008 until June 2011 with the start date of the liaison nurse post in January 2010. Overall, there were 4,327 patient discharges to hospital wards (before group $=2,063$, after group $=2,264$ ). The odds of experiencing a delay in discharge $>4$ hours were 3.2-fold higher in the before group compared to the after group $(95 \% \mathrm{Cl}=2.808$ to $3.717, P<0.0001)$. Accumulated discharge delays decreased by $23 \%$ from 1,116 (before group) to 864 days (after group) despite an increase in patient turnover of $10 \%(n=201)$. The median delay time was 7.2 hours (IQR 5.0 hours, 10.4 hours) in the before group and 5.3 hours in the after group (IQR 2.7 hours, 9.0 hours). See Figure 1. Conclusion Our analysis suggests that the introduction of a liaison nurse post within intensive care significantly reduced the length of delays in the discharge process despite an increase in patient turnover.

P514

Assessing demand for intensive care services: the role of

readmission rates

RA O'Leary, B O'Brien

Cork University Hospital, Cork, Ireland

Critical Care 2012, 16(Suppl 1):P514 (doi: 10.1186/cc11121)

Introduction Irish ICUs typically have bed occupancy rates approaching $100 \%$, with 75 to $80 \%$ being the recommended level [1]. Detection of excessive demand from simple databases can thus be difficult: expedited turnover and cancellations of elective surgery often ensue, leaving occupancy rates unchanged. We hypothesised that excessive demand would produce higher readmission rates, thus illustrating the strain imposed on ICU resources during the $\mathrm{H} 1 \mathrm{~N} 1$ influenza pandemic. Methods The GICU database was examined from 1 March 2010 to 1 March 2011. The H1N1 pandemic was recognised as a period of strain on the ICU and this period was estimated as 24 December 2010 to 21 January 2011. All ICU readmissions during the same hospital stay were noted. Transfers between GICU, cardiac ICU and theatre recovery were excluded as patients were still being treated by the intensive care team. Patients readmitted after transfer for extracorporeal membrane oxygenation (ECMO) were also excluded.

Results The number of GICU admissions during the period was 422 . There were 19 readmissions (readmission rate of $4.6 \%$ ). However, this rate increased to $8.6 \%$ during the period of high activity encompassing the H1N1 pandemic (Figure 1). Hospital mortality was $36.8 \%$ in the readmission group, higher than the average, $24.6 \%$, for the whole GICU population. This is in keeping with previous research showing up to an 11 -fold increase in relative risk of mortality in patients readmitted to the ICU [2]. 

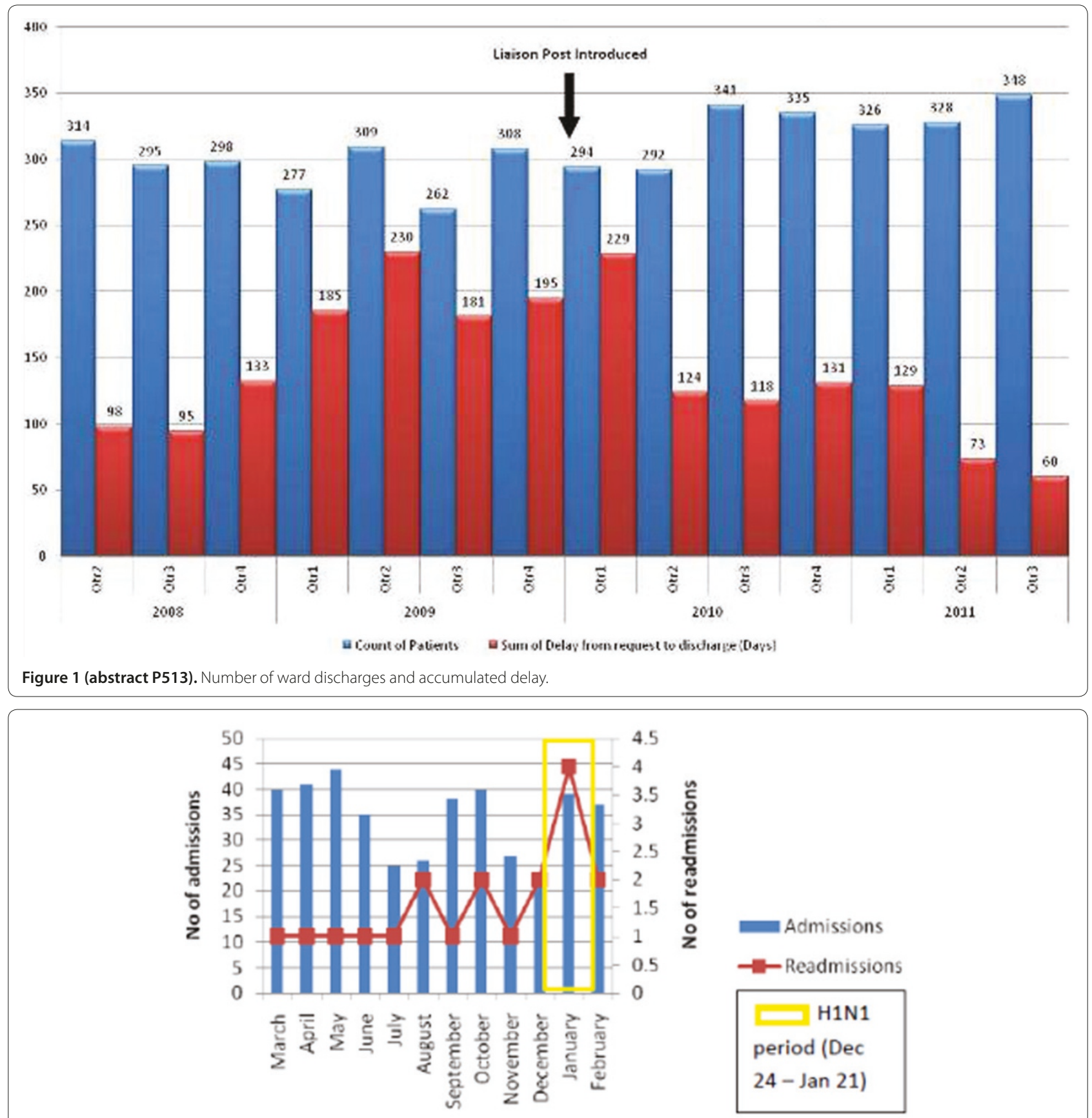

Month

Figure 1 (abstract P514). Readmissions over time.

Conclusion The annual readmission rate for our unit was acceptable [3]. A clear spike was noted during the period of the H1N1 pandemic. Whilst this is a pattern we hope to address, it is a useful indicator of increased demand. Our study suggests that readmission trends in a single institution may be helpful when analysing the severity of epidemics, planning staffing needs, and comparing periods of heightened demand.

\section{References}

1. Intensive Care Society: Standards for Intensive Care Units. London: ICS; 1997.

2. Rosenberg AL, et al:: Crit Care Med 2001, 29:511-551.

3. Rosenberg AL, et al:: Chest 2000, 118:492-502.
P515

Management of cardiac drugs in a critical care setting

M Mallick', J Walkington', A Gratrix', R Pretorius²

${ }^{1}$ Hull Royal Infirmary, Hull, UK; ${ }^{2}$ York Teaching Hospital, York, UK

Critical Care 2012, 16(Suppl 1):P515 (doi: 10.1186/cc11122)

Introduction ICU admissions may lead to discontinuation of longstanding evidence-based therapies. A recent study demonstrated how such medications have been discontinued for patients even after their ICU stay [1]. Evidence has shown the beneficial role of $\beta$-blockers in the perioperative period [2], and roles for other drugs such as 
angiotensin-converting enzyme inhibitors (ACE-I) and statins have been demonstrated. The aim of the current study was to examine 30day mortality and complication rates in the critical care population who were on cardiac medications and did not receive these medications during their ICU stay.

Methods We looked retrospectively at the last 80 patients admitted to the ICU or HDU in York, 2011. The patients' case notes were examined to assess if they were on cardiac medications and if those drugs were omitted during their admission. The cardiac medications assessed were $\beta$-blockers, ACE-I and statins. We also reviewed any cardiac complications incurred during their stay, alongside 30-day mortality. Results A total of $29.6 \%$ of patients on $\beta$-blockers received them, whilst $67.8 \%$ did not. Complication and mortality rates for medications given versus not given were $12.5 \%$ versus $68.4 \%$ and $0 \%$ versus $42.1 \%$ $(P=0.003$ and $P=0.007)$ respectively. A total of $17.6 \%$ of patients on ACE-I received them, whilst $82.3 \%$ did not. Complication and mortality rates for medications given versus not given were $0 \%$ versus $9.0 \%$ and $0 \%$ versus $35.7 \%(P=0.004$ and $P=0.055)$ respectively. A total of $31.6 \%$ of patients on statins received them, whilst $68.4 \%$ did not. Complication and mortality rates for medications given versus not given were $25.0 \%$ versus $42.3 \%$ and $8.3 \%$ versus $38.5 \%(P=0.256$ and $P=0.02$ respectively). The global complication and mortality rates for medications given versus not given were $28 \%$ versus $55.2 \%$ and $11.5 \%$ versus $51.7 \%(P=0.0648$ and $P=0.0039)$ respectively. Omission of $\beta$-blockers resulted in significantly higher complication and mortality rates. Omission of ACE-I resulted in higher complication rates and of statins in higher mortality rates. Omission of cardiac medications resulted in a significantly higher mortality rate.

Conclusion The study does highlight a trend associated with patients who are on medications who do not receive them to either develop higher complication rates or higher mortality rates or both. Further research involving larger numbers is required to produce validated opinions.

References

1. Bell CM: JAMA 2011, 306:840-847.

2. $2009 \mathrm{ACCF} / \mathrm{AHA}$ focused update on preoperative beta blockade: a report of the American College of Cardiology Foundation/American Heart Association task force on practice guidelines. Circulation 2009, 120:2123-2151.

P516

Pharmacists and fastidiousness improve compliance with guidelines for stress ulcer prophylaxis

S Sanders, KC Shelley, AJ Marsh

Frenchay Hospital, Bristol, UK

Critical Care 2012, 16(Suppl 1):P516 (doi: 10.1186/cc11123)

Introduction This audit assessed compliance with guidelines for the use of stress ulcer prophylaxis (SUP) in our mixed general/neurosurgical ICU. These patients are at increased risk of gastrointestinal bleeding with clinically important bleeding occurring in about $3.5 \%$ of patients ventilated for 48 hours or more [1]. SUP guidelines: all patients at risk of stress ulceration (coagulopathy/IPPV $>48$ hours/nasogastric (n.g.) feed not absorbed) or already on ant acids should receive ranitidine, enterally where possible. Exceptions are patients on a proton pump inhibitor (PPI) prior to ICU admission. PPIs should continue enterally if possible as lanzoprazole, or as omeprazole i.v.

Methods Data were collected from May to August 2010 (Period 1). Results from this were discussed and the following interventions adopted prior to further data collection (Period 2: August to November 2011): prescription of SUP in all ventilated patients on admission to the ICU; discontinuation of SUP after 48 hours if n.g. feeding tolerated; documented daily review of SUP including consideration of discontinuation, drug, route and dose used; and the presence of the ICU pharmacist on ward rounds, briefed specifically to prompt correct SUP use.

Results Period $1(n=86)$ revealed excess use of SUP, excess use of PPIs when ranitidine was indicated, unnecessary i.v. administration and failure to discontinue prophylaxis appropriately. Period $2(n=71)$ demonstrated: no fall in SUP use in those with indications ( $93 \%$ vs. $97 \%, P=0.65$ ); increased prescription accuracy in terms of drug, dose and administration route ( $40 \%$ vs. $84 \%, P=0.0001)$; no increased unindicated SUP use; and reduction in inappropriate i.v. administration ( $23.1 \%$ vs. $0 \%, P=0.0024)$.

Conclusion Emphasis on the guidelines for SUP to all members of the team, especially the pharmacist, improves compliance. Inclusion in SUP prescriptions of the intended discontinuation date may further reduce excessive duration of treatment. Re-audit will occur after implementation of new guidelines which acknowledge the diminishing benefit from SUP and the not-insignificant risks associated with its use. Reference

1. Cook DJ, et al.: Crit Care 2001, 5:368-375.

\section{P517}

Healthcare workers' experience when using an electronic medical order entry and bar-code technology in an ICU

R Fumis, I Souza, V Pizzo, G Schettino

Hospital Sírio-Libanês, São Paulo, Brazil

Critical Care 2012, 16(Suppl 1):P517 (doi: 10.1186/cc11124)

Introduction Medication errors are frequent in the ICU and may occur during medical ordering, transcription or administration of drugs. A system consisting of a computerized physician order entry (CPOE) with bar-code verification of medications (TASY; Web Sistemas, Brazil) has been described as a tool to improve medication safety [1], but few data are available about the satisfaction of healthcare workers with the use of this new technology in the ICU.

Methods We conducted a survey to evaluate the satisfaction of healthcare workers when using a CPOE with bar-code verification of medications in a tertiary 40-bed adult ICU in Sao Paolo, Brazil 6 months after implementing the system. A satisfaction questionnaire which consisted of items in a numeric scale type from 1 (low satisfaction) to 10 (high satisfaction) was filled out by physicians $(n=42)$, nurses $(n=58)$, nurses technicians $(n=84)$ and other professionals $(n=66)$.

Results Most subjects were female (66\%), below 36 years of age (69\%) and used the computer daily at home (81\%). On average, respondents were satisfied with the CPOE system (score $5.74 \pm 2.14$ ) and believed it improved safety (score $7.64 \pm 2.42$ ). Satisfaction was lower among physicians (score $4.62 \pm 1.79$ ) when compared to other professionals (score $5.97 \pm 2.14 ; P<0.0001$ ). The ease to place the first medical order and to copy the order form the previous day scored $5.41 \pm 2.05$ and $6.39 \pm 1.93$. The visualization of the medical order with the bar-code verification of drugs administration scored $5.95 \pm 2.51$ by the nurses. On average, physicians found the system less user-friendly (score $3.88 \pm 1.85)$ than other professionals $(6.40 \pm 2.29 ; P<0.0001)$.

Conclusion Although most of the ICU staff believe that the CPOE and bar-code has the potential to improve medication safety and the quality of care for critically ill patients, our survey showed a low level of satisfaction 6 months after implementing the system, particularly for physicians who consider the system unfriendly.

Reference

1. Poon EG, et al:: N Eng/ J Med 2010, 362:1698-1707.

\section{P518}

Safer ICU trainee handover: a service improvement project

E Godfrey', I Hassan', A Carson-Stevens², AG Saayman

'University Hospital of Wales, Cardiff, UK; ${ }^{2}$ Cardiff University, Cardiff, UK

Critical Care 2012, 16(Suppl 1):P518 (doi: 10.1186/cc11125)

Introduction Quality handover between team members within the ICU is vital for patient safety. Critically ill patients are at high risk of medical errors; these complex patients are exposed to high-risk interventions, medical and procedural [1]. Distractions are known to be particularly prevalent within critical care [2]. This can compromise handover efficiency, interrupt information-giving and may ultimately lead to poorer patient outcomes [3]. We sought to demonstrate the capability of junior physicians to lead change to their practices that benefit the quality of patient care in a large critical care unit. We present an improvement project that has transformed handover quality in our ICU. Methods Participant observation of handover practices took place within a high-occupancy 33-bed adult ICU. Quantitative assessment of handover criteria as per Royal College of Anaesthetists guidelines 
[4] was performed at baseline (handovers: $n=6$, patients: $n=119$ ) and 3 months post-intervention (handovers: $n=4$, patients: $n=108$ ). Interventions included presentation of data at multiprofessional departmental meetings, education of team members regarding frequency of handover interruptions and development and utilisation of an electronic handover tool.

Results Provision of patient details during handover was substandard. Utilisation of a structured handover sheet significantly improved the number of patient details provided; in particular, patient age (18\% vs. $100 \%)$, duration of stay ( $29 \%$ vs. $79 \%)$ and medical management plan $(53 \%$ vs. $93 \%)$. Frequent handover interruptions seen on initial observation significantly improved $(100 \%$ vs. $25 \%$ of handover periods interrupted) following our collaboration with the senior nurse, physiotherapist and other team leads regarding the number and nonurgent nature of interruptions; at re-audit, interruptions occurred for clinically urgent requests only.

Conclusion Simple measures instituted by junior doctors, such as team education and use of a structured handover tool, can aid high-quality handover within critical care. Evidence suggests that high-quality handover within critical care will translate into improved clinical care for patients.

References

1. Reader TW, et al:: Curr Opin Crit Care 2007, 13:732-736.

2. Horn J, et al:: Anaesthesia 2004, 59:658-663.

. Nimmo G, et al:: J/CS 2008, 9:240-242.

4. McQuillan P, et al:: In The Royal College of Anaesthetists. Raising the Standard: A Compendium of Audit Recipes. 2nd edition; 2006:218-219 [http://www.rcoa. ac.uk/docs/ARB-section10.pdf].

P519

ICU handover: are we forgetting something? A preliminary study

T Aslanidis, IL Chytas, A Kontos, I Soultati, A Efthmiou, E Geka,

$\checkmark$ Ourailoglou, E Anastasiou, M Giannakou-Peftoulidou

G.H. AHEPA, Thessaloniki, Greece

Critical Care 2012, 16(Suppl 1):P519 (doi: 10.1186/cc11126)

Introduction The aim of this ongoing study is to review the process of handover in a university teaching hospital ICU, highlight areas of special interest and deficiency during the process, and improve current practice. Clinical handover, defined as a process of transferring authority and responsibility for providing care of patients from departing caregiver to named recipient, is a basic part of clinical practice. Failure to exchange essential information and focus on the important may have disastrous consequences for the patient.

Methods A prospective observational study was undertaken over a 22-day period to examine the quality and content of clinical handover by nightshift doctor to the medical team. Key aspects expected to be handed over included patient details, diagnosis, system - treatment domains and communication with relatives. Additional data collected also included duration of handover and frequency of interruptions.

Results A total of 207 sets of patients were collected during the study period. All handovers were supervised by a consultant intensivist. Clinical information handed over verbally covered reason for admission in $12 \%$ of cases, working diagnosis in $13 \%$ and current management plan in $29 \%$ (100\% in these three in new admissions). Medical comorbidities where also poorly covered (8\%). The handover was rather focused on special aspects of clinical information like the respiratory system (86\%), fluid balance and laboratory findings (68\%), infections status (67\%), CNS (56\%) and hemodynamics (54\%), while nutrition and GI was poorly covered (20\%). Only $26 \%$ of handovers covered significant changes in the last shift, $21 \%$ commented on the interventions made and $32 \%$ had a proposed plan for the forthcoming day discussed. Of the allocated 30 minutes, the duration of the handover varied from 20 to 50 minutes (average 28 minutes). There was a total of 34 interruptions over 22 days of the audited period. Reasons for interruption included telephone calls and requests from visiting teams and nurses.

Conclusion Our study identified that the structure of the handover was rather focused on a system-based approach. Difficulty in concentration due to fatigue or frequent interruptions prolongs its duration and disturbs the right flow of information. The senior clinician must ensure that handover should be a focused but educational experience for the trainee with appropriate feedback.

\section{References}

1. Patterson ES, et al.: It Comm J Qual Patient Saf 2010, 36:52-61.

2. Brenier G, et al:: Crit Care 2011, 15:491.

P520

Quality and value of intensive care discharge summaries for general practitioners

F Daruwalla, FJ Lamb, CA Mearns

Surrey and Sussex Healthcare NHS Trust, Redhill, UK

Critical Care 2012, 16(Suppl 1):P520 (doi: 10.1186/cc11127)

Introduction Good communication between healthcare professionals is required to provide continuity of care for patients being discharged from the ICU [1]. It is our unit's practice to send a copy of a patient's computerized ICU discharge summary to both the hospital team with ongoing responsibility and to their general practitioner (GP). The aim of this study was to establish and compare the quality and value of the summaries as judged by ICU doctors and GPs.

Methods Discharge summaries for patients admitted in July 2011 were obtained from the ICU WardWatcher ${ }^{\oplus}$ database. These were scored independently by two ICU consultants and a trainee doctor using a predefined rating scale. The GPs were sent postal questionnaires regarding their perceptions of the quality and value of the summaries. A comparison was made between the ratings made by the ICU team and the responses to the GP questionnaires.

Results Sixty patients were admitted during the study period. All 60 summaries were independently rated by three ICU doctors and good inter-rater reliability was demonstrated (Cronbach's $a=0.89$ ). There was a strong correlation between the ratings given by the ICU consultants and the trainee doctor (Spearman's $=0.91$ ). Twenty-eight per cent achieved an acceptable score of 6 out of 10 or greater (median score 5 , interquartile range 3 to 6 ). Fifty-four postal questionnaires were sent to GPs and 36 were returned (response rate 67\%). Seventy-six per cent achieved an acceptable score of 16 out of 25 or greater (median score 18 , interquartile range 16 to 25 ). Sixty-nine per cent of GPs found the discharge summary helpful and $86 \%$ wanted to be sent this type of summary in future. Correlation between the ICU team rating and the GP score for the summaries was weakly positive (Spearman's $=0.15$ ). Conclusion Although only $28 \%$ of discharge summaries achieved an acceptable or higher rating from the ICU team, GPs valued the majority of discharge summaries issued by our ICU. Further research is needed to explain the difference between ICU doctors' perception of discharge summary quality and the value provided by them to GPs.

Reference

1. National Institute for Health and Clinical Excellence: Clinical Guideline 83: Rehabilitation after Critical IIIness. London: National Institute for Health and Clinical Excellence; 2009 [www.nice.org.uk/CG83].

P521

Volume-outcome relationship in critical care: a systematic review DJ Wallace', YL Nguyen², L Trinquart², DC Angus', P Ravaud², JM Kahn' 'CRISMA Center, University of Pittsburgh School of Medicine, Pittsburgh, PA, USA: ${ }^{2}$ Centre d'épidémiologie clinique, CHU Hôtel Dieu, Paris, France Critical Care 2012, 16(Suppl 1):P521 (doi: 10.1186/cc11128)

Introduction The relationship between provider volume and patient outcome has been demonstrated for many medical and surgical services, including critical care. This relationship is used as one rationale for regionalization of adult intensive care. However, the volumeoutcome relationship is not always consistent across studies, and it has not been explicitly evaluated in a heterogeneous population. We performed a systematic review of studies that assessed the association between volume and outcome among critically ill adult patients.

Methods We searched the MEDLINE and EMBASE databases for articles published between January 2001 and December 2011 using medical subject heading terms and text words for conditions related to critical illness in adults. Trauma studies were excluded. Two study investigators independently reviewed titles, abstracts and articles identified from the search algorithm and abstracted study-specific data using a standardized abstraction form. Variables of interest included study 
characteristics, patient characteristics, study period, volume definition, primary and secondary outcomes, risk-adjustment methodology, statistical analyses, results, risk of bias and funding body.

Results We reviewed 80 studies, of which 27 (34\%) met all inclusion criteria. Studies were excluded most commonly when the majority of the patients did not require critical care $(n=46)$, the study was presented only in abstract form $(n=4)$, data were duplicative $(n=2)$ or an outcome measure was not assessed $(n=1)$. One publication included three different patient populations; these were counted as separate studies. The final 29 studies represented seven clinical categories: respiratory $(n=9)$, postoperative $(n=7)$, cardiovascular $(n=4)$, general admissions $(n=3)$, sepsis $(n=2)$, neurological $(n=2)$ and gastrointestinal $(n=2)$. Eighteen studies (62\%) demonstrated a statistically significant association between higher patient volume and better health outcomes, although the magnitude of the relationship varied across diagnoses. No study showed a statistically significant association between higher volume and poorer outcomes.

Conclusion The majority of studies evaluating the volume-outcome relationship in critically ill patients demonstrated better outcomes with higher clinical volumes. There was variability in the association across diagnostic categories, indicating that quality improvement efforts based on the volume-outcome relationship such as regionalization of care may be more successful in specific patient subsets.

Acknowledgements Supported by NIH T32-HL07820.

P522

Radiation doses in young ICU patients: a cause for concern?

RA O'Leary, C Houlihane, P McLaughlin, M Maher, D Breen

Cork University Hospital, Cork, Ireland

Critical Care 2012, 16(Suppl 1):P522 (doi: 10.1186/cc11129)

Introduction The aim of this study was to quantify the radiation dose in young ICU patients to determine if it approached a clinically significant level. lonising radiation is a well-recognised risk factor for development of cancer. The risk is dose-related and there is no lower threshold at which the dose can be considered clinically irrelevant. The availability of computed tomography (CT) scanning has led to a significant increase in exposure to ionising radiation of patients over the last decade. Children and young adults are particularly at risk. This is partly because there is a longer lifetime in which radiation effects may be manifest but also because children are up to 10 times more sensitive to radiation than adults. In view of these issues it is important to quantify the risk to young ICU patients.

Methods The general ICU database was examined from 1 March 2010 to 1 March 2011. The overall radiation exposure was quantified using the cumulative effective radiation dosage (CED) in millisieverts ( $m S v)$. The CED was calculated for all of the procedures performed during the stay in the ICU using average procedure-specific effective doses published by the UK National Radiation Protection Board. A cohort of patients $<30$ years of age were selected for subanalysis.

Results There were 403 patients admitted to the general ICU during the period of interest. The number of patients $<30$ years of age was 75 with a mean age of 19 (range 0.5 to 30 years). The mean CED was 10.84 $\mathrm{mSv}(\mathrm{SD}=15.08)$ with 10 patients receiving $>30 \mathrm{mSv}$. The mean CED for patients who did not undergo CT examination was $0.063 \mathrm{mSv}(n=31$, $\mathrm{SD}=0.062)$. Trauma patients received a far higher dose $(21.86 \mathrm{mSv})$ than either medical (3.1 mSv) or postoperative surgical ( $3.96 \mathrm{mSv}$ ) admissions. Conclusion CT is a useful and necessary tool in our diagnostic and therapeutic armoury. However, our results show that young patients can potentially be exposed to significant doses of ionising radiation in an ICU setting mainly due to $C T$. In view of the lifetime risk of cancer to these patients we should try to minimise radiation exposure by more judicious utilisation of $\mathrm{CT}$ and by use of other imaging modalities.

References

1. Hart D, Wall B: Radiation Exposure of the UK Population from Medical and Dental X-ray Examinations. Chilton: National Radiological Protection Board; 2002. Kinsella SM, et al.: Kidney Int 2010, 78:789-793.

3. Dawson P: Br J Radiol 2004, 77(Spec No 1):S10-S13.

4. Slovis T: Radiology 2002, 223:5-6.

5. Cardis E, et al: Radiat Res 2007, 167:396-416.

6. Cascade PN, et al: AJR Am J Roentgenol 1998, 1770:561-564.
P523

Accuracy of height and weight estimation by critical care staff

KDunne, S Hickey

Forth Valley Royal Hospital, Larbert, UK

Critical Care 2012, 16(Suppl 1):P523 (doi: 10.1186/cc11130)

Introduction Patient's height and weight measurements are used regularly within the critical care setting whether for calculation of drug doses, nutritional intake, ventilator settings or calibration of cardiac output monitoring [1]. In sedated patients these parameters are often obtained via estimation by critical care staff. Errors in these estimations have the potential to cause harm either from errors in drug calculations [2], inappropriate ventilatory settings or underfeeding or overfeeding. Methods We asked members of the critical care team (medical, nursing staff, physiotherapists and dieticians) to anonymously estimate the heights and weights of patients within the unit at that time. Following this we obtained accurate measurements by measuring height with a measuring tape and patients' weight with the Scotweigh weighing machine. The results were then collated and the percentage inaccuracy of estimate compared to actual measurement was calculated.

Results There were 330 estimations made by 30 members of staff. Height estimation was accurate $\pm 10 \%$ for 291 patients $(88.4 \%$ ). Inaccuracy in height estimation ranged from $-9.5 \%$ to $+25 \%$ with a mean inaccuracy of $4.75 \%$. Weight estimation was accurate $\pm 10 \%$ for 123 patients (38.4\%). Inaccuracy of weight estimation ranged from $-48.9 \%$ to $+40.3 \%$ with a mean inaccuracy of $16.4 \%$. There was a tendency to underestimate weight with only 33 estimates (10\%) greater than $10 \%$ of actual weight and 174 estimates $(52.7 \%)$ less than $10 \%$ of actual weight. See Figure 1.

Conclusion Although height estimation was measured to within 10\% accuracy in the majority of cases, staff were considerably less reliable at estimating an accurate patient weight and on more than one-half of all estimates underestimated the weight by greater than $10 \%$. We therefore strongly discourage the practice of weight estimation in situations where clinical decisions are being based on an often unreliable value, and alternative means of obtaining an accurate weight measurement should be sought.

References

1. Wigfull J, et al:: Critical assessment of haemodynamic data. Contin EduC Anaesth Crit Care Pain 2005, 5:84-88.

2. Mahajan RP: Medication errors: can we prevent them? Br J Anaesth 2011, 107:3-5.

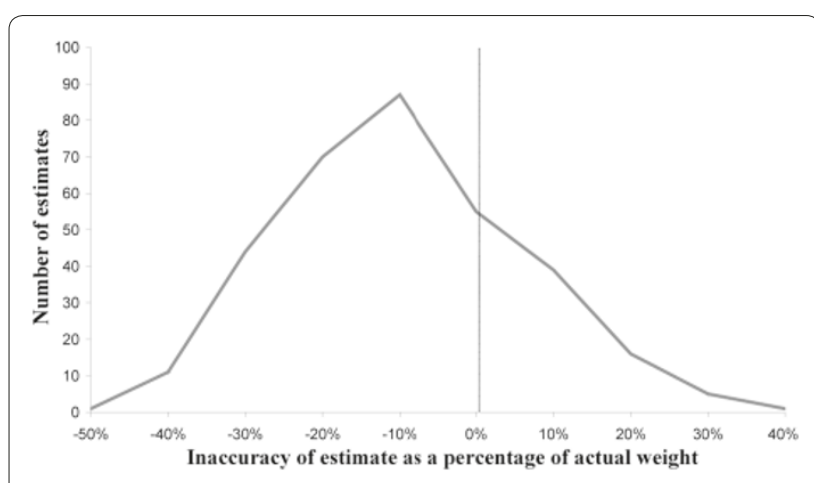

Figure 1 (abstract P523). Accuracy of weight estimation by critical care staff.

P524

Implementation of evidence-based care bundles in the ICU

G Juknevicius, E Balakumar, A Gratrix

Hull Royal Infirmary, Hull, UK

Critical Care 2012, 16(Suppl 1):P524 (doi: 10.1186/cc11131)

Introduction Implementation of an evidence-based care bundle in critically ill patients has been shown to improve outcome. Use of care bundles to reduce ventilator-associated pneumonia and other ICU complications has been increasing in critical care practice. 
Methods We conducted a prospective audit on implementation of a care bundle after audit approval. We collected data for 101 patient days from all patients admitted to Hull Royal Infirmary ICU during the month of November 2011. We collected information regarding stress ulcer prophylaxis, deep vein thrombosis (DVT) prophylaxis, ventilator care bundle, blood glucose control, daily assessment of need for a central line, sedation score assessment and delirium score assessment at least twice a day.

Results All patients received stress ulcer prophylaxis. At least $95 \%$ of patients received DVT prophylaxis, adequate blood glucose control and appropriate sedation need assessment. There was further scope for improvement in areas of sedation hold practice and assessing daily need for a central line. Poor clinical practice was identified in delirium score assessment and head elevation to reduce VAP. See Table 1.

Table 1 (abstract P524)

\begin{tabular}{lc}
\hline Intervention in eligible patients & Adherence, $n$ (\%) \\
\hline Stress ulcer prophylaxis & $101 / 101(100)$ \\
DVT/PE prophylaxis & $94 / 97(97)$ \\
Head elevation 30\% in ventilated patients & $62 / 75(83)$ \\
Daily sedation hold & $28 / 32(88)$ \\
Blood glucose control & $96 / 101(95)$ \\
Need for central line assessed & $73 / 85(86)$ \\
Sedation score assessment & $98 / 101(97)$ \\
CAM-ICU score at least twice a day & $29 / 101(28)$ \\
\hline
\end{tabular}

Conclusion It is very challenging to implement care bundles despite evidence showing that they improve outcome. A recent study suggests that doing a daily quality rounds checklist (QRC) will improve long-term compliance, thereby reducing potential complications for intensive care patients [1]. We have implemented QRC in our practice and will be re-auditing in 6 months to ensure continued adherence.

Reference

1. DuBose et al: Measurable outcomes of quality improvement in the trauma intensive care unit: the impact of a daily quality rounding checklist. $J$ Trauma 2008, 64:22-29.

P525

Awareness of difficult airway equipment on the ICU

A Wozniak, A lyer

Royal Liverpool University Hospital, Liverpool, UK

Critical Care 2012, 16(Suppl 1):P525 (doi: 10.1186/cc11132)

Introduction It is widely recognised that critically ill patients can be difficult to intubate, requiring the use of advanced airway skills and equipment. The range of airway equipment necessary for patients on the ICU has recently been recommended [1]. Our ICU has a comprehensive difficult airway trolley (DAT) which is regularly maintained. With a high turnover of trainees, we were keen to determine if there was a training need to be met regarding airway management in ICU patients. The objectives were to determine awareness of the DAT, assess knowledge of its contents and ascertain confidence in its use.

Methods We audited against previously described standards [1] using a short questionnaire, disseminated to trainees and consultants working on the ICU in November 2010: 100\% of clinicians should be aware of the location and contents of the DAT; $100 \%$ of anaesthetists should have had difficult airway equipment training.

A re-audit was conducted in June 2011 to complete the audit cycle.

Results One hundred per cent of clinicians were aware of the DAT. Only $35 \%$ had read the folder detailing its contents with instructions. Ninety per cent could confidently name the equipment which should be readily available for difficult intubations but only $70 \%$ were confident to use it unaided. Fifty per cent would request the presence of an operating department practitioner (ODP) for an unplanned intubation on the ICU. Twenty-eight per cent were not airway trained. Re-audit showed $100 \%$ of respondents were aware of the equipment. Sixty per cent had confidence in its use, a similar proportion to the original audit. Eighty per cent would have an ODP for unplanned intubations. One hundred per cent were airway trained. Outcomes A designated consultant was assigned to teach difficult airway management at quarterly departmental induction sessions which included equipment location and algorithms. Trainees and consultants underwent simulation and mannequin training, including tracheostomy and surgical airway management. Regular updates and case-based teaching sessions were implemented. Airway proficiency assessments were conducted at induction.

Conclusion This audit highlights our variable workforce. The presence of junior, nonairway-trained staff on the ICU calls for regular, compulsory airway teaching sessions for all, regardless of grade. Airway competency must be formally assessed at the start of an ICU attachment. Airway instructions for challenging patients should be clearly documented with advice on access to senior assistance for emergencies.

\section{Reference}

1. Jeanrenaud P, et al:: Difficult airway trolleys for the critical care unit. JICS 2010, 11:98-103.

P526

A new patient mobilization scoring system in the ICU: what is the degree of similarity in scores between assessors in daily use? M Vogel, CW Casteleijn, P Bruins, AJ Meinders

St Antonius Ziekenhuis, Nieuwegein, the Netherlands Critical Care 2012, 16(Suppl 1):P526 (doi: 10.1186/cc11133)

Introduction Inactivity and immobility in ICU patients have significant deleterious physiologic effects, including atelectasis, pressure ulcers, and increased susceptibility to aspiration and pneumonia. A new trend on the ICU is early mobilization of critically ill adult patients. However, evidence of when to start mobilization is missing. Casteleijn developed a new scoring system, the Patient Mobilization Frame (PMF), to improve early mobilization in the ICU. The framework is based on a multidisciplinary agreement. The aim of this study was to evaluate interobserver agreement using the PMF.

Methods A prospective observational study in 47 critically ill patients in the ICU was performed. The PMF categorizes patients into one of three stages of possible training using a scoring system based on 14 items. Various factors influencing individual stage are used including circulation, respiration, infection, kidney function, wounds and neurology. Stage A (critically ill) permits only passive physical examination. Whereas stage B (stable) and stage C (nearly recovered) permit (guided) active mobilization and functional training, respectively. Two staff members and one resident obtained 47 independent observation series of the PMF. All observations were at the same date and time and were compared.

Results Interobserver reliability of observers 1,2 and 3 proved to be adequate. Kappa for observers 1 and 2 was 0.9. Kappa for observers 1 and 3 was 0.6 . Kappa for observers 2 and 3 was 0.6 . The value of kappa can range from 0 (disagreement) to 1 (perfect agreement). Kappa larger than 0.6 was regarded as substantial agreement.

Conclusion Casteleijn's PMF proved to be a reliable scoring system as both resident and staff members had comparable results for staging the physical abilities of the critically ill patient in the ICU.

\section{P527}

Motor and respiratory intensive rehabilitation in bedridden patients

E Canedo, V Nunes Velloso, L Calejman, N Leidi

Hospital de Infecciosas F.J. Muñiz, Buenos Aires, Argentina

Critical Care 2012, 16(Suppl 1):P527 (doi: 10.1186/cc11134)

Introduction Inability to play significant social roles due to a pattern of motor disability affects the quality of a person's life, and is where the motor and respiratory rehabilitation process takes fundamental importance. This disability prevents one to function independently in basic tasks such as dressing and feeding and in more complex tasks such as handling in public and/or work. It can also be a constraint for the 
dependent patient in personal care activities. The objective of a motor rehabilitation plan is to reduce the impact caused by this alteration of motor ability, facilitating the restoration of functional patient capacity so they can effectively engage in occupations, reaching the highest level of functional independence possible.

Methods A cross-sectional retrospective descriptive and observational study of rehabilitation of bedridden patients in hospital from January 2010 to June 2011. The programme is implemented in Section 30 (21,9, and 20 rooms). The inclusion criteria for the rehabilitation programme were patients of both sexes, without age limit, inpatient of Hospital F.J. Muñiz coming from the ICU, in bedridden condition (limitation or motor disability in which the patient cannot move or perform activities of daily living and must depend on the care of others), with Barthel scale value 0 to 35 with total or severe dependence and stability hemodynamics.

Results We included patients who met the inclusion criteria. The program presented an intensive character in terms of the frequency of weekly sessions as the number of exercises implemented in the form was specified according to the pathology of the patient. Ninety percent of patients were male. The median age was 41 years. The predominant infectious pathology was pulmonary tuberculosis $(90 \%)$, cerebral toxoplasmosis $(50 \%)$, spastic paraplegia $(6 \%)$, bilateral pneumonia $(6 \%)$, and fumigares aspergillosis (6\%). The profit was $100 \%$ of kinesic treatment adherence, $94 \%$ of cases won full independence valued on the Barthel scale with a value of 100 , and a single case achieved independence moderated by the presence of spastic paraplegia.

Conclusion The intensive rehabilitation programme presented a great benefit for hospitalized patients; taking them from being bedridden to total independence in the AVD, the outpatient had better social and labor conditions.

P528

Severity of electrophysiological alterations correlates with severity of illness in the early phase of critical illness polyneuropathy

R Nemes, Z Fülep, B Fülesdi

University of Debrecen, Hungary

Critical Care 2012, 16(Suppl 1):P528 (doi: 10.1186/cc11135)

Introduction We aimed to investigate the early characteristics of critical illness polyneuropathy in surgical patients in a 5-day follow-up setting. Methods Twenty critically ill patients were enrolled showing signs of systemic inflammatory response, sepsis or multiorgan failure featuring APACHE II score $\geq 12$ on admittance aged 26 to 86 years. Routine noninvasive nerve conduction study of bilateral median and ulnar nerves was performed on a two-channel portable Keypoint Medtronic apparatus. Nerve conduction studies were performed on five consecutive days starting within at most 2 days after admittance, then weekly follow-up was carried out. Electrophysiological findings were compared to age-matched control group parameters.

Results On first examination, within 2 days following admission 17 of $20(85 \%)$ patients showed signs of axonal type sensory-motor polyneuropathy. Medians of compound muscle action potential (CMAP) and sensory nerve action potential (SNAP) amplitudes of all nerves showed a significant decrease compared to control values $(P<0.001)$. During the 5-day study period four patients showed improvement. Sensory nerve fibres were less severely affected than motor fibers. The consecutive measurements revealed negative correlation with the severity of peripheral interstitial oedema determined by circumference of the elbow. Changes in CMAP and SNAP amplitudes also showed a negative correlation with daily rated APACHE II and SAPS II severity scores, and thus with patients' general condition.

Conclusion Electrophysiological alterations appear early after the development of critical illness [1-4]. Early electrophysiological investigations are advisory although results should be evaluated cautiously, as it is hard to differentiate between definitive lesions and temporary disorder caused by bioenergetic failure [3,5-6] of the nerve which tend to improve with normalisation of patients' condition.

\section{References}

Tennilä A, et al:: Intensive Care Med 2000, 26:1360-1363.

Khan J, et al: Neurology 2006, 67:1421-1425.

Latronico N, et al:: Crit Care 2007, 11:R11.
4. Mohammadi B, et al.: J Neurol 2008, 255:265-272.

5. Latronico N, et al:: Lancet Neurol 2011, 10:931-941.

6. Bolton CF, et al:: Crit Care Med 1996, 24:1408-1416.

P529

Muscle strength assessment of critically ill patients is associated with functional ability and quality of life at hospital discharge

G Sidiras, I Patsaki, M Dakoutrou, E Karatzanos, V Gerovasili, A Kouvarakos, A Kardara, K Apostolou, S Dimopoulos, V Markaki, S Nanas

University of Athens, Greece

Critical Care 2012, 16(Suppl 1):P529 (doi: 10.1186/cc11136)

Introduction Patients with critical illness after hospital discharge often exhibit poor functional ability and quality of life as a consequence of acquired muscle weakness. The Medical Research Council (MRC) strength score and hand-grip dynamometry (HGD) are reliable and valid methods to detect clinically significant muscle weakness. The objective of this study is to examine the correlation of these instruments to functional ability and quality-of-life questionnaires at hospital discharge.

Methods Two hundred and sixty-six consecutive patients who had been discharged from the ICU were evaluated and 37 of them were eligible (inclusion criteria: in mechanical ventilation $>72$ hours, a cognitive status that allows assessment) for the study (mean \pm SD: age $55 \pm 15$; APACHE $14 \pm 5$; SOFA $8 \pm 3$; length of ICU stay $22 \pm 22$ days; duration of mechanical ventilation $17 \pm 19$ days). Muscle strength was evaluated with the MRC score and HGD every 7 days until discharge from the hospital. The Functional Independence Measure (FIM) was used to evaluate the functional ability while health-related quality of life was assessed by the Nottingham Health Profile (NHP).

Results At hospital discharge the MRC scale and HGD were significantly correlated with FIM $(r=0.69, P<0.001$ and $r=0.58, P<0.001$, respectively). There seems to be a good correlation of the MRC scale $(r=-0.57, P<0.001)$ with the section of mobility of the NHP. There is also certain association among the domain of mobility and energy of the NHP with the FIM $(r=-0.88, P<0.001$ and $r=-0.61, P<0.05$, respectively).

Conclusion The significantly reduced muscle strength of critically ill survivors could have detrimental effects on their mobility and quality of life. By this study it was shown that muscle strength assessment was well associated with functional ability. We assume that this might be a possible significant prognostic role.

\section{P530}

Functional dependency in the direct post-ICU phase in patients with prolonged mechanical ventilation

S Vossenberg' ${ }^{1}$ I Drogt ${ }^{2}$, N Bruins ${ }^{2}$, C De Jager ${ }^{2}$, EC Boerma², M Tijkotte 'Zorggroep Noorderbreedte, Leeuwarden, the Netherlands; ${ }^{2}$ Medical Centre Leeuwarden, the Netherlands

Critical Care 2012, 16(Suppl 1):P530 (doi: 10.1186/cc11137)

Introduction Prolonged mechanical ventilation and length of stay (LOS) in the ICU is associated with long-term impaired functional capacity. However, little is known about functional dependency in the direct post-ICU phase. Therefore the timing and location for optimal post-ICU rehabilitation programs remain to be established.

Methods In this single-centre observational study we aimed to quantify functional dependency at three different time points: discharge from ICU (DI), discharge from hospital (DH) and discharge from nursing home rehabilitation unit (DR). To this end we retrospectively assed Barthel scores (BS) for individual patients [1], with a duration of mechanical ventilation $>48$ hours. Data are presented as median (IQR). Comparison between time points was performed with nonparametric tests for paired data and repeated measurements. $P<0.05$ was considered significant.

Results Thirty-four patients were included. Baseline characteristics: APACHE II score 20 (17 to 25 ), age 68 ( 55 to 73 ) years, LOS ICU 22 (8 to 36) days, mechanical ventilation 8 ( 2 to 17 ) days, LOS hospital 21 (14 to 30) days, LOS rehabilitation unit 53 (31 to 85 ) days. Median BS at DI was 2 (1 to 3), indicating total functional dependency. In comparison 
to baseline, BS increased to 8 ( 2 to 12$)$ at $\mathrm{DH}(P<0.001)$, indicating severe dependency, and finally to 16 (11 to 18$)$ at $D R$, indicating independency with some disabilities $(P<0.001)$. The absolute increase in BS was significantly greater during the stay in the rehabilitation unit, as compared to the general hospital ward $(P<0.001)$.

Conclusion ICU patients with prolonged mechanical ventilation remain severely functionally dependent after ICU discharge, but dependency reduces significantly during rehabilitation in hospital and in a nursing home rehabilitation unit.

Reference

1. Mahoney Fl, Barthel DW: Functional evaluation: the Barthel Index. Md Med J $1965,2: 61-65$ 